\title{
Using low-frequency earthquakes to monitor slow tectonic deformation in the central Southern Alps, New Zealand
}

by

Laura-May Baratin Wachten

A thesis

submitted to Victoria University of Wellington in fulfilment of the requirements for the degree of Doctor of Philosophy in Geophysics 

"Pa kapab lé mor san éséyé"

Proverbe créole de La Réunion 



\section{Abstract}

This thesis involves the study of low-frequency earthquakes (LFEs) in the central Southern Alps. The Alpine Fault is the principal locus of deformation within the Australia-Pacific plate boundary in the South Island of New Zealand and it is late in its typical $~ 300$-year seismic cycle. Surveying the seismicity associated with slow deformation in the vicinity of the Alpine Fault may provide constraints on the stresses acting on a major transpressive margin prior to an anticipated great $(\geq \mathrm{M} 8$ ) earthquake. Here, we use 8 years of data from the Southern Alps Microearthquake Borehole Array (SAMBA) (amongst those, 3 years of data were collected as part of this project) in order to: (1) generate an updated LFE catalogue using an improved matched-filter technique that incorporates phaseweighted stacking; (2) compute LFE focal mechanisms and invert them to infer the crustal stress field on the deep extent of the Alpine Fault; (3) expand the LFE catalogue to cover a wider range of spatial/temporal behaviours; (4) study LFE families' characteristics to identify periods where slow slip might happen.

We first use fourteen primary LFE templates in an iterative matched-filter and stacking routine, which allows the detection of similar signals and produces LFE families sharing common locations. We generate an 8-yr catalogue containing 10,000 LFEs that are combined for each of the 14 LFE families using phaseweighted stacking to produce signals with the highest possible signal-to-noise ratios. We find LFEs to occur almost continuously during the 8-yr study period and we highlight two types of LFE distributions: (1) discrete behaviour with an inter-event time exceeding 2 minutes; (2) burst-like behaviour with an inter-event time below 2 minutes. The discrete events are interpreted as small-scale frequent deformation on the deep extent of the Alpine Fault and the LFE bursts (corresponding in most cases to known episodes of tremor or large regional earthquakes) are interpreted as brief periods of increased slip activity indicative of slow slip. We compute improved non-linear earthquake locations using a 3D velocity model and find LFEs to occur below the seismogenic zone at depths of $17-42 \mathrm{~km}$, on or near the hypothesised deep extent of the Alpine Fault. We then compute the first estimates of LFE focal mechanisms associated with continental faulting. Focal mechanisms, in conjunction with recurrence intervals, are consistent with quasi-continuous shear faulting on the deep extent of the Alpine Fault. 
We then generate a new catalogue that regroups hundreds of LFE families. This time 638 synthetic LFE waveforms are generated using a 3D grid and used as primary templates in a matched-filter routine. Of those, 529 templates yield enough detections during the first iteration of the matched-filter routine $(\geq 500$ detections over the 8-yr study period) and are kept for further analysis. We then use the best $25 \%$ of correlated events for each LFE family to generate linear stacks which create new LFE templates. From there, we run a second and final iteration of the matched-filter routine with the new LFE templates to obtain our final LFE catalogue. The remaining 529 templates detect between 150 and 1,671 events each totalling 300,996 detections over the 8-yr study period. Of those 529 LFEs, we manage to locate 378 families. Their depths range between 11 and $60 \mathrm{~km}$ and LFEs locate mainly in the southern part of the SAMBA network. We finally examine individual LFE family rates and occurrence patterns. They indicate that LFE sources seem to evolve from an episodic or 'stepped' to a continuous behaviour with depth. This transition may correspond to an evolution from a stick-slip to a stable-sliding slip regime. Hence, we propose that the distinctive features of LFE occurrence patterns reflect variations in the in-situ stress and frictional conditions at the individual LFE source locations on the Alpine Fault.

Finally, we use this new extensive catalogue as a tool for in-depth analyses of the deep central Alpine Fault structure and its slip behaviour. We identify eight episodes of increased LFE activity between 2009 and 2017 and provide time windows for further investigations of tremor and slow slip. We also study the spatial and temporal behaviours of LFEs and find that LFEs with synchronous occurrence patterns tend to be clustered in space. We thus suggest that individual LFE sources form spatially coherent clusters that may represent localised asperities or elastic patches on the deep Alpine Fault interface. We infer that those clusters may have a similar rheological response to tectonic forcing or to potential slow slip events. Eventually, we discover slow (10 km/day) and rapid ( $20-25 \mathrm{~km} / \mathrm{h})$ migrations of LFEs along the Alpine Fault. The slow migration might be controlled by slow slip events themselves while the rapid velocities could be explained by the LFE sources' intrinsic properties. 


\section{Acknowledgments}

I would like to thank John for providing me with the great opportunity to study in New Zealand and to work on such an exciting subject for the past three years. John and Martha, I am extremely grateful for your time, help and insight on this project over the past 3 years. Many thanks to Calum for his precious help with this thesis' work all along the past 3 years and a half. Thanks also to William for his crucial advice, for sharing his experience with me, and also for welcoming me at MIT. I'm grateful for the financial support, that I have received throughout my PhD, from the Royal Society of New Zealand (through the Marsden Fund), Victoria University of Wellington and the American Geophysical Union.

I would like to thank Carolin and Calum for setting up the SAMBA network, and especially Calum for teaching me how to properly service/maintain it (and also for teaching me how to properly say 'chopper'). Incredibly grateful to everybody who helped me on the field (special poops to Kostas and Emily), and to the Department of Conservation and the people on the West Coast (notably the helicopter pilots who always managed to keep us safe).

Huge thanks to my officemates and great friends, Rachel, Jesse and Francesco, for the hours spent laughing, geeking and sharing cake in CO505. Carpe Aptenodytes! Thanks to my fellow post-grad students/post-docs, Laurine, Hannah, Dom, Kostas, Lucie, Weiwei, Chet, Konrad, Hubert, Kenny, Katrina, Holly, Cécile, Emily, Juergen, Marcel and Pegah for the many laughs and overall good times!

Huge thanks to T.S for coming up with the best feel good tracks ever. So much of my writing/coding inspiration and motivation came from listening to you. Look what you made me do! Also, huge thanks to J.K.R for providing me with happiness even in the darkest of times. Words are, indeed, our most inexhaustible source of magic.

Merci à Stéphane Mazzotti, et à Jean Chéry pour votre précieuse aide avec mon tout premier papier. Et un merci tout particulier à Stéphane, pour le bon tuyau de la thèse en Nouvelle-Zélande avec du terrain en hélicoptère !!

À mes amis en France, Tomtom, Marush, Poilu, Marine, Stephen, Père Castor, 
mes Proussettes (Marjocien et Panoramix), Aurel, Les Hippies de L'IPGP (Soso, Seb, Popo, Flo): nos conversations m'ont toujours donné le sourire et votre soutien continuel m'a fait chaud au coeur. Merci à mes 3 BFF: Sarita, Audrey et Adélaïde pour vos rires et vos sourires et pour avoir cru en moi ces 3 dernières années, j'ai eu l'impression de ne jamais vous avoir quittées. Pensée spéciale à Adé et Fer: vos messages à l'improviste ont tellement de fois égayé ma journée en me faisant rire à gorge déployée..! À ma belle-famille, merci pour vos messages et photos qui m'ont permis d'avoir l'impression d'être près de vous pendant ces 3 longues années. À mon frérot Thomas, merci pour tes rares interventions qui ont toujours réussi à me faire rire...

À mes deux soeurettes chéries: Aude et Marie, merci pour votre soutien ces 3 dernières années et merci d'avoir toujours cru en moi ! Marie, merci pour les nombreuses fois où tu m'as accompagnée au téléphone sur le chemin de la fac. Aude, il t'aura fallu 3 ans pour comprendre le décalage horaire entre la France et la Nouvelle-Zélande, mais merci de n'avoir jamais compris quand je te disais que demander à une thésarde comment se passe sa thèse c'était un sujet tabou. Puisque du coup, ça m'a fait rire à chaque fois que tu me l'as demandé !

Un merci tout particulier à mes parents, Alain et Brigitte. Tout d'abord, merci de vous être occupés de mon démon de chat... Bounty pendant mon exil en Terre du Milieu ! Merci de m'avoir donné le goût du voyage et de l'aventure dès mon plus jeune âge. Merci d'avoir toujours cru en moi et de m'avoir aidée à réaliser un de mes rêves: être docteur. Votre support moral et financier lors de mes études a permis ma réussite jusqu'à présent. Vous êtes absolument incroyables ! Et ces travaux de thèse, je vous les dédie.

Et finalement, je tiens à remercier Yann, mon mari (le meilleur des meilleurs des meilleurs avec mention). Sans ton soutien continuel et ta présence, ce travail n'aurait pas été possible. Tu devrais figurer en tant que co-auteur sur cette thèse! Tes encouragements dans les moments les plus durs ont été tellement précieux. Je suis consciente et infiniment reconnaissante des sacrifices que tu as dû faire pour que je puisse accomplir cette thèse et les mots me manquent pour te transmettre mon immense gratitude et mon amour. Tout ce que je peux te dire, c'est que j'ai hâte que nous nous "envoilions" vers de nouvelles aventures... 


\section{Contents}

Abstract

Acknowledgments

Contents $\quad$ v

List of Figures $\quad$ xi

List of Tables $\quad$ xvii

1 Introduction 1

1.1 Motivations and thesis objectives .............. 1

1.2 Slow earthquakes .................... 4

1.3 Tectonic setting ..................... 8

1.4 Geological background . . . . . . . . . . . . . . . . . . . 12

1.5 Seismicity in the central Southern Alps . . . . . . . . . . . . 15

1.5.1 Rupture history in the Alpine Fault region . . . . . . . . . 15

1.5.2 Previous seismicity studies . . . . . . . . . . . 17

1.5.3 Tectonic tremor and LFEs in the Southern Alps . . . . . . 21

1.6 Lithospheric structure and Alpine Fault geometry . . . . . . . . 25

1.7 Thesis outline . . . . . . . . . . . . . . . . . . . . . 28

2 Research Methodology 31

2.1 Data acquisition . . . . . . . . . . . . . . 31

2.1.1 The Southern Alps Microearthquake Borehole Array . . . 31

2.1.2 Data collection and processing . . . . . . . . . . 34 
2.2 Low-frequency earthquakes detection . . . . . . . . . . . . 37

2.2.1 Matched-filter and cross-correlation . . . . . . . . . 37

2.2.2 Phase-Weighted Stacking . . . . . . . . . . . . . . . . 39

2.2.3 Synthetic templates generation . . . . . . . . . . . 41

2.2.4 Computational implementation . . . . . . . . . . . . 42

2.3 LFE locations . . . . . . . . . . . . . . . . . . 43

2.3.1 Linear earthquake locations . . . . . . . . . . . 43

2.3.2 Non linear earthquake locations . . . . . . . . . . . 45

2.4 Focal mechanisms and stress estimate . . . . . . . . . . . 46

2.4 .1 Focal mechanisms . . . . . . . . . . . . . . . 46

2.4.2 Stress inversion . . . . . . . . . . . . . . . . 49

3 An 8-yr LFE catalogue for the central Southern Alps 51

3.1 Introduction . . . . . . . . . . . . . . . . . . . 52

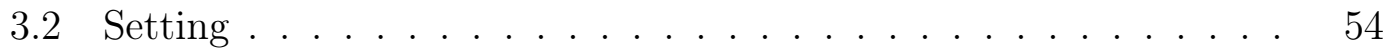

3.3 Methods ........................ 56

3.3.1 LFE detection . . . . . . . . . . . . . . 56

3.3.2 LFE location . . . . . . . . . . . . . . 57

3.3.3 Focal mechanisms estimation and stress inversion . . . . . 59

3.4 Observations . . . . . . . . . . . . . . . . . . . . 59

3.4 .1 LFE catalogue . . . . . . . . . . . . . . . . . 59

3.4.2 LFE locations . . . . . . . . . . . . . . . . . . . 69

3.4.3 LFE focal mechanisms and stress estimate . . . . . . 73

3.5 Discussion . . . . . . . . . . . . . . . . . . . . . 77

3.5.1 Catalogue improvements . . . . . . . . . . . . 77

3.5.2 LFE interevent timing and triggering . . . . . . . . 79

3.5.3 Alpine Fault rheology and geometry . . . . . . . . . . 87

3.6 Conclusions . . . . . . . . . . . . . . . . . . . . . . . . . . . 88 
4 Using synthetics to build a continuous LFE catalogue 89

4.1 Introduction . . . . . . . . . . . . . . . . . . . 90

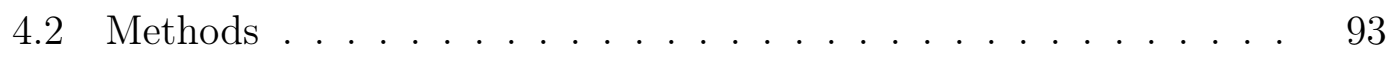

4.2.1 Real LFE template generation . . . . . . . . . . . . 93

4.2 .2 Catalogue generation . . . . . . . . . . . . . 94

4.2 .3 LFE locations . . . . . . . . . . . . . . . . . . . . . 96

4.3 Results . . . . . . . . . . . . . . . . . . 96

4.3 .1 Performance of synthetic LFEs . . . . . . . . . . 96

4.3.2 Continuous LFE catalogue . . . . . . . . . . . . 101

4.3 .3 LFE locations . . . . . . . . . . . . . . . 107

4.4 Discussion . . . . . . . . . . . . . . . . . . . . . . 111

4.4.1 LFE families behavioural styles . . . . . . . . . . . 111

4.4 .2 Implications for deep Alpine Fault . . . . . . . . . . 116

4.5 Conclusions . . . . . . . . . . . . . . . . . . . . 119

5 Characteristics of LFEs in the Southern Alps 121

5.1 Introduction . . . . . . . . . . . . . . . . . 122

5.2 Methods ........................ 124

5.2 .1 LFE episodes . . . . . . . . . . . . . . . . 124

5.2 .2 LFE migrations . . . . . . . . . . . . . . . . 125

5.2 .3 LFE family redundancy . . . . . . . . . . . . . 125

5.3 Results . . . . . . . . . . . . . . . . . . 127

5.3.1 LFE activity temporal variations . . . . . . . . . 127

5.3.2 Spatio-temporal variations . . . . . . . . . . 136

5.3 .3 LFE Clustering . . . . . . . . . . . . . . . 145

5.4 Discussion . . . . . . . . . . . . . . . . . . . . . 146

5.4.1 LFE patterns and migrations . . . . . . . . . 146

5.4 .2 Slow slip investigation . . . . . . . . . . . . 155 
5.5 Conclusions . . . . . . . . . . . . . . . . . . . 157

6 Summary and Conclusions $\quad 159$

6.1 Summary of findings . . . . . . . . . . . . . . . . . 159

6.1.1 Extended LFE catalogue . . . . . . . . . . . . . . . . . 159

6.1.2 Continuous LFE catalogue . . . . . . . . . . . . . . . . 161

6.1.3 LFE characteristics . . . . . . . . . . . . . . . . . 162

6.2 Tremor and LFEs in a global context . . . . . . . . . . . . . 165

6.3 Slow deformation in the central Southern Alps . . . . . . . . . . 166

6.4 Recommendations for future studies . . . . . . . . . . . . . . 168

6.4.1 Slow slip investigation . . . . . . . . . . . . . 168

6.4 .2 Magnitudes . . . . . . . . . . . . . . . 170

6.4.3 Automatic detections of LFE migrations . . . . . . . . . . 170

6.4 .4 Crustal Anisotropy . . . . . . . . . . . . . . . 172

6.4 .5 Triggering .................... 173

6.4.6 Very low-frequency earthquakes . . . . . . . . . . . . 174

6.5 Concluding statement . . . . . . . . . . . . . . . . . 175

$\begin{array}{ll}\text { Appendices } & 177\end{array}$

$\begin{array}{lr}\text { A LFE catalogues } & 179\end{array}$

A.1 3-yr catalogue . . . . . . . . . . . . . . . . . . . . 179

A.2 Extended catalogue . . . . . . . . . . . . . . . . . 185

A.2.1 Waveforms . . . . . . . . . . . . . . 185

A.2.2 Focal mechanisms . . . . . . . . . . . . . . . . 200

A.3 Continuous catalogue . . . . . . . . . . . . . . . . 202

A.3.1 Waveforms . . . . . . . . . . . . . . . . 202

A.3.2 Locations . . . . . . . . . . . . . . . 205

A.3.3 LFE families redundancy . . . . . . . . . . . . . . . 223 
A.3.4 Anisotropy . . . . . . . . . . . . . . . . . . 227

B Deep Fault Drilling Project - 2 229

B.1 Real time seismic monitoring . . . . . . . . . . . . . . . . . 229

$\begin{array}{ll}\text { C Field work reports } & 235\end{array}$

C.1 September $2015 \ldots \ldots \ldots$. . . . . . . . . . . . . . . 235

C.1.1 Summary ..................... 236

C.1.2 Daily $\log \ldots \ldots \ldots \ldots . \ldots . \ldots . . \ldots 236$

C.1.3 Memo for next servicing . . . . . . . . . . . . . . 244

C.2 April $2016 \ldots \ldots \ldots \ldots$. . . . . . . . . . . . . . . . . . . 245

C.2.1 Summary . . . . . . . . . . . . . . . . 245

C.2.2 Daily $\log \ldots \ldots \ldots \ldots \ldots$. . . . . . . . . . . . . . 246

C.2.3 Update on Godley Valley . . . . . . . . . . . . . . 253

C.2.4 Memo for next servicing . . . . . . . . . . . . . 254

C.3 October $2016 \ldots \ldots \ldots$

C.3.1 Summary . . . . . . . . . . . . . . . 255

C.3.2 Daily $\log \ldots \ldots \ldots \ldots \ldots$. . . . . . . . . . . . 256

C.3.3 Memo for next servicing . . . . . . . . . . . 263

$\begin{array}{ll}\text { Bibliography } & 265\end{array}$ 


\section{List of Figures}

1.1 Historic seismicity in the South Island of New Zealand. . . . . . . 3

1.2 Schematic model for slip. . . . . . . . . . . . . . . . . . 5

1.3 Scaling law for slow earthquakes. . . . . . . . . . . . . . . 6

1.4 Brownian walk for slow earthquakes. . . . . . . . . . . . . . 7

1.5 Map of New Zealand. . . . . . . . . . . . . . . . . . . . . . . 9

1.6 Segments of Alpine Fault. . . . . . . . . . . . . . . . . . . 11

1.7 Geology of New Zealand. . . . . . . . . . . . . . . . . . . 13

1.8 Historic ruptures of Alpine Fault. . . . . . . . . . . . . . . . . 16

1.9 Seismicity in the study area. . . . . . . . . . . . . . . . 19

1.10 Tremor location. . . . . . . . . . . . . . . . . 22

1.11 LFEs identified within tremor. . . . . . . . . . . . . . 23

1.12 Lithospheric structure beneath the Southern Alps. . . . . . . . . 25

1.13 Alpine Fault possible geometries. . . . . . . . . . . . . . . 27

2.1 SAMBA network. . . . . . . . . . . . . . . 32

2.2 Data continuity for this study. . . . . . . . . . . . . . . 36

2.3 Steps overview for a matched-filter run. . . . . . . . . . . . . 38

2.4 Phase stack illustration. . . . . . . . . . . . . . . . 40

2.5 Comparison between linear and phase-weighted stacks. . . . . . . 41

2.6 Example of focal mechanisms. . . . . . . . . . . . . . . . . . . . 47

2.7 Example of a focal mechanism computation output. . . . . . . . . 48

2.8 Example of output from stress inversion codes. . . . . . . . . . . 50

3.1 Map of study area. . . . . . . . . . . . . . . 55 
3.2 Comparison between original template, linear stack and phaseweighted stack traces. . . . . . . . . . . . . . . . 58

3.3 LFE families individual temporal evolution. . . . . . . . . . . . 60

3.4 Waveforms and spectrograms for an LFE and a typical earthquake detection. . . . . . . . . . . . . . . . . 62

3.5 Waveforms and spectrograms for family ID:60070. . . . . . . . 63

3.6 Cumulative detections for family ID:37575 . . . . . . . . . . . 64

3.7 Inter-event times for family ID:37575 . . . . . . . . . . . 65

3.8 Stacks and cross-correlation sum values for family ID:60070. . . . 66

3.9 Linear stacks for three consecutive iterations for family ID:37575. 67

3.10 Example waveforms and spectrograms for the final stack of family

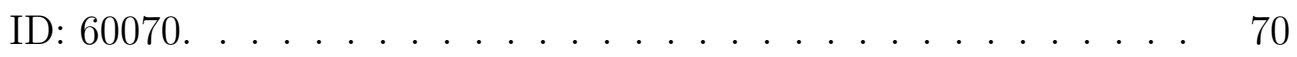

3.11 Example waveforms and spectrograms for the final stack of family

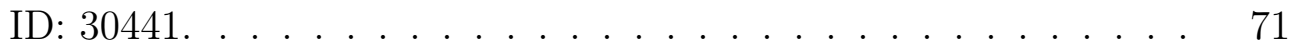

3.12 LFE hypocentres for our 14 LFE families. . . . . . . . . . . . . . 72

3.13 Focal mechanism solutions of 8 LFE families. . . . . . . . . . . . 74

3.14 Focal mechanisms and principal stress directions. . . . . . . . . 76

3.15 Original and extended catalogues comparison. . . . . . . . . . . 79

3.16 Detrended cumulative detections and energy density. . . . . . . . 81

3.17 Inter-event times for a subset of LFE families. . . . . . . . . . . 82

$3.18 \mathrm{LFE}$ detections for a selection of large regional earthquakes. . . . 84

3.19 Temporal evolution and inter-event times of LFEs. . . . . . . . . 86

4.1 Map of study area . . . . . . . . . . . . . . . . . 91

4.2 Evolution of LFE template ID:169.8_-43.5795_26. . . . . . . . . 94

4.3 Example of detections made with a real LFE template. . . . . . . 95

4.4 Comparison of earthquake spectra. . . . . . . . . . . . . . 97

4.5 Comparison between real and false detections for family 169.641_$43.729 \_44 \_1 . \ldots \ldots \ldots \ldots . \ldots \ldots \ldots$ 
4.6 Detections overlap between the synthetic and extended catalogues. 100

4.7 Number of LFEs per day. . . . . . . . . . . . . . . . . . 102

4.8 Example of detections made by a single LFE template. . . . . . . 103

4.9 Cumulative detections and LFEs per day. . . . . . . . . . . . . . . 104

4.10 Comparison between linear and phase-weighted stacks. . . . . . . 106

4.11 Example of waveforms and spectrograms for high SNR and low SNR stacks. . . . . . . . . . . . . . . . . 107

4.12 Map of LFE locations. . . . . . . . . . . . . . . . . . . . . 109

4.13 Comparison of initial and final depths. . . . . . . . . . . . . 111

4.14 Cumulative detections for a selection of LFE families. . . . . . . . 113

4.15 Detrended cumulative detections and inter-event times. . . . . . . 115

4.16 Map of event rate COVr. . . . . . . . . . . . . . . . 117

5.1 Map of study area. . . . . . . . . . . . . . . . . 123

5.2 Evolution of the LFE catalogue in time. . . . . . . . . . . . 128

5.3 LFE daily rates and LFE episodes over time. . . . . . . . . . . . . 130

5.4 Comparison between LFE episode ID:4 and the quiet period. . . . 133

5.5 Comparison between stacked waveforms of a single LFE family. 135

5.6 Comparison of spectrograms between an LFE episode and the quiet period. . . . . . . . . . . . . . 137

5.7 LFE catalogue in time and space along strike. . . . . . . . . . 138

5.8 LFE catalogue in time and space orthogonally to the fault. . . . . 139

5.9 LFE rates for an LFE episode . . . . . . . . . . . . . . . . . 142

5.10 LFE rates for the quiet period . . . . . . . . . . . . 143

5.11 LFE families redundancy. . . . . . . . . . . . . . . . 147

5.12 Spatial and temporal variation for an LFE episode (a) and the quiet period (b). . . . . . . . . . . . . . . 151

5.13 Slow and fast migration during an LFE episode. . . . . . . . . . . 153

5.14 Fast migration of LFEs along strike. . . . . . . . . . . . . . . 154 
6.1 Map of average LFE density. . . . . . . . . . . . . . . . . . . . . 164

6.2 Summary diagram. . . . . . . . . . . . . . . . . . . 169

6.3 Algorithm scheme. . . . . . . . . . . . . . . . . . 171

A.1 3-yr catalogue cumulative detections. . . . . . . . . . . . 180

A.2 3-yr catalogue final stacks for six LFE families. . . . . . . . . . . 181

A.3 3-yr catalogue stacks for family IDs:17208 and 55115. . . . . . . . 182

A.4 3-yr catalogue stacks for family ID:55115 . . . . . . . . . . . . 183

A.5 3-yr catalogue location map. . . . . . . . . . . . . . . . . . 184

A.6 LFE family $17208 . \quad \ldots \ldots \ldots \ldots$

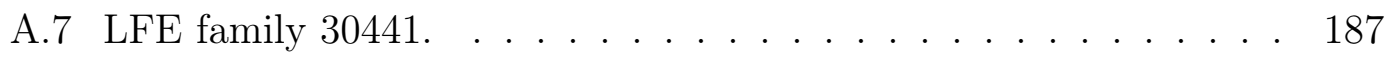

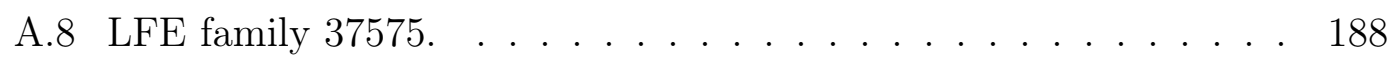

A.9 LFE family 54905. . . . . . . . . . . . . . . . . . . . . 189

A.10 LFE family 55115. . . . . . . . . . . . . . . . . . . . . . 190

A.11 LFE family $55200 . \ldots \ldots \ldots 19 . \ldots \ldots$

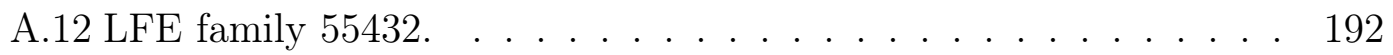

A.13 LFE family 59966. . . . . . . . . . . . . . . . . . . . . 193

A.14 LFE family 60070. . . . . . . . . . . . . . . . . . . . . 194

A.15 LFE family 60905. . . . . . . . . . . . . . . . . . . . 195

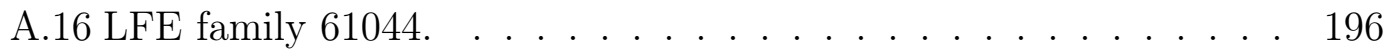

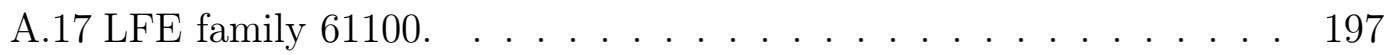

A.18 LFE family 61220 . . . . . . . . . . . . . . . . . . . . . 198

A.19 LFE family 63877. . . . . . . . . . . . . . . . . . . . . 199

A.20 Focal mechanisms for the remaining 6 LFE families. . . . . . . . . 201

A.21 Example of linear stack for family ID:169.999_-43.5637_18_1. . . 203

A.22 Example of linear stack for family ID:169.733_-43.7912_16_1. . . 204

A.23 Example of P- and S-picks. . . . . . . . . . . . . . . . . 205

A.24 Another example of P- and S-picks. . . . . . . . . . . . . . . 206 
A.25 Example of P- and S-picks on linear stacks. . . . . . . . . . . . 207

A.26 Example of P- and S-picks on phase-weighted stacks. . . . . . . . 208

A.27 Another example of P- and S-picks on linear stacks. . . . . . . . . 209

A.28 Another example of P- and S-picks on phase-weighted stacks. . . . 210

A.29 Example of preliminary location in SEISAN. . . . . . . . . . . . . 211

A.30 NonLinLoc locations with clouds of uncertainties. . . . . . . . . 212

A.31 Stacks comparison. . . . . . . . . . . . . . . . 225

A.32 Stack SNR comparison. . . . . . . . . . . . . . . . 226

A.33 LFE individual detection example. . . . . . . . . . . . . . . 227

A.34 LFE stack example. . . . . . . . . . . . . . . . . . . . . . . . 228

B.1 Traffic light system. . . . . . . . . . . . . . . . . 230

B.2 Flow diagram. . . . . . . . . . . . . . . 231

B.3 Seismicity recorded during DFDP-2. . . . . . . . . . . . . . 232

C.1 Tasman Lake from helicopter. . . . . . . . . . . . . . 235

C.2 Fox glacier from helicopter. . . . . . . . . . . . . . . . 238

C.3 Failed attempt to service COVA. . . . . . . . . . . . . . . . 239

C.4 Gravity measurements on Mt. Adams. . . . . . . . . . . . . . 239

C.5 DFDP-2 solar panel installation. . . . . . . . . . . . . . . 242

C.6 Tasman River from helicopter. . . . . . . . . . . . . . . . 242

C.7 GOVA servicing. . . . . . . . . . . . . . . . 243

C.8 Southern Alps viewed from helicopter. . . . . . . . . . . . . . . 245

C.9 Shattered solar panel at WMSZ . . . . . . . . . . . . . . 247

C.10 River viewed from the new diverted tourist path. . . . . . . . . . 248

C.11 WHYM servicing. . . . . . . . . . . . . . . . 249

C.12 LARB servicing. . . . . . . . . . . . . . . . . . 250

C.13 COVA servicing. . . . . . . . . . . . . . . . 250 
C.14 Data screenshot for MTBA station . . . . . . . . . . . . 251

C.15 PhD students digging out a sensor. . . . . . . . . . . . . . 252

C.16 LABE servicing. . . . . . . . . . . . . . . . . . . . 253

C.17 GOVA servicing. . . . . . . . . . . . . . . . . 254

C.18 Mt. Cook from helicopter. . . . . . . . . . . . . . . . 255

C.19 A bit of a pond at the Quarry site. . . . . . . . . . . . . 257

C.20 New station being installed . . . . . . . . . . . . . . 258

C.21 PhD Students installing a new seismometer. . . . . . . . . . . . 258

C.22 COVA station from helicopter. . . . . . . . . . . . . . 259

C.23 COVA servicing. . . . . . . . . . . . . . . 260

C.24 Servicing of Lake Hawea. . . . . . . . . . . . . . . . . . . . . . 261

C.25 Our means of transportation - Glentanner Aerodrome. . . . . . . 261

C.26 State of GOVA station. . . . . . . . . . . . . . . 262 


\section{List of Tables}

2.1 SAMBA site locations. . . . . . . . . . . . . . . . 33

2.2 Current inventory of deployed SAMBA equipment and status. . . 35

3.1 Characteristics of LFE families. . . . . . . . . . . . . . . 61

5.1 LFE episode characteristics. . . . . . . . . . . . . . . . 131

A.1 LFE family locations. . . . . . . . . . . . . . . . . . . . 222

A.2 LFE family redundancy. . . . . . . . . . . . . . . . . . . 224 


\section{Introduction}

\subsection{Motivations and thesis objectives}

One of the biggest challenges in seismology is to understand how the recurring behaviour of large faults is linked to small-scale deformation processes within the earthquake machine. Our understanding of seismogenesis is generally limited by our ability to observe and detect earthquakes across a wide range of magnitudes. Large earthquakes $\left(M_{w}>7-8\right)$ are for example not easy to observe because of their relatively long recurrence interval. Small earthquakes $\left(M_{w}<2\right)$ occur more frequently, however detecting them is challenging because of (1) noisy environments and/or (2) the attenuating properties of the crust, resulting in low signal to noise records. Other than recording for longer periods, not much can be done regarding large earthquakes. However, for small earthquakes, a lot can be done to improve our ability to detect them and consequently our understanding of their role in the earthquake machine.

This thesis is part of the Marsden-funded project 'Locked and loaded? Effects of deep seismic and aseismic deformation on Alpine Fault earthquakes'. The project's overall goal is to assess how slow tectonic deformation is loading the Alpine Fault toward a major earthquake by addressing the following research topics:

- Detection, location and characterisation of seismic deformation near the Alpine Fault;

- Refining models of upper crustal velocity structure;

- Understanding where deep deformation is occurring.

Why are we studying the Alpine Fault? The Alpine Fault accommodates the relative motion between the Australian and Pacific plates in the South Island of New Zealand (Norris and Cooper, 2001) and is thought to be late in its earthquake cycle (Sutherland et al., 2007). The Alpine Fault has not ruptured in historic times, but paleoseismic records indicate typical $M_{w}$ 7-8 earthquakes with $\mathrm{a} \sim 300$ yr mean recurrence time, the last one being in $\sim 1717 \mathrm{AD}$ (Wells et al., 
1999; Berryman et al., 2012; Howarth et al., 2016), thus indicating that the Alpine Fault is overdue for a next 'big one'. As illustrated by the recent Canterbury and Kaikoura earthquake sequences (Gledhill et al., 2011; Quigley et al., 2012; Bannister and Gledhill, 2012; Hamling et al., 2017), damaging earthquakes occur on faults possessing a wide range of size, geometry and slip-rates. Understanding as much as we possibly can about the phenomena governing earthquake rupture is therefore essential to better estimate the hazards posed by such large faults. Studying the Alpine Fault is especially relevant as we know that it is late in its cycle of stress accumulation and release, which makes it an ideal candidate to study the conditions under which earthquakes occur in the South Island of New Zealand.

Why specifically the central part of the Alpine Fault? This project builds on recent studies conducted at Victoria University of Wellington (VUW) documenting lithospheric deformation and seismicity on and near the central part of the Alpine Fault (e.g. seismic anisotropy (Bourguignon et al., 2007), microseismicity (Boese et al., 2012), tectonic tremor (Wech et al., 2012) and low-frequency earthquakes (Chamberlain et al., 2014). In recent years, many studies have focused on studying the central Southern Alps to assess the seismic potential of the central part of the Alpine Fault (e.g. Deep Fault Drilling Project, Phase 1, Townend et al. (2009b); Sutherland et al. (2012); Townend et al. (2013); Toy et al. (2015) and Phase 2, Sutherland et al. (2017); Toy et al. (2017)). Paleoseismic and geologic evidence show a gap in recent seismicity in the central part of the Alpine Fault (Fig. 1.1). Rapid uplift as well as extremely high heat flow measurements and geothermal gradient are measured in this area suggesting that the rapid exhumation along the Alpine Fault perturbs isotherms at shallower depths (Sutherland et al., 2017). Sutherland et al. (2007) suggest that a large earthquake $\left(M_{w} 7-8\right)$ is likely to occur in the central section of the Alpine Fault, thus making the central Southern Alps an optimal area to study seismic hazard associated with the New Zealand Alpine Fault.

Why slow deformation in particular? Understanding the processes behind slow deformation is essential to assess plate boundary dynamics. Slow earthquakes are a class of earthquakes that occur unusually slowly (Beroza and Ide, 2011). These recently discovered seismic phenomena (Obara, 2002) play a critical part in the seismic cycle as they involve stress transfers between the locked and stably sliding portions of a fault (Wech and Creager, 2011). To monitor slow slip, tectonic tremor (a weak, long duration, emergent seismic signal) can be used. Tectonic tremor can be decomposed as a superposition of low-frequency earthquakes (Shelly 


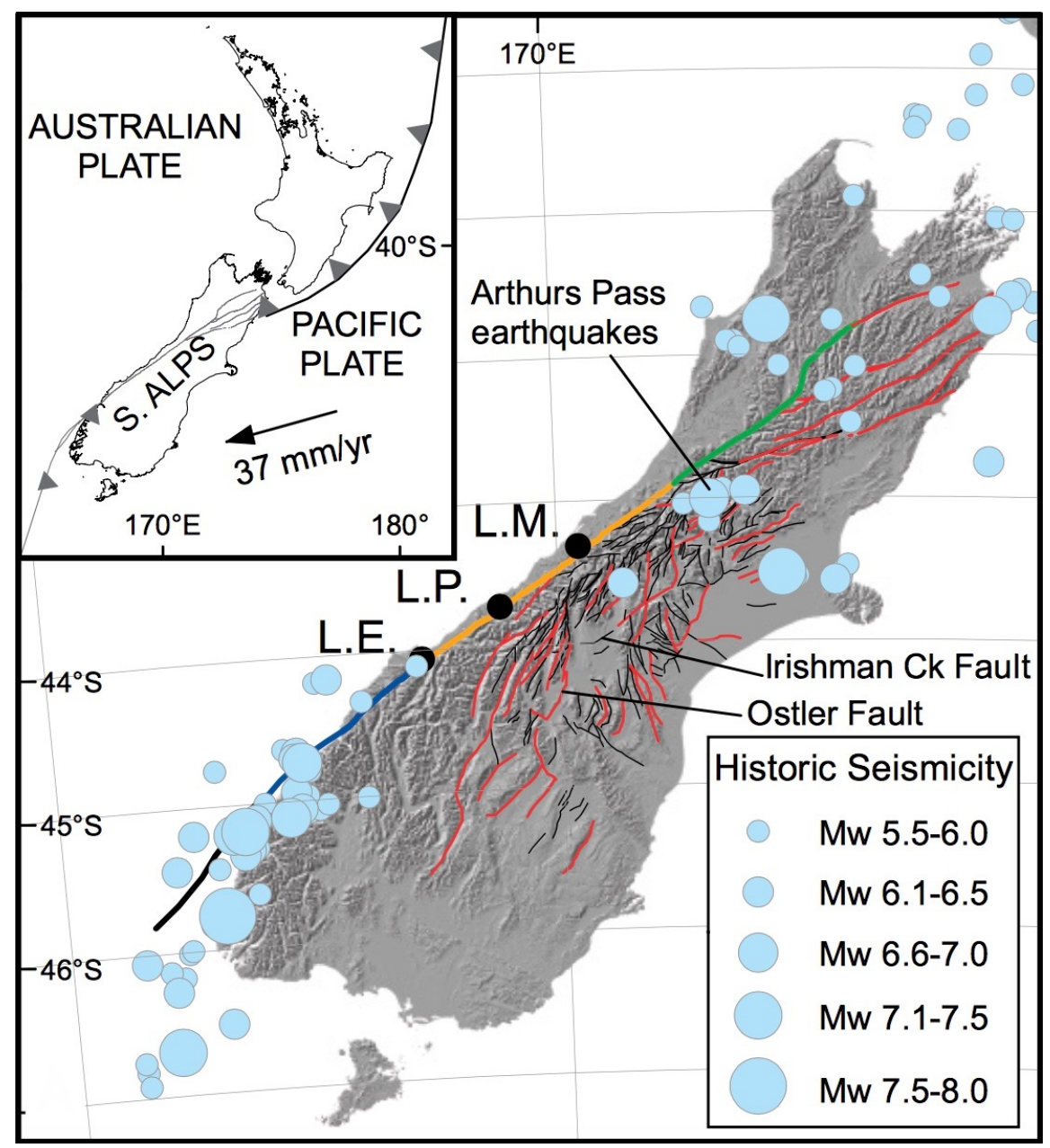

Figure 1.1: Historic seismicity in the South Island of New Zealand. From Howarth et al. (2016). Map showing the active and potentially active faults in the Southern Alps (in red: $M_{\max } \geq 7$ and in black: $M_{\max } \leq 7$ ), as well as the historic seismicity $M_{w} \geq 5.5$ between 1960 and present time (historic earthquake data from GeoNet). Inset shows the wider tectonic setting of New Zealand. L.M. corresponds to Lake Mapourika; L.P. to Lake Paringa and L.E. to Lake Ellery.

et al., 2007). Several studies worldwide suggest that tectonic tremor is a seismic signature of slow slip on the deep extent of faults. Indeed, the presence of tectonic tremor and low-frequency earthquakes occurring in the central Southern Alps, has been inferred to indicate slow slip on the deep extent of the Alpine Fault (Wech et al., 2012; Chamberlain et al., 2014). Several studies (e.g. Gomberg et al., 2008; Thomas et al., 2009; Hill, 2012) have shown that tectonic tremor is susceptible to being triggered by small changes in stress. Therefore understanding deep slow slip is crucial to understand spatial and temporal patterns of stress redistribution. Tremor can also be used to estimate the down-dip extent of the seismogenic zone. Furthermore, studying tremor can also be of utmost importance to assess seismic hazard (Kato et al., 2012; Ito et al., 2013; Ruiz et al., 2014). Indeed, Mazzotti 
and Adams (2004) suggested that an increase in tremor activity might signify an increase in probability of a large earthquake rupture.

This thesis focuses on a particular class of slow earthquake phenomena: lowfrequency earthquakes, on and near the central part of the New Zealand Alpine Fault. The main goals of this project are: (1) to detect, locate and characterise low-frequency earthquakes in the central part of the Southern Alps; (2) to understand how slow tectonic deformation is loading the Alpine Fault toward an earthquake rupture and (3) to provide new understanding of the geometry and rheology of the Alpine Fault and of the stress field prevailing in the central Southern Alps. To investigate these topics, we use the Southern Alps Microearthquake Borehole Array (SAMBA), a station network designed for the detection of microearthquakes in the central Southern Alps Boese et al. (2012) and described in more details in Section 2.1. By understanding how these frequent and smaller earthquakes influence -or are influenced- by the seismic cycle of large earthquakes, we might be able to pinpoint the behaviour of large earthquakes and their repetitive patterns. Hence, we might be able to find predictive models for large earthquakes.

\subsection{Slow earthquakes}

Slow earthquakes are transient phenomena that may play a crucial role in the seismic cycle. Slow earthquakes comprise a wide range of recently discovered phenomena (e.g. slow-slip events, tectonic tremor, low-frequency earthquakes) whose principal feature is their long duration:

- From several days or weeks to months or years for slow slip events, (e.g. Dragert et al., 2001; Schwartz and Rokosky, 2007; Wallace and Beavan, 2010);

- From minutes to days or weeks for tremor, (e.g. Obara, 2002; Rogers and Dragert, 2003; Nadeau and Dolenc, 2005; Obara and Hirose, 2006; Peng and Chao, 2008);

- From seconds to minutes for low-frequency earthquakes, (e.g. Shelly et al., 2006; Frank et al., 2013; Brown et al., 2013; Royer and Bostock, 2014; Shelly, 2017).

Slow earthquakes are inferred to involve stress transfers between the locked and stably sliding portions of a fault (Wech and Creager, 2011). This transition of 
faults, from locked to stably sliding with depth, is presumably a gradual process, with a diversity of possible slip mechanisms (Fig. 1.2) from rapid large earthquake ruptures to slow aseismic slip (Wech and Creager, 2011).

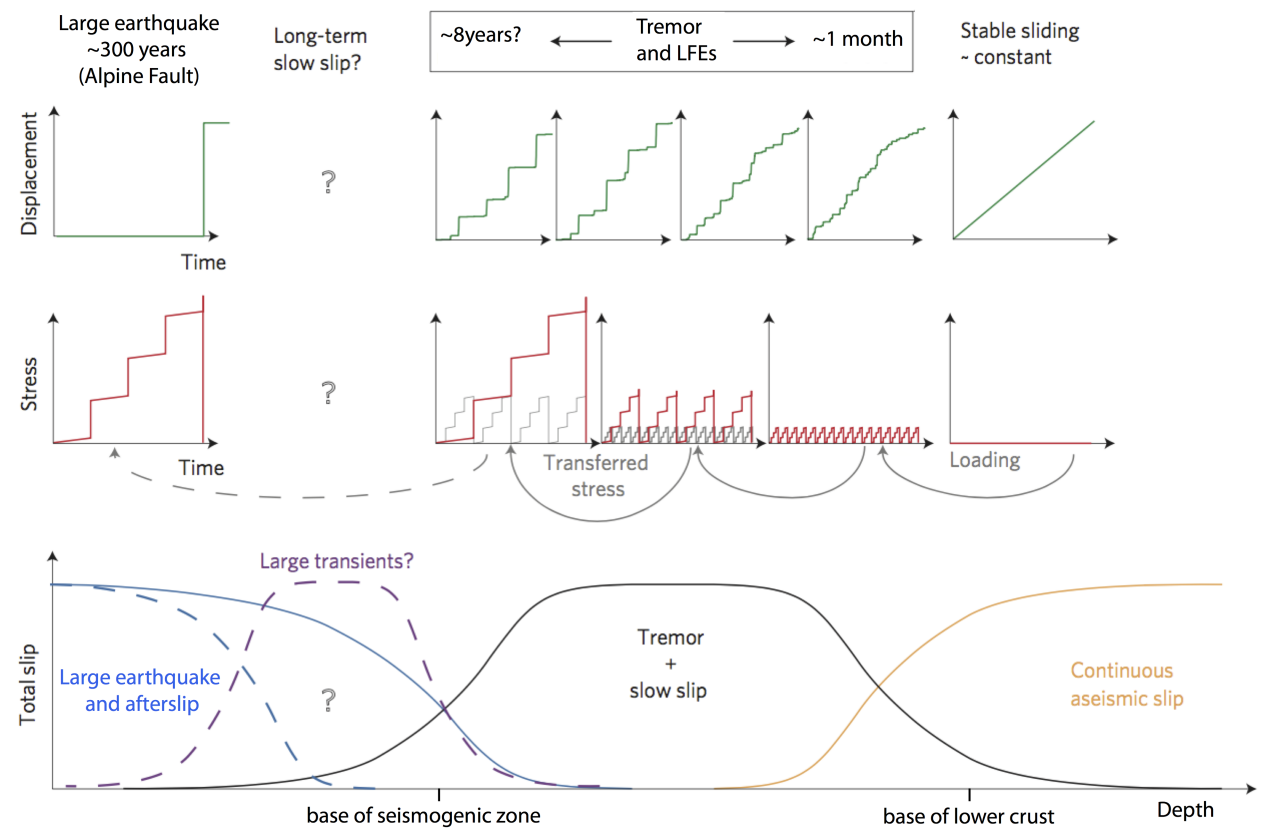

Figure 1.2: Schematic model for slip. After Wech and Creager (2011). Schematic model showing how stress and displacement evolve with time and depth, depending on the type of earthquakes.

Slow earthquakes generally manifest as geodetically observed slow-slip events (Dragert et al., 2001) and seismically observed tectonic tremor (Obara, 2002). All different types of slow earthquakes share similar scaling characteristics that are different from those of typical earthquakes (Fig. 1.3). Indeed, Ide et al. (2007b) show that the seismic moment of ordinary earthquakes increases with the cube of their duration, while the seismic moment of slow earthquakes increases linearly with their duration (Fig. 1.3). This scaling behaviour highlights the fact that slow earthquakes represent different manifestations of the same slow deformation phenomena and that they constitute a new earthquake category (Ide et al., 2007a).

Some statistical features of slow earthquakes can be modelled kinematically using a simple stochastic model described by Ide (2008): the Brownian slow earthquake model (Fig. 1.4). In this model, a slow earthquake is approximated by a circular zone corresponding to shear slip on a circular fault (blue patches in Fig. 1.4). A variation of the parameter values in the Brownian model (e.g.radius of the patch, diffusion coefficient or slip rate coefficient) could partly explain the differences in slow-slip events behaviours worldwide. For example, if we look at the 


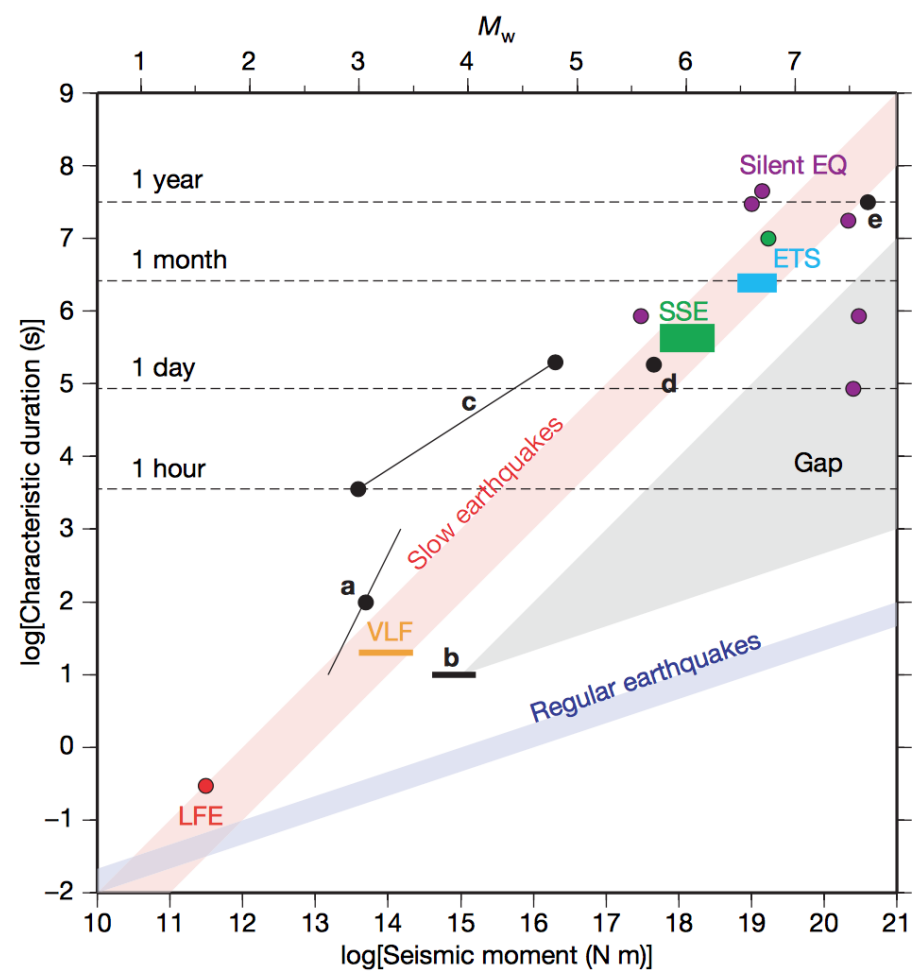

Figure 1.3: Scaling law for slow earthquakes. From Ide et al. (2007a). The typical scaling relation for shallow earthquake is shown with a highlighted blue area. The overall slow earthquake scaling range is highlighted in red. LFEs are indicated with a red circle. Slow slip events (SSE) are indicated with a green box.

slow-slip event that occurred without tectonic tremor in the Tokai region, the observed migration velocity in the shallower extension of the tremor zone was inferred to be of the order of $10 \mathrm{~km}$ per day (Hirose and Obara, 2006). If now, the damping coefficient is decreased by one order, the evolution of the slow earthquake becomes invisible in the seismological frequency range (hence the absence of detected tectonic tremor). Although this Brownian walk model can explain for example, the linear relation between the seismic moment and the duration of the slow earthquake, it is too simple to properly describe the complete spectrum of slow earthquake activity (e.g. their sensitivity to tidal stress).

Despite the slow release of seismic energy radiated by slow-slip events, the slip accumulated over their duration can represent as much elastic energy as those of large earthquakes (e.g in Cascadia, where the energy release from the first slow-slip event ever identified was estimated to correspond to a $M_{w} 6.7$ earthquake, Dragert et al., 2001). Furthermore, Mazzotti and Adams (2004) have estimated the conditional probabilities of a great earthquake occurring in the Cascadia subduction zone based on estimations of earthquake recurrence intervals. They estimated 


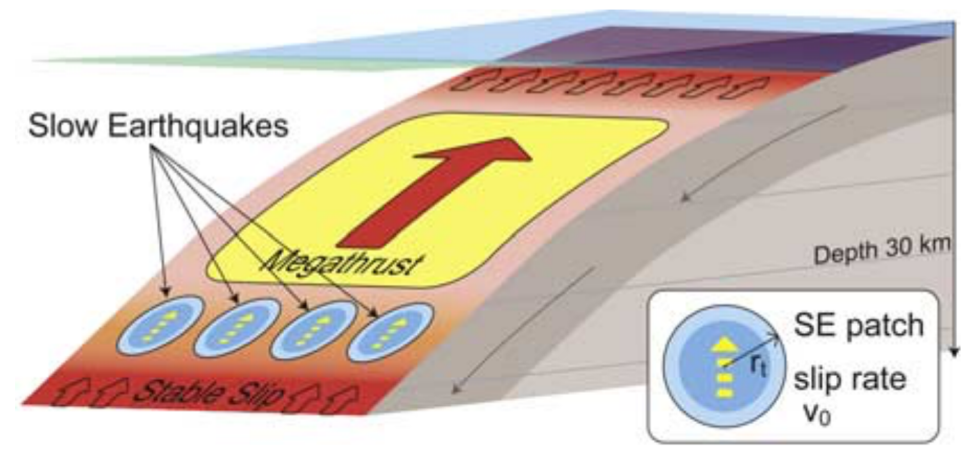

Figure 1.4: Brownian walk for slow earthquakes occurring on a subducting plate. From Ide (2008). Schematic illustration of a Brownian walk model for slow earthquakes. Ide (2008) assumes circular patches of slow earthquakes on the subducting plate interface, between the locked area and the deeper stable-sliding zone. The radius $\left(r_{t}\right)$ of the slow earthquake patch is governed by the Langevin equation and three parameters: (1) a damping coefficient; (2) a diffusion coefficient; and (3) a slip rate coefficient $\left(v_{0}\right)$. The seismic moment rate $\left(M_{0}\right)$ of the slow earthquake is estimated as follows: $M_{0}=\pi \mu v_{0} r_{t}^{2}$ where $\mu$ is the rigidity.

that slow-slip events on the deep extension of the plate interface in Cascadia may increase the probability of a great earthquake by a factor of $30 \%$ during the 2 week period over which they occur, thus highlighting the importance of studying slow earthquakes to better understand seismic hazard.

Tectonic tremor is a weak, long duration, emergent seismic signal without clear impulsive P- and S- wave arrivals. Tectonic tremor was first observed in subduction zones (Obara, 2002; Rogers and Dragert, 2003) but later found along major strike-slip faults (Nadeau and Dolenc, 2005; Wech et al., 2012; Aiken et al., 2013). Some tremor signals, found to correlate spatially and temporally with slow slip events (Episodic Tremor and Slip), were documented on the deeper interface of the Cascadia subduction zone (Rogers and Dragert, 2003). However, slow slip has also been observed without the occurrence of tectonic tremor, in Cascadia as well as in other areas (Wech and Bartlow, 2014; Delahaye et al., 2009). Similarly, tremor has been detected without observable slow slip (Shelly, 2009). Overall, the relationship between tectonic tremor and slow slip is insufficiently understood and the mechanisms involved in their generation remain poorly constrained. Several studies (Liu and Rice, 2005; Segall et al., 2010; Beroza and Ide, 2011) agree that tectonic tremor is located at the plate interface in the transition zone from a stickslip (in the brittle seismogenic zone) to a stable-sliding (in the ductile downdip portion of the fault) regime. Two simple mechanisms could explain the weakening 
of the plate interface and its consequent transition to a viscously slipping downdip portion of the fault: (1) an increase in temperature with depth; (2) trapped fluids at the plate interface and their resulting high pore pressures (Shelly et al., 2006; Audet and Burgmann, 2014; Frank et al., 2015). Numerous studies also report that tectonic tremor can be triggered instantaneously during the passing of surface waves of teleseismic events (Miyazawa and Mori, 2006; Rubinstein et al., 2007; Gomberg et al., 2008; Peng and Chao, 2008; Miyazawa and Brodsky, 2008), thus highlighting the importance of studying tremor triggering.

A low-frequency earthquake is a type of slow earthquakes that possess discernible, emergent S-wave arrivals, and lack clear P-wave arrivals. Low-frequency earthquakes were first observed in Shikoku, Japan (Shelly et al., 2006). Their waveforms generally have low amplitudes and look 'noisy' compared to ordinary earthquakes. Their dominant frequency is clearly lower than that of ordinary earthquakes as they are typically observed in the $1-10 \mathrm{~Hz}$ frequency range. Tectonic tremor can be decomposed as a swarm of discrete low-frequency earthquakes (Shelly et al., 2006). The principal feature of an LFE is its repetitive nature: each LFE source in space generates many events in time, forming what is called an LFE family. Low-frequency earthquakes sources have been modelled by Beeler et al. (2013) to infer the rheological properties of rocks producing creep (hence to infer the rheology of the fault at depth). They model the LFE sources as small and constant regions, repeatedly failing during the shear of a considerably larger and otherwise aseismically creeping region of a fault.

\subsection{Tectonic setting}

New Zealand is located at the boundary between the Australian and Pacific plates (Fig. 1.5). The Alpine Fault, an $850 \mathrm{~km}$-long transpressional fault, is the principal structure defining the Australian/Pacific plate boundary in the South Island. This major active crustal-scale fault links two subduction zones with opposite dips (Berryman et al., 1992):

- The Kermadec trench and its southern continuation, the Hikurangi trough, situated along the northwest dipping margin of the North Island and northern South Island, where subduction of the Pacific plate beneath the Australian plate is observed;

- the Puysegur trench located offshore Fiordland at the southwest of the South Island presenting an east-dipping subduction of the Australian plate. 
The average strike of the Alpine Fault is $055^{\circ}$ at an angle of $\sim 18^{\circ}$ to the $\mathrm{Pa}$ cific-Australia relative plate motion vector at a latitude of $-43.5^{\circ}$ (Little, 2004). An average of $70 \%$ of total plate displacement (equivalent to a strike-slip rate of $\sim 27 \pm 4 \mathrm{~mm} / \mathrm{yr}$ ) is accommodated by the Alpine Fault (Norris and Cooper, 2001). The rates of interplate displacement vary with distance along the plate boundary (Norris and Toy, 2014). Dip-slip also changes considerably along strike, from $10 \mathrm{~mm} / \mathrm{yr}$ in the central section of the Alpine Fault to $0 \mathrm{~mm} / \mathrm{yr}$ at its southern end (Norris and Cooper, 2001).

The Alpine Fault can be divided into three distinctive sections with different dips, structural styles, slip rates and deformation widths (Sutherland et al., 2000), as described below (Fig. 1.6).

In the north of the South Island, the Alpine Fault splays into four major faults in a broad region ( $\sim 200 \mathrm{~km}$ wide) of strike-slip deformation: the Malborough Fault System. Spaced $\sim 30 \mathrm{~km}$ apart, those predominantly dextral strike-slip faults with parallel strike directions are steeply-dipping. The Malborough Fault System accomodates $80-100 \%$ of the plate motion (Holt and Haines, 1995). Slip partitioning (i.e. partitioning of total slip into normal and shear components) takes place between those faults which, in turn, transfer motion to the west-dipping Hikurangi subduction zone (Little and Jones, 1998).

From Hokitika to Haast, the Alpine Fault appears as a straight fault trace within an $80 \mathrm{~km}$ wide zone of deformation. The central Alpine Fault dips to the southeast with a dip of $60^{\circ}$ (Sibson et al., 1981). Seismic reflection studies by Kleffmann et al. (1998) and Okaya et al. (2007) infer that the fault becomes subhorizontal at mid-crustal depth (i.e. listric fault). A transition from west-dipping subduction to continental collision is observed in this area (Cox and Sutherland, 2007). Offsets of late Quaternary features and kinematic indicators suggest that this section of the Alpine Fault displays oblique displacement with both dextral strike-slip and reverse dip-slip components (Norris and Cooper, 2001).

Oblique thrusting is predominantly observed on short sections of a few kilometers with a northerly strike whereas dextral strike-slip sections mostly exhibit an easterly strike (Norris and Cooper, 1995). The central Alpine Fault accomodates $70-75 \%$ of the fault-parallel interplate motion (Norris and Cooper, 2001). Beavan et al. (2002) estimate the current rate of plate motion in the central South Island to be around $40 \mathrm{~mm} / \mathrm{yr}$, oriented between $065^{\circ}$ and $068^{\circ}$. Partitioning of this displacement results in $39 \mathrm{~mm} / \mathrm{yr}$ strike-parallel motion and $7-9 \mathrm{~mm} / \mathrm{yr}$ convergent motion on the central part of the Alpine Fault. The convergent motion results 


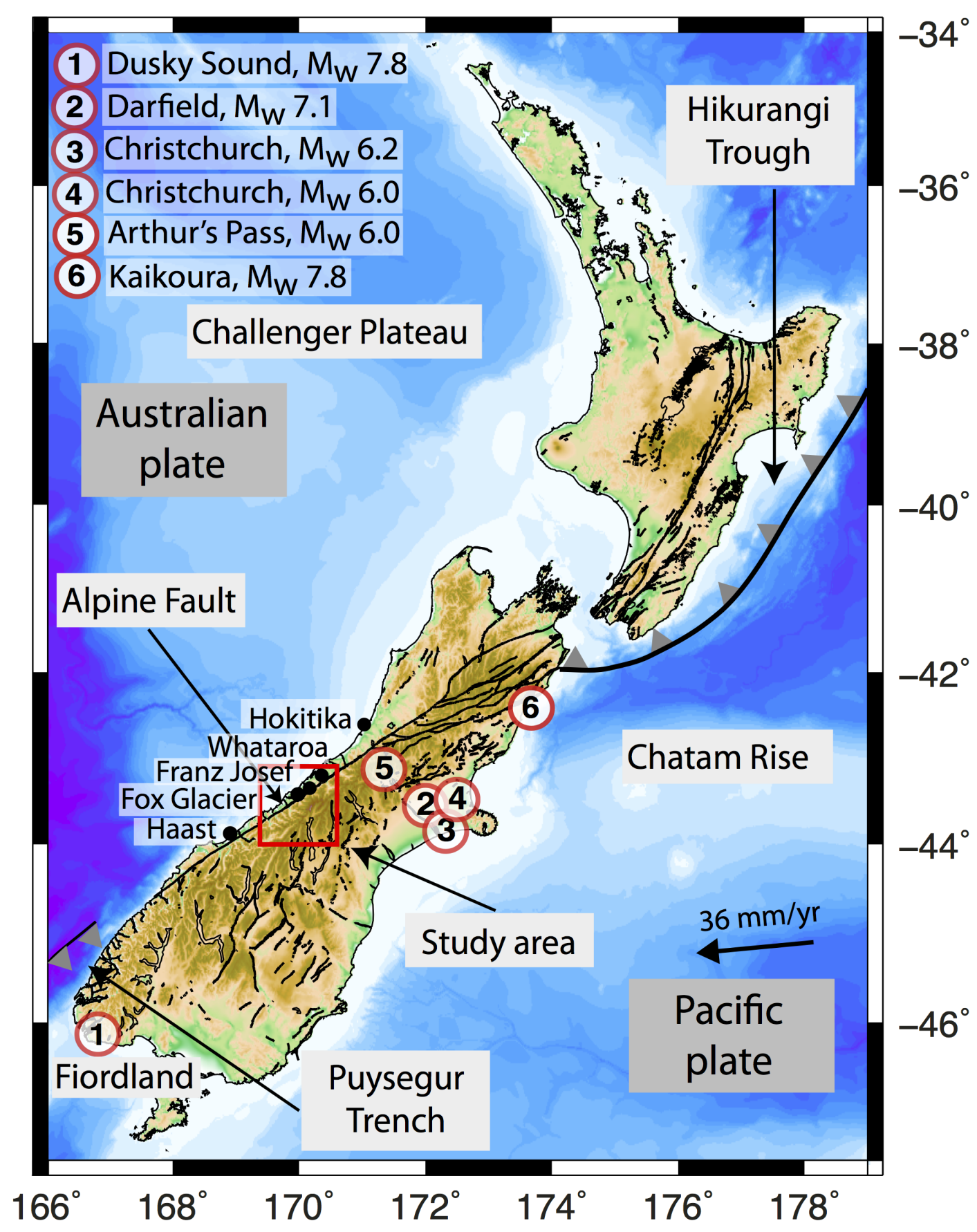

Figure 1.5: Map of New Zealand. Regional map highlighting the plate boundary between the Australian and Pacific plates through New Zealand. Numbers correspond to large regional earthquakes that will be mentioned throughout this thesis.

in an area of enhanced uplift (with rates of 5-8 mm/yr, Houlié and Stern, 2012) that corresponds to regions of highest peaks in the central Southern Alps (Little et al., 2005). 


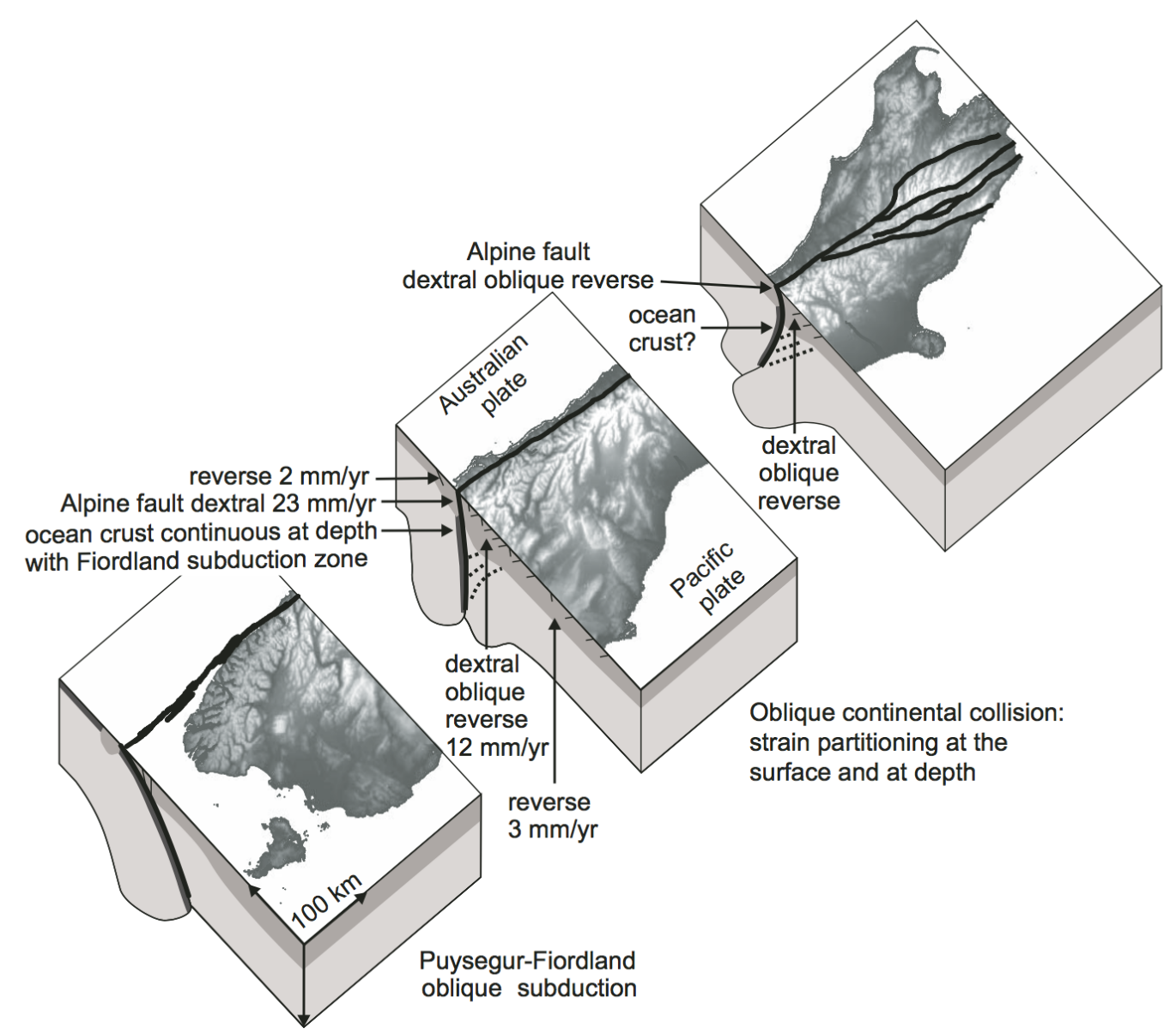

Figure 1.6: Segments of the Alpine Fault. From Sutherland et al. (2006). Kinematic model for southern New Zealand. Left: The Australian plate oceanic crust is obliquely subducted at the Puysegur subduction zone. The steeply dipping plate boundary is inferred to be continuous at depth with $23 \mathrm{~mm} / \mathrm{yr}$ of strike-slip motion on the Alpine Fault. Middle: In the central South Island, the crustal plate motion occurs by oblique reverse movement on the Alpine Fault. The central Alpine Fault accomodate $70-75 \%$ of the fault-parallel interplate motion (with a current rate of plate motion of $\sim 40 \mathrm{~mm} / \mathrm{yr}$ partioned into $39 \mathrm{~mm} / \mathrm{yr}$ of strikeparallel motion and 7-9 mm/yr of convergent motion). Right: In the north of the South Island, the Alpine Fault splays into four major faults in a broad region of strike-slip deformation: the Malborough Fault System. The Malborough Fault System accommodates $80-100 \%$ of the plate motion with slip partioning taking place between those faults.

South of Haast, the fault is straight with little segmentation and is steeply dipping (70-90 ${ }^{\circ}$ (Berryman et al., 1992). In the southern section, the fault becomes purely strike-slip (Sutherland et al., 2000). Approximately $65 \%$ of the interplate displacement is accomodated on the fault. According to Sutherland et al. (2006) the slip rate on this section of the fault is estimated to $23 \pm 2 \mathrm{~mm} / \mathrm{yr}$. 
Drilling into the hanging wall of the Alpine Fault was undertaken as part of the Deep Fault Drilling Project to better understand the ambient conditions, rock properties, and geophysical phenomena within an active fault zone prior to a large earthquake (Townend et al., 2009b, 2013; Sutherland et al., 2017). The most recent phase of drilling revealed a pore fluid pressure gradient of $9 \pm 1 \%$ above hydrostatic levels and an extremely high geothermal gradient of $125 \pm 25^{\circ} \mathrm{C} / \mathrm{km}$ within the fault hanging wall, reinforcing the hypothesis that rapid uplift and exhumation near the Alpine Fault have raised isotherms, thus weakening the crust and focusing deformation along the fault (Sutherland et al., 2017). Those extreme hydrothermal conditions affect the frictional-mechanical processes associated with seismogenesis, controlling the distribution (e.g. depth) of earthquakes.

Recent work by Bourguignon et al. (2015) revealed a change in seismogenic thickness across the Alpine Fault. They linked this observation to a variation in temperature across the fault and concluded that temperature had a crucial control on seismogenic depth near the Alpine Fault. To this date, little is known about the footwall of the Alpine Fault below seismogenic depths. The footwall will likely play a critical role in future Alpine Fault earthquakes, possibly as a source of fluids which might in turn weaken the fault, thus allowing for tremor to occur (Wech et al., 2012).

\subsection{Geological background}

New Zealand is a fragment of the Gondwana Supercontinent which was adjacent to Australia and Antarctica before the Late Cretaceous sea floor spreading. The majority $(\sim 90 \%)$ of the continental crust in the greater New Zealand region (termed Zealandia by Luyendyk, 1995) is submerged, with only roughly $10 \%$ of the crust above water as the North and South Islands. The formation of New Zealand's main crustal components happened via accretion onto the Gondwana Supercontinent plate margin during the Paleozoic and Mesozoic. No Precambrian craton is exposed onland New Zealand. In fact, the New Zealand basement is composed of a series of sedimentary terranes and igneous suite of varying age (Fig. 1.7, Mortimer, 2004; Cox and Sutherland, 2007). The New Zealand's Precambrian to Jurassic basement can be overall described using:

- Ten major volcano-sedimentary terranes, amongst them present on the South Island: the Eastern (Permian to Jurassic in age, Mortimer, 2004) and Western (dated from Precambrian to late Mesozoic, Cox and Barrell, 2007) 
Provinces. The Eastern Province on the South Island is made up of (1) the Caples (in grey in Fig. 1.7), Maitai (in purple in Fig. 1.7), Murihiku (in blue in Fig. 1.7) and Brook Street (in yellow in Fig. 1.7) Terranes that can be found in the upper north and lower south of the South Island; (2) the Rakaia Terrane (in light grey in Fig. 1.7) that makes up most of the central part of the South Island; and (3) the Pahau Terrane that can be found on the northeastern part of the South Island. As for the Western Province, it is made up of: (1) the Buller Terrane (in brown in Fig. 1.7) which can be found west of the Alpine Fault and (2) the Takaka Terrane (in brown in Fig. 1.7);

- Two composite regional batholiths : the Median Batholith (red in Fig. 1.7) and the Karamea, Paparoa and Hohonu Batholith that can be found in the northwestern part of the Alpine Fault (in orange in Fig. 1.7);

- Three regional metamorphic and tectonic belts overprinting the terranes and batholiths, all present in the South Island: the Esk Head and Whakatane Mélanges (found in the northeastern part of the South Island), the Haast Schist (found in the central part of the South Island), and the Gneiss (found in the southwest).

During the Paleozoic, the basement rocks of the Western Province formed sediments along the margin of the Gondwana Supercontinent. These sediments were later (Devonian-Cretaceous) metamorphosed and intruded by igneous rocks (i.e. granites and granitoids) when rifting occurred during the break-up phase of the Gondwana Supercontinent. The Australian-Pacific plate boundary developed during the Paleogene and Neogene (Cox and Sutherland, 2007). The Southern Alps are the result of the Neogene transpression accross the Australian-Pacific plate boundary and more precisely of the oblique continental collision between the Chatham Rise and Campbell Plateau to the east and the Challenger Plateau to the west. Uplift of the Southern Alps started to accelerate in the late Miocene and early Pliocene following an increase of the oblique compression of the relative Australian-Pacific plate motion (e.g. Walcott, 1978, found an increase in convergence rates between $11 \mathrm{Ma}$ and $6 \mathrm{Ma}$ leading to shortening in the central South Island by a maximum amount of $\sim 90-110 \mathrm{~km}$ ).

The Alpine Fault is the major onland feature forming the Australian-Pacific plate boundary and it constitutes the western boundary of the Southern Alps. The rocks of the Western Province form the footwall of the current Alpine Fault and they become adjacent to rocks from the Eastern Province at the fault. Rocks from 


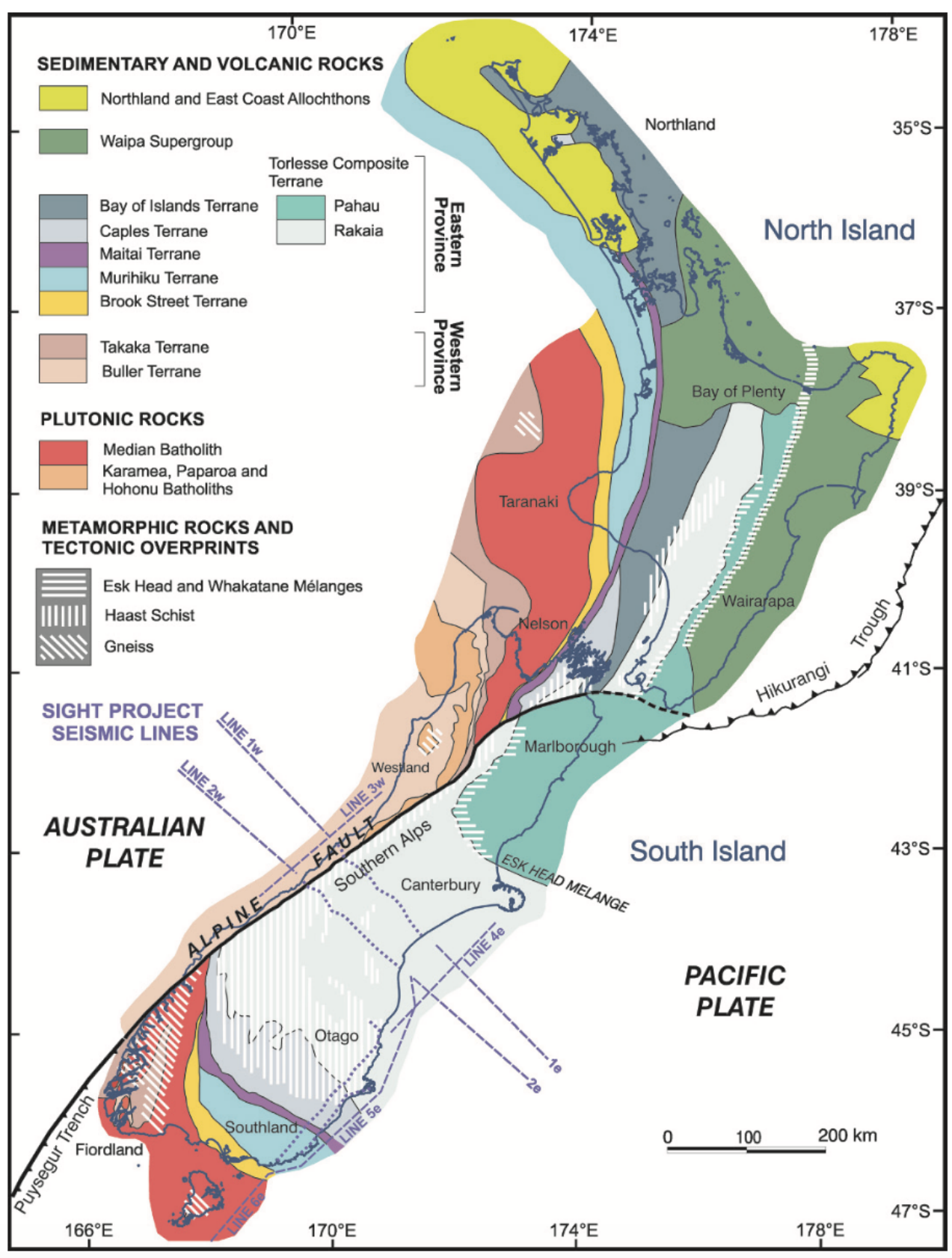

Figure 1.7: Geology of New Zealand. From Cox and Sutherland (2007). Map of the basement of New Zealand showing that the Alpine Fault clearly offsets the Maitai Murihiku and Brook Street Terranes (amongst others) as well as the Median Batholith from Fiordland to the northern South Island.

the Western Province are mainly made up of quartz sediments (Buller Terrane, in brown in Fig. 1.7) which have subsequently been metamorphosed and intruded by plutonic rocks (Cox and Barrell, 2007). Eastern Province rocks were formed following the deposition of sediments (e.g. sandstone, mudstone and volcanic rocks) in tubidity currents along the eastern coast of Gondwana. These sedimentary 
rocks (mainly lithic and feldspathic greywackes) are now being rapidly uplifted along the Alpine Fault. Estimates of the total uplift during the last 5 Ma varies between 25-30 km (e.g. Little et al., 2002; Norris and Cooper, 2003). In the central part of the South Island (i.e. our study area), the zone of deformation is $\sim 80 \mathrm{~km}$ wide (Cox and Barrell, 2007). On a transect from Canterbury toward the central Alpine Fault (e.g. Line 1w in Fig. 1.7), the Eastern Province rocks forming part of the Rakaia Terrane become increasingly metamorphosed and deformed as they transition from greywacke to foliated schists. Within $2 \mathrm{~km}$ of the fault, the Alpine Fault schists transition into protomylonites, mylonites and ultramylonites (Norris and Cooper, 2007; Toy et al., 2015). The width of the Alpine schist is narrowest in the central part of the Southern Alps ( $8 \mathrm{~km}$ between Franz Josef and Fox Glacier versus a maximum of $26 \mathrm{~km}$, according to Little et al., 2005; Cox and Sutherland, 2007). Contiguous to the Alpine Fault principal slip zone, mylonites in the hanging-wall are separated from the fault gouge by cataclastic units. The cataclastic rocks in the hanging-wall are primarily formed by protoliths, even though there is some evidence of a footwall source (Pacific plate) for some of the cataclasites (Toy et al., 2015). Drilling into the Deep Fault Drilling Project-1 boreholes (Townend et al., 2009b; Sutherland et al., 2012; Townend et al., 2013), at Gaunt Creek in the central part of the Alpine Fault, revealed particularly high coefficient of friction $\left(\mu \sim 0.61-0.76\right.$ for $\left.T=70-350{ }^{\circ} \mathrm{C}\right)$ and normal stress $(\sigma=31-156 \mathrm{MPa})$ for the fault gouge (Boulton et al., 2014). These frictional values are thoroughly different to the low values of $\mu$ found in the southern portion of the Alpine Fault ( $\mu=0.12-0.37$, Barth et al., 2012) or in similar areas worldwide (e.g in Parkfield along the San Andreas Fault where $\mu=0.1-0.15$ according to Lockner et al., 2011).

\subsection{Seismicity in the central Southern Alps}

\subsubsection{Rupture history in the Alpine Fault region}

Several large earthquakes have occurred in the South Island in historic times: south of the central Southern Alps, in the Puysegur subduction zone (e.g. 1918 $M_{w} 6.7$ and $1945 M_{w} 7.7$ Puysegur Bank earthquakes, Doser et al., 1999); near Arthur's Pass, in the northern portion of the central Southern Alps (with the March $1929 M_{w} 7$ Arthur's Pass earthquake, Doser et al., 1999), in the North Canterbury region, east of our area of interest (e.g. two large $\left(M_{w}>7\right)$ earth-

quakes identified by dating surface ruptures and landslides in the last 2500 years 
by Cowan et al., 1996), to the north of our study area, in the Malborough Fault system (with three large earthquakes occurring since 1840; the $1888 M_{w}>7$ Hope Fault earthquake, Doser et al., 1999; Cowan, 1991) and in the Buller region (e.g. $1929 M_{w} 7.3$ Buller earthquake, Doser et al., 1999; Anderson et al., 1993). A few large earthquakes have been observed more recently in the Arthur's Pass region (1994 $M_{w} 6.7$ Arthur's Pass earthquake followed by the $1995 M_{w} 6.2$ Cass earthquake, $\sim 30 \mathrm{~km}$ to the east Robinson and McGinty, 2000; Gledhill et al., 2000); in the Puysegur subduction zone (e.g the recent $M_{w} 7.82009$ Fiordland earthquake, Beavan et al., 2010), in the Canterbury region (with the $M_{w} 7.1$ September 2010 Darfield earthquake followed by the $M_{w} 6.2$ February and $M_{w} 6.0$ June 2011 Christchurch earthquakes, Gledhill et al., 2011; Quigley et al., 2012; Bannister and Gledhill, 2012), or even in the Kaikoura Peninsula (with the 14 November $2016 M_{w} 7.8$ Kaikoura earthquake, Hamling et al., 2017).

The Alpine Fault exhibits an average late Quaternary surface displacement rate of $2-3 \mathrm{~cm} / \mathrm{yr}$ (Sutherland et al., 2006) and was shown to have offset basement rocks by $\sim 470 \mathrm{~km}$ and deposits of the last glaciation (18-80 ka) by $0.4-2.0 \mathrm{~km}$ (Wellman, 1953; Norris and Cooper, 2001; Sutherland et al., 2006). In spite of high deformation rates, the Alpine Fault has not ruptured since the early European settlement $\sim 200$ years ago (Wells et al., 1999; Evison, 1971). In the central Southern Alps, only two $M_{w}>6$ earthquakes have been reported since then (Doser et al., 1999; Anderson et al., 1993): the $1946 M_{w} 6.7$ Lake Coleridge earthquake and the 1984 $M_{w} 6.1$ Godley Valley earthquake. The two earthquakes exhibited strike-slip motion on steeply-dipping fault planes and both events occurred at distances of 40 and $55 \mathrm{~km}$ from the surface trace, to the southeast of the Alpine Fault.

Paleoseismic records date the most recent rupture of the Alpine Fault to $1717 \mathrm{AD}$ (Wells et al., 1999; Berryman et al., 2012). Evidence for this event spans $\sim 400 \mathrm{~km}$ (Sutherland et al., 2007, from Te Anau to Taramakau River in Fig. 1.8) and is associated with 8-9 m offsets in some areas between Milford Sound and Haast River. To infer the year of the last Alpine Fault earthquake, Wells et al. (1999) used: (1) estimated ages of forest disturbance at 1700-1730 AD near the Alpine Fault, correlated with (2) terrace abandonment at Karangarua River (Fig. 1.8) dated at 1710-1720 AD and (3) a notable tree growth suppression event at 1717 AD (Wells et al., 1999). Two other major Alpine Fault earthquakes (Fig. 1.8) have been identified and dated to $1620 \mathrm{AD}$ and $1430 \mathrm{AD}$ from data of aggradation terraces, landslides, trenches across the Alpine Fault trace, forest disturbance and tree-ring suppression (Wells et al., 1999; Yetton, 1998; Berryman et al., 1998; Cullen et al., 2003; Sutherland et al., 2007). Studies of paleoseimic records allowed estimate of 
the mean recurrence time for an Alpine Fault earthquake to be $\sim 300$ years. Several studies (Norris and Cooper, 2001; Sutherland et al., 2007; Berryman et al., 2012; Howarth et al., 2014) suggest that failure of the Alpine Fault during those episodes resulted in large surface displacements along broad sections of the fault. The $M_{w} 7.91717 \mathrm{AD}$ event is inferred to have ruptured approximately $300-500 \mathrm{~km}$ length of fault; the $M_{w} 7.61620 \mathrm{AD}$ event close to $200-300 \mathrm{~km}$ and the $M_{w} 7.9$ 1430 AD event about 350-600 km (Sutherland et al., 2007).

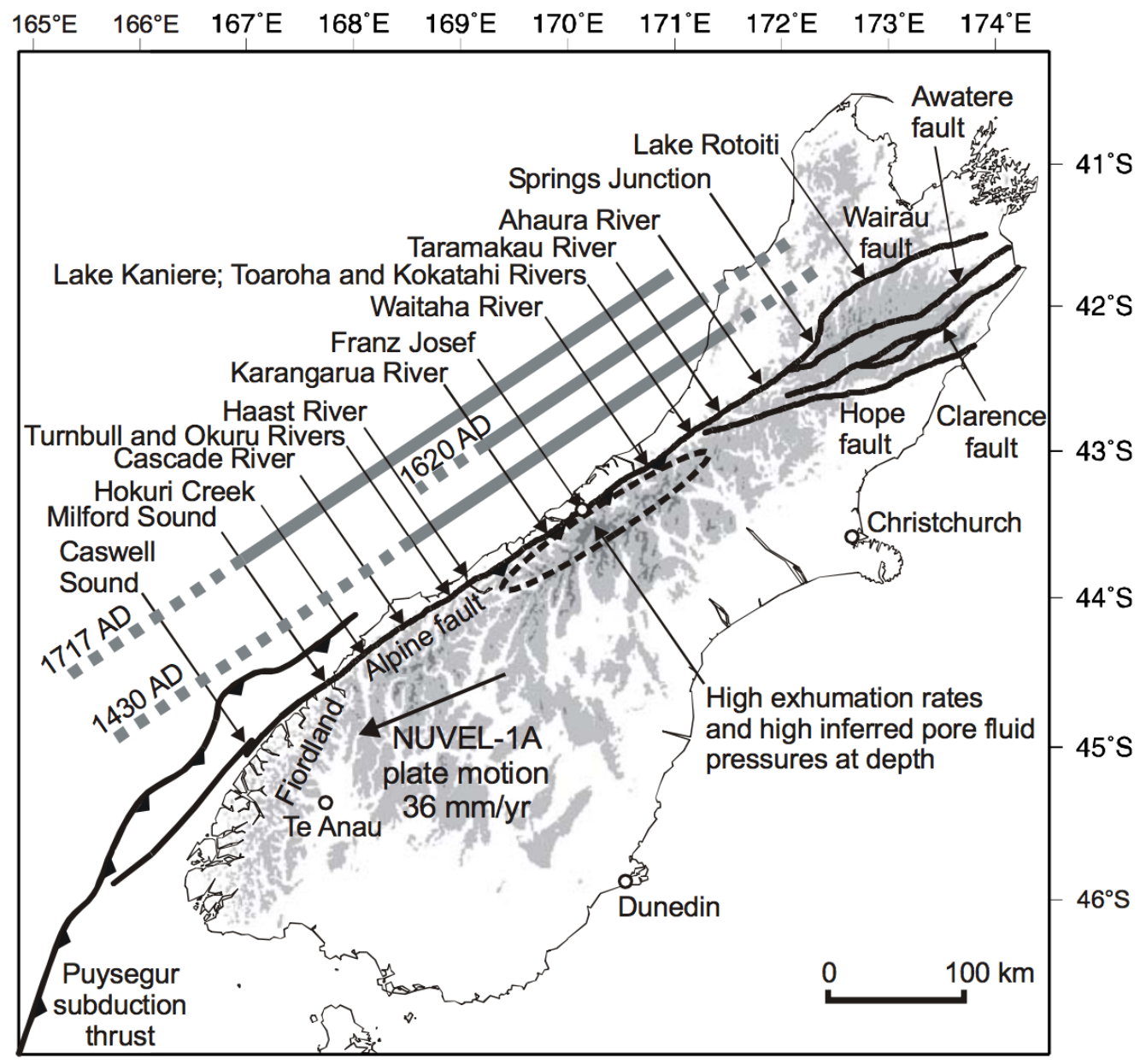

Figure 1.8: Historic ruptures along the Alpine Fault. From Sutherland et al. (2006). Location and inferred extent of historical Alpine Fault ruptures. The topography $\leq 800 \mathrm{~m}$ is shown with a light shade and relief $\geq 1800 \mathrm{~m}$ with a dark shade.

More recent work by Howarth et al. (2016) estimated a 2000 years rupture history of the Alpine Fault from lake sediments and slightly younger ages for previous Alpine Fault ruptures (e.g. 1726-1690 AD; 1594-1549 AD; 1407-1388 AD and 1213-1008 AD), thus reducing the average returning time of an Alpine Fault earthquake to $\sim 230$ years over the last millenium. 


\subsubsection{Previous seismicity studies}

The first seismicity study in the South Island was undertaken by Evison (1971) who presented a sparse catalogue of Alpine Fault earthquakes of moderate magnitudes $\left(M_{L} \leq 4\right)$. He was the first to observe the lack of seismicity in the central Alpine Fault.

Microseismicity along the Alpine Fault was first discussed by Scholz et al. (1973) who documented a rather diffuse seismicity throughout the region. They observed microearthquakes occurring in the central Alpine Fault between depths of 2-20 km. They noticed that the rates of seismicity in the central Alpine Fault were distinctly lower than further South (south of Haast).

Between 1975 and 1983, a network of 9 seismometers was installed around Lake Pukaki (grey triangles in Fig. 1.9) to monitor changes in microseismicity linked with the damming of the lake for hydroelectric power generation (Reyners, 1988). During the 8 years of operation, the network recorded 2,825 earthquakes of $M_{L} \geq$ 0.8 (with a completeness magnitude of $M_{L}=1.8$ ) (Reyners, 1988). Of those earthquakes, only $2 \%$ occurred at depth greater than $15 \mathrm{~km}$ - the majority of them being located between 5 and $9 \mathrm{~km}$ depth.

Eberhart-Phillips (1995) used the New Zealand National Seismic Network (NZNSN, installed in 1990 and now operated by GeoNet) to document seismicity in the central South Island between 1991 and 1994. At that time, the network was sparse with only a few stations located in the central Southern Alps (yellow triangles in Fig. 1.9). Eberhart-Phillips (1995) relocated 122 events (completeness magnitude of $\left.M_{L}=2.6\right)$ with a $1 \mathrm{D}$ velocity model and found an increase in the average earthquake depth from $10 \mathrm{~km}$ near the fault, to $20 \mathrm{~km}$ at distances of $\sim 60 \mathrm{~km}$ from the fault. However the depth uncertainties for all those events were substantial due to the sparsity of the network.

The Southern Alps Seismic Passive Experiment (SAPSE) was deployed between 1995 and 1996 in the central South Island to monitor seismicity across the Southern Alps (Anderson, 1997). This network (blue triangles in Fig. 1.9) comprised 26 broadband and 14 short-period sensors with and average station spacing of 30-50 km. Leitner et al. (2001) used the SAPSE temporary network along with 15 permanent NZNSN stations and the Lake Pukaki network to conduct a thorough analysis of seismicity in the central Southern Alps. Leitner et al. (2001) recorded 5,491 earthquakes with magnitudes of $2 \geq M_{L} \leq 4.2$ (blue circles in Fig. 1.9). Using a subset of 195 earthquakes, a simultaneous inversion was done in order to 
determine hypocentre locations and a 1D P-wave velocity structure. From those earthquakes, 53 well-constrained focal mechanisms were computed using first motion picks and amplitude ratios; which showed predominantly oblique strike-slip and thrust mechanisms. They inverted those mechanisms for a uniform stress field and found a $S_{H \max }$ oriented at $60^{\circ}$ to the Alpine Fault $\left(S_{H \max }=120^{\circ}\right)$. Leitner et al. (2001) observed a consistent maximum seismogenic depth of $12 \pm 2 \mathrm{~km}$ throughout the Southern Alps, with the exception of its central portion, where they found the seismogenic cut-off depth to be $3-4 \mathrm{~km}$ shallower. Leitner et al. (2001) noticed the existence of a triangular seismic gap near the Alpine Fault (between the Wanganui River, $40 \mathrm{~km}$ southwest of Hokitika, and Franz Josef Glacier in Fig. 1.9). They linked this zone of low seismicity $\left(M_{L}<2\right)$ to a transition zone where deformation occurred on a single structure along the Alpine Fault and was then partitioned across multiple splays of the fault.

In order to improve the depth constraints of microearthquakes in the central Southern Alps, denser networks were set up in this area. The first one, deployed between September 2006 and March 2007 by O'Keefe (2008), consisted of 8 seismometers located between Harihari and Fox Glacier (purple triangles in Fig. 1.9). O'Keefe (2008) recorded over 400 earthquakes of $M_{L} \geq 1.6$ (purple circles in Fig. 1.9) during this study period with a maximum seismogenic depth of $15 \mathrm{~km}$ for the majority of the study area (and a slightly shallower depth of $10 \mathrm{~km}$ in the Fox Glacier area). O'Keefe (2008) also highlighted an area of low seismicity between the Wanganui River and Franz Josef and they described it as possessing a 'horseshoe' pattern rather than the triangular shape noticed by Leitner et al. (2001).

Boese et al. (2012) deployed the Southern Alps Microearthquake Borehole Array (SAMBA) network between November 2008 and June 2009 (red triangles in Fig. 1.9). At this time, the SAMBA network consisted of 10 short-period seismometers with a station spacing of $8 \mathrm{~km}$ on average. A total of 1,791 earthquakes (red circles in Fig 1.9) were recorded by Boese et al. (2012) between November 2008 and December 2009 with a completeness magnitude of $M_{L}=1.4$ (with events as small as $M_{L}-0.3$ and as big as $M_{L} 4.2$ ). Boese et al. (2012) found seismogenic depths of $10 \pm 2 \mathrm{~km}$ near the Alpine Fault trace (similar to those of Leitner et al., 2001), decreasing to $8 \pm 2 \mathrm{~km}$ within $20 \mathrm{~km}$ of the fault trace and deepening to $15 \pm 2 \mathrm{~km}$ further from the fault. Boese et al. (2012) reported infrequent seismicity for earthquakes of magnitudes $M_{L} \geq 2.5$ in the Mt. Cook area (i.e. region of highest uplift). They also computed focal mechanisms and stress inversions for their catalogue and their results were in agreement with those of Leitner et al. 


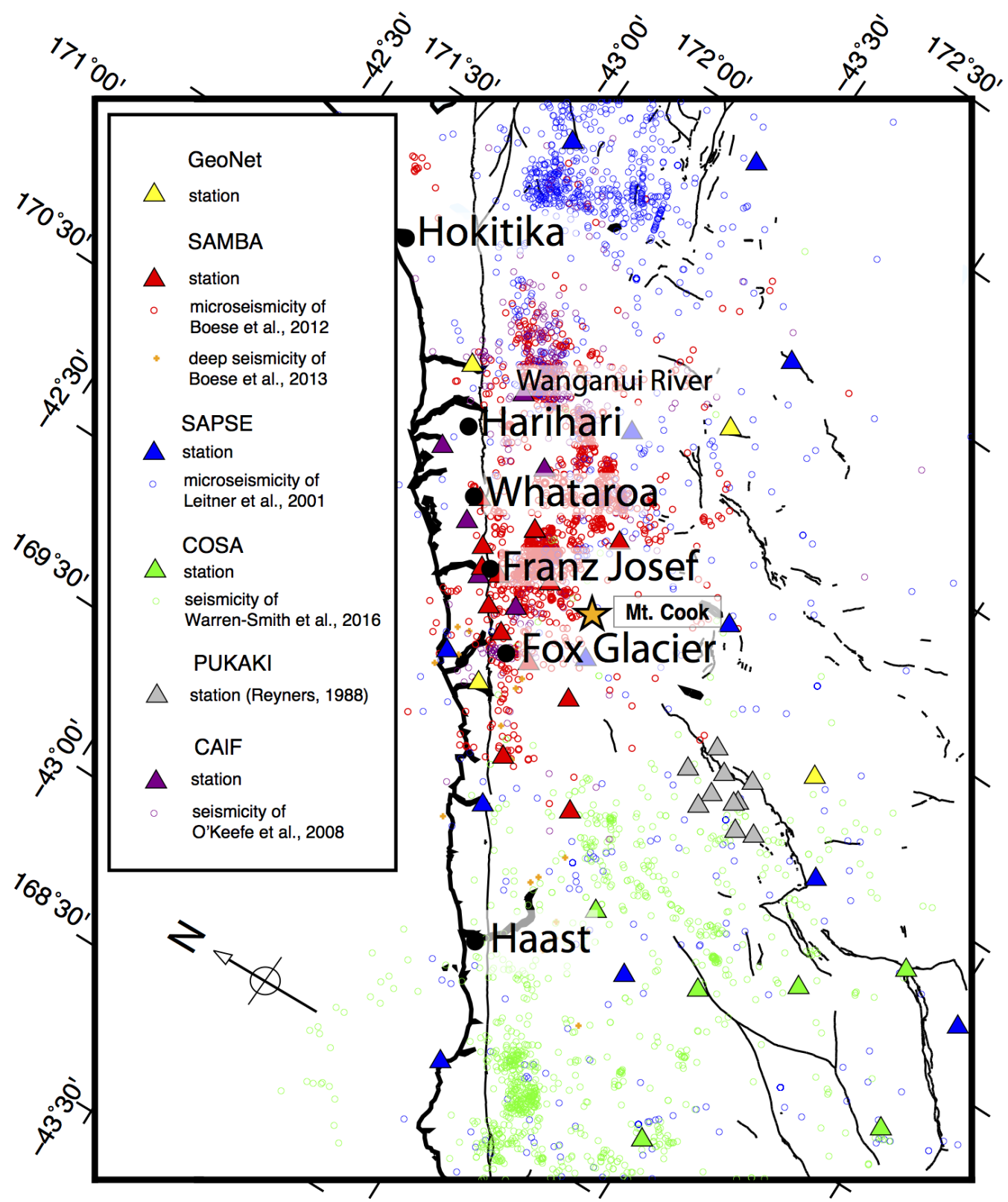

Figure 1.9: Seismicity in the area recorded by several studies. Temporary (SAPSE, PUKAKI, CAlF) and permanent (GeoNet, SAMBA, COSA) networks are plotted with coloured symbols according to the legend on the map. Five previous seismicity studies are also plotted here. The map's focus is on the central part of the Southern Alps, so only a subset of stations are plotted for the GeoNet, SAPSE and COSA networks. Earthquake catalogues are incomplete as well on this map for the SAPSE and COSA networks.

(2001). They found that the Alpine Fault as a whole is oriented at an angle of $59^{\circ}$ to the direction of maximum horizontal compressive stress $\left(S_{H \max }=115 \pm 10^{\circ}\right.$ at the $80 \%$ confidence level). Boese et al. (2012) also observed a seismicity gap 
between the Whataroa and Wanganui River valleys which they attributed to the existence of a strong unfractured block surrounded by high-attenuating fracture zones.

Boese et al. (2013) located 20 subscrustal earthquakes at depths of $47-74 \mathrm{~km}$ between December 2008 and February 2012 (orange cross in Fig. 1.9). Their analysis produced robust hypocentre locations for all 20 earthquakes as well as reliable local magnitudes and focal mechanisms (determined for only 14 of those events) yielding a stress field favouring oblique-reverse faulting in the region. Boese et al. (2013) identified three distinct seismotectonic zones with specific characteristics: (1) a region at the northern and southern ends of the Alpine Fault (Hikurangi and Puysegur margins) defined by intermediate and deep earthquakes associated with the subduction of oceanic lithosphere; (2) a region of sparse intermediate and subcrustal seismicity in a continental collision area (extending from the southern limit of the Hikurangi subduction zone to Mt. Cook); and (3) a sharp transition region consisting of tremor and moderate subcrustal seismicity extending south of Mt. Cook to the Puysegur subduction zone. Boese et al. (2013) inferred that subcrustal earthquakes locating between $30-50 \mathrm{~km}$ depth occurred within the Pacific plate and that only deeper earthquakes could be attributable to distributed shortening of the passive margin of the Australian lithosphere. The zone of intermediate-depth earthquakes adjacent to the Alpine Fault seems to correspond to the termination of a partially subducted Eocene passive margin (Sutherland et al., 2000). This region of the crust could potentially be rich in fluids, thus providing an area of fault weakening at depth able to produce tremor and slow-slip events (e.g. Wech et al., 2012).

Boese et al. (2014) provided evidence for both background and triggered swarms in the central Southern Alps consisting of low-magnitude earthquakes $\left(M_{L} \leq 2.8\right)$. Eight background swarms were documented between November 2008 and April 2010 as well as five seemingly triggered swarms that occurred after large regional earthquakes: the July $2009 M_{w} 7.8$ Dusky Sound and the September $2010 M_{w} 7.1$ Darfield earthquakes. The triggered swarms showed a delay of several hours between the passage of the surface waves of the large regional earthquake and the beginning of the triggered swarms (hence termed triggered-delayed swarms). This delay indicates that the dynamic stress changes associated with the earthquakes' surface waves themselves are unlikely to have triggered the swarms directly. Instead, Boese et al. (2014) suggest that the delays of the triggered swarms as well as their locations are more consistent with triggering by pore fluid diffusion.

Bourguignon et al. (2015) analysed some temporary network data in an area north 
of the region examined by Boese et al. (2012) (i.e. north of the Wanganui River). They catalogued over 1,300 earthquakes locating at depth of $4-11 \mathrm{~km}$ with an overall magnitude of completeness of $M_{L}=1.5$. Bourguignon et al. (2015) used high precision earthquake relocations to compute a tomographic model of the area. They also computed focal mechanisms for 148 of those events. Bourguignon et al. (2015) found clear evidence for variations of seismicity rates, perpendicular to the Alpine Fault. They observed low seismicity rates (constrained to depths of $4-11 \mathrm{~km}$ ) within $9 \mathrm{~km}$ of the Alpine Fault to the southeast of their study area. At greater distances of the fault, higher rates of seismicity are observed (with earthquakes generally occurring shallower than $7 \mathrm{~km}$ ). This change in seismicity coincides with regions of high horizontal strain rates (Lamb and Smith, 2013), thus suggesting that these structures are active.

More recently, Chamberlain et al. (2017b) analysed seismicity in the vicinity of the DFDP-2 drilling site (Sutherland et al., 2015, 2017; Toy et al., 2017) using 63 templates events in a matched-filter detection routine. Chamberlain et al. (2017b) used three temporary passive seismic deployments: (1) the SAMBA network, operating continuously since late 2008; (2) the WIZARD network active between January 2012 and January 2014 (Feenstra et al., 2016); and (3) the DFDP-10 network installed in February 2013 and consisting of four shallow borehole sites; as well as data from GeoNet. They constructed a high-precision catalogue of 283 earthquakes locating within $5 \mathrm{~km}$ of the central Alpine Fault trace in an otherwise seismically quiet zone (magnitude of completeness of $M_{L}=1.8$ ). They inferred that seismicity in this area is distributed throughout a highly fractured zone. They computed 13 focal mechanisms exhibiting predominantly strike-slip faulting, consistent with mechanisms observed further from the fault (e.g. Boese et al., 2012). They concluded that, in this region, the Alpine Fault is locked throughout the seismogenic zone and is accumulating stress.

\subsubsection{Tectonic tremor and LFEs in the Southern Alps}

Tremor was first documented on the transpressive Alpine Fault, New Zealand by Wech et al. (2012) using data recorded by the Southern Alps Microearthquake Borehole Array (SAMBA) (Boese et al., 2012). Wech et al. (2012) reported tremor occurring in 12 separate episodes between March 2009 and October 2011. Their location showed that tremor was occurring at shallow depth below the seismogenic zone and more precisely in the lower crust (between $25-45 \mathrm{~km}$ ) south of the SAMBA network (Fig. 1.10). They showed tremor to coincide with a zone of high 
P-wave attenuation (i.e. low Qp as seen of Fig. 1.10) and high reflectivity. They associated tremor with slow slip occurring on the deep extent of the Alpine Fault in a region of elevated fluid content.

LFEs were subsequently manually identified in the vicinity of the Alpine Fault by Chamberlain et al. (2014), by visual inspection of seismic data spanning periods of tremor (Fig. 1.11). Fourteen LFEs were identified and used as primary templates in a matched-filter routine (technique detailed in Section 2.2.1 of Chapter 2) to detect similar events occurring throughout a 36 month period from 26 March 2009 to 2 April 2012. Following an initial detection run, the $10 \%$ of detections with the highest signal-to-noise ratios were stacked linearly to generate a new template. This process was repeated until the number of detections stabilised. Despite this, few P- and S- wave arrival times could be picked accurately, and P-wave polarities could not be determined. As a consequence of the resulting large uncertainties in hypocentral depths and the absence of source parameters, interpreting Alpine Fault tremor and LFE observations in terms of slip on the fault itself remained ambiguous and overall inconclusive.

Here, we will try to overcome those uncertainties by using refined signals with higher signal-to-noise ratios, allowing accurate arrival time and polarity picks and thus refined hypocentre locations and reliable focal mechanisms computation.

We aim to provide the longest continuous documentation of LFEs occurring over an 8-yr period in the New Zealand Southern Alps. By obtaining robust hypocentre locations and focal mechanisms, we aim to demonstrate whether LFEs locate on the deep extent of the Alpine Fault and whether they represent shear slip on the fault interface. 


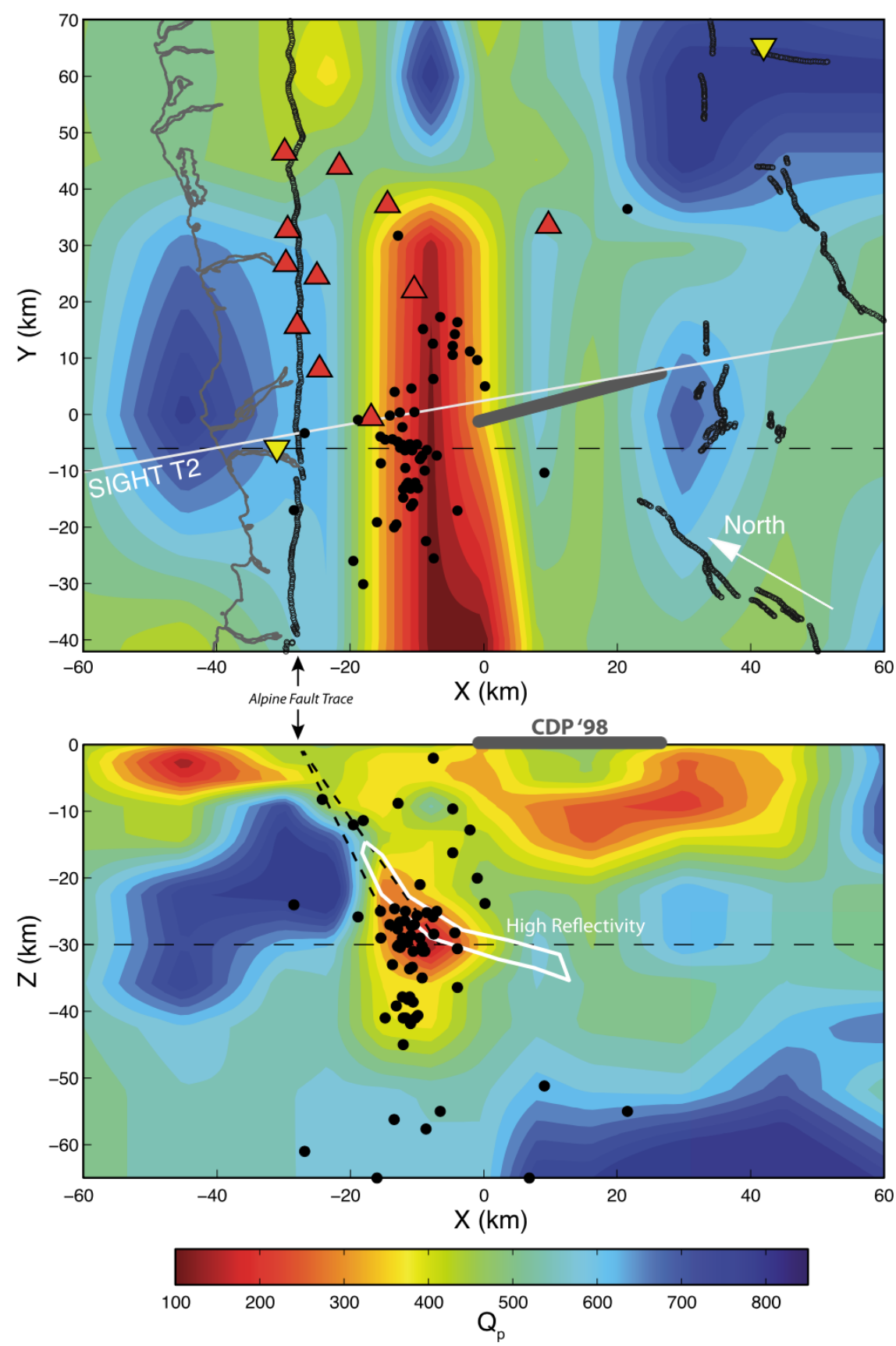

Figure 1.10: Tremor location in the central Southern Alps. After Wech et al. (2012). Tremor (black dots) and Qp values from regional tomography of Eberhart-Phillips et al. (2008). Low Qp corresponds to high attenuation. We can see here that tremor locations correlate with areas of high reflectivity (white box). Top: Horizontal section at $30 \mathrm{~km}$ depth. Bottom: Profile through tremor region with the white area outlining a region of high reflectivity by Stern et al. (2007). The two dashed-lines emanating from the Alpine Fault trace represent $45^{\circ}$ and $60^{\circ}$ dipping faults 


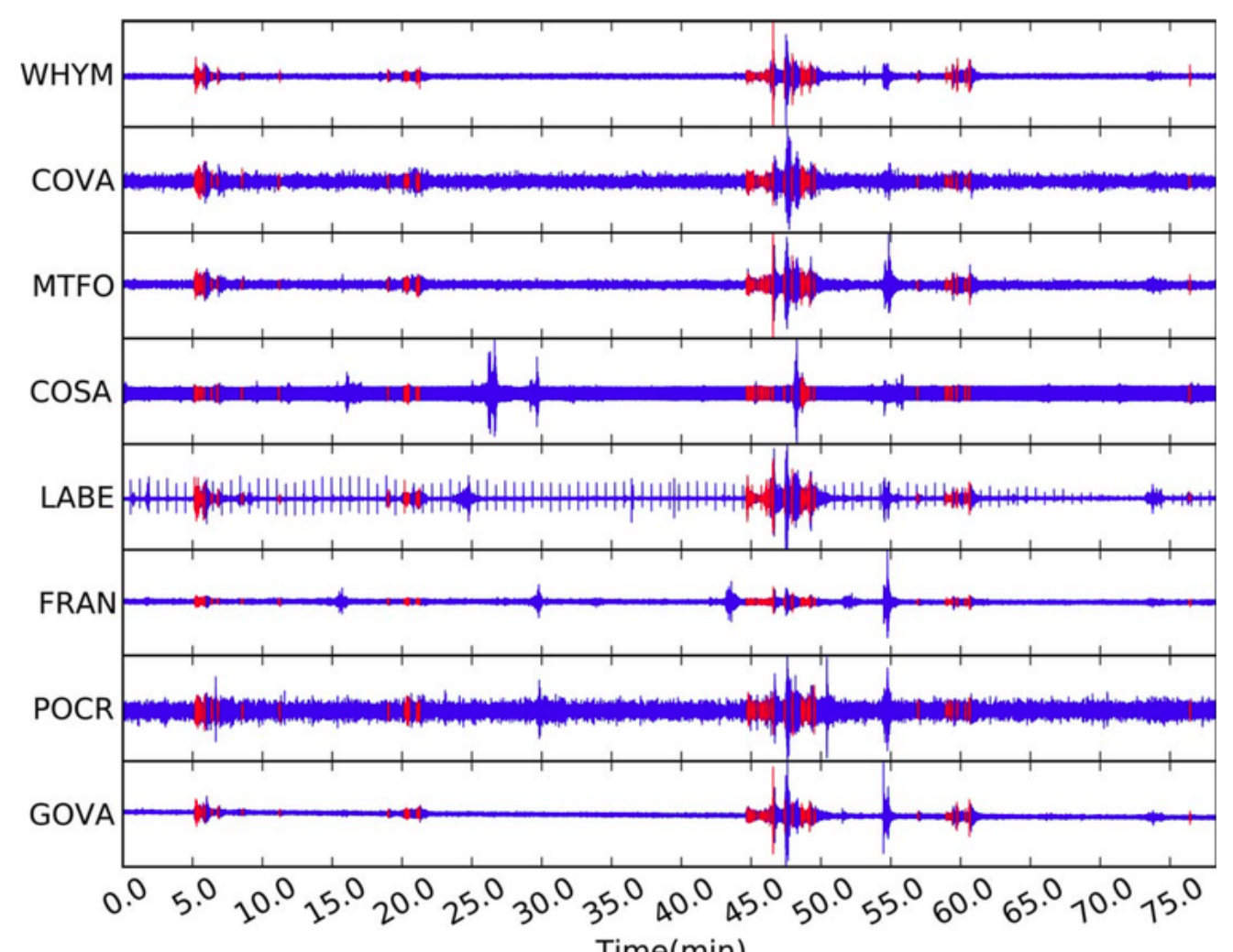

Figure 1.11: Low-frequency earthquakes identified within tremor bursts. From Chamberlain et al. (2014). An example of a tremor burst, first identified by Wech et al. (2012), can be seen at around $45 \mathrm{~min}$. Several episodes of LFEs were later manually identified (in red) within this tremor burst by Chamberlain et al. (2014).

\subsection{Lithospheric structure and Alpine Fault geometry}

A key factor in trying to understand the depth of faulting and the possible slip planes for a future earthquake is the lithospheric structure beneath the Southern Alps. The Southern Alps crustal structure has been investigated by passive-source (e.g. the Southern Alps Passive Seismic Experiment (SAPSE), Anderson, 1997) and by active-source seismic studies (e.g. the South Island Geophysical Transect (SIGHT), Davey et al., 1995). The results of Okaya et al. (2007) from velocity profiles confirmed a complex structure under the central South Island. This was also confirmed by Lamb and Smith (2013) who inferred a prevailing thickened crust under the Southern Alps and a general crustal discontinuity associated with the Alpine Fault (Fig. 1.12). 


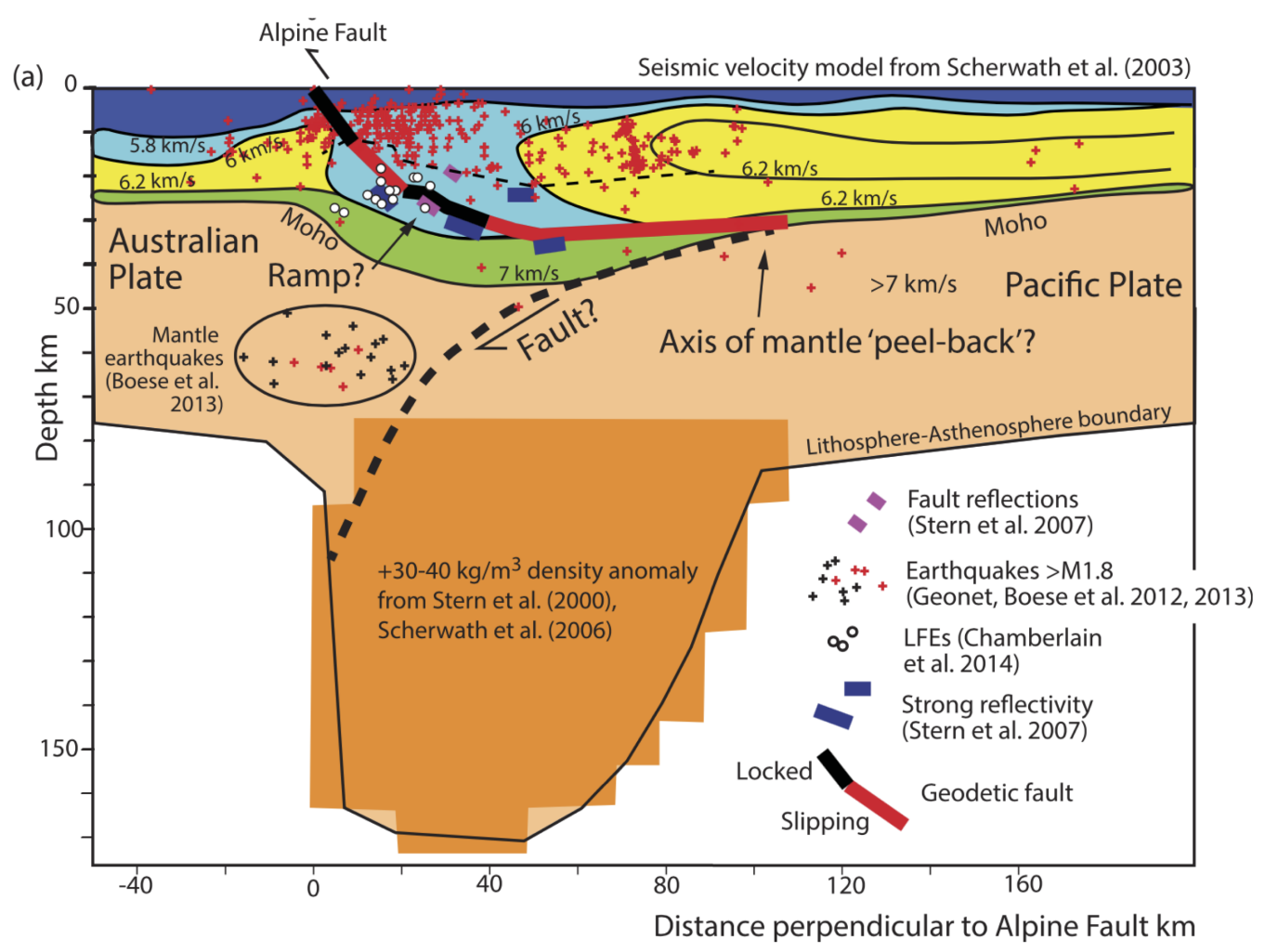

Figure 1.12: Lithospheric structure beneath the Southern Alps. After Lamb et al. (2015). Lithospheric and crustal structure through the Southern Alps based on a compilation of published seismic data combined with a new interpretation of 5-10 years of GPS measurements (Lamb et al., 2015, and references therein).

Eberhart-Phillips and Bannister (2002) used both SIGHT and SAPSE data to model in 3D the crustal structure in the Southern Alps and found that the crustal root was deepest $80 \mathrm{~km}$ south of Mount Cook and that it had an asymmetric shape. A broad negative Bouguer anomaly in the Southern Alps also shows evidence of an extensive crustal root under the Southern Alps. This gravity anomaly is larger than what would be expected from a crustal root balancing the Southern Alps isostatically, thus suggesting a deficit of mass in the mantle (Bourguignon et al., 2007) and the presence of a dense body in the upper mantle. Several models are proposed to explain the crustal thickening and discontinuities observed under the Southern Alps: for instance, Stern et al. (2000) suggested that the thickening might be due to a pull of thickened mantle lithosphere directly below the crustal root. According to Sutherland et al. (2000), overthrusting of ocean lithosphere beneath the southern and central Southern Alps might have caused a thickening of the crust which might be compatible with an oblique reverse fault continuously dipping to the south-east in the upper mantle. They also inferred that the 
lithospheric discontinuity was most likely controlled by an Eocene passive margin. Davey et al. (2007) proposed that the asymmetry in the crustal deformation might be linked either to an initial contrast in lithospheric rheology or to an inherited suture from previous plate motion (Fig. 1.12). They also suggested that the crustal root was formed of a thickened middle crust overlying a delaminated lower crust (resulting from the delamination of the Pacific plate).

Little is known with regards to the geometry of the Alpine Fault, a major continental transform fault, at depth. Several models for the deep structure of continental transform faults were summarised by Norris and Toy (2014). Generally, continental transforms accommodate a high proportion of intraplate slip either (1) on a single surface fault that becomes more or less diffuse with depth or (2) on a narrow zone of interlinked faults. Norris and Toy (2014)'s preferred model for the Alpine Fault is based on an interpretation by Koons et al. (2003), in which the Alpine Fault partitions at depth into a steep zone extending into the mantle with largely fault-parallel motion and a flat ductile décollement in the lower crust. They suggested that in its central part, the Alpine Fault extends through the upper crust (up to $10-12 \mathrm{~km}$ depth) from a narrow single frictional fault zone (corresponding to the seismogenic zone failing in $M w 7-8$ earthquakes every $\sim 300$ years) into a few-km-wide area of highly localised ductile shear within an otherwise broad zone of distributed deformation. They proposed that the shear zone flattens into a lower crustal detachment to the east, with a majority of the strike-slip component possibly becoming partitioned onto a steep shear area on the western side of the root. In other words, they proposed that the central Alpine Fault corresponds to a single east-dipping oblique structure in the upper crust and that at the base of the crust, the fault is partitioned between a westward steep ductile strike-slip fault and an eastward flat ductile detachment. Two examples of Alpine Fault geometries are shown in Fig.1.13: Norris and Toy (2014)'s preferred model as well as one proposed by Lamb et al. (2015).

By studying slow deformation and especially LFEs, we aim to provide new constraints on the central Southern Alps crustal structure and Alpine Fault geometry. Indeed, obtaining LFE hypocentre depths and focal mechanisms will help discern which geometry is more suitable for the deep central Alpine Fault. 


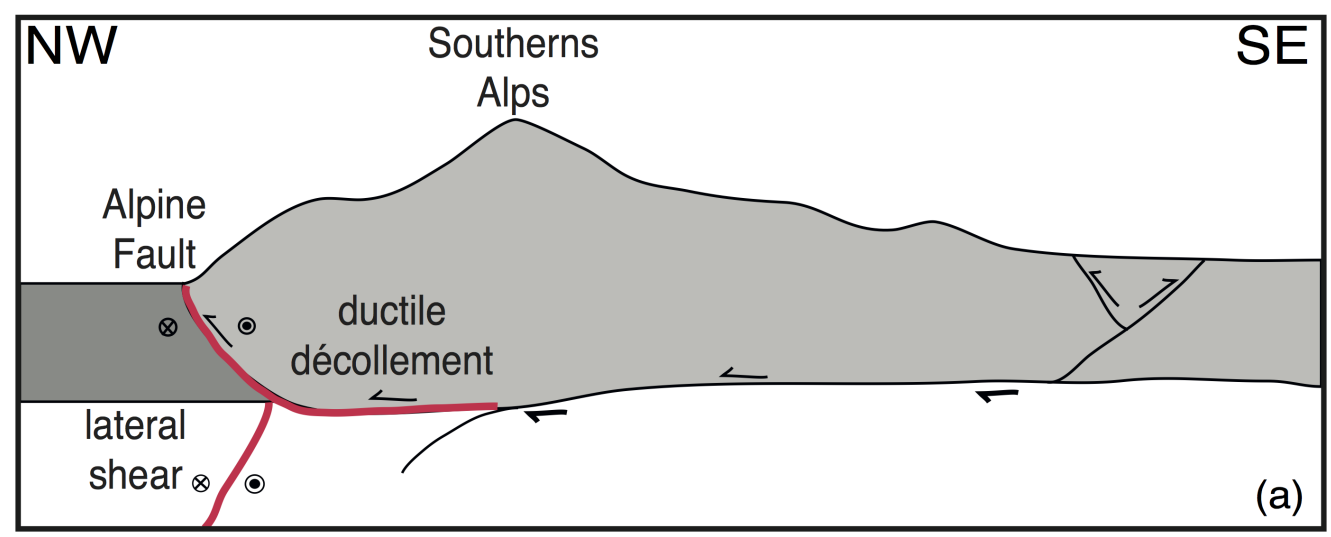

After Koons et al., 2003

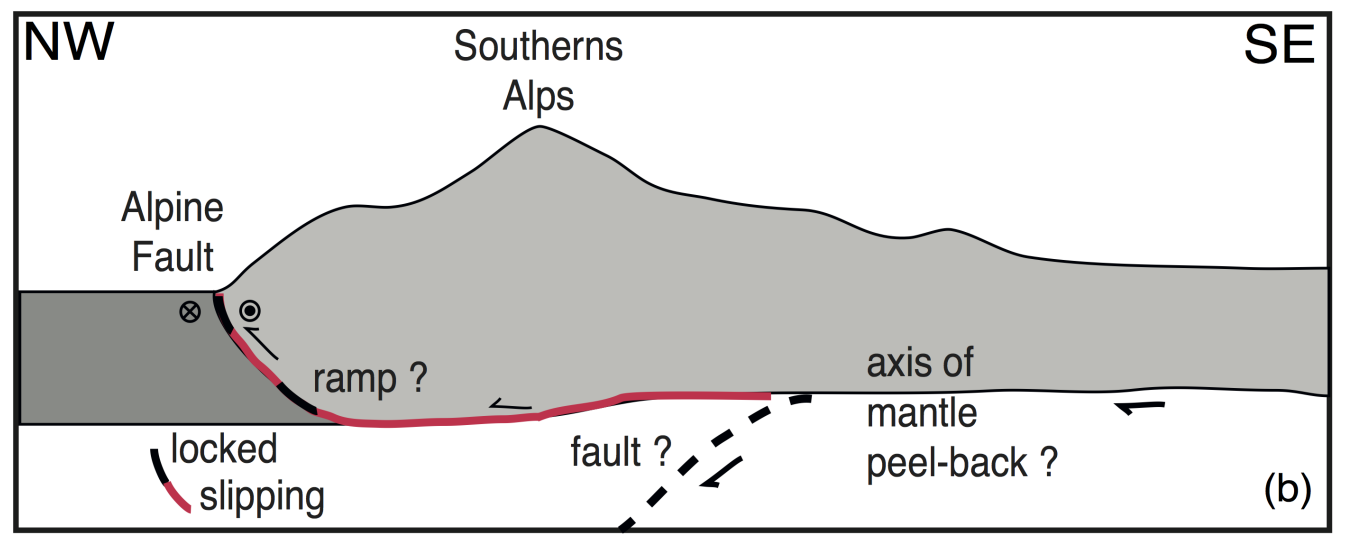

After Lamb et al., 2015

Figure 1.13: Alpine Fault possible geometries. Cartoons showing different models for the deep structure of continental transforms. To this day, the structure of the Alpine Fault in the lower crust is poorly constrained. Here we show two hypotheses for the geometry of the deep Alpine Fault. (a): This figure represents a model for obliquely convergent zones by Koons et al. (2003) which is the preferred model for the Alpine Fault according to Norris and Toy (2014). (b): Model of the Alpine Fault after Lamb et al. (2015)

\subsection{Thesis outline}

This Introduction Chapter is followed by a theoretical background in Chapter 2 that starts with an overview of the SAMBA station network and a description of the data processing. Techniques to efficiently detect and locate LFEs are then described in detail. Finally, we present a quick overview of how focal mechanisms and stress estimation are computed.

Chapter 3 discusses the finding from $\sim 7$ years of LFE detections, including locations, family characteristics, inter-event times and the first LFE focal mechanisms 
associated with continental faulting anywhere.

Chapter 4 complements this work by using an automated template generation method, allowing the creation of a spatially and temporally continuous 8-yearlong LFE catalogue for the central Southern Alps.

Chapter 5 builds on the previous Chapter by presenting a detailed study of the spatio-temporal characteristics of LFEs in the central Southern Alps.

These Chapters are connected throughout their study of slow deformation phenomena, but they all have their own introductions and methodologies, as the focus and techniques employed in each Chapter varied and as Chapters 3 and 4 have been written in the form of papers for publications. All the introductions and methodologies outlined in Chapters 3-5 are complementary to those of Chapters 1 and 2. Chapter 3 has already been published as a letter in Earth and Planetary Science Letters under Baratin et al. (2018), Focal mechanisms and inter-event times of low-frequency earthquakes reveal quasi-continuous deformation and triggered slow slip on the deep Alpine Fault. Chapter 4 is ready for submission to Geochemistry, Geophysics, Geosystems. As for Chapter 5, a slightly modified version of the chapter will be prepared for submission to Journal of Geophysical Research.

During the course of this work, I also contributed to the following papers (as part of the DFDP-2 project, described in more detail in Appendix B):

- Chamberlain et al. (2017a), Real-Time Earthquake Monitoring during the Second Phase of the Deep Fault Drilling Project, Alpine Fault, New Zealand, Seismological Research Letters;

- Sutherland et al. (2017), Extreme hydrothermal conditions at an active platebounding fault, Nature;

- Toy et al. (2017), Bedrock geology of DFDP-2B, central Alpine Fault, New Zealand, New Zealand Journal of Geology and Geophysics;

- Townend et al. (2017), Petrophysical, Geochemical, and Hydrological Evidence for Extensive Fracture-Mediated Fluid and Heat Transport in the Alpine Fault's Hanging-Wall Damage Zone, Geochemistry, Geophysics, Geosystems.

All of the work presented in this thesis is original work that has been conducted by me under the supervision of John Townend and Martha Savage, unless explicitly 
stated. All co-authors of Baratin et al. (2018) provided valuable feedback on the work described in Chapter 3, which ultimately led to its publication. The creation of synthetics described succinctly in Chapter 4 was conducted by Calum Chamberlain as part of his own thesis. However, the real low-frequency earthquakes templates used within the detection routine to generate the final LFE catalogue are my own work. William Frank provided some valuable feedback on the generation of the LFE continuous catalogue presented in Chapters 4 and 5. All the analysis in Chapter 5 was conducted solely by me, with editorial feedback from Martha Savage and John Townend. To be consistent with the format of Chapter 3 (published) and Chapter 4 (in the process of submission), I have chosen to write the rest of this thesis in the first person plural, which is also a reflection of the guidance that I received from my supervisors during the entirety of this project. 


\section{Research Methodology}

\subsection{Data acquisition}

\subsubsection{The Southern Alps Microearthquake Borehole Array}

In the central section of the Southern Alps, the New Zealand national seismograph network (GeoNet) provides sparse coverage with only three broadband stations spaced $80-100 \mathrm{~km}$ apart. In order to investigate the seismicity in the central section of the Southern Alps in detail, the Southern Alps Microearthquake Borehole Array (SAMBA) was installed in 2008 by Carolin Boese of VUW under the supervision of Tim Stern, John Townend and Euan Smith (Boese et al., 2012). The current geometry of the network (Table 2.1 and Fig. 2.1) allows for accurate hypocentre locations for events located within the network. Following the discovery of tremor south of the network by Wech et al. (2012), the array was extended to the south by Calum Chamberlain of VUW between 2013 and 2014 . The addition of three stations (SOLU, MTBA and LARB) somewhat filled the gap between the SAMBA and Central Otago Seismic Array (COSA, deployed and maintained by Emily Warren-Smith during her PhD at VUW under the supervision of Tim Stern and Simon Lamb) networks and will provide better constraints on tremor and LFE locations. The SAMBA network (Fig. 2.1) now comprises thirteen stations, all equipped with short-period seismometers. Of those thirteen stations, nine have their seismometers deployed in boreholes in order to improve signal-to-noise ratios. Additional details on the location and characteristics of each SAMBA site used in this project can be found in Table 2.1. 


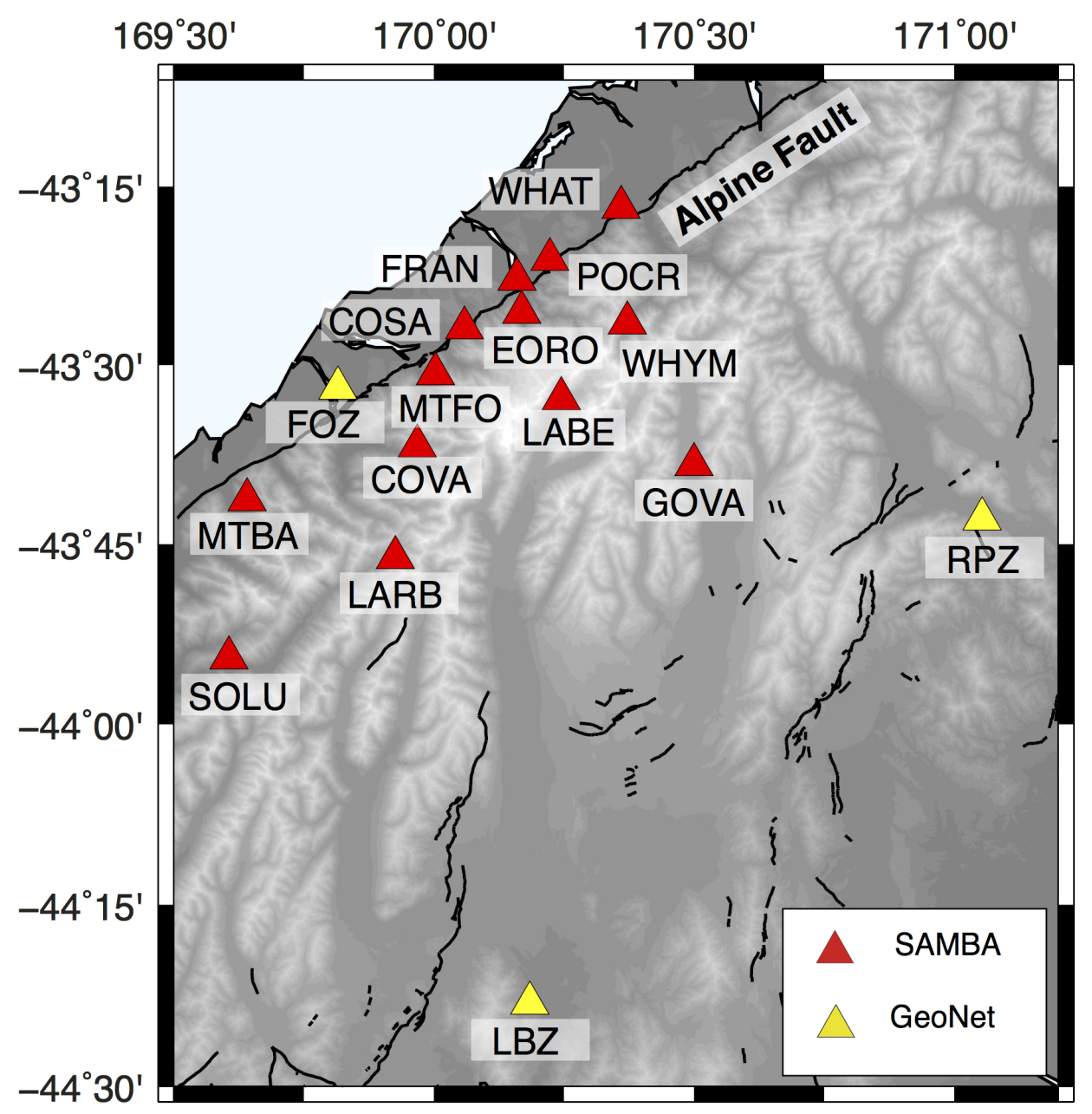

Figure 2.1: SAMBA network location map. Locations of the short-period SAMBA stations (red triangles) and the GeoNet broadband stations (yellow triangles) used in this study.

In addition to seismic data collected from the SAMBA network, we are using data of three broadband stations belonging to the GeoNet network (RPZ, LBZ, FOZ, yellow triangles in Fig. 2.1) and freely available on their website (http:// geonet.org.nz/). Those stations were included in the second part of this project (Chapter 4) in order to pinpoint the spatial extent of low-frequency earthquakes in the central Southern Alps. The entirety of the SAMBA data is now archived and available on request on the IRIS website (http://ds.iris.edu/ds/nodes/ $\mathrm{dmc} /$ data/\#requests). 


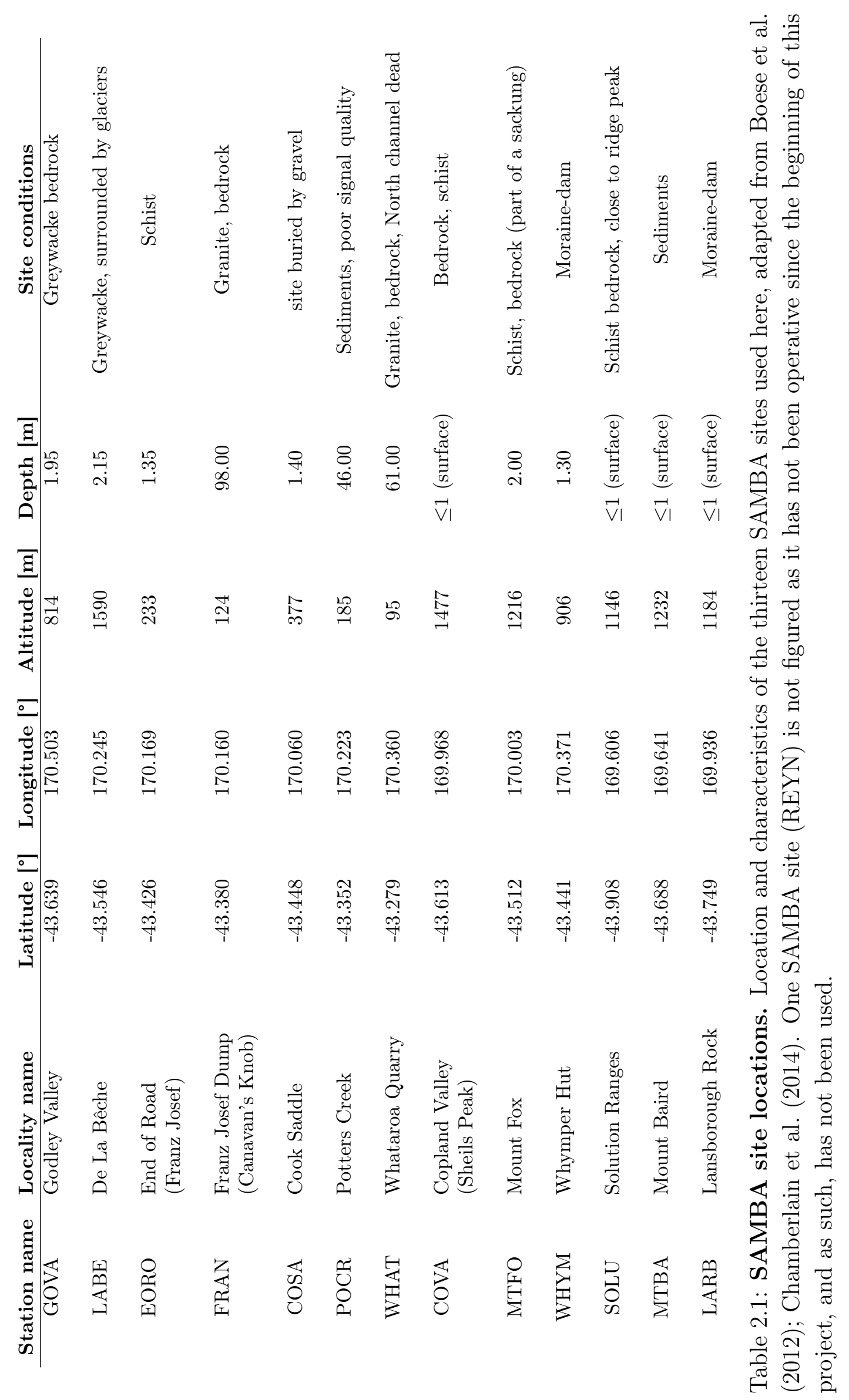




\subsubsection{Data collection and processing}

Most of the data presented here have been collected using the SAMBA network. A detail of the instrumentation deployed for each site can be seen on Table 2.2. The biannual maintenance and data collection of this network formed a large part of my project as I have personally been in charge of this network between March 2015 and March 2017. Additionally, five sensors were installed as part of the DeepFault Drilling Project, for which the routine maintenance and data collection was also done as part of this project's field trips. During our service trips, we were also in charge of collecting data from three Japanese seismometers, installed by our colleagues at the Earthquake Research Institute of Tokyo. In addition to those stations, those of the COSA network (supervised by Emily Warren-Smith) were added for joint maintenance while I was in charge of the SAMBA network. A detailed overview of the field reports can be viewed in Appendix C.

Data are recorded via RefTek RT-130 dataloggers on SD or RefTek cards (varying in size between 4-16 GB). Data are stored in a RefTek compression format, itself based on the miniseed Steim-2 format. The data files collected during this project were mainly hour-long files. During each field trip, in order to back-up the data and make sure that no major problems were detected at a particular station, several steps had to be followed:

- After collecting the flash disks from their respective sites, we transferred the data to a hard drive and we then converted the data to the miniseed format (data conversion was done using the Pascal tool: rt2ms, while still on the field);

- A quick check of the data was performed daily on the field using a different Pascal tool: logpeek;

- Once we were back from the field, the recently collected raw data were copied to a VUW server (Taranaki01), where they were stored by station and then by day; as for the miniseed files, they were copied to a different server (GeoPhysics09), stored in that case, by year then by day and finally by stations. 


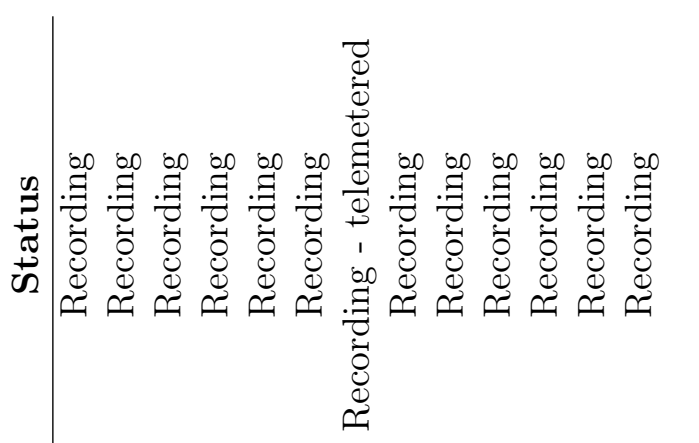

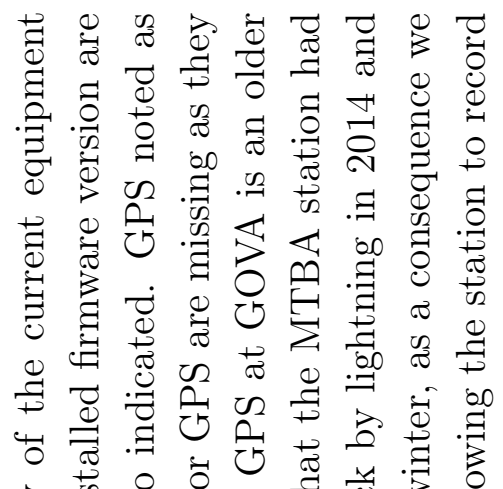

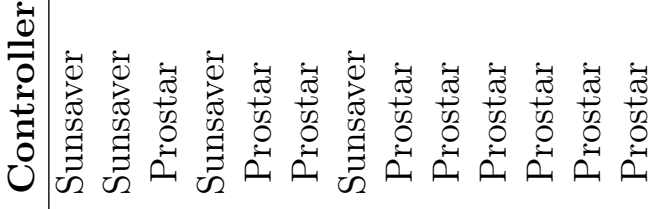

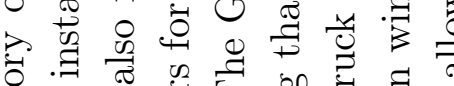

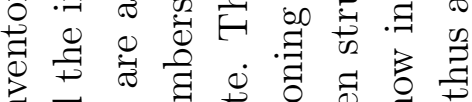

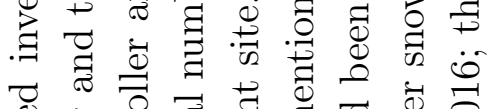

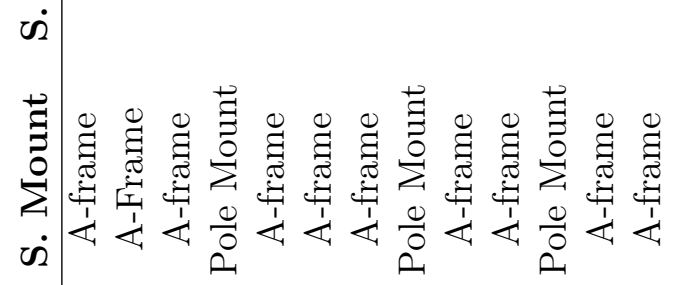

它

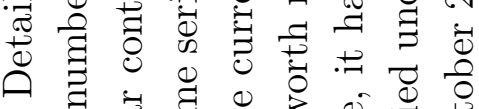

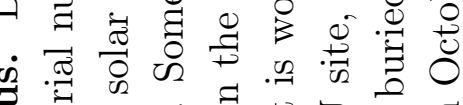

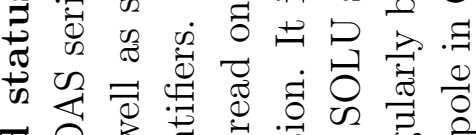

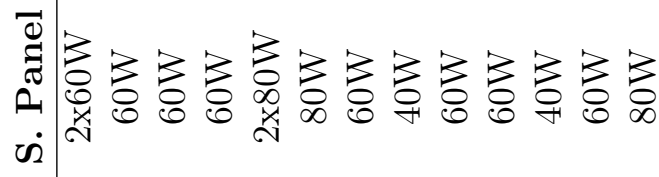

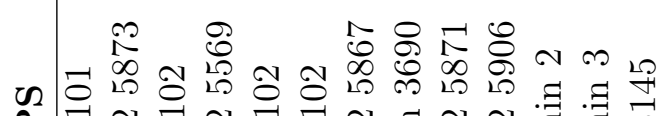

v m

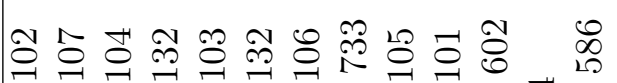

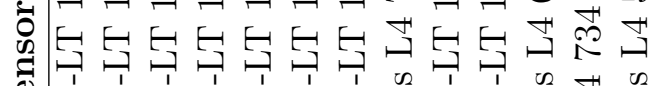

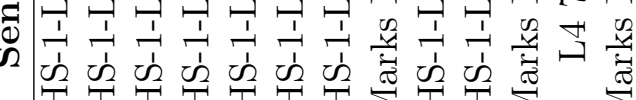

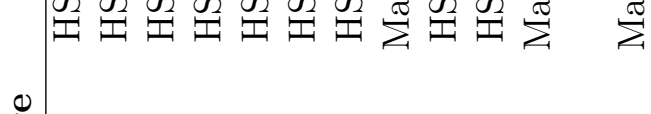

สี.

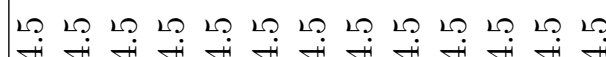

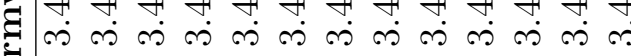

庄

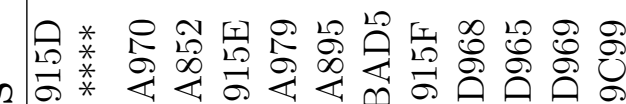

崔

ये

至

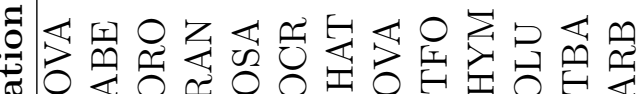

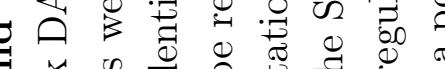

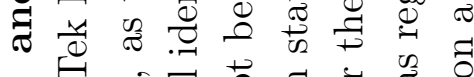

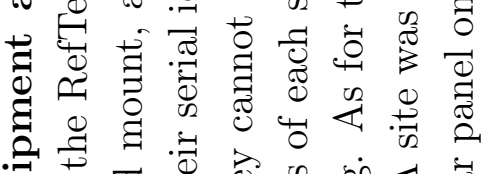

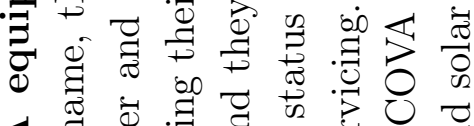

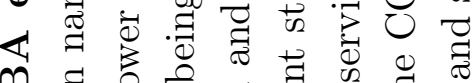

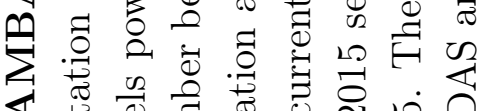

纤

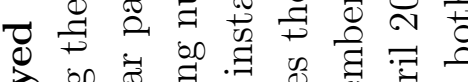

完

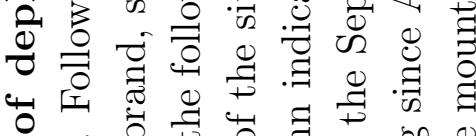

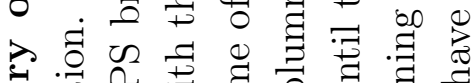

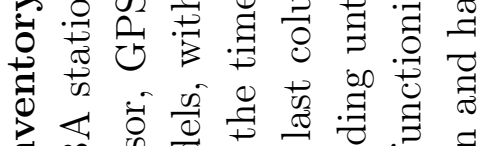
$\Xi \vartheta$

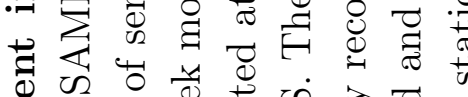

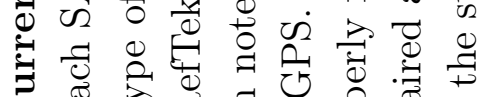
ป ठ) i

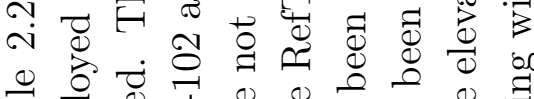

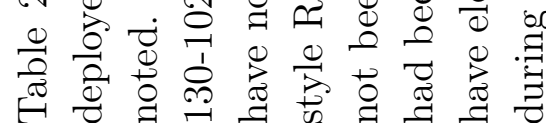




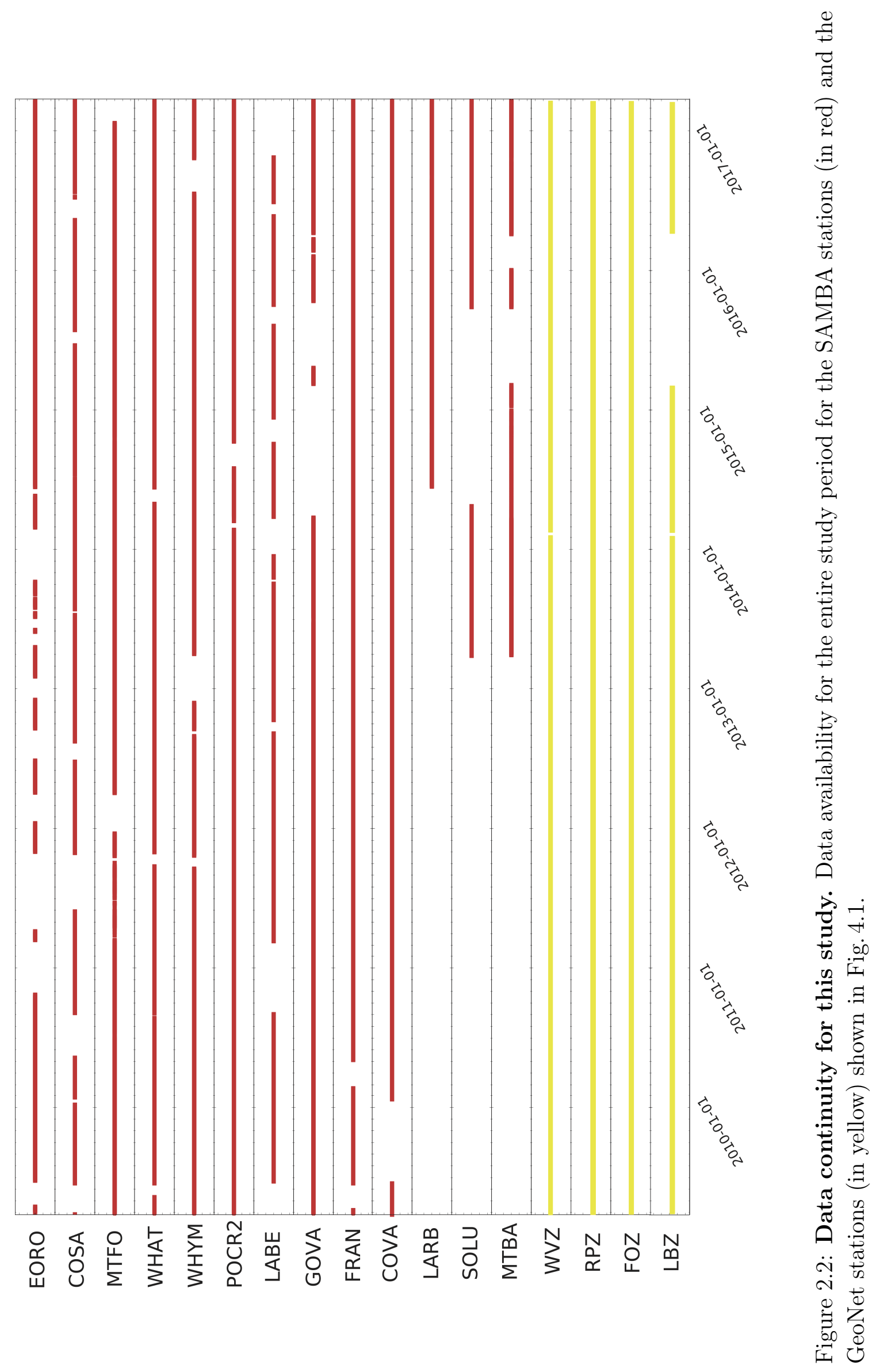


Here, we use data collected between March 2009 and March 2017 (the available data for this study is shown in Fig. 2.2 for each station) from the SAMBA and GeoNet networks to generate two LFE catalogues: (1) an extended LFE catalogue regrouping 14 LFE families, from March 2009 to October 2016 for which hypocentre locations and fault plane solutions were determined (Chapter 3); and (2) a new continuous catalogue (from March 2009 to March 2017), regrouping hundreds of LFE families, hence forming the first spatially and temporally continuous catalogue of LFEs for the central Southern Alps (Chapter 4).

\subsection{Low-frequency earthquakes detection}

\subsubsection{Matched-filter and cross-correlation}

A powerful operation in the analysis of time series (such as seismological data) is the correlation $C_{x y}(t)$ of two functions $x(t)$ and $y(t)$. If those two functions are identical, the correlation is termed 'auto-correlation' whereas if the operation is performed on two different functions, it is then called 'cross-correlation'. Crosscorrelation in the time domain describes the process of: (1) shifting the function $\mathrm{y}(\tau)$ relative to $\mathrm{x}(\tau)$ by $\mathrm{t}$; (2) multiplying those two functions; and finally (3) integrating their product as seen on equation 2.1 .

$$
C_{x y}(t)=\int_{-\infty}^{+\infty} x(\tau) y(\tau+t) \mathrm{d} \tau
$$

A normalised cross-correlation coefficient $C_{x y}^{N}(t)$ is obtained by normalising the cross-correlation function by the square-root of the two auto-correlation functions. This coefficient is a direct measure of similarity between two time series. The closer this coefficient is to 0 , the more dissimilar the functions are. If $C_{x y}^{N}(t)=1$, then the functions are identical and if $C_{x y}^{N}(t)=-1$, the functions are of opposite polarity but otherwise identical.

$$
C_{x y}^{N}(t)=\frac{\int_{-\infty}^{+\infty} x(\tau) y(\tau+t) \mathrm{d} \tau}{\sqrt{C_{x x}(t) C_{y y}(t)}}
$$

Here, we utilise cross-correlation as a tool to extract a weak signal from noisy data. An overview of the different steps for a matched-filter technique is shown in Fig. 2.3. Firstly, we use the waveforms of fourteen LFEs identified by Cham- 
berlain et al. (2014) in the Southern Alps and in a second time, the waveforms of 638 synthetic LFEs, as 'primary templates' in a matched-filter technique similar to the one used by Shelly et al. (2007). This technique allows us to search within a continuous dataset for similar signals. For this project, each template has been recorded at a minimum of 5 stations (Chamberlain et al., 2014). The cross-correlation coefficients are summed for these stations in order to produce a network cross-correlation sum (ccsum). The cross-correlation sum serves as a detection statistic: an LFE is detected when the ccsum exceeds a threshold value, determined by synthetic testing, of eight times the Median Absolute Deviation (MAD) (Shelly et al., 2007). Following the initial detection run, the best $20 \%$ of detections are stacked (see section 2.2.2) to generate a new template with a higher signal-to-noise ratio. This new template is in turn used in the stacking and network cross-correlation routine and this process is iterated until the signal-tonoise ratio of our signal is deemed sufficient to allow reliable picking of polarities and arrival times on the waveforms. This technique creates, for each primary template, an LFE family with similar locations and focal mechanisms.

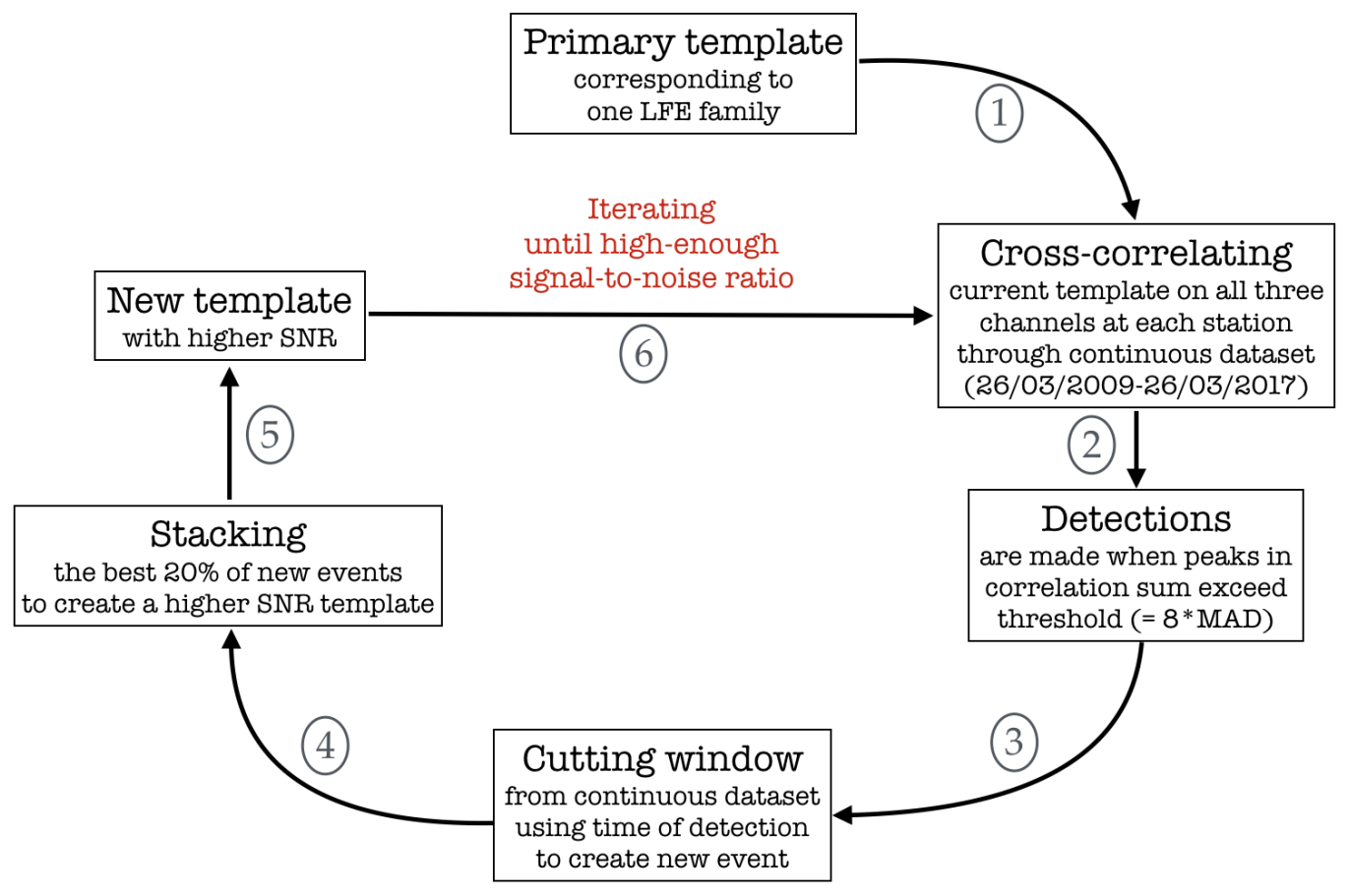

Figure 2.3: Steps overview for running a matched-filter routine. Description of the steps needed for the first run of matched-filtering. 


\subsubsection{Phase-Weighted Stacking}

The majority of location methods are based on the distinctive impulsive character of an earthquake wave arrival. An inherent obstacle in detecting and locating LFEs is the emergent character of their wave arrivals (Shelly et al., 2007). Here, we use phase-weighted stacking (PWS) to deal with the emergent character of LFE wave arrivals and to improve the extraction of signal from ambient noise (Schimmel and Paulssen, 1997; Thurber et al., 2014). PWS involves the weighting of the linear stack with a time-dependent measure of phase coherency (Schimmel and Paulssen, 1997). Weak phases can only be detected by their coherent appearance on different traces (Schimmel and Gallart, 2007). Therefore PWS should be an effective technique for picking LFE arrivals.

The PWS is calculated using the Hilbert transform to give an analytic signal (i.e. complex signal). In complex trace analysis, an analytic signal $S_{j}(t)$ combines a real part corresponding to each seismic trace $s_{j}(t)$ and an imaginary part equal to its Hilbert transform $H_{j}(s(t))$. This complex trace can also be rewritten in terms of time-dependent amplitude $A_{j}(t)$ and instantaneous phase $\phi_{j}(t)$. Index $j$ in equations (2.3) to (2.6) refers to the number of traces used $N$.

$$
S_{j}(t)=s_{j}(t)+i H\left(s_{j}(t)\right)=A_{j}(t) \mathrm{e}^{i \phi_{j}(t)}
$$

Stacking is an important procedure in seismic data processing as it can enhance the signal-to-noise ratio. We obtain the linear stack $g_{l s}(t)$ by taking the real part of the sum of the analytic traces. The linear stack can be computed as an average of the $N$ traces $s_{j}(t)$ and is obtained by the following equation:

$$
g_{l s}(t)=\frac{1}{N} \sum_{j=1}^{N} s_{j}(t)
$$

PWS achieves a much higher signal-to-noise ratio than the equivalent linear stack as it suppresses incoherent signals in the stack (Thurber et al., 2014). PWS involves the weighting of the linear stack with a time-dependent measure of coherency known as the phase stack $C(t)$. The phase stack described in equation (2.5) is a direct measure of instantaneous phase coherency between multiple signals and acts as a filter on the linear stack. The amplitudes of the phase stack range between 0 and 1 (Figure 2.4). The instantaneous phases of the signals are coherent when the amplitude equals to 1 . An amplitude of 0 means that the signals summed up completely destructively (Schimmel and Gallart, 2007). 
A

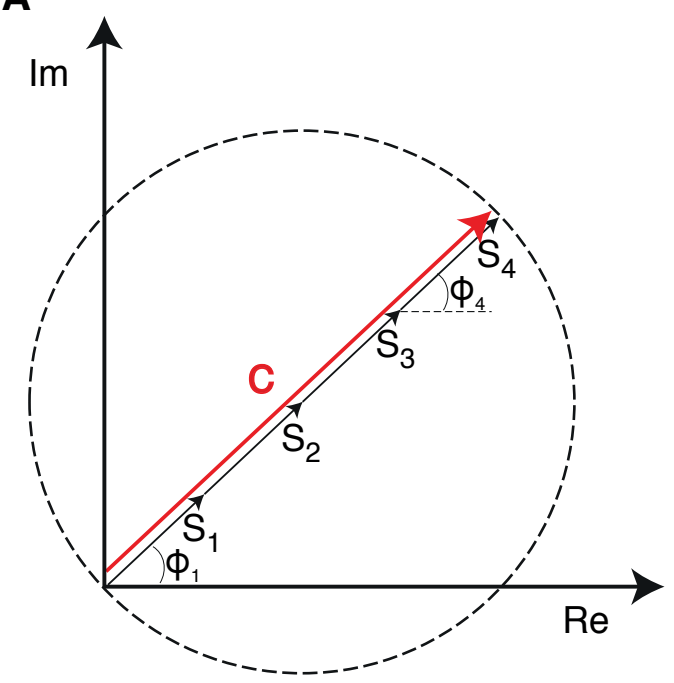

B

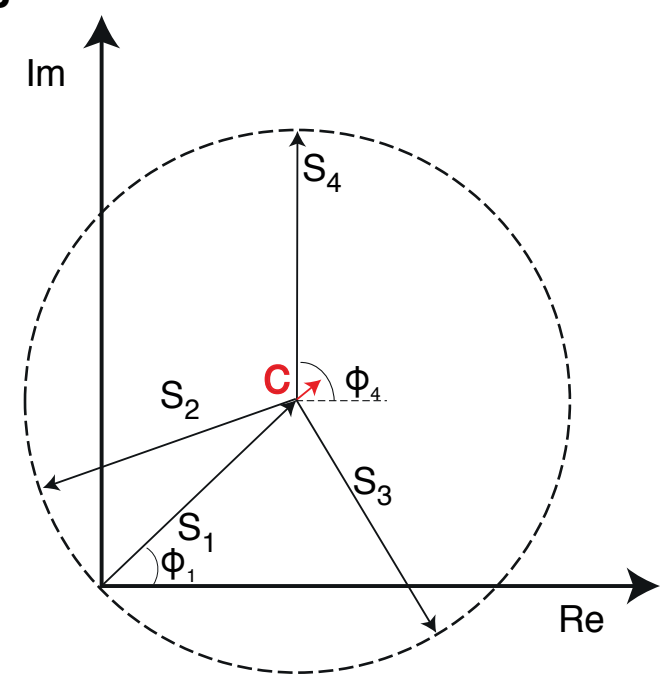

Figure 2.4: Phase stack illustration. Illustration of the summation of four analytic traces in the complex plan after Schimmel and Gallart (2007) and Thurber et al. (2014). A : Summation of four signals with the same instantaneous phase showing that their phase sum is equal to 4 and the subsequent phase stack $\mathrm{C}$ is equal to 1. B : Summation of four signals with instantaneous phase varying randomly showing that phase stack is close to 0 in that case.

$$
C(t)=\frac{1}{N}\left|\sum_{j=1}^{N} \mathrm{e}^{i \phi_{j}(t)}\right|
$$

The weight of the phase stack is controlled by the phase exponent $\nu$. A value of $\nu=2$ is commonly used in seismology (e.g. Schimmel and Gallart, 2007; Thurber et al., 2014) to remove the incoherent noise in the PWS: $g_{P W S}(t)$.

$$
g_{P W S}(t)=g_{l s}(t) C^{\nu}(t)=\frac{1}{N} \sum_{j=1}^{N} s_{j}(t)\left|\frac{1}{N} \sum_{j=1}^{N} \mathrm{e}^{i \phi_{j}(t)}\right|^{\nu}
$$

During this project, PWS is used for the final iteration of the network crosscorrelation to improve the template quality. We use PWS only in the last iteration of the matched-filter routine as we found using linear stacking within matchedfilter iterations to produce more stable LFE stacks. This technique generates a clearer signal than linear stacking or unstacked data and allows the picking of wave arrivals in order to determine reliable locations, focal mechanism solutions and stress orientation for our LFE catalogues. 

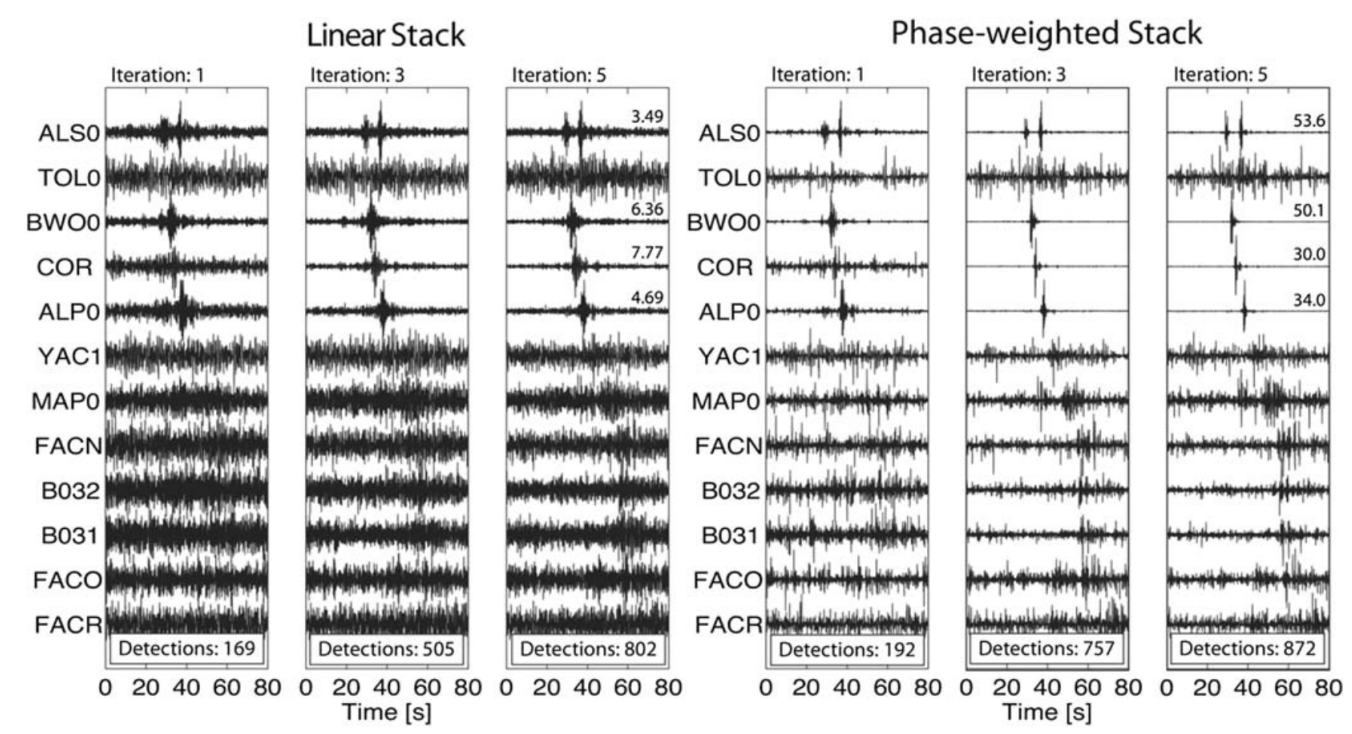

Figure 2.5: Comparison between linear and phase-weighted stacks. From Thurber et al. (2014). LFE template evolution during five iterations of the matched-filter routine using linear stacking (left) and PWS (right). Each panel shows the horizontal component of the seismogram for stations located in central Oregon. We notice here that the use of PWS results in a cleaner template with higher signal-to-noise ratios than the linear stack.

\subsubsection{Synthetic templates generation}

We use the method developed by Chamberlain (2016) for swarms and repeating seismicity based on the simulation of a real earthquake waveform by convolving assumed source properties with an appropriate Green's function and we apply this method to low-frequency earthquakes. This way, a series of 'naive' synthetics (i.e. extremely simple approximation of a real low-frequency earthquake) are created.

We initially depict the LFE seismic waveform as a single alternating-polarity spike corresponding to the $\mathrm{P}$ arrival and a series of alternating-polarity spikes representing the S-arrival and its coda. The S-P time is set using the 3D velocity model of Eberhart-Phillips et al. (2008) (described in more details below). We then convolve the spiked time-series with a $50 \mathrm{~Hz}$ decaying sine wave. We chose this value as it represents the Nyquist frequency of the lowest sampling-rate site used in the network. This process generates a high-frequency signal that is then filtered in the $2-8 \mathrm{~Hz}$ frequency band which corresponds to the dominant frequency content of tremor and LFE signals in the central Southern Alps (Wech et al., 2012; Chamberlain et al., 2014; Baratin et al., 2018). We then cut a 6-s window around the S-arrival to imitate the original LFE templates of Chamberlain et al. (2014) 
and to keep the synthetics as close as possible to the original real LFE templates. This process is then repeated to generate a multi-component template. The most important characteristic of this synthetic template is its lack of similarity with the ambient noise. Here, we capitalise on seeking a signal that not only matches the synthetic, but also that is different from the background noise.

In order to detect LFEs originating from diverse source locations, a series of templates is needed. Here, the synthetic templates have been generated for a predefined set of known sources situated within our area of interest. The source region of LFEs for the central Southern Alps is relatively well known and has been shown to be anti-correlated in depth with shallow seismicity (Wech et al., 2012; Chamberlain et al., 2014; Baratin et al., 2018). Here, we select a grid encompassing the LFE and tremor source region (cyan box in Fig. 4.1) that extends from 16 to $44 \mathrm{~km}$ depth, with a node spacing of $2 \mathrm{~km}$. For each node in the grid, travel-times have been calculated at all stations using the 3D velocity model of EberhartPhillips et al. (2008) and the NonLinLoc ray-tracing algorithm of Lomax et al. (2000). This process yields 638 synthetic templates.

\subsubsection{Computational implementation}

To use the matched-filter routine explained in Section 2.2.1, the python package EQcorrscan has been used (Chamberlain et al., 2017c). EQcorrscan is an open-source Python package (largely based on ObsPy routines) developed for the detection and analysis of repeating seismicity. The package's goal is to provide a reliable and well-tested tool for large-scale earthquake detection and analysis routines. In essence, EQcorrscan rests upon efficient matched-filter routines where detection of new earthquakes can be carried out on servers and super-computers.

The step that generally takes most time in a matched-filter run is the calculation of many discrete time-step cross-correlations. When dealing with a large dataset (for example several years) and a large number of templates (>20), parallel computation has to be implemented for time efficiency reasons. In order to optimise our running time, we have used the New Zealand eScience Infrasctructure (NeSi) high perfomance computer cluster: PAN. PAN possess $\sim 400$ nodes, the majority of which have 16 cores and $128 \mathrm{~GB}$ of RAM. This allows the computation of the full 8 years of data, corresponding to $\sim 5,000$ hours of computation, in less than 14 hours when distributed across 48 nodes with 100 templates. Using PAN and its ability to generate a full catalogue in a couple of days (requiring several iterations of a matched-filter run), we were able to test a wide range of parameters 
(e.g. number of detections kept for stacking) and to check the evolution of the catalogue between iteration (e.g. looking at individual detections and their frequency content). Those tests were crucial in helping us obtain high signal-to-noise stacks and were not entirely possible with the previous Matlab codes that were initially used for this project (the results from that work are detailed in Appendix A.1).

\subsection{LFE locations}

The parameters commonly used to describe an earthquake are hypocentre coordinates (geographic latitude and longitude of epicentre, and focal depth), origin time and energy release. Those parameters are essential as they reflect the earthquake source properties which in our case correspond to the motion of faults. The hypocentre coordinates refer to a point on the fault where the rupture starts and the origin time relates to the time of initiation of rupture. Hereafter, the term 'earthquake location' will refer to the process of determining the hypocentre parameters (3D spatial location and origin time).

Earthquake location involves (1) the forward problem of computing theoretical travel-times from a given velocity model and (2) the inverse problem of determining the unknown hypocentre parameters.

\subsubsection{Linear earthquake locations}

The accurate location of earthquakes is of critical concern to this project. If we assume a simple homogeneous half-space, with a constant wave speed $v$, the earthquake wave arrival time $t$ at the $i^{\text {th }}$ recording station can be calculated as followed:

$$
t_{i}=T+\frac{1}{v} \sqrt{\left(X-x_{i}\right)^{2}+\left(Y-y_{i}\right)^{2}+\left(Z-z_{i}\right)^{2}}
$$

with $T$ being the origin time and $(X, Y, Z)$ the hypocentre coordinates in the Cartesian coordinate system. This forward calculation can not only be performed by assuming an initial hypocentre $\left(X_{0}, Y_{0}, Z_{0}, T_{0}\right)$ but also by calculating the travel times at all stations for all possible hypocentre parameters. The latter is achieved by gridding a specific region and by calculating the travel times at each grid node.

The calculated arrival time is then compared with the observed arrival time at each station in order to determine the true hypocentre coordinates. The best location is the one that minimises the arrival time residuals $r_{i}$ (i.e the differences 
between observed and calculated arrival times):

$$
r_{i}=t_{o b s}^{i}-t_{c a l c}^{i}
$$

The basis of numerical methods for hypocentre determination consists in the linearisation of equations depicting arrival times and their solutions using leastsquare methods (Geiger, 1910). Geiger's location method uses the inverse approach of the above forward problem. The arrival times are considered as nonlinear functions of the station coordinates and of the hypocentre parameters. The problem is linearised using a first-order Taylor series about a set of initial hypocentre parameters $\left(X_{0}, Y_{0}, Z_{0}, T_{0}\right)$ assumed to approximate the true hypocentre. If the initial hypocentre estimate is close to the actual hypocentre, the arrival time residuals $r_{i}$ can be expressed as followed :

$$
r_{i}=\frac{\delta t_{i}}{\delta X} \Delta x+\frac{\delta t_{i}}{\delta Y} \Delta y+\frac{\delta t_{i}}{\delta Z} \Delta z+\Delta t_{0}
$$

where $t_{0}$ is the origin time, $\frac{\delta t_{i}}{\delta X}, \frac{\delta t_{i}}{\delta Y}, \frac{\delta t_{i}}{\delta Z}$ are the partial derivatives of the travel time for station $i$ with respect to the Cartesian coordinates of the hypocentre $(x, y, z)$ and finally, the term $\Delta$ describes the correction needed to adjust the hypocentre model. The first order partial derivatives are the following :

$$
\begin{aligned}
\frac{\delta t_{i}}{\delta X} & =\frac{X-x_{i}}{v S} \\
\frac{\delta t_{i}}{\delta Y} & =\frac{Y-y_{i}}{v S} \\
\frac{\delta t_{i}}{\delta Z} & =\frac{Z-z_{i}}{v S}
\end{aligned}
$$

where all terms are the same as for equations (2.7) and (2.9) and $S$ is the path length. At least four observations of arrival times are required to constrain the hypocentre parameters. Often more than four stations record an earthquake, thus the linear system is overdetermined, leading to a decrease in earthquake location uncertainties.

This linear approximation is reasonable for weakly nonlinear problems where all variables are independent (Tarantola and Valette, 1982). This is the case when stations are close to the earthquake hypocentre and when slowly varying seismic velocities are observed between the hypocentre and the stations. However if the stations are too far away from the hypocentre, the observations are not sufficient to fully constrain the spatial location meaning that the linear inversion becomes 
unstable. The reason for this instability lies in the fact that the least-squares technique requires all variables to be independent while hypocentre locations and origin times are co-dependent.

We use this linear approach in order to get initial locations of low-frequency earthquakes within the SAMBA network. Keeping in mind that the velocity is rarely constant within the Earth, we require a known velocity structure for our study area. We use two different velocity models for the Southern Alps: (1) the 1D velocity structure of O'Keefe (2008) and (2) a recently available 3D velocity structure (personal communication with Boese). The preliminary locations are obtained with the SEISAN software (Havskov and Ottemoller, 1999).

\subsubsection{Non linear earthquake locations}

In order to produce a more stable earthquake location for distant hypocentres, we include the second order partial differential in equation (2.9) in the style of Thurber (1985). The second derivative terms can be calculated analytically as follows (with similar terms for $\mathrm{X}$ and $\mathrm{Y}$ ):

$$
\begin{aligned}
& \frac{\delta^{2} t}{\delta Z^{2}}=\frac{1}{v S}\left[1-\frac{\left(Z-z_{i}\right)^{2}}{S^{2}}\right] \\
& \frac{\delta^{2} t}{\delta Y \delta Z}=-\frac{\left(Y-y_{i}\right)\left(Z-z_{i}\right)}{v S^{3}} \\
& \frac{\delta^{2} t}{\delta X \delta Z}=-\frac{\left(X-x_{i}\right)\left(Z-z_{i}\right)}{v S^{3}}
\end{aligned}
$$

It is now clear that the linear terms do not always dominate. Specifically, in the case of a shallow source, when the source and receiver depths are equal $\left(Z=z_{i}\right)$ the first order partial derivative $\frac{\delta t}{\delta Z}$ vanishes while the second order term $\frac{\delta^{2} t}{\delta Z^{2}}$ is maximised (Thurber, 1985). The increased stability in the nonlinear location method relies on the greater sensitivity of the second order term at shallow depth.

Describing accurately hypocentre locations and understanding their uncertainties (e.g. event-station geometry, arrival-time errors) are primary concerns in this study. To further increase the location method stability, the nonlinear method described above can be improved by adding a probabilistic hypocentre uncertainy represented by a probability density function over the unknown hypocentre parameters (Tarantola and Valette, 1982). This uncertainty takes the form of a Posterior Density Function (PDF) that can be depicted as a volume in space. The PDF solutions can then be plotted and the minimum misfit hypocentre (i.e. the 
optimal hypocentre) corresponds to the point of maximum likelihood in the PDF solutions.

After having obtained initial linear locations as described in section 2.3.1, LFE events are relocated using the NonLinLoc algorithm (Lomax et al., 2000) based on the probabilistic nonlinear inversion method of Tarantola and Valette (1982). The NonLinLoc algorithm produces an estimate of the PDF for each hypocentre for each cell on a grid by (1) generating a synthetic travel time grid for each source and by (2) comparing the phase arrival picks to the grid travel time using a Metropolis-Gibbs approach (Metropolis et al., 1953). Gaussian uncertainties are given for the optimal hypocentre in the form of a $68 \%$ spatial confidence ellipsoid (Lomax et al., 2000).

\subsection{Focal mechanisms and stress estimate}

\subsubsection{Focal mechanisms}

The focal mechanism describes the geometry of faulting during an earthquake. More precisely, it describes the orientation of the fault and the slip on the fault relative to a geographical coordinate system. Focal mechanisms use the characteristic radiation patterns of seismic energy that form $\mathrm{P}$ and $\mathrm{T}$ quadrants. The polarity and amplitude of motion of a seismic wave depend on the position of the station with regards to the earthquake source. During an earthquake, the surrounding seismic stations record an initial 'up' or 'down' motion, respectively corresponding to the $\mathrm{P}$ and T quadrants, referred to as the 'first motion'. The separation between quadrants occurs along two planes, known as the nodal planes: the fault plane and the auxiliary plane, orthogonal to the fault plane. In order to easily describe in $2 \mathrm{D}$ the geometry of a fault, the concept of focal sphere is used. A focal sphere results from the projection of the two nodal planes onto a lower-sphere, creating what is called a 'beach ball' diagram. The beach ball appearance provides enough information to determine whether a fault is normal, reverse, strike-slip or a combination of mechanisms, e.g. oblique reverse (Fig. 2.6). In order to resolve which one of the nodal planes is the fault plane, additional geologic or geodetic information is needed as simple earthquake first motions are not sufficient (Stein and Wysession, 2009).

Only one of the nodal planes is actually needed to fully determine a focal mechanism as the remaining nodal plane is orthogonal to the known nodal plane. All 

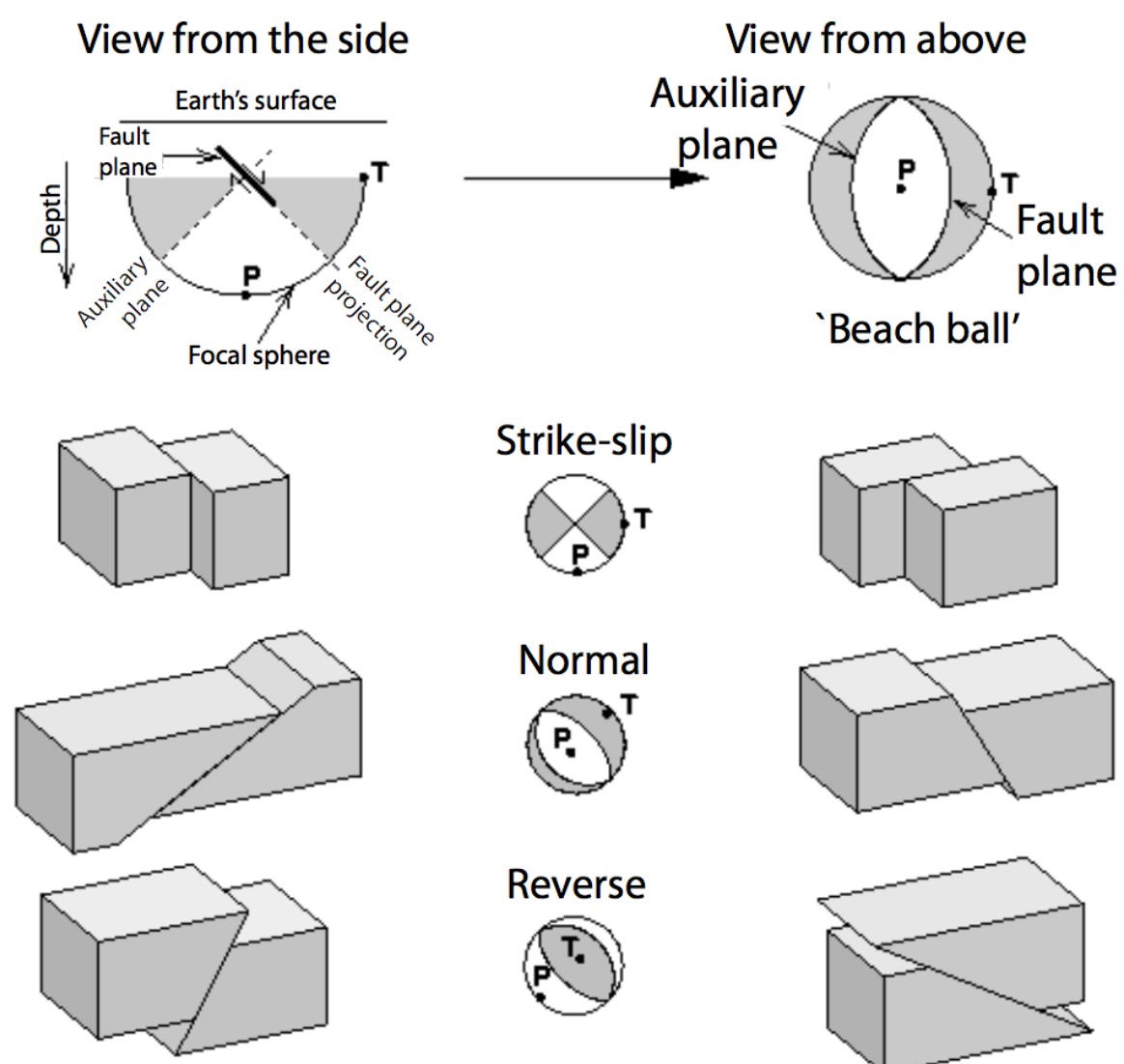

Reverse

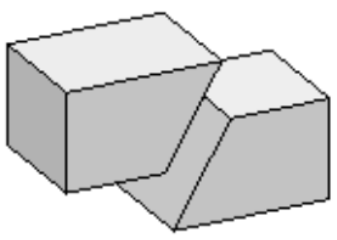

\section{Oblique reverse}
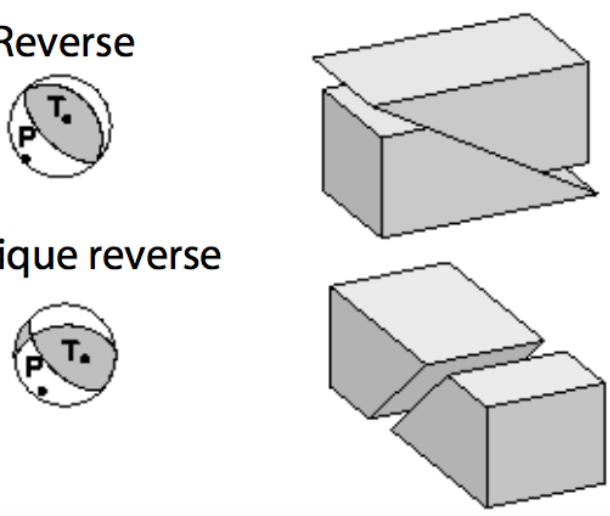

Figure 2.6: Example of focal mechanisms. Beach ball diagrams for the three main mechanisms, (strike-slip, reverse or thrust and normal) as well as an intermediate value (oblique reverse) are presented here. After a diagram by USGS, 1996.

focal mechanism values in this thesis refer to either the fault plane or its auxiliary plane. No specific technique was used in the process of selecting one nodal plane over the other. To define the fault plane, we employ the convention of Aki and Richards (2002) that uses the term strike, dip and rake, defined as follows:

- The strike angle describes the direction of the fault with respect to True North. It is measured from True North, clockwise, and ranges from 0 to $360^{\circ}$;

- The dip angle describes the inclination of the fault plane from the horizontal and it ranges from 0 to $90^{\circ}$. A dip of $0^{\circ}$ corresponds to an horizontal plane 
and a dip of $90^{\circ}$ to a vertical one;

- The rake angle describes the relative motion of the hanging wall with respect to the footwall and its value ranges between $-180^{\circ}$ and $180^{\circ}$. It is measured on the plane fault, from the strike direction. Negative values correspond to a downward motion of the hanging wall (normal faulting in Fig. 2.6) and positive values indicate an upward motion of the hanging wall (reverse faulting in Fig. 2.6).

We compute focal mechanisms using the P-wave first motions (upward motion in red and downward motion in blue in Fig. 2.7) of the LFE stacked waveforms following the Bayesian approach of Walsh et al. (2009). This method accommodates uncertainties in hypocentre location, velocity model, and P-wave polarities. For each event, the joint posterior probability density function of the three focal mechanism parameters (strike, dip, and rake) is calculated (e.g. Fig. 2.7). This distribution is then approximated using Matrix Fisher distributions which are in turn used to determine the standard deviation of the errors in strike, dip and rake. These errors are then used to parametrise the probability density contours of the $\mathrm{P}$ (orange in Fig. 2.7) and T (green in Fig. 2.7) axes.
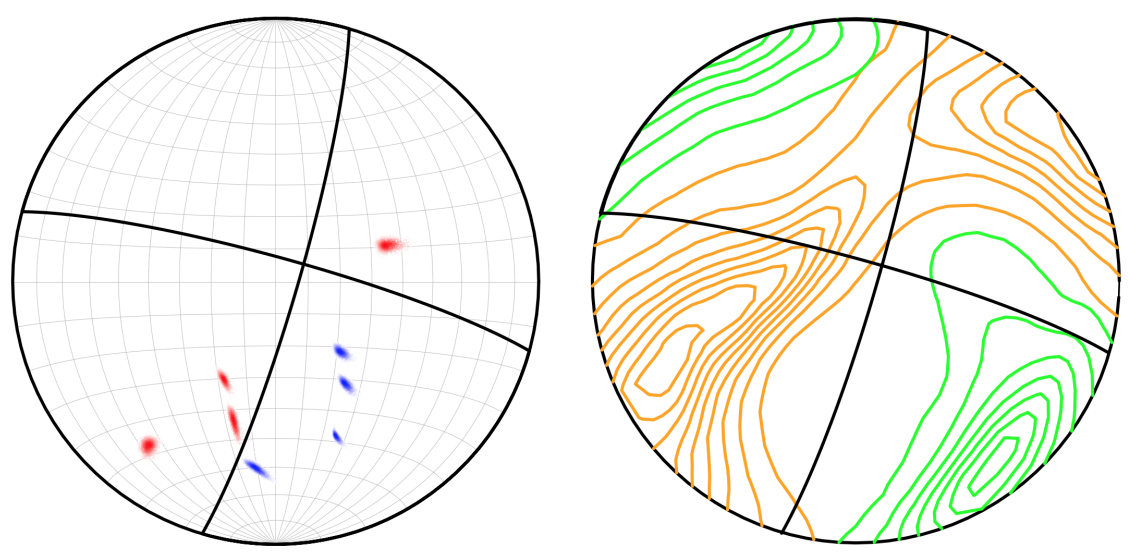

Figure 2.7: Example of a focal mechanism computation output for this study. Focal mechanism of a low-frequency earthquake and P and T-axes computed using the Bayesian approach of Walsh et al. (2009). Left: Polarity picks plotted with their uncertainty scatter obtained from the non-linear location of a low-frequency earthquake. Red stations indicate a downward first motion and blue stations indicate an upward first motion. Right: Pressure (P) and Tension (T) axes with their uncertainty contours, plotted respectively in orange and green.

The overall reliability of a focal mechanism depends on several factors:

- The number of first motions picked at the available seismic stations; 
- The number of available seismic stations as well as their distribution around an earthquake source;

- Systematic errors in first motion picks, resulting from wrongly wired field equipment (e.g. at the COVA and WHAT stations of the SAMBA network, the polarities are reversed on the seismograms);

- An earthquake hypocentre location and takeoff angles. Inaccurate hypocentre locations can be caused, for example, by errors in velocity model or poorly picked events.

\subsubsection{Stress inversion}

Stress acting on rocks is characterised by a symmetric $3 * 3$ tensor with six independent values. Stress can also be described by three orthogonal principal stresses: $\sigma_{1}$ (maximum); $\sigma_{2}$ (intermediate) and $\sigma_{3}$ (minimum). In most failure criterion (i.e Mohr-Coulomb) for brittle failure in homogeneous rocks, $\sigma_{2}$ lies in the fault plane and slip occurs in the direction orthogonal to $\sigma_{2}$, that is to say that slip occurs at the intersection of the fault plane with the plane containing $\sigma_{1}$ and $\sigma_{3}$ (Ranalli, 1995).

To predict the direction of slip on pre-existing fault planes, the Wallace-Bott hypothesis is generally used (Wallace, 1951; Bott, 1959). This hypothesis states that a fault will slip parallel to the direction of the maximum resolved shear stress in the fault plane. For a comparable ambient stress field, different fault planes will have different resolved shear stress directions. By using numerous fault planes, randomly oriented and independent of one another, we can constrain the orientation of the principal stress axis by calculating what is called the misfit angle. The misfit angle corresponds to the difference between the fault slip vector and the direction of maximum resolved shear stress in the fault plane and it is a value estimated by many modern stress inversion algorithms (e.g. Gephart and Forsyth, 1984; Rivera and Cisternas, 1990; Arnold and Townend, 2007).

Assuming that individual focal mechanisms represent a uniform state of stress and are driven by the same stress field, regional stress estimates can be derived by inverting a set of independent focal mechanism observations (Arnold and Townend, 2007). In this project, we apply the Bayesian approach of Arnold and Townend (2007) to estimate tectonic stress parameters from the LFE focal mechanisms (an example of this is shown in Fig. 2.8). This formalism of the stress inversion problem enables uncertainties in each focal mechanism's strike, dip and rake pa- 
rameters, as well as nodal plane ambiguity, to be taken into consideration. We presume that the individual focal mechanisms collectively represent a single, spatially uniform state of stress. We then invert our focal mechanism observations to obtain a regional stress estimate.
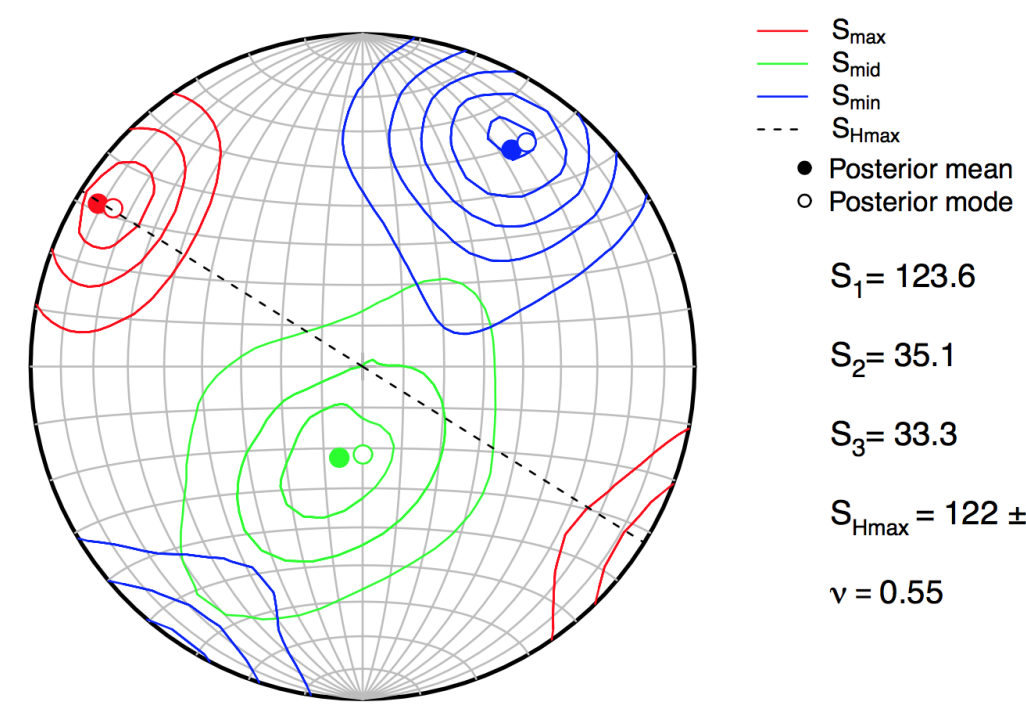

$S_{1}=123.6$

$\mathrm{S}_{2}=35.1$

$\mathrm{S}_{3}=33.3$

$\mathrm{S}_{\mathrm{H} \max }=122 \pm 25^{\circ}$

$v=0.55$

Figure 2.8: Example of output from the stress inversion codes of Arnold and Townend (2007). Stress inversion computed for this study, from 8 LFE focal mechanisms, following the approach of Arnold and Townend (2007). Principal stress direction $\left(S_{\text {min }}\right.$ or $S_{1}<S_{\text {mid }}$ or $S_{2}<S_{\text {max }}$ or $\left.S_{3}\right)$ and maximum horizontal compressive stress $\left(S_{\operatorname{Hax}}\right)$. 


\section{Focal mechanisms and inter-event times of low-frequency earthquakes reveal quasi-continuous deformation and triggered slow slip on the deep Alpine Fault*}

This Chapter summarises the results of our low-frequency earthquakes (LFEs) data analysis over $\sim 7$ years (March 2009 - October 2016). Characterising the seismicity associated with slow deformation in the vicinity of the Alpine Fault may provide constraints on the stresses acting on a major transpressive margin prior to an anticipated great $(\geq \mathrm{M} 8)$ earthquake. Here, we use recently detected tremor and low-frequency earthquakes to examine how slow tectonic deformation is loading the Alpine Fault late in its typical 300-year seismic cycle. We analyse a continuous seismic dataset recorded between 2009 and 2016 using a network of 10-13 short-period seismometers, the Southern Alps Microearthquake Borehole Array. Fourteen primary LFE templates are used in an iterative matched-filter and stacking routine, allowing the detection of similar signals corresponding to LFE families sharing common locations. This yields an 8-yr catalogue containing 10,000 LFEs that are combined for each of the 14 LFE families using phaseweighted stacking to produce signals with the highest possible signal-to-noise ratios. We show that LFEs occur almost continuously during the 8-yr study period and highlight two types of LFE distributions: (1) discrete behaviour with an interevent time exceeding 2 minutes; (2) burst-like behaviour with an inter-event time below 2 minutes. We interpret the discrete events as small-scale frequent deformation on the deep extent of the Alpine Fault and LFE bursts (corresponding in most cases to known episodes of tremor or large regional earthquakes) as brief periods of increased slip activity indicative of slow slip. We compute improved non-linear earthquake locations using a 3D velocity model. LFEs occur below the

*This Chapter has been published as a letter in Earth and Planetary Science Letters under Baratin et al. (2018), Focal mechanisms and inter-event times of low-frequency earthquakes reveal quasi-continuous deformation and triggered slow slip on the deep Alpine Fault, 483:111123. 
seismogenic zone at depths of 17-42 km, on or near the hypothesised deep extent of the Alpine Fault. The first estimates of LFE focal mechanisms associated with continental faulting, in conjunction with recurrence intervals, are consistent with quasi-continuous shear faulting on the deep extent of the Alpine Fault.

This Chapter has been marginally altered with regards to the published version so as to fit into the context of this thesis. The first catalogue of Chamberlain et al. (2014) will be referred to as 'original catalogue' and this study's catalogue as 'extended catalogue'. The Results section has been slightly modified to include (1) the improvements made on our catalogue between iterations (cf. the subsection 'Variations between iterations' of Section 3.4.1 which is based on the Supplementary material of the published version); (2) a slightly more detailed analysis of the differences between families (cf. subsection 'Variations between families' of Section 3.4.1). The Discussion has also been modified to include further talks on the improvements made to the extended catalogue with regards to the original catalogue (cf. subsection 3.5.1 entitled 'Catalogue improvements' which contains material from the original discussion in Baratin et al. (2018) as well material from the Supplementary material).

\subsection{Introduction}

Slow earthquakes are transient-slip phenomena hypothesised to play an important role in the seismic cycle as they involve stress transfers between stably sliding and locked portions of a fault (Wech and Creager, 2011). Slow earthquakes generally manifest as short- to long-term (days-months) geodetic transients (Dragert et al., 2001) with (e.g. Dragert et al., 2004; Ohta et al., 2006; Frank et al., 2015) or without contemporaneous non-volcanic tremor (e.g. Delahaye et al., 2009). Tremor signals correlated both spatially and temporally with slow slip events were first documented on the deep portion of the Cascadia subduction zone (Rogers and Dragert, 2003) and interpreted to be the seismic manifestation of deep slow slip on the fault interface (Obara, 2002; Shelly et al., 2007). However, slow slip has been observed without concurrent tremor, in Cascadia and elsewhere (Delahaye et al., 2009, and references therein); and tremor has also been detected without observable slow slip (e.g. Guilhem and Nadeau, 2012). Even though incomplete detection of tremor or slow-slip might explain those occurrences, this nonetheless emphasises that the mechanisms involved in tremor generation require further elucidation. 
Low-intensity, long-duration tremor signals without impulsive P- and S-wave arrivals were first observed at subduction zones (Obara, 2002; Rogers and Dragert, 2003) but later reported at major strike-slip fault zones (e.g. Nadeau and Dolenc, 2005; Wech et al., 2012; Aiken et al., 2013). Several studies show tremor to occur on the plate interface in the rheological transition zone between stick-slip (in the brittle seismogenic zone) and stable sliding (in the ductile downdip portion of the fault; e.g. Beroza and Ide, 2011). Two principal mechanisms have been proposed to explain the inferred weakening of the plate interface at depth: (1) an increase in temperature or (2) trapped fluids at the plate interface and resulting high pore pressures (Shelly et al., 2006).

Shelly et al. (2007) demonstrated that tremor can be decomposed into a swarm of discrete low-frequency earthquakes (LFEs). LFEs are commonly interpreted as the rupture of small asperities within otherwise aseismically slipping regions of a fault (e.g. Ide, 2008). Each LFE source generates multiple events constituting an LFE family. In this study, we capitalise on the repeating nature of LFEs to construct a continuous catalogue of LFE seismicity associated with the central Alpine Fault using an iterative matched-filter routine (Shelly, 2017). In this context, matched-filtering is the process of correlating the waveforms of a known template event (e.g. the phases of an LFE) with continuous seismic data to detect near-repeats of the template event. In the following we refer to all the detections made by a single template as being part of a single 'family' of detections.

Tremor was first documented on the Alpine Fault by Wech et al. (2012) using data recorded by the Southern Alps Microearthquake Borehole Array (SAMBA) (Boese et al., 2012). Wech et al. (2012) reported tremor occurring in 12 separate episodes between March 2009 and October 2011 in the lower crust (between 25$45 \mathrm{~km}$ ) south of the SAMBA network. They interpreted tremor to represent slow slip occurring on the deep extent of the Alpine Fault in a region of elevated fluid content. LFEs were subsequently identified by Chamberlain et al. (2014) by visual inspection of seismic data spanning periods of tremor. Fourteen LFEs were identified and used as primary templates in a matched-filter routine to detect similar events occurring throughout a 36 month period (26 March 2009-2 April 2012). Following an initial detection run, the $10 \%$ of detections with the highest signal-to-noise ratios were stacked linearly to generate a new template. This process was repeated until the number of detections stabilised. Despite this, few $\mathrm{P}$ - and S-wave arrival times could be picked accurately, and P-wave polarities could not be determined. As a consequence of the resulting large uncertainties in hypocentral depths and the absence of source parameters, interpreting Alpine 
Fault tremor and LFE observations in terms of slip on the fault itself remained inconclusive.

Here, we use an additional 4 years of data and present the longest continuous record of LFEs beneath the New Zealand Southern Alps to date (spanning an almost 8-yr period). Using templates manually identified by Chamberlain et al. (2014) and a matched-filter detection technique, we detect 10,000 events occurring in 14 families between 26 March 2009 and 22 October 2016. We implement a phase-weighted stacking method (Schimmel and Paulssen, 1997; Thurber et al., 2014) instead of linear stacking within the matched-filter routine to emphasise coherent signals resulting in higher signal-to-noise ratio stacks. This facilitates more accurate picks, which in turn allow for the generation of refined hypocentre locations and the calculation of reliable focal mechanism parameters with which to examine the state of stress in the vicinity of the LFEs. We study inter-event times and document an almost-constant background rate of LFE generation punctuated by distinctive short-term rate increases corresponding to known periods of tremor or large $\left(M_{w}<5\right)$ regional earthquakes.

\section{$3.2 \quad$ Setting}

The Alpine Fault is an $850 \mathrm{~km}$-long dextral-reverse transpressional fault and is the principal locus of deformation within the Australia-Pacific plate boundary in the South Island of New Zealand (Fig. 3.1). An average of 70-75\% of total plate displacement (equivalent to a fault-parallel slip rate of $39 \mathrm{~mm} / \mathrm{yr}$ and convergence rate of $7-9 \mathrm{~mm} / \mathrm{yr}$ ) is accommodated by the central part of the Alpine Fault (Norris and Cooper, 2001). The convergent motion results in an area of enhanced uplift (5-8 mm/yr, Beavan et al., 2002; Lamb and Smith, 2013) that corresponds to regions of highest peaks in the central Southern Alps (Little et al., 2005).

Paleoseismic studies suggest that $M_{w} 7-8$ earthquakes rupture the central Alpine Fault every $271 \pm 73$ years (Sutherland et al., 2007; Howarth et al., 2016), with the most recent major event inferred to have occurred in 1717 A.D. (Wells et al., 1999). In other words, the Alpine Fault is late in its typical earthquake cycle, making it an ideal place to study the processes leading up to a large earthquake. 


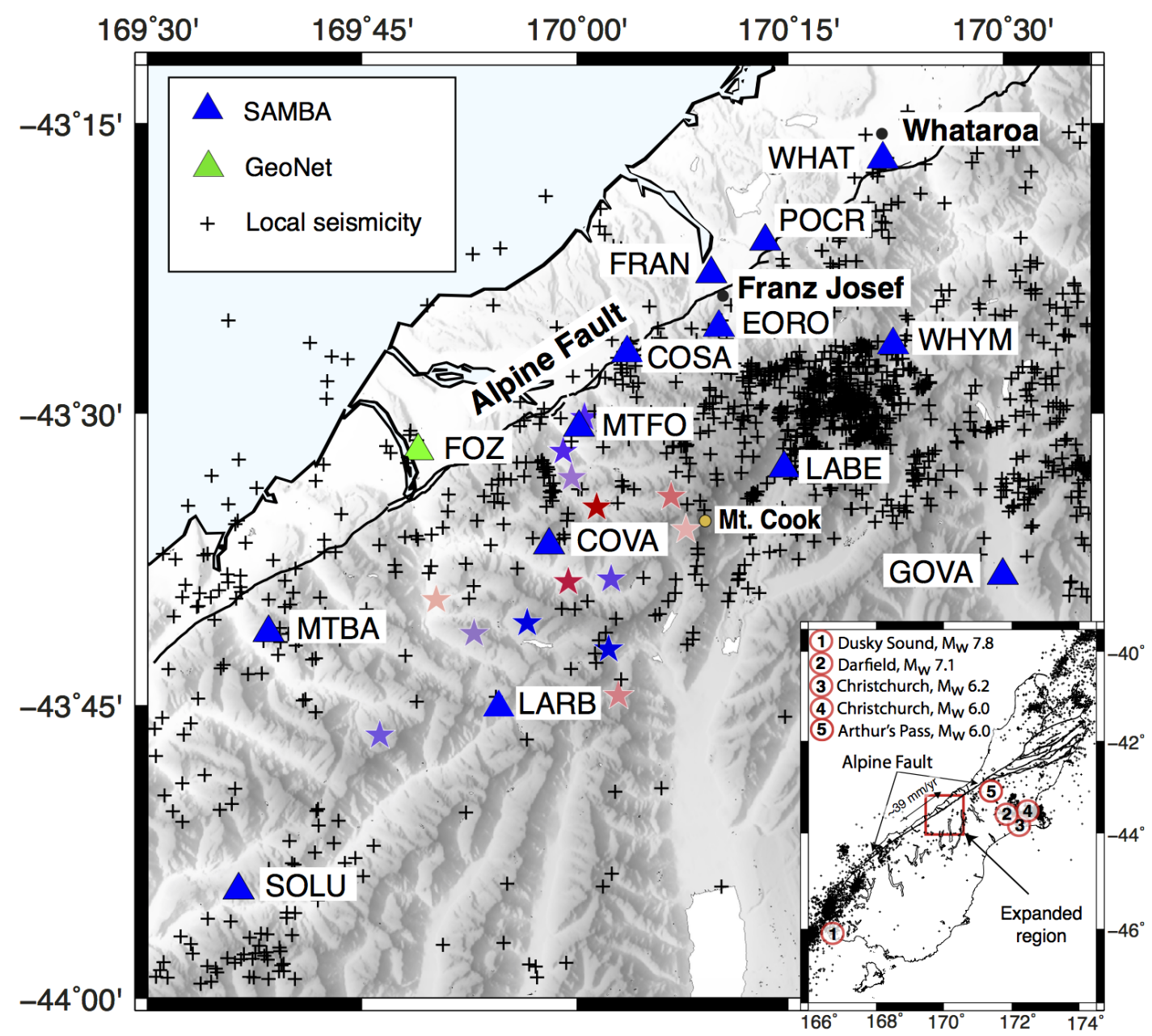

Figure 3.1: Map of study area. Locations of the short-period SAMBA stations (blue triangles) and GeoNet broadband station (green triangle) with local seismicity (black crosses) from Boese et al. (2012) covering the year 2009 and from the GeoNet catalogue covering our entire study period (3/2009-10/2016). The coloured stars show LFE locations from this study. LFE hypocentres are coloured by average LFE detection rates, according to the legend in Fig. 3.3. Inset: location of the study area in the central Southern Alps in South Island, New Zealand. Black crosses show the background seismicity from the GeoNet catalogue $\left(M_{w} \geq 4\right)$ during the study period. Numbers 1-5 reference large regional earthquakes; respectively the July 2009 Dusky Sound earthquake $\left(M_{w} 7.8\right)$, the 4 September 2010 Darfield earthquake $\left(M_{w} 7.1\right)$, the 22 February and 11 June 2011 Christchurch earthquakes $\left(M_{w} 6.2\right.$ and $\left.M_{w} 6.0\right)$ and the 6 January 2015 Arthur's Pass earthquake $\left(M_{w} 6.0\right)$. 
Drilling into the hanging wall of the Alpine Fault was undertaken as part of the Deep Fault Drilling Project to better understand the ambient conditions, rock properties, and geophysical phenomena within an active fault zone prior to a large earthquake (e.g. Townend et al., 2013; Toy et al., 2015; Sutherland et al., 2017; Toy et al., 2017). The most recent phase of drilling revealed a pore fluid pressure gradient exceeding $9 \pm 1 \%$ above hydrostatic levels and an extreme geothermal gradient of $125 \pm 55^{\circ} \mathrm{C} / \mathrm{km}$ within the fault hanging wall (Sutherland et al., 2017). Those observations reinforce the hypothesis that rapid uplift and exhumation near the Alpine Fault have raised isotherms, thus weakening the crust and focusing deformation along the fault.

\subsection{Methods}

\subsubsection{LFE detection}

We use the waveforms of fourteen LFEs manually identified by Chamberlain et al. (2014) as primary templates in an iterative stacking and cross-correlation routine to look for similar events (following the approach used by Shelly, 2017). We use the EQcorrscan Python package to compute matched-filter detections (Chamberlain et al., 2017c). Each template is constructed using a $6 \mathrm{~s}$ window beginning $0.5 \mathrm{~s}$ before the S-pick to ensure that it encompasses the S-phase arrival and coda. Each template and the dataset as a whole are resampled to $100 \mathrm{~Hz}$ and bandpass-filtered at $2-8 \mathrm{~Hz}$, which corresponds to the LFEs' peak frequency band (Chamberlain et al., 2014). We correlate each template with the data recorded at each station on all three channels at $0.01 \mathrm{~s}$ intervals. The minimum inter-event trigger time for individual families is fixed at $6 \mathrm{~s}$. A detection is made when the cross-correlation sum across the network exceeds a threshold value. Here, we use a threshold of eight times the median absolute deviation of the day-long cross-correlation sum, which has to be composed of correlations from at-least 15 channels. This threshold minimises the number of falsely detected events per day per template to 0-3 (Chamberlain et al., 2014). After an initial detection run, we linearly stack the $20 \%$ of detections with the highest cross-correlation sum to generate a refined template with higher signal-to-noise ratio. We then employ each new template in the matched-filter routine and iterate until the final template has a sufficiently high signal-to-noise ratio for us to pick reliable arrival times (requiring 2-4 iterations, depending on the template). After each iteration, the number of detections for most families increases significantly. Furthermore, the use of a higher signal-to- 
noise ratio template results in the detection of a greater number of relatively higher signal-to-noise ratio events between each iteration. This change in signalto-noise ratio is reflected in the $20 \%$ best correlated detections that includes a larger number of high signal-to-noise ratio detections after each iteration, thus refining the template between iterations. We extend the final stacked waveform to 20 s-length to include both $\mathrm{P}$ and $\mathrm{S}$ arrivals for picking purposes (the resulting stack being a 20 s-long record with the S-arrival at $\sim 10 \mathrm{~s}$ ). Finally, we apply phase-weighted stacking, described below, to the last iteration's detections.

\subsubsection{LFE location}

The majority of earthquake location methods are based on the arrival times of distinctive impulsive seismic phases. An inherent obstacle in locating LFEs is the emergent character of their P- and S-wave arrivals (Shelly et al., 2007). In this study, we use phase-weighted stacking (Schimmel and Paulssen, 1997) to improve the signal-to-noise ratio of signals of interest to allow us to more precisely pick the onset of phases. Phase-weighted stacking involves the weighting of the linear stack by a time-dependant measure of phase coherency, thereby suppressing incoherent signals in the stack. It has been successfully used with LFEs previously and was shown to yield a higher signal-to-noise ratio than the equivalent linear stack (Thurber et al., 2014; Plourde et al., 2015). Here, we use phase-weighted stacking exclusively for picking purposes, and therefore only in the last iteration of the matched-filter routine. Indeed, we found using linear stacking within matchedfilter iterations to produce more stable LFE stacks. Our final template catalogue is composed of highly improved stacks compared with the original catalogue of Chamberlain et al. (2014) (e.g. Fig. 3.15 in section 3.5.1). By applying phaseweighted stacking on the last iteration stacks, we have significantly improved the clarity of our stacked signals compared to the original template and the equivalent linear stack (Fig. 3.2); the cleaner phase arrivals of apparently impulsive nature allow more accurate phase and polarity picks.

We compute hypocentre locations and their associated uncertainties using the NonLinLoc software (Lomax et al., 2000) and a 1D velocity model described by Boese et al. (2012). The NonLinLoc algorithm produces a probabilistic estimate of each hypocentre's location by first comparing synthetic travel times calculated for each potential source within a 3D grid and then comparing the observed and calculated phase arrival times using a Metropolis-Gibbs approach. 


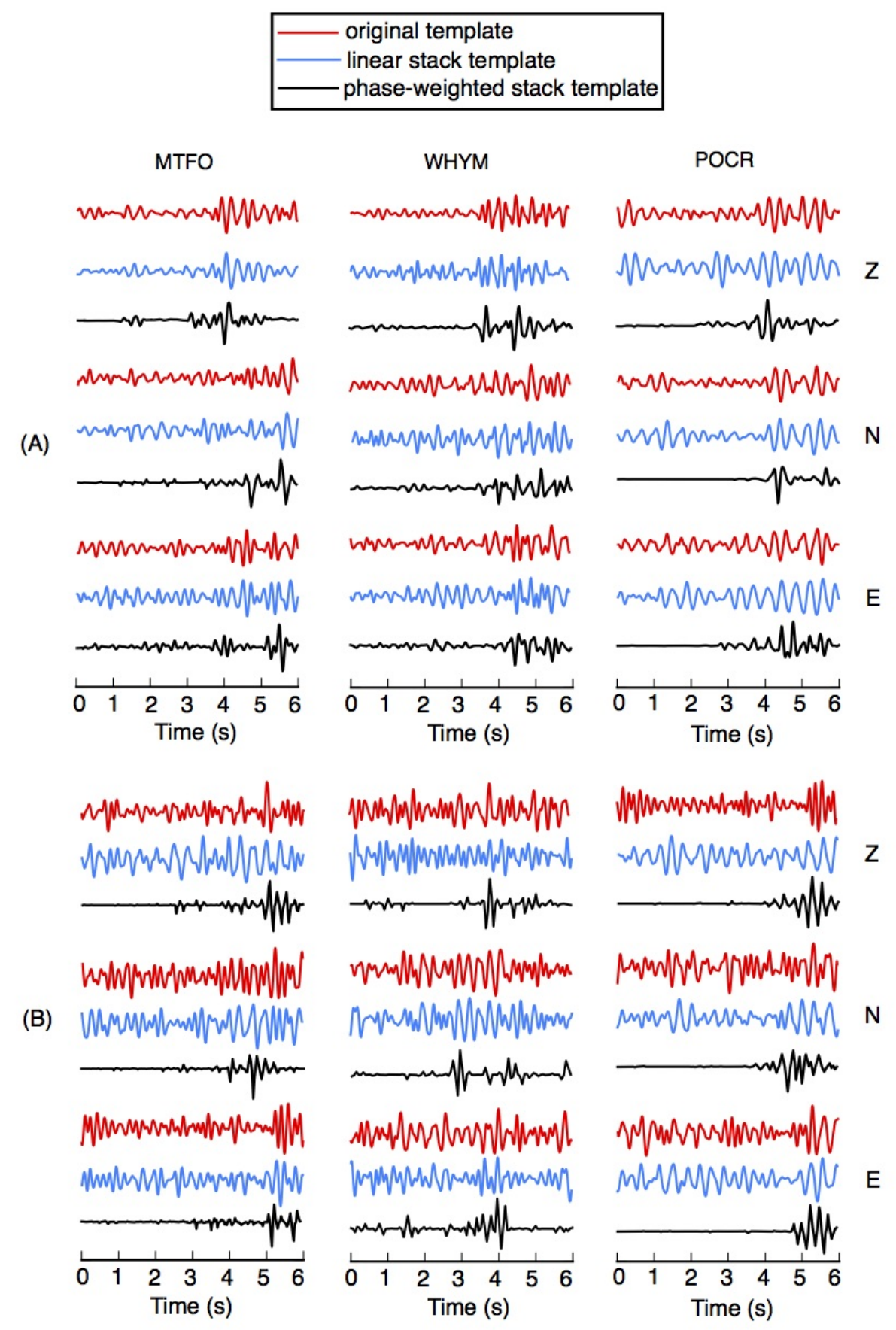

Figure 3.2: Comparison between original template, linear stack and phase-weighted stack traces. Primary template and stacked traces of LFE family IDs: $17208(\underline{\mathrm{A}})$ and $54905(\underline{\mathrm{B}})$. The red, blue and black traces represent respectively the original template, the linear stack and the phase-weighted stack obtained during the last iteration of the matched-filter routine; they are plotted, from left to right, for stations MTFO, WHYM and POCR and from top to bottom, for the vertical, north and east components. 


\subsubsection{Focal mechanisms estimation and stress inversion}

We compute focal mechanisms using the P-wave first motions of the LFE stacked waveforms with the Bayesian approach of Walsh et al. (2009). This method accommodates uncertainties in hypocentre location, velocity model, and P-wave polarities. For each event, the joint posterior probability density function of the three focal mechanism parameters (strike, dip, and rake) is calculated. This distribution is then approximated using Matrix Fisher distributions.

We first pick polarities on the phase-weighted stacks for templates exhibiting distinct first motions. The posterior probability density function of the hypocentre locations and take-off angles for each station, generated with NonLinLoc, and the polarity picks are then used to estimate the distribution of focal mechanism parameters.

We then apply the Bayesian approach of Arnold and Townend (2007) to estimate tectonic stress parameters from the LFE focal mechanisms. This formalism of the stress inversion problem enables uncertainties in each focal mechanism's strike, dip and rake parameters, as well as nodal plane ambiguity, to be taken into consideration. We presume that the individual focal mechanisms collectively represent a single, spatially uniform state of stress. We then invert our focal mechanism observations to obtain a regional stress estimate.

\subsection{Observations}

\subsubsection{LFE catalogue}

\section{Catalogue overview}

Using the 14 final LFE templates, a total of 10,000 events were detected between March 2009 and October 2016 in the central Southern Alps, constituting the longest record of LFEs in New Zealand to date. The final number of detections for each family varies between $\sim 300$ and $\sim 1,300$ over the eight year study period (Table 3.1). LFE activity is frequent during the study period (Fig. 3.3), yielding an overall long-term rate of 4 LFEs per day. 


\section{(1) (2) (3) (4)} (5)

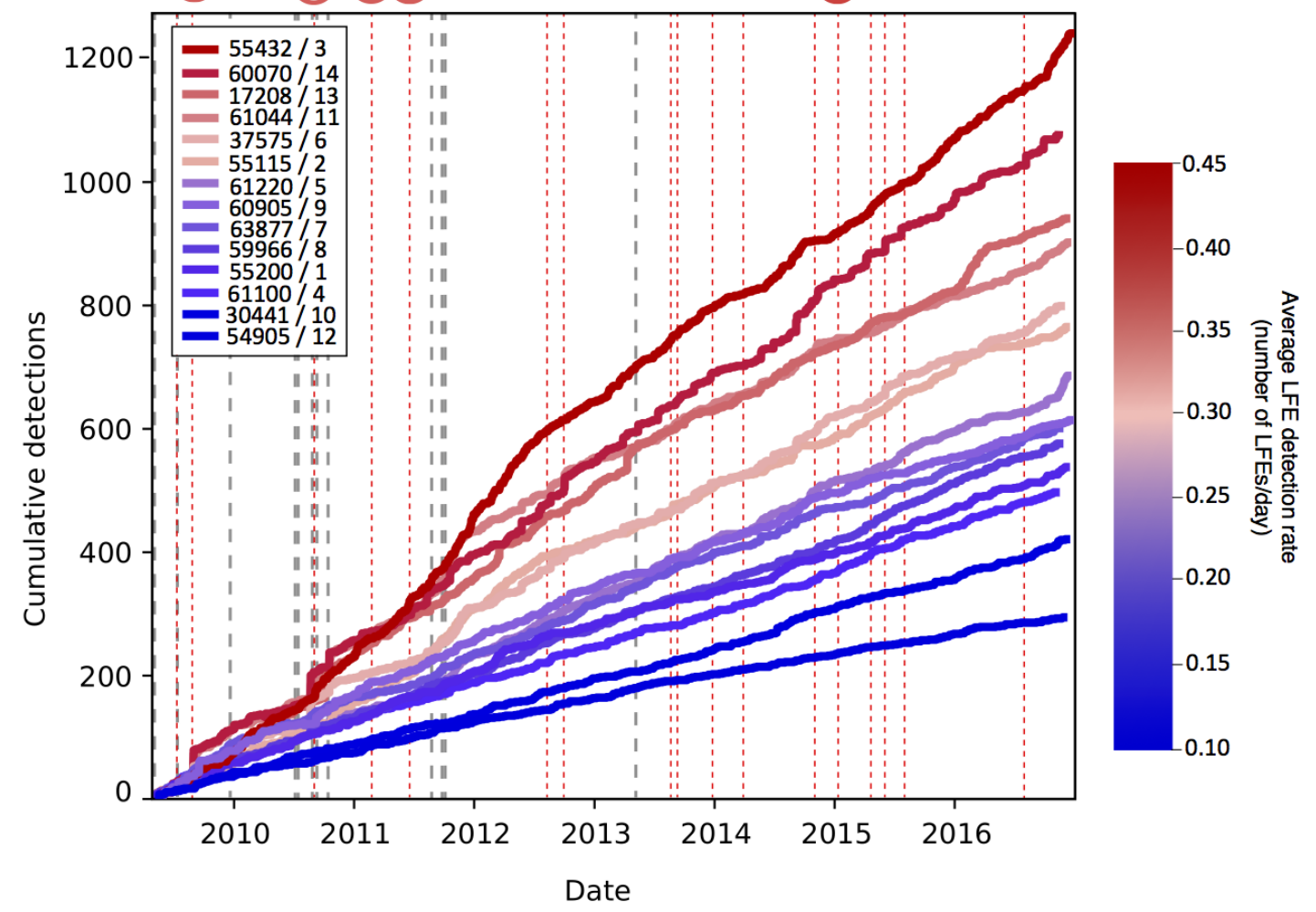

Figure 3.3: Temporal evolution of individual families. Cumulative plot showing the number of detections for each family over the study period. The colour scale indicates the long-term rate for each individual LFE family. The dashed red lines show the main earthquakes that are thought to have triggered LFE episodes. Those earthquakes are taken from the GeoNet catalogue within events of $M_{w} \geq 5$ and locating up to $50 \mathrm{~km}$ depth; most of them are within $50 \mathrm{~km}$ of the Alpine Fault, with the exception of earthquakes occurring in the Canterbury area (locating within $150 \mathrm{~km}$ of the Alpine Fault). The grey dashed lines represent tremor periods during which LFEs have been reported (Wech et al., 2012; Chamberlain et al., 2014); tremor analysis has not been undertaken beyond 2013.

We check the frequency content of our LFE stacks to make sure that the detected events used in the stack are different from regular earthquakes (Fig. 3.4). We find our LFEs to have frequency peaks between $2-8 \mathrm{~Hz}$ as shown by the spectra of the linear and phase-weighted stacks of a representative LFE in Fig. 3.5 (spectral peak shown in red corresponding to the LFE S-wave arrival). This is in agreement with other studies demonstrating that tremor and LFEs are enriched in low frequencies $(1-10 \mathrm{~Hz})$ and depleted in higher frequencies compared to nearby ordinary earthquakes (e.g. Obara, 2002; Shelly et al., 2007; Wech et al., 2012). 


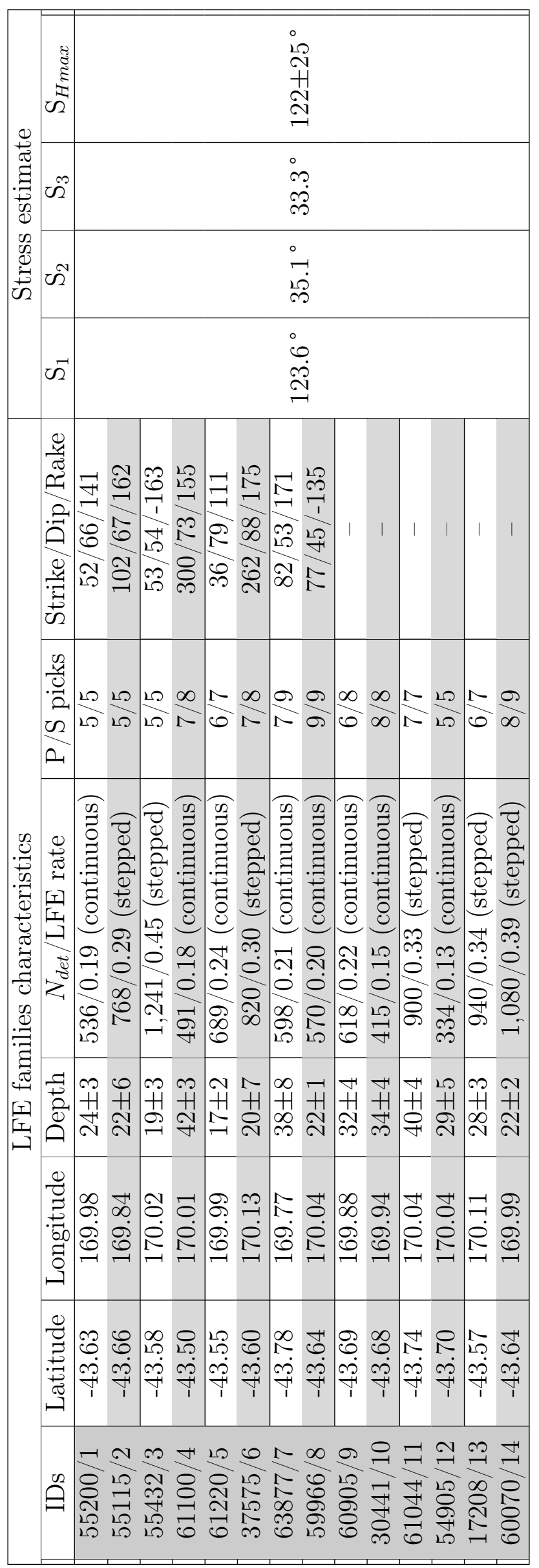

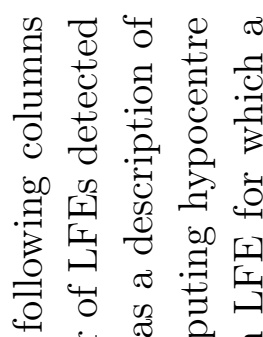

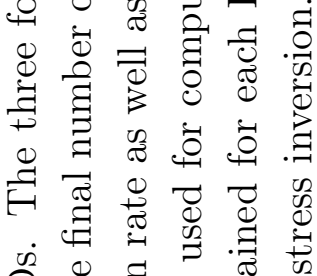

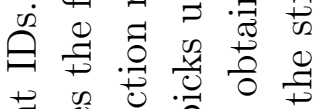

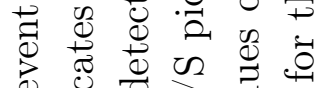

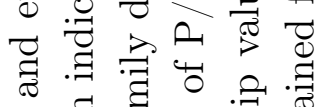

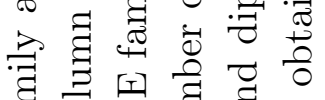

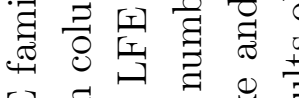

II

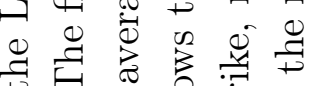

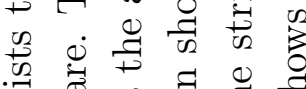

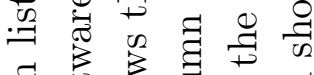

节

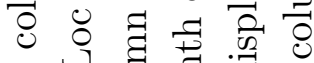

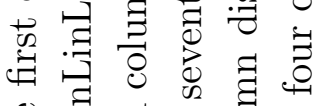

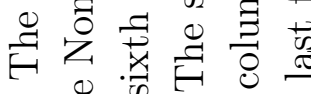

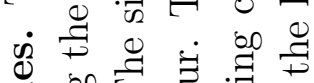

:

भ

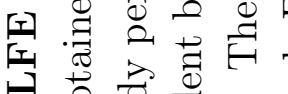

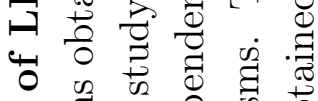

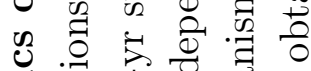

.

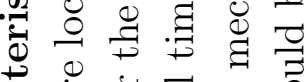

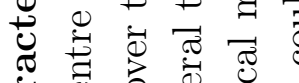

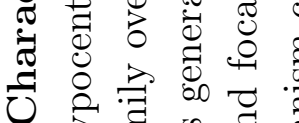

৩

궈

0 ॠ

范 
Moreover, we observe for all the families in our catalogue that the phase-weighted stacks provides cleaner phase arrivals than the linear stacks (Figs. 3.2 and 3.5). Our final catalogue yields phase-weighted stacks with high signal to noise ratios allowing us to reliably pick most S-wave arrivals and some P-wave arrivals - an essential step in the obtention of accurate locations.
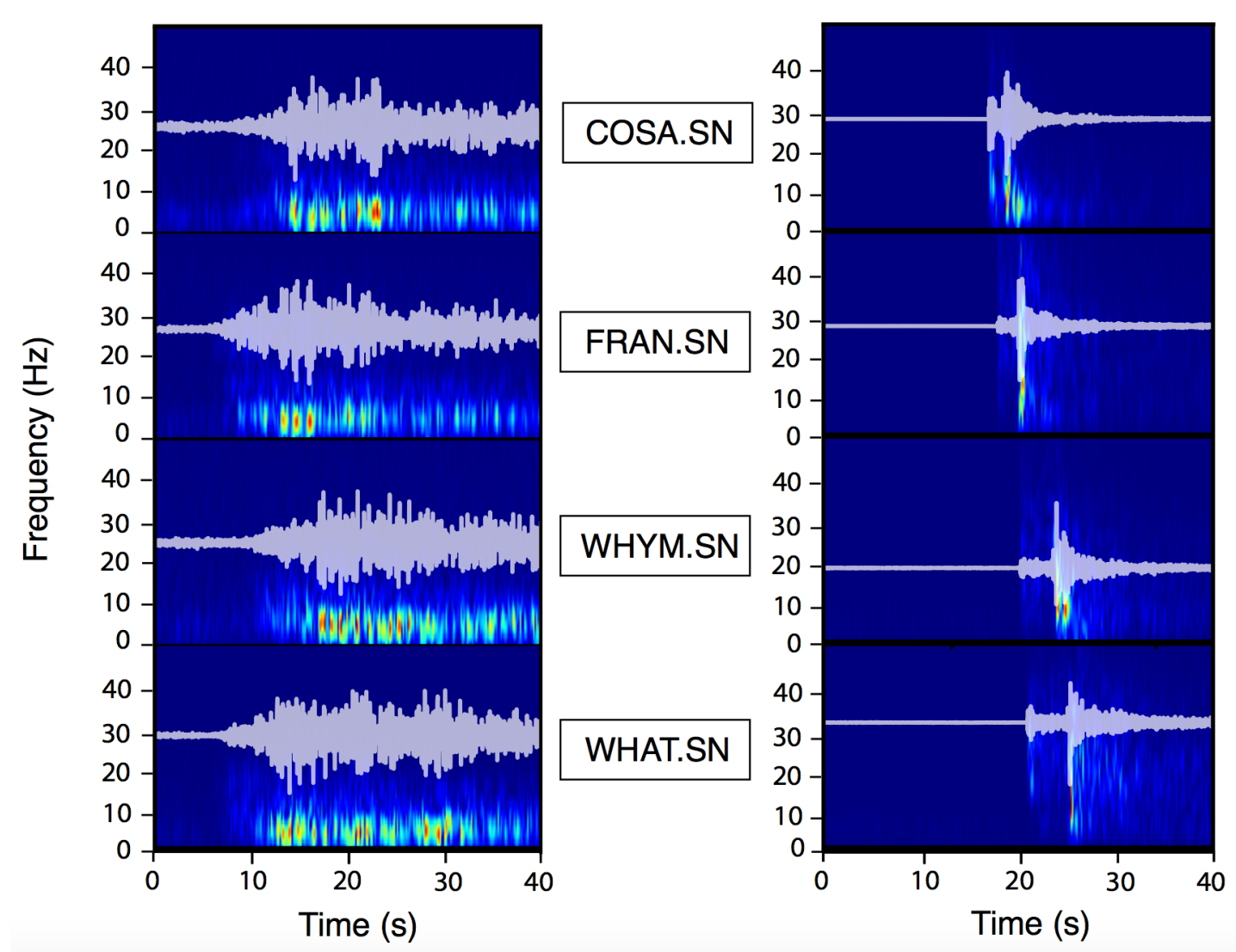

Figure 3.4: Waveforms and spectrograms for an LFE and a typical earthquake detection. Spectrograms and waveforms resampled to $100 \mathrm{~Hz}$ and filtered between 2-49 Hz for an LFE detection (left) and a typical earthquake detection (right). Left: LFE detections occurring on 2013/02/15 at 18:17:26. An emergent, tremor-like arrival can be seen on all channels at $\sim 10 \mathrm{~s}$. There are clear spectral peaks between 2 and $10 \mathrm{~Hz}$ that correspond to a low-frequency earthquake spectra. Right: typical earthquake detection occurring on 2010/09/10 at 05:30:24. Spectral peaks can be seen between 5 and $35 \mathrm{~Hz}$ (higher frequencies than a lowfrequency earthquake). We can also easily distinguish the impulsive arrival at $\sim 20 \mathrm{~s}$. 


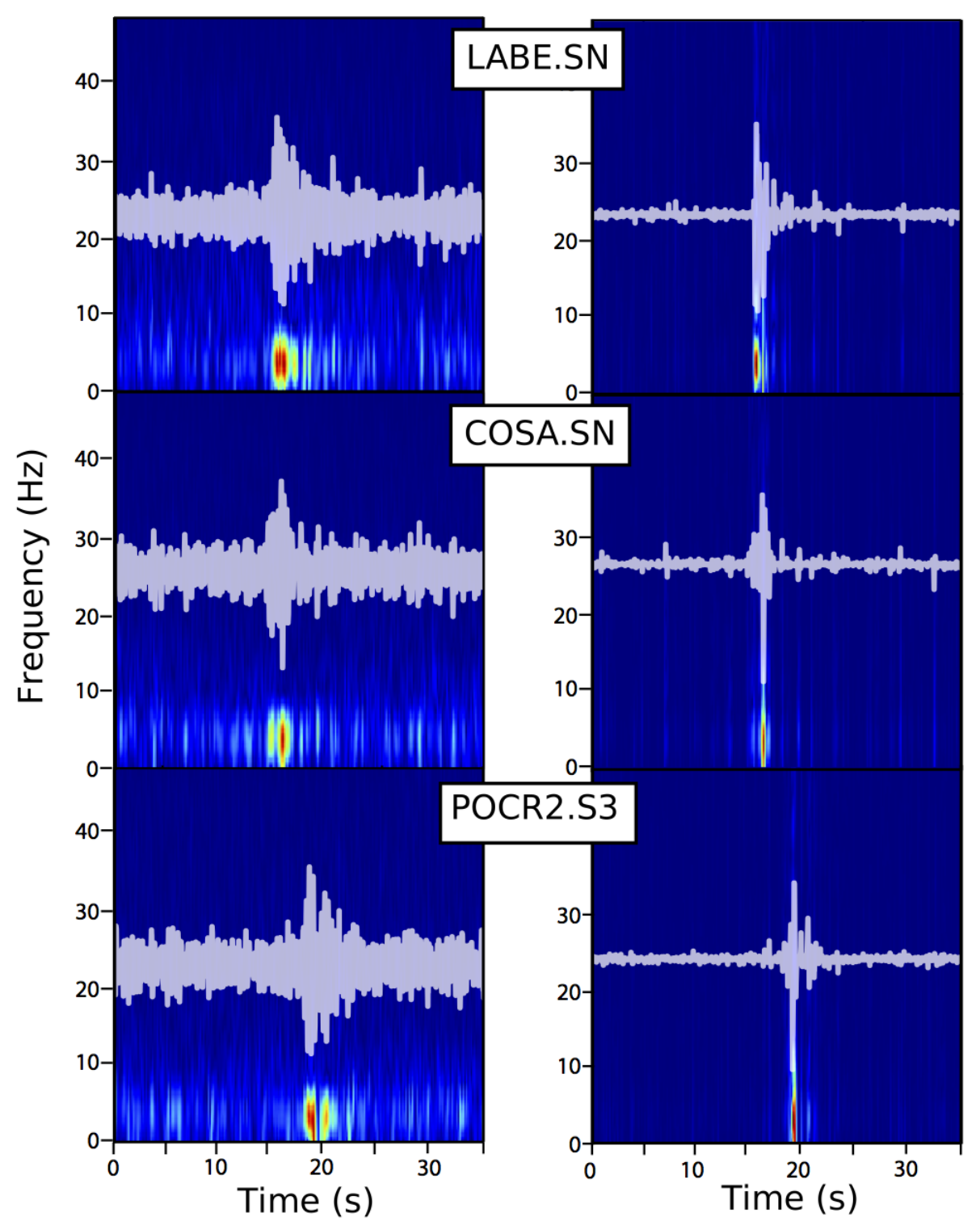

Figure 3.5: Waveforms and spectrograms for the final stack of family ID:60070. Left: linear stack. Right: phase-weighted stack. We are showing here the spectrograms and waveforms for an LFE family with a high signal to noise ratio. Waveforms are resampled to $100 \mathrm{~Hz}$ and filtered between $2-49 \mathrm{~Hz}$ to remove long-period noise while retaining signal outside the tremor band to show the consistent dominant energy in the $2-8 \mathrm{~Hz}$ band. There are clear spectral peaks between 2 and $10 \mathrm{~Hz}$ that correspond to a low-frequency earthquake. 


\section{Variations between iterations}

The goal behind iterating within the matched-filter routine is twofold. This process aims to significantly increase the number of detected events for most families (e.g. family ID:37575 in Figs. 3.6 and 3.7), as well as to increase the average signalto-noise ratios for the LFE families (e.g. Fig. 3.8). For example, for family ID:37575 (Fig. 3.6), 712 events are detected during the first iteration of the matched-filter routine, 806 events during the second iteration and 820 events during the last iteration. This emphasises a regular increase in the number of detected events between iterations for this family. By looking at inter-event times for that same family (Fig. 3.7), we also note that the process of iterating appears to increase the number of bursts. We define a burst as consecutive detections separated by less than 2 minutes (i.e. inter-event time $\leq 10^{-3}$ days in Fig. 3.7). We notice that for most families, the process of iterating through the matched-filter routine increases the number of bursts detected over the study period.

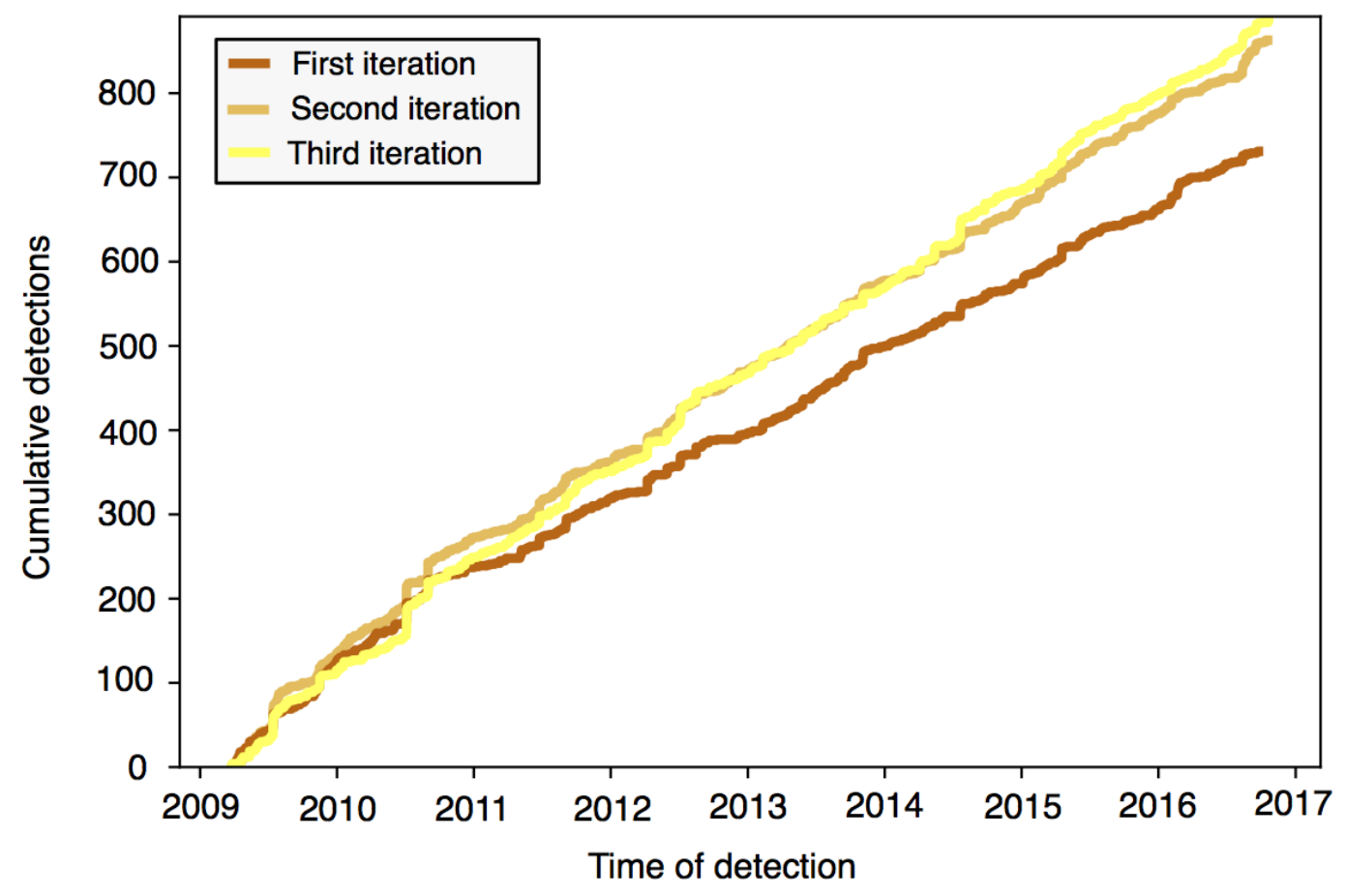

Figure 3.6: Cumulative detections for family ID: 37575. Cumulative detections for the first, second and third iterations of LFE family ID: 37575 are shown respectively in brown, ochre and yellow. We show here that the process of iterating systematically increases the number of detected events between iterations. 

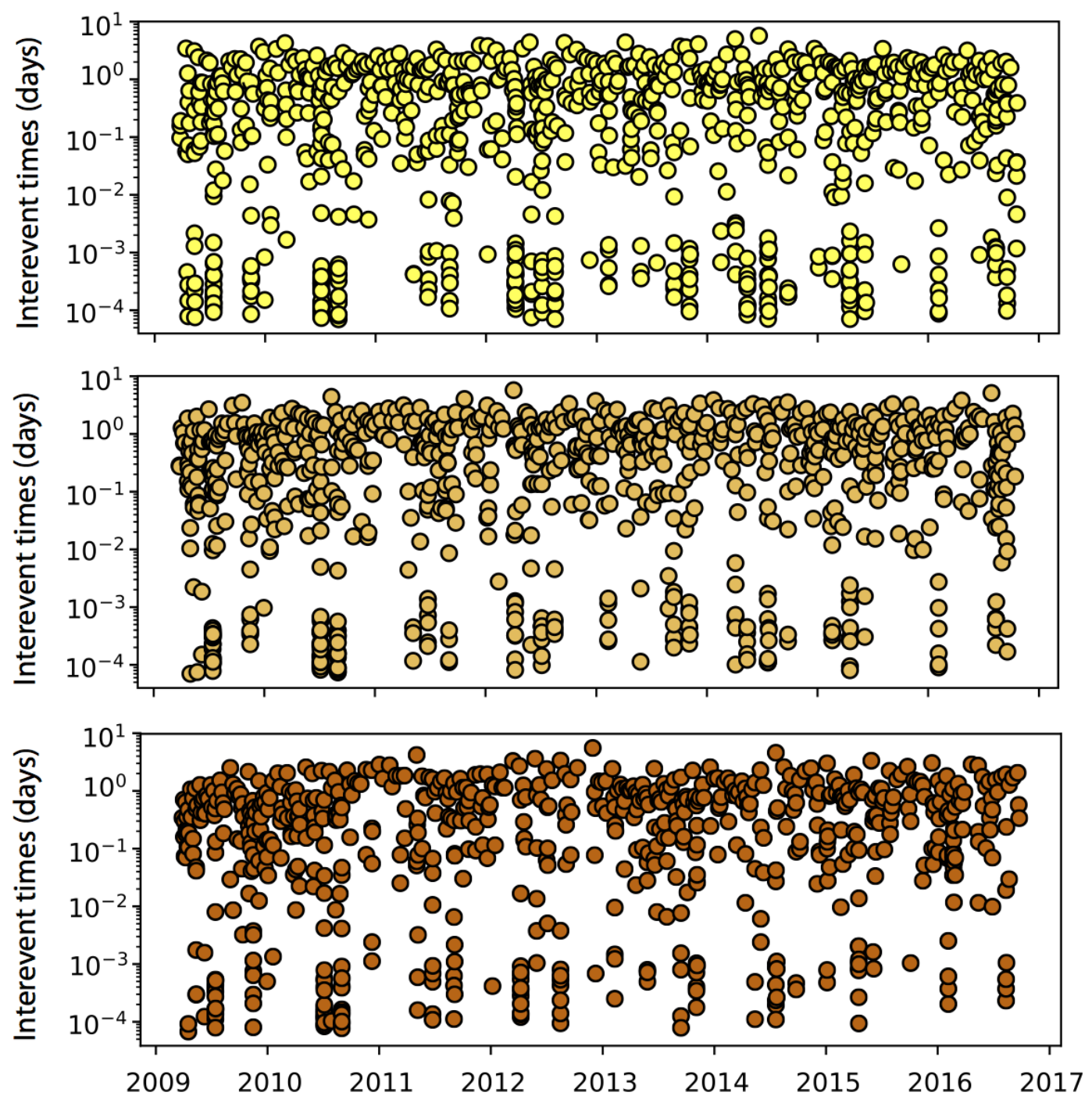

Figure 3.7: Inter-event times for family ID: 37575. Inter-event times during the first, second and third iterations (from bottom to top) of the matched-filter routine are shown respectively in brown, ochre and yellow (same colour coding as in Fig 3.6) for LFE family ID: 37575. We can see that the more we iterate, the more we highlight the burst-like behaviour of LFEs (inter-event time $\leq 10^{-3}$ days).

The process of iterating systematically improves the signal to noise ratios of the LFE families between iterations (e.g. family IDs:60070 and 37575 respectively in Figs. 3.8 and 3.9). This improvement is measured by an increase of the mean threshold of detection between iterations (Fig. 3.8) which reflects the overall quality of the stack. For example, family ID:60070 corresponds to one of the LFE families possessing the highest signal-to-noise ratios, which is shown with the el- 
evated cross-correlation detection values obtained for that family (blue circles for the first iteration and red crosses for the second iteration in Fig. 3.8). We notice that the second iteration stacks (red seismograms in Fig. 3.8) are obtained with detections possessing higher cross-correlation detection values (red crosses in Fig. 3.8) in comparison with the first iteration detections. As a consequence, the second iteration stack is less emergent and retain a higher signal-to-noise ratio compared with the first iteration's stack (blue seismograms in Fig. 3.8). This is roughly true for all consecutive iterations of the matched-filter routine (e.g. Fig. 3.9 where we can see the stacks of three consecutive iterations for family ID:37575).
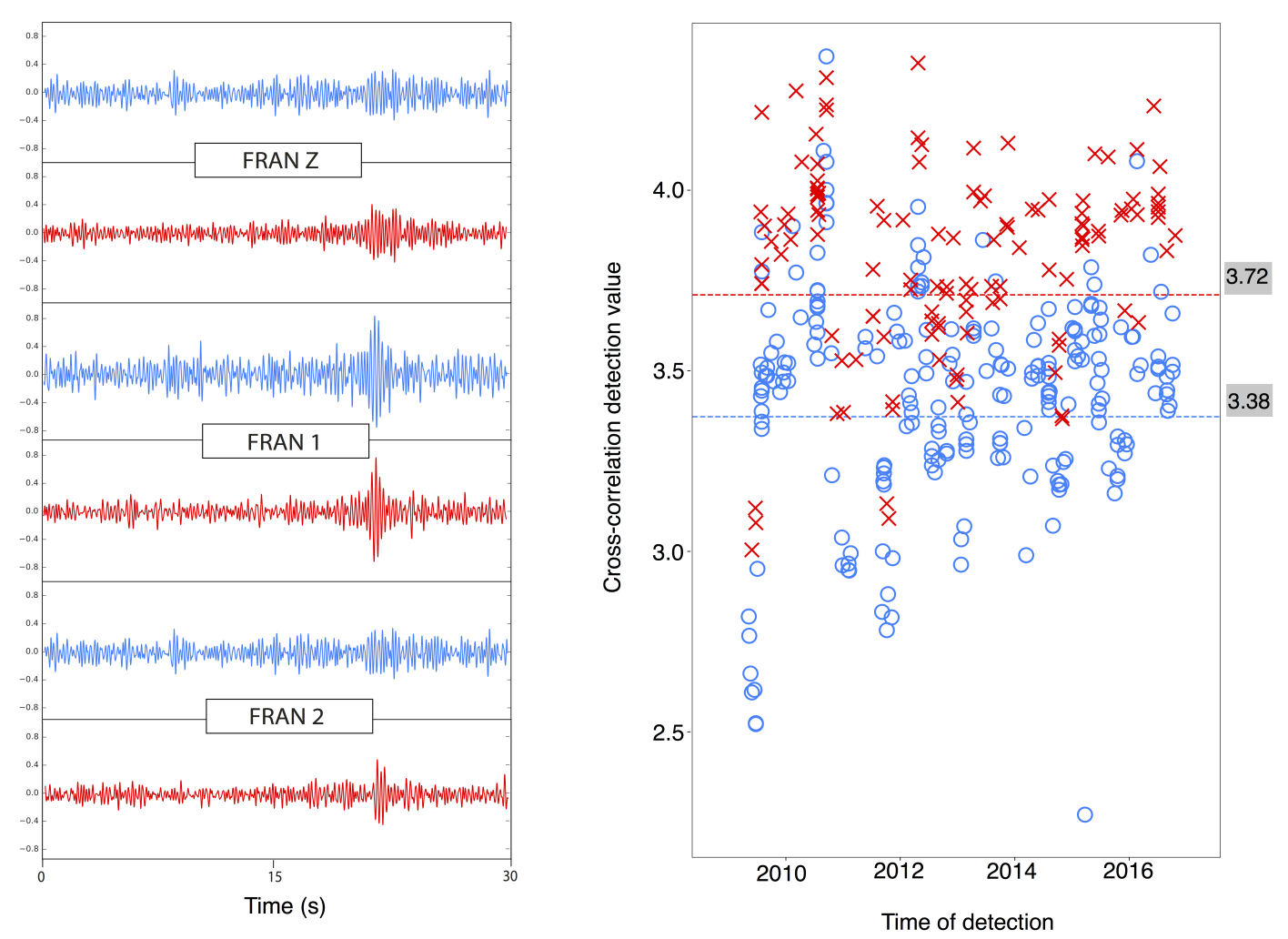

Figure 3.8: Stacks and cross-correlation sum values of stacked events for the first and second iteration for family ID:60070. Left: Linear stacks are plotted for all three components of the FRAN station. We show in blue the first iteration stacks and in red the stacks for the second iteration of the matchedfilter routine. Right: Cross-correlation sums are plotted in blue circles for the detections used in the stacks of the first iteration of the matched-filter routine and in red cross for those of the second iteration. The blue and red line represent respectively the mean threshold of detection used in each case. Family ID:60070 is one of our families with the highest signal to noise ratio which is shown with the higher thresholds obtained for the detections used in the first and second iteration stacks. 
Between the first iteration stacks (black seismograms in Fig. 3.9) and the last iteration stacks (light grey seismograms in Fig. 3.9), the signals become clearer and more impulsive, with a discernible amount of noise being cancelled out. This improvement is generally observed for all LFE families, on all stations and across all channels. We also observe that the process of iterating is particularly efficient when starting with a low signal to noise ratio signal (for example, channel $\mathrm{Z}$ of the MTFO station in Fig. 3.9).

MTFO

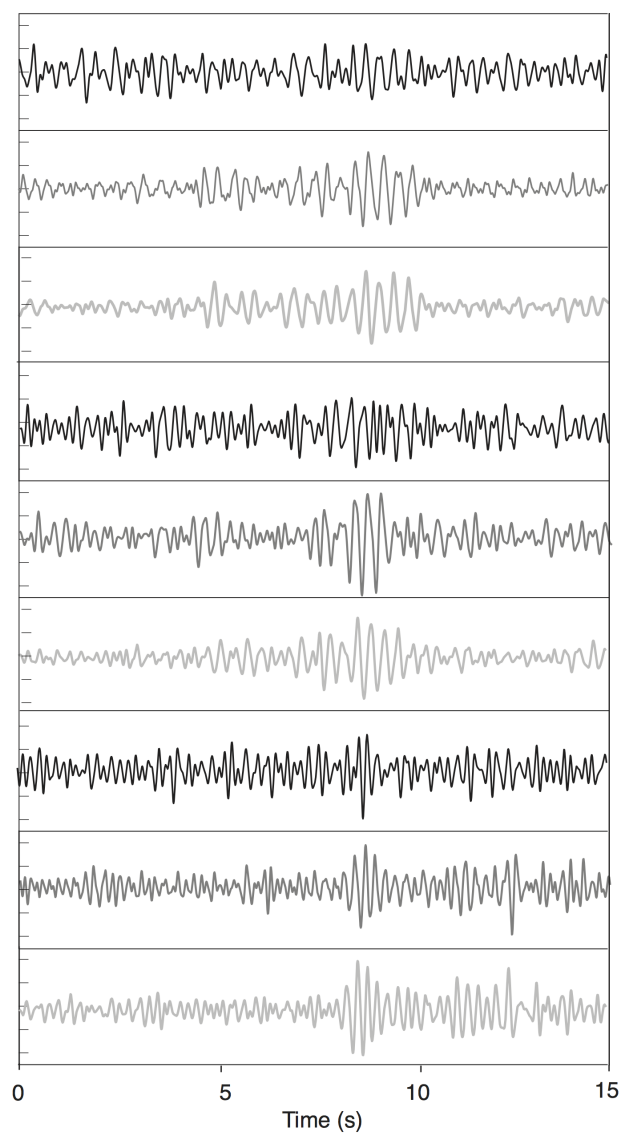

POCR

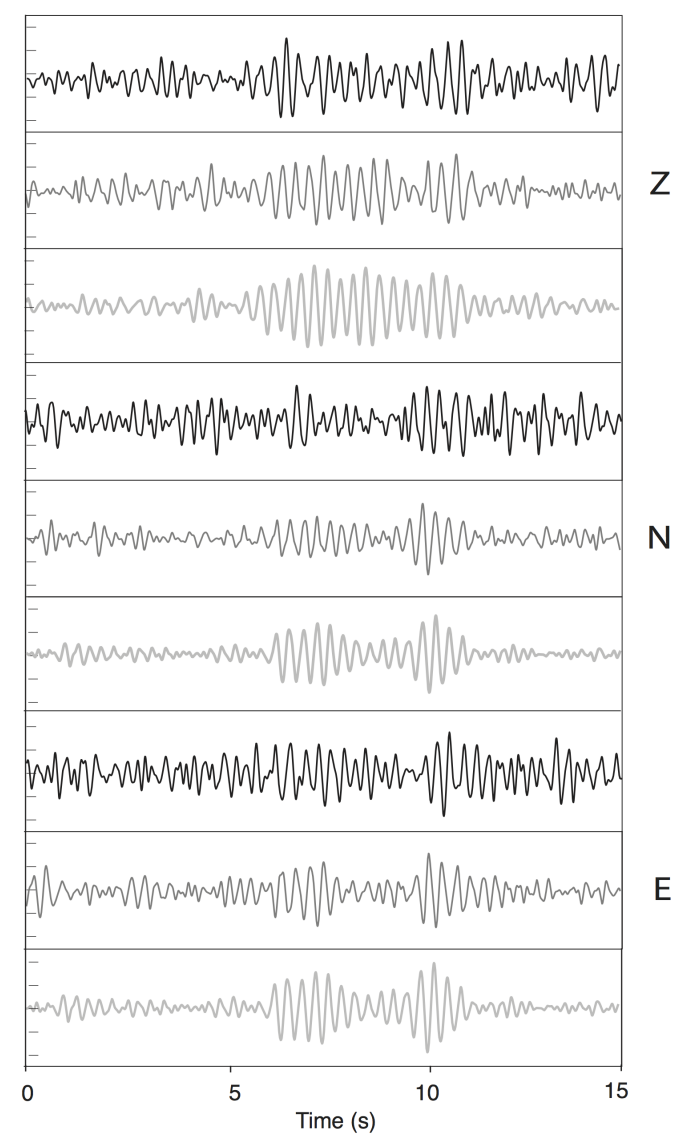

Figure 3.9: Linear stacks for three consecutive iterations for family ID:37575. First iteration (black), second iteration (dark grey) and third iteration (light grey) stacks for family ID:37575 are shown here at stations MTFO and POCR. We see here that the process of iterating increases the overall clarity of the stacks.

Overall, we notice that by using the iterative cross-correlation and stacking routine, we are able to increase the average signal-to-noise ratios for all the LFE families. 


\section{Variations between families}

We can divide the 14 LFE families into two groups: (1) families exhibiting an approximately linear accumulation of detections with time ('continuous', depicted by cold colours in Fig. 3.3), occurring at a long-term rate of $<0.3$ LFEs per day and producing less than $\sim 700$ detections in eight years; and (2) families exhibiting episodic accumulations of detections with time ('stepped', depicted by warm colours in Fig. 3.3), which have a long-term rate $>0.3$ LFEs per day. In the latter group, two families contain greater than 1,000 detected events over the study period (Fig. 3.3). The observed periods of increased LFE activity commonly coincide with known periods of tremor or large $(\mathrm{Mw}>5)$ regional earthquakes (grey dashed and red lines respectively in Fig. 3.3).

The LFE families respond differently to tremor and regional earthquakes (Fig. 3.3). For example, families 37575 and 55115 exhibit mostly similar responses, with the exception of the $M_{w} 7.1$ September 2010 Darfield earthquake (earthquake no. 2 in Figs. 3.1 and 3.3 Quigley et al., 2012) when family 37575 shows an increase in LFE rate and family 55115 does not. We also see evidence of different responses over time for the two families with the highest number of detections, families 55432 and 60070. For family 55432, the majority of events occur closely in time, with a noticeable rate increase after the September 2011 tremor episodes (with more than $20 \%$ of the family's constituent events occurring in the following 9 months). In contrast, family 60070, exhibits more evenly distributed rate increases over the 8-yr period with strong response to the July 2009 Dusky Sound (Beavan et al., 2010) and September 2010 Darfield earthquakes (earthquakes numbers 1 and 2 in Figs. 3.1 and 3.3) and limited response to the 2011 Christchurch earthquakes (earthquakes numbers 3 and 4 in Figs. 3.1 and 3.3; Holden, 2011; Bannister and Gledhill, 2012). Family 30441, on the other hand, has the lowest number of detections and seems to behave more linearly over time, with an almost-constant rate of 1 LFE per week.

Despite systematically improving of our signals between iterations, we still observe some substantial differences between families in the final LFE stacks (Figs. 3.10 and 3.11). By comparing the waveforms (white seismograms in Figs. 3.10 and 3.11) of one of our families possessing the highest number of detections (family ID: 60070; 1,080 detections; Fig. 3.10) with the family possessing the lowest number of detections (family ID:30441, 415 detections; Fig. 3.11), we find that family ID:60070 shows clear LFE S-wave arrivals between $50 \mathrm{~s}$ and $60 \mathrm{~s}$ across all stations in Fig. 3.10 whereas the waveforms are distinctly more emergent for family 
ID:30441 in Fig. 3.11. Those two families are however slightly more consistent when looking at spectral peaks. We can see that the frequency content is mostly comprised between $1-10 \mathrm{~Hz}$, with clear spectral peaks (in red in Figs. 3.10 and 3.11) corresponding to the LFE S-wave arrivals. However, we note that for families with lower signal to noise ratios, e.g. family ID:30441, the frequency peaks in the $2-8 \mathrm{~Hz}$ band and clear S-wave arrivals are not seen systematically across all stations rendering the picking of arrival times and polarities slightly less accurate.

\subsubsection{LFE locations}

LFEs occur beneath the southern portion of the SAMBA network, mostly confined to an area southwest of Aoraki/Mt Cook. They are distributed within an area of $54 \times 32 \mathrm{~km}$ and a $25 \pm 2 \mathrm{~km}$ depth extent (Table 3.1 and Fig. 3.12). The fourteen LFE families locate below the seismogenic zone defined by regular microseismicity (0-10 $\pm 2 \mathrm{~km}$ as determined by Boese et al., 2012) at depths of 17-42 km. These depths are approximately the same as those reported for tremor by Wech et al. (2012) and the original LFE depths reported by Chamberlain et al. (2014). Some tremor appears to occur deeper than the LFEs detected in this study (blue circles vs coloured stars in Fig. 3.12). Wech et al. (2012) assumed there to be large uncertainties in their tremor depths due to their epicentral positions and to the geometry of the seismic network, which could explain the differences with our results. Another possibility is that the fourteen LFE families represent an incomplete sample of low-frequency earthquake activity in the central Southern Alps and thus we could be missing some deeper tremor occurring in the area. We consider this a less-likely explanation given (1) the much longer time interval analysed here than analysed by Wech et al. (2012) and (2) the fact that our original LFE templates were taken from tremor periods detected by Wech et al. (2012).

The locations of the LFEs we have computed concur with the interpretation made by Chamberlain et al. (2014) that almost all LFE families are located on or near the proposed deep extent of the Alpine Fault inferred from GPS measurements (Lamb and Smith, 2013), deep seismic reflectors (Okaya et al., 2007) and activeand passive-source tomography (Stern et al., 2001; Feenstra et al., 2016).

The majority of the LFEs are clustered around the inferred change in dip of the Alpine Fault from a steep $\left(50-60^{\circ}\right)$ stably sliding fault in the upper crust to a moderately dipping $\left(15-30^{\circ}\right)$ and partially locked fault in the lower crust (the dashed and full black lines in Fig. 3.12, respectively; Lamb and Smith, 2013; Feenstra et al., 2016). 


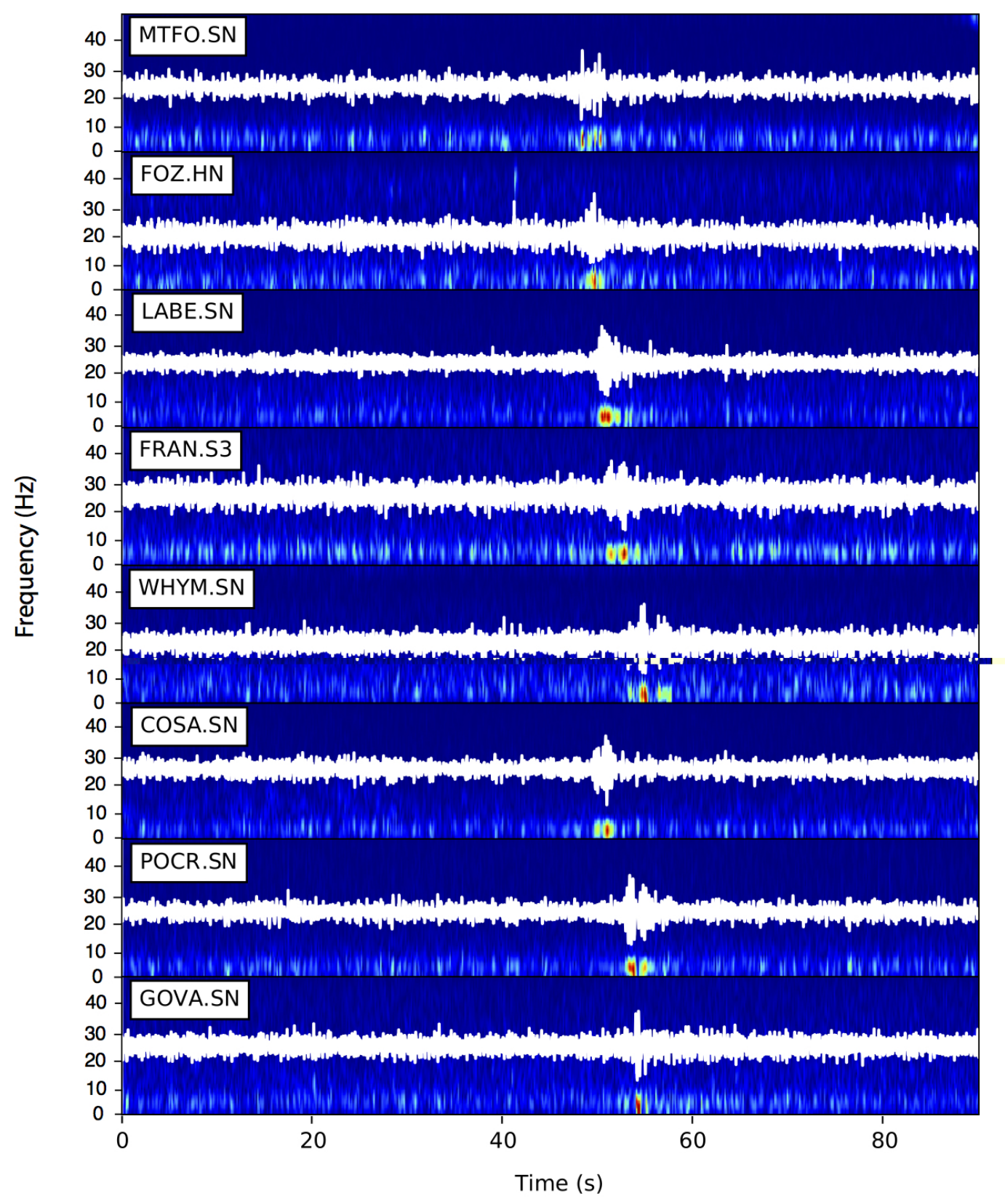

Figure 3.10: Example waveforms and spectrograms for the final stack of family ID:60070. We are showing here the spectrograms and waveforms (in white) for a family with a high signal-to-noise ratio. Waveforms are resampled to $100 \mathrm{~Hz}$ and filtered between $2-49 \mathrm{~Hz}$ to remove long-period noise on the broadband sites, while retaining signal outside the tremor band to show the consistent dominant energy in the $2-8 \mathrm{~Hz}$ band. There are clear spectral peaks between 2 and $10 \mathrm{~Hz}$, which is characteristic of low-frequency earthquakes activity. 


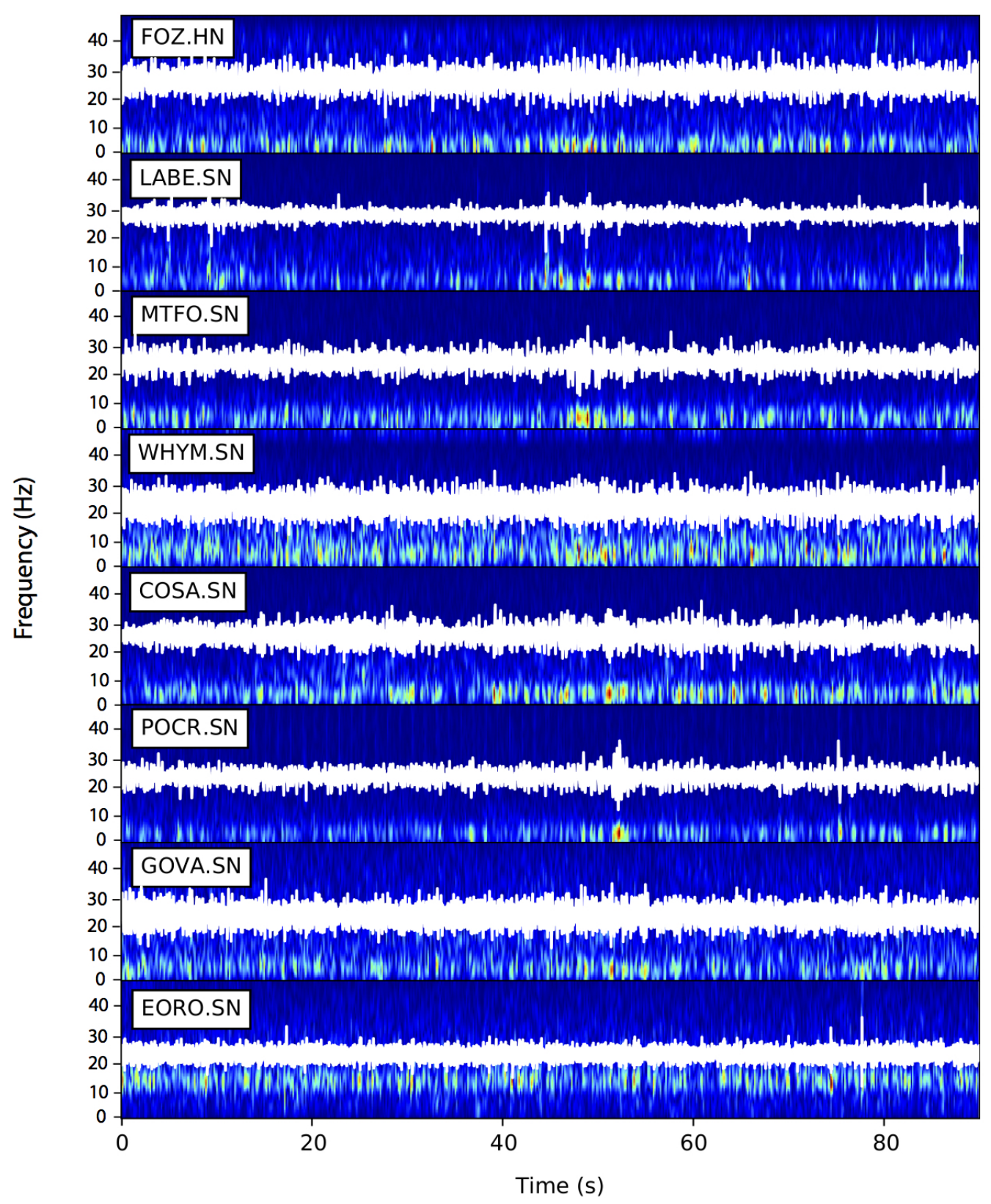

Figure 3.11: Example waveforms and spectrograms for the final stack of family ID:30441. We are showing here the spectrograms and waveforms (in white) for a family with a low signal-to-noise ratio. Waveforms are resampled to $100 \mathrm{~Hz}$ and filtered between $2-49 \mathrm{~Hz}$ to remove long-period noise on the broadband sites, while retaining signal outside the tremor band to show the consistent dominant energy in the $2-8 \mathrm{~Hz}$ band. Clear spectral peaks between 2 and $10 \mathrm{~Hz}$ can still be seen on some channels, but the emergent waveform characteristic of a low-frequency earthquake is not that easily seen. However the spectral peaks provide indications to determine the timing of the LFE S-wave arrival. 


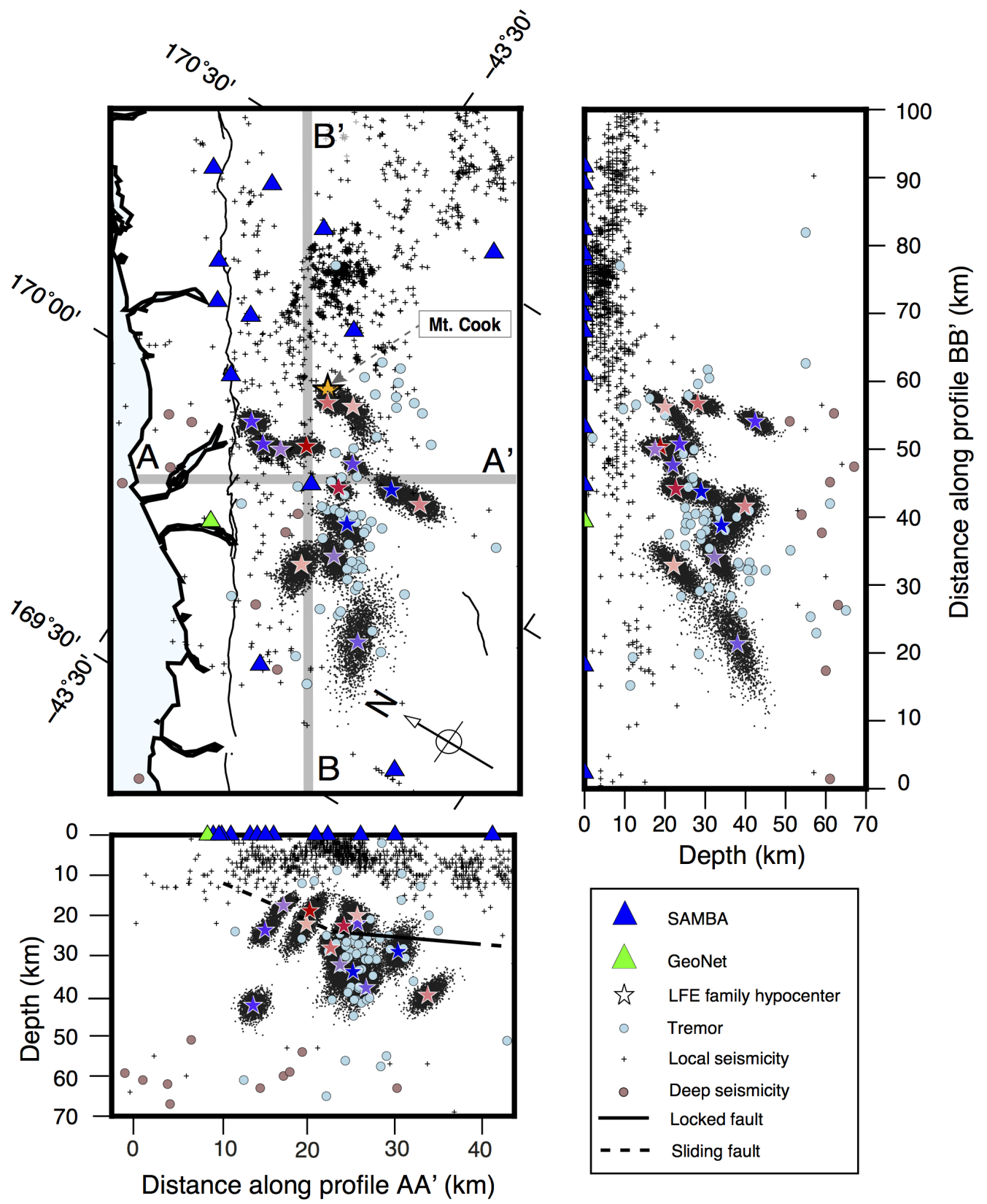

Figure 3.12: NonLinLoc LFE hypocentres of the 14 families identified in this study, with uncertainties plotted as black cloud of probability density function (68\% confidence level). Local seismicity (Boese et al., 2012), deep seismicity (Boese et al., 2013) and tremor (Wech et al., 2012) are plotted with the symbols shown in the legend. LFE hypocentres are coloured by average LFE detection rates, according to the legend in Fig. 3.3. Top left: rotated map view. Top right: cross section, parallel to fault, along profile BB'. Bottom left: cross section, orthogonal to fault, along profile AA', with a GPS modelled fault after Lamb and Smith (2013). 
We note that LFE families lying within $5 \mathrm{~km}$ of the inferred position of the Alpine Fault typically exhibit higher rates of LFE generation (warm coloured stars in Fig. 3.12) than families located further away from the fault. Moreover, the same LFE families appear to exhibit more marked short-term increases in activity, commonly coinciding with known periods of tremor or large $\left(M_{w}>5\right)$ regional earthquakes (Fig. 3.3). This suggests that LFE families located closer to the fault produce more bursts (i.e. with an inter-event time below 2 minutes) and are thus more susceptible to triggering by transient stresses.

\subsubsection{LFE focal mechanisms and stress estimate}

We have been able to constrain the focal mechanisms of eight of the fourteen LFE families (Fig. 3.13 and Table 3.1 for strike/dip/rake values). Most focal mechanisms are poorly constrained due to the small number of picks arising from a combination of the low signal-to-noise ratios of the LFE waveforms and the observing network's geometry. (Figs. 3.13 and 3.14). The majority of mechanisms have one nodal plane striking subparallel to the Alpine Fault and exhibit dextral strike-slip motion with subordinate reverse slip. This indicates that LFEs involve motion consistent with shear on the deep extent of the Alpine Fault. We have managed to estimate the focal mechanism of one LFE family (61100) occurring on the northwest, hanging-wall side of the Alpine Fault (event ID:4 in Figs. 3.13 and 3.14). This LFE family has a focal mechanism with a steeply northeast-dipping plane ( $\sim 80^{\circ}$ dip) exhibiting mostly dextral strike-slip motion, and is consistent with the suggestion by Norris and Toy (2014) of the existence of partitioned displacement in the lower crust between a steeply-dipping branch of the Alpine Fault, and a flat-lying detachment to the east. However, the determination of more LFE focal mechanisms in this region is needed to confirm this hypothesis.

Focal mechanisms compatible with shear slip on the central Alpine Fault are expected to exhibit thrust and oblique strike-slip mechanisms, with one nodal plane striking subparallel to the Alpine Fault ( $\left.55^{\circ}\right)$ (e.g. Boese et al., 2012). However, two mechanisms in this study (family IDs: 55432 and 59966, respectively event IDs:3 and 8 in Figs. 3.13 and 3.14) exhibit dextral transtension, on the nodal plane that is compatible with an expected Alpine Fault earthquake. These two LFEs mechanisms are amongst the ones that possess the highest uncertainties of focal mechanism solutions (with minimum rotation angles between the focal mechanism solutions of $\sim 36^{\circ}$ as defined by Walsh et al., 2009). 


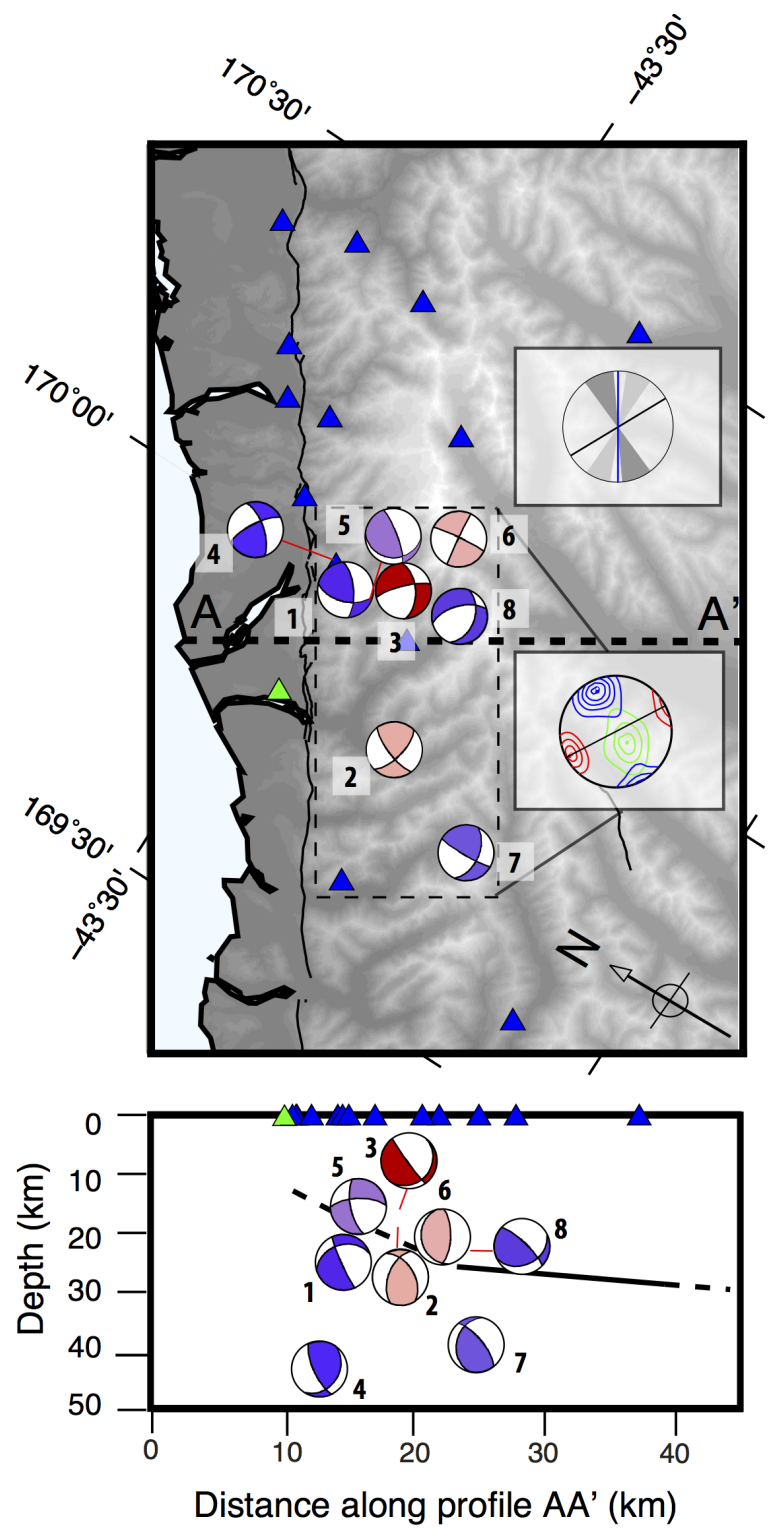

Figure 3.13: Focal mechanism solutions of LFE families. Focal mechanisms are numbered from 1 to 8; each corresponding LFE family is in Table 3.1 and they are coloured by average LFE detection rates, according to the legend in Fig. 3.3. Detailed information about each focal mechanisms is listed in Table 3.1. Top: rotated map view. The upper box shows the stress estimates for the region from Boese et al. (2012), with the azimuth of maximum horizontal compressive stress $S_{\text {Hmax }}=115 \pm 10^{\circ}$ shown in black, as well as the strike of the Alpine Fault $\left(\sim 55^{\circ}\right)$ in blue and its respective thrust and strike-slip segments (in dark and light grey). The bottom box shows the stress estimate from this study with: (1) the principal stress directions: S1, S2 and S3 respectively featured in red, green and blue (trend/plunge: $\mathrm{S} 1=123.6^{\circ} / 7.1^{\circ}, \mathrm{S} 2=35.1^{\circ} / 66.9^{\circ}, \mathrm{S} 3=33.3^{\circ} / 21.9^{\circ}$ ); and $(2)$ the maximum horizontal compressive stress $S_{H \max }=122 \pm 25^{\circ}$ in black. Bottom: cross section, orthogonal to the Alpine Fault, along profile AA', with a GPS modelled fault after Lamb and Smith (2013). 
We also remark that family ID:55432 (event ID:3 in Figs. 3.13 and 3.14) is obtained with the lowest number of $\mathrm{P}$-wave polarity picks (5 picks). A possible explanation for the computation of this dextral transtension mechanism could be linked with the paucity of available picks for this event. Family ID:59966 (event ID:8 in Figs. 3.13 and 3.14) is obtained with the highest number of picks, however the focal sphere coverage is poor for this particular event; thus making it a poorly constrained event.

The prevailing directions of the principal stresses and the maximum horizontal compressive stress (SHmax) obtained from inverting our 8 focal mechanisms is shown in Fig. 3.13. We observe a NW-SE maximum compressive stress direction with a value of $S_{H \max }=122 \pm 25^{\circ}$ at the $80 \%$ confidence interval. We also notice a slight overlap of the contours of S2 and S3 (Fig. 3.13).

The direction of $S_{H \max }$ is consistent with previous stress measurements from the region which show an average value of $S_{H \max }=115 \pm 10$ at the $80 \%$ confidence level (Boese et al., 2012, and references therein). However, since our inversion was done using a low variety of focal mechanisms resulting from our small number of events, the derived uncertainties in tectonic stress parameters are substantial.

Boese et al. (2012) showed that the Alpine Fault as a whole is oriented at an angle of $59^{\circ}$ to the direction of maximum horizontal compressive stress (Fig. 3.13) and that the high topography in the central Southern Alps has little effect on the overall stress field. Here, we find that the central part of the Alpine Fault is oriented at a high angle of $67^{\circ}$ to the direction of maximum horizontal compressive stress, thus suggesting high normal stress acting on the fault plane.

Even though our LFEs occur deeper than the micro-earthquakes and larger regional earthquakes used for previous stress inversions in the central Southern Alps (both of which are mostly confined to the crust, Boese et al., 2012, and references therein), no significant differences in stress orientations are observed. The stress field obtained from shallow crustal earthquakes also appears to be relatively uniform in neighbouring regions of the central Southern Alps (e.g. Southern Lakes region: $S_{H \max }=114 \pm 10^{\circ}$ at the $95 \%$ confidence level, after Warren-Smith et al., 2017). We see no rotation of stress with depth here as opposed to observations of Boese et al. (2013) that showed a rotation in principal stress orientations in the central Southern Alps (of $60^{\circ}$ around the maximum compressive stress) for sub-crustal earthquakes ( $47-74 \mathrm{~km}$ depth, hence deeper than LFEs). This is not entirely unusual as LFE mechanisms and their stress estimates have previously been shown to correlate with those of typical seismicity and to match the expected 
stress regime in a specific area (e.g. Ide et al., 2007b; Frank et al., 2013).
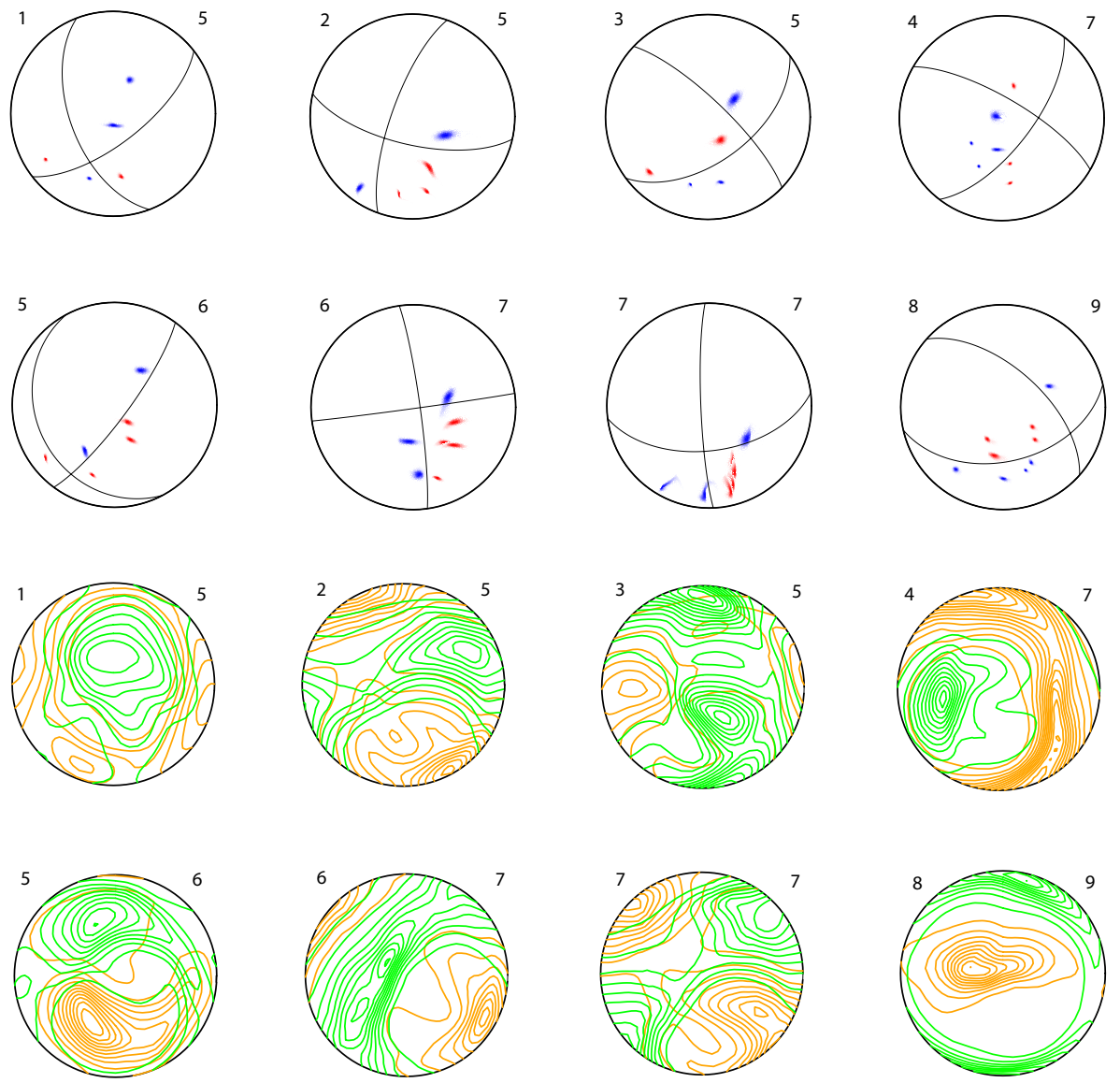

Figure 3.14: Focal mechanism solutions and principal stress directions. Focal mechanisms are numbered from 1 to 8 on the left side and each corresponding LFE family is in Table 3.1. The number on the right side of the mechanism solution represents the number of polarity picks used in the computation. Detailed information about each focal mechanism is listed in Table 3.1. Top: P-wave polarities and focal mechanisms solutions for our $8 \mathrm{LFE}$ families. Bottom: Probability density contours of P- (orange) and T-axes (green) of the focal mechanism solution for our 8 LFE families. 


\subsection{Discussion}

\subsubsection{Catalogue improvements}

Several key analytical steps, taken in order to improve our final stacks and overall catalogue in comparison with those of Chamberlain et al. (2014), are summarised below:

(1) We used a new Python package to cut down the processing time and generate our catalogue faster (Chamberlain et al., 2017c);

(2) We optimised our codes to run them on a supercomputer based in Auckland, the NeSi Pan Cluster (detailed in Section 2.2.4), to further cut down the processing time - from 3-4 weeks to a couple of days to generate a full catalogue;

(3) We thoroughly tested the sensitivity of several parameters (e.g. the number of detections kept for each stack, the minimum number of stations used for a detection, the detection threshold) to find the optimal ones - this process was made easier as the overall running time had been significantly cut down;

(4) We used the entire available dataset (8 years of data) to increase the total number of high signal-to-noise ratio detections utilised in the stacks;

(5) We retained the best $20 \%$ of detections in our stacks (as opposed to the best $10 \%$ of detections for Chamberlain et al. (2014) - which amounted to only 30 detections in some cases). The testing undertaken for this study has shown that to retain a stack with a sufficiently high signal-to-noise ratio, a minimum of at least 50 detections had to be used;

(6) We used phase-weighted stacking in the style of Thurber et al. (2014) instead of linear stacking for our final iteration stack, which provided an overall more impulsive signal.

Each LFE family in our extended catalogue detects between $\sim 300$ and $\sim 1,300$ over the eight-year study period (Table 3.1). In comparison, Chamberlain et al. (2017c) obtained final numbers of detections varying between $\sim 200$ and $\sim 1,700$ over a three-year study period (from March 2009 to April 2012 - equivalent to the first three years of our study period). We assume that using higher signalto-noise ratio LFE templates in the matched-filter routine results in fewer false 
detections, thus explaining why our final number of detection for each family is not significantly higher than those of Chamberlain et al. (2014), even though ours were obtained on a much longer study period. Overall, we detected more events than Chamberlain et al. (2014) for most of our LFE families. Two LFE families (IDs: 37575 and 61044) detected fewer events in this study than in the original analysis by Chamberlain et al. (2014). A possible explanation for this is that those excessive detections were lost here as they constituted false LFE detections. It is likely that the templates used by Chamberlain et al. (2014) had been contaminated by aftershocks following large regional earthquakes (e.g. Mw 7.8 July 2009 Dusky Sound earthquake). This contamination effect would be muted here, as the stacks for subsequent iterations of LFE templates are generated using detections from a much longer study period.

By using the iterative cross-correlation and stacking routine we are able to increase the number of detections for most families, and the average signal-to-noise ratios of all families (e.g. Figs. 3.8 and 3.9). We found this method to be stable between iterations, with each LFE family stacks systematically converging toward more coherent signals (e.g. Fig. 3.9). This appears to be a significant improvement from the original LFE catalogue where only a couple of families were consistent between iterations (e.g. family IDs:17208 and 55115 in Appendix A.1).

Our extended catalogue yields highly improved stacks in comparison with the original catalogue of Chamberlain et al. (2014), as seen in Fig. 3.15, which allows us to pick reliably most S-wave arrivals as well as many $\mathrm{P}$-wave arrivals and polarities - an essential step in the obtention of accurate locations and reliable focal mechanisms.

Finally, our individual LFE families' epicentres on the whole differ from those computed by Chamberlain et al. (2014), and the events are located $4 \mathrm{~km}$ deeper on average (Fig. 3.12). However, if we consider the location of the highest signalto-noise ratio LFE family (17208) published by Chamberlain et al. (2014), we find it to be similar to the corresponding hypocentre found here $\left(-43.52^{\circ} ; 170.06^{\circ}\right.$; $28 \pm 2 \mathrm{~km}$ in the first case, versus $-43.57^{\circ} ; 170.11^{\circ} ; 28 \pm 3 \mathrm{~km}$ in the second, corresponding to an epicentral difference of $6.8 \mathrm{~km}$ ). We therefore infer that the differences in location observed for the 14 families as a group arise mainly from the use by Chamberlain et al. (2014) of low signal-to-noise ratio linear stacks. Notwithstanding this, the uncertainties we compute are slightly larger than those obtained by Chamberlain et al. (2014). This can be explained by our use of a relative measure of uncertainty (or weight) applied to the picks. The use of a pick-weighting parameter was made possible by the generally high signal-to-noise 
Family ID: 60070
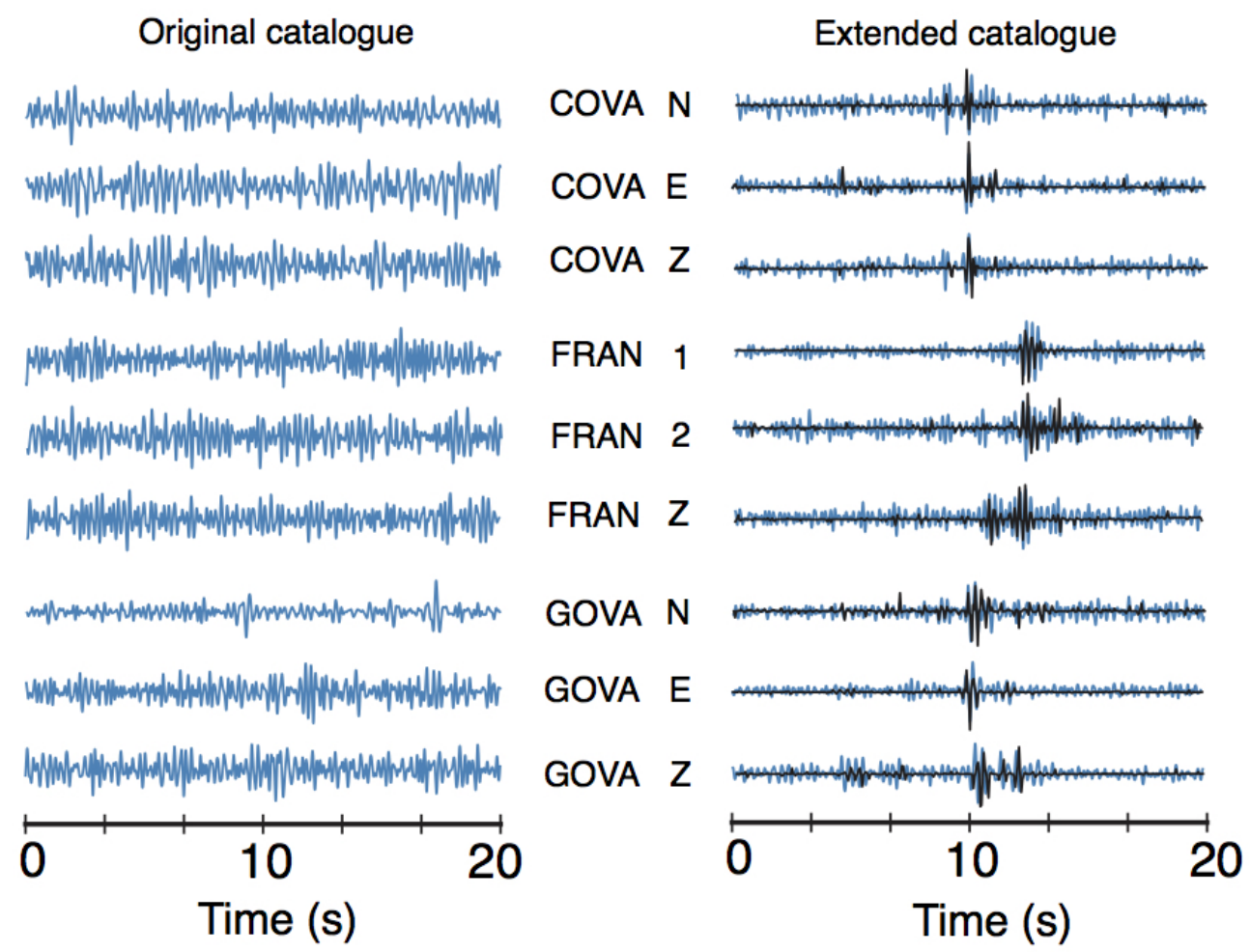

Figure 3.15: Comparison between stacks for the original catalogue of Chamberlain et al. (2014) and the extended catalogue (this study). Linear stacks of LFE family ID:60070 for the last iteration of the matched-filter are plotted for all three components for stations COVA, FRAN and GOVA. All traces are normalised by their respective absolute maximum amplitude. Left: Linear stacks for the short original catalogue (Chamberlain et al., 2014). Right: Linear stacks (in blue) and phase-weighted stacks (in black) for the extended catalogue. The extended catalogue yields better stacks for the linear and phase-weighted methods and both stacks are coherent (1) across channels and (2) between themselves. With the extended catalogue, all of the 14 families show some coherence across channels. That was not the case for the original catalogue, where only $50 \%$ of the LFE families showed some sort of coherency across channels.

ratios of the templates, which enabled us to estimate a confidence for each pick and downweight ambiguous phase arrivals accordingly. This weight was then included in the NonLinLoc locations, resulting in broader probability density distributions. Despite the location uncertainties being somewhat larger than those of Chamberlain et al. (2014), our locations are more reliable as we have better constrained the uncertainties, and therefore they are more realistic. 


\subsubsection{LFE interevent timing and triggering}

We have documented nearly constant rates of LFE generation throughout the 8-yr study period, interspersed with sudden increases in rate coinciding with episodes of tremor and/or large regional earthquakes (Fig. 3.16).

All of the tremor periods between May 2009 and September 2011 that were identified by Wech et al. (2012) correspond to abrupt increases in LFE rate (Figs. 3.3 and 3.16). More than $15 \%$ of the LFEs detected in the time period 2009-2011 (previously analysed by Wech et al., 2012) occurred on days containing tremor. We also observe substantial increases in LFE rate outside that period that do not clearly correspond to triggering by regional seismicity. We interpret those bursts to be newly detected tremor periods (black arrows in Fig. 3.16). Further analysis is required to confirm this interpretation but is beyond the scope of this paper.

We observe no clear threshold in the earthquake energy density (defined after Wang and Manga, 2010, as the maximum seismic energy available in a unit volume) above which LFEs respond and below which they do not (Fig. 3.16). For example, if we examine periods with the highest regional earthquake energy density, we notice five episodes (green arrows in Fig. 3.16) with an earthquake energy density above $10^{-11} \mathrm{~J} \mathrm{~m}^{-3}$. Of these, one period (May 2015) does not visibly incite an LFE response. Of the four remaining periods, the one with the lowest earthquake energy density value (July 2009) actually incites the strongest LFE response. It is also interesting to point out that the average earthquake energy density in July 2009 is only slightly less (by a factor of 0.9) than that in May 2015 when no response in LFEs count can be seen.

Notwithstanding the quasi-continuous activity observed for the LFE catalogue as a whole during the 8-yr study period, detrended cumulative counts and interevent times show significant variations between individual families (Figs. 3.16 and 3.17). In general, more productive families exhibit more burst-like behaviour (e.g. family 60070 in Figs. 3.16 and 3.17). However, two families generating almost the same number of events, families 59966 and 55200 (located $12 \mathrm{~km}$ away from each other), do not behave similarly in time (Figs. 3.16 and 3.17), with family 59966 detecting more bursts than family 55200. During the last iteration of the matched-filter routine, we even observed some families with close to no burst detections (e.g. family 55200 in Fig. 3.17). In most cases, however, the process of iterating through the matched-filter routine increased the number of bursts detected over the study period. 


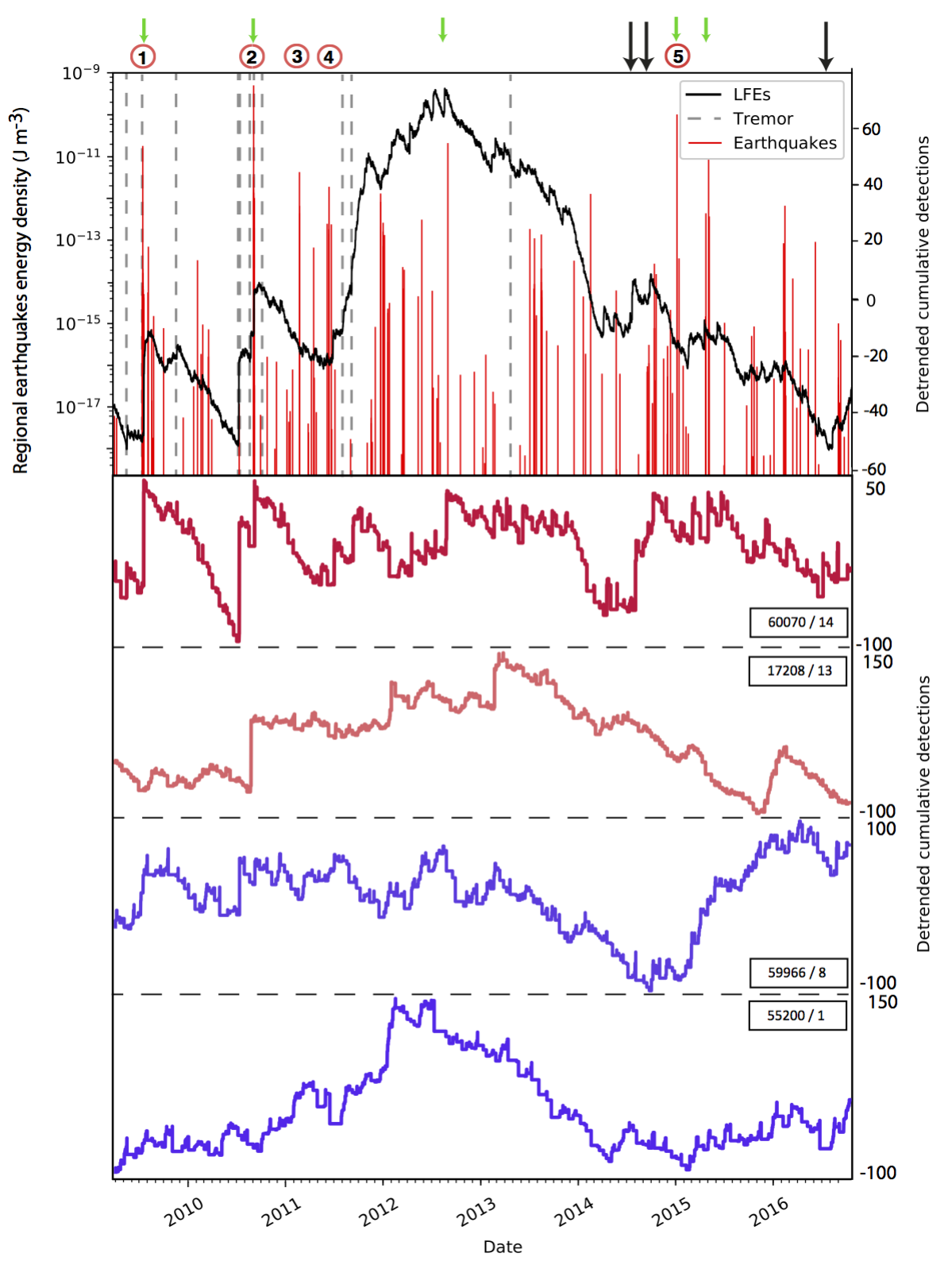

Figure 3.16: Detrended cumulative LFE detections and regional earthquake energy density. Top: The detrended cumulative detections are plotted alongside tremor events (grey dashed lines) identified between 2009 and 2011 by Wech et al. (2012) and possible new tremor periods (black arrows at the top). The numbers refer to big regional earthquakes, located in Fig. 3.1. The green arrows correspond to periods with the highest regional earthquake energy density, respectively July 2009, September 2010, September 2013, January 2015 and May 2015. The energy density is calculated for all earthquakes in the GeoNet catalogue for a previously determined LFE source region after Wang and Manga (2010) (see red box in Fig. 3.1). Bottom: Detrended cumulative detections, respectively for families 60070, 17208, 59966, 55200. Each family is coloured with its average long-term LFE detection rate with regards to legend in Fig. 3.3. 

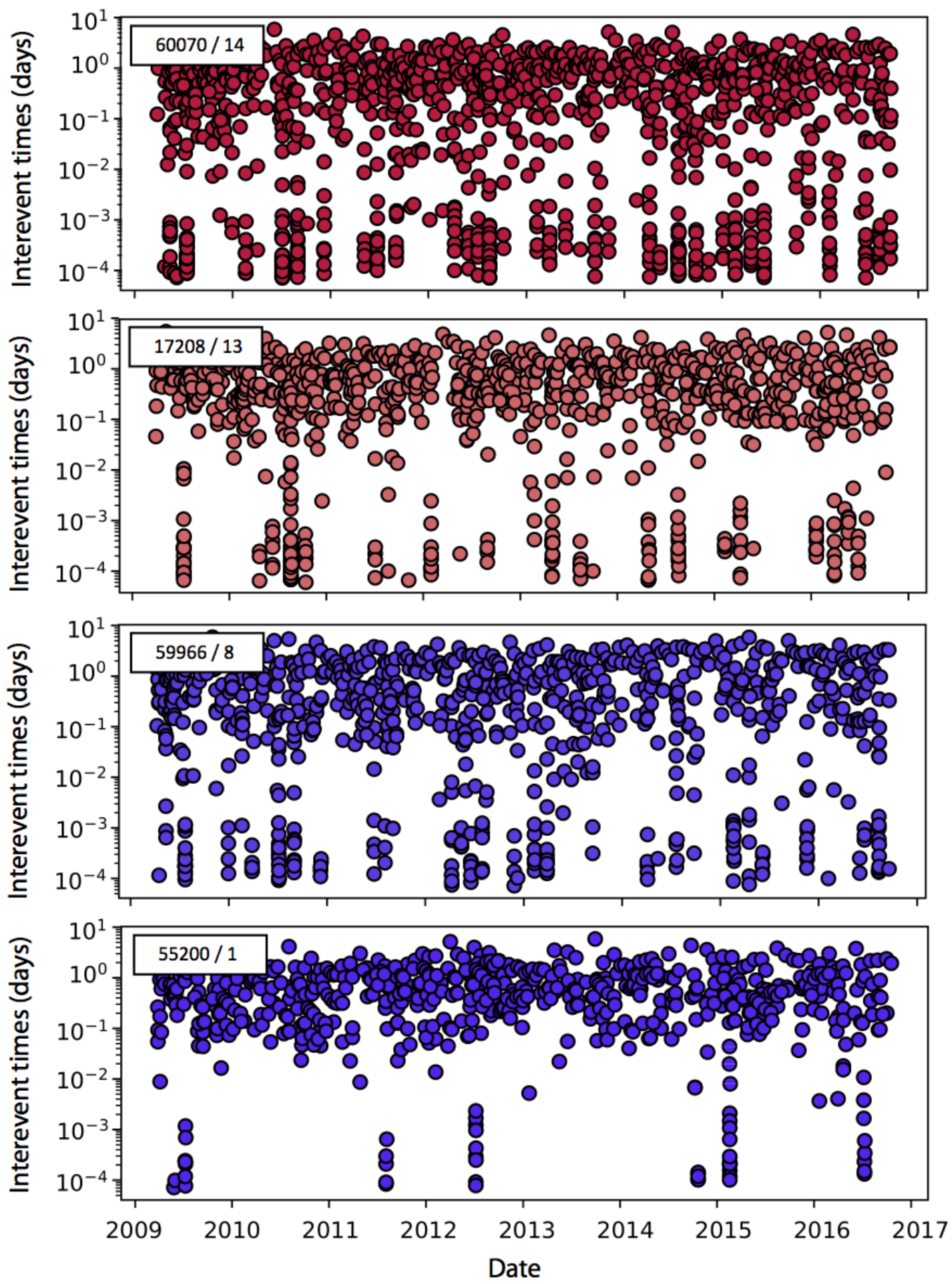

Figure 3.17: Inter-event times for a subset of LFE families. Inter-event times during the last iteration of the matched-filter, respectively for families 60070 , 17208, 59966, 55200. Each family is coloured with its average long-term LFE detection rate with regards to legend in Fig. 3.3. 
Several large regional earthquakes $\left(M_{w}>5\right)$ occurred during the 8-yr study period (Figs. 3.3, 3.16 and 3.18), some of which coincided with increases in LFE activity rates. The highest increase in LFE rate concomitant with a large regional earthquake happened near the time of the 15 July $2009 M_{w} 7.8$ Dusky Sound earthquake (Beavan et al., 2010, earthquake number 1 in Figs. 3.1, 3.3 and 3.16). Wech et al. (2012) identified tremor occurring on the 14 and 15 July 2009, and in this study we detected over 200 LFEs on the 14 July 2009, followed by an additional 100 events within the following two days (Fig. 3.18). This suggests positive feedback between typical earthquakes (i.e. large regional earthquakes) and the generation of tremor and LFEs over distances of $\sim 200 \mathrm{~km}$.

LFEs appear to react differently to earthquakes belonging to the same mainshock/aftershock sequence (and thus occurring in similar positions relative to the LFEs). In addition, we observe distinct responses within individual LFE families with regards to a particular event (middle plots in Fig. 3.18). An example of this is the 2010-2012 Canterbury earthquake sequence that commenced with the 4 September $2010 M_{w} 7.1$ Darfield earthquake, followed by the 22 February 2011 $M_{w} 6.2$ and 13 June $2011 M_{w} 6.0$, Christchurch earthquakes (earthquakes numbers 2-4 in Figs. 3.1, 3.3 and 3.18; Holden, 2011). Following the 4 September 2010 $M_{w} 7.1$ Darfield earthquake (Fig. 3.18a), fewer than 20 detections were made on the day of the earthquake and just over 30 detections in the following 10 days (with only three LFE families: 17208, 55115 and 61220, seemingly reacting directly to the earthquake). In contrast, we did not notice an increase in LFE activity in the days preceding the 22 February $2011 M_{w} 6.2$ Christchurch earthquake (Fig. 3.18b). However, we observed a somewhat muted increase (relative to the Darfield earthquake) in response to this event, with $\sim 20$ LFEs detected in the two days following the event (with minor responses from LFE families: 55432, 60070, 60905 and 59966). In comparison, a slightly more pronounced response was shown to the 13 June $2011 M_{w} 6.0$ Christchurch earthquake (Fig. 3.18c) with $\sim 20$ LFEs detected on the day of the event alone, with families 55432, 61044 and 61100 especially reacting to the event. No particular reaction is seen in the LFE activity rates besides a possibly weak response from family 61100 , following the 6 January $2015 M_{w} 6.0$ Arthur's Pass earthquake (Fig. 3.18d), which is the closest to our network of the large regional earthquakes during this period (earthquake no. 5 of Figs. 3.1, 3.3 and 3.16); 39 LFEs were detected in a period of 10 days surrounding the event, consistent with the number expected given the long-term rate. 


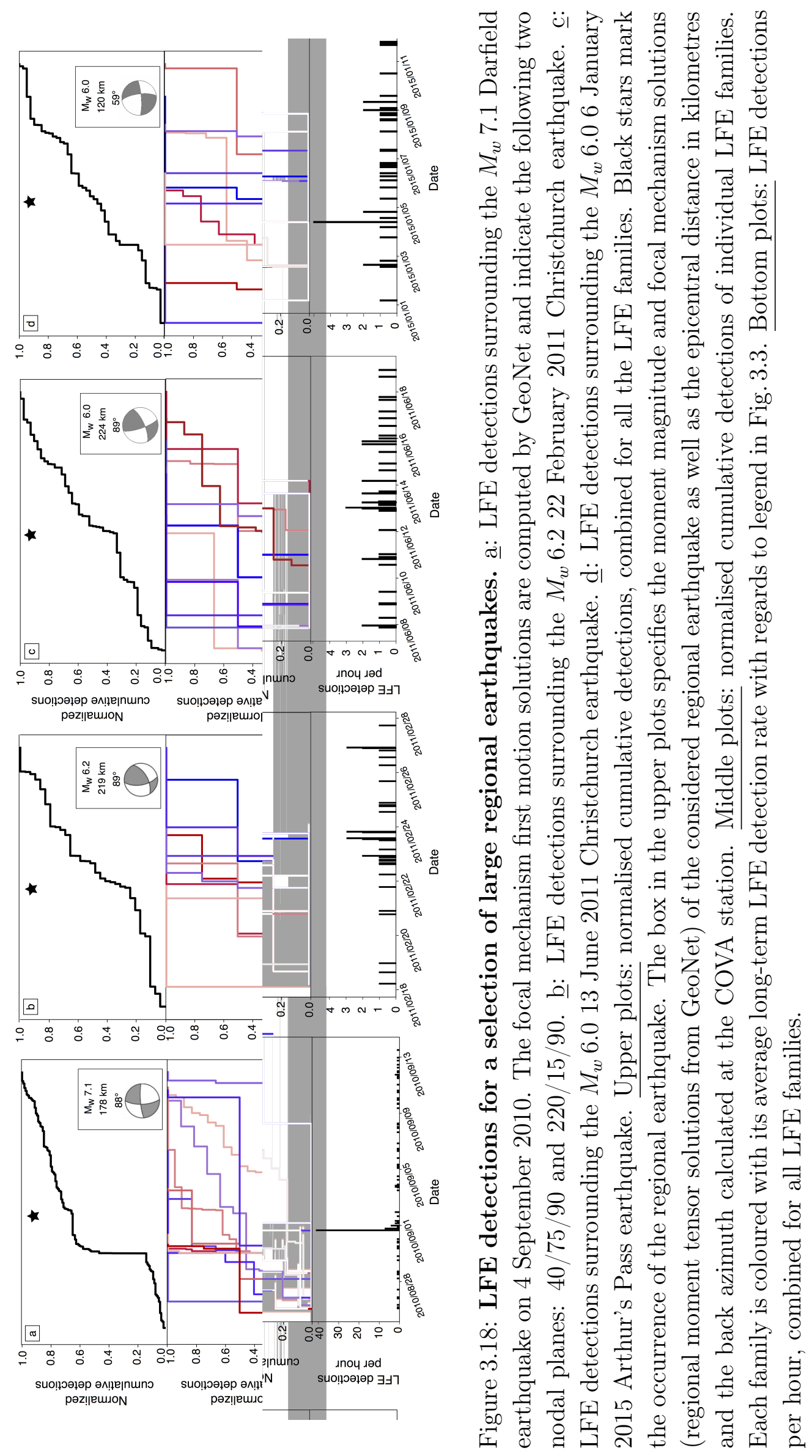


We also notice an interesting behaviour in the days preceding the 4 September $2010 M_{w} 7.1$ Darfield earthquake (Fig. 3.18a). A rapid increase in the number of detected LFEs is observed, with over 70 LFEs being detected in the 3 days preceding the earthquake $(\sim 6$ times more detections than expected for the longterm rate), which we think to be a coincidence.

The sudden increases in LFE rates that we observe just after large regional earthquakes, may be indicative of LFE triggering. However, some variations in LFE rates could not be directly related to regional seismicity. This could indicate that either other unrecognised sources of farfield or endogenous triggering are in play. Several studies report tremor and LFEs being highly sensitive to small stress perturbations: (1) triggering of tremor and LFEs by teleseismic waves imposing stress transient of only a few kilopascals (e.g. Gomberg et al., 2008); (2) triggering of tremor by dynamic stresses of Love and Rayleigh passing surface waves (Hill, 2012); (3) strong sensitivity of tremor and LFEs to tidal stress perturbation of fractions of a kilopascal (e.g. Thomas et al., 2009). Additional work is underway in the region to better understand the different behaviours between LFE families and to study tectonic tremor and LFEs triggering in more detail.

We recognise two distinct distributions of LFE inter-event times: (1) isolated events when the inter-event time exceeds 2 minutes; and (2) event bursts when the inter-event time is less than 2 minutes (Fig. 3.19). We also note a gap in detections at around 15 minutes' inter-event time that could be explained by an incomplete spatial representation of LFEs in our catalogue (our original templates being picked manually through periods of tremor located exclusively in an area south of Mt. Cook).

A total of $21 \%$ of the entire catalogue is formed of LFE bursts. Overall, $3 \%$ of detections have inter-event times lower than $9 \mathrm{~s}$ (with the minimum inter-event time within individual families being fixed at $6 \mathrm{~s}$ ). The clustering of inter-event times around a few seconds typically coincides with tremor periods or large earthquakes (arrows in Fig. 3.19). However, the majority of the events in our catalogue ( $\sim 79 \%$ of total detections) exhibit discrete behaviour, with large inter-event times (of between a couple of days and 15 minutes for $\sim 73 \%$ of total detections and between 15 minutes and 2 minutes for $\sim 6 \%$ of total detections).

Discrete episodes of LFEs in subduction zones have been interpreted using concomitant geodetic observations as infrequent small-scale ruptures, occurring without larger-scale slow slip on an otherwise locked region of a fault (Frank, 2016). However, the occurrence of LFE bursts in conjunction with large episodes of slow 


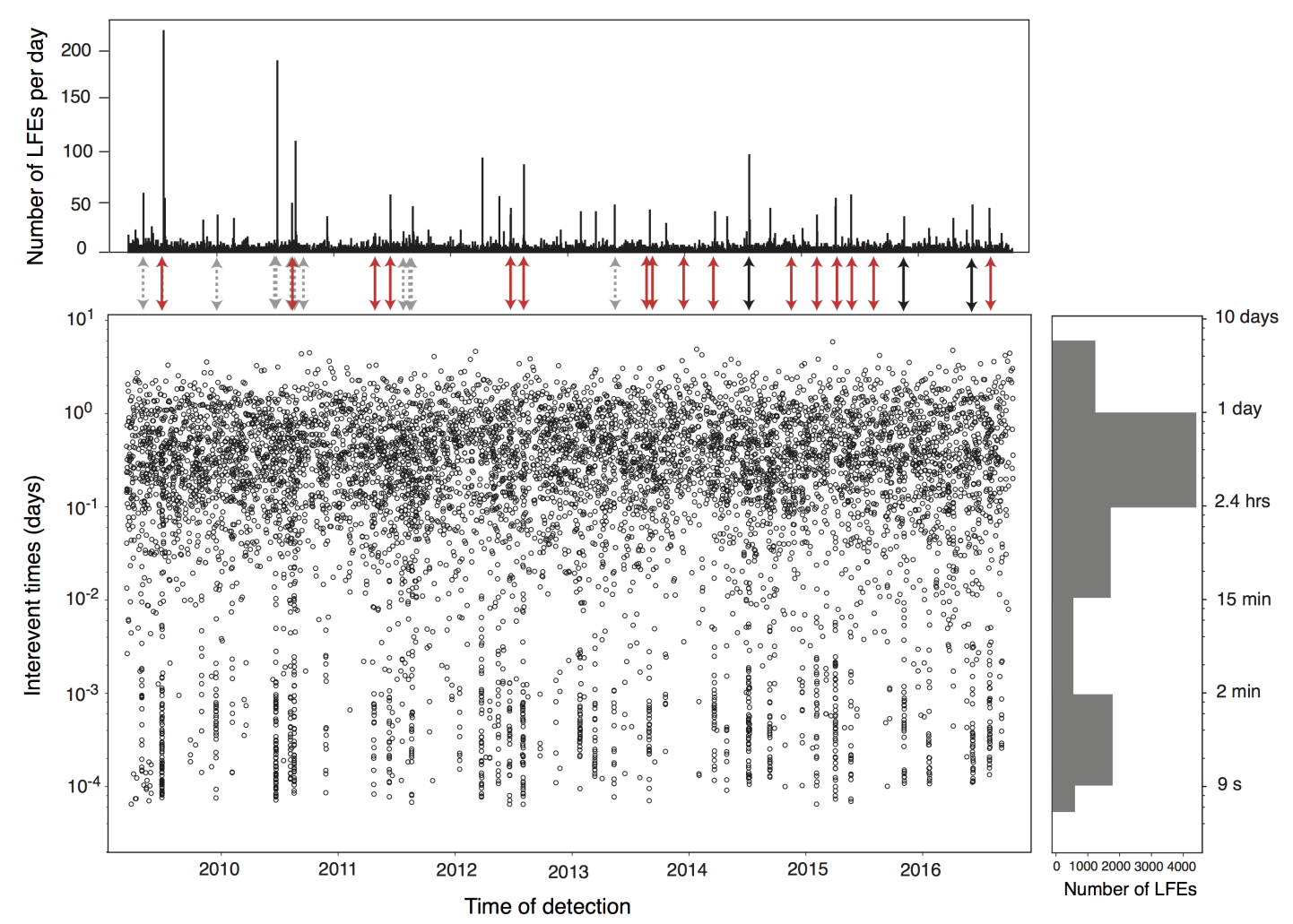

Figure 3.19: Temporal evolution and inter-event times of LFEs. Dashed grey arrows represent tremor occurrence (Wech et al., 2012; Chamberlain et al., 2014), red arrows represent large regional earthquakes $\left(M_{w} \geq 5\right)$ and black arrows represent possible new tremor periods. Top: total number of LFEs (all families combined) detected per day over the study period. Bottom-left: inter-event times shown for our entire catalogue (10,000 detected events over our 8-yr study period). Bottom-right: Histogram showing the distribution of inter-event times for our catalogue.

slip could indicate that discrete episodes of LFEs occur at the same time as frequent small-scale and geodetically undetectable slip at depth. Here we suggest that those discrete LFEs might occur simultaneously with slip on localised patches of the fault at depth.

We interpret these discrete events as small-scale frequent slip on the deep extent of the Alpine Fault and the LFE bursts as brief periods of increased slip-rates indicative of episodic slow slip (Frank et al., 2016). However, geodetic evidence is needed to confirm whether slow slip is indeed occurring simultaneously with periods of LFEs activity on the Alpine Fault.

We note that since our catalogue is predominantly composed of events near the detection threshold and located to the southwest of the network where station 
coverage is poor, we may be missing some detections and it is possible that some of those discrete events could actually represent LFE bursts.

\subsubsection{Alpine Fault rheology and geometry}

LFEs are thought to be the seismic manifestation of slow shear slip on crustal faults (Ide et al., 2007b). We suggest that discrete LFEs represent frequent localised slip (e.g. Shelly et al., 2006) on the deep Alpine Fault and that LFE bursts depict times of accelerated and more distributed slip (e.g. Shelly et al., 2007) in the same region. Here, we provide the first direct evidence of shear slip on the deep extent of the Alpine Fault by computing LFE focal mechanisms compatible with such slip.

Geophysical and geological evidence suggests that the geometry of the Alpine Fault changes in the area beneath Mt. Cook from a sub-vertical structure in the north to a listric fault south of Mt. Cook (e.g. Stern et al., 2001; Little et al., 2005; Okaya et al., 2007; Feenstra et al., 2016). Thirteen of the LFE families appear to be clustered around a modelled change in dip of the Alpine Fault and they locate within $15 \mathrm{~km}$ of the GPS inferred Alpine Fault (Lamb and Smith, 2013). All seven focal mechanisms computed for LFEs occurring within this cluster show one nodal plane striking subparallel to the Alpine Fault and most exhibit dextral strike-slip motion with some reverse slip component. This observation verifies the assumptions of Wech et al. (2012) and Chamberlain et al. (2014) that tectonic tremor and LFEs involve motion consistent with shear on the deep extent of the Alpine Fault. The remaining LFE family occurs southwest of the proposed deep Alpine Fault and the focal mechanism for this family is consistent with a steeply-dipping plane. We thus assume that this LFE family occur on a more steeply-dipping branch of the fault, the existence of which is suggested by other studies that propose a partitioning of motion near the base of the crust $(\sim 30 \mathrm{~km}$ depth) between a steep strike-slip shear zone and a flat-lying detachment to the east (Norris and Toy, 2014, and references therein).

This change in the geometry of the Alpine Fault seems to coincide with a change in seismicity. LFEs in the central Southern Alps appear to fill a vertical gap in seismicity, left by both shallow (0-12 km) and deep seismicity (50-100 km) (Boese et al., 2012, 2013; Wech et al., 2012). The LFEs occur at depths at which 'typical' earthquakes have not been detected (Fig. 3.12) in a zone of broad and strong seismic reflectivity (Okaya et al., 2007), low seismic-wave speeds (Stern et al., 2001), high P-wave attenuation (Eberhart-Phillips et al., 2008) and increased fluid 
pressure (Stern et al., 2001; Wech et al., 2012). The region of low velocities and low resistivity has been interpreted previously to be associated with elevated fluid pressure and fault weakness (Stern et al., 2001; Eberhart-Phillips et al., 2008). The presence of high fluid pressure implies a decrease in the effective normal stress, which in turn makes shear failure on a fault possible at lower differential stress.

\subsection{Conclusions}

We have obtained the longest continuous record of LFEs beneath New Zealand's Southern Alps and show using LFE inter-event times and focal mechanisms that the LFEs are the seismic manifestation of quasi-continuous deformation on the deep extent of the Alpine Fault, interspersed with increased rates of deformation corresponding to tremor periods and slow slip. We have provided evidence that LFEs do indeed represent localised slow shear slip on the deep extent of the Alpine Fault: (1) the simultaneous detection of tremor and LFEs; (2) LFE focal mechanisms being indicative of shear slip on the Alpine Fault; and finally (3) LFE locations being consistent with the location of the deep extent of the Alpine Fault inferred by seismic reflection (Okaya et al., 2007), by velocity models (Stern et al., 2001) and by GPS inversion (Lamb and Smith, 2013).

Our results show that LFEs occur almost continuously during the 8-yr study period, with punctuated episodes of increased rates, corresponding to known episodes of tremor or large regional earthquakes. We have located fourteen LFE families and found that they occur at similar depths to tremor $(15-45 \mathrm{~km})$. Of the fourteen families, thirteen lie within $15 \mathrm{~km}$ of the Alpine Fault. The remaining family is located to the southwest of the proposed deep extent of the Alpine Fault.

We have computed the first LFE focal mechanisms associated with the Alpine Fault, or indeed with continental plate-boundaries anywhere. Our results reveal mostly dextral strike-slip motion with a subordinate reverse component suggesting that LFEs occur mainly as shear faulting on the deep extent of the Alpine Fault. We have also determined the focal mechanism of an LFE family occurring to the SW of the Alpine Fault's presumed position at depth. This focal mechanism involves reverse slip on a steeply-dipping fault plane $\left(\sim 80^{\circ} \mathrm{dip}\right)$, with a minor component of dextral strike-slip motion. These kinematics are consistent with the preferred model of Norris and Toy (2014) of a more steeply-dipping western branch of the Alpine Fault. Nevertheless, an extensive search of this region for further 
LFEs and the determination of additional LFE focal mechanisms are required to reinforce this observation.

Further work is underway to extend our catalogue by looking for new LFE families, with the particular aim of better constraining the spatial and temporal evolution of LFEs in the area and the lower-crustal geometry of the Alpine Fault. 


\section{Using synthetic templates to build a spatially and temporally continuous catalogue of low-frequency earthquakes*}

This Chapter summarises the construction of a spatially and temporally continuous low-frequency earthquakes (LFEs) catalogue for the central Southern Alps. To gain insight into deep fault slip behaviour, a detailed study of the spatial and temporal evolution of low-frequency earthquakes (LFEs) is required. Here, we generate a catalogue of LFEs spanning the years 2009 to 2017 using a different technique than that presented in Chapter 3/Baratin et al. (2018) in order to detect LFEs more efficiently. This time 638 synthetic LFE waveforms are used as primary templates in a matched-filter routine. Of those, 529 templates yield enough detections during the first iteration of the matched-filter routine $(\geq 500$ over the 8 -yr study period) and are kept for further analysis. We then use the best $25 \%$ of correlated events for each LFE in a linear stack to create 'real LFE templates' (i.e. made of real LFE events). From there, we run a second and final iteration of the matched-filter routine with the real LFE templates to obtain the final LFE catalogue. The remaining 529 templates detect between 150 and 1,671 events each, totalling 300,996 detections over the 8-yr study period. This technique paired up with phase-weighted stacking greatly increases the number of detected events, roughly thirtyfold when compared to the previous catalogue detailed in Chapter 3/Baratin et al. (2018). Of those 529 LFE families, we manually pick $\mathrm{P}$ and S-arrivals and locate 378. Their depths range between 11 and $59 \mathrm{~km}$ and LFEs locate mainly in the southern part of the network. Individual LFE family rates and occurrence pattern indicate that LFE sources seem to evolve from an episodic or 'stepped' to a continuous behaviour with depth. This transition may correspond to an evolution from a stick-slip to a stable-sliding slip regime. Hence, we propose that the distinctive features of LFE occurrence patterns reflect variations in the in-situ stress and frictional conditions at the individual LFE source

\footnotetext{
*In the process of being submitted to Geochemistry, Geophysics, Geosystems. The version presented in this Chapter is only slightly altered from the one that will be submitted to G-cubed so as to fit in the context of this thesis (i.e. the references to Chapters/Appendices are removed and some Figures are combined and/or moved to the Supporting Information).
} 
locations on the Alpine Fault.

The creation of the synthetic templates described succinctly in Section 4.2.1 of this Chapter was led by Calum Chamberlain and is the subject of a forthcoming publication to Geophysical Journal International. However, the work relating to the creation of the low-frequency earthquakes templates (referred to as 'real' LFE templates), that are used within the detection routine to generate the continuous LFE catalogue presented in this Chapter, was conducted by me as part of this Thesis. Lengthy discussions with William Frank during a 3-week visit to the Massachusetts Institute of Technology have also helped in optimising the generation of this continuous catalogue.

\subsection{Introduction}

Low-frequency earthquakes are characterised by long duration, emergent, and overall low signal-to-noise ratio (SNR) signals which make the analysis of single events difficult. Low-frequency earthquakes have also been observed to occur in a persistent manner with, for example, multiple sources episodically producing large numbers of LFEs within a narrow timeframe (e.g. Shelly et al., 2006; Frank et al., 2014; Lengliné et al., 2017; Baratin et al., 2018). For that reason, the detection of low-frequency earthquakes is undertaken using matched-filter techniques that capitalise on the repeating nature of LFE sources (e.g. Shelly, 2017). Matchedfiltering relies on correlating the waveform of a known event (primary template) with continuous seismic data to detect repeats of the primary template. Hereafter, we refer to all the detections made by a primary template as being part of a single LFE 'family'. A major flaw of the matched-filter approach is its dependence on a-priori information. Matched-filter searches are indeed incapable of detecting events that have not been previously characterised by a primary template event. Without an established catalogue of primary LFE templates, finding new LFE templates to use in the matched-filtering can be challenging.

Our first catalogue for the central Southern Alps used 14 LFE primary templates to detect 10,000 events over $\sim 7$ years (Baratin et al., 2018, cf. Chapter 3). In comparison, Shelly (2017) was able to detect 1 million LFEs over 15 years using 88 LFE templates on the deep San Andreas fault. Frank and Shapiro (2014) detected nearly 2 million LFEs over 2 years using 1,120 LFE templates, in Guerrero, Mexico. Those extensive catalogues have demonstrated the spatially and temporally continuous character of LFE activity. Possessing a spatially and temporally con- 
tinuous LFEs catalogue is primordial to better understand the spatio-temporal characteristics of LFEs. For example, Frank and Shapiro (2014) used their LFE catalogue to geodetically identify repeating transient slip events that were previously hidden within the ambient noise (Frank et al., 2015; Frank, 2016). Shelly (2017) used the relatively high temporal and spatial resolutions of his LFE catalogue to provide new insights in tremor migration, recurrence and triggering by static and dynamic stress perturbations. In order to construct a similar catalogue for the central Southern Alps, a method to identify appropriate LFE templates is needed.

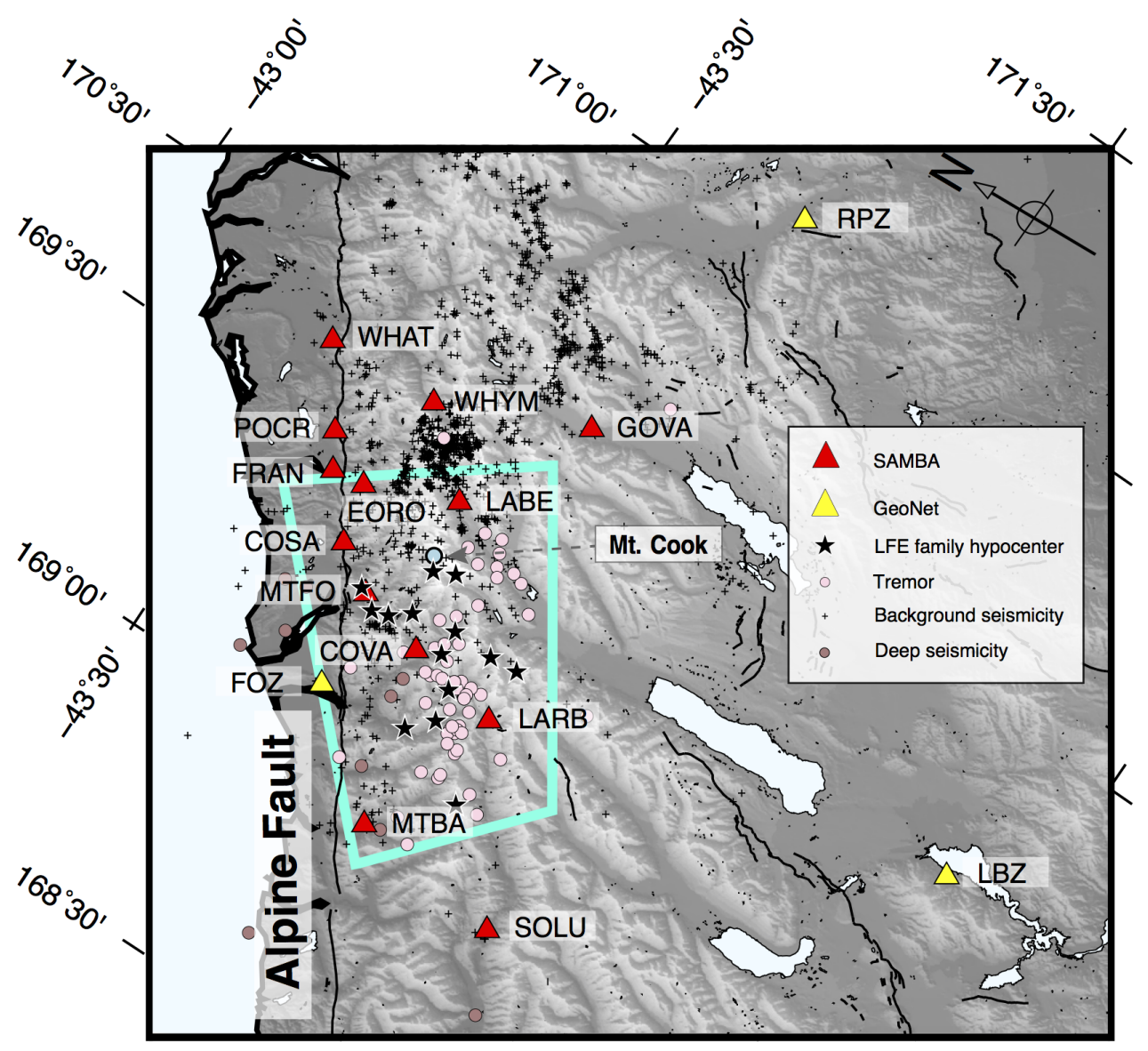

Figure 4.1: Map of study area. Study area in the central Southern Alps showing: the target area for the generation of synthetic LFEs (in cyan); the two networks used here: the SAMBA network (red triangles) and the GeoNet network (yellow triangle); the previous LFE locations (black stars) of Chapter 3/Baratin et al. (2018); the background seismicity (black crosses) of Boese et al. (2012) recorded between 2009 and 2010 and the background seismicity from GeoNet recorded since 2009; the tremor of Wech et al. (2012) recorded between 2009 and 2011 (blue circles) and the deep earthquakes of Boese et al. (2013). 
The common approach to identify LFE templates is through visual inspection of periods of tremor (Shelly et al., 2007; Frank et al., 2013; Chamberlain et al., 2014). However, this method restricts the detection of templates to events that are visible above the tremor signal, and is somewhat a subjective and time-consuming approach. For noisy environments in which tremor is sparse (such as the Southern Alps) this method is not ideal.

A number of other methods can be used to automatically detect new templates:

(1) an auto-correlation method that relies on the repetitiveness of an LFE waveform at a given seismic station (e.g. Brown et al., 2008, 2013; Bostock et al., 2012; Royer and Bostock, 2014);

(2) a cross-station correlation method based on the comparison of waveforms from the same time-window at widely separated stations (e.g. Rubin and Armbruster, 2013; Peng et al., 2015; Savard and Bostock, 2015);

(3) the PageRank algorithm, based on research in data mining, that uses all the temporal links between LFEs to identify time windows that most likely represent LFE signals (Aguiar and Beroza, 2014);

(4) a brightness method relying on the response of the entire seismic network to a theoretical source (Frank and Shapiro, 2014);

(5) a full-waveform detection method based on time-reversal principles (Solano et al., 2017).

All these techniques have their own pecularities that either prevent their applicability or reduce their efficiency in the central Southern Alps. The auto-correlation technique is useful when looking for discrete tremor events, but is computationally costly with a computing time that increases with the catalogue duration (Frank et al., 2014). Hence using this technique to develop a continuous set of LFE templates would be unfeasibly slow for an 8-yr catalogue. The cross-station correlation method relies on cross-station coherence (e.g. Rubin and Armbruster, 2013; Peng et al., 2015; Savard and Bostock, 2015). However, we observe a weak cross-station coherence in the $2-8 \mathrm{~Hz}$ tremor band in the current network configuration, thus making this technique poorly suited to our dataset. The PageRank approach is also inappropriate for the Southern Alps. Indeed, the presence of noisy stations in the currently deployed network results in low SNR for tremor and LFEs and this greatly impedes the detection statistics used in this algorithm. Chamberlain 
(2016) extensively tested the brightness approach of Frank and Shapiro (2014) for the central Southern Alps as part of PhD research at Victoria University of Wellington. He found that it was difficult to distinguish the ambient noise from the actual signal; with the noise often being detected instead of the signal. Finally, the full-waveform detection of non-impulsive seismic events based on time-reversal methods relies on the knowledge of a diversity of LFE mechanisms. Since we are trying to find new LFE events (beyond the 14 LFE families described in Chapter 3/Baratin et al. (2018), for which eight mechanisms were found), this renders the method unsuitable for our study area. Unfortunately, none of these methods are appropriate for the central Southern Alps, thus motivating the use of a different approach to objectively generate multiple LFE templates for this study area.

Here we focus on generating the largest number possible of LFE templates by using synthetic waveforms as preliminary templates. We use 638 synthetic waveforms in a large-scale cross-correlation detection routine, following the approach of Chamberlain (2016). We use data recorded by the Southern Alps Microearthquake Borehole Array (SAMBA) network (Fig. 4.1) between the $26^{\text {th }}$ of March 2009 and the $26^{\text {th }}$ of March 2017 to build a spatially and temporally continuous catalogue of LFEs in the central Southern Alps. We use the synthetic templates as preliminary templates in a first matched-filter iteration. We then stack the events for each LFE family yielding enough detections (amounting to 529 families) to create what we refer to as 'real LFE templates'. The real LFE templates are then used as primary templates in a new matched-filter iteration to generate a spatially and temporally continuous catalogue of LFEs spanning 8 years. Of those 529 LFE families, 378 families yield enough energy to manually pick P- and S-arrivals. We locate those 378 events using the NonLinLoc software of Lomax et al. (2000) and a $3 \mathrm{D}$ velocity model. In the following, we use the term 'real' to refer to recorded data, whereas the term 'synthetic' is used to refer to manually and automatically constructed waveforms.

\subsection{Methods}

\subsubsection{Real LFE template generation}

We use the 638 synthetic templates, described in Chapter 2, Section 2.2.3, as preliminary templates in a large-scale cross-correlation detection routine in order to 
detect real LFE events. We first cross-correlate the synthetic templates continuously through an 8-yr dataset (from 26 March 2009 to 26 March 2017). All 638 families are observed to detect a large number of events, with a number of detections per family varying between $\sim 400$ and $\sim 800$. We then linearly stack the best $25 \%$ of detections for each family. We individually check a subset of families detecting less than $\sim 500$ events over the 8-yr study period and estimate a rate of false detections of $\sim 30 \%$. By looking at the stacks for those families, we see relatively low SNR stacks, with a significant number of false detections being included in the final stacks. Therefore, we decided to keep families that detect more than 500 events during the 8 yr study period for further analysis and are left with 529 families. We also tried stacking the best $10 \%$ and $50 \%$ of detections but found that using $25 \%$ of detections in the stack yielded the best SNR in the stacks. We finally use these stacks as real LFE templates (i.e. LFE templates made from stacking real LFE events) in a new matched-filter iteration. An example of the evolution of an LFE template through the matched-filter process can be seen in Fig. 4.2.

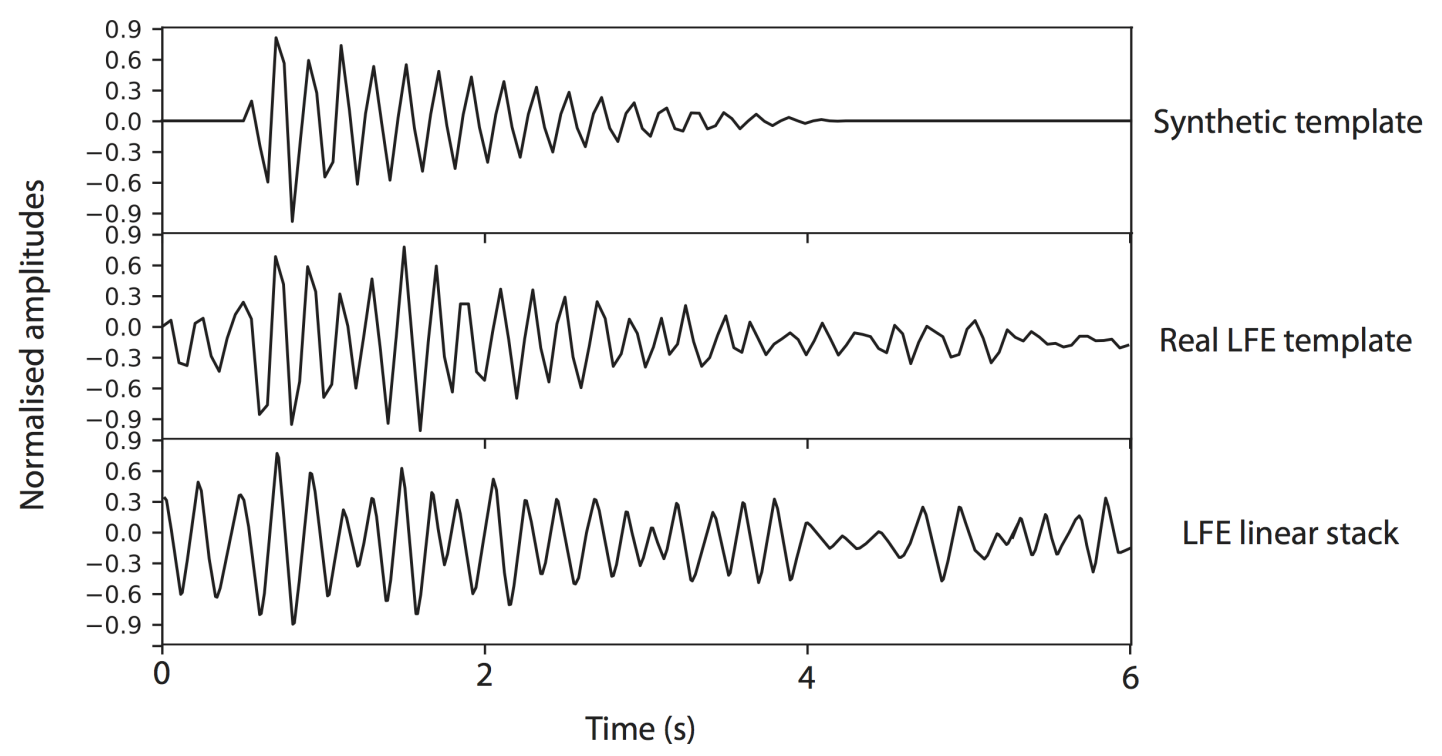

Figure 4.2: Evolution of LFE template ID:169.8_-43.5795_26. Example of the evolution of an LFE template (from top to bottom): LFE synthetic template, real LFE template and the final linear stack for LFE family ID:169.8_$43.5795 \_26$. 


\subsubsection{Catalogue generation}

We employ the waveforms of the 529 real LFE templates as primary templates in a large-scale cross-correlation routine to look for similar events (Fig. 4.3), using the same method as the one described in Chapter 3/Baratin et al. (2018). We employ the EQcorrscan Python package to compute matched-filter detections (Chamberlain et al., 2017c).

Each template and the dataset are resampled to $20 \mathrm{~Hz}$ and bandpass-filtered at 2-8 Hz (corresponding to the LFEs' peak frequency band). We correlate each template with the data recorded at each station on all three channels at $0.05 \mathrm{~s}$ intervals. The minimum inter-event trigger time for individual families is fixed at $6 \mathrm{~s}$ similarly to the previous LFE catalogues of Chamberlain et al. (2014) and Chapter 3/Baratin et al. (2018). A detection is made when the cross-correlation sum across the network exceeds a threshold value. Here, we use a threshold of eight times the median absolute deviation of the day-long cross-correlation sum, which is composed of correlations from at least 9 channels. This threshold was chosen through synthetic testing and was found to be robust to limit outliers expected to arise from real event detections. The use of synthetic templates generates a significant amount of false detections in the final catalogue (with brief testing on a selection of templates showing between 15 and $40 \%$ of false detections depending on the template). Therefore, at the end of the detection run, we establish an SNR cutoff in order to minimise false detections in the stacks (detailed in Section 4.3.1). We then stack the best $25 \%$ of detections (i.e. detections with the highest cross-correlation sums) using phase-weighted stacking (Schimmel and Paulssen, 1997; Thurber et al., 2014) to generate a refined waveform with a higher SNR. We extend the final stacked waveform to 30 s-length to include both $\mathrm{P}$ - and S-arrivals for picking purposes (the resulting stack is a $30 \mathrm{~s}$-long record with the $\mathrm{S}$-arrival at $\sim 15 \mathrm{~s}$ ).

\subsubsection{LFE locations}

We have previously demonstrated the efficiency of phase-weighted stacking to enhance the impulsive character of a waveform with a low SNR (Baratin et al., 2018, cf. Chapter 3). Here we use phase-weighted stacking on the last iteration's stacks to allow the precise picking of the onset of phases. We manually pick 378 LFE waveforms using the SEISAN software (Havskov and Ottemoller, 1999) and retain only those where at least 1 reliable P-pick and 5 reliable S-picks can be 


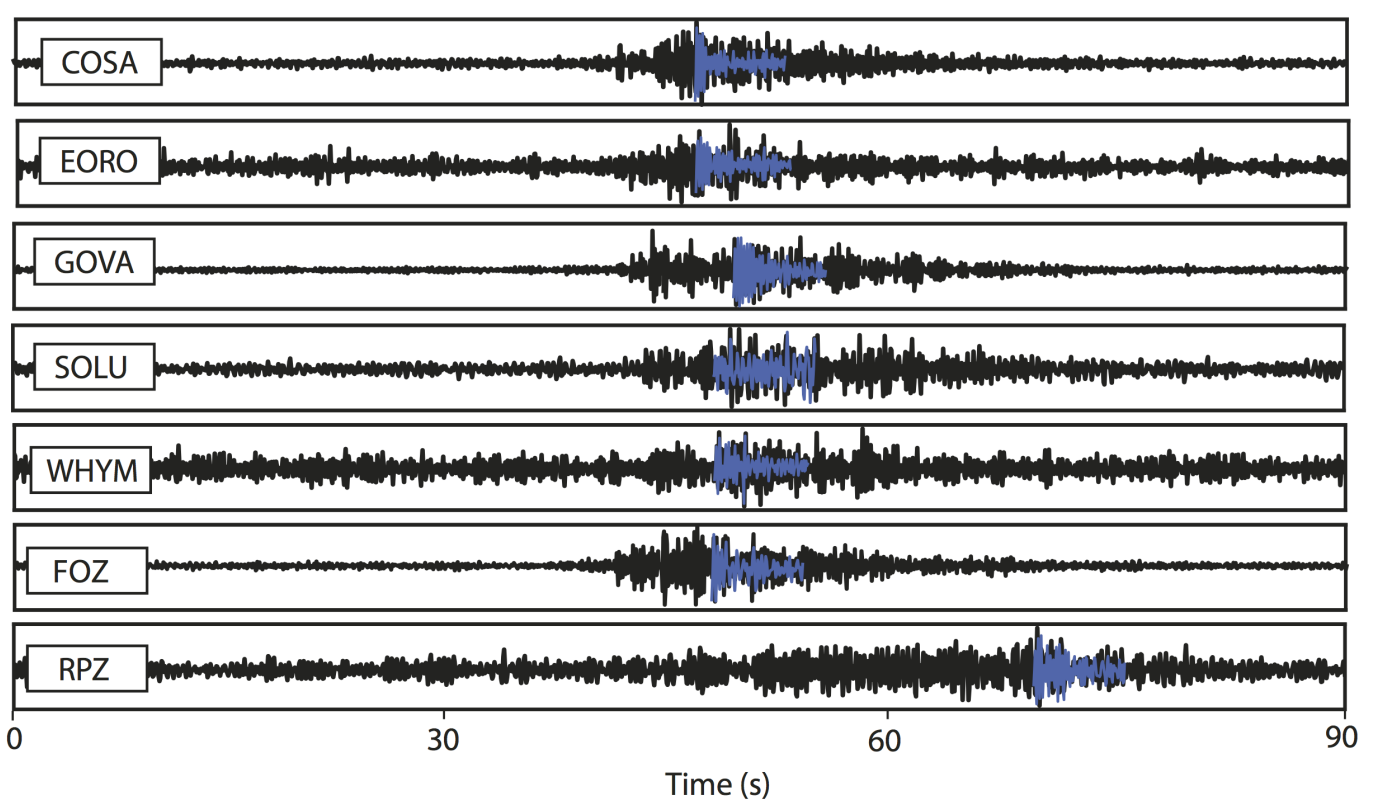

Figure 4.3: Real LFE template plotted on top of detections. Real LFE template ID: 169.597_-43.6853_32_1 (in blue) plotted on top of detected event on 2015-11-04 at 12:09:43 (in black), for the north component of several SAMBA (COSA, EORO, GOVA, SOLU, WHYM) and GeoNet (FOZ, RPZ) stations.

identified (i.e. 378 LFE families). We compute hypocentre locations and their associated uncertainties using the NonLinLoc software (Lomax et al., 2000) and a 3D velocity model. The NonLinLoc algorithm is a probabilistic earthquake location method that uses synthetic travel times generated in a $3 \mathrm{D}$ model to examine the locations and uncertainties given by an exhaustive grid-search. It uses a Metropolis-Gibbs approach to compare the observed and calculated phase arrivals and to produce comprehensive uncertainties represented by a probability density function (Lomax et al., 2000).

\subsection{Results}

\subsubsection{Performance of synthetic LFEs}

We compare the spectra of an LFE template generated in Chapter 3/Baratin et al. (2018) to the spectra of (1) an initial synthetic LFE template and (2) a real LFE template (Fig. 4.4). The synthetic template (green spectrum in Fig. 4.4), that was used to create the real LFE template, appears to be a good approximation of the 
LFE templates found in the Southern Alps (purple spectra in Fig. 4.4). We notice that the real LFE template produced by this study is almost identical to the LFE template of Chapter 3/Baratin et al. (2018), respectively blue and purple spectra in Fig. 4.4. Indeed, both spectra show similar frequency content, between $2-8 \mathrm{~Hz}$, which coincide to the peak frequencies of tremor and LFEs in the central Southern Alps (Wech et al., 2012; Chamberlain et al., 2014; Baratin et al., 2018). We can also see that the spectrum of an LFE is drastically different from the spectrum of a microearthquake (purple and blue spectra vs orange spectrum in Fig. 4.4).

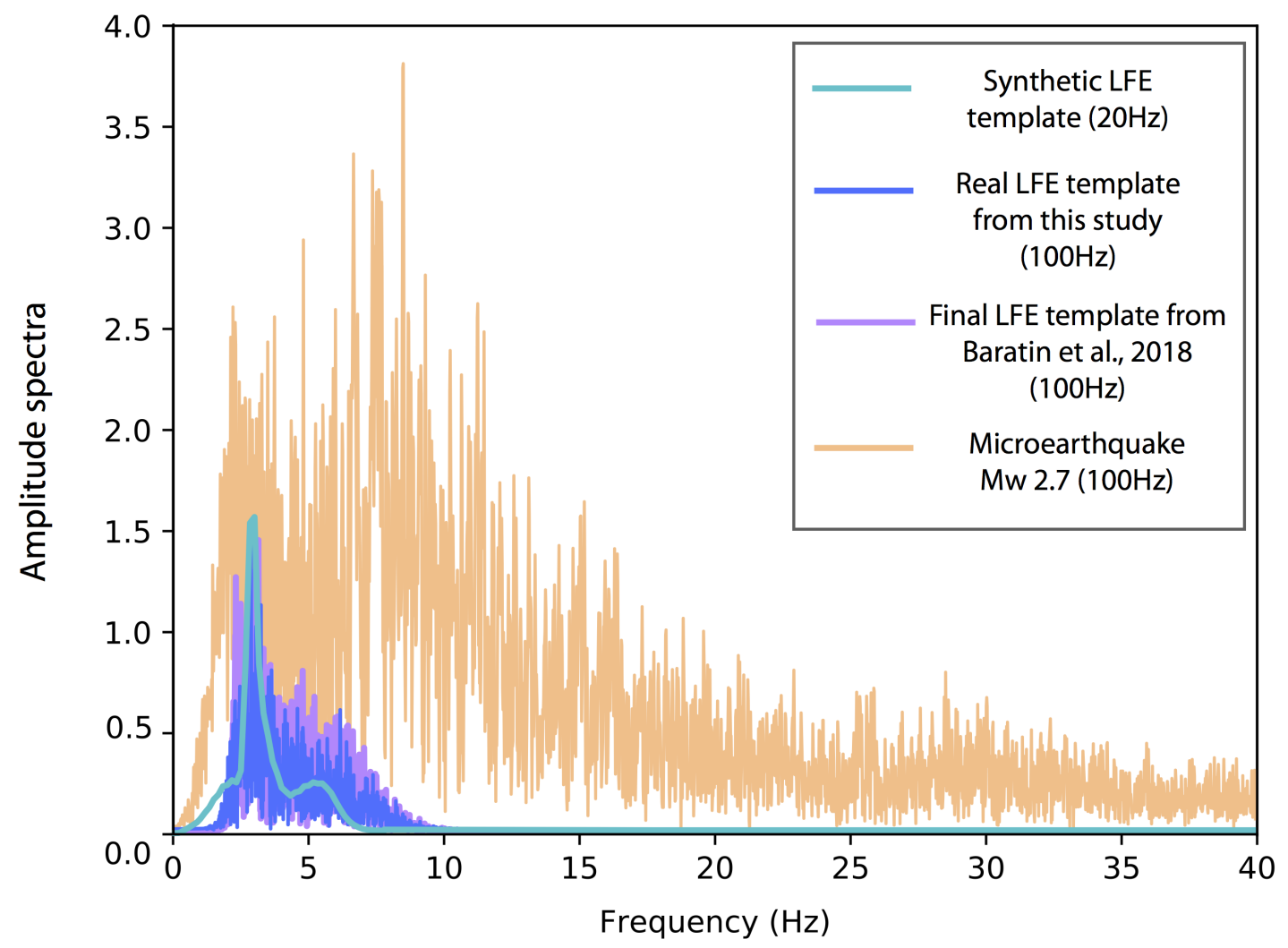

Figure 4.4: Comparison of earthquakes spectra. Comparison of spectra showing the similarities between the final LFE template generated in Chapter 3/Baratin et al. (2018) and the LFEs of this study. The spectra of a $M_{w} 2.7$ microearthquake occurring in the study area on the $3^{\text {rd }}$ May 2009 (Konstantinos Michailos, VUW PhD candidate, personal communication) is also shown in order to highlight the differences between LFEs and typical earthquakes found in the area.

Using synthetic templates generates a larger amount of false detections than when using real LFE templates (an average of $25 \%$ for the continuous catalogue against $\sim 20 \%$ for the catalogue of Baratin et al., 2018, cf. Chapter 3). By examining a 
subset of $20 \mathrm{LFE}$ families representative of the diversity of locations and depths found within the templates, we found, for a few families, almost twice as many false detections as Chamberlain et al. (2014) reported. To minimise the number of false detections incorporated in the stacks, we establish an SNR cutoff by discarding detections with an SNR lower than 4 (most likely noise) and higher than 15 (most likely an earthquake). Those thresholds were chosen after extensive testing of the subset of LFE families as they were found to yield the best stacks. This testing showed that a threshold lower than 4 would still include detections in the final stacks that were likely noise. As for a threshold above 15, it produced stacks that looked too impulsive to be LFEs. We find this additional processing step to significantly improve most of the stacks.

Examples of a typical LFE detection and two false detections can be seen in Fig. 4.5 for LFE family ID: $169.641 \_-43.729 \_44 \_1$. A real detection typically looks like an emergent signal that is seen on most stations with an SNR generally comprised between $\sim 4.5$ and $\sim 11.5$. In addition, its frequency content is that of LFEs $(2-8 \mathrm{~Hz})$. A false detection, however, can either have a low $\operatorname{SNR}(\leq 4.5$; e.g. middle plot in Fig. 4.5) or a high SNR ( $\geq 11.5$; e.g. right plot in Fig. 4.5). Furthermore, the frequency content of the signal is not restrained to $2-8 \mathrm{~Hz}$, but instead covers a greater range of frequencies (2-49 Hz for the examples in Fig. 4.5). For this particular template, we estimate a percentage of $\sim 20 \%$ of false detections through visual observation of 216 detections before applying the SNR cutoff. This estimation has been brought down to $\sim 10 \%$ after application of the SNR cutoff.

We compare the detections of the extended catalogue of Chapter 3/Baratin et al. (2018) to the detections made by the continuous catalogue of this study in order to see if the detections from the original 14 families overlap with this new catalogue. For each detection from the extended catalogue, we calculate the number of detections that are represented in the new catalogue within a 2-minute window (i.e. corresponding to the duration of an LFE burst in the extended catalogue). 


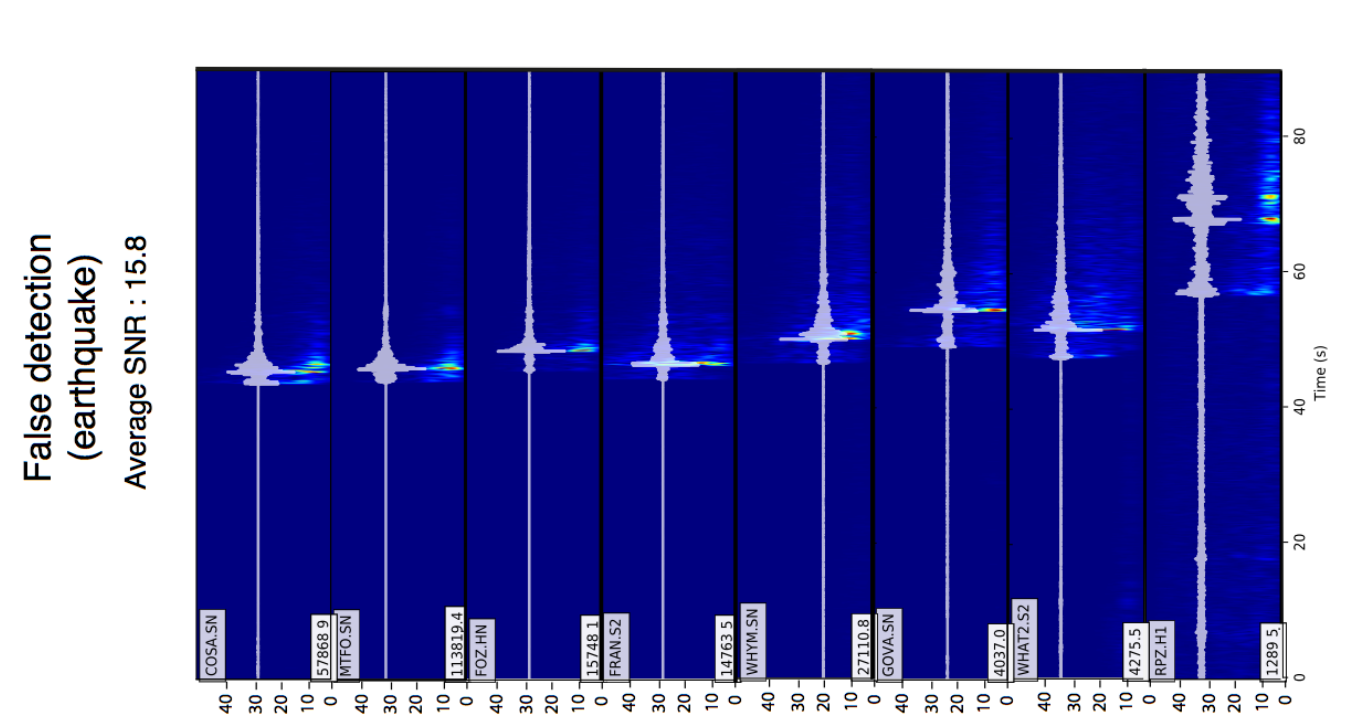

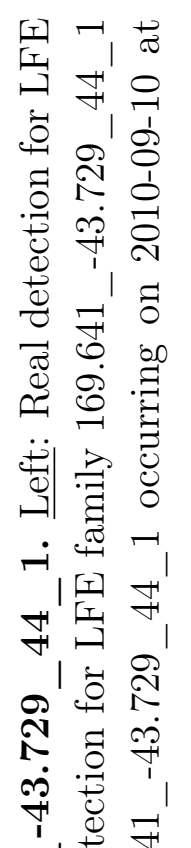

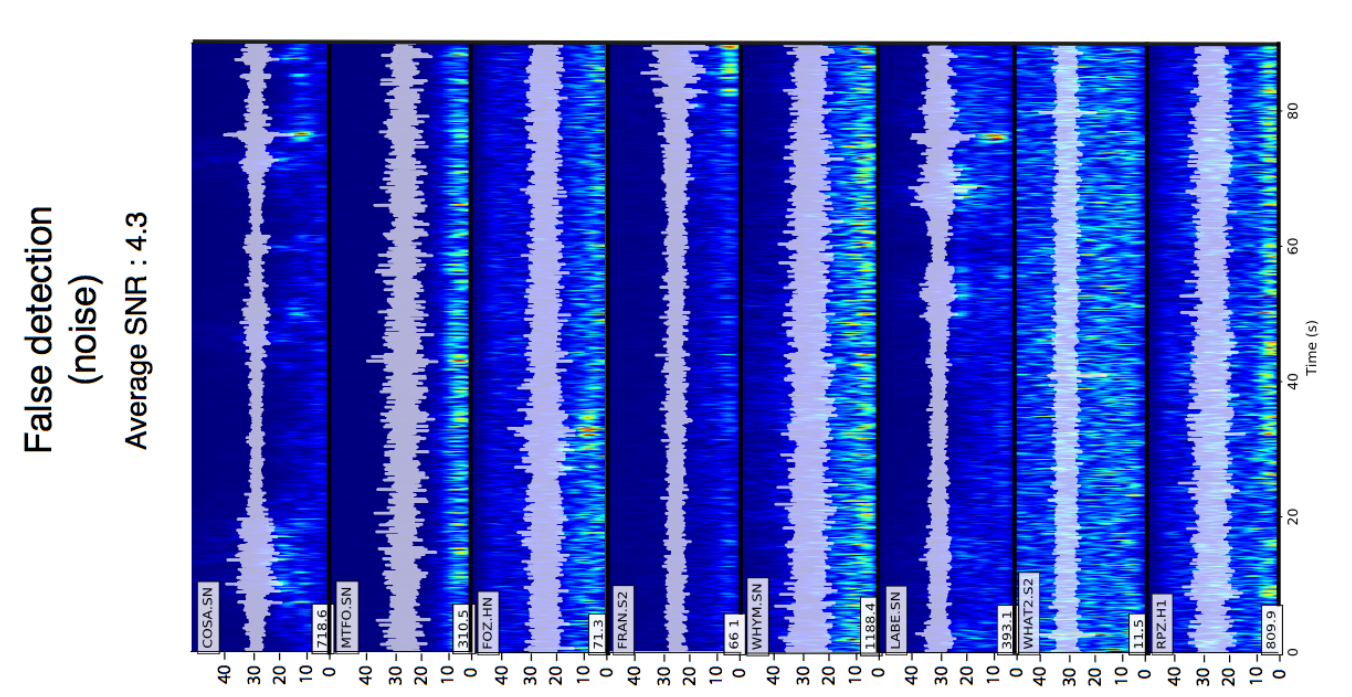

I

تี

ठำ

그 임

결 矛

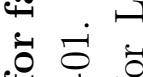

थ

잉 곡

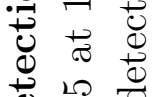

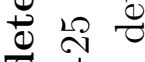

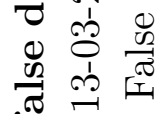

స్

중

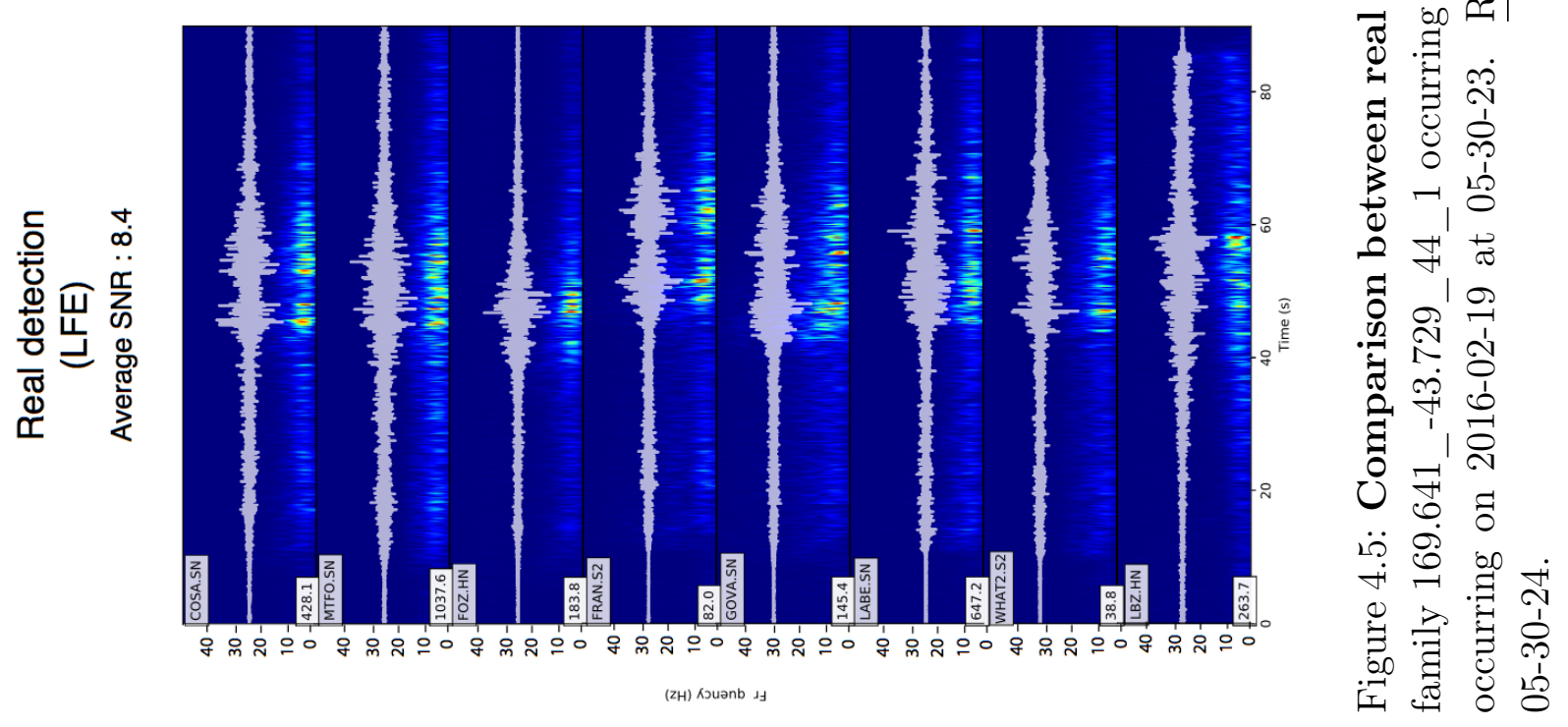




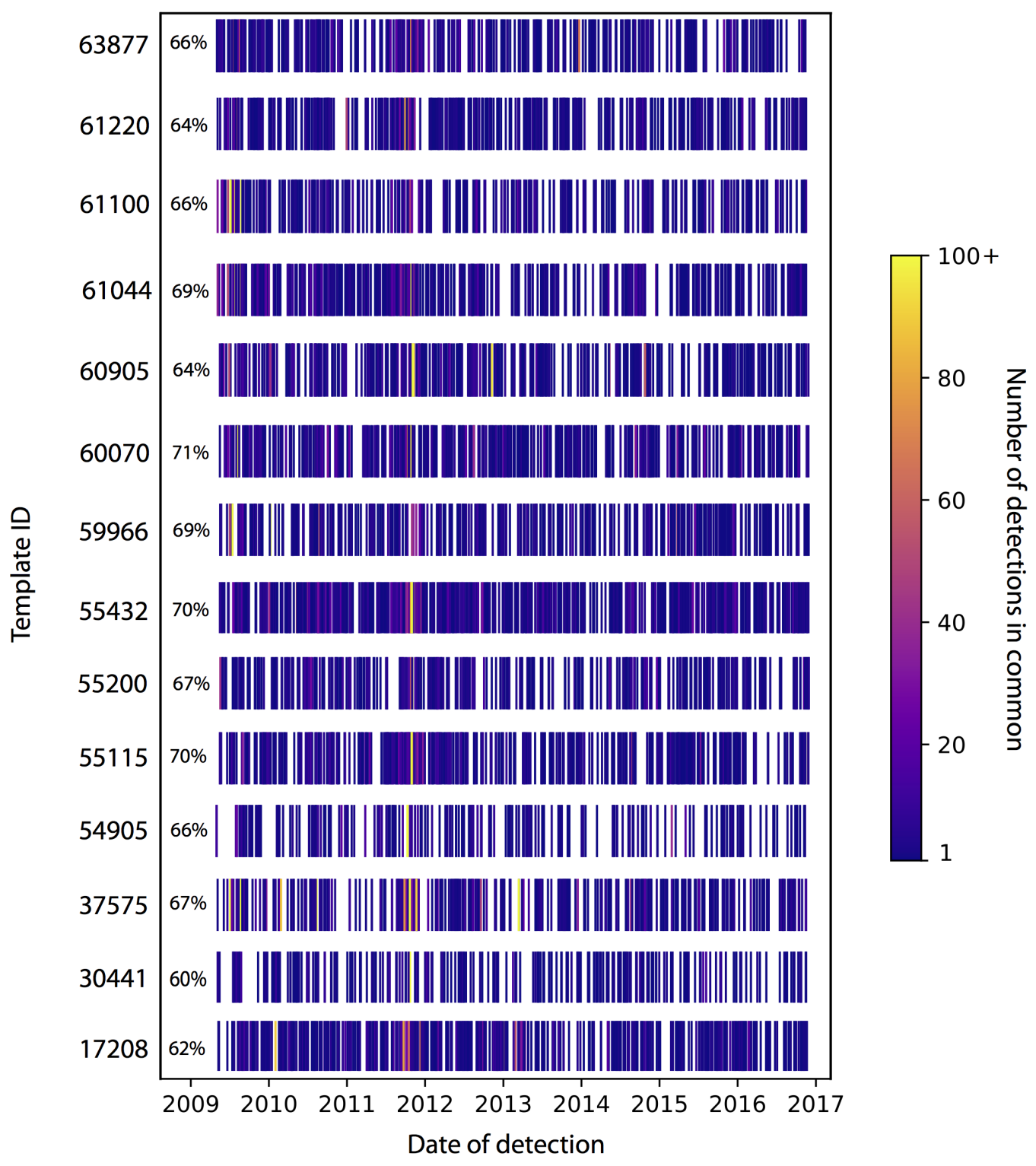

Figure 4.6: Detections overlap between the synthetic and extended catalogues. Comparison of detections from the extended catalogue of Chapter 3/Baratin et al. (2018) and detections from the continuous catalogue of this study. The extended catalogue template ID that is being compared to the new catalogue is indicated on the left side of the plot. The colour scale shows the number of detections from the new continuous catalogue coinciding with detections in the extended catalogue. White colour equals no detections. This comparison is performed up to September 2016 as the extended catalogue is 6 months shorter than the new continuous LFE catalogue. 
We find that overall $67 \%$ of the LFE catalogue from Chapter 3/Baratin et al. (2018) is represented in the new continuous catalogue. By looking at individual families (Fig. 4.6), we find that despite their distinct behaviours over the 8-yr study period, the percentage of catalogues overlap is consistent between families $(\sim 60$ $70 \%$ ). In other words, the new LFE catalogue is missing $\sim 33 \%$ of the detections found in the extended catalogue.

\subsubsection{Continuous LFE catalogue}

Using the 529 real LFE templates, 300,996 events are detected between March 2009 and March 2017 in the central Southern Alps, yielding the longest continuous record of LFEs in New Zealand. This amounts to a long-term rate of 103 LFEs per day (namely 30 times more than in the original catalogue of Baratin et al., 2018, cf. Chapter 3). If we look at events detected at only 5 stations (i.e. detection made with a cross-correlation sum across the network exceeding the threshold value for a total of 5 stations), the median number of LFEs detected per day amounts to $\sim 100$ (Fig. 4.7). This number decreases to $\sim 65$ for events detected at 9 stations. Those observations indicate that a significant majority of analysed days in the continuous catalogue yield a number of detections consistent with the long-term rate, regardless of the number of stations used in the detections.

The 529 LFE families total 128 to 1,671 detections each (depending on families) over the 8-yr study period. The diverse time signatures of LFE families is indicative of a large scope of LFE behaviours that we will investigate in more detail in Section 4.4.1.

A significant number of false detections arises from the use of templates containing only the S-phase. However, the long duration of the dataset enables us to mitigate the number of false detections used in the stacks, providing that we are indeed detecting mostly real LFEs. This is confirmed by visual inspection of a group of templates for which the best $25 \%$ of detections were plotted. Most of the detections examined during visual inspection looked like emergent tremor/LFE signals (e.g. individual detections made by template ID: 169.661_-43.7184_42_1 in Fig. 4.8). We also observe some coherence in the onsets of S-wave energy across channels and stations as well as between signals detected all along the study period (Fig. 4.8). We estimate a rate of $10-20 \%$ of false detections in the observed stacks (after the application of the SNR cutoff) depending on families.

Cumulative detections (panels C and D in Fig. 4.9) show continuous detections 


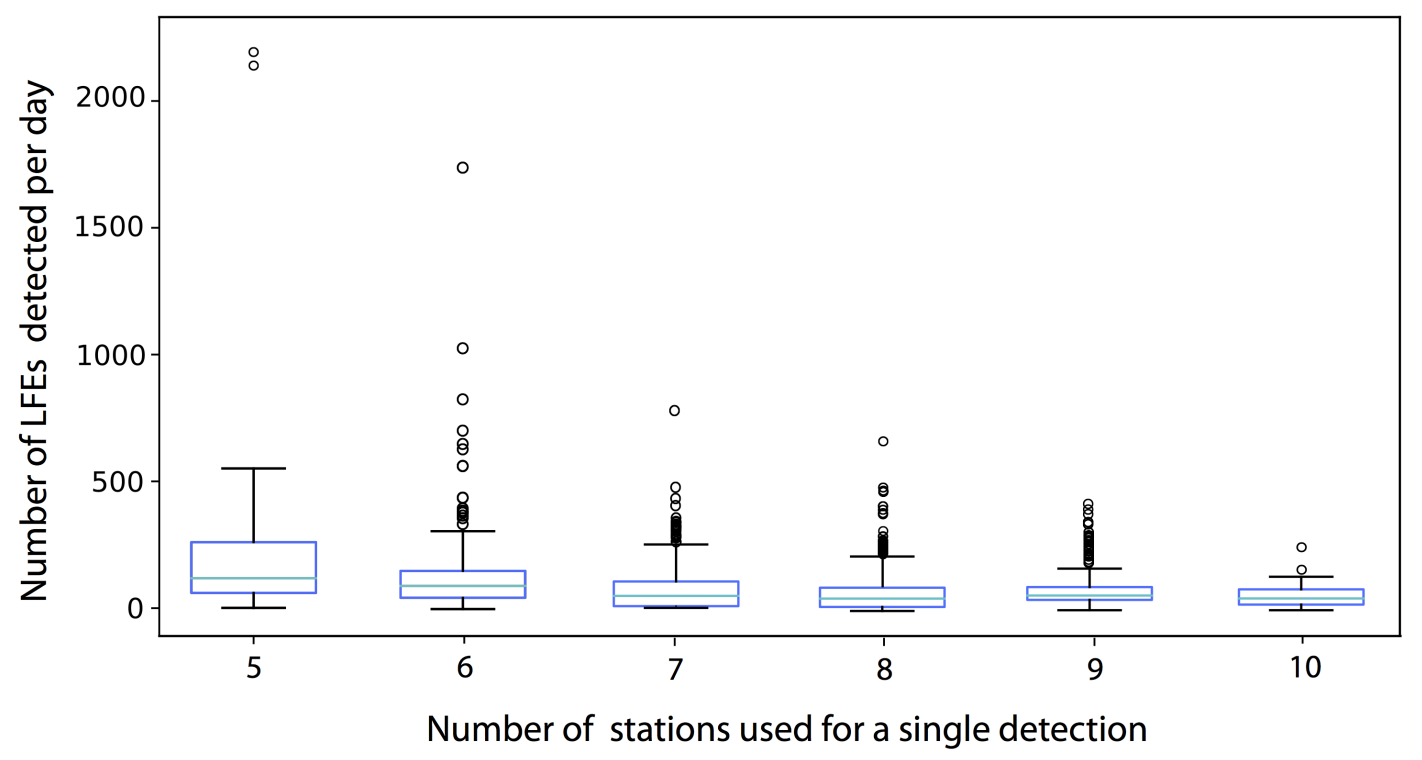

Figure 4.7: Number of LFE detection per day as a function of the number of stations used in the detection routine. Each box and whiskers plot represents the number of LFEs detected per day as a function of the corresponding number of stations used in the detection routine. The blue box represents the interquartile range (25\% quantile to $75 \%$ quantile). The green line represents the median. The whiskers extend to \pm 1.5 interquartile range. The black circles represent any outliers farther than the whiskers.

punctuated by increases in LFE rate (i.e. increases in the number of LFEs generated per day, panels A and B in Fig. 4.9) coinciding in some cases with known tremor events and large $\left(>M_{w} 5\right)$ regional earthquakes (similarly to what was observed in Baratin et al., 2018, cf. Chapter 3).

We make considerably more detections from August to October 2011 than at any other point in the 8-yr study period (pink box in Fig. 4.9). This 'step' of $\sim 50,000$ detections, coincides with a decrease in the number of functioning stations. This can be seen when comparing the cumulative number of detections made by using at least 9 channels (black curve in panel D of Fig. 4.9) which amounts to 50,000 detections, to the cumulative number of detections made by using at least 15 channels (blue curve in panel D of Fig. 4.9) which amounts to only $\sim 25,000$ detections. Consequently, $\sim 25,000$ detections are lost at that time (August -October 2011) by increasing the number of channels required to exceed the detection threshold from 9 to 15. This could indicate that the huge 'step' that we see in the cumulative detections at that time might be mostly composed of false detections. 


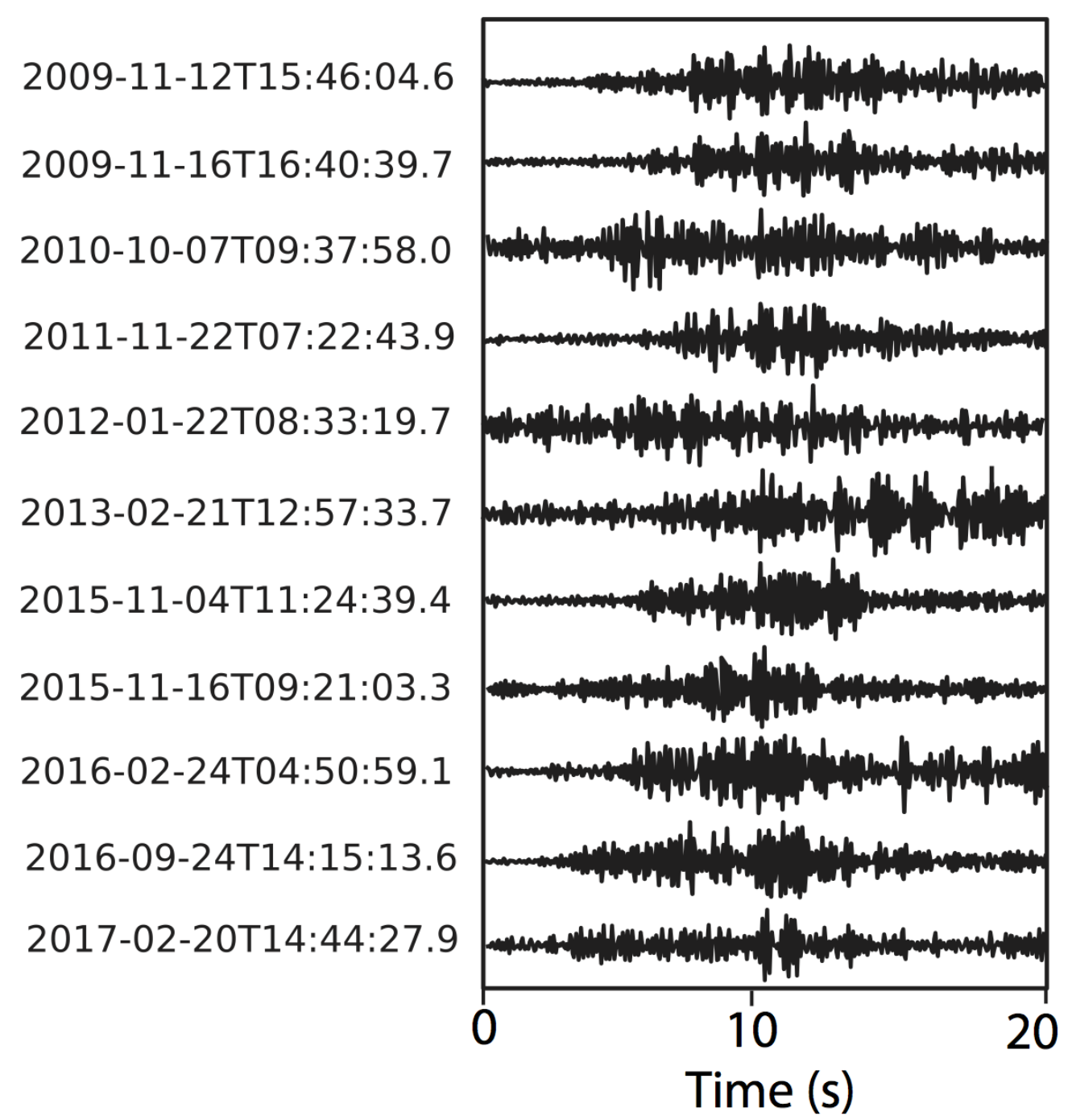

Figure 4.8: Example of detections made by a single LFE template. Examples of real individual detections made by LFE template ID: 169.661_43.7184 42_ 1 over the $8 \mathrm{yr}$ study period. All seismograms are recorded on the northern component of the COSA station. We can see an emergent arrival characteristic of tremor and LFEs lasting $\sim 10$ s.

However, a thorough inspection of this 'step' shows that it is itself made up of distinctive smaller steps indicative of several consecutive episodes of increased LFE activity (Lengliné et al., 2017). The magnitude of this event ( 50,000 LFEs generated over 3 months) may indicate that a large-scale slow slip event is occurring at that time (as seen in Frank et al., 2014, 2016). As a matter of fact, amongst the 12 days where Wech et al. (2012) identified tremor, three episodes of tremor occured between August and September 2011 (respectively 03-08, 02-09 and 04-09), thus supporting our findings of increased LFE rates during this time. 


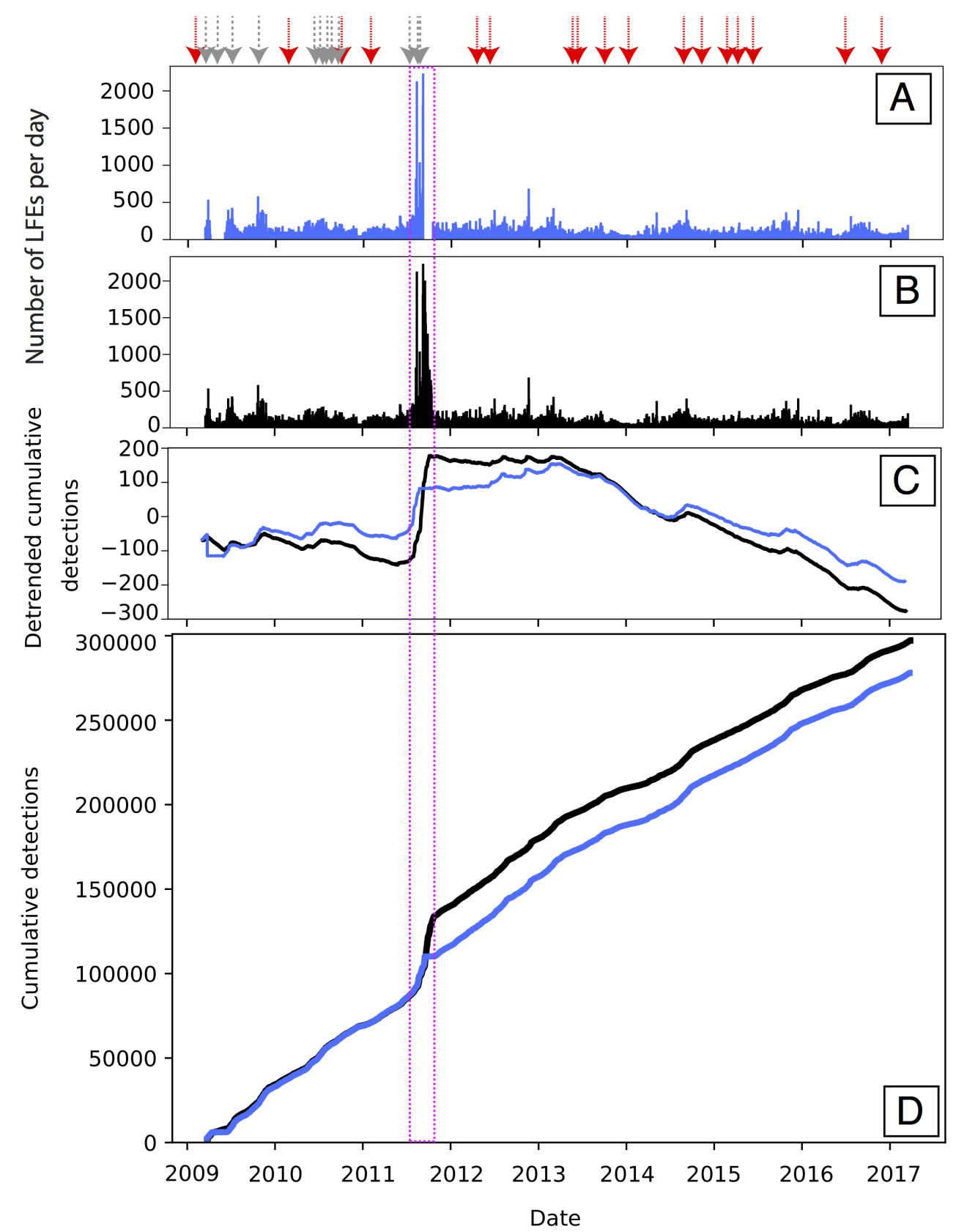

Figure 4.9: Cumulative detections and LFEs per day. Number of LFEs detected per day ( $\underline{\mathrm{A}}$ and $\underline{\mathrm{B}})$, detrended cumulative detections $(\underline{\mathrm{C}})$ and cumulative detections $(\underline{D})$ shown for a catalogue constructed with a detection made on at least 3 stations or 9 channels (in black) and 5 stations of 15 channels (in blue). Red arrows represent large regional earthquakes taken from the GeoNet catalogue (above $M_{w}>5$ ) that happened within $\sim 300 \mathrm{~km}$ of the network. Grey arrows represent tremor periods identified by Wech et al. (2012). No tremor analysis has been undertaken beyond September 2011. The pink box indicates the occurrence of a potential large-scale slow slip. 
Finally, we can rule out false detections as being responsible for this 'step' for two reasons: (1) by taking a closer look at detections made during that period, we observed mainly waveforms characteristics of LFEs; (2) a similar increase is seen in the detrended cumulative count of the 14 original families at the same period (Fig. 3.16 in Section 3.5.1 of Chapter 3; cf. Baratin et al., 2018). However, this needs further investigating and will be addressed in Chapter 5.

Many of the LFE families exhibit coherent, impulsive S-wave arrivals across all stations in their final linear stacks. Other families, however, display linear stacks that are still too emergent to allow precise picking of the onset of the S-wave arrivals (e.g. left panel in Fig. 4.10). We have previously demonstrated how phase-weighted stacking improves the impulsive character of an LFE wave arrival (Baratin et al., 2018, cf. Chapter 3). Here we once again use phase-weighted stacking to increase the SNR of all of families in order to facilitate reliable hypocentre estimations. Phase-weighted stacking appears to be particularly beneficial when starting with an emergent signal (e.g. right panel in Fig. 4.10); indeed phase-weighted stacking helps in precisely picking onsets of the S-wave arrivals and especially in distinguishing some P-wave arrivals in most of the stacks.

When examining the LFE stacks frequency content, we observe that the majority of LFEs have the highest power between $2-8 \mathrm{~Hz}$ (e.g. left panel in Fig. 4.11). This is in agreement with other studies demonstrating that tremor and LFEs are enriched in low frequencies in the $1-10 \mathrm{~Hz}$ frequency band and depleted in higher frequencies as opposed to nearby ordinary earthquakes (Shelly et al., 2007; Wech et al., 2012; Chamberlain et al., 2014; Baratin et al., 2018).

For example, we can see that the frequency content is mostly comprised between $1-10 \mathrm{~Hz}$, with clear spectral peaks (in red in Fig. 4.11) corresponding to the LFE S-wave arrivals for family ID:170.025_-43.528_40_1. However, for families with lower SNR, e.g. family ID:169.728_-43.8164_42_1, frequency peaks in the $2-8 \mathrm{~Hz}$ band and clear S-wave arrivals are only seen across a few stations (e.g. COSA, LABE, FRAN and MTFO in Fig. 4.11). Therefore, we choose to discard families that do not have at least 5 clear S-wave arrival as they would not provide reliable locations and are left with 378 families to locate. 
Linear stack

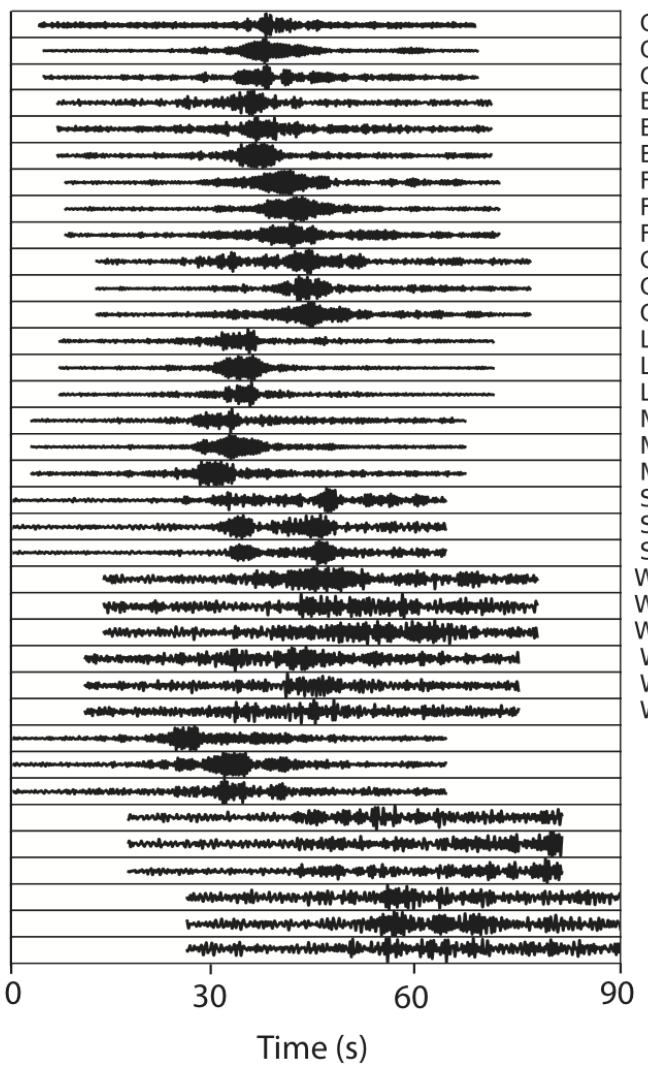

Phase-weighted stack

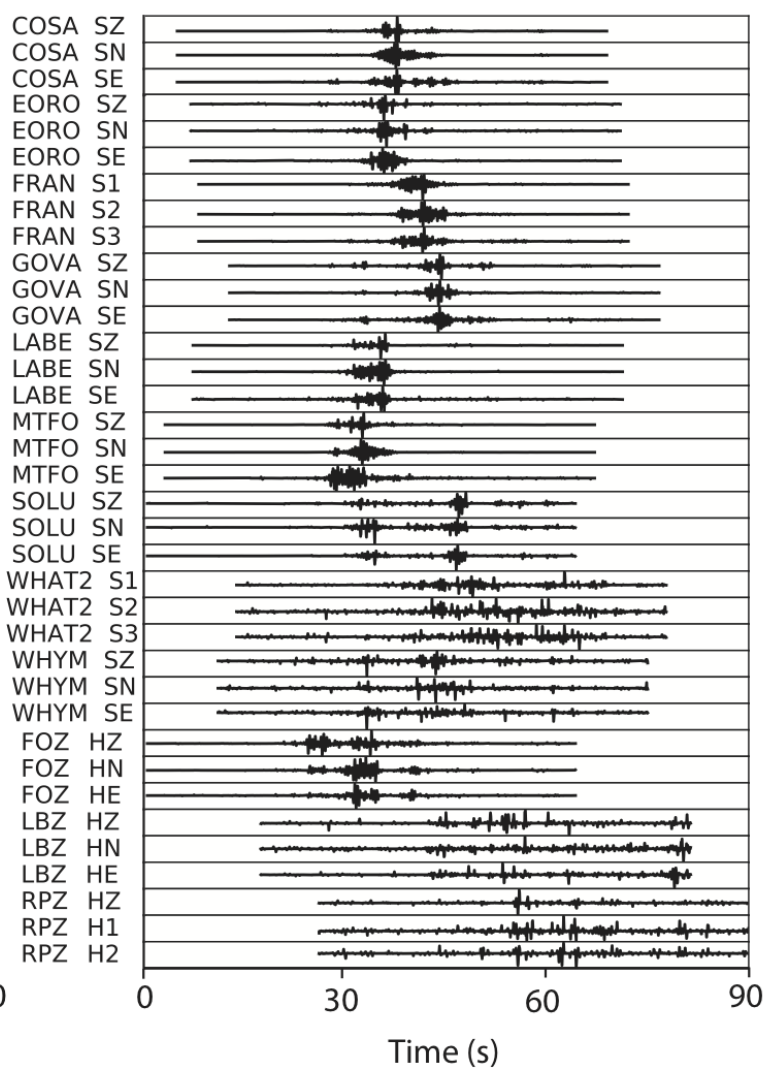

Figure 4.10: Comparison between linear and phase-weighted stacks for template ID: $\mathbf{1 6 9 . 6 2 6} \mathbf{- 4 3}^{\mathbf{4}} \mathbf{7 1 4 4} \mathbf{3 8}_{-} \mathbf{1}$. Linear stack $\underline{\underline{\text { Left }}}$ ) and phaseweighted stacks (Right) for synthetic template ID: $169.626 \_-43.7144 \_38 \_1$ made by stacking 290 individual detections (corresponding to $25 \%$ of the best detections for that family). 

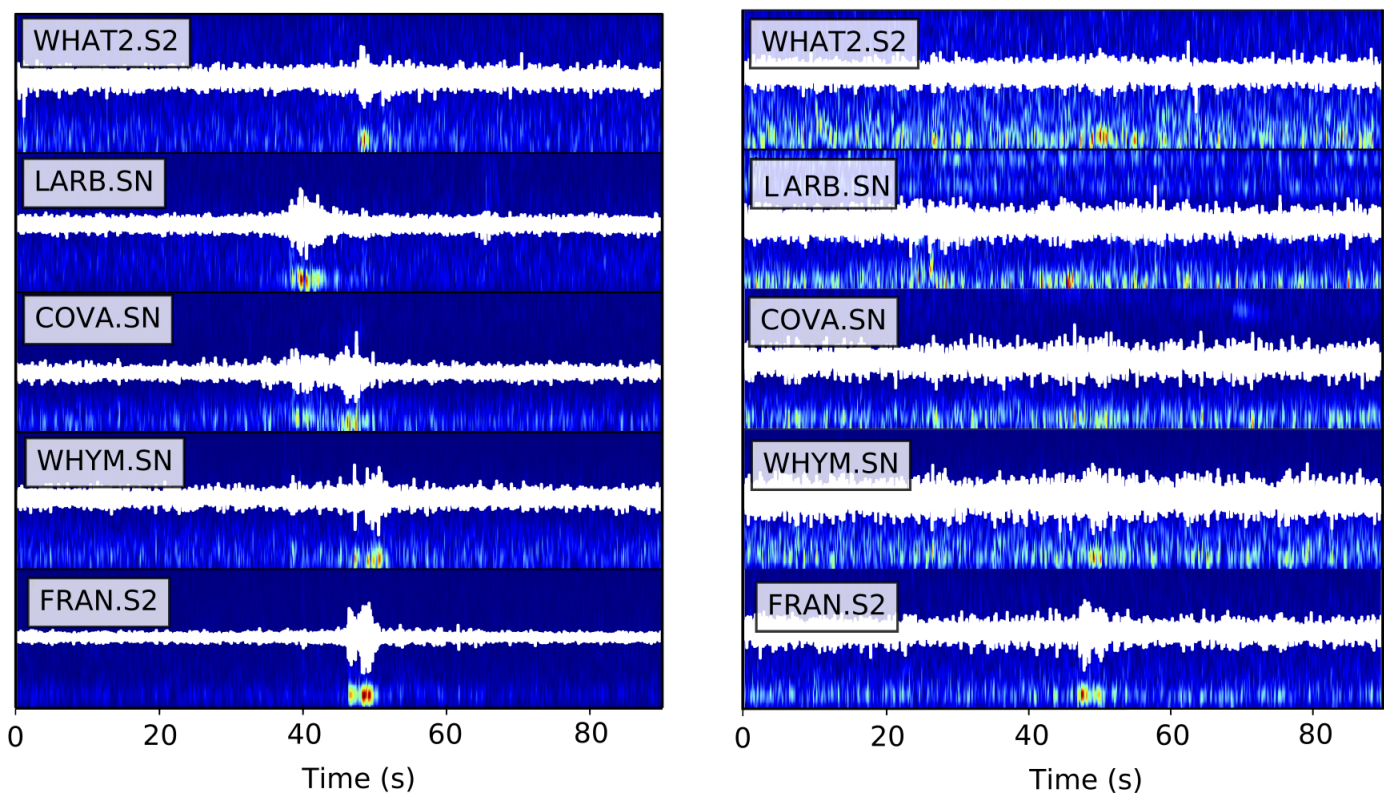

Figure 4.11: Example of waveforms and spectrograms for high SNR and low SNR stacks. Left: Waveforms of linear stack (white) and spectrograms (coloured scale) of LFE family ID:170.025_-43.528_40_1. This family possess a high SNR (=8.52) and was part of the 378 families that we managed to locate. A clear arrival for the S-wave is seen on most stations. Right: Waveforms of linear stack (white) and spectrograms (coloured scale) of LFE family ID:169.728_43.8164_42_1. This family possess a low SNR (=4.32) was part of the 127 families that we could not locate. A clear arrival for the S-wave is seen on a few stations (e.g. FRAN); however this was not sufficient to obtain a reliable location.

\subsubsection{LFE locations}

LFEs occur beneath the southern portion of the SAMBA network, most of them confined to an area located west of Aoraki/Mt. Cook (see Table A.1 in Appendix A.3 for a complete list of locations). LFE locations are distributed within an area of $60 \times 55 \mathrm{~km}$ and a $50 \pm 2 \mathrm{~km}$ depth extent (Fig. 4.12). This area encompasses the previous LFE locations of Chapter 3/Baratin et al. (2018). The 378 LFE families locate at depths of 11-59 km; hence below the seismogenic zone defined by regular microseismicity (0-10 $\pm 2 \mathrm{~km}$ as determined by Boese et al., 2012). Those depths are approximately the same as those reported for tremor by Wech et al. (2012) and deeper than the original LFE depths reported by Chamberlain et al. (2014) and the updated LFE depths of Chapter 3/Baratin et al. (2018). 
In contrast to the previous catalogue of Chapter 3/Baratin et al. (2018), which was only composed of 14 families, this new catalogue encompasses hundreds of families and allows us to better analyse the extent of LFEs within the study area. Some events locate outside of the starting model grid (e.g. to the NE of the grid; top left panel of Fig. 4.12); however the majority of LFEs are located within the starting model grid boundary, as expected. Most of the LFE families with reliable locations can be found between 25 and $45 \mathrm{~km}$ depth (cold coloured stars in Fig. 4.12), which reflects our starting model grid to some extent (Fig. 4.13). The model grid started with a higher number of synthetic families at depths of $18-20 \mathrm{~km}$ than what was finally found in our study area (green vs blue curve on Fig. 4.13). Noticeably, the depth distribution of the real LFE families in the area seems more homogenous than the one of the starting model, which might indicate that we have efficiently sampled the real LFE families in the area.

We remark that the LFE families with the biggest uncertainties are mainly located at depths greater than $45 \mathrm{~km}$, i.e. outside of the starting model grid (red stars in Fig. 4.12). However, the most reliable LFE families, locate within an area that encompass the previous LFE locations of Chapter 3/Baratin et al. (2018) (black stars vs cold coloured stars in Fig. 4.12). Hence, we remain confident in the locations of the LFE families that are situated within the starting model grid (i.e. locations within the cyan boxes in the top right and bottom left panels in Fig. 4.12).

The entirety of the LFE families locate in the southern part of the SAMBA network between Franz Josef and Mt. Baird (respectively FRAN and MTBA in Fig. 4.12). Two families locate near the De La Bêche site (LABE in Fig. 4.12) at the northeastern extent of the grid, however the remaining families locate South of Franz Joseph (FRAN in Fig. 4.12). Interestingly, the majority of events occur in a radius of $15 \mathrm{~km}$ of Aoraki/Mt. Cook. Beyond this radius, we notice that less than $10 \%$ of the families locate southeast of Mt. Fox (FOZ in Fig. 4.12). It is worth noting that no detections were made in the southern section of the original synthetic grid (near the MTBA station), hence hinting at the boundary for the southern extension of low-frequency earthquake activity in the Southern Alps (e.g. few families are actually found in the grid between 29 and $34 \mathrm{~km}$ distance along profile aa' in bottom left panels of Fig. 4.12) 

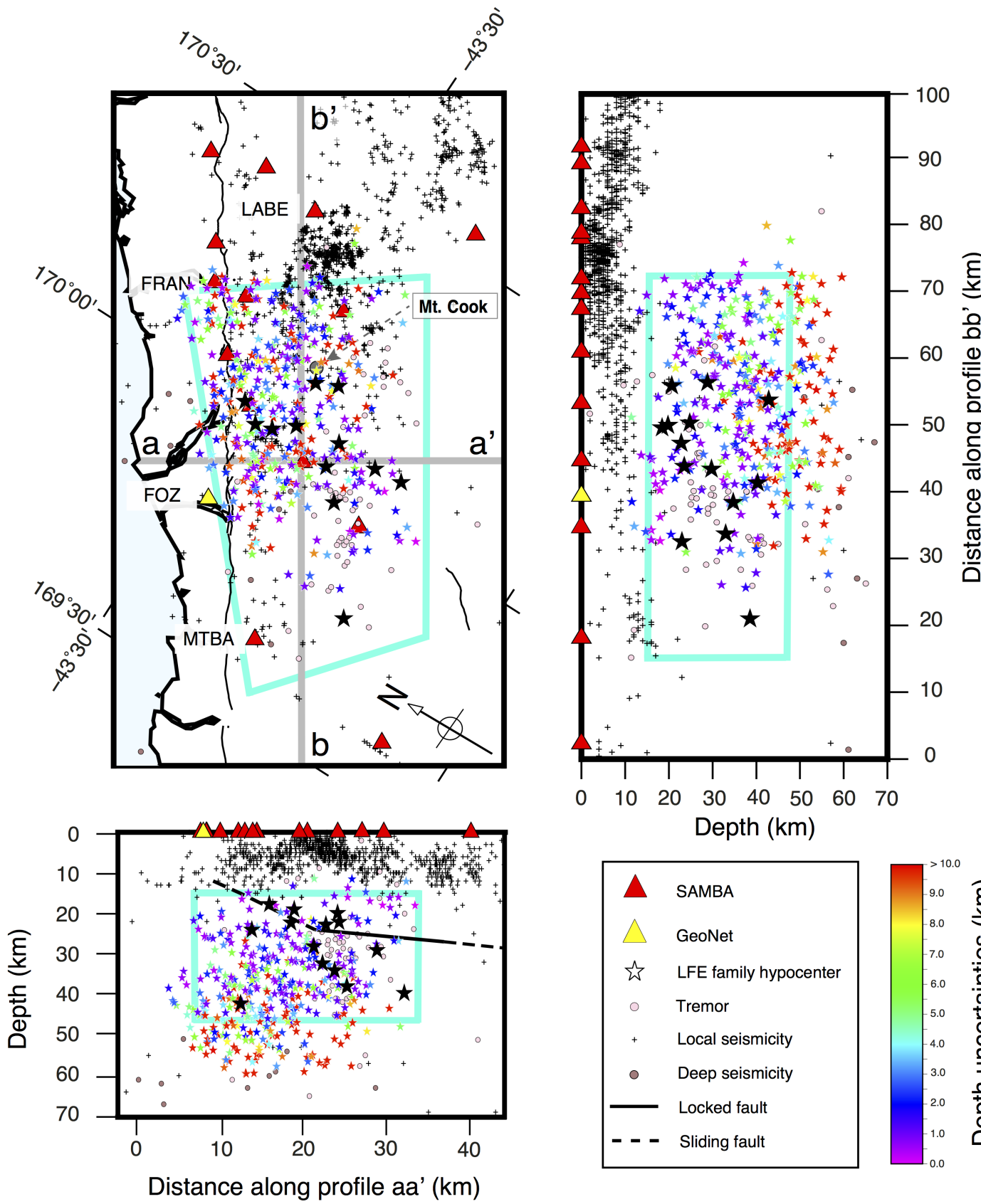

Figure 4.12: Map of LFE locations. Local seismicity (Boese et al., 2012), deep seismicity (Boese et al., 2013) and tremor (Wech et al., 2012) are plotted with the symbols shown in the legend. Black stars correspond to the 14 LFE families of Baratin et al. (2018)/Chapter 3. The 378 LFE hypocentres from this study are coloured by depth uncertainties, according to the legend on the figure. Top left: rotated map view. Top right: cross section, parallel to fault, along profile bb'. Bottom left: cross section, orthogonal to fault, along profile aa', with a GPS modelled fault after Lamb and Smith (2013). 
Few LFEs families locate on the proposed deep extent of the Alpine Fault inferred by GPS measurements by Lamb and Smith (2013) (black line in bottom left panel of Fig. 4.12), yet the ones that do are amongst our most reliable locations. In addition, a small proportion (approximately $\sim 15 \%$ ) of the LFE families seem to be clustered (within a $10 \mathrm{~km}$ radius) around the inferred change in dip of the Alpine Fault, from a steep $\left(45-60^{\circ}\right)$ stably sliding fault to a moderately dipping (15-20 $)$ and partially locked fault (respectively dashed and full black lines in Fig. 4.12). Below this inferred change in dip, we also observe concomitant locations of tremor and LFEs (pink circles vs coloured stars in the bottom left panel of Fig. 4.12). In addition, the uncertainties for the LFE families located in that area are amongst the lowest ( $0-3 \mathrm{~km}$ of depth uncertainty), therefore we infer that the locations of the LFE families occurring near the inferred change in dip are quite reliable.

The majority of the LFE families are located mainly to the SW of the proposed Alpine Fault deep extent (black line in bottom left panel of Fig. 4.12). The families located in that area exhibit fairly reliable locations up to $50 \mathrm{~km}$ depth. Those locations coincide with a previous LFE family location of Baratin et al. (2018)/Chapter 3 (black star to the SW of the proposed Alpine Fault in bottom left panel of Fig. 4.12). As inferred in Chapter 3)/Baratin et al. (2018), we suggest that those LFE families may occur on a more steeply dipping branch of the Alpine Fault as proposed by (Norris and Toy, 2014) although focal mechanisms would be required to corroborate this hypothesis.

Even though most families showed few clear P-wave arrivals, the depths are relatively well constrained for the majority of the LFEs; with the exception of the deepest LFE (over $40 \mathrm{~km}$ depth) families (see Appendix A, Fig. A.30 for an additional location map with hypocentre uncertainties). However, some of the deepest families show relatively low depth uncertainties $(\leq 3 \mathrm{~km})$, hence allowing us to remain fairly confident in the LFE locations for those particular families. 

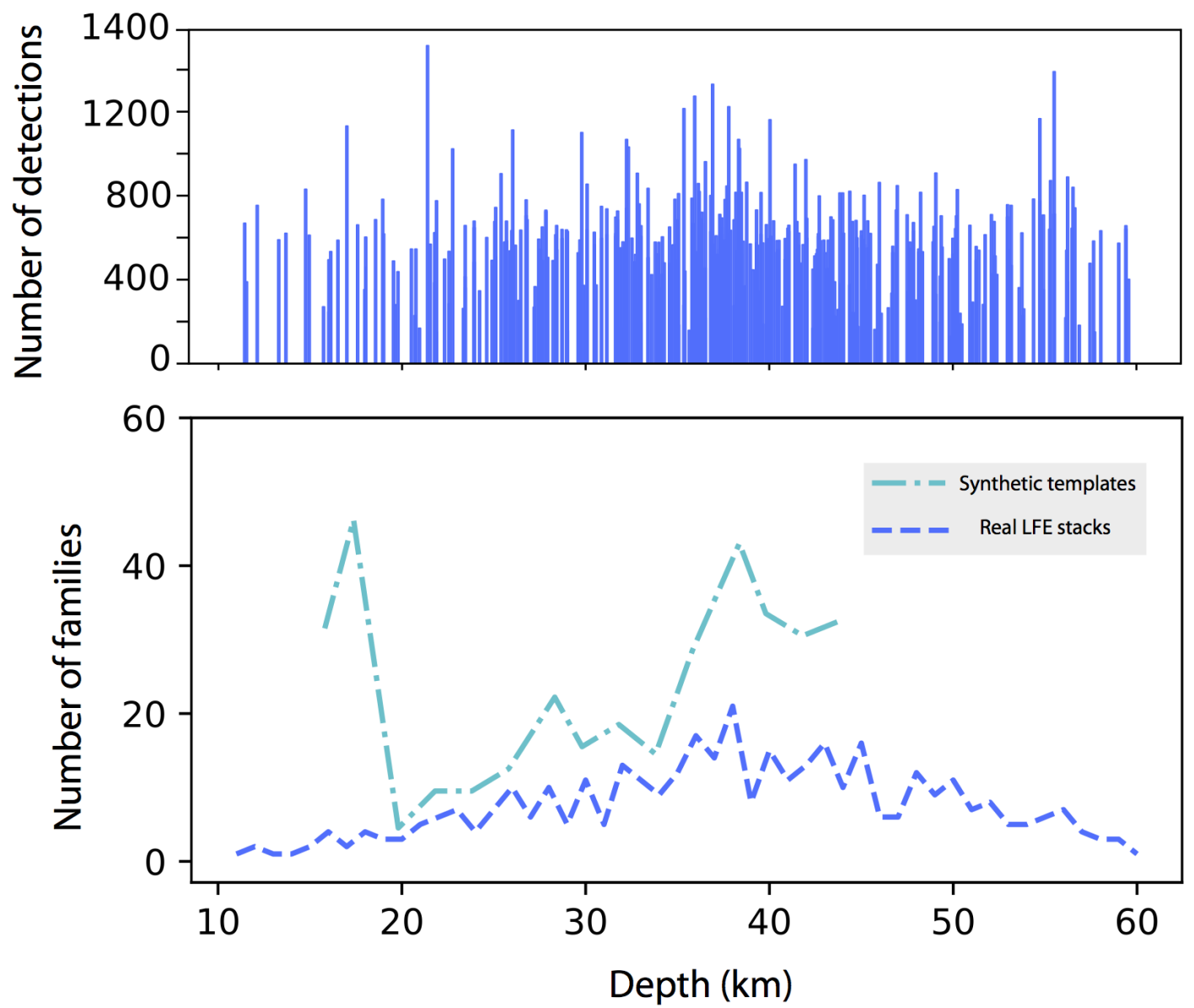

Figure 4.13: Comparison of initial and final depths. Top: Number of events detected in function of their depths for the continuous catalogue. Bottom: Comparison between the depths of the original synthetic templates (input templates for the LFE families that could be located, i.e., 378 templates) and the depths of the final LFE stacks (real LFE stacks output), respectively in green and blue.

\subsection{Discussion}

\subsubsection{LFE families behavioural styles}

We observe some substantial differences between families in the final LFE stacks. Indeed most of the families' stacks (75\%) present at least 5 stations showing clear S-arrival and at least 1 station showing a noticeable P-wave arrival (e.g. LFE family on the left panel of Fig. 4.11). However, 25\% of the LFE families' final stacks are still too noisy (e.g. LFE family on the right panel of Fig. 4.11). Most LFE families display a high SNR, generally above $\sim 4.5$. By comparing the waveforms 
of a high SNR family and a low SNR family (white seismograms in Fig. 4.11), we find that the LFE family with the high SNR shows clear LFE S-wave arrivals between $40 \mathrm{~s}$ and $60 \mathrm{~s}$ across all stations on the left panel of Fig. 4.11 whereas the waveforms are distinctly more emergent on most stations for the LFE family with the low SNR on the right panel of Fig. 4.11. The linear stacks of the LFE families that we managed to locate had an average SNR $>4.5$. The LFE families that we did not manage to locate had either a low SNR or still appeared too emergent to confidently pick enough $\mathrm{P}$ - and S-wave arrivals. In addition, we note that the overall number of detected events for each family does not seem to play a huge role in the overall quality of the stack. For example, family ID:169.999_43.5637_18_1 which detected 814 events, during the 8-yr study period, produced a stack too emergent to reliably pick enough P- and S-wave arrivals. Conversely, family ID:169.733_-43.7912_16_1 which only detected 218 events over the 8-yr study period gave a stack clear enough to obtain a reliable location (see Figs. A.21 and A.22 in Appendix A.3 for examples of the waveforms).

Cumulative counts and inter-event times show significant variations between individual families (Figs. 4.14 and 4.15). The most active families generally exhibit more burst-like behaviour (e.g. family IDs: G and E in Figs. 4.14 and 4.15), similarly to our findings from Baratin et al. (2018)/Chapter 3. For example, two families generating almost the same number of events, family IDs: D and F (respectively located at $33 \mathrm{~km}$ and $23 \mathrm{~km}$ depths), behave fairly similarly in time (Figs. 4.14 and 4.15), with both families detecting the same major burst near the end of 2011. Other families, less active over time, do not seem to contribute much to the big 'step' in detections occurring at the end of 2011 (e.g. family IDs: C and $\mathrm{H}$ in Figs. 4.14 and 4.15).

The diversity of the LFE families' cumulative temporal characteristics highlights the different styles of occurrence pattern for the LFE sources. As noted in Figs. 4.14 and 4.15, the event rate of some LFE sources is stationary, while for others, LFE event rates are highly episodic, with burst-like sequences of LFE events separated by long quiet periods. Overall, the findings of this study agree with those of Baratin et al. (2018)/Chapter 3, where some LFE families exhibit a 'stepped' (or episodic) behaviour and others exhibit a more 'continuous' behaviour over time.

This difference in behaviours could potentially be explained by variations in the insitu stress and frictional conditions at the individual LFE source locations (Johnson et al., 2012, 2013; Trugman et al., 2015). Trugman et al. (2015) inferred, through quantitative comparison to analogous laboratory shear slip experiments 


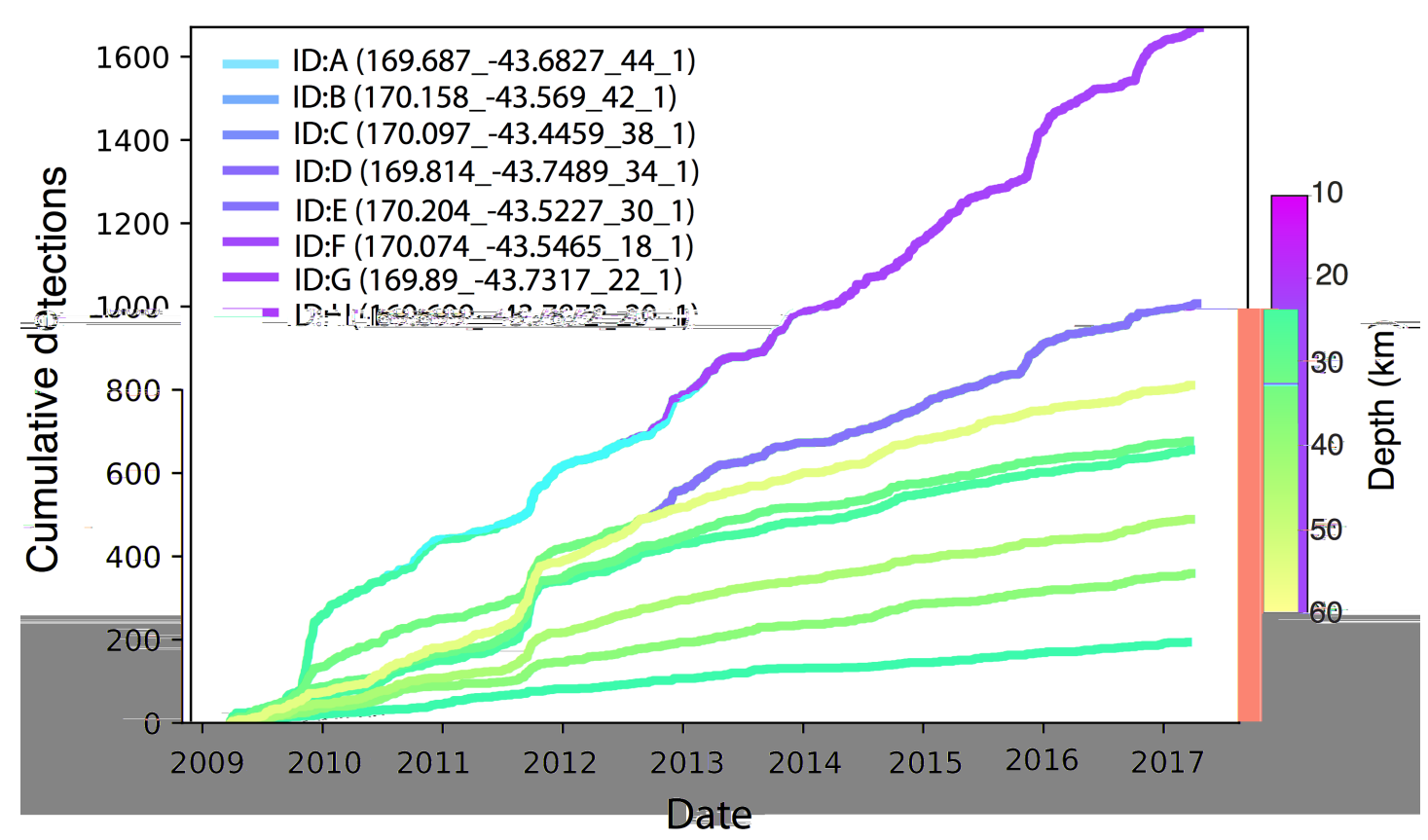

Figure 4.14: Cumulative detections for a selection of LFE families. Cumulative detections are shown for a subset of 8 LFE families, chosen at random locations and depths over the study area. The colour scale indicate the depth of each LFE family source.

on fault gouge-like materials, that the distinctive features of LFE occurrence patterns reflected an evolution from an episodic slip regime (for the 'stepped' families tending to occur in burst-like sequences) to a continuous slip regime (for the 'continuous' families). In their study, Trugman et al. (2015) observed a clear transition of acoustic emission (i.e.slip events that they hypothesised to be analogous to LFEs) from episodic to continuous occurrence with decreasing normal stress. This transition may correspond to an evolution from a stick-slip slip regime to a stable-sliding slip regime (Marone, 1998).

The rheology and physical conditions of the deep Alpine Fault are unquestionably more complex than those of any laboratory experiments. However, the physical processes controlling the occurrence styles in laboratory shear slip experiments (e.g. normal stress, fault gouge composition, slip rate) may explain the singular features in the patterns of LFE occurrence (Johnson et al., 2012, 2013; Trugman et al., 2015). 

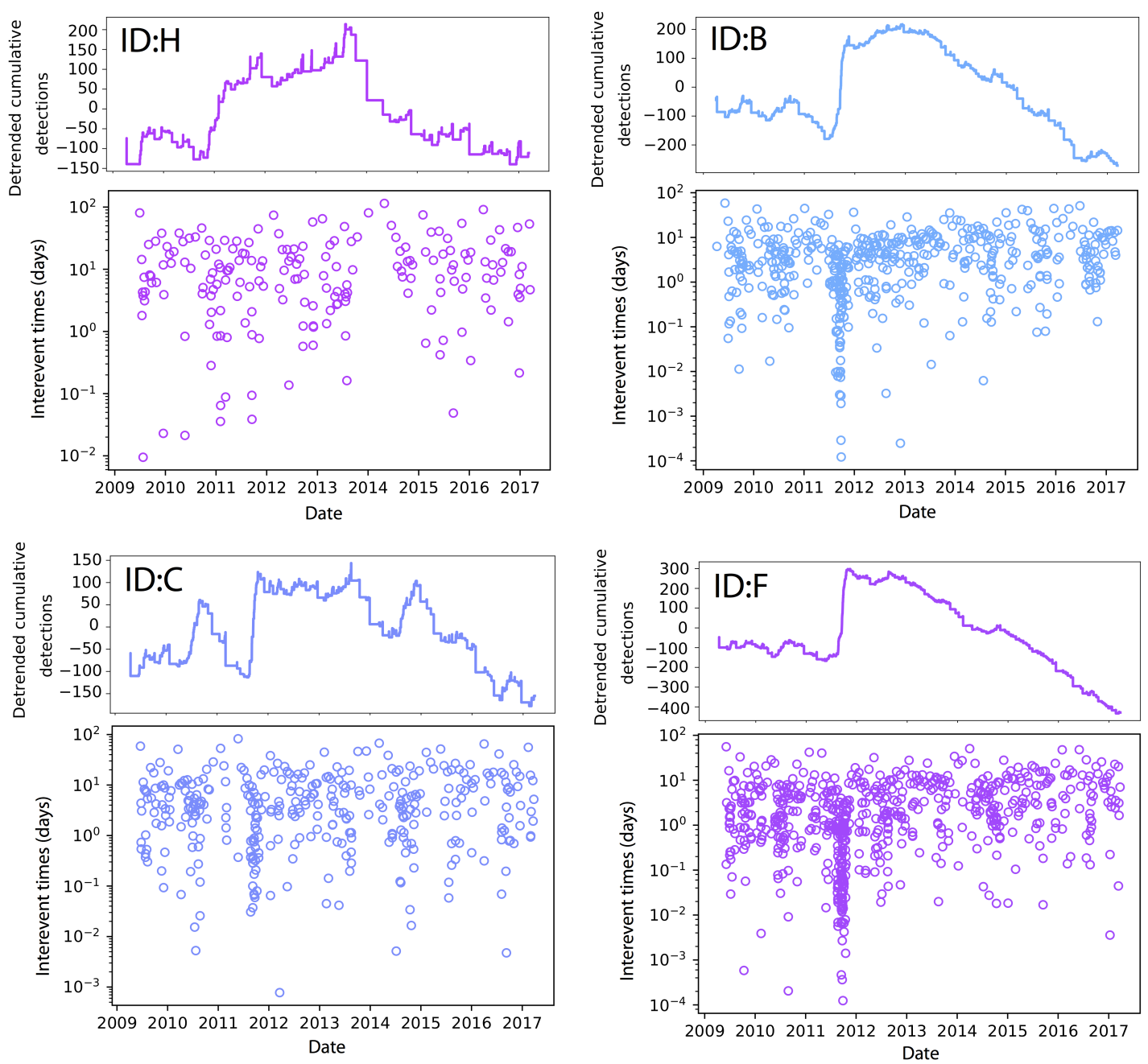

(a) Detrended cumulative detections and inter-event times for LFE family IDs: $\mathrm{H}$

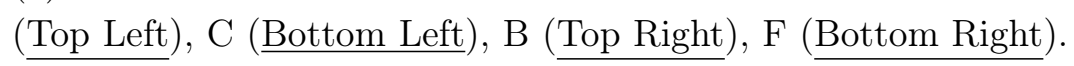



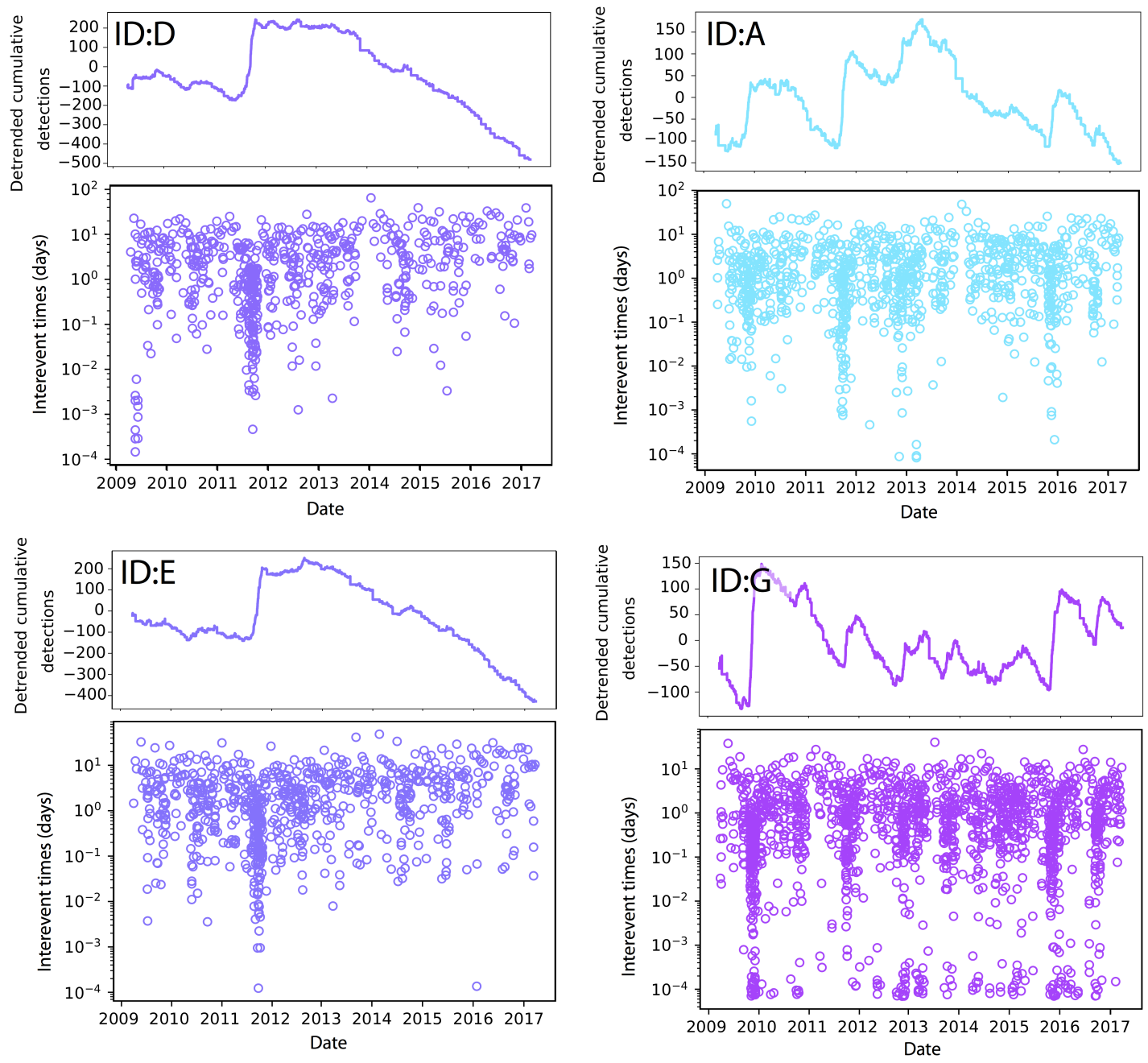

(b) Detrended cumulative detections and inter-event times for LFE family IDs: D

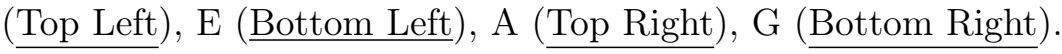

Figure 4.15: Detrended cumulative detections and inter-event times for a subset of 8 LFE families. LFE families are coloured by depth as per legend in Fig. 4.14. Upper pannel: Detrended cumulative detections. Lower pannel: Interevent times. 
If we take the example of LFE source regions where the local fault conditions include lower effective stress (e.g. higher pore pressure) or finer grained fault gouge material (e.g. mylonite clay gouge), we might expect those areas to display continuous styles of LFE occurrence (the equivalent of a stable-sliding regime). Now, if we look at LFE source regions where higher effective stress or coarser gouge material are found, they might tend to display episodic LFE occurrence (i.e. a stick-slip regime).

To further quantify the style of LFE occurrence for each individual source, we compute the coefficient of variation of the daily event rate $r$ in the style of Trugman et al. (2015), $C O V_{r}$ :

$$
C O V_{r}=\frac{\operatorname{std}(r)}{\operatorname{mean}(r)}
$$

$C O V_{r}$ corresponds to the standard deviation in the daily rate divided by the mean daily rate (for each LFE source), hence LFE families that are 'continuous' have low $C O V_{r}$, while those that are episodic (or 'stepped') have high $C O V_{r}$.

We observe that shallower LFE sources tend to possess a high $C O V_{r}$ whereas deeper events show lower $C O V_{r}$ values (Fig. 4.16). This implies that LFE sources seem to evolve from episodic or 'stepped' (high $C O V_{r}$, warmer colours in Fig. 4.16) to continuous (low $C O V_{r}$, colder colours in Fig. 4.16) with depth. This observation is consistent with findings in the San Andreas region where similar LFE patterns are observed (e.g. Trugman et al., 2015).

\subsubsection{Implications for deep Alpine Fault}

The LFE locations highlight a broad area of slow deformation near the deep Alpine Fault (from depths of 11 to $59 \mathrm{~km}$ ). LFEs hence occur just below the seismogenic zone defined by microseismicity (0-10 $\pm 2 \mathrm{~km}$, Boese et al., 2012). However, because of the bias stemming from the use of our starting model grid (extending from depths of 16 to $44 \mathrm{~km}$ ), we have not looked for the existence of shallower LFEs (i.e. between 0-10 km). Boese et al. (2012) established a strong correlation between the thickness of the seismogenic zone and the shape of a high-resistivity zone (Wannamaker et al., 2002) and argued that crustal microearthquakes were clustered in those high-resistivity areas; no 'typical' earthquakes being generated at depths greater than $20 \mathrm{~km}$ in the deeper lower resistivity regions. Deep earthquakes were however recorded by Boese et al. (2013) mostly to the west of Mt. 


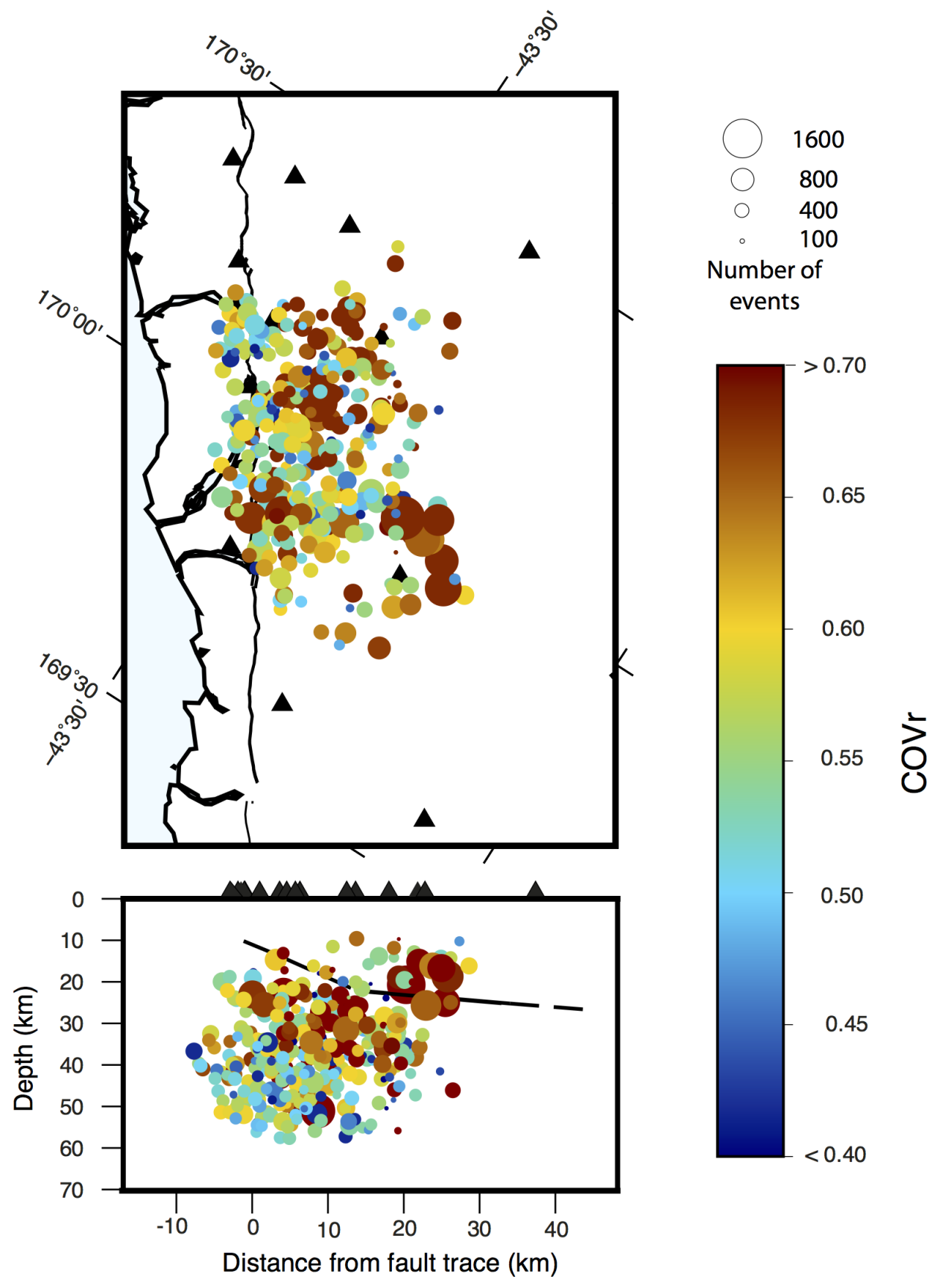

Figure 4.16: Map of event rate $C O V_{r}$. The colour scale indicate the value of $C O V_{r}$ calculated after the definition of Trugman et al. (2015). Circles are sized by the total number of events for that particular family. Top: Rotated map view. Bottom: Projection along profile aa' of Fig.4.12.

Cook and in the southwest portion of the SAMBA network; roughly in the same area as some of the LFEs but at slightly greater depths (brown circles vs coloured 
stars in Fig. 4.12). Our final locations reflect our starting model grid to some extent, with LFEs filling a gap left by both shallow (0-12 km, Boese et al., 2012) and deep seismicity (50-100 km, Boese et al., 2013) in the central Southern Alps.

We have made the first observation of LFEs northeast of Mt. Cook, which is where other authors (Boese et al., 2012, 2013; Wech et al., 2012) observed a noticeable change in the shallow and deep seismicity pattern along strike (along profile bb', Fig. 4.12). This change in seismicity seems to coincide with a change in fault geometry. Geophysical and geological evidence suggest that the geometry of the Alpine Fault changes in the area beneath Mt. Cook from a subvertical structure in the north to a listric fault south of Mt. Cook (Stern et al., 2001; Little et al., 2005; Okaya et al., 2007). Close to $15 \%$ of the LFE families (amongst our most reliable locations) are clustered around the presumed change in dip and locate within $10 \mathrm{~km}$ of the GPS inferred Alpine Fault (Lamb and Smith, 2013). We also find that the LFEs closer to the fault have higher generation rates (similarly to the extended LFE catalogue of Baratin et al., 2018, cf. Chapter 3) than the ones away from the fault.

Another interesting new feature is that $~ 50 \%$ of the LFE families are located to the SW of the proposed deep extent of the Alpine Fault. The majority of those families is accurately located, and locate between $25 \mathrm{~km}$ and $45 \mathrm{~km}$ depth. Moreover, those locations coincide with a previous LFE family location of Baratin et al. (2018)/Chapter 3. The position of those LFE families could help us discern the geometry of the Alpine Fault in this area provided that some focal mechanisms are obtained for them. We assume that those LFE families may occur on a more steeply-dipping branch of the fault as suggested by other studies (Koons et al., 2003; Norris and Toy, 2014), but in-depth analysis of focal mechanisms would be needed to confirm this.

We note that the majority of the LFEs occur in areas of high reflectivity (Okaya et al., 2007), low seismic wave speeds (Stern et al., 2001), high P wave attenuation (low $Q_{p}$, Eberhart-Phillips et al., 2008) and increased fluid pressure (Stern et al., 2001; Wech et al., 2012). Yet, for the first time, we observe some LFEs occurring in areas of high $Q_{p}$ (Fig. 1.10 in Chapter 1 for a map of $Q_{p}$ Eberhart-Phillips et al., 2008). Those distinctive local fault conditions might explain the diversity of LFE behaviours.

Finally, we observe a variation of LFE time cumulative signature patterns with depth, with LFE sources evolving from episodic or 'stepped' behaviour to continuous. This finding suggests an evolution from an unstable stick-slip behaviour to 
more stable-sliding and ductile behaviour with depth (Johnson et al., 2012, 2013; Trugman et al., 2015).

Similar behaviours have been documented in tremor zones elsewhere (e.g. in Japan (Obara, 2010), in Cascadia, (Wech and Creager, 2011) and in San Andreas (Trugman et al., 2015)). Those observations may have broader implications for seismic hazard in the Alpine Fault area as LFEs may play a role in loading the locked portion of the Alpine Fault, hence influencing the nucleation of an eventual large earthquake.

\subsection{Conclusions}

We present a new method for generating real LFE templates by using synthetics in a matched-filter routine. We have proven the method's effectiveness in detecting LFEs in New Zealand's central Southern Alps. We have built the longest record (spanning 8 years) of LFEs in New Zealand to date as well as the first spatially and temporally continuous catalogue of LFEs beneath the central Southern Alps. Using 638 synthetics templates in a matched filter routine, we create 529 real LFE templates to use in a new matched-filter iteration. This process allows the detection of 300,996 events between 26 March 2009 and 26 March 2017 (i.e. 30 times more events than in the extended catalogue of Baratin et al., 2018, cf. Chapter 3), amounting to a long-term rate of 103 LFEs per day. We find a $67 \%$ overlap between the extended LFE catalogue and this study's catalogue (i.e. we miss $1 / 3$ of the previous catalogue's detections), thus suggesting that we are indeed mainly detecting real events in the new continuous LFE catalogue.

We manually pick and locate 378 families and thus obtain the locations of hundreds of LFE families which allows us to discuss the extent of LFEs within our study area. The LFEs locate at depths of $11-59 \mathrm{~km}$ (roughly at similar depths to tremor). We also show that LFEs mainly occur in (but are not entirely bound by) an area of low resistivity (Eberhart-Phillips et al., 2008), low seismic-wave speeds (Stern et al., 2001) and increased fluid pressure (Stern et al., 2001; Wech et al., 2012).

Cumulative detections show continuous detections punctuated by increases in LFE rate coinciding in some cases with known tremor events and large $\left(>M_{w} 5\right)$ regional earthquakes. We also observe an interesting episode at the end of 2011, with $\sim 50,000$ LFEs generated in only 3 months, which could indicate that a largescale slow slip event occurred at that time (Frank et al., 2016). These observations 
suggest that accelerated rates of LFEs generation might represent episodic slow slip on or near the deep extent of the Alpine Fault.

Finally, we observe a variation of LFE time cumulative signatures patterns with depth, with LFE sources evolving from episodic or 'stepped' behaviour to continuous. This finding suggests an evolution from an unstable slip behaviour (stick-slip) to a more stable, ductile (stable-sliding) slip behaviour with depth. 


\section{Spatio-temporal characteristics of low-frequency earthquakes in the central Southern Alps*}

This Chapter summarises the spatio-temporal analysis of the low frequency earthquakes (LFE) catalogue generated in Chapter 4. A detailed study of the spatial and temporal evolution of low-frequency earthquakes in the central Southern Alps is required to better understand the behaviour of the LFE sources and the inferred slip behaviour of the deep Alpine Fault. To achieve this goal, we use an extensive continuous catalogue of more than 300,000 LFEs detected along the central Alpine Fault from 2009 to 2017. These LFEs have been detected using a multichannel matched-filter search, via cross-correlations of 529 different templates with a continuous seismic dataset (of those templates, 378 have been located). Here, we use the high temporal and spatial resolutions of the continuous catalogue, to provide new insights into LFEs recurrence time, occurrence pattern and migration as well as to identify potential episodes of slow slip. We first use LFEs occurrence pattern to identify periods of uncommonly high LFE activity. We find that all but one of the LFE episodes identified in this study from 2009-2011 occur concomitantly with tremor identified by Wech et al. (2012). We thus infer that those periods could be indicative of slow slip events. We then compare LFE behaviours between an episode of high LFE activity and a period of low activity. We find that during a period of low LFE activity, only $\sim 30 \%$ of the LFE families are active and the LFE sources with the highest activity are located closer to the Alpine Fault. However, during an episode of increased activity, we observe that all the LFE families become active, with intermittent extreme LFE rate increases. This behaviour might reflect a rate-dependent friction on the fault, with the increase in LFE rates being indicative of an external increase in loading. Alternatively, this behaviour could also be indicative of possible triggering occurring between LFE families. We also discover slow $(10 \mathrm{~km} /$ day $)$ and rapid $(\sim 20-25 \mathrm{~km} / \mathrm{h})$ migrations of LFEs along the Alpine Fault, with velocities similar to what has been found

\footnotetext{
*In preparation for submission to Journal of Geophysical Research. The clustering analysis represents preliminary work intended to facilitate further analysis of LFEs in the study area, and as such, will not be included in the submitted manuscript.
} 
elsewhere (e.g. Mexico, San Andreas). The slow migration might be controlled by slow slip events themselves while the rapid velocities could be explained by the LFE sources' inherent properties. For example, the rapid velocities could be explained by increased average slip rates linked with slip-induced dilatancy in the LFE source area. The static stress transfers occurring during repeated episodes of slip, might for instance generate a series of LFEs propagating along the fault zone, whose duration and speed could depend on the overall fluid diffusion time.

\subsection{Introduction}

Extensively studying the spatio-temporal characteristics of LFEs might help better understand the behaviour of a fault prior to a large earthquake, hence improving our comprehension of seismic hazard. Indeed, large and damaging earthquakes typically originate in the upper crust, where a brittle frictional regime usually prevails. However, understanding the behaviour of the upper crust is not enough in itself to characterise seismic hazard. Lower crustal slip (occurring beneath the seismogenic zone) can prompt seismicity in the upper crust and lead to the nucleation of large earthquakes (e.g. Scholz, 2002; Nadeau and Guilhem, 2009; Shelly, 2009; Segall and Bradley, 2012). LFEs have been found to accompany slow slip events occurring on the deep extent of faults in Cascadia (Rogers and Dragert, 2003; Bostock et al., 2012), in Japan (Obara et al., 2004; Shelly et al., 2006), in California (Nadeau and Dolenc, 2005; Shelly, 2009) and in Mexico (Frank et al., 2015). As a consequence, LFEs (i.e. slip events that occur repeatedly at source locations within the lower crust) can be used to elucidate the slip behaviour of the lower crust (Rubinstein et al., 2009b).

Mechanisms for LFEs worldwide reveal that LFEs involve dominantly shear slip on the deep extent of major faults (e.g. Shelly et al., 2007; Ide et al., 2007b; Frank et al., 2013; Baratin et al., 2018). This suggests that LFEs can be monitored in order to semi-qualitatively detect occurrences of slip, especially if the slip is too small to be observed geodetically. This approach has been used successfully in the Mexican and Cascadia subduction zones where the repeating nature of LFEs occurrence was used to enhance geodetic signals in order to detect repeating episodes of slow slip (Frank et al., 2015; Frank, 2016). Therefore, episodes of increased LFE activity may be used to identify slow slip events and hence, to further investigate the rheology and slip behaviour of the lower crust. 


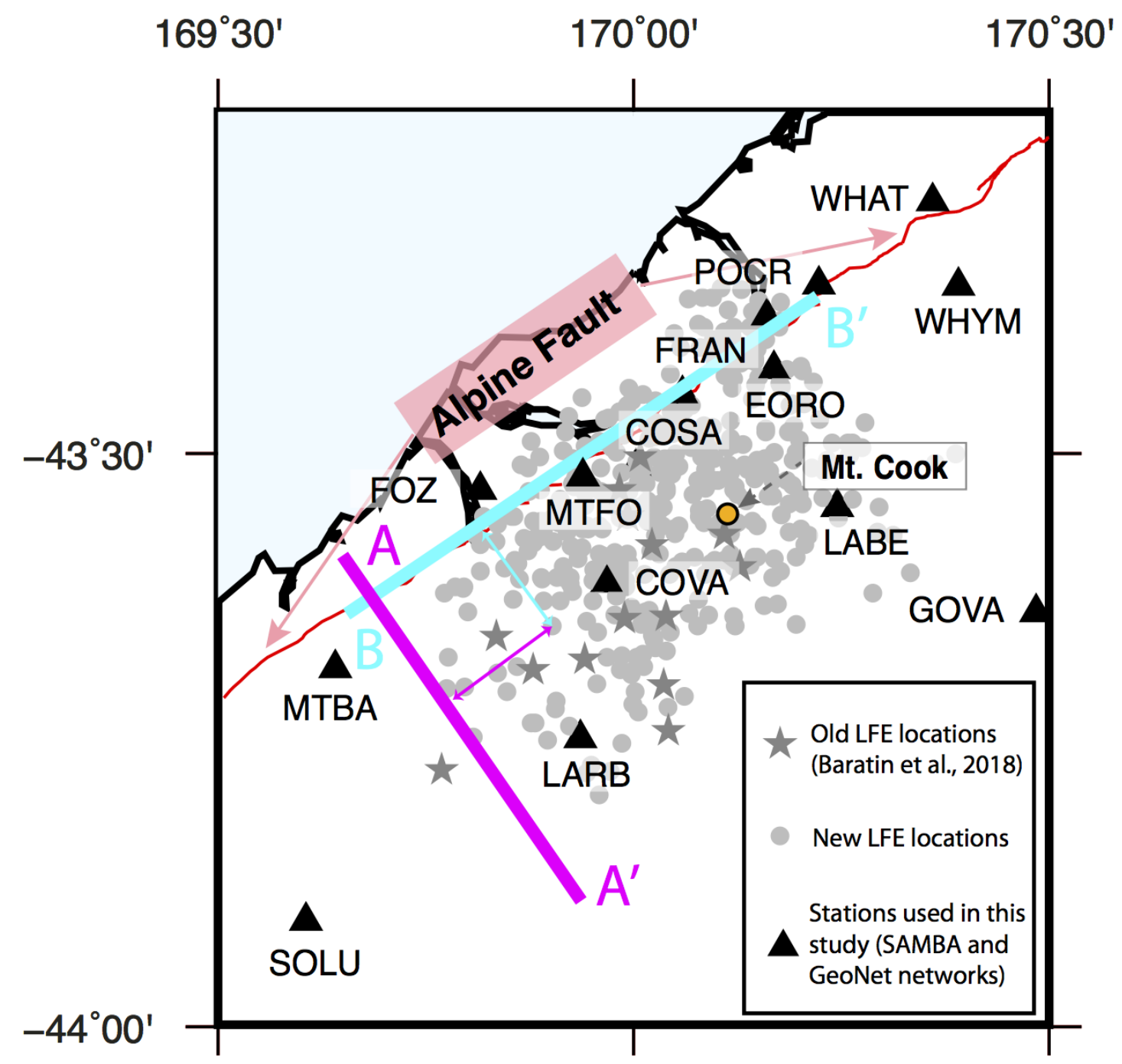

Figure 5.1: Map of study area. Map of the central Southern Alps showing the previous LFE locations of Baratin et al. (2018)/Chapter 3 as well as the new LFE locations obtained in Chapter. 4. Throughout this Chapter, LFE locations projected onto profile AA' will be referred as 'distance along strike' and LFE locations projected onto profile BB' will be referred as 'distance from surface fault trace'.

Here, we use a continuous catalogue of 300,996 LFEs distributed along the central Alpine Fault. These LFEs have been detected by cross-correlation of 529 different templates with a continuous seismic dataset between March 2009 and March 2017 (see Chapter 4 for a detailed description of the generation of the continuous LFE catalogue). We locate 378 LFE families (Fig. 5.1) and find that the LFE sources range in depth from 11 to $59 \mathrm{~km}$ and span an area of $60 \times 55 \mathrm{~km}$ along the central Alpine Fault. LFE individual family rates and occurrence pattern indicate that LFE sources seem to evolve from an episodic behaviour (subject to many bursts) 
to a continuous behaviour (linear over time) with depth. This transition may correspond to an evolution from a stick-slip slip regime to a stable-sliding slip regime with depth (Johnson et al., 2013; Trugman et al., 2015). Here, we use the high temporal and spatial resolutions of this catalogue, to (1) provide new insights into LFEs recurrence time, occurrence pattern and migration; as well as to (2) further understand the slip behaviour of the deep Alpine Fault.

\subsection{Methods}

\subsubsection{LFE episodes}

To start examining the space-time correlations in the LFEs occurrence pattern, we first need to identify periods in which all LFE families are particularly active. An intuitive approach to track up periods of increased LFE activity is the visual inspection of the number of bursts. We define a burst as consecutive detections separated by less than 120 s (i.e. inter-event time or recurrence time $<120$ s) as done in Chapter 3/Baratin et al. (2018). A high number of bursts usually coincide to an intense period of LFE activity.

Here, we attempt to establish an improved method to identify particularly active periods of LFEs within the whole catalogue. We begin by breaking down the observation interval (a total of 2,923 days from the $26^{\text {th }}$ March 2009 to the $26^{\text {th }}$ March 2017) into discrete 1-day time epochs. We then calculate the sum of all the LFEs detected for each epoch (all families taken together) yielding a 2,923component vector, with each component per time epoch representing the LFE daily rate.

We next compare all the daily rates in the vector by studying the degree of variance in the data, using a whisker plot of the daily rates and looking for outliers. We empirically establish a threshold over which the LFE daily rate is considered as extreme. We choose this threshold as being equal to 3 standard deviations above the mean daily rate (i.e. highest daily rate value still within the 1.5 interquartile range of the upper quartile). This threshold was chosen as any value above it is considered an outlier.

To establish the duration of a period in which LFE families are extremely active, we require that the LFE daily rate exceeds the chosen threshold for at least 5 days in a 10-day sliding window. This way, we obtain the start and end-dates corresponding to the outer bounds of the increased LFE activity period. In the 
remainder of this Chapter, we will refer to those periods as 'LFE episodes'. The threshold values ( 5 and 10 days) were chosen after extensive testing, as their combination optimised the number of peaks in the LFE daily rate that were part of an LFE episode.

\subsubsection{LFE migrations}

To conduct a detailed study of the spatio-temporal evolution of the LFE activity, we only use the LFE families that could be located. Since each LFE family is presumed to correspond to a single source, we ascribe the same location to each of the individual detections in a given family.

To study LFE migration patterns, we start by converting each LFE family's location to:

(1) a distance along strike, by projecting the individual LFE location along profile AA' (Fig. 5.1);

(2) a distance from the surface fault trace, by projecting the individual LFE location along profile BB' (Fig. 5.1).

We then plot the LFE locations in time and space for every LFE in the catalogue. Since each LFE family has been assigned a unique distance along strike (or from the surface fault trace), each LFE family can be found on a distinct horizontal line with respect to the time of detection on such a plot.

We finally calculate migration velocities of the LFE clusters. To do so, we select periods of increased LFE activity (i.e. LFE episodes as defined in Section 5.2.1) and plot the LFE detections for different time scales (e.g. a few days to a few weeks). We visually inspect each LFE cluster to check its inclination. We then calculate the migration velocity of the cluster (i.e.corresponding to the slope of the cluster) which gives us an estimate of the speed for LFE migration in the area.

\subsubsection{LFE family redundancy}

Because the LFEs detection method used here can detect distinct events in time originating from the same location, it is essential to check the redundancy of the LFE families that we managed to locate to see if we can identify families that are very similar. 
To check the redundancy of the LFE families, we use the method developed by Frank et al. (2014). Frank et al. (2014) start by comparing the detection times of one LFE family, described as a vector of detection times $t_{l}$ of length $L$, to the detection times of all other LFE families. In this study, we consider that any event that is detected within $3 \mathrm{~s}$ (i.e. half the duration of an LFE template) of another family's has been detected at the same time. We use a $3 \mathrm{~s}$ time-window to take into account the fact that the start of the event's waveform within the $6 \mathrm{~s}$ long template window is not automatically similar for each template. The next step is to calculate the percentage of events of an LFE family that are presumed to have been detected at the same time as the events of another LFE family. This percentage is what Frank et al. (2014) call the 'family redundancy', $R$, and it is defined for each unique pair of LFE families $i$ and $j$ as:

$$
R_{j}^{i}=\frac{\sum_{l}^{L} r^{j}\left(t_{l}^{i}\right)}{L}
$$

where $l$, is the detection number for LFE family $i$ of total length $L, t_{l}^{i}$ is the time of detection and $r^{j}\left(t_{l}^{i}\right)$ is defined as:

$$
r^{j}\left(t_{l}^{i}\right)= \begin{cases}1, & \text { if }\left|t_{l}^{i}-t_{l^{\prime}}^{j}\right|<=3 \\ 0, & \text { otherwise. }\end{cases}
$$

where $l^{\prime}$ is the detection number for family $j$.

If the redundancy is greater than a certain threshold for a given pair of LFE families, the waveform similarity of the two LFE families' stacked waveforms is consequently calculated. We choose this threshold to be equal to 0.1 here, which corresponds to $10 \%$ of similarity between the detections of an LFE pair. The waveform similarity corresponds to the mean of the correlation coefficients for the stacked waveforms of the families $i$ and $j$, calculated for each station, and component by component. If the waveform similarity is also above a certain threshold (estimated at 0.2 ), then LFE families $i$ and $j$ can be grouped together into a new composite family, yielding a new LFE family with a higher signal-tonoise ratio (SNR) stack (Frank et al., 2014). 


\subsection{Results}

\subsubsection{LFE activity temporal variations}

\section{Identifying LFE episodes}

We use the LFE catalogue described in Chapter 4 to analyse the LFE space-time correlations. This catalogue contains $\geq 300,000$ LFEs occurring between March 2009 and March 2017 and distributed along the central Alpine Fault. Here, we use the LFEs occurrence pattern to identify periods of uncommonly high LFE activity.

We first study the evolution of the LFE detections over time by looking at (from top to bottom in Fig. 5.2): (1) detrended LFE count, (2) LFE recurrence time (i.e. inter-event times) and (3) LFE daily rate. The detrended LFE cumulative count (top panel of Fig. 5.2) shows a pronounced increase or 'step' in LFE detections between July and October 2011, as well as several smaller steps (e.g. October 2009, 2015). We then look at recurrence interval to see if the LFEs behaviour is comparable to that of the extended catalogue of Baratin et al. (2018)/Chapter 3. Inter-event times show two distinct types of periods: (1) overly active periods, where several consecutive bursts (i.e. inter-event time $\leq 10^{-3}$ days in the middle panel of Fig. 5.2) are observed and (2) quieter periods, where almost no bursts are seen. This type of behaviour was not as easily seen in the extended catalogue of Baratin et al. (2018)/Chapter 3, due to the paucity of detected events and overall small number of bursts. Additionally, Baratin et al. (2018)/Chapter 3, observed a gap in detections at an inter-event time of $\leq 10^{-2}$ days (i.e. 15 minutes). They attributed this gap to an incomplete spatial representation of the LFE sources in the catalogue (the original 14 templates being picked manually through periods of tremor located exclusively in an area south of Mt. Cook). This hypothesis is confirmed with the continuous catalogue as no gap is observed around that time, and as this catalogue is using a considerably higher number of LFE families with a much larger spatial extension. We notice that abrupt daily rate increases (warm colours in bottom panel of Fig. 5.2 and sharp steps in the cumulative detection curve in top panel of Fig. 5.2) typically coincide with an increase of the number of bursts (middle panel of Fig. 5.2). 


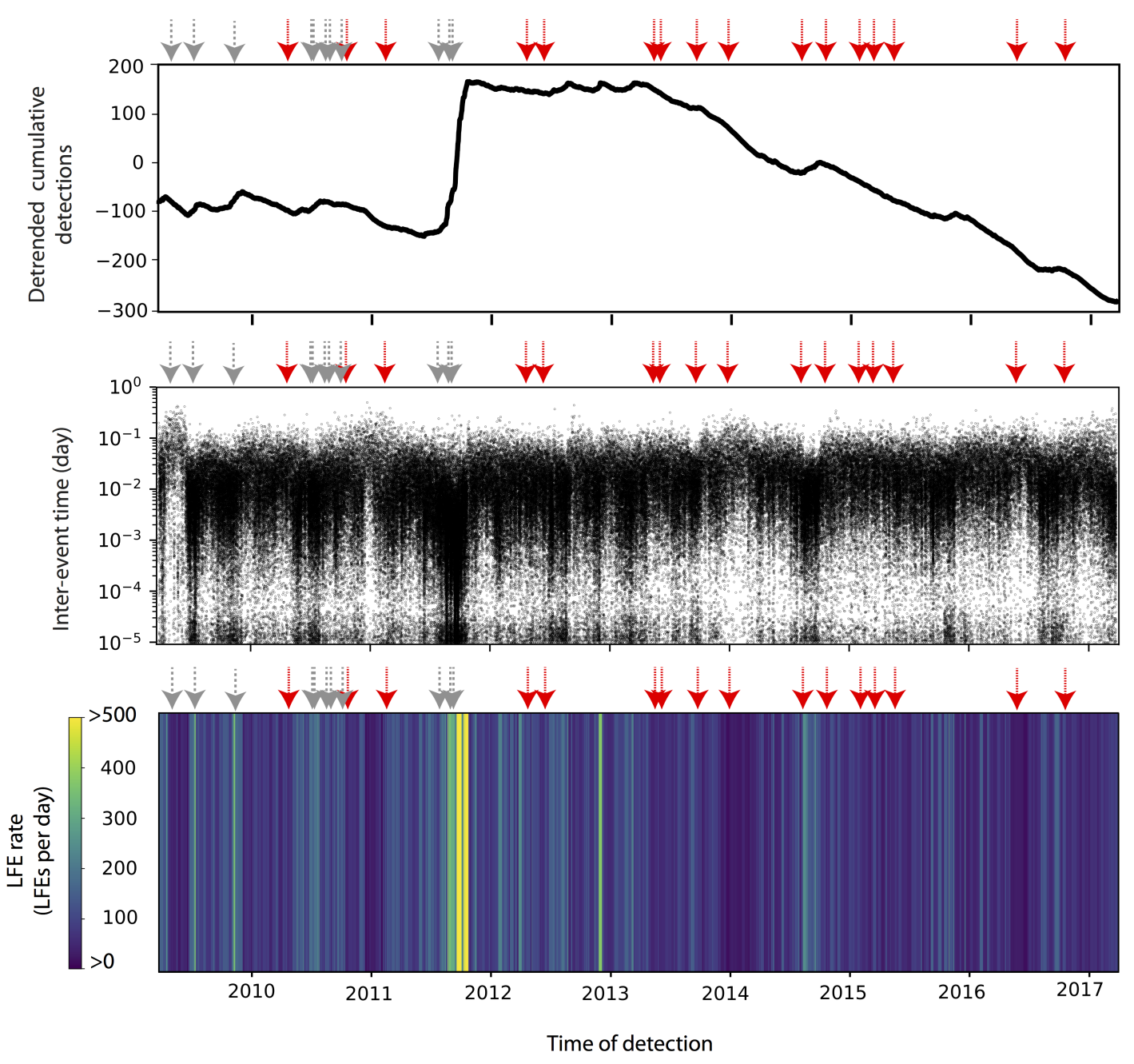

Figure 5.2: Evolution of the LFE catalogue in time. The gray and red arrows correspond respectively to tremor identified by (Wech et al., 2012) and large regional earthquakes in the study area. Top: Detrended cumulative number of detections for the entire synthetic LFE catalogue. Middle: Inter-event times for the entire catalogue. Bottom: Daily rates of LFEs per day over the study period. 
To identify LFE episodes, we set a threshold of 3 standard deviations above the mean LFE daily rate which amounts to a threshold value of 219 LFEs/day (Fig. 5.3). If this threshold is exceeded for 5 days in a 10-day sliding window period, we obtain the start and end-dates corresponding to the outer bounds of the increased LFE activity period. This way, we identify 8 episodes of LFE increased activity (Fig. 5.3 and Table 5.1) between March 2009 and March 2017. Table 5.1 lists all the characteristics of the 8 LFE episodes identified in this study. We find, for these episodes, an overall average daily rate equal to $234 \mathrm{LFEs} /$ day, with LFE counts varying between 1,888 and 47,093 detections, depending on the episode. The mean duration of the identified LFE episodes is 26 days. The largest LFE episode (ID:4 in Fig. 5.3 and Table 5.1) has a duration of 95 days and $\sim 47,000$ events are detected over this period, with an average daily rate of $496 \mathrm{LFEs} /$ day. This amounts to $17 \%$ of the entire catalogue being detected over a period equivalent to $3 \%$ of the total catalogue duration. A detailed study of this particular episode is carried out later in this Section.

We find that almost all of the LFE episodes identified in this study from 20092011 occur concomitantly with tremor detected by Wech et al. (2012). Wech et al. (2012) found tremor on 3 days in August 2011 and September 2011 (no tremor analysis was undertaken beyond September 2011) which is when we observe the biggest LFE episode (ID:4 in Table 5.1). Out of the 12 days where tremor was found, 5 are part of the LFE episodes. The only episode that doesn't occur concurrently with tremor (between 2009-2011), starts on the $17^{\text {th }}$ of July 2010 and lasts until the $29^{\text {th }}$ of July 2010 (episode ID:3 in Table 5.1). However, Wech et al. (2012) happened to observe two episodes of tremor occurring on the $5^{\text {th }}$ of July and on the $14^{\text {th }}$ of July 2010, as well as two other episodes of tremor on the $20^{\text {th }}$ and $31^{\text {th }}$ of August 2010. Hence, it is possible that either tremor occurred during this LFE episode and its detection was missed, or that the threshold we chose for the identification of the LFE episode was too strict and that this episode actually lasted longer (thus including all 4 days of tremor).

Ultimately, of the total 11 episodes of tremor that were identified between 2009 and 2011, 5 of them occurred during the LFE episodes, 2 of them occurred within days of an LFE episode and 2 others occurred in the next two weeks following an LFE episode. 

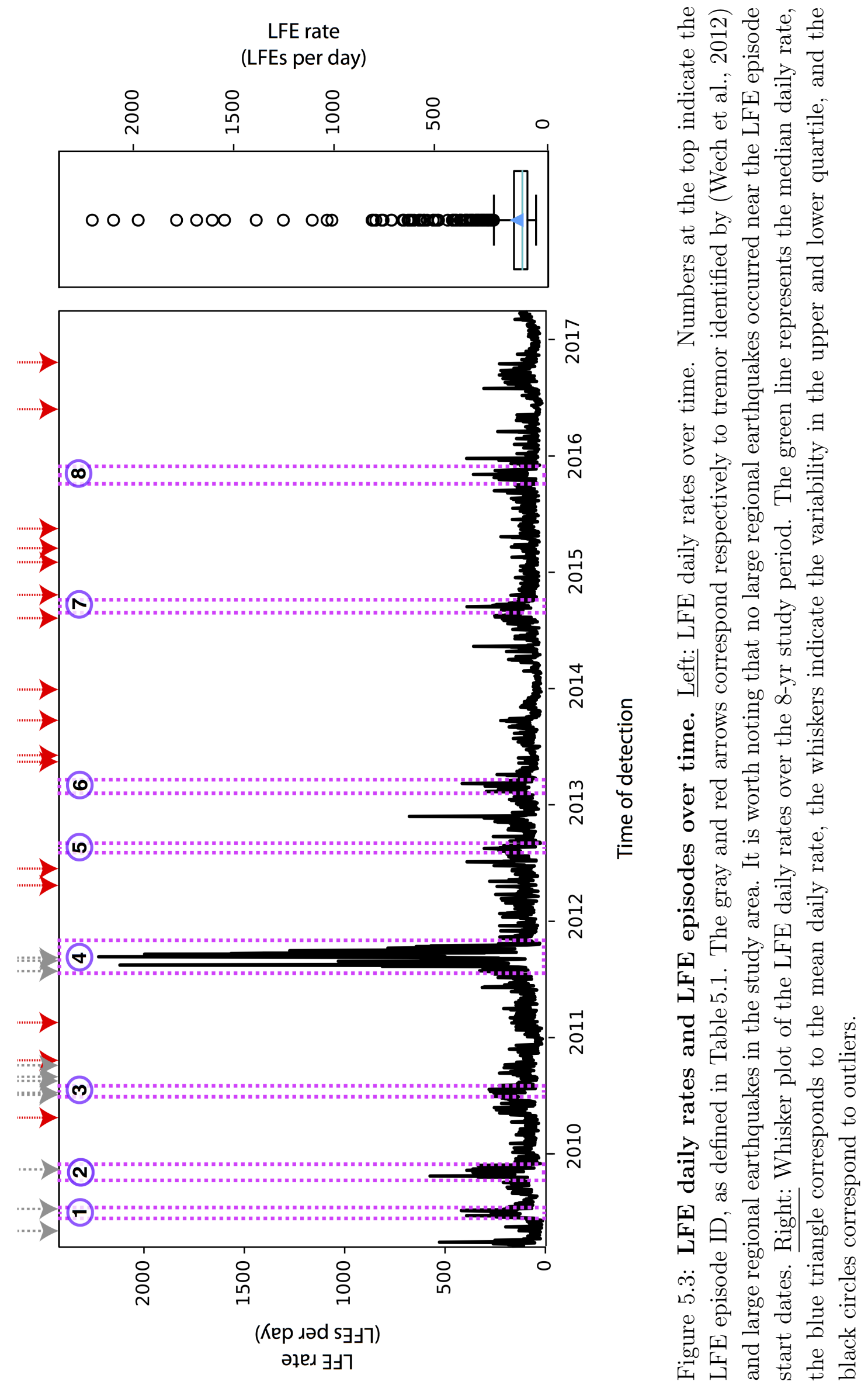

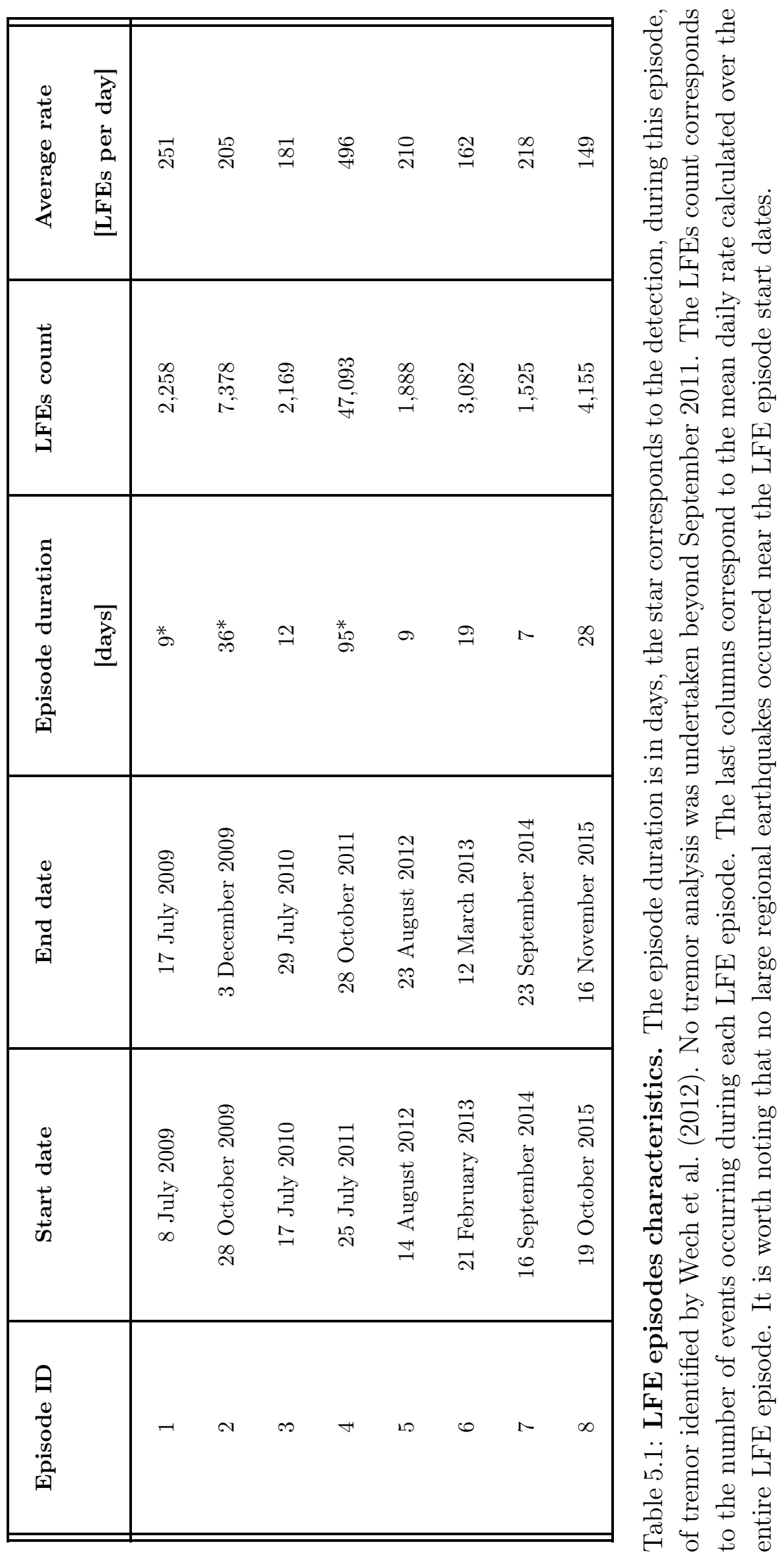
We thus infer that all of the periods that we identified as LFE episode could be indicative of occurrences of tremor as well as slow slip events. Interestingly, Wech et al. (2012) noticed a certain periodicity in tremor occurrence between 2009 and 2011, with tremor being observed every year around the months of July/August. We see a similar pattern with the LFE episodes identified here. Therefore, it is possible that a seasonal phenomena (occurring during winter time) might influence tremor/LFE generation. However, additional work on tremor/LFE triggering is required to corroborate this hypothesis.

\section{LFE activity behavioural styles}

The study of LFE inter-event times has highlighted two distinct types of periods: (1) some extremely active periods, where several consecutive bursts are observed and (2) some quieter periods, where almost no bursts are seen. Here, we compare two distinct periods having the same duration (95 days): an extremely active period, LFE episode ID:4 (Table 5.1) lasting from the $25^{\text {th }}$ July 2011 to the $28^{\text {th }}$ October 2011; and a background activity-like period, that we refer to as the 'quiet period', lasting from the $13^{\text {th }}$ November 2013 to the $16^{\text {th }}$ February 2014 (respectively red and blue boxes in Fig. 5.4A).

By looking at the cumulative event counts for all the LFE families during the two selected 3-month windows (Fig. 5.4B), two different types of behaviour are clearly illustrated. During the quiet period, the LFEs occur at an approximately constant rate (blue curve in Fig. 5.4B). However, during the LFE episode (red curve in Fig. 5.4B), the events occur mainly as bursts, which are seen as sharp steps in the cumulative event counts. A zoomed view of these sharp steps indicates that the large steps (or bursts) are often made of many smaller steps, which could be indicative of a scale-invariant time clustering (Frank et al., 2016). In this study, we use the definition of clustering described by Frank et al. (2016) as relating to the timing of any given event being: (1) dependent on events that precede it or influenced by events that follow it and (2) not randomly distributed in time.

We then try to quantitatively characterise the observed clustering, during the LFE episode and during the quiet period, following the approach of Frank et al. (2016). We start by converting the catalogued events for each period, and for every LFE family (each corresponding to a unique source), into a discrete event count signal. We then compartmentalise both periods into 1-minute time epochs, regrouping each event into the time step during which it is detected. We next compute an autocorrelation function for both time series (Fig. 5.4C). 

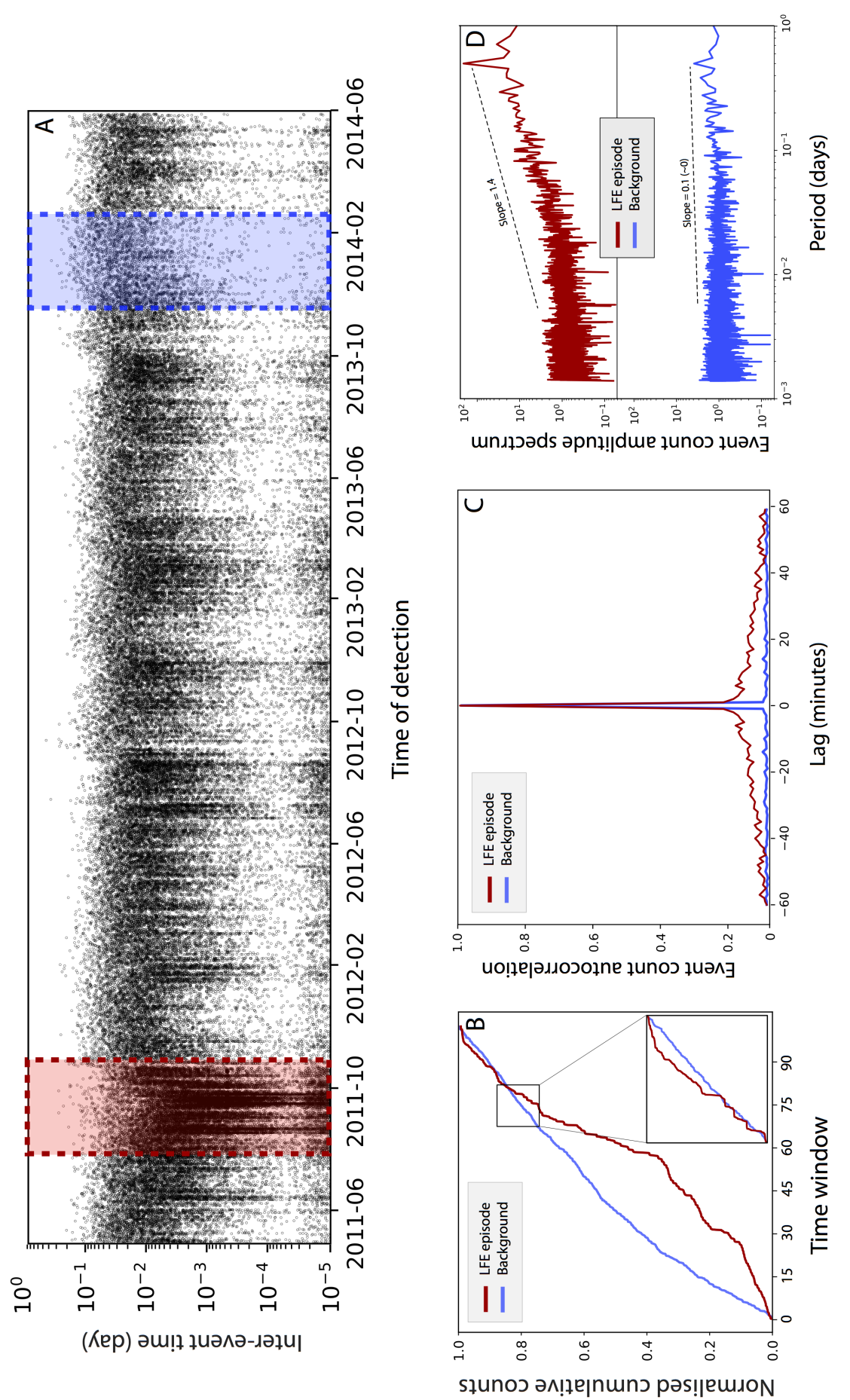

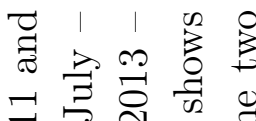

尺ิ

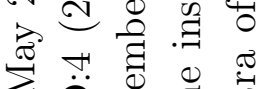

光草邑

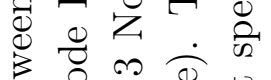

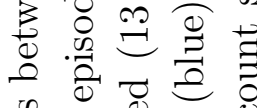

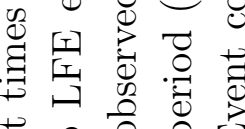

궁요

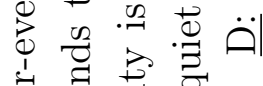

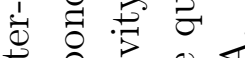

.尹

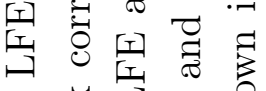

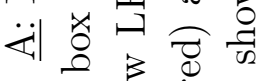

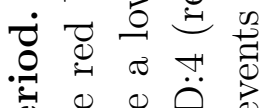

过包它

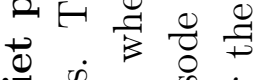

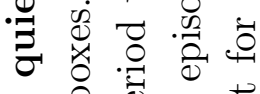

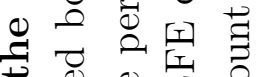

む怘 \&

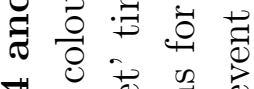

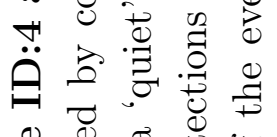

焉

.

일

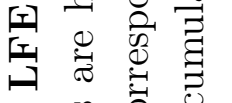

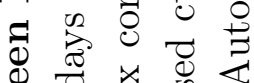

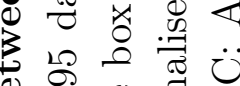

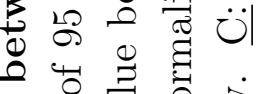

궁

象

สิ

范

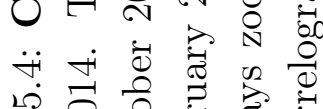

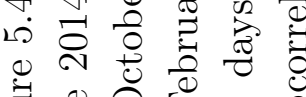

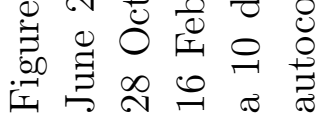


For the quiet period, the autocorrelation function appears like a Dirac at zero lag time (blue curve in Fig. 5.4C). This shows that during this period, the LFEs seem to occur as a Poisson process (Lowen and Teich, 2005). However, the autocorrelation function for the LFE episode displays a steady decrease from zero lag time (red curve in Fig. 5.4C). This behaviour is indicative of short-term correlations (or clustering) between the events during this period. Lastly, we compute the Fourier spectra for each autocorrelation functions and plot the resulting spectra in log-log space (Fig. 5.4D). We find that during the quiet period, the amplitude spectra is mostly flat with a slope equal to $\sim 0$, which is what we would expect for a Poisson-like behaviour, where event occurrences are uncorrelated in time (blue curve in Fig. 5.4D). Yet, during the LFE episode, the amplitude spectrum increases with the period, illustrating a scale-invariant time clustering (red curve in Fig. 5.4D). To quantitatively estimate the degree of clustering occurring during an LFE episode, we can measure the power law exponent, which corresponds to the linear slope of the spectrum (in log-log space). We calculate the power law exponent (or slope) for this particular LFE episode to be equal to 1.4. This value being significantly greater than 0, we suggest that during LFE episodes, a strong clustering of events in time is occurring.

A concern that was raised in Chapter 4, is that the huge 'step' that we see in the cumulative detections (corresponding to LFE episode ID:4) could be mostly composed of false detections. In order to address this, we choose an LFE family that is occurring not only during the LFE episode but also during the quiet period (LFE family ID:170.052_-43.8019_44_1). The goal is to thoroughly look at the individual detections made during those times and check that the same LFE source is active during both periods.

We therefore stack the best $25 \%$ of events occurring during the LFE episode for LFE family ID:170.052_-43.8019_44_1 (red waveforms in Fig. 5.5) and all of the events occurring during the quiet period for that same family (blue waveforms in Fig. 5.5). We decide to stack all of the events for the quiet period (and not just the best $25 \%$ ) as there is a limited number of events to start with. We use 31 detections in the LFE episode stack (for a total number of 126 events detected for this family during the LFE episode) against 15 events for the stack of the quiet period. Keeping in mind that we are stacking detections from a short period of time (95 days), and that we are using a reduced number of events, we anticipate an overall low SNR for the stacks. 


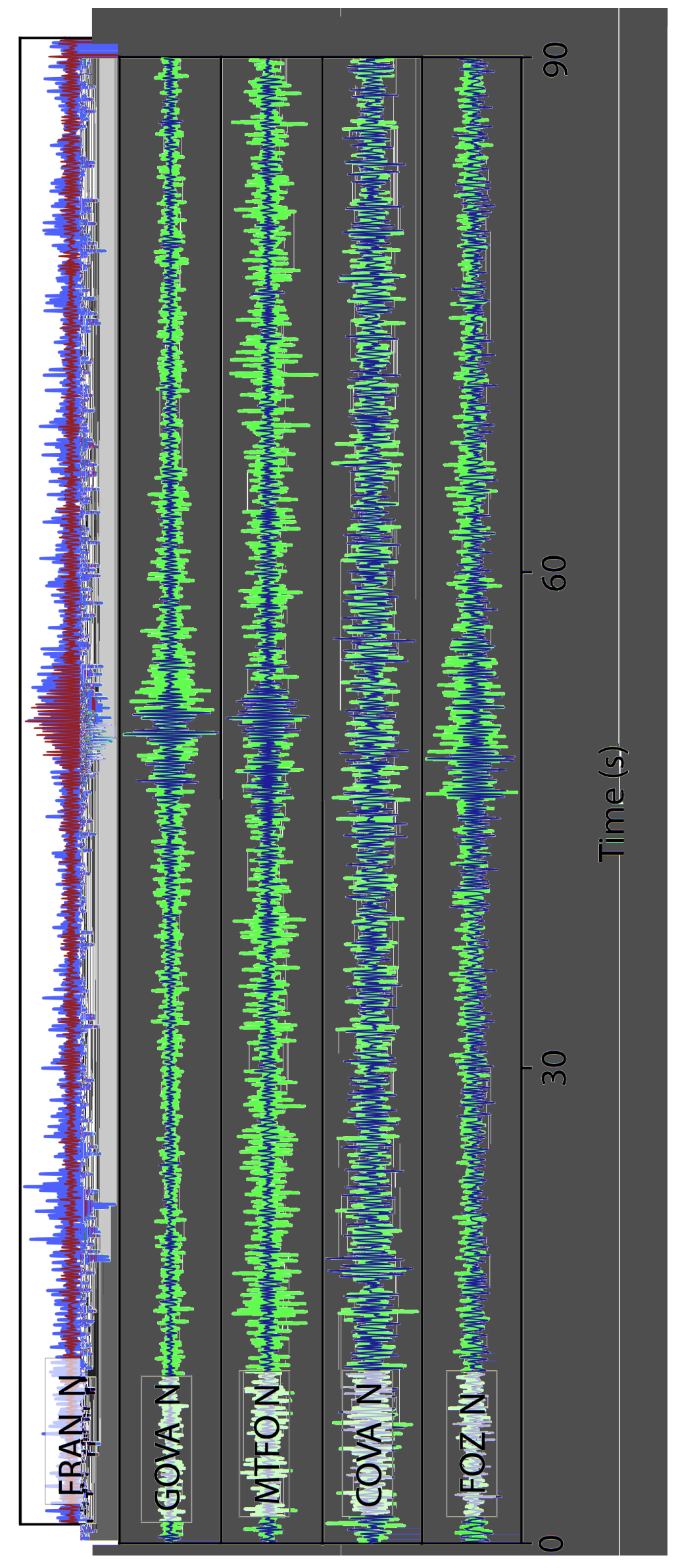

氖

ఠ $\Xi \Xi$

ఫิ

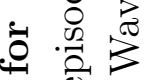

急项

ชี ซี

番

일.

되

告寻

ส

-10 ठ

궁

है है

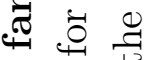

玨总壱

(c)

:

is -1

* $\exists$

४⿻

द 00

$\infty$

육

\% 1.

เ ํํำ

ర용

讨

的至

ఫृ శ్ష

$3 \stackrel{\pi}{\pi}$

पै

- छ

ठี

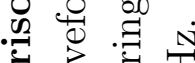

สี

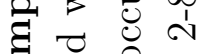

हृ

范范

वे

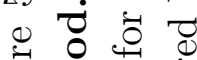

$\exists$.

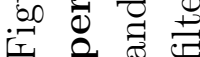


However, even with the low SNR, we are able to see that the stacks are very similar for both periods (Fig. 5.5). The quiet period stack (blue waveforms in Fig. 5.5) appears noisier, which is expected as a lesser number of 'good' signals is used in the stack. Yet, we can still observe similar arrival times and waveforms for the two stacks, thus suggesting that the same LFE source is being detected during both periods.

This is confirmed by looking at the spectrograms of the stacked waveforms for the quiet period (left panel in Fig. 5.6) and by comparing it with the spectrograms of the stacked waveforms for the LFE episode (right panel in Fig. 5.6). Even though the spectrograms for the quiet period are noisier (e.g. COVA station), both spectrograms show a fairly similar frequency content, with frequency peaks between $1-10 \mathrm{~Hz}$ (e.g. FRAN station), which is what we expect for LFEs in the Southern Alps (Wech et al., 2012; Chamberlain et al., 2014; Baratin et al., 2018). The spectrograms and the stacks are consistent with a real LFE signal for both periods. This confirms that during the quiet period, not only are real LFEs detected, but some of the same LFE sources are active during both periods. This finding also highlights the fact that this particular LFE family happens to behave differently over time, alternating between periods of intense activity (depicted by numerous bursts) and quieter periods.

\subsubsection{Spatio-temporal variations}

To examine the LFE families evolution in time and space, we only work with LFE sources that could be located, which amounts to 378 families for a total of 234,948 detections. As each LFE family originates from a single source, the same location is attributed to each individual LFE detection for a given family. Each LFE family can therefore be found on a horizontal line with respect to the time of detection on a spatio-temporal plot. This way, we can visualise the entire LFE catalogue in space and time on the same 2D plot (Figs. 5.7 and 5.8).

We start by analysing the evolution in time and space of the LFE catalogue by projecting the LFE family locations orthogonally to the surface fault trace (along profile AA' in Fig. 5.1). This approach allows us to study the evolution of the LFEs along strike (Fig. 5.7).

Few families are found SW of the LARB station (Fig. 5.1) in the southern portion of the SAMBA network (i.e., between 0 and $12 \mathrm{~km}$ along strike in Fig. 5.7). Similarly, hardly any family occurs in the northern portion of the SAMBA network, 

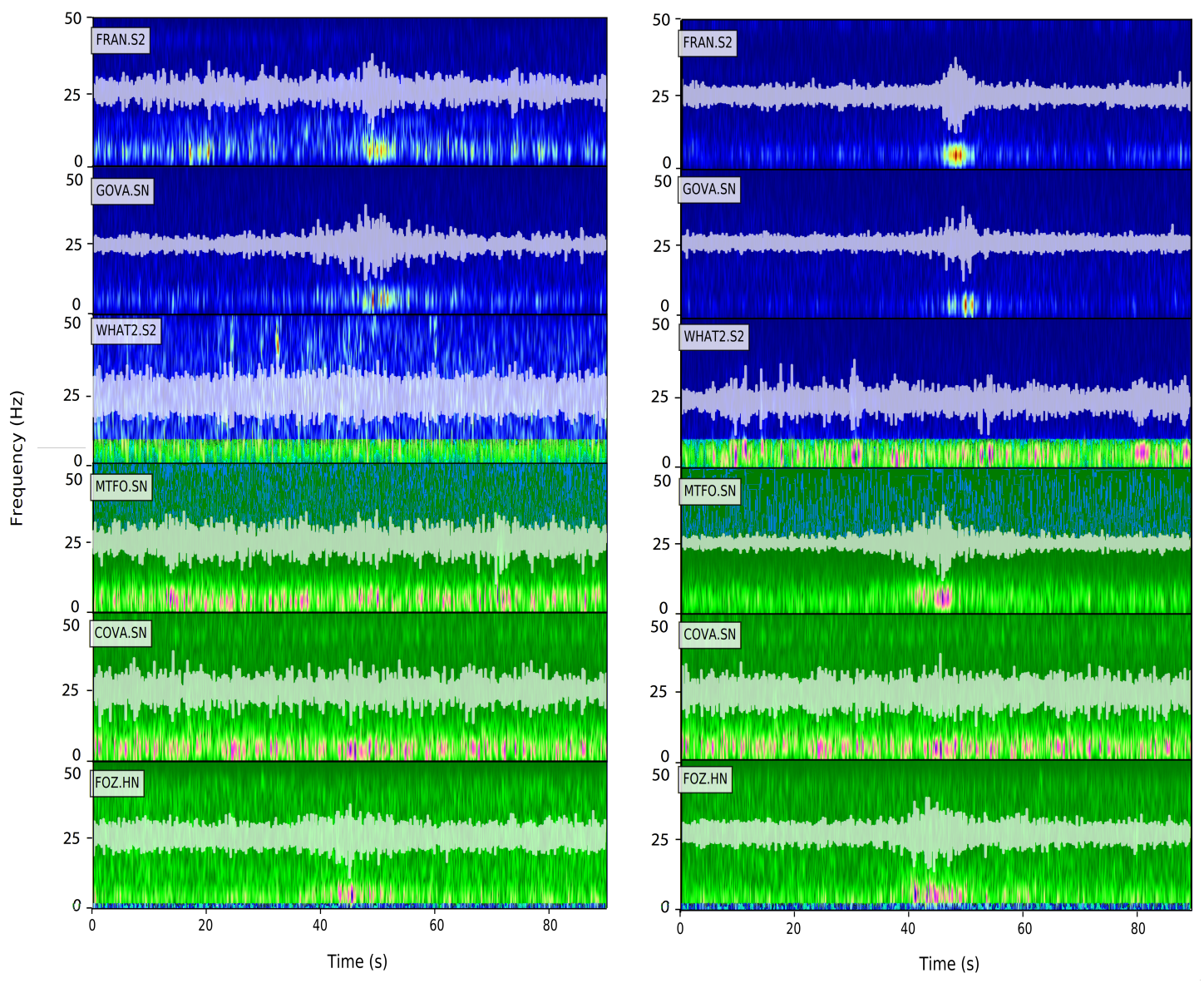

Figure 5.6: Spectrograms of stacked waveforms during an LFE episode and during the quiet period. Comparison between the spectrograms of the stacked waveforms of family ID:170.052_-43.8019_44_1 for events occurring coLFE episode (Right) and for events occurring during an inter-episode time period ( $\underline{\text { Left }})$. Only the North component is shown here. Waveforms are filtered between $2-49 \mathrm{~Hz}$. For both spectrograms, we see that the frequency content is mainly comprised between $1-10 \mathrm{~Hz}$ which coincides with the LFE peak frequency.

NE of the FRAN station in Fig. 5.1 (i.e. above $48 \mathrm{~km}$ along strike in Fig. 5.7). The sparse families that occur at the network extremities, seem overall less active with large gaps in detections (i.e. white periods on a horizontal line, synonym of no detections for the given LFE family). Yet, all those families appear continuously active during LFE episode ID:4 in Fig. 5.7. This highlights a disparate behaviour across the study period for those particular LFE families, with long periods of inactivity, interspersed by increases in event counts. 


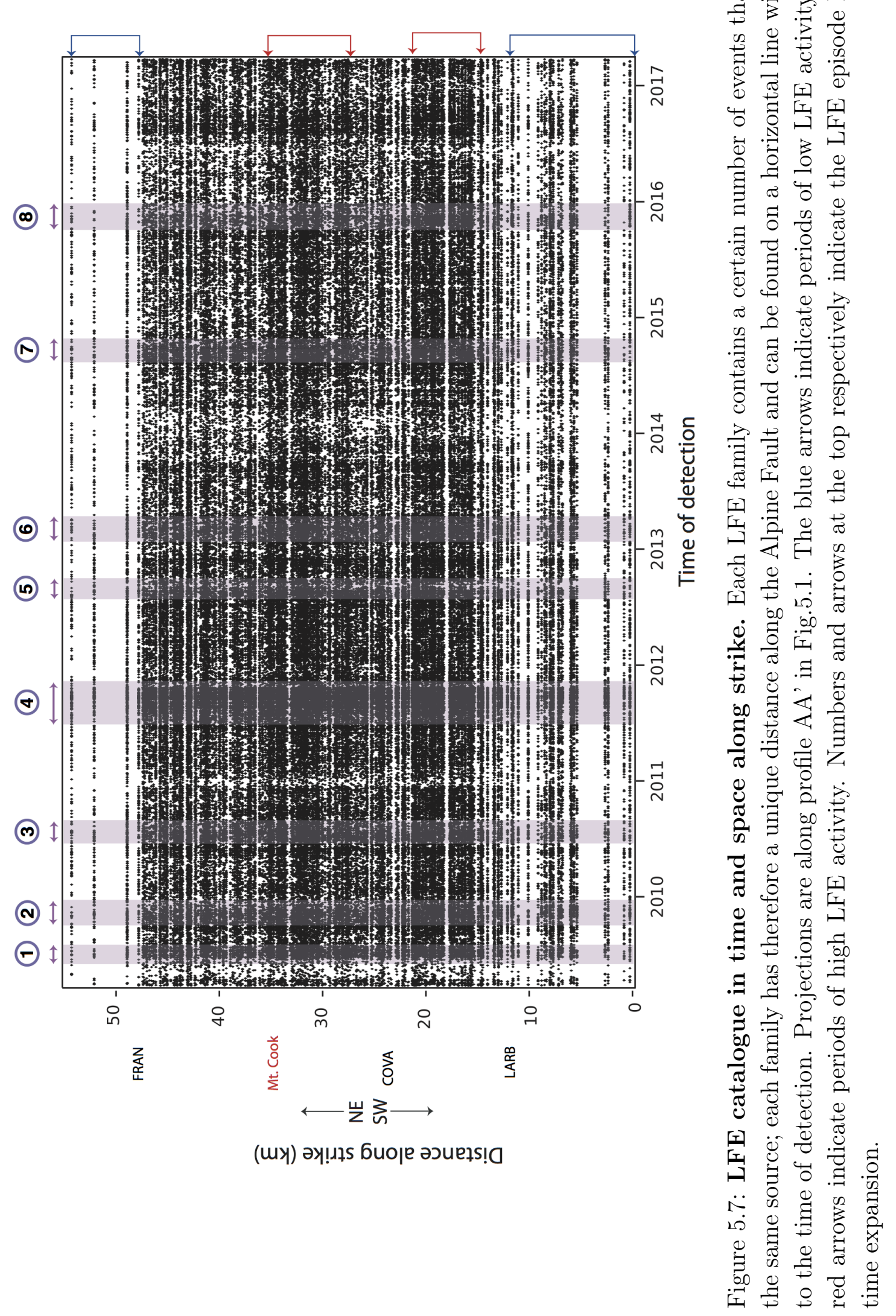

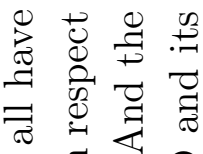

$\rightarrow$ 군

3.5

वै:

苇厌

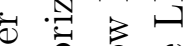

๙

구 용

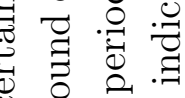

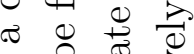

శี

चี च्व

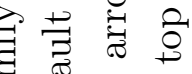

青

尝过

过

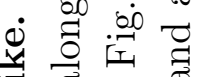

i $0 . \exists$

유 要

o 焉

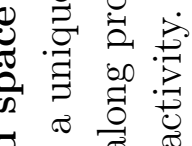

ส

过氙

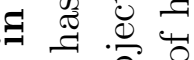

$\Rightarrow \triangleq$

ส․ ॠ्ञ

ช

되 \&

$\rightarrow \exists$

$\leftarrow$ o

焉

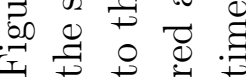



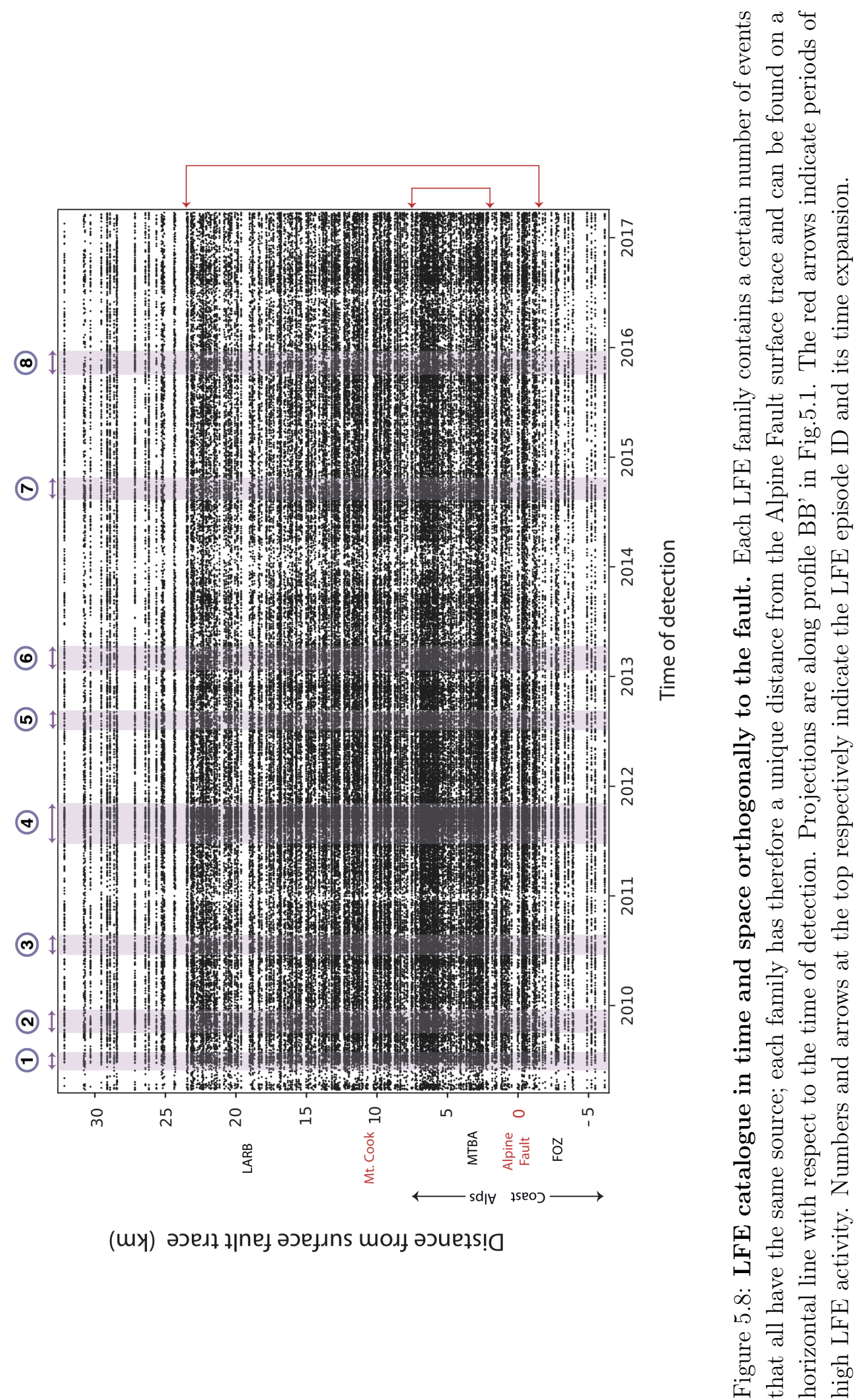

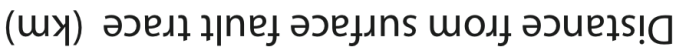


The most active areas of LFE families are observed between $15-21 \mathrm{~km}$ and 28 $35 \mathrm{~km}$ distance along strike (extremely dense black horizontal bands delimited by red arrows in Fig. 5.7). Those LFE families appear continuously active for most of the study period, with almost no periods of inactivity. Interestingly, both areas exhibit a lack of regular seismicity, with clusters of microearthquakes observed further North (Boese et al., 2012) and some deep earthquakes observed further South (Boese et al., 2013). The first band (15-21 km) gathers LFE sources occurring in the area where Wech et al. (2012) observed the highest density of tremor. The second band $(28-35 \mathrm{~km})$ coincides with an area where the second highest density of tremor was found (Wech et al., 2012). Most LFEs in this area are located in a $15 \mathrm{~km}$-circular area surrounding Aoraki/Mt. Cook. We hypothesise that a migration phenomenon might occur; with LFEs located in the central part of the study area (in the first and second bands) triggering LFE sources in the more distant areas.

We then examine the evolution in time and space of the LFE catalogue by projecting the LFE family locations along the surface fault trace (along profile BB' in Fig. 5.1). This way, the evolution of LFE across the Southern Alps can be investigated (Fig. 5.8).

One compact horizontal band ( $5 \mathrm{~km}$-width) of increased family activity is seen between $2.5-7.5 \mathrm{~km}$ distance from the surface fault trace (delimited by red arrows in Fig. 5.8). The highest density of LFE families activity is therefore observed close to the surface fault trace. Hereafter, a relatively steady LFE families activity is observed until $24 \mathrm{~km}$ distance from the surface fault trace. This $\sim 25 \mathrm{~km}$-width band coincides with the tremor extension described by Wech et al. (2012). Notwithstanding the $25 \mathrm{~km}$-width band $(-1-24 \mathrm{~km})$ where a persistent activity is observed, scarcely any LFE families are observed in the portion of the Alpine Fault footwall located to the NW of the surface fault trace (i.e. $-7-0 \mathrm{~km}$ from the surface fault trace in Fig. 5.8). In addition, few families are observed to the southeastern extremity of the surface fault trace (i.e. $24-32 \mathrm{~km}$ from the surface fault trace in Fig. 5.8). The majority of those families appear overall less active across the study period, yet, their activity seems to surge during LFE episodes.

In order to study, in detail, the time clustering and the LFE families recurrence behaviour over time, we examine the week-by-week variations in LFE rates for all the LFE families during: (1) an LFE episode (Fig. 5.9); and (2) a quiet period (Fig. 5.10).

We observe that during an LFE episode, all LFE families are active, with some 
families displaying a surge in activity for certain weeks (e.g. warm colour patches in Fig. 5.9 for Weeks 5 and 9). This behaviour highlights the existence of intermittent periods of extremely increased activity, within an already high activity episode. This recurrence pattern (i.e. cascading chain reaction) hints at time clustering, thus indicating that the occurrence of LFEs is not randomly distributed in time and is dependent on events that occur before it and affect events after it in neighbouring families within this time window (Shelly, 2015; Frank et al., 2016).

We try to assess whether the surges in rate are correlated to tremor occurrence. Tremor was found to occur on 12 days between May 2009 and September 2011 (Wech et al., 2012). Amongst those 12 occurrences, 3 occurrences happened to be comprised within the first 6 weeks of the LFE episode presented here. Tremor analysis was not conducted after the beginning of September 2011, so it is likely that additional tremor might have occurred during this LFE episode. The first tremor occurrence was observed on August, $3^{\text {rd }} 2011$ (Week 2 of LFE episode ID:4). The second and third occurrences were observed respectively on September, $2^{\text {nd }}$ and $4^{\text {th }} 2011$ (Week 6 of LFE episode ID:4). The two weeks following the observed tremor exhibit a high percentage of active LFE families (respectively $88 \%$ and $96 \%$ for Week 3 and 7 ). We also notice, that in both cases, the tremor occurrence coincides with gradual increases in LFE rates, leading to surges in the LFE rate which last a few weeks (e.g. Weeks $3-5$ and $7-9$ in Fig. 5.9). This pattern hints at possible triggering occurring between LFE families, with the activity of certain LFE families during tremor leading (1) to the generation of other LFEs; and consequently to (2) an episode of extreme LFE activity.

When no surge is observed, the LFE activity is more or less steady, with the most active families tending to be located at depths of $25-45 \mathrm{~km}$ (e.g. Week 7 in 5.9). When a surge is occurring, all families are highly active, regardless of their locations (e.g. Week 9 in 5.9). This behaviour also supports the possibility of triggering occurring between LFE families before and/or during an episode of extreme activity.

The majority of the active LFE sources of this study appears to be located in a diffuse area to the southwest of the Alpine Fault's flat lying detachment (i.e. southwest of the GPS-inferred deep Alpine Fault by Lamb and Smith (2013) in Fig. 5.9). Baratin et al. (2018) (cf. Chapter 3) computed the focal mechanism for one LFE family located in this area and found a mechanism consistent with a steeply-dipping plane (cf. Fig. 3.13 in Chapter 3). 


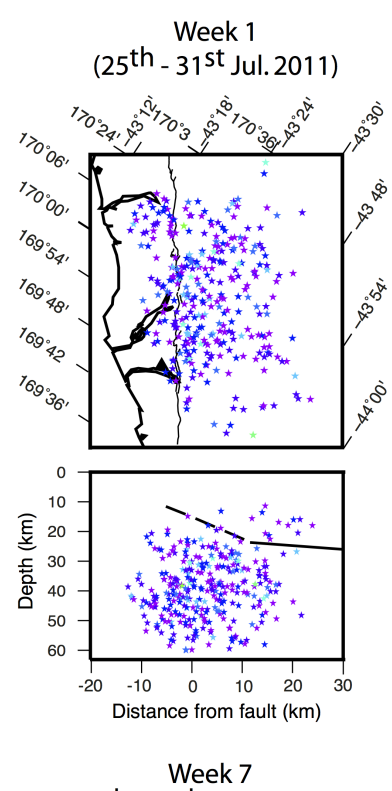

$\left(5^{\text {th }}-11^{\text {th }}\right.$ Sept. 2011)
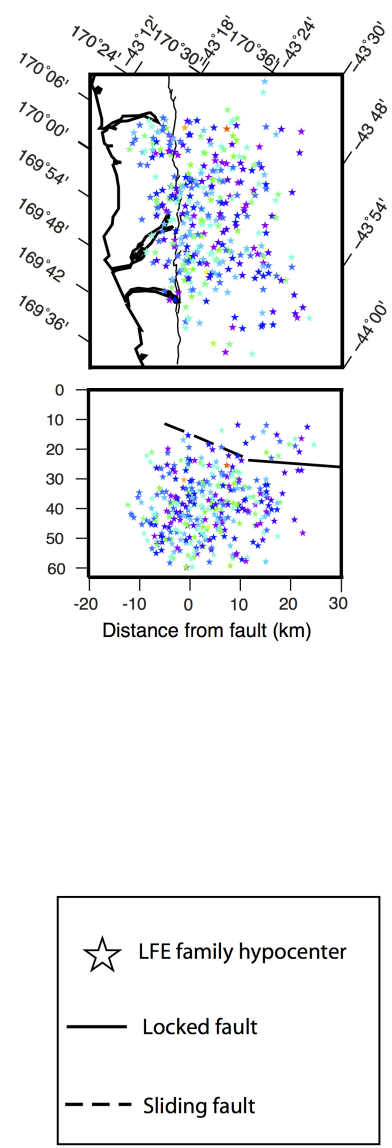
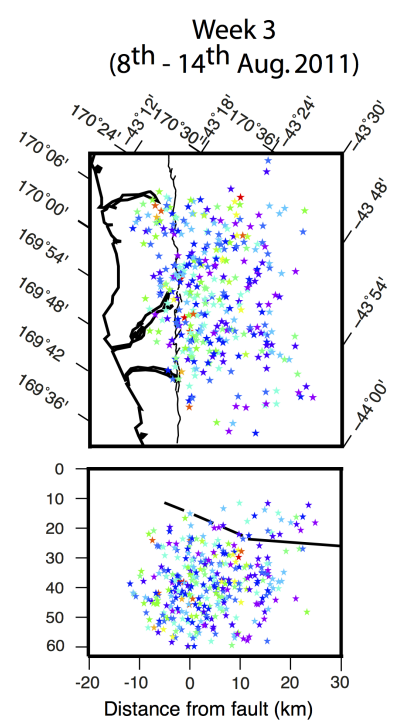

Week 9

(19th $-25^{\text {th }}$ Sept. 2011)
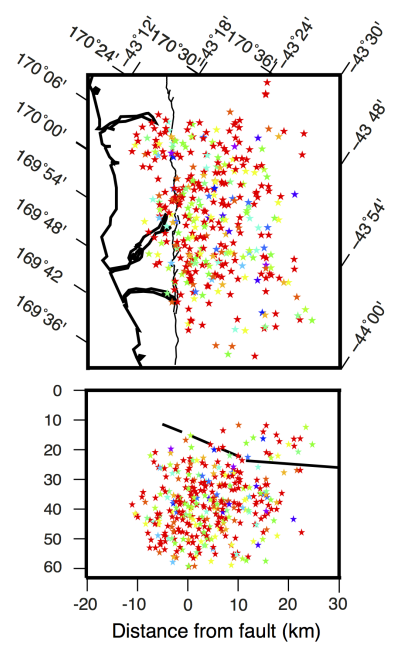

Week 13

$\left(17^{\text {th }}-23^{\text {rd }}\right.$ Oct. 2011)
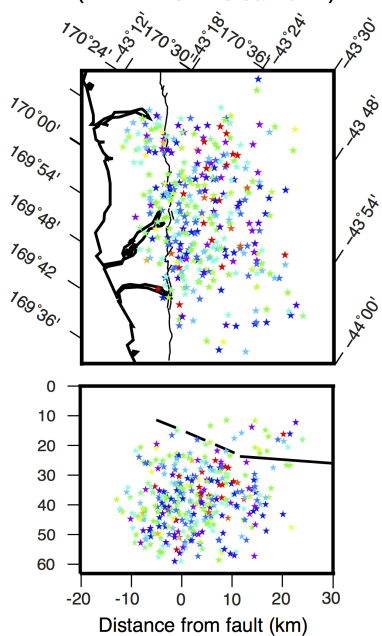

Week 5

(22 th $-28^{\text {th }}$ Aug. 2011)
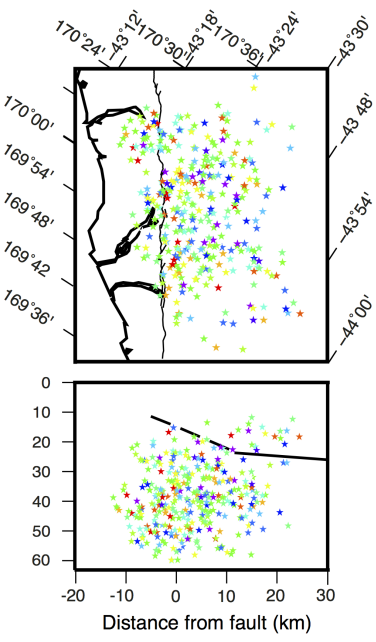

Week 11

$3^{\text {rd }}-9^{\text {th }}$ Oct. 2011)
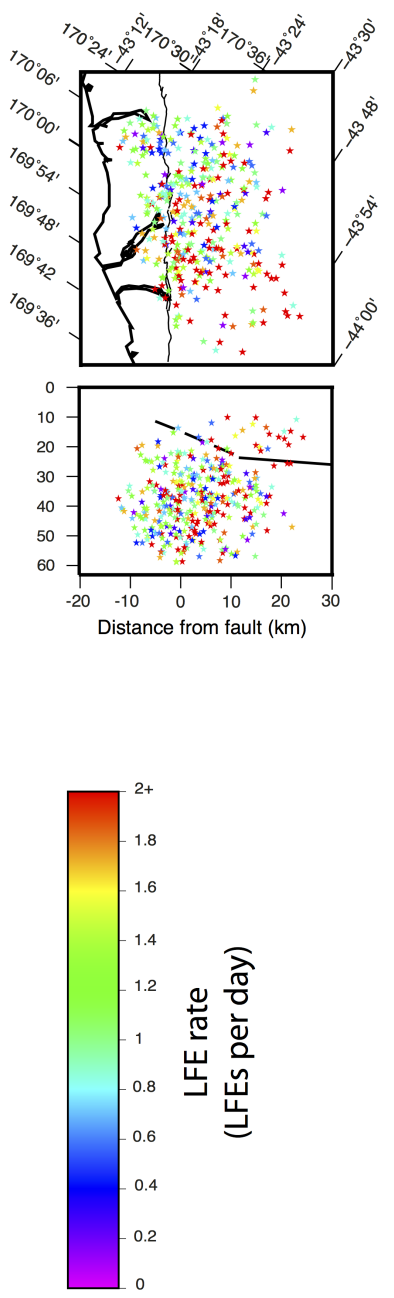

Figure 5.9: LFE rates for LFE episode ID:4 (25/07/2011 - 23/10/2011). Weekly spatial variation of LFE activity (Week 1-13). LFEs families are coloured by their daily rates calculated over a one-week period. 


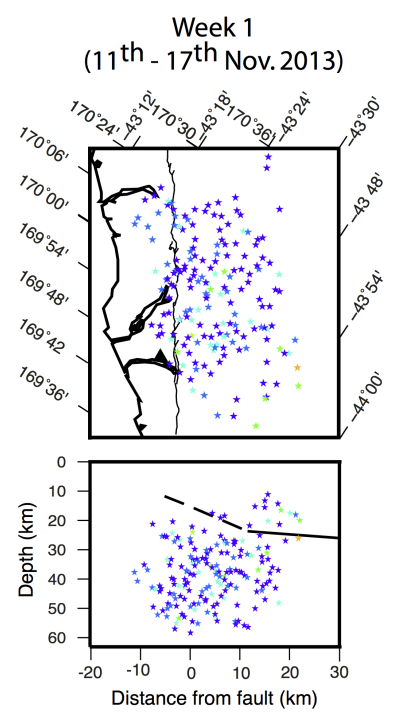

Week 7

(23 ${ }^{\text {rd }}-29^{\text {th }}$ Dec. 2013)
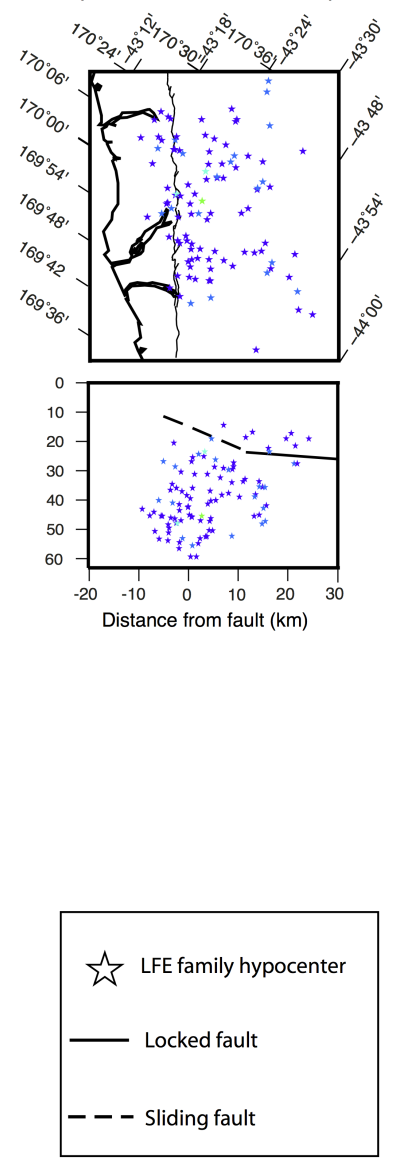

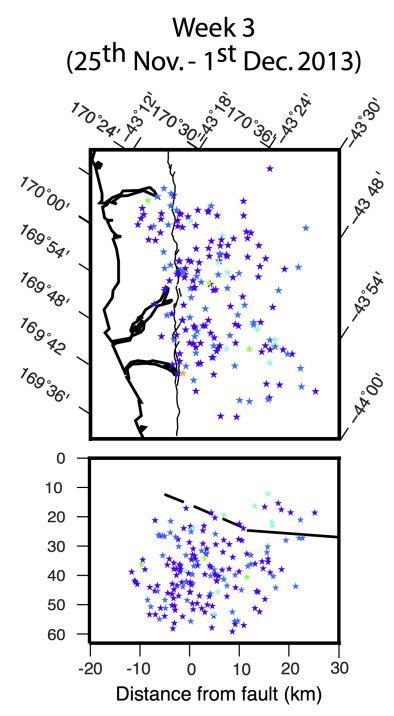

Week 9

$\left(6^{\text {th }}-12^{\text {th }}\right.$ Jan. 2014)
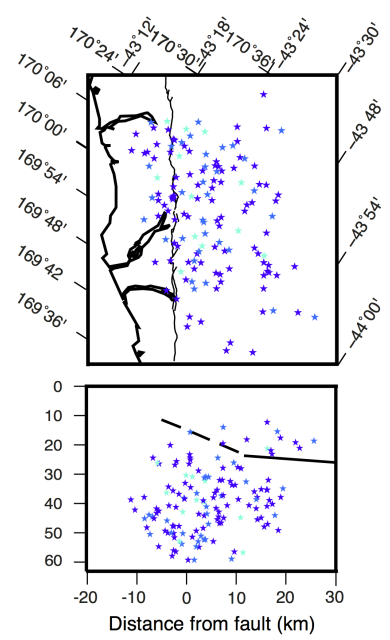

Week 13

(3rd $-9^{\text {th }}$ Feb. 2014)

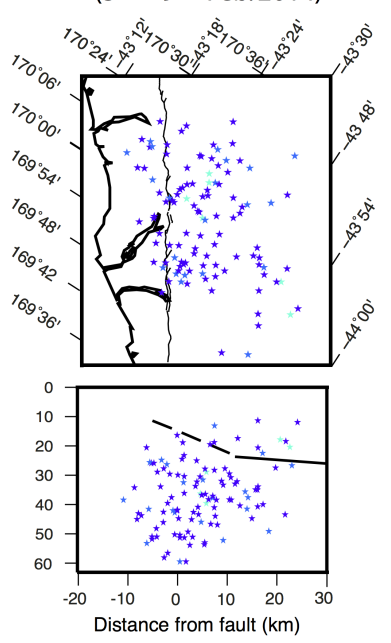

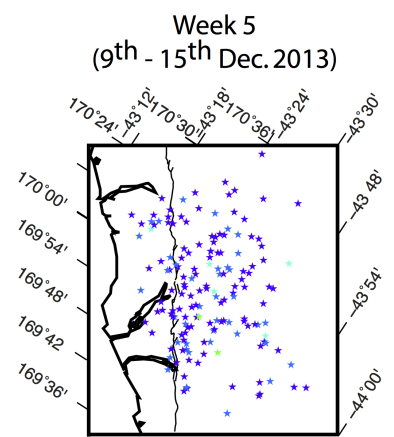

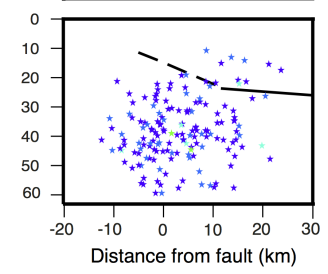

Week 11

(20 $0^{\text {th }}-26^{\text {th }}$ Jan. 2014)
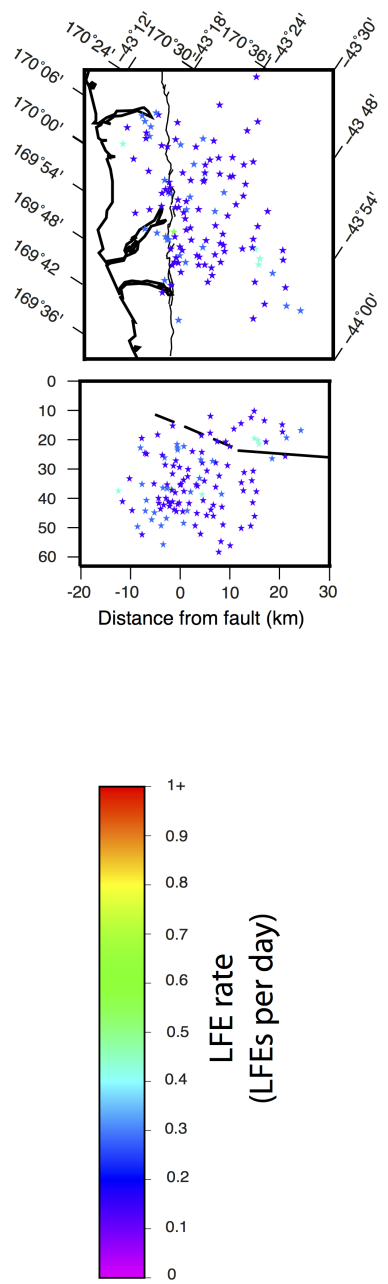

Figure 5.10: LFE rates for the quiet period $(11 / 11 / 2013-16 / 02 / 2014)$. Weekly spatial variation of LFE activity (Week 1-13). LFEs families are coloured by their daily rates calculated over a one-week period. 
It was therefore assumed that this LFE family occurred on a more steeply-dipping branch of the Alpine Fault, the existence of which is suggested by other studies. Indeed, Koons et al. (2003) and Norris and Toy (2014) propose a partitioning of motion near the base of the crust $(\sim 30 \mathrm{~km}$ depth) between a steep strike-slip shear zone to the west and a flat-lying detachment to the east. This could indicate that the LFEs might be located on/near a second branch of the Alpine Fault. In any case, LFE sources located in that area are amongst the ones that possess the highest rates of LFE generation.

Finally, we don't observe clear patterns of spatial migration during the LFE episode when looking at the week-by-week evolution of rate. However, we find that LFEs with contemporaneous occurrence patterns have a tendency to be clustered in space (e.g. same colour patches for Week 9 in Fig. 5.9). We thus suggest that individual LFE sources form spatially coherent clusters that may represent localised asperities or elastic patches on the deep Alpine Fault interface (Ide, 2008; Trugman et al., 2015). We infer that those clusters may have a similar rheological response to tectonic forcing or to potential slow slip events (Lengliné et al., 2017). In addition, previous focal mechanisms computed for LFEs in that same area are consistent with shear failure on the deep Alpine Fault (Baratin et al., 2018, cf. Chapter 3). With that in mind, we could argue that LFEs represent discrete dynamic ruptures in a slowly slipping interface, and that a generalised surge in LFE rates coincides with a larger-scale slow slip event.

By studying the evolution of the week-by-week rate during the quiet period, we notice that a smaller number of LFE families appear to be active. The observed families exhibit rates that are generally $50 \%$ lower than those observed during the LFE episode. Moreover, we do not observe surges in detections during the quiet period. On the contrary, we observe relatively constant rates for the LFE families that do manifest. The only change stems from the number of families that are active each week. We generally observe a gradual increase (e.g. Week 1-3 in Fig. 5.10) followed by a steady decrease (e.g. Week 5-8 in Fig. 5.10) in the number of LFE families that are manifesting.

This behaviour is drastically different than the one observed during the LFE episode, where most families are continuously active and occasionally experience surges in their activity. For example, if we look at Week 7 of the quiet period in Fig. 5.10, only $26 \%$ of the total LFE families are active, with all the present families exhibiting relatively low rates (maximum rate of $0.6 \mathrm{LFEs} /$ day per family). In contrast, for Week 9 of the LFE episode in Fig. 5.9, we observe that $99 \%$ of the 
LFE families are active, and that most families present a surge in rates at that time (with the highest rate equal to $6.5 \mathrm{LFEs} /$ day per family).

The tendency of some LFE families to display a variability in recurrence behaviour over time was first documented by Shelly (2017) for his LFE catalogue on the deep San Andreas Fault. Shelly (2017) suggested that this behaviour might reflect a rate-dependent friction on the fault, with the increase in LFE rates being indicative of an external increase in loading. Alternatively, during an LFE episode, the likely changes in stress and/or friction and/or pore pressure (Trugman et al., 2015) might allow localised triggering to occur between LFE families, thus explaining why some quieter families become more active at that time.

\subsubsection{LFE Clustering}

To identify LFE families that are very similar, we calculate for each successfully located LFE family, its redundancy with all of the other LFE families in the catalogue. We then plot a symmetrical matrix showing, for every LFE family, a mesh coloured by the redundancy value calculated with each of the other LFE families (Fig. 5.11). If the redundancy is greater than or equal to 0.1, the mesh is coloured in red in Fig. 5.11.

We note that overall, most LFE families appear non-redundant, with redundancy values well below 0.04 (displayed by the majority of cold colours in the redundancy matrix in Fig. 5.11). Some families even appear quite unique, with no other events detected at the same time (e.g. family ID:169.906_-43.5015_28_1, which can be seen at $\sim 35.5 \mathrm{~km}$ along strike). Indeed, most families located from $\sim 41 \mathrm{~km}$ along strike going north-eastward, exhibit extremely low redundancy values or even no redundancy at all. Those families are located in the northern section of the SAMBA network, where the station coverage is denser. We therefore presume that those families have some of the most reliable locations in the catalogue.

On the other hand, the highest redundancy values are found for families in the southwest section of the study area. Those families are located at a short distance along strike going to the northeast (from 0-20 km along strike). They occur in the southern edge of the SAMBA network, where there is sparse station coverage. It is thus possible that those families are incorrectly located and that their individual detections are indeed part of other LFE families.

To check whether families with a high redundancy (i.e.greater than 0.1 for a given pair of LFE families, which is equivalent to a $10 \%$ similarity between the 
detections of both families) should be grouped together, we calculate the waveform similarity of the two LFE families' stacked waveforms. If the waveform similarity is superior to a threshold set to 0.2 , we consider that those families are redundant. Those families are then grouped together for further analysis.

With a waveform similarity threshold of 0.2 , we observe 57 LFE families that are redundant, which can then be grouped into 17 new families (see Table A.2 in Appendix A.3.3 for a complete list of the redundant families). Regrouping redundant families allows the computation of higher SNR stacks (e.g. Fig. A.32 in Appendix A.3); corresponding to an increase of $\sim 7 \%$ of the stacks' SNRs. This may lead (for those particular LFE families) to more reliable picking of P- and Sarrival times and polarities which ultimately could lead to more accurate locations together with the computation of focal mechanisms.

\subsection{Discussion}

\subsubsection{LFE patterns and migrations}

To examine in more detail the LFE patterns in the central Southern Alps, we compare the spatio-temporal variations between detections occurring during LFE episode ID:4 and those occurring during the quiet period (respectively red and blue boxes in Fig. 5.4). Because each LFE family has a unique distance from the surface fault trace (or along strike), each LFE family can be found on a horizontal line with respect to the time of detection in panels A and B of Fig. 5.12. Firstly, we notice a striking contrast in occurrence patterns between the LFE episode and the quiet period. Indeed, many bursts occur during the LFE episode (panels A, $\mathrm{B}$ and $\mathrm{C}$ in Fig. 5.12(a)), whereas only a few bursts are observed during the quiet period (panels A, B and C in Fig. 5.12(b)). 


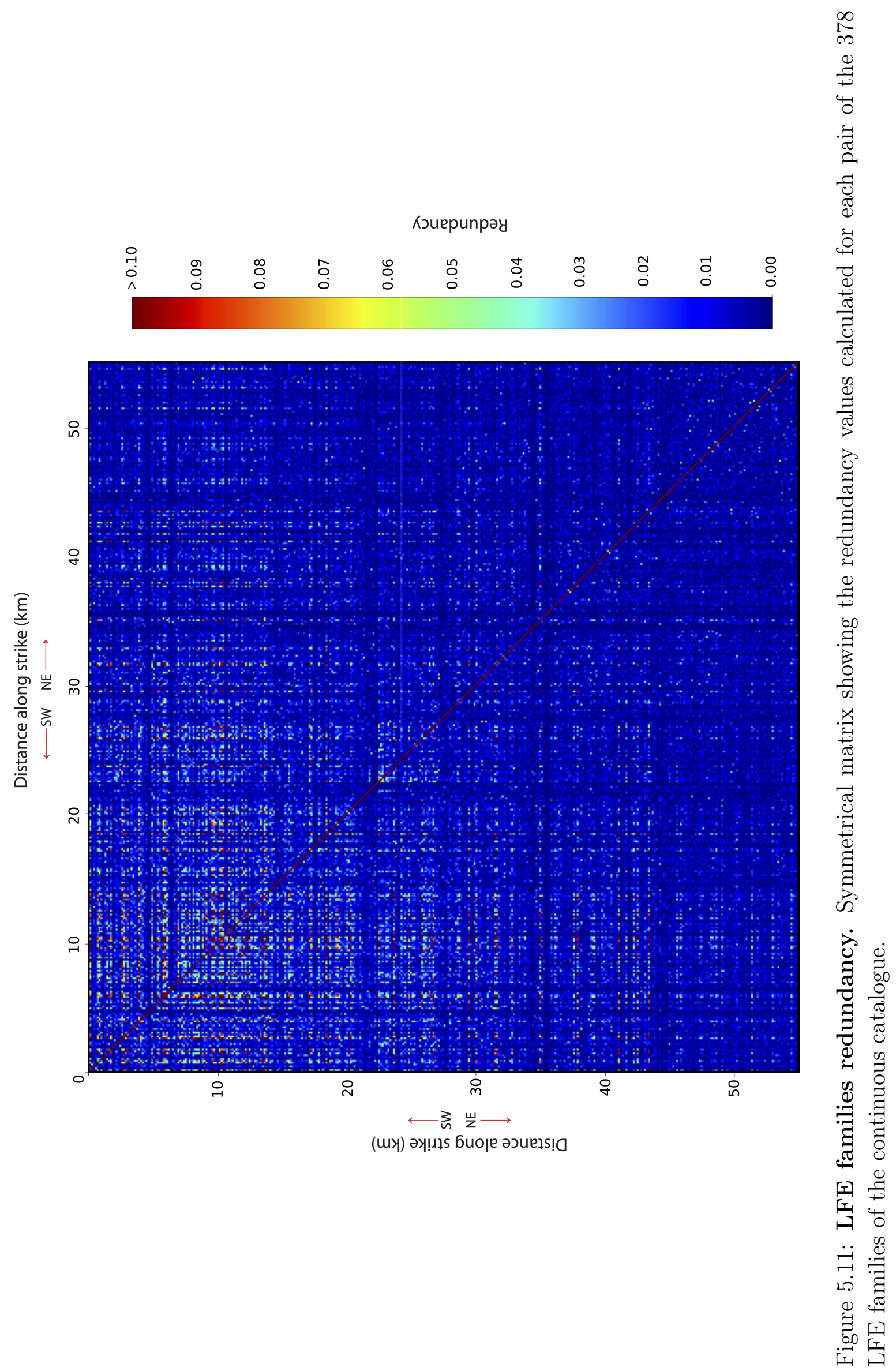


In addition, we remark differences in occurrence patterns, not only within the LFE episode itself; but also within the same families depending on the time of observation. For example, if we examine families located between 5 and $8 \mathrm{~km}$ distance from the surface fault trace, we find that they are more or less continuously active during the 95 days of the LFE episode (panel A in Fig. 5.12(a)) and that they appear to contribute to bursts (panel C in Fig. 5.12(a)). However, the same families exhibit a disparate behaviour during the quiet period where almost no bursts are observed, with large periods of gaps where no detections are observed for those particular families (panels A and C in Fig. 5.12(b)).

This behaviour supports the observations that were made in Section 5.3.2, for the tendency of some LFE families to display a variability in recurrence behaviour over time. Those observations confirm that this type of behaviour might reflect a ratedependent friction on the fault, with the increase in LFE bursts being indicative of an external increase in loading during the LFE episode (Shelly, 2017).

We estimate the regional earthquake activity using the seismic energy density metric, defined by Wang and Manga (2010) as 'the maximum seismic energy available in a unit volume to do work on rock'. The earthquake energy density (plotted in grey in panel D in Fig. 5.12) is calculated for earthquakes of $M_{w}>1$ taken from the GeoNet catalogue in an area of $250 \times 320 \mathrm{~km}^{2}$ encompassing the study area. During the LFE episode, we observe two 'steps' (i.e. periods of increased LFE activity) that seem to coincide with the highest values of elevated earthquake energy density (23-24 $4^{\text {th }}$ August 2011 and $24-26^{\text {th }}$ September 2011). However, we observe other periods of high energy density that do not necessarily initiate clear responses in the LFEs count $\left(8-10^{\text {th }}\right.$ August 2011). Hence, we cannot establish a clear-cut relation between the LFEs activity and the regional earthquake energy density using just those observations. The only unequivocal observation we can make is that, during the LFE episode, we notice a higher regional earthquake energy density than during the quiet period (panels D in Fig. 5.12(a) and (b)). Thus, it is possible that during an LFE episode small changes in in-situ stress, produced by regional earthquakes, might trigger a higher LFE activity. Indeed, several studies report LFEs being extremely sensitive to small stress perturbations: for instance, the triggering of LFEs by the dynamic stresses of Love and Rayleigh passing surface waves (Hill, 2012). A future study of LFEs in the area involving a more quantitative analysis would be crucial in order to better understand the relation between earthquake activity and LFE triggering.

Migration of tremor and LFEs has been documented in multiple areas: in subduction zones for instance, in Japan (Shelly et al., 2007), in Cascadia (Ghosh et al., 
2010) and along the Guerrero subduction zone in Mexico (Frank et al., 2014); but also along major strike-slip faults for instance, along the deep San Andreas Fault (Shelly, 2017).

To find migration velocity, we examine individual clusters to inspect their inclination. We could not identify migration velocities during the quiet episode as no definite clusters were observed during this period. For LFE episode ID:4, the search for migration velocities was done by visual inspection of individual clusters during this period. The clusters that were chosen are representative of this particular LFE episode. But it is important to note that those velocities might change if we look at a different LFE episode and might not reflect the average cluster velocity for the entire catalogue. They however are representative of the velocities uncovered during the biggest LFE episode identified within the study period (i.e. between 2009 and 2017).

We first examine the migration patterns orthogonally to the Alpine Fault (distances projected along profile BB' in Fig. 5.1). We identify several clusters moving at high-velocities $(\sim 20 \mathrm{~km} / \mathrm{h}$, e.g. Fig. $5.13 \mathrm{~A})$. Those velocities are within the order of the high-speed LFE migration velocities found along strike elsewhere: for instance, $20-40 \mathrm{~km} / \mathrm{h}$ in Guerrero, Mexico (Frank et al., 2014); and $15-90 \mathrm{~km} / \mathrm{h}$ in the Parkfield area of the San Andreas Fault, in California (Shelly, 2017). Yet, we also observe clusters exhibiting much slower velocities of $10 \mathrm{~km} /$ day, across the Alpine Fault (Fig. Fig5.4.13B). This range of slow velocities has previously been reported in Japan and Cascadia and along the San Andreas Fault for tremor and LFE migration (Obara, 2002; Dragert et al., 2004; Shelly et al., 2007; Shelly, 2017).

We remark that for some periods, more detections are made during the day than at night-time, although this observation is not systematic (e.g. fewer detections around 12am in Fig 5.13B). A possible explanation for this phenomenon could be the difference in noise level between day-time and night-time (e.g. linked with climatic conditions or anthropogenic activities). 

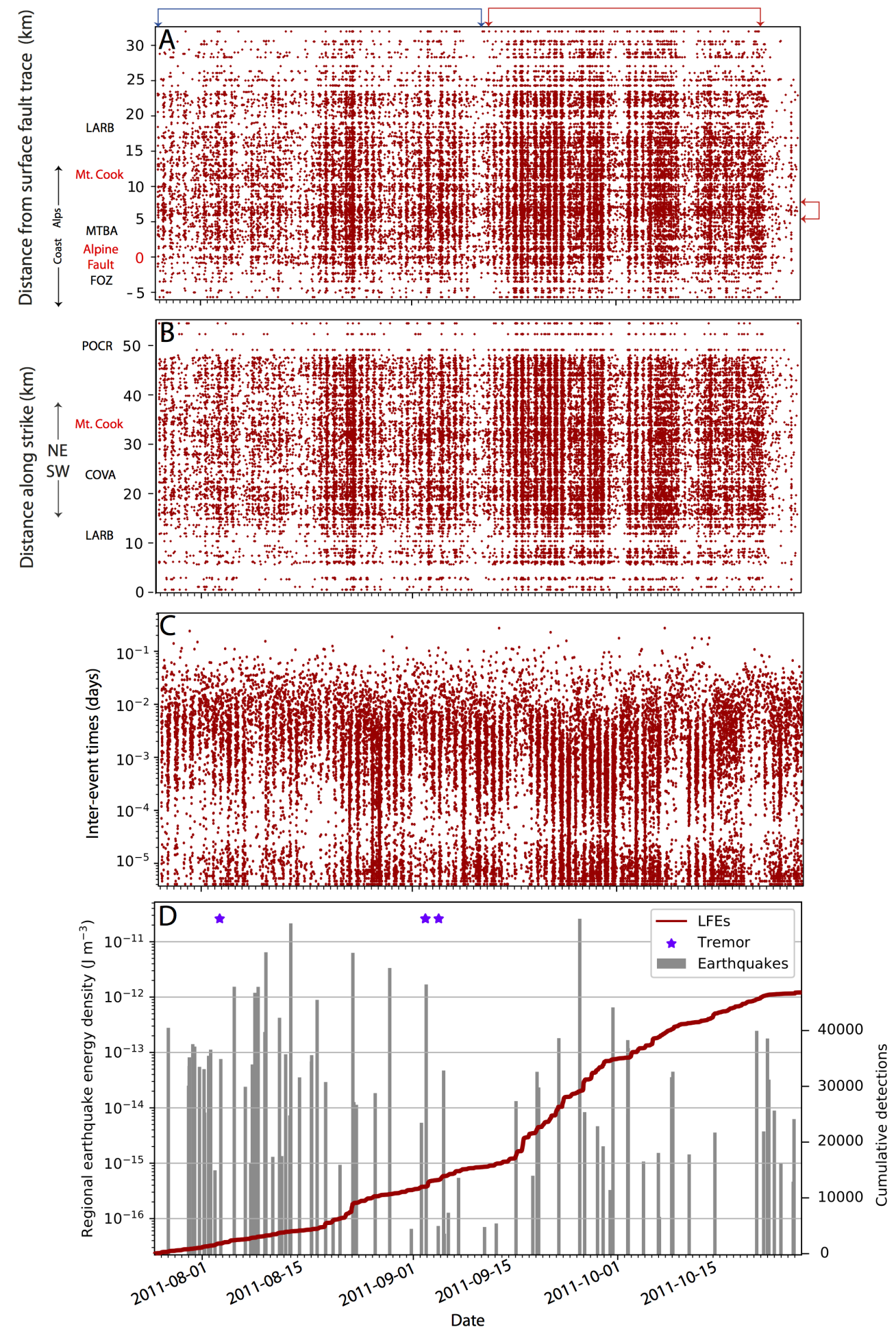

(a) LFE episode ID:4 (25 July 2011-28 October 2011) identified in Fig. 5.4. 

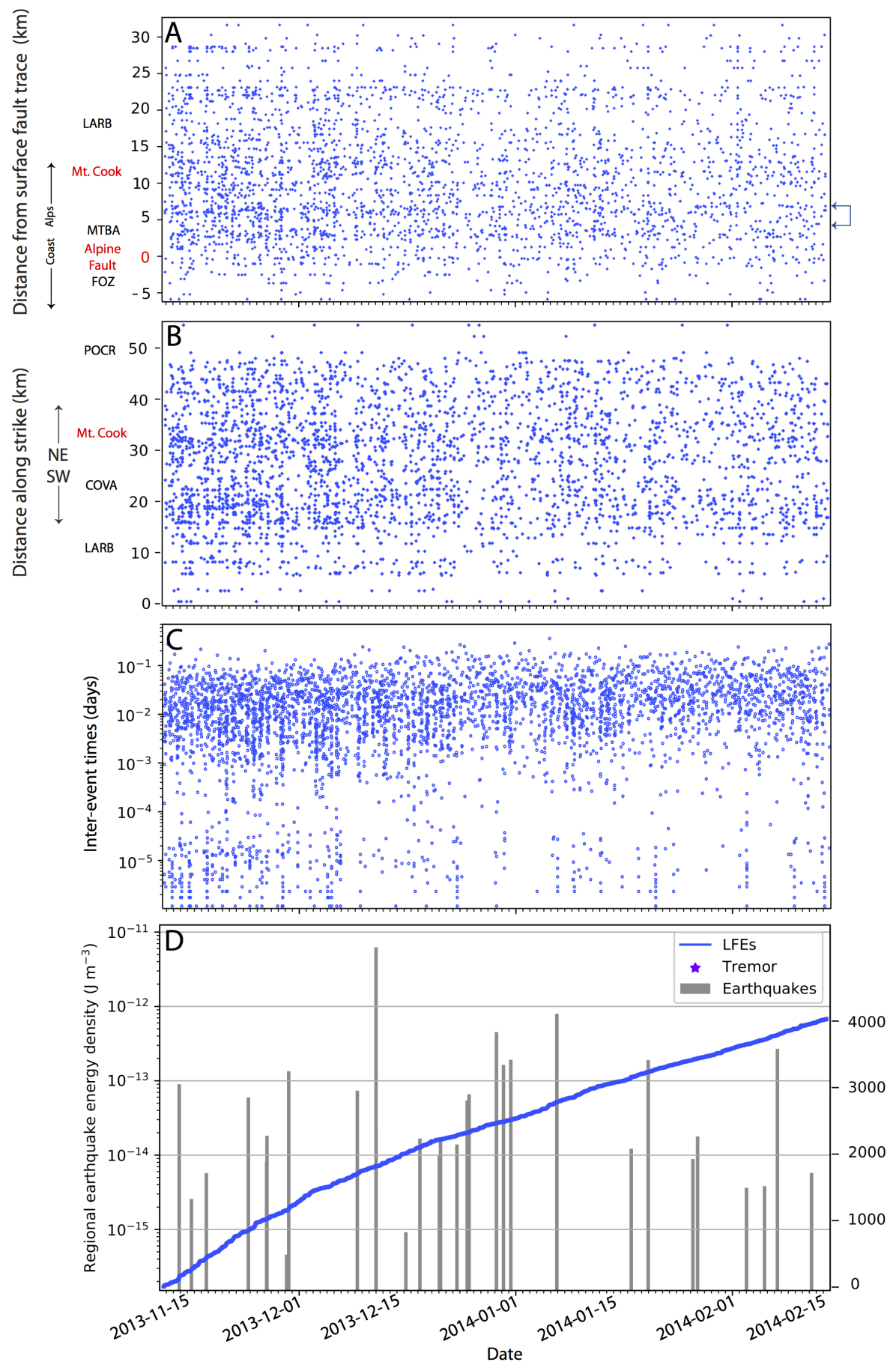

(b) Background LFE activity (11 November 2012-15 February 2014) identified in Fig. 5.4.

Figure 5.12: Spatial and temporal variation for an LFE episode and the quiet period. Evolution in time and space of LFEs $(\underline{\mathrm{A}-\mathrm{B}})$ and inter-event times $(\underline{\mathrm{C}})$ are shown for a period of 95 days. Earthquake energy density $(\underline{D})$ is shown in grey for earthquakes of $M_{w}>1$ (taken from the GeoNet catalogue in an area of $250 \times 320 \mathrm{~km}^{2}$ encompassing the study area). Cumulative detections (므) are also shown for this period. Red and blue time intervals respectively indicate periods of low and high activity. Date is in UTC-time. 
However, as demonstrated in Chapter 4, the matched-filtering technique used to build this catalogue is robust enough to surmount that type of noise fluctuations. Another possibility to explain the diurnal variation in the number of detections could be linked with the network and stations limitations. In fact, during several maintenance runs, we uncovered some power supply issues at several stations (e.g. EORO, see Appendix $\mathrm{C}$ for a detailed overview of the field work reports). The SAMBA stations are powered by solar panels charging a $12 \mathrm{~V}$ battery and weak batteries are expected to disturb the continuous recording of data on memory cards, hence causing data loss. Indeed, while on field, a systematic check of the stations' log files (containing information recorded every 30 minutes, such as battery voltage, disk usage, GPS operation, etc...) was performed and we found that the voltage was lower at night-time than during the day. Hence, more power outages are expected at night-time, leading to fewer detections. This behaviour is also observed in the microseismicity recorded within the SAMBA network (Oestreicher, 2018). Oestreicher (2018) thoroughly analysed this phenomenon for microearthquakes and found that by only taking into account the voltage of the battery, the higher rate of seismicity during the day could not be explained in its entirety. This observation hence highlights an interesting behaviour in LFEs that would require further investigation in future studies of slow deformation in the Southern Alps.

We now look at the migration patterns along strike (distances projected along profile AA' in Fig. 5.1) and identify some high-speed migration velocities. Indeed, we identify velocities of $\sim 20-25 \mathrm{~km} / \mathrm{h}$ for two clusters observed on the $18^{\text {th }}$ and $19^{\text {th }}$ September 2011 (Fig. 5.14).

Overall, we identify episodes of high-speed migration when the LFE activity is extreme (e.g. September 2011 in Figs. 5.13A and 5.14 and Week 9 in Fig. 5.9), whereas the slower migration are observed during period of slightly less elevated LFE activity (e.g. August 2011 in Fig. 5.13B and Week 3 in Fig. 5.9).

This phenomenon of slow versus fast migration has also been encountered elsewhere: along the San Andreas Fault (Shelly, 2017). Indeed, Shelly (2015) suggested that the slow migration phenomenon was seemingly controlled by slow slip events along the San Andreas Fault, whereas the rapid migration was a reflection of the dynamic processes involved at the tremor and LFE sources themselves. For example, the migration velocity of a slow slip event, in a similar strike-slip area, has been found to be close to $\sim 10 \mathrm{~km} /$ day (e.g. in San Andreas, Shelly, 2015). This speed coincides with the slow migration velocities of tremor and LFEs found here and elsewhere (Obara, 2002; Dragert et al., 2004; Shelly, 2017). 

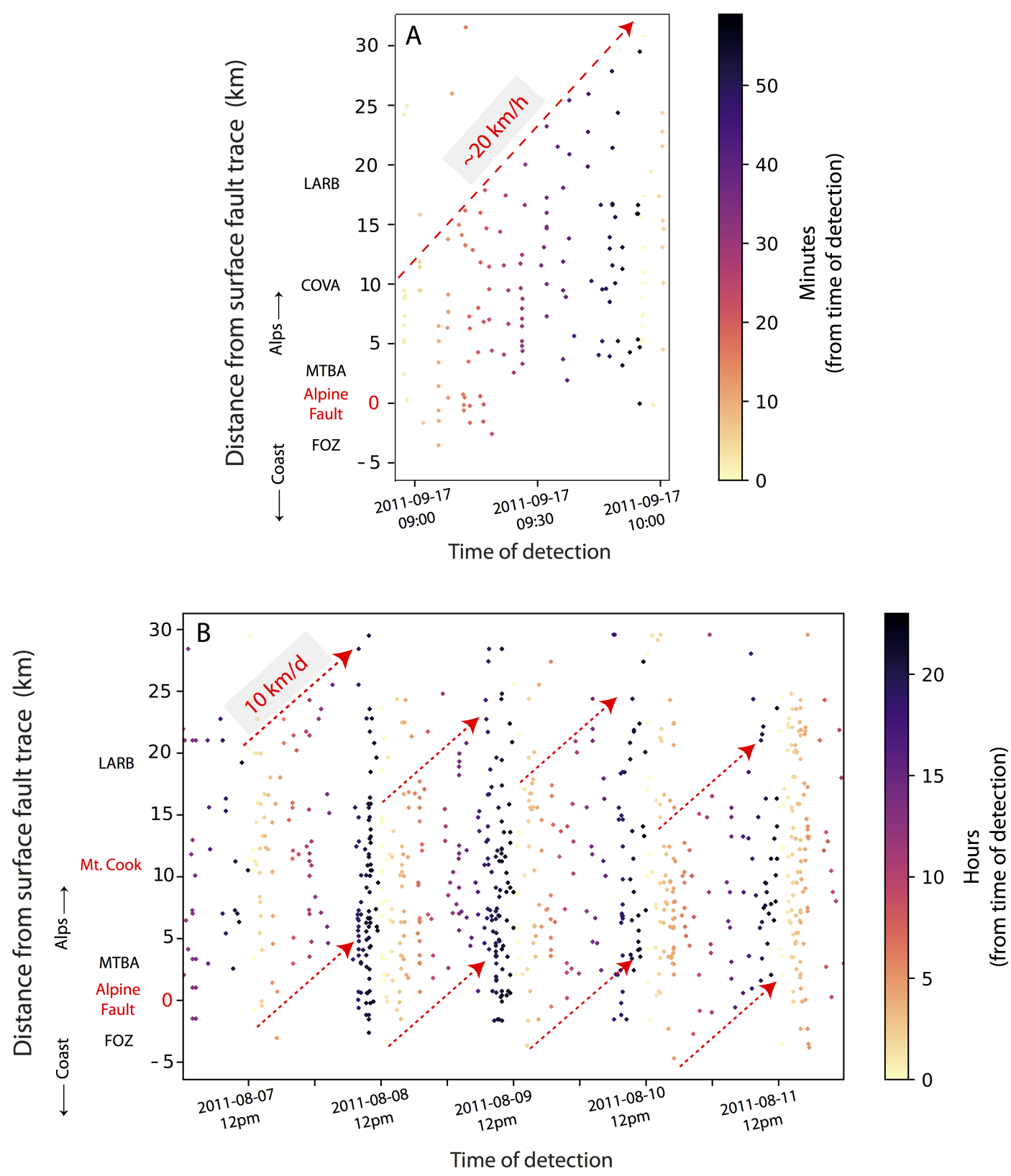

Figure 5.13: Slow and fast migration during LFE episode ID:4. A: Example of a rapid LFE migration occurring in September 2011. Time of detection is in NZST. Detections are coloured by minute of detection. The red arrow indicate the migration direction as well as the velocity of the cluster. B: Example of a slow LFE migration occurring in August 2011. Zoomed in view of Fig. 5.12(a) between the $7^{\text {th }}$ and $11^{\text {th }}$ of August 2011. Time of detection is in NZST. Detections are coloured by hour of detection. The red arrow indicate the migration direction as well as the velocity of the cluster.

As for the high-speed migration, it could be linked with: (1) a large-scale fluid flow (Ghosh et al., 2010); (2) an actual velocity of considerably slower propagation 


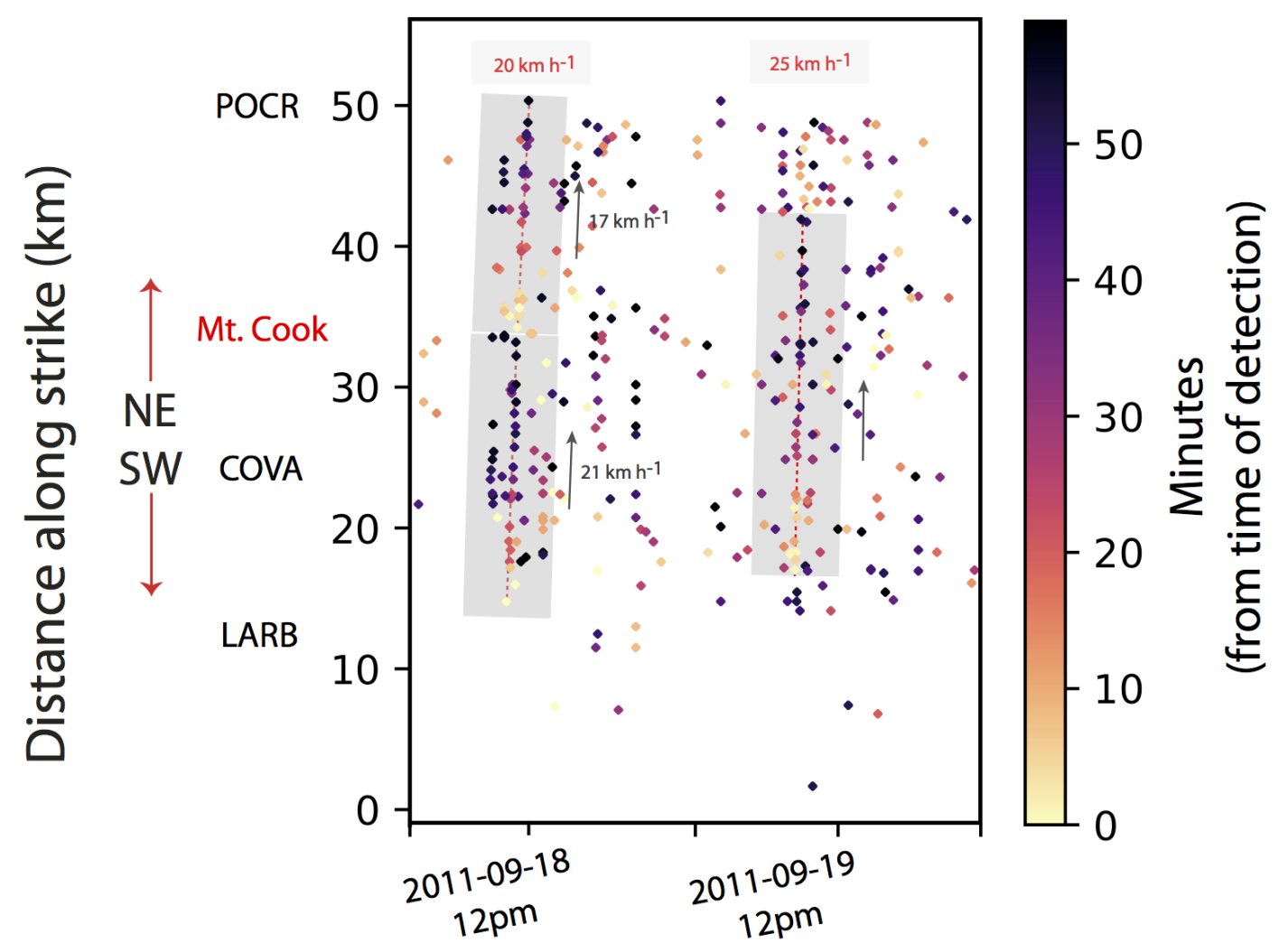

\section{Time of detection}

Figure 5.14: Fast migration of LFEs along strike during 2 days of LFE episode ID:4. Zoomed in version of Fig. 5.12(a) between the $18^{\text {th }}$ and $19^{\text {th }}$ September 2011. Time of detection is in NZST. Average velocity of cluster is indicated in a box at the top of the cluster. Each grey box is equivalent to one hour of elapsed time. The red dashed line indicates the cluster for which the velocity has been calculated. The direction of migration for each cluster is shown with a grey arrow.

(Ando et al., 2010); or (3) smaller stress drops and/or increased average slip rates (Rubin, 2011; Rubin and Armbruster, 2013). A recent study by Shelly (2015) discovered that LFEs generation along the San Andreas fault was stimulated by the same factors that incite high-speed slip propagation, thus pointing to an increase in slip-rate as the most likely explanation for the LFE high-speed migration velocities.

Furthermore, laboratory experiments and numerical modelling infer that slipinduced dilatancy might play a crucial role in regulating tremor (hence lowfrequency earthquakes) and slow slip (Samuelson et al., 2009; Segall et al., 2010; 
Yamashita, 2013). Slip dilatancy corresponds to the phenomenon occurring when an increasing shear rate leads the fault gouge to dilate. Slip dilatancy has been proposed as a credible regulating mechanism to explain the high tremor and LFE migration velocities (e.g. Segall et al., 2010; Shelly, 2015; Trugman et al., 2015). Indeed, if we assume that an LFE forms as an unsteady rupture on an asperity nested in the deep portion of the fault; while slip accelerates, the fault zone dilates, hence decreasing the pore pressure, which in turn produces an increase in the frictional resistance (Shelly, 2015). Subsequent ruptures are then likely to occur somewhere else, consequently broadening the slip zone into neighbouring areas which have previously been stressed but which have not ruptured thus far. Increased along-fault permeability possibly favours accelerated fluid pressure diffusion along the fault (Yamashita, 2013), which might explain the high-speed migrations along-strike. In addition, ongoing surrounding creep on the deep extent of the fault might encourage repeated fault patch ruptures (Yamashita and Suzuki, 2011). Lastly, the static stress transfers occurring during an LFE episode could generate a series of LFEs propagating along the fault zone, whose duration and speed would depend on the overall fluid diffusion time (Shelly, 2015).

\subsubsection{Slow slip investigation}

Slow earthquakes are transient phenomena that generally manifest as geodetically observed aseismic slip (or slow-slip events, Dragert et al., 2001) and seismically observed tectonic tremor and low-frequency earthquakes (Obara, 2002; Shelly et al., 2006). In Cascadia (Rogers and Dragert, 2003), in Japan (Obara et al., 2004) and more recently in Mexico (Frank et al., 2013), tectonic tremor consisting of a swarm of low-frequency earthquakes was shown to occur at the same time and at similar depths as geodetically detected slow slip events. These observations suggest that tremor and LFEs might represent the seismic signature of slow slip events (Ide et al., 2007b).

Transient strain episodes (i.e. slow slip events) occur on the deep portions of plate interfaces and large-scale episodes are often observed, with limited sensitivity and resolution, on GPS stations installed at the surface (Lengliné et al., 2017). Slow slip events can last from several days to months, hence the slip released during a slow slip event can represent an elastic release that is equivalent to that of a $M_{w} \geq 7$ earthquake (Beroza and Ide, 2011). Studying in detail slow slip events and their associated stress release is critical to assess seismic hazard and earthquake triggering. However, most of the time, the deformation associated with slow slip 
is not detected. Indeed, the dislocation along the fault at depth does not often generate a geodetic signal greater than the observational noise level (Frank et al., 2015; Frank, 2016), therefore making the detection of slow slip laborious.

In contrast, tremor and LFEs are more precisely recorded, and in recent years, many spatially and temporally continuous LFE catalogues were built (e.g. this study's catalogue; Frank et al., 2014; Shelly, 2017). The contemporaneous detections of slow slip and increase in tremor and LFE activity suggest that both signals share the same mechanical origin (Ide et al., 2007b; Lengliné et al., 2017). This means that LFE records can be used to constrain the evolution of slow slip events, and particularly of the small geodetically undetected ones.

Indeed, in order to improve the SNR in the geodetic signal, the timing of catalogued LFE and tremor activity can be used to extract slow slip signals from the geodetic noise. Frank et al. (2015) developed a technique based on aligning GPS time series with episodes of low-frequency earthquake activity and on stacking the GPS time series in order to uncover a previously unobserved, repeating transient slip signal. This technique was successful in revealing geodetic slow slip signals, previously hidden in the noise, occurring simultaneously with LFEs in the Mexican subduction zone (Frank et al., 2015), in the Cascadia subduction zone (Frank, 2016) and in the deep portion of the San Andreas Fault (Lengliné et al., 2017). This way, Frank (2016) discovered, for instance, a previously unobserved slow slip event producing less than a millimetre $(\sim 0.6 \mathrm{~mm})$ of surface deformation in Cascadia. Those recent studies demonstrate not only that (1) LFEs can be used as effective monitors of slow slip at depth; but also that (2) a lot of small episodes of transient aseismic slip are hidden in the noise and they occur over a broad spectrum of amplitudes and time scales; and finally that (3) the plate interface where slow slip, tremor and LFEs occur, is strongly coupled.

Here, we infer that the big 'step' in LFE detections (Fig. 5.2), corresponding to LFE episode ID:4 in Table 5.1, might be indicative of a large-scale slow slip event occurring in the area. This assumption is supported by the detection of 3 days of tremor in the first 6 weeks of the LFE episode. Consequently, $25 \%$ of the tremor observed between May 12t $\mathrm{t}^{\text {th }}, 2009$ and September $4^{\text {th }}, 2011$ occurred in the first half of this LFE episode. The concomitant increase of tremor and LFE activity hints that a significant phenomenon of slow deformation is occurring at that time. No tremor analysis was undertaken beyond the first 6 weeks of the LFE episode, hence it is possible that additional undetected tremor is occurring in the second half of the LFE episode. In any case, we suggest that this potential slow slip event occur at least between the $25^{\text {th }}$ July 2011 and the $28^{\text {th }}$ October 2011 
(concomitantly with the increased tremor and LFE activity, happening during LFE episode ID:4). During that time, we observe increased generation rates for all of the LFE families in the study area. Hence we suggest that, similarly to other studies (e.g. Rogers and Dragert, 2003; Obara et al., 2004; Frank et al., 2013), this slow slip event might occur at similar depth than LFEs, i.e. between depths of $\sim 11-59 \mathrm{~km}$. We estimate the LFE rupture zone to $\sim 36,000 \mathrm{~km}^{3}(\sim$ $40 \mathrm{~km}$ length $\times 30 \mathrm{~km}$ width $\times 30 \mathrm{~km}$ depth-extent). Therefore, this probable slow slip event might involve slip on a consequent portion of the plate interface. The other smaller-scale LFE episodes might represent small amplitude slow slip events, also invisible in GPS records. Therefore, looking for episodes of increased LFE activity constitutes preliminary work which has implications to identify (1) periods to look for slow slip in GPS records; or (2) periods beyond September 2011 to look for new tremor.

To unequivocally demonstrate the relation between LFEs and slow slip events in the central Southern Alps, and to quantify the amount of slip occurring during a transient, GeoNet GPS times series can be used to extract the geodetic signature of slow slip events using the discrete timing of LFEs as a guide. Indeed, now that periods of elevated LFEs activity have precisely been identified, it is possible to correlate LFEs, spatially and temporally, with geodetically observed slow slip by stacking GPS time series during LFE episodes following the approach of Frank et al. (2015). This method will allow a deeper look into the relationship between LFEs and slow slip generation as well as into the loading/unloading occurring on the deep portion of the Alpine Fault.

\subsection{Conclusions}

We use an extensive continuous catalogue of more than 300,000 LFEs detected along the central Alpine Fault from 2009 to 2017 to carry out a detailed study of the spatial and temporal evolution of low-frequency earthquakes (LFEs) in the central Southern Alps. We take advantage of the high temporal and spatial resolutions of this continuous catalogue to provide new insights into LFEs recurrence time, occurrence pattern and migration phenomena.

We first exploit the LFEs recurrence time to identify periods of uncommonly high LFE activity. We find that all but one of the LFE episodes identified in this study from 2009-2011 occur systematically on days where Wech et al. (2012) identified tremor. We thus infer that those periods could be indicative of small or larger- 
scale slow slip events.

We then compare the occurrence pattern of LFES observed during an LFE episode to the ones observed during a quiet period. We find that during the quiet period, only $\sim 30 \%$ of the LFE families are active and the LFE sources with the highest activity are located closer to the fault. However, during an episode of increased activity, we observe that all LFE families become active, with intermittent surges in LFE rate. This behaviour could indicate a rate-dependent friction on the fault, with the surge in LFE activity being indicative of an external increase in loading. Alternatively, this behaviour could also be indicative of possible triggering occurring between LFE families.

We also find that LFEs with synchronous occurrence patterns tend to be clustered in space. We thus suggest that individual LFE sources form spatially coherent clusters that may represent localised asperities or elastic patches on the deep Alpine Fault interface. We infer that those clusters may have a similar rheological response to tectonic forcing or to potential slow slip events.

We finally discover slow (10 km/day) and rapid $(\sim 20-25 \mathrm{~km} / \mathrm{h})$ migrations of LFEs along the Alpine Fault, with velocities similar to what has been found elsewhere (e.g. San Andreas, Mexico). The slow migration might be controlled by slow slip events themselves while the rapid velocities could be explained by the LFE sources' intrinsic properties. For example, the rapid velocities could be explained by increased average slip rates linked with slip-induced dilatancy in the LFE source area. The static stress transfers occurring during repeated episodes of slip, might for instance generate a series of LFEs propagating along the fault zone, whose duration and speed could depend on the overall fluid diffusion time. 


\section{Summary and Conclusions}

\subsection{Summary of findings}

\subsubsection{Extended LFE catalogue}

As described in Chapter 3, we have used recently detected tremor and low-frequency earthquakes (LFEs) to examine how slow tectonic deformation is loading the Alpine Fault late in its typical 300-year seismic cycle. We have analysed a continuous seismic dataset recorded between 2009 and 2016 using a network of 10-13 short-period seismometers, the Southern Alps Microearthquake Borehole Array. We have obtained the longest continuous record of LFEs beneath New Zealand's Southern Alps.

The aim of this work was to generate an improved LFE catalogue in comparison with that of Chamberlain et al. (2014), in order to provide better constrained locations and to compute focal mechanisms. Several key analytical steps, were taken to achieve this goal:

(1) We used a new Python package to cut down the processing time and generate our catalogue faster (Chamberlain et al., 2017c);

(2) We optimised our codes to run them on a supercomputer, the NeSi Pan Cluster, to further cut down the processing time;

(3) We thoroughly tested the sensitivity of several parameters (e.g. the number of detections kept for each stack, the minimum number of stations used for a detection, the detection threshold) to find the optimal ones;

(4) We used the entire dataset available to us (8 years of data) to increase the overall number of high signal-to-noise ratio detections used in the stacks;

(5) We retained more detections in our stacks, which provided higher signal-tonoise ratio stacks than those of Chamberlain et al. (2014);

(6) We used phase-weighted stacking instead of linear stacking for our final iteration stack, which provided an overall more impulsive signal. 
To generate this improved catalogue, we used fourteen primary LFE templates in an iterative matched-filter and stacking routine, allowing the detection of similar signals corresponding to LFE families sharing common locations. This routine yielded an 8-yr catalogue containing 10,000 LFEs that were combined for each of the 14 LFE families using phase-weighted stacking to produce signals with the highest possible signal-to-noise ratios.

We then computed improved non-linear earthquake locations using a 3D velocity model. We find LFEs to occur below the seismogenic zone at depths of $17-42 \mathrm{~km}$, on or near the hypothesised deep extent of the Alpine Fault. We find that the LFE families occur at similar depths to tremor $(15-45 \mathrm{~km})$. Of the fourteen families, thirteen lie within $15 \mathrm{~km}$ of the Alpine Fault. The remaining family is located to the southwest of the proposed deep extent of the Alpine Fault.

We have computed the first LFE focal mechanisms associated with the Alpine Fault, or indeed with continental plate-boundaries anywhere. Focal mechanisms, in conjunction with recurrence intervals, are consistent with quasi-continuous shear faulting on the deep extent of the Alpine Fault. Our results reveal mostly dextral strike-slip motion with a subordinate reverse component suggesting that LFEs occur mainly as shear faulting on the deep extent of the Alpine Fault. We have also determined the focal mechanism of an LFE family occurring to the SW of the Alpine Fault's presumed position at depth. This focal mechanism involves reverse slip on a steeply-dipping fault plane $\left(\sim 80^{\circ} \mathrm{dip}\right)$, with a minor component of dextral strike-slip motion. These kinematics are consistent with the preferred model of Norris and Toy (2014) of a more steeply-dipping western branch of the Alpine Fault. Nevertheless, an extensive search of this region for further LFEs and the determination of additional LFE focal mechanisms are required to reinforce this observation.

Finally, we have shown that LFEs occur almost continuously during the 8-yr study period, with punctuated episodes of increased rates, corresponding to known episodes of tremor or large regional earthquakes. We have also highlighted two types of LFE distributions: (1) discrete behaviour with an inter-event time exceeding 2 minutes; (2) burst-like behaviour with an inter-event time below 2 minutes. We interpret the discrete events as small-scale frequent deformation on the deep extent of the Alpine Fault and the LFE bursts (corresponding in most cases to known episodes of tremor or large regional earthquakes) as brief periods of increased slip activity indicative of slow slip.

To conclude, we have shown using LFE inter-event times and focal mechanisms 
that the LFEs are the seismic manifestation of quasi-continuous deformation on the deep extent of the Alpine Fault, interspersed with increased rates of deformation corresponding to tremor and potentially to slow slip. We have provided evidence that LFEs do indeed represent localised slow shear slip on the deep extent of the Alpine Fault: (1) the simultaneous detection of tremor and LFEs; (2) LFE focal mechanisms being indicative of shear slip on the Alpine Fault; and finally (3) LFE locations being consistent with the location of the deep extent of the Alpine Fault inferred by seismic reflection (Okaya et al., 2007), by velocity models (Stern et al., 2001) and by GPS inversion (Lamb and Smith, 2013).

\subsubsection{Continuous LFE catalogue}

To gain insight into deep fault slip behaviour, a detailed study of the spatial and temporal evolution of low-frequency earthquakes was required. For this, an extensive catalogue regrouping hundreds of LFE families was needed. In Chapter 4, we have presented a new method for generating real LFE templates by using synthetics in a matched-filter routine. Using this method, we have successfully built the longest record (spanning 8 years) of LFEs in New Zealand to date as well as the first spatially and temporally continuous catalogue of LFEs beneath the central Southern Alps.

We have generated a new catalogue of LFEs spanning the years 2009 to 2017 using a different technique than that presented in Chapter 3 in order to detect LFEs more efficiently. This time, 638 synthetic LFE waveforms were used as primary templates in a matched-filter routine. Of those, 529 templates yielded enough detections during the first iteration of the matched-filter routine $(\geq 500$ over our 8-year study period) and were kept for further analysis. We then used the best $25 \%$ of correlated events for each LFE family in a linear stack to create new LFE templates. From there, we ran a second and final iteration of the matchedfilter routine with the new LFE templates to obtain a final LFE catalogue. The remaining 529 templates detected between 150 and 1,671 events each, totalling 300,996 detections over the 8-year study period. We established a long-term rate of 103 LFEs per day. Each LFE template and its subsequent detections hence formed an LFE family, itself associated with a single source and hypocentre location. This technique paired up with phase-weighted stacking greatly increased the number of LFE families and overall detected events roughly thirtyfold when compared to the previous catalogue detailed in Chapter 3.

Of the 529 templates, we have manually picked the $\mathrm{P}$ and S-arrivals and located 
378. We therefore have obtained the locations of hundreds of LFE families which allowed us to discuss the extent of LFEs within our study area. Our LFEs locate below the seismogenic zone defined by regular microseismicity, at depths of 11$59 \mathrm{~km}$ (roughly at similar depths to tremor). We have demonstrated that LFEs in the central Southern Alps fill a gap in seismicity and that they occur at depths where 'typical' earthquakes have not been detected. We have also shown that LFEs mainly occur in (but are not entirely bound by) an area of low resistivity (Eberhart-Phillips et al., 2008), low seismic-wave speeds (Stern et al., 2001) and increased fluid pressure (Stern et al., 2001; Wech et al., 2012).

We have shown looking at cumulative detections continuous LFE detections over the study period, punctuated by increases in LFE rate coinciding in some cases with known tremor events and large $\left(>M_{w} 5\right)$ regional earthquakes. We also observe an interesting episode at the end of 2011, with 50,000 LFEs generated in only 3 months, which could indicate that a large-scale slow slip event occurred at that time (Frank et al., 2016). Our observations suggest that accelerated rates of LFEs generation might represent episodic slow slip on or near the deep extent of the Alpine Fault.

Finally, we have observed a variation of LFE time signature patterns with depth, with LFE sources evolving from episodic or 'stepped' behaviour to continuous. This transition may correspond to an evolution from a stick-slip slip regime to a stable-sliding slip regime with depth. Hence, we have proposed that the distinctive features of LFE occurrence patterns might reflect variations in the in-situ stress and frictional conditions at the individual LFE source locations.

To conclude, we have found a $67 \%$ overlap between the extended LFE catalogue in Chapter 3 and this study's catalogue, thus suggesting that we have indeed mainly detected real LFE events in the continuous LFE catalogue. We have therefore proven the use of synthetic templates to effectively detect LFEs in New Zealand's central Southern Alps. We provided, through this new extensive catalogue, a valuable tool for in-depth analyses of the deep central Alpine Fault structure and its slip behaviour.

\subsubsection{LFE characteristics}

In Chapter 5, we have conducted a detailed study of the spatio-temporal evolution of low-frequency earthquakes in the central Southern Alps. This type of study is essential to better understand the behaviour of the LFE sources and the inferred 
slip behaviour on the deep extent of faults.

To carry out this detailed study of the spatial and temporal evolution of lowfrequency earthquakes in the central Southern Alps, we have used the extensive continuous catalogue of more than 300,000 LFEs detected along the central Alpine Fault from 2009 to 2017, detailed in Chapter 4. We have taken advantage of the high temporal and spatial resolutions of this catalogue, to provide new insights into LFEs recurrence time, occurrence pattern and migration, as well as to identify potential episodes of slow slip.

We have first exploited the LFEs recurrence time to identify periods of uncommonly high LFE activity. This way, we identified 8 episodes of increased LFE activity between March 2009 and March 2017. During those episodes, we have observed an average daily LFE rate equal to $234 \mathrm{LFEs} /$ day, with LFE counts varying between 1,888 and 47,093 detections, depending on the episode. The mean duration of an LFE episode was found to be 26 days. We identified the largest LFE episode between July 25 ${ }^{\text {th }}$, 2011 and October $28^{\text {th }}$, 2011 (duration of 95 days) and its average daily rate was calculated to be $496 \mathrm{LFEs} /$ day. With this finding, we established that $17 \%$ of the entire catalogue was detected over a period equivalent to $3 \%$ of the total catalogue duration. We have found that all of the LFE episodes identified in this study from 2009-2011 occurred systematically on days where Wech et al. (2012) identified tremor. We thus inferred that those periods could be indicative of large-scale slow slip events.

We have then compared LFE occurrence patterns observed during an LFE episode to the patterns observed during a quiet period. We have found that during the quiet period, only $\sim 30 \%$ of the LFE families are active and the LFE sources with the highest activity are located closer to the fault (Fig 6.1). However, during an episode of increased activity, we have observed that all LFE families become active, with intermittent surges in LFE rate. This behaviour could indicate a ratedependent friction on the fault, with the surge in LFE activity being indicative of an external increase in loading. Alternatively, this behaviour could also be indicative of possible triggering occurring between LFE families.

We have also remarked that LFEs with synchronous occurrence patterns tended to be clustered in space (same colour patches in Fig6.1). We thus suggested that individual LFE sources formed spatially coherent clusters that may represent localised asperities or elastic patches on the deep Alpine Fault interface. We inferred that those clusters may have a similar rheological response to tectonic forcing or to potential slow slip events. 


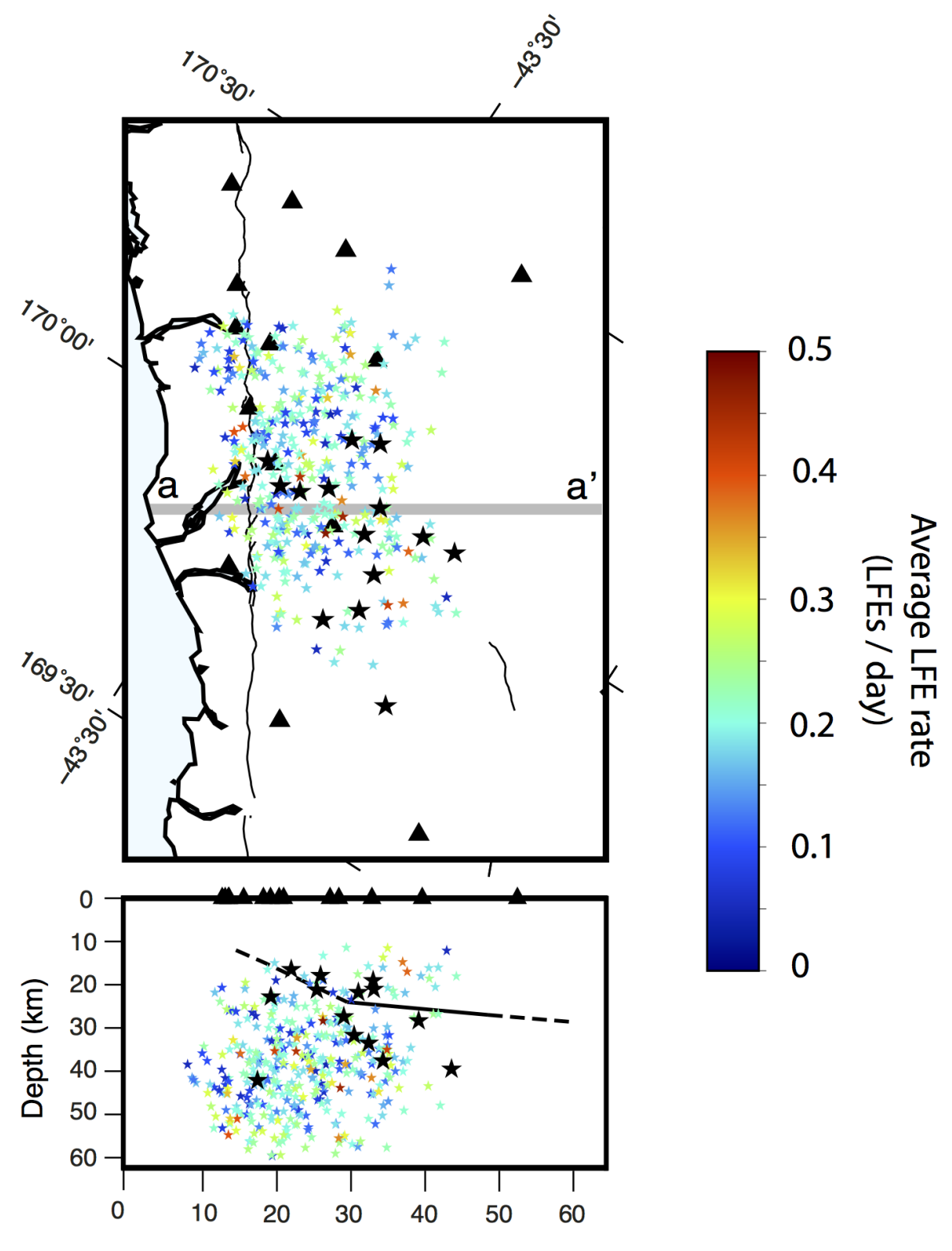

Distance along profile aa' $(\mathrm{km})$

Figure 6.1: Map of average LFE daily rate. LFE families are coloured according to their average density (i.e. average number of LFE detections per day per family). Top: rotated map view. Bottom: cross section, orthogonal to fault, along profile aa', with a GPS modelled fault after Lamb and Smith (2013). The black stars correspond to the previous LFEs of Chapter 3/Baratin et al. (2018). The LFE family of Chapter 3/Baratin et al. (2018), located to the SW of the GPS inferred fault (black line), could potentially be located on a second branch of the Alpine Fault, similarly to what was described by (Norris and Toy, 2014).

We finally have also discovered slow $(10 \mathrm{~km} /$ day $)$ and rapid $(\sim 20-25 \mathrm{~km} / \mathrm{h}) \mathrm{mi}$ grations of LFEs along the Alpine Fault, with velocities similar to what has been 
found elsewhere (e.g. Mexico, San Andreas). The slow migration could be controlled by slow slip events themselves while the rapid velocities could be explained by the LFE sources' inherent properties. For example, the rapid velocities could be explained by increased average slip rates linked with slip-induced dilatancy in the LFE source area. The static stress transfers occurring during repeated episodes of slip, could for example generate a series of LFEs propagating along the fault zone, whose duration and speed might depend on the overall fluid diffusion time.

\subsection{Tremor and LFEs in a global context}

Deciphering the rheological and physical properties of a fault at depth is extremely challenging. This is notably due the variety of fault slip behaviour (i.e. various spatio-temporal scales) that can be found on a fault interface (Lengliné et al., 2017). A common theoretical concept to explain the diversity of fault slip behaviour consists of a model of a locked fault interface - composed of unstable patches able to nucleate earthquakes - embedded in a more stable region of the fault capable of transient aseismic slip. The heterogeneity in rheological properties between these two components of the fault interface lead to a diversity of phenomena, such as slow slip (Radiguet et al., 2011) or even creep episodes (Rousset et al., 2016).

A possible way to gain more insight into fault slip behaviour (hence fault properties) at depth, it to use the characteristics of slow slip, and more precisely of the seismic signals that are known to accompany it: tectonic tremor corresponding to a superposition of low-frequency earthquakes.

Tectonic tremor has several interesting properties that are systematically found in any tectonic setting and that can be used to probe the properties of a fault at depth. For example, Obara (2002) was the first to observe that tectonic tremor was episodic. He observed large periods of little to no tremor activity, interspersed by periods with abundant tremor activity. In fact, concentrated tremor activity has been intrinsically linked in several areas worldwide with the presence of large quantity of fluids (Beroza and Ide, 2011). For example, tectonic tremor in the Parkfield area of the San Andreas Fault, was found correlate with heterogeneous and localised large water release originating from the breakdown of certain minerals (Fagereng and Diener, 2011). In the Southern Alps, Wech et al. (2012) also supported the idea that tectonic tremor occurred in a fluid-rich region marked by high attenuation and high reflectivity. 
The physical properties of tectonic tremor are not yet understood for all tectonic settings. However, low effective-stress conditions, originating from metamorphic fluid production within the fault zone are typically inferred to explain the presence of tremor in subduction zones (Audet and Burgmann, 2014). In contrast, tremor occurring on the San Andreas Fault (i.e. in a strike-slip tectonic regime, similar to what is found in the New Zealand Southern Alps) was found to be rather related either to mantle-derived fluids (originating from below the area defined by tectonic tremor) or to fault zone weakening occurring as phyllosilicates replaced more granular and competent minerals (Fagereng and Diener, 2011).

Detailing the behaviour of low-frequency earthquakes plays a critical role in deciphering the mechanisms of deep tectonic tremor, especially since low-frequency earthquakes are easier to detect than tremor. Low-frequency earthquakes are found to behave similarly across all tectonic settings, with temporal and spatial behaviours being consistent for LFEs occurring in strike-slip areas, but also for LFEs occurring in subduction zones (e.g. Shelly et al., 2007; Ide et al., 2007b; Frank et al., 2013; Baratin et al., 2018). Our understanding of low-frequency earthquakes so far seems to indicate that the LFE focal mechanisms and locations are consistent with shear failure occurring on the plate interface (Shelly et al., 2007; Ide et al., 2007b; Frank et al., 2013, e.g.). Low-frequency earthquakes in the Southern Alps are consistent with findings worldwide and support the model described above where LFEs are occurring as discrete dynamic ruptures in an otherwise slowly slipping plate interface.

Low-frequency earthquakes sources can be modelled, similarly to the work done by Beeler et al. (2013), to infer the rheological properties of rocks producing creep (hence to infer the rheology of the fault at depth). Beeler et al. (2013) modelled the LFE sources as small and constant regions, repeatedly failing during the shear of a considerably larger and otherwise aseismically creeping region of the fault. That way, they managed to quantify the loading of the LFE source patches and to estimate the shear stress and the seismicity rate in their study area. Implementing this type of approach in the Southern Alps is crucial to quantify how low-frequency earthquakes are loading the Alpine Fault but also to better comprehend the stress field in the area. 


\subsection{Slow deformation in the central Southern} Alps

LFEs in the central Southern Alps (Fig. 6.2) appear to fill a vertical gap in seismicity, left by both shallow $(0-12 \mathrm{~km})$ and deep seismicity $(50-100 \mathrm{~km})$ (Boese et al., 2012, 2013, 2018; Wech et al., 2012). The low-frequency earthquakes locations obtained to date, while highlighting a broad area of slow deformation, enable us to infer that semi-brittle behaviour extends to depths greater than previously thought (0-12 km depth, Boese et al., 2012). The LFEs occur at depths at which 'typical' earthquakes have not been detected in a zone of broad and strong seismic reflectivity (Okaya et al., 2007), low seismic-wave speeds (Stern et al., 2001), high P-wave attenuation (Eberhart-Phillips et al., 2008) and increased fluid pressure (Stern et al., 2001; Wech et al., 2012). The region of low velocities and low resistivity has been interpreted previously to be associated with elevated fluid pressure and fault weakness (Stern et al., 2001; Eberhart-Phillips et al., 2008). The presence of high fluid pressure implies a decrease in the effective normal stress, which in turn makes shear failure on a fault possible at lower differential stress.

The limited spatial resolution provided by surface data hinders our ability to probe the dynamics of the Alpine Fault at depth. In order to gain insight into the loading cycle of the deep Alpine Fault, the spatial and temporal characteristics of slow slip events can be used. Because inferring small-amplitude displacements at depth is not possible as of yet in the Southern Alps, we exploit the temporal behaviour of signals that are known to accompany slow slip: low-frequency earthquakes. Lowfrequency earthquakes can indeed be considered as markers of the local slip rate on the fault interface (Frank, 2016).

In this study, we have highlighted two distinct types of LFE behaviours (Fig. 6.2):

(1) Disparate spatio-temporal behaviour depending on LFE families;

(2) Disparate temporal behaviours within the same LFE family;

This first type of LFE temporal behaviour implies that there could be variations in the in-situ stress and frictional conditions at the individual LFE source locations on the Alpine Fault. The distinctive features of LFE occurrence patterns reflect an evolution from an episodic slip regime to a continuous slip regime with depth. This transition from episodic to continuous also seems to coincide with decreasing normal stress on the Alpine Fault (Stern et al., 2001; Eberhart-Phillips et al., 
2008). Here we infer that this transition may correspond to an evolution from a stick-slip slip regime to a stable-sliding slip regime. This transition might also be a reflection of the Moho depth, which agrees with other studies in the area inferring the Moho depth based on seismicity (e.g. Lamb et al., 2015). Those observations are supported by other studies worldwide. Indeed, at most plate boundaries, tectonic tremor and low-frequency earthquakes are inferred to be associated with slow shear-slip failure in the transition zone between the stick-slip and stable sliding portion of a fault (Shelly et al., 2006; Shelly, 2009; Wech and Creager, 2007).

The second type of LFE temporal behaviour highlights variations in the local slip regimes over time, with periods where no time clustering is observed between events (i.e. events occurring independently of one another) and periods where the occurrence of events is strongly clustered (i.e. strong interactions between LFEs, indicative of slow slip events). These variations in local slip regimes can also be inferred by looking at migration velocities (i.e. slow versus fast migration phenomena). Indeed, Shelly (2015) suggested that the slow migration phenomenon was seemingly controlled by slow slip events along the San Andreas Fault. In contrast, the rapid migration might be a reflection of the dynamic processes involved at the tremor and LFE sources themselves which could be linked with a large-scale fluid flow (Ghosh et al., 2010) or with smaller stress drops and/or increased average slip rates (Rubin, 2011; Rubin and Armbruster, 2013).

A crucial question raised by Rubin (2011) is whether or not tectonic tremor and low-frequency earthquakes act as a passive indicator of increased slip rate or if they rather play an essential role in sustaining the slip. (Kao et al., 2010) found the geodetic moment of slow slip to be several orders of magnitudes higher than that of tremor; hence leading Kao et al. (2010) to infer that tremor was generally passive on a broad scale (i.e. tremor occurring during episodes of slow LFE migrations). However, Trugman et al. (2015) found that this might not be true for small-scale rapid tremor (i.e. tremor occurring during episodes of rapid LFE migrations). Indeed, Trugman et al. (2015) found that tremor propagated more effectively in regions of elevated tremor production, incidentally corresponding to regions where strong clustering of LFE families is observed. Similarly, episodes of rapid migrations coincide with continuous tremor and LFE activity (Shelly, 2015; Trugman et al., 2015; Baratin et al., 2018). Eventhough this analogy does not necessarily prove that LFE rupture is essential for high-speed migration, it implies at the very least that LFE generation is encouraged by the same factors that spur high-speed slip migration. Because an LFE rupture is stretched out in time when 
compared to a typical earthquake rupture, a 'braking' mechanism is required in all cases Shelly (2015); Trugman et al. (2015). In addition, this mechanism must be applicable in a variety of tectonic settings and in a variety of spatial scales, as similar behaviour are observed in subduction zones but along major transform boundaries (e.g. Shelly, 2017; Frank, 2016).

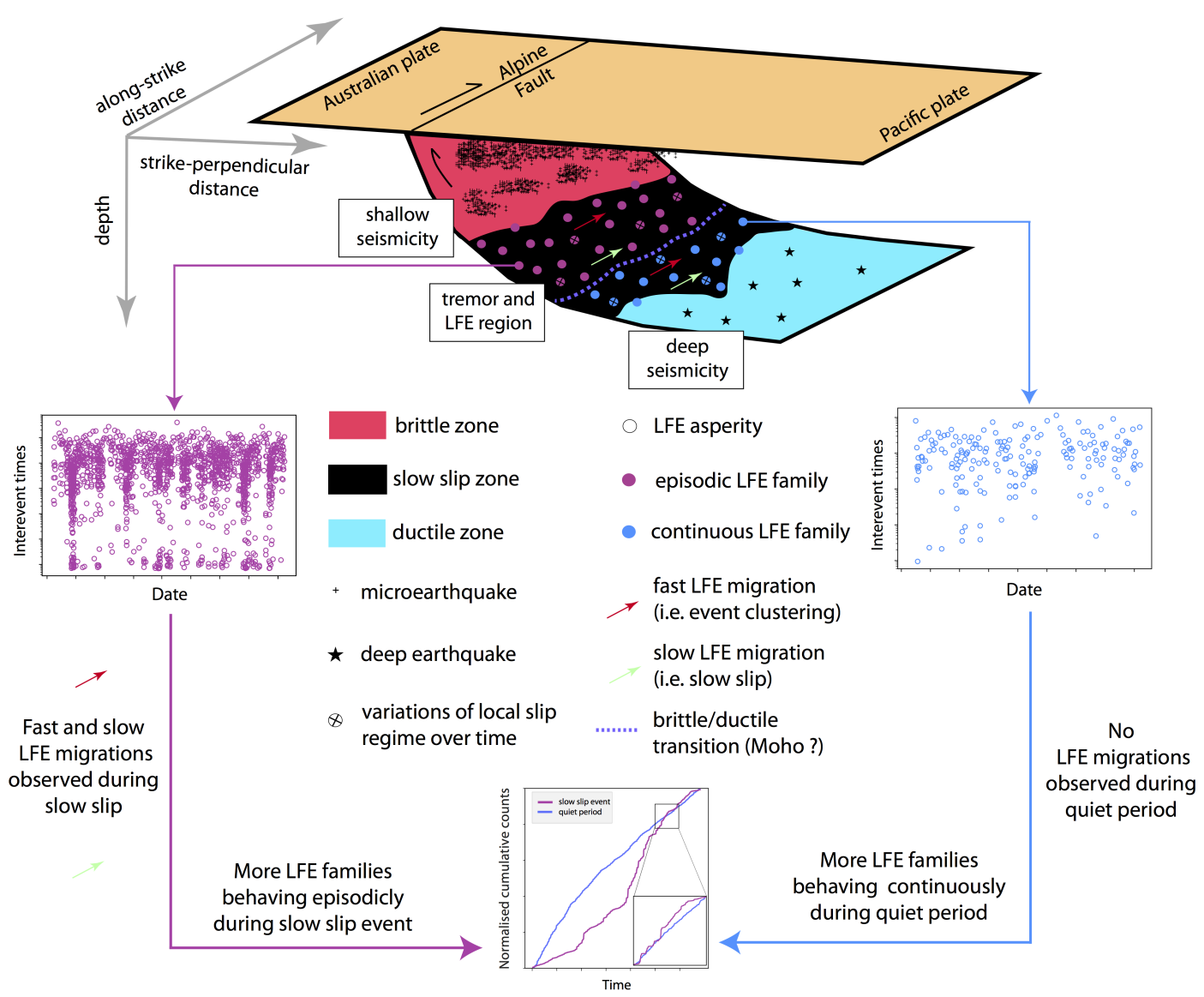

Figure 6.2: Summary diagram. Schematic model for LFE generation in the Southern Alps. The transition from the shallow seismogenic zone to the stable sliding transition at depth in characterised by the presence of tremor and LFE (Wech et al., 2012). We assume that LFE are generated on small patches (or asperities) within an otherwise stably sliding region of the fault. Two types of LFE behaviours are identified on this diagram (episodic versus continuous). We infer that this change in behaviour coincides with the brittle/ductile transition, similarly to what was found by Trugman et al. (2015) in the San Andreas region. 


\subsection{Recommendations for future studies}

Now that extended LFE catalogues have been built for the central Southern Alps, detailed studies of these catalogues may provide a greater understanding of several key processes occurring on a major transform fault that is late in its earthquake cycle, as well as key properties of both the shallow and the deep portion of the fault.

\subsubsection{Slow slip investigation}

In Cascadia (Rogers and Dragert, 2003), in Japan (Obara et al., 2004) and more recently in Mexico (Frank et al., 2015), tectonic tremor consisting of a swarm of low-frequency earthquakes was shown to occur at the same time and at similar depths as geodetically detected slow slip events (SSEs). Frank (2016) provided the first geodetic evidence of slow slip occurring at the same time as bursts of LFEs. Studying tremor and slow slip in detail and their associated stress release is critical to assess seismic hazard and earthquake triggering. Slow slip events can last from several days to months, hence the slip released during an SSE can represent an elastic release that is equivalent to that of a $M_{w} \geq 7$ earthquake (Beroza and Ide, 2011). However, the deformation associated with slow slip is not easy to detect especially if the displacement at depth does not produce a geodetic signal greater than the observed noise (Frank et al., 2015). Frank et al. (2015) demonstrated how GPS time series from different episodes of LFEs could be stacked together to increase the signal-to-noise ratio of geodetic data. They hypothesise that each low-frequency earthquake episode represents a recurring slow slip event. Hence any coherent signal in the GPS time series will constructively stack, while the random noise will sum incoherently. Using this method, they managed to extract a relatively small motion associated with slow slip occurring during episodes of high LFE activity in the Mexican subduction zone.

A GPS transect currently runs across the Alpine Fault and the Southern Alps, just above the region documented to generate tremor and LFEs. In this study, we managed to identify 8 episodes of LFEs that might be concomitant with slow slip. Those can be used as a guide to stack GPS time series. Therefore, it might be possible to implement this method in the central Southern Alps. Properly preparing the GPS data would be key here, as the signals of interest will be hidden under the noise prior to stacking. However, we have to keep in mind that the GPS data in the Southern Alps are not all of high quality, which may result 
in low quality stacks (Hreinsdottir 2015, personal communication). Overall, this analysis could (1) help us understand how LFEs and deep slow slip relate to the locking of the Alpine Fault at depth and (2) provide more constraints on the states of stress and strain prevailing in the brittle-ductile transition zone.

\subsubsection{Magnitudes}

Magnitudes calculation may clarify certain properties of the asperities (or small patches) that fail during LFE rupture (e.g.rupture area, rupture length). An understanding of these properties as well as of the stress drops may help quantify the amount of coseismic slip associated with LFE failure. LFEs do not represent the whole area that is slipping during slow slip, hence these estimates would likely be considerably less than any geodetically measurable slip (Aguiar et al., 2009; Frank, 2016).

\subsubsection{Automatic detections of LFE migrations}

The occurrence of tremor and LFEs during slow slip events is not incidental. An essential point raised by Rubin (2011) is whether tremor and low-frequency earthquakes act, following a slow slip event, as a passive indicator of increased slip rate, or if they play a crucial role in sustaining the actual slip. Seeing as the geodetic moment of slow slip is found to be several orders of magnitude higher than the cumulative seismic moment of tremor, tremor is generally assumed to be passive on a broad scale (Kao et al., 2010). However, this might not be the case for small-scale, rapidly propagating tremor (Shelly, 2015).

When looking at large-scale slow slip events (potentially what is occurring during the LFE episode ID:4), spatially and temporally coherent migrations of tremor and LFEs have been observed within the slipping section of numerous faults after the 'main slip front', associated with the slow slip event, had elapsed (e.g. Houston et al., 2011; Royer et al., 2015; Bletery et al., 2017). These migration patterns are inferred to correspond to 'secondary slip fronts', which occur through a broad range of distances and durations.

Bletery et al. (2017) developed an algorithm to automatically detect 'secondary slip fronts' or migrations of low-frequency earthquakes. This algorithm (detailed in Fig. 6.3) is based on the analysis of LFE migration patterns in 3-D using: (1) the distance along dip; (2) the distance along strike; and (3) time. 


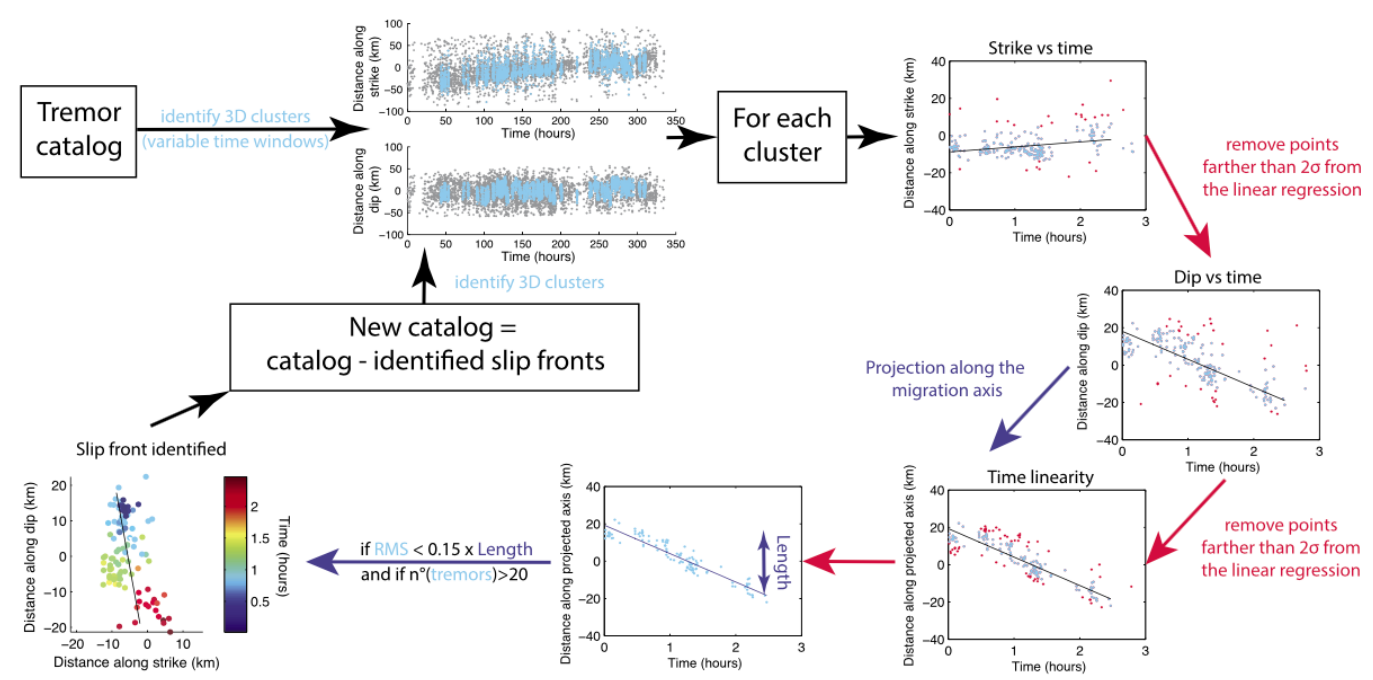

Figure 6.3: Algorithm scheme of Bletery et al. (2017). Algorithm scheme for automatic detections of LFE migration fronts at different time scales from (Bletery et al., 2017).

The idea behind automatically identifying LFE migration patterns is to use them as a tool to asses secondary slip fronts which happen to propagate faster than the main front (Houston et al., 2011). Using clusters of tremor and LFEs, Bletery et al. (2017) calculated linear regressions, for several durations, for each cluster for: (1) the distance along strike as a function of time; and (2) the distance along dip as a function of time. The events were then projected along the best fitting migration axis as a function of time. Finally, if the number of events in the cluster is above a certain threshold, and if the root mean square of the events distance to the last regression is smaller than a certain percentage of the migration length, then the cluster is classified as a 'secondary slip front'.

This type of analysis helps fill the gaps between seismically and geodetically detectable slow earthquake processes. It would also provide an order of magnitudes for stress drops and slip rates in the central Southern Alps. The biggest challenge to implement this technique in our study area, would be the lack of a reliable model for the deep Alpine Fault. Indeed, this method requires to project the LFE locations on the fault at depth (along dip), hence it requires a well-know geometry for the fault in the area.

\subsubsection{Crustal Anisotropy}

Seismic anisotropy refers to the property of a medium where the velocity of a seismic wave depends on its direction of propagation and its polarisation (Savage, 
1999). When shear waves travel in an anisotropic medium, the component polarised parallel to the fast direction travels faster than the orthogonal component. The difference in speed causes the waves to separate; this phenomenon is called shear wave splitting. Shear wave splitting can be used to investigate deformation in the lithosphere.

High quality LFE stacks have been used to investigate crustal anisotropy in Northern Cascadia (a subduction zone forearc; Matharu et al., 2014). Indeed, LFE waveforms show significant uniformity when plotted at an individual station, with particle motions that are mainly horizontal and that manifest as an up-going wavefield composed principally of S-waves (Bostock and Christensen, 2012). This behaviour is indicative of easily reproducible polarisation directions and of a source mechanism that is persistent all over the study area (Matharu et al., 2014). Hence, under those observations, LFE waveforms can be interpreted as empirical Green's function (Bostock and Christensen, 2012). Matharu et al. (2014) therefore used their LFE stacks to do S-wave splitting analyses across Vancouver Island and northern Washington. This study allowed them to find the presence of a heterogeneous distribution of crustal anisotropy for the northern part of the American plate.

Given the high signal-to-noise ratio of some of our stacks, we believe this type our approach to be possible in the central Southern Alps. Furthermore, some preliminary analysis indicates that we are beginning to see S-phase delays between the north and east components of some stations on our LFE individual detections and stacks (e.g. template ID: 169.641_-43.729_44_1 on channels 1 and 2 of station FRAN, Figs. A.33 and A.34 in Appendix A.3). Measurements of crustal anisotropy from LFEs could provide useful ray-paths for analysis of splitting directions in the crust.

\subsubsection{Triggering}

Studies of tremor and LFEs have shown the critical impacts of large transient stresses (e.g. Rubinstein et al., 2007; Miyazawa and Brodsky, 2008; Peng et al., 2009; Ghosh et al., 2009), but also of very small changes in stress (e.g. Rubinstein et al., 2008; Thomas et al., 2009, 2012), on tremor and LFE activity. Better comprehending the physics initiating the triggering of tremor and LFEs is essential as it can provide evidence of the processes governing slip along major plate boundary faults in the transition zone from brittle failure (in the seismogenic upper crust) to aseismic deformation (at depth). Acknowledging that quasi-static stresses (as- 
sociated with solid earth and ocean tides) and dynamic stresses (associated with passing seismic waves) are able to trigger or modulate tremor and LFEs, render the calculation of these stress oscillations possible (e.g. Rubinstein et al., 2007; Nadeau and Guilhem, 2009; Hill, 2012). Therefore, tremor and LFEs can be used as tools to examine in-situ fault properties and the state of stress in their source area.

Remote triggering of tremor and low-frequency earthquake by seismic passing waves has been extensively studied in the past, in Japan (e.g. Miyazawa and Brodsky, 2008), in Cascadia (e.g. Rubinstein et al., 2007), and in the San Andreas area (e.g. Nadeau and Guilhem, 2009; Peng et al., 2009). For instance, Peng et al. (2009) identified 10 teleseismic events between 2001 and 2009 (amongst the 31 events of $M_{w} \geq 7.5$ that occurred during that time) that were associated with clear triggering of tremor. Hill (2012) inferred that surface waves could trigger tremor by transiently increasing the ambient stress on existing fault planes towards some Coulomb failure threshold.

Furthermore, tidal modulation of tremor was initially observed in subduction zones (e.g. in Japan, or Cascadia, Nakata et al., 2008; Rubinstein et al., 2008). Thomas et al. (2009) established an apparent correlation between small amplitude shear stress induced by tides and tremor activity in a strike-slip context (near Parkfield, California, on the deep San Andreas Fault). Thomas et al. (2012) were the firsts to actually study the influence of solid earth and ocean tides on lowfrequency earthquakes activity in a strike-slip context. Analysing the response of LFEs and tremor to small stress perturbations, prompted by the Earth's tidal deformation, enables the estimation of the stress orientation and magnitude that prevails during tremor and LFE nucleation. This type of study hence gives a better understanding of the frictional processes that control tremor and LFE nucleation at depth (Thomas et al., 2009, 2012).

In the central Southern Alps, the sudden increases in LFE rates that we observe just after large regional earthquakes, may be indicative of LFE triggering. However, we did not manage to robustly link variations in LFE rates to the regional seismicity. This observation could indicate that either (1) other unrecognised sources of farfield or (2) endogenous triggering are in play.

Several options are worth considering in order to investigate the other potential source of triggering in the central Southern Alps:

- LFEs could be triggered by teleseismic waves that impose stress transient of only a few kilopascals (e.g. Gomberg et al., 2008; Peng et al., 2009); 
- LFEs could be triggered by dynamic stresses of Love and Rayleigh passing surface waves from microearthquakes or typical earthquakes occuring in the South Island (Miyazawa and Brodsky, 2008; Hill, 2012);

- LFEs could be triggered by perturbation of fractions of a kilopascal in response to tidal stress (e.g. Thomas et al., 2009, 2012);

- Some LFE families could be triggered by neighbouring LFE families or tremor (Shelly, 2015).

\subsubsection{Very low-frequency earthquakes}

Detecting the signal of slow deformation in the $0.1-1.0 \mathrm{~Hz}$ frequency band is laborious as microseism noise is mostly dominant. It is however possible to detect very low-frequency earthquakes (VLFE) in this frequency band (Kaneko et al., 2018). Very low frequency earthquakes are enriched in low frequency energy in the $0.01-0.1 \mathrm{~Hz}$ frequency band (i.e with periods of $10-100$ s Ito et al., 2007). Kaneko et al. (2018) looked at different slow earthquakes phenomena (i.e. tremor, LFEs, VLFE, slow slip events), in several frequency bands, and inferred similar locations and timing for the different events thus suggesting that these signals all originate from a common source. This means that it is possible, using LFEs, to investigate VLFE signals. Indeed, by aligning broadband records on the origin times of the different LFE signals and by stacking those records, we might be able to extract some VLFE signals. For instance, we could use the GeoNet broadband stations that are in or near our network (e.g. JCZ, FOZ, RPZ, LBZ), and look for VLFE signals during the LFE episode ID:4. To do so, we would need to take all of the detections originating from one family during this episode, to align the GeoNet's broadband records on each of their origin times and then to stack the broadband records. 


\subsection{Concluding statement}

This study provides the longest record of LFEs along the Alpine Fault as well as the first LFE focal mechanisms computed on a continental plate boundary anywhere. These LFE catalogues provide insight into contemporary slow tectonic deformation on the deep extent of the Alpine Fault, which is likely to have implications for (1) understanding the state of stress of a fault prior to a large earthquake; (2) resolving the geometry of the fault at depth; (3) inferring velocity structure models; (4) seismic hazard assessment; and (5) potentially earthquake forecasting. This work provides a strong basis for further study of LFEs, slow deformation and especially slow slip in the central Southern Alps. 
Appendices 


\section{A. LFE catalogues}

\section{A.1 3-yr catalogue}

The first year of this project was spent working on a much shorter catalogue spanning 3 years, from March 2009 to April 2012. The aim of this study was to improve the overall signal-to-noise ratio of the stacks obtained by Chamberlain et al. (2014). The whole process was supposed to provide a signal clear enough to allow a reliable pick of both $\mathrm{P}$ - and S-arrival phases, and subsequent locations. The ultimate goal for processing the data differently was to obtain LFE focal mechanisms. In order to achieve this, the idea was to use the same catalogue duration and the same codes as Chamberlain et al. (2014). The main difference stemmed from the use of phase-weighted stacking (described in detail in Section 2.2.2 of Chapter 2) instead of linear stacking within iterations of the matched-filter.

We first implemented phase-weighted stacking within the Matlab codes provided by David Shelly (USGS) and used by Chamberlain et al. (2014) to generate their original LFE catalogue. Several weeks of processing time were needed to generate a full catalogue over a 3-yr study period (March 2009 - April 2012). Testing the parameters' sensitivity was laborious because of (1) the total running time; (2) the lack of possibility to change parameters and (3) the impossibility to check the stacks between iterations. We used phase-weighted stacking for all iterations of the matched-filter routine. We obtained a catalogue of $\sim 8,000$ events. We plotted the cumulative detections of all 14 families and found out that increases in LFE rates (corresponding to steps in Fig. A.1) coincided with tremor and/or large regional earthquakes.

However, even though our final phase-weighted stacks were more impulsive and less noisy than the linear stacks of the original catalogue of Chamberlain et al. (2014) as seen in Fig. A.2, we observed that the majority of stacks were not coherent across all stations and channels (e.g. Family ID:552200 in Fig. A.3) as well as between iterations (Fig. A.4). An hypothesis for the lack of coherence between iterations and stations is that a high number of false detections might have been included in the stacks, thus resulting in poor quality stacks (as was the case for Chamberlain et al. (2014)'s catalogue). This issue has been overcome in the 
extended catalogue by including more data (longer duration catalogue).

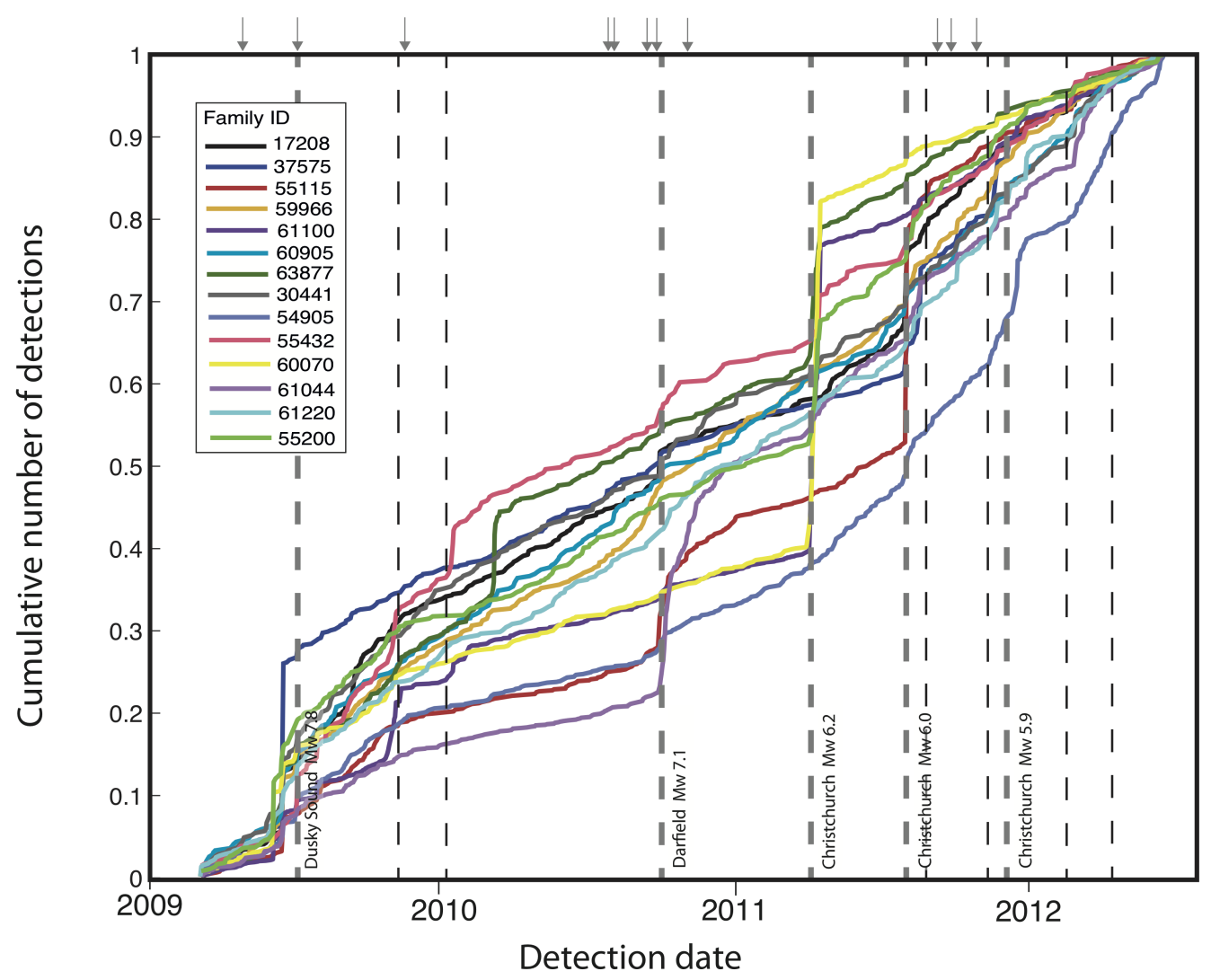

Figure A.1: 3-yr catalogue normalised cumulative detections for all 14

families. Grey arrows correspond to tremor periods identified by Wech et al. (2012). Grey dashed lines correspond to large regional earthquakes $(\mathrm{M}>6)$ and black dashed lines corresponds to regional earthquake $(\mathrm{M}>5)$. 


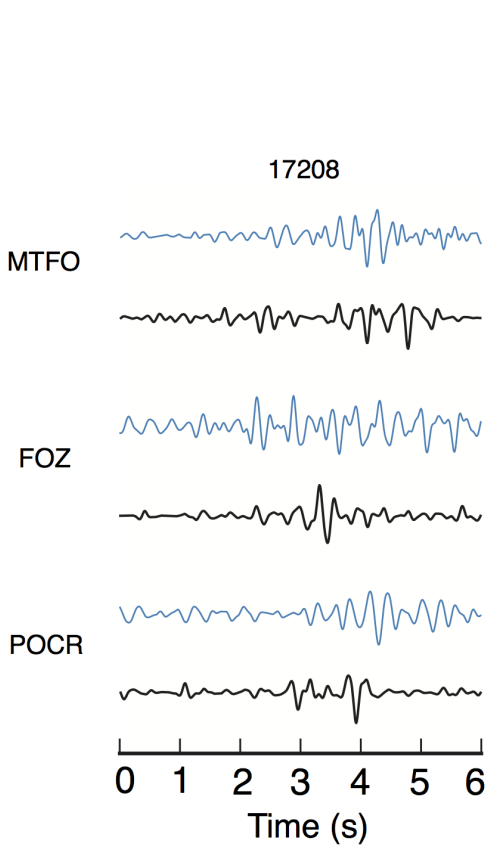

59966

MTFO

FOZ

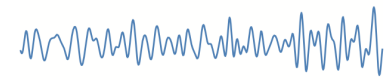

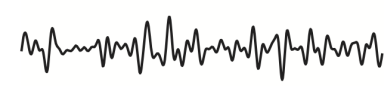

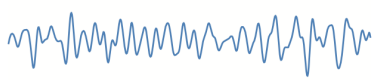

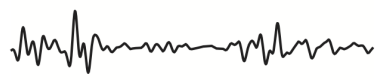
POCR

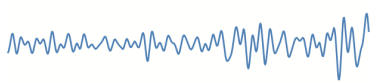

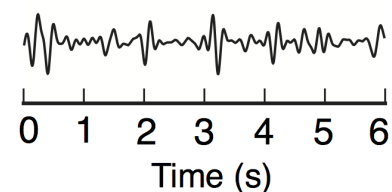

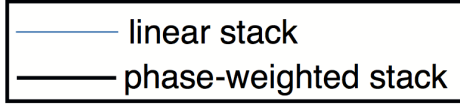

phase-weighted stack

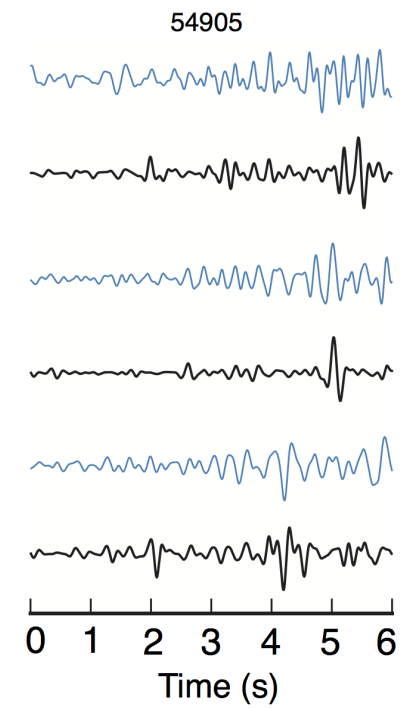

60905

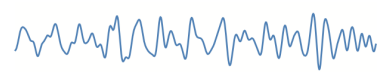

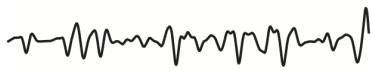

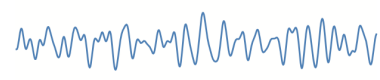

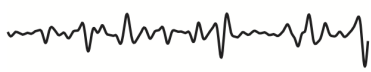

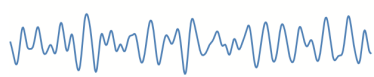

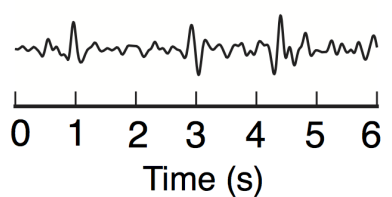

55115

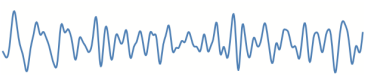

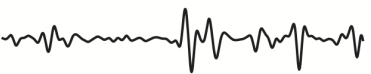

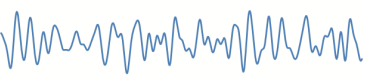

numpupphompunsum

wompropproms

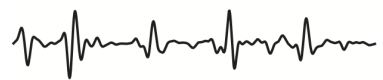

\begin{tabular}{llllllll}
\hline 0 & 1 & 2 & 3 & 4 & 5 & 6
\end{tabular}

Time (s)

63877

monumblywown

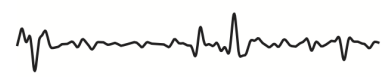

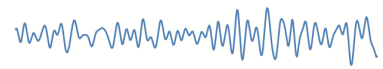

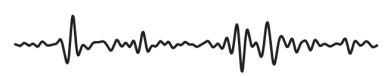

monuming phom

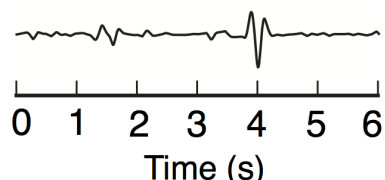

Figure A.2: 3-yr catalogue final stacks for 6 LFE families. Comparison of the final stacks for family IDs:17208, 54905, 55115, 59966, 60905 and 63877. The vertical component is plotted for 3 stations (MTFO, FOZ and POCR) with the phase-weighted stacks obtained in this study in black versus the linear stacks of Chamberlain et al. (2014) in blue. We can see here that the phase-weighted stacks provides clearer arrivals than the linear stacks and some coherent arrivals can be seen across stations. However a minority of stations in the stacks exhibited this behaviour.

We managed to pick some $\mathrm{S}$ arrivals and a few $\mathrm{P}$ arrivals on all templates. However, when locating them, the uncertainties in the picks were too big for the lo- 

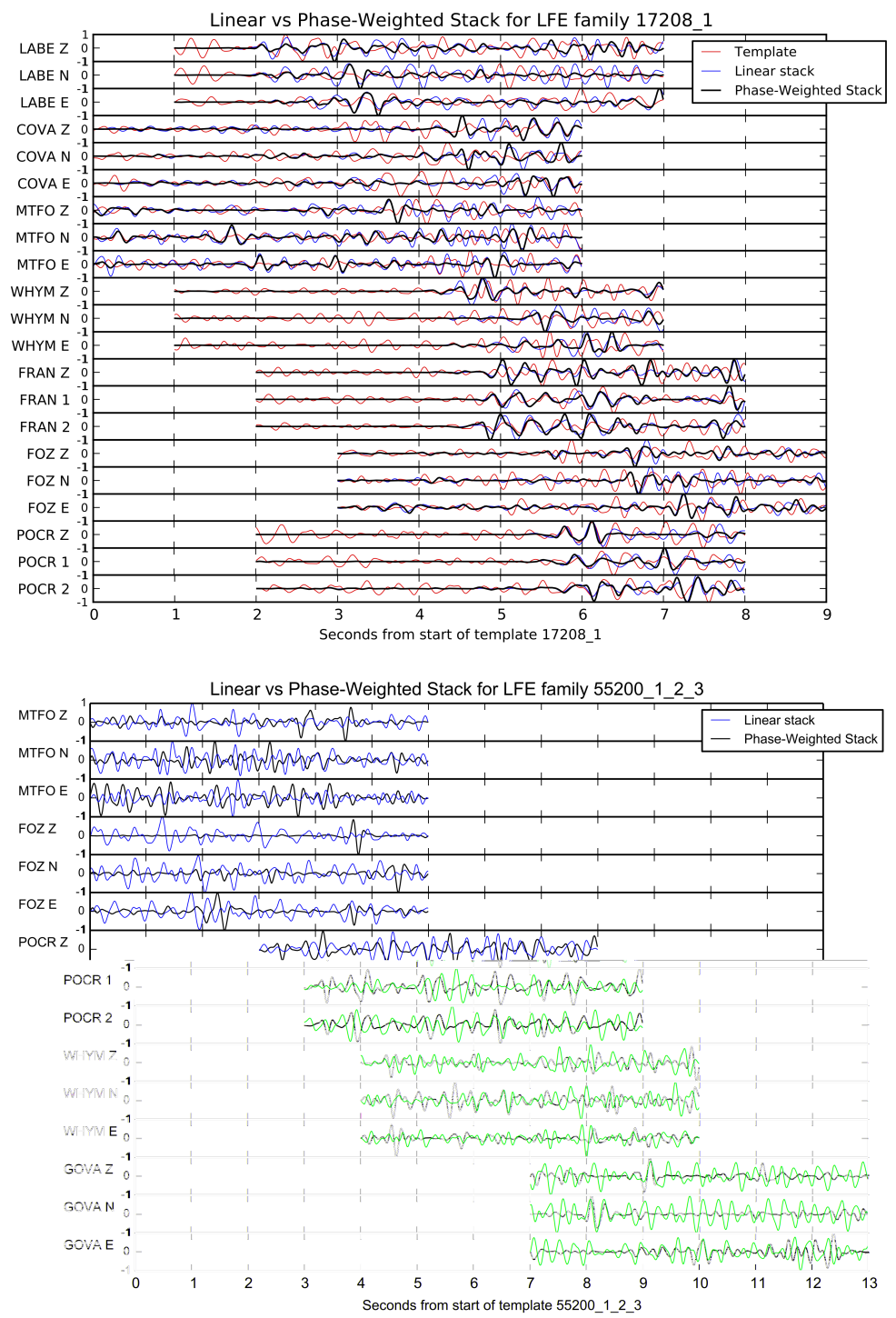

Figure A.3: 3-yr catalogue stacks for family IDs:17208 and 55115. Top: Comparison of the initial template (in red) and the final stacks for family ID:17208, with the phase-weighted stacks of this study in black versus the linear stacks of Chamberlain et al. (2014) in blue. This family shows coherence across stations and channels, but was one of the few families to do so. Bottom: Comparison of the final stacks for family ID:55200, with the phase-weighted stacks of this study in black versus the linear stacks of Chamberlain et al. (2014) in blue. We can see here, e.g. at the GOVA station, that the linear stacks are slightly coherent between channels. However, the phase-weighted stacks shows no coherence across channels for this station. The majority of stacks behaved similarly to this family in the 3-yr catalogue. The initial template has not been plotted here for clarity purposes. 


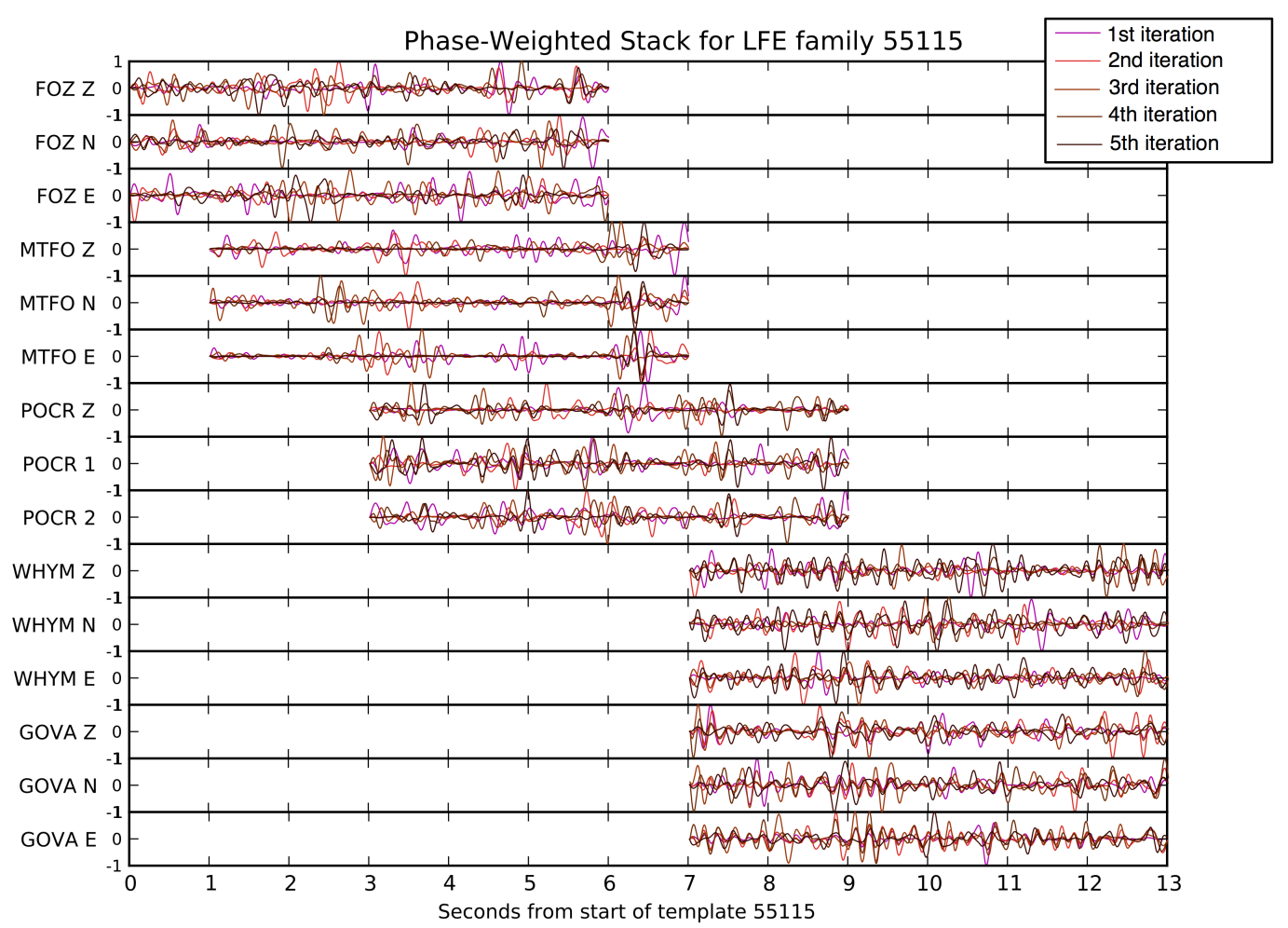

Figure A.4: 3-yr catalogue stacks for family ID:55115. Comparison of the stacks for family ID:55115 for all 5 iterations of the matched-filter routine. We can see here that there is no real coherence between the stacks of consecutive iterations. We do not see the stack converging towards a clearer phase arrival. A phase-shift seems to be occurring as well (e.g. MTFO station).

cations to be reliable (Fig. A.5). Because of these issues, arrival times (especially P-waves arrival times) and polarities could not be picked reliably on most stations and we did not manage to achieve our goals of obtaining accurate locations and reliable focal mechanisms.

We decided to (1) change the way we processed the data (by using phase-weighted stacking only in the last iteration of the matched-filter routine instead of for all iterations); (2) to use different codes (detailed in Chapter 2, Section 2.2.4); as well as to (3) include the entire dataset (made possible with the use of a supercluster and parallelisation tools) and thus generated the extended LFE catalogue. 


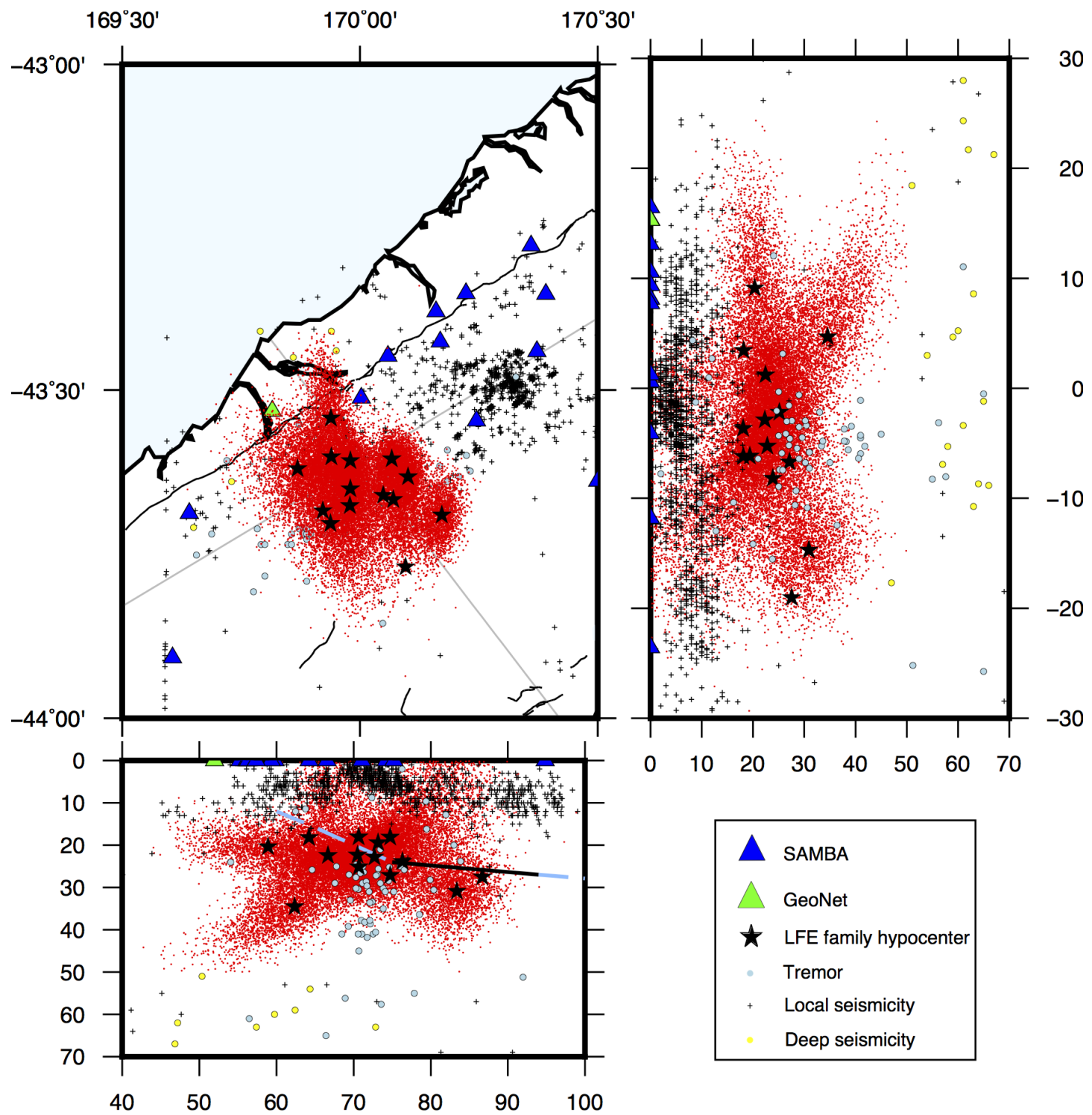

Figure A.5: 3-yr catalogue location map. Locations are computed with a 1D velocity model and the NonLinLoc software of Lomax et al. (2000). Uncertainties are plotted in red. The uncertainties for those locations were too significant for the locations to be deemed reliable. 


\section{A.2 Extended catalogue}

We present in this section the complementary material to Chapter 3.

\section{A.2.1 Waveforms}

Here we show the final waveforms for the $14 \mathrm{LFE}$ families presented in Chap. 3. The waveforms shown below (Figs. A.6 - A.19) correspond to the linear stacks (in blue) and the phase-weighted stacks (in black) obtained in the final iteration of the matched-filter for each LFE family. The linear stacks are downsampled to $20 \mathrm{~Hz}$ and normalised. The phase-weighted stacks are sampled at $100 \mathrm{~Hz}$ and normalised. Only the North component is shown for clarity. 


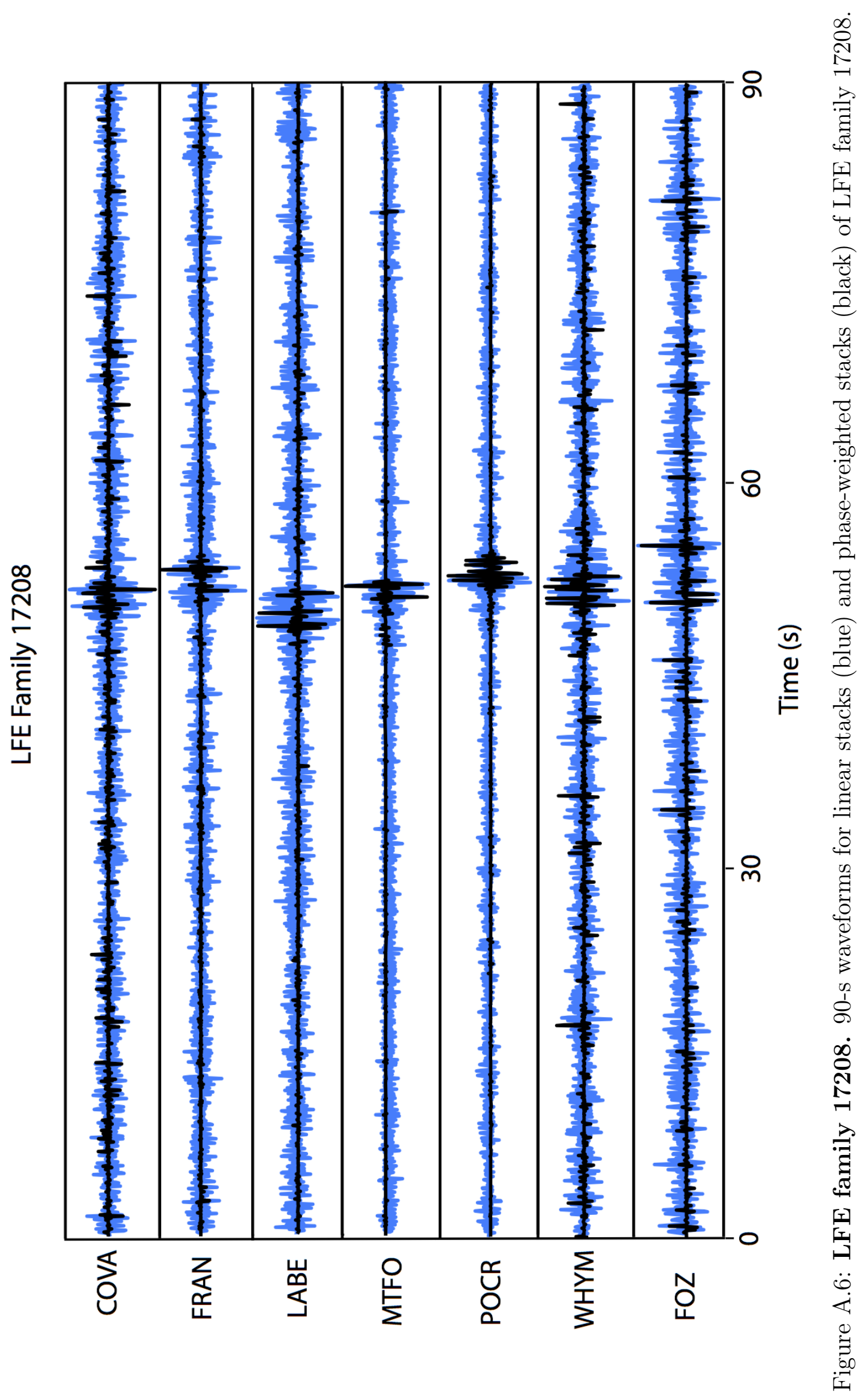




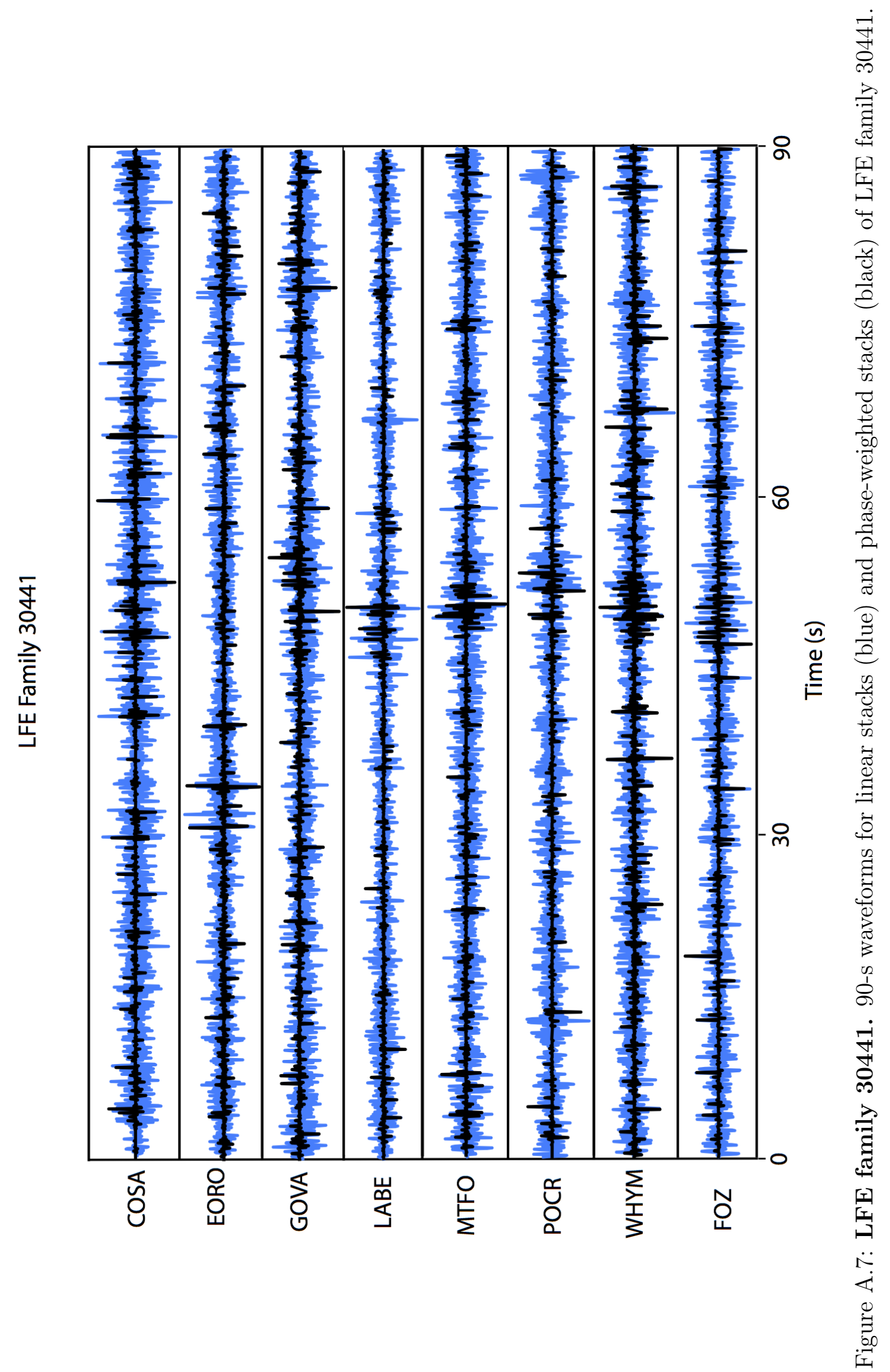




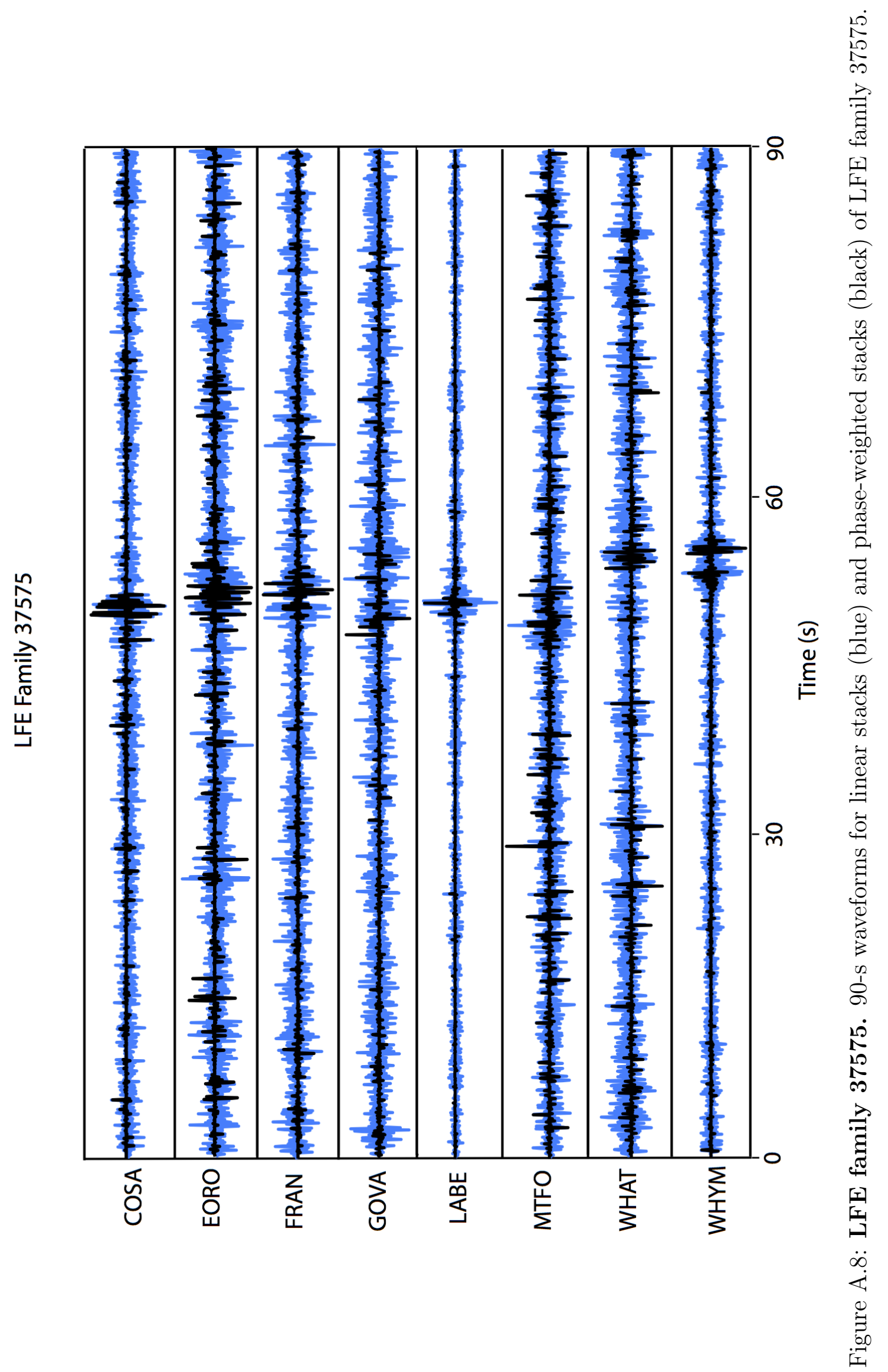




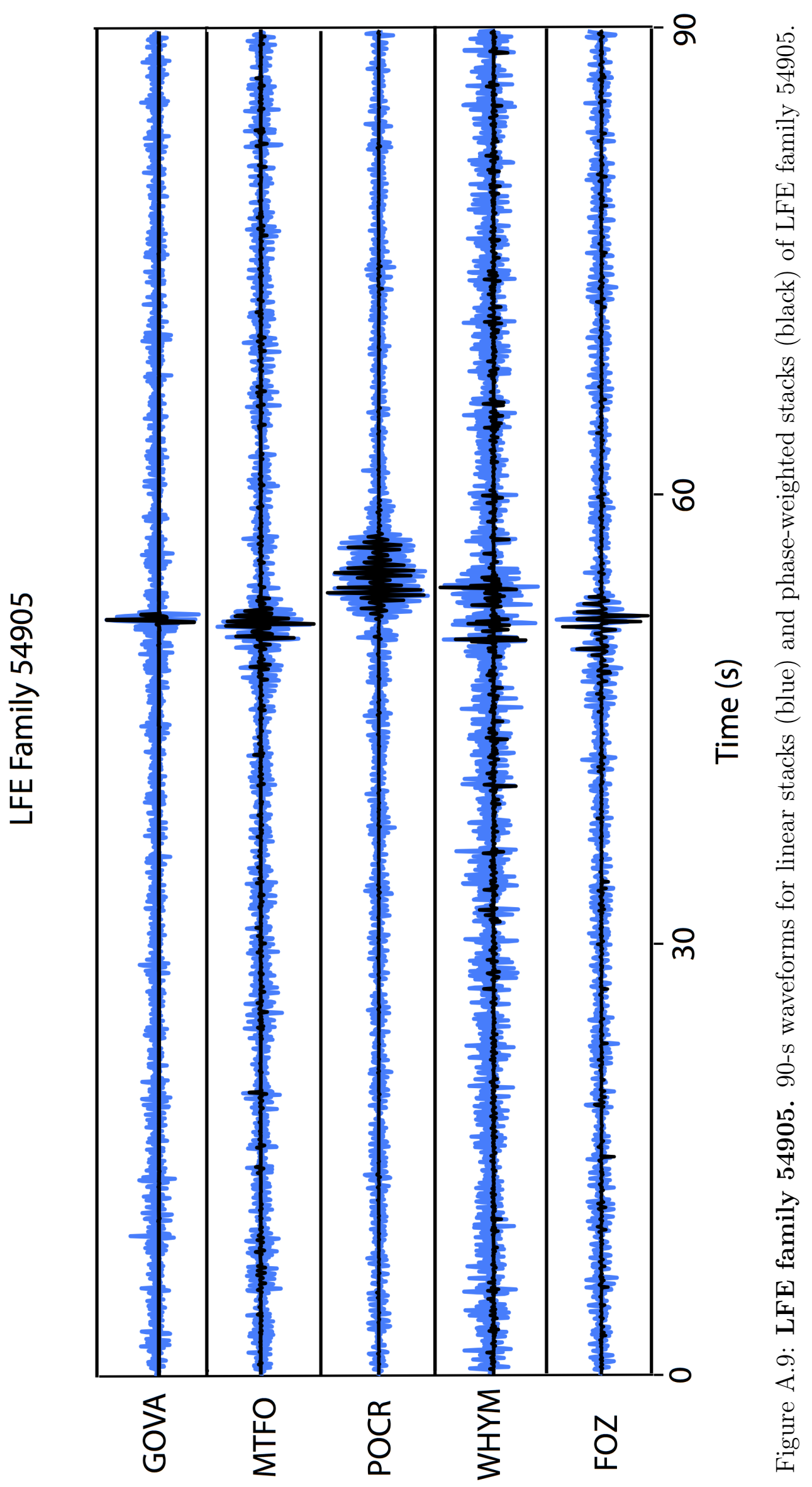




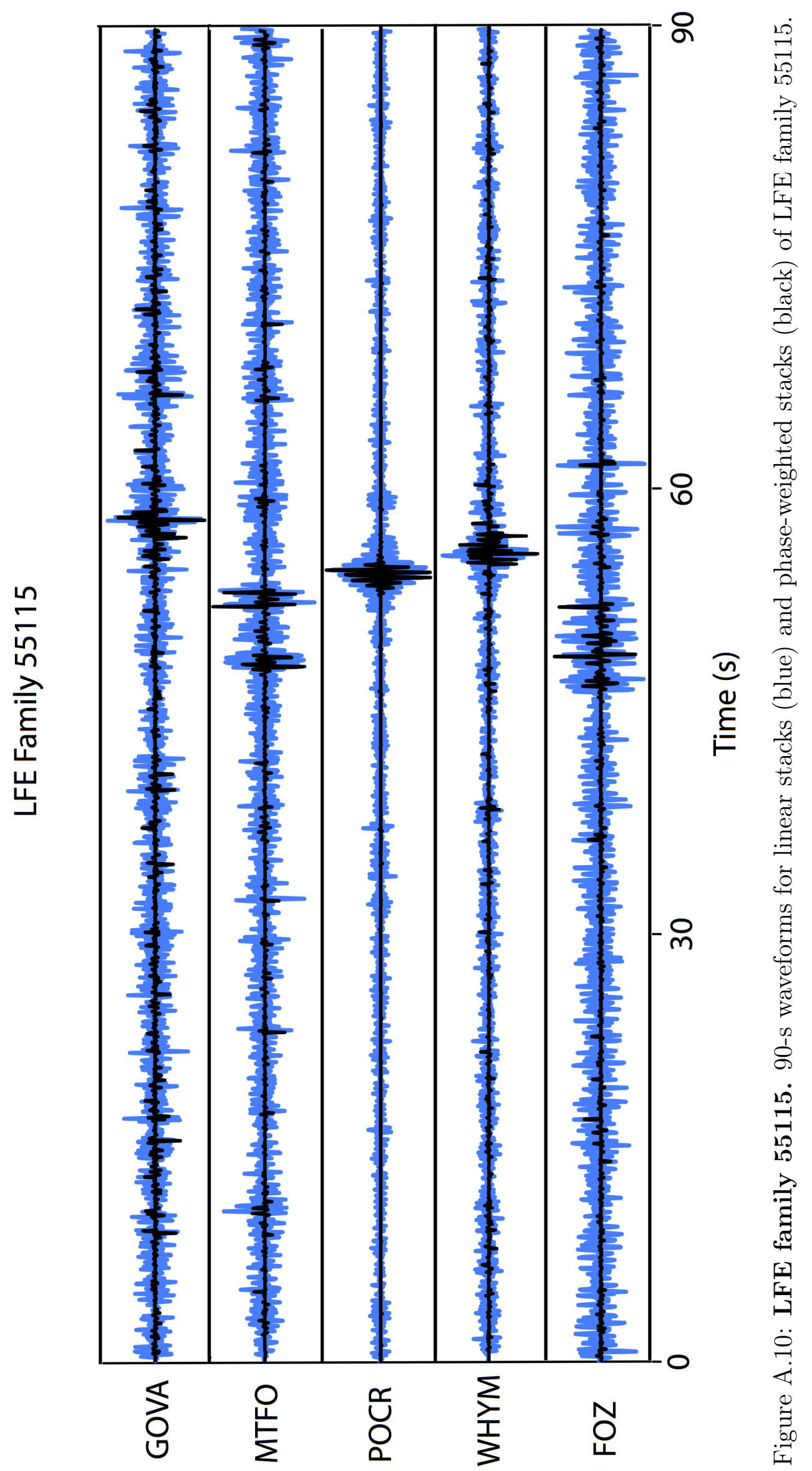




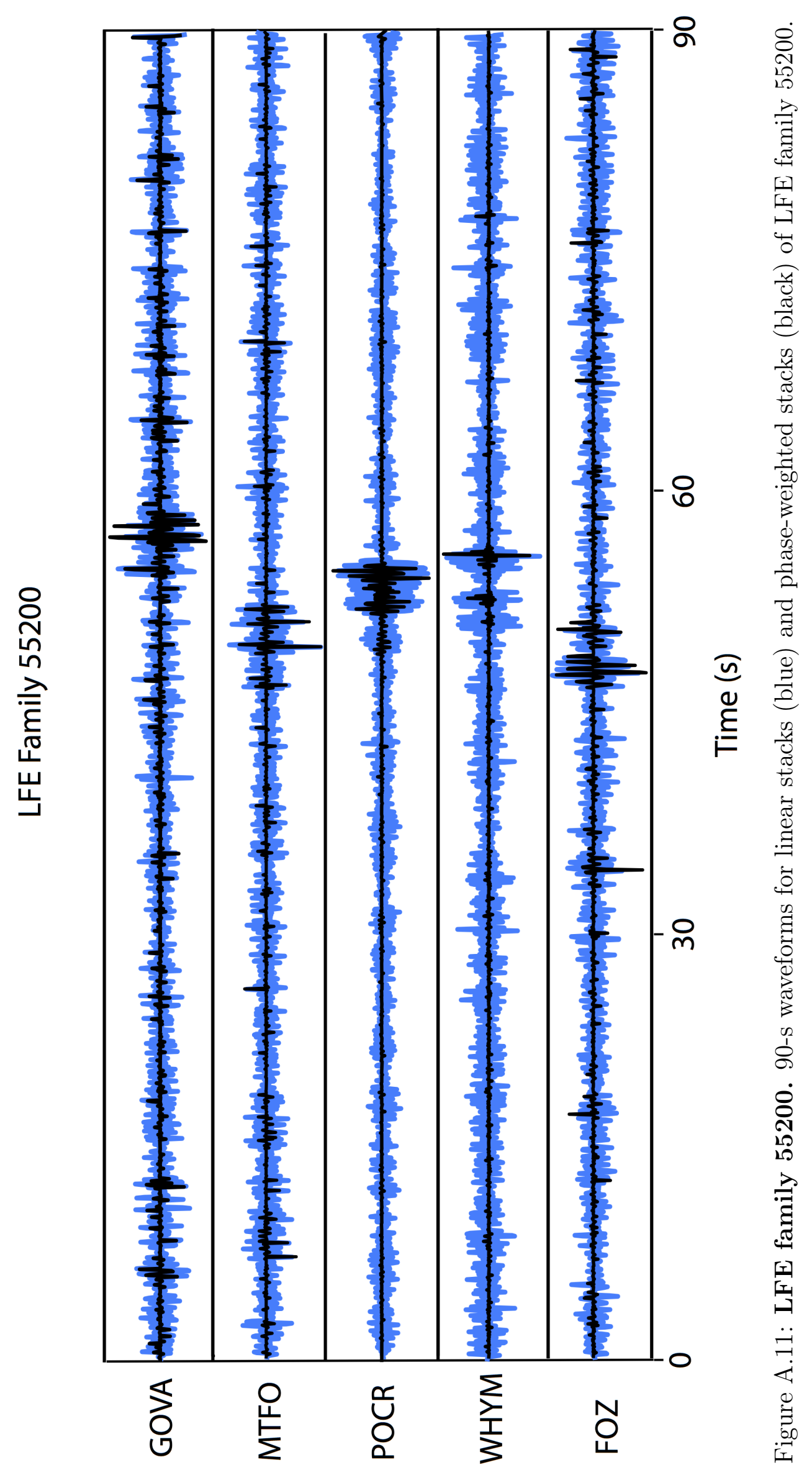




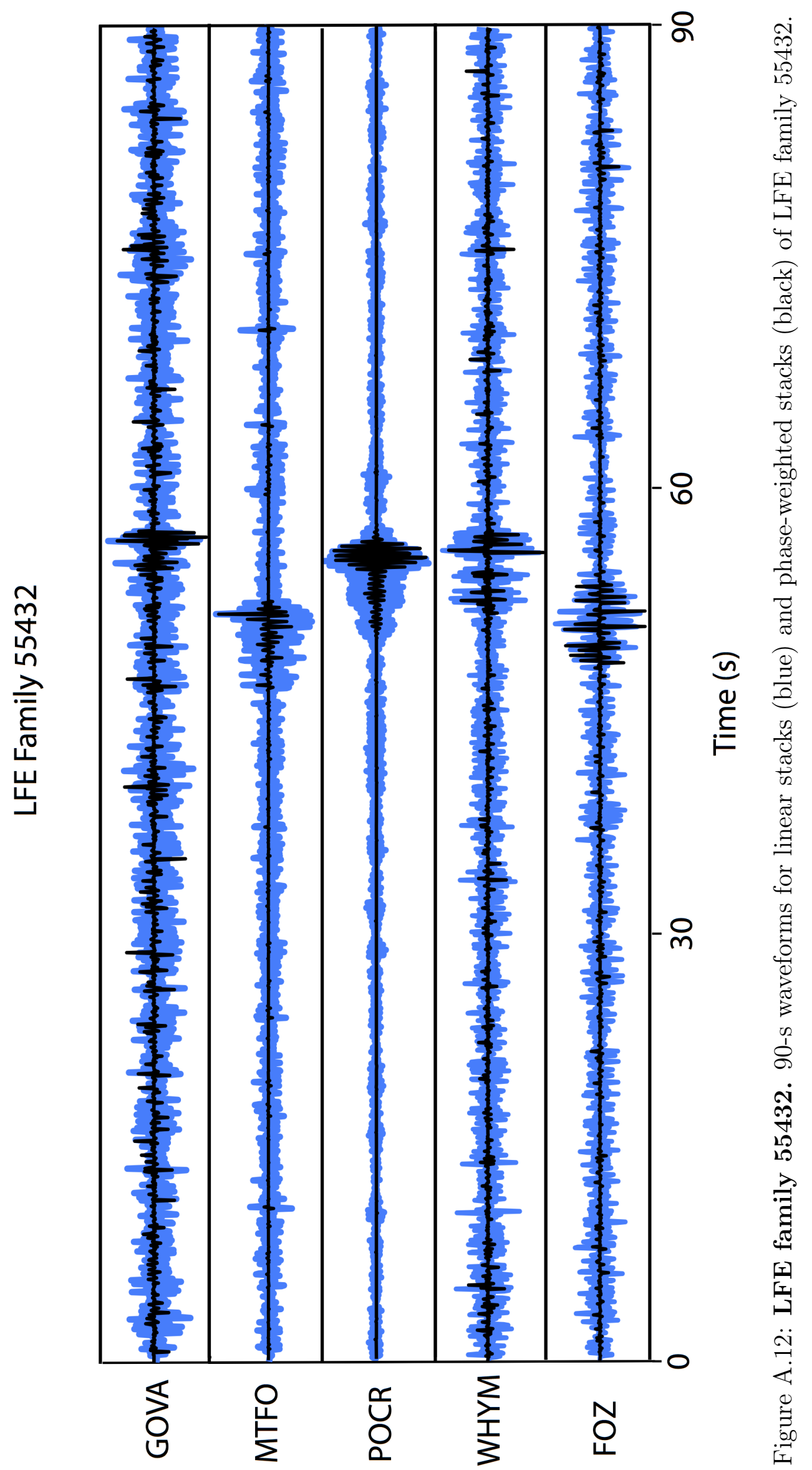




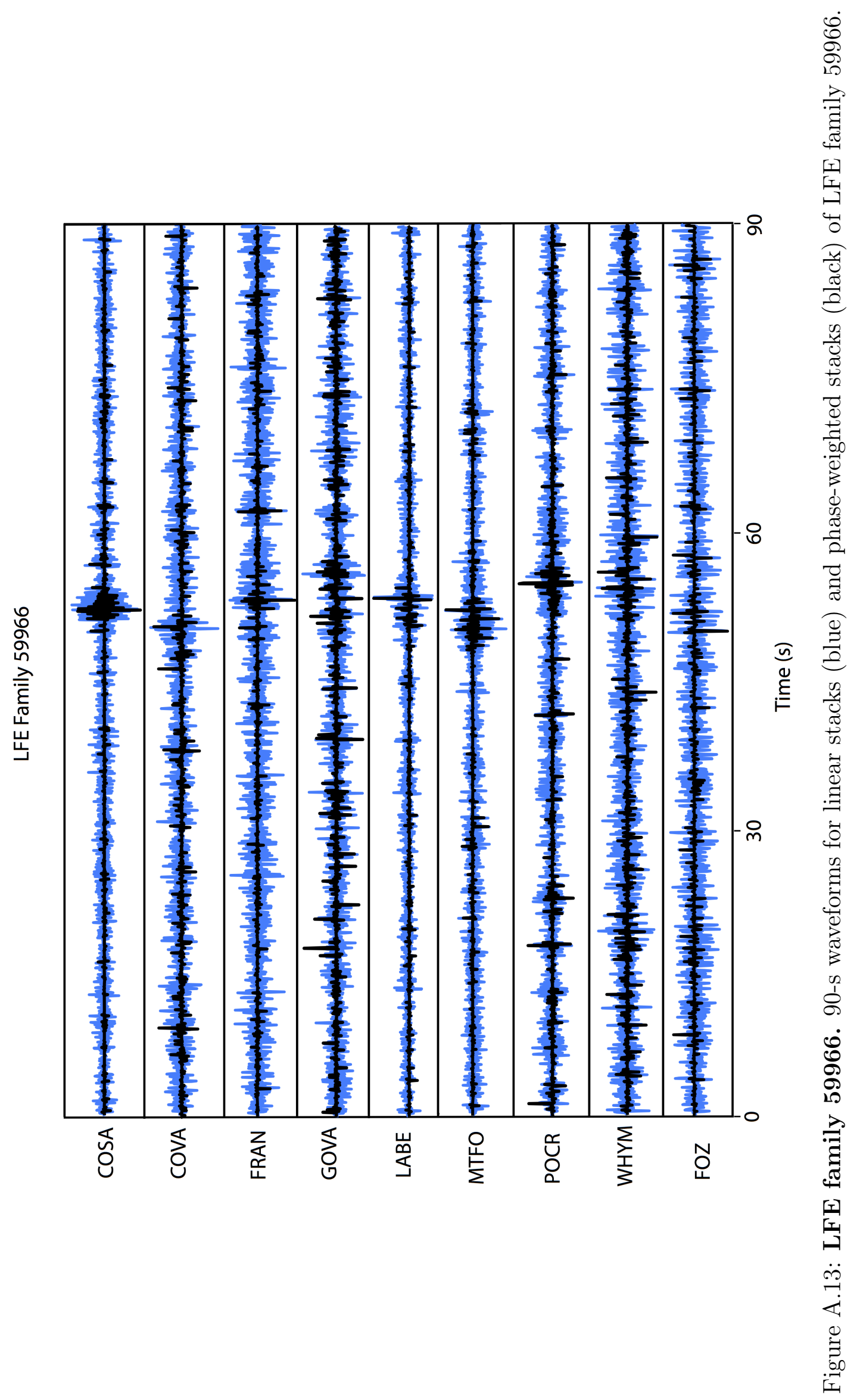




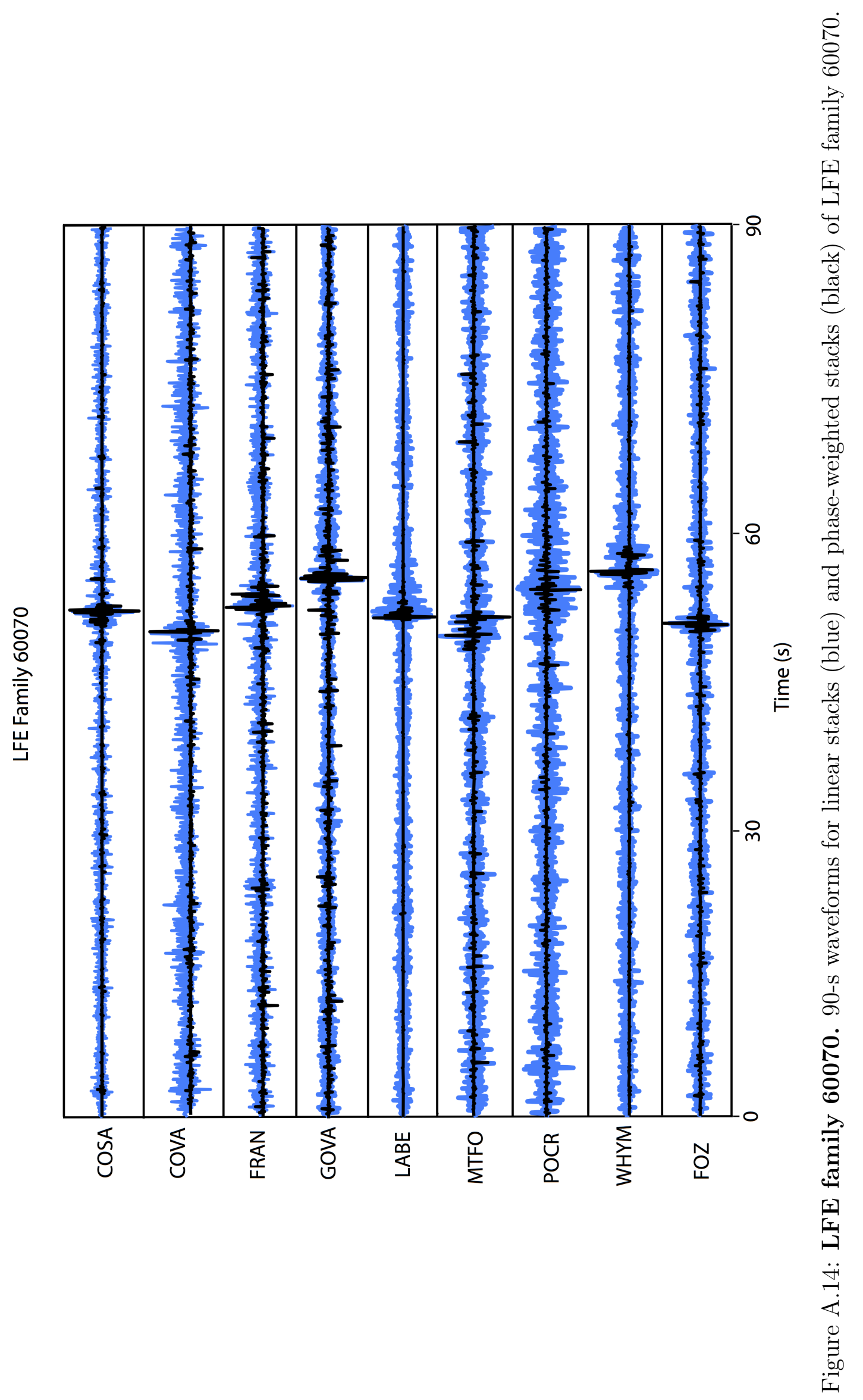




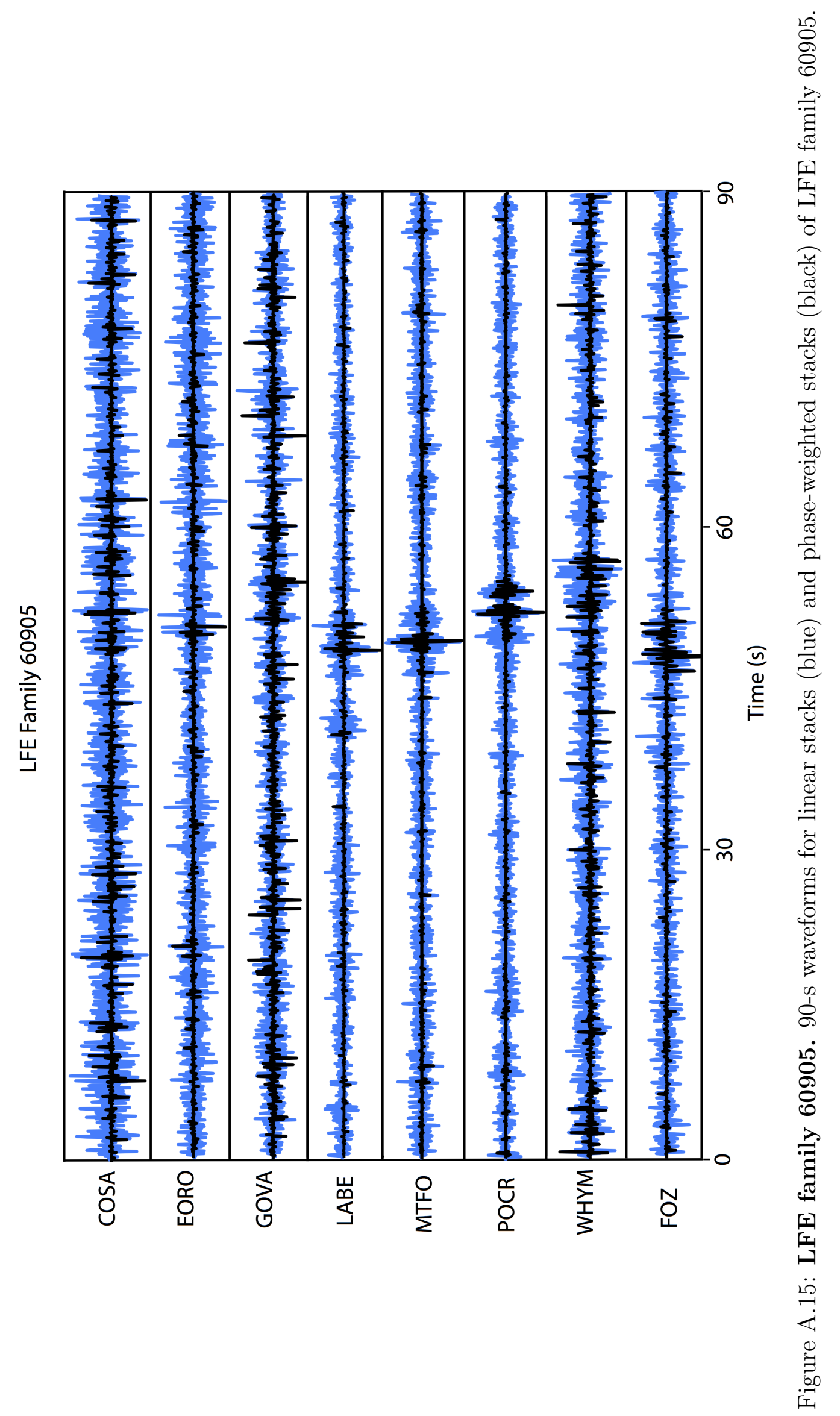




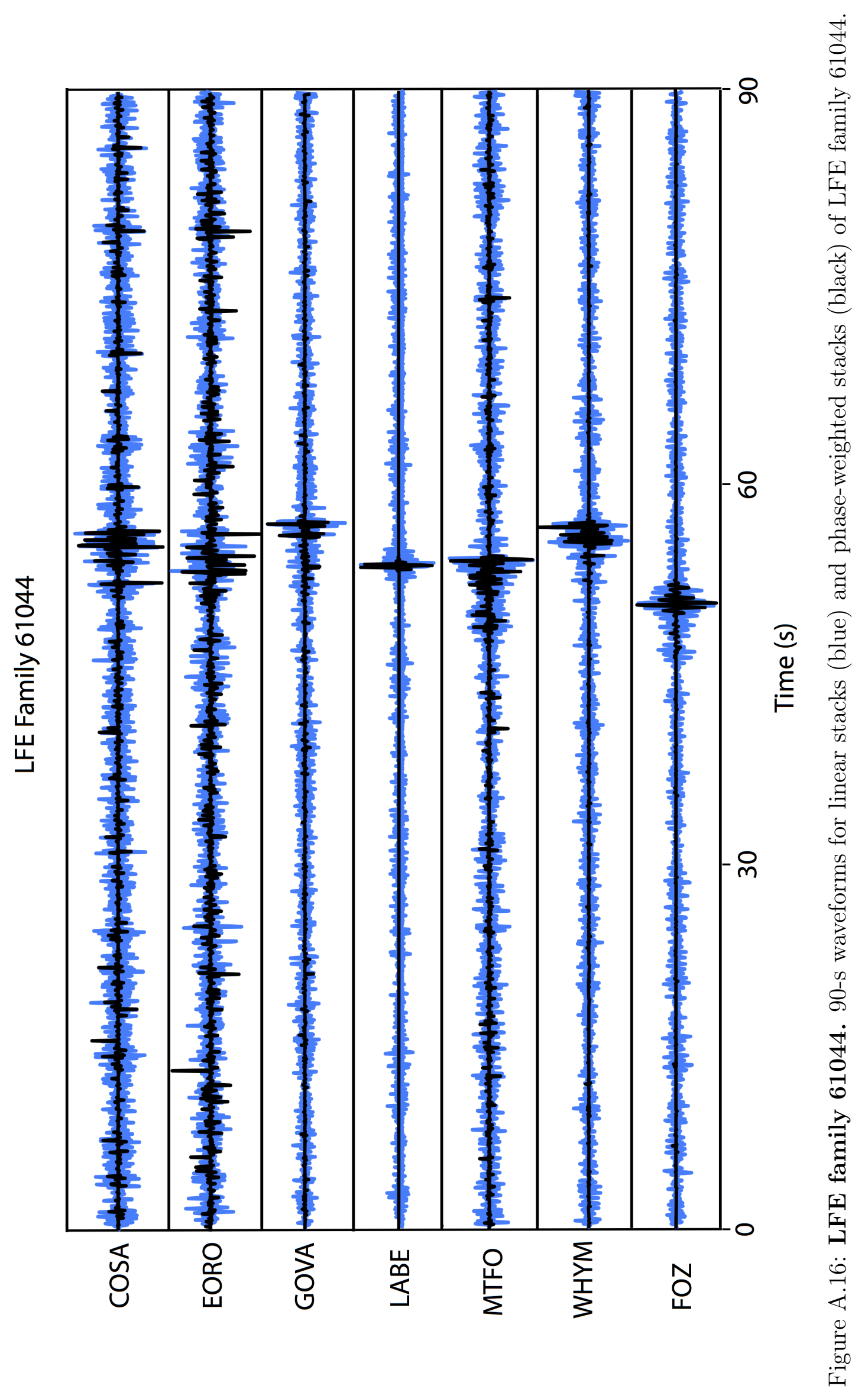




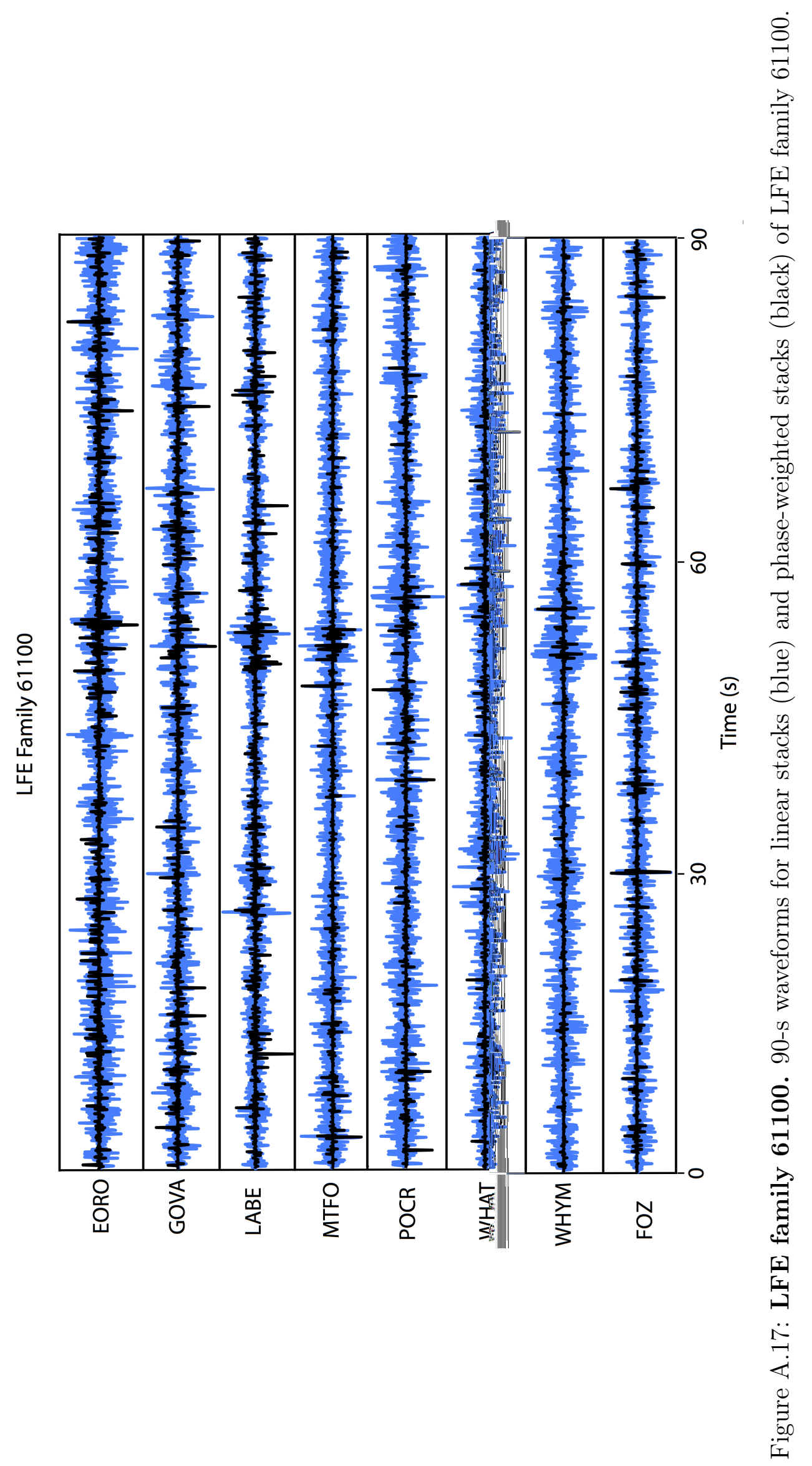




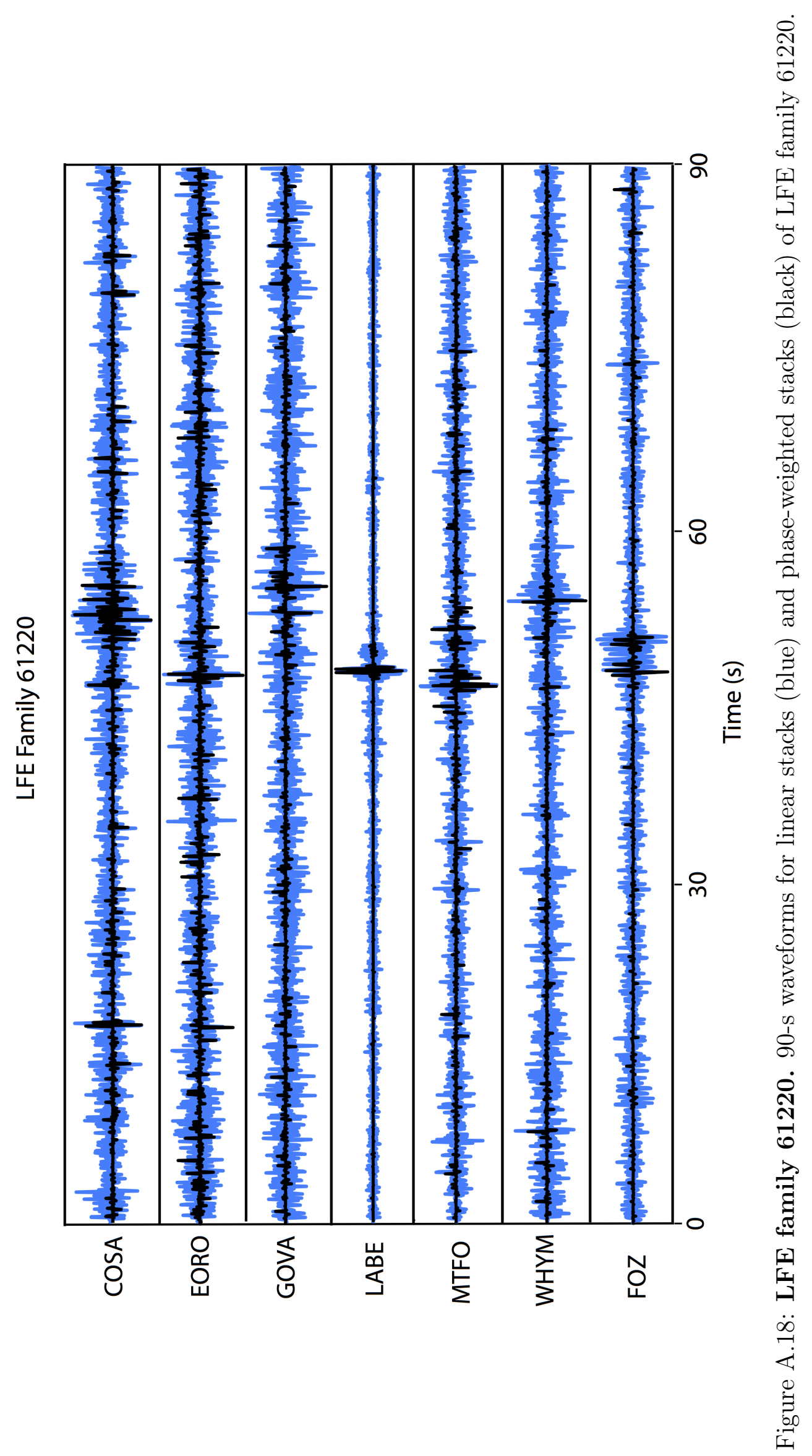




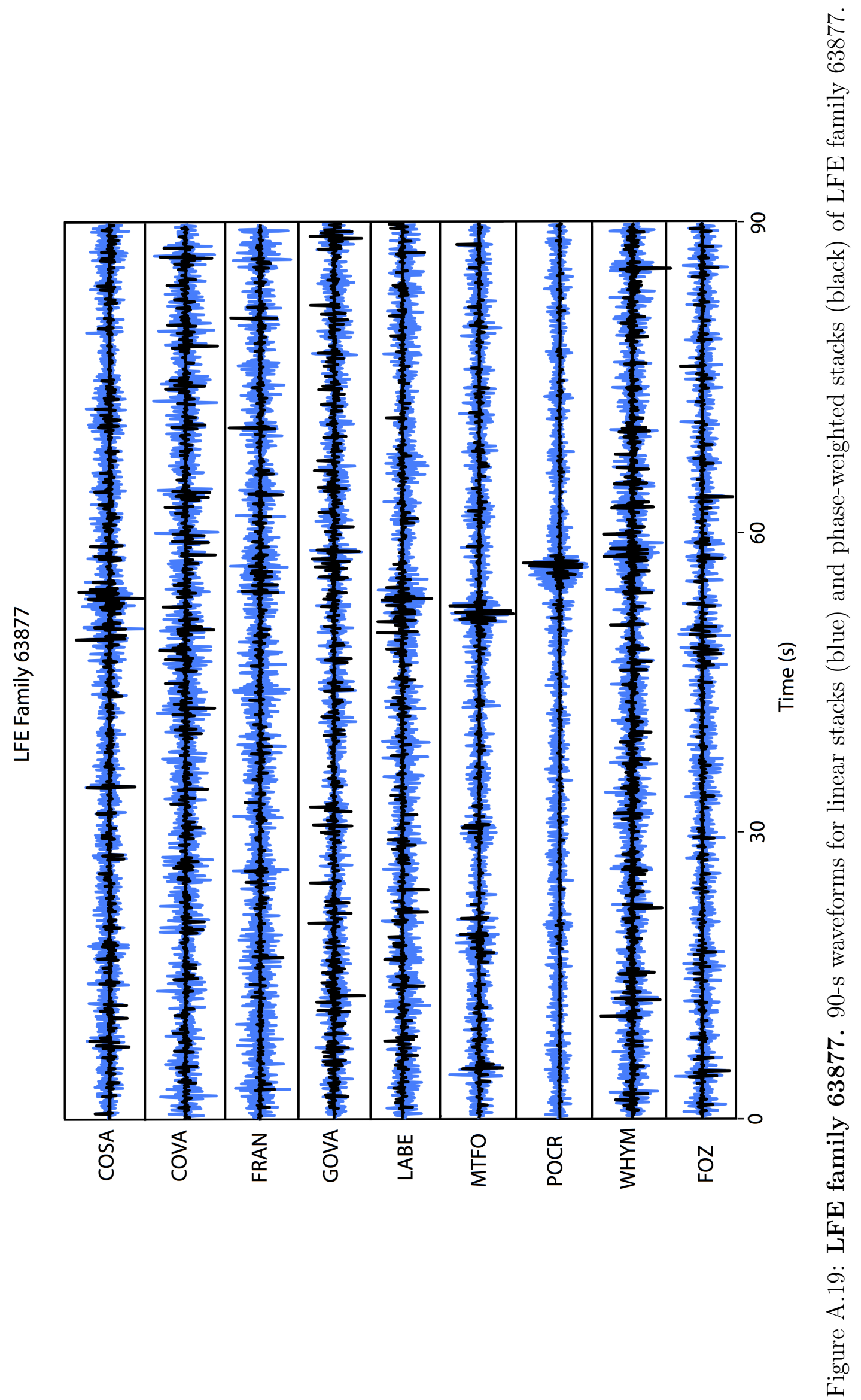




\section{A.2.2 Focal mechanisms}

Out of the $14 \mathrm{LFE}$ families obtained with this catalogue, we generated reliable focal mechanisms for 8 . We however computed the focal mechanisms solutions for all 14 families. The remaining 6 solutions either had poor coverage on the focal sphere (e.g. LFE family 60905 in Fig. A.20) and/or not enough polarity picks (e.g. LFE family 54905 in Fig. A.20). The focal mechanisms solutions for those 6 families can be seen in Fig. A.20. 

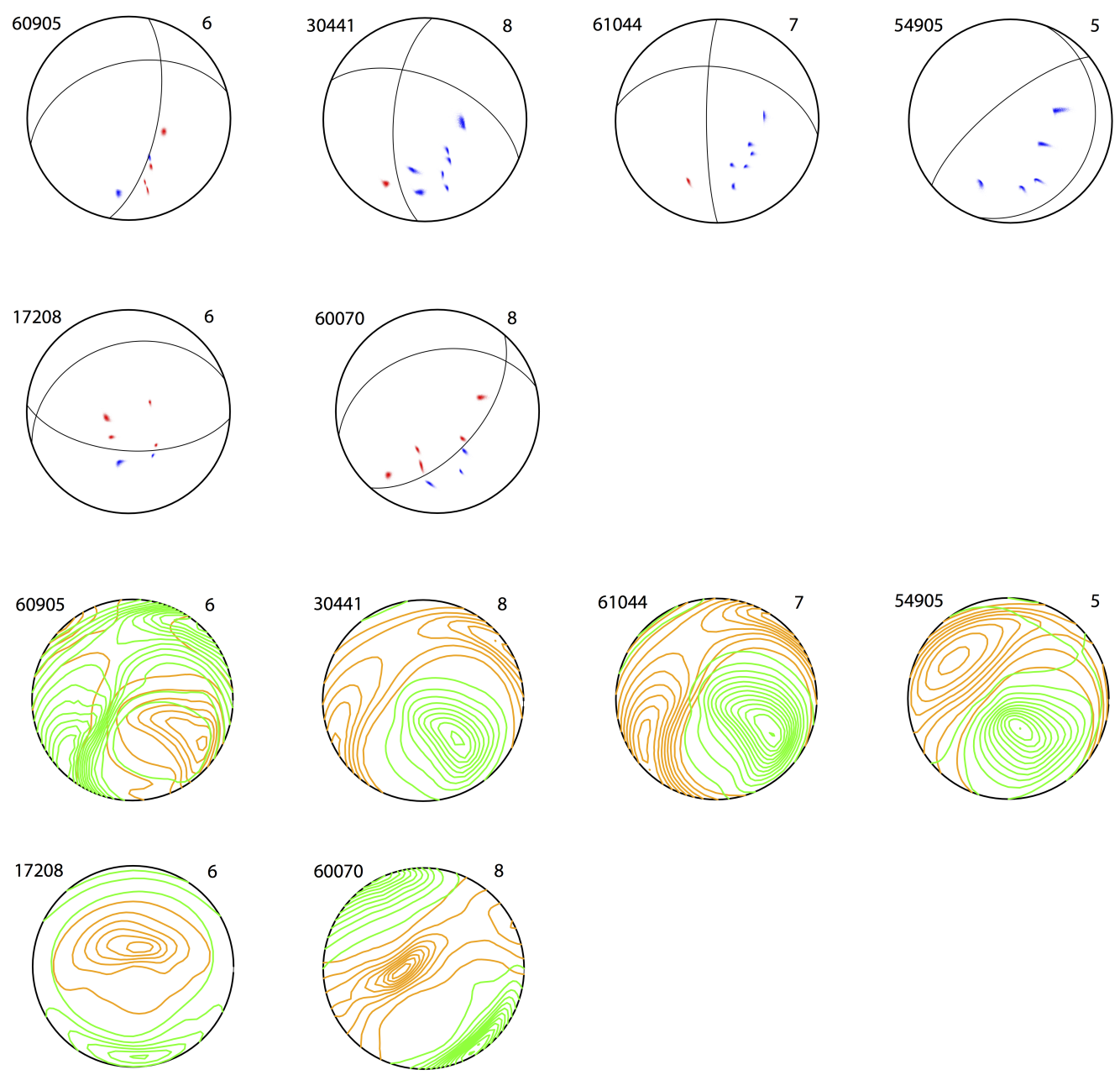

Figure A.20: Focal mechanisms for the remaining 6 LFE families. The number on the left side of the mechanism solution corresponds to the ID of the LFE family. The number on the right side of the mechanism solution represents the number of polarity picks used in the computation. Top: P-wave polarities and focal mechanisms solutions for our 6 LFE families. Bottom: Probability density contours of P- (orange) and T-axes (green) of the focal mechanism solution for our 6 LFE families. Those focal mechanisms were discarded either because of the paucity of available polarity picks or because of the poor coverage of the focal sphere. 


\section{A.3 Continuous catalogue}

We present in this section the complementary material to Chapters 4 and 5 .

\section{A.3.1 Waveforms}

Below are two examples of waveforms from LFE families that either had (1) numerous detections, but that we still could not reliably locate (Fig.A.21); or (2) few detections, but that we managed to reliably locate (Fig.A.22). 


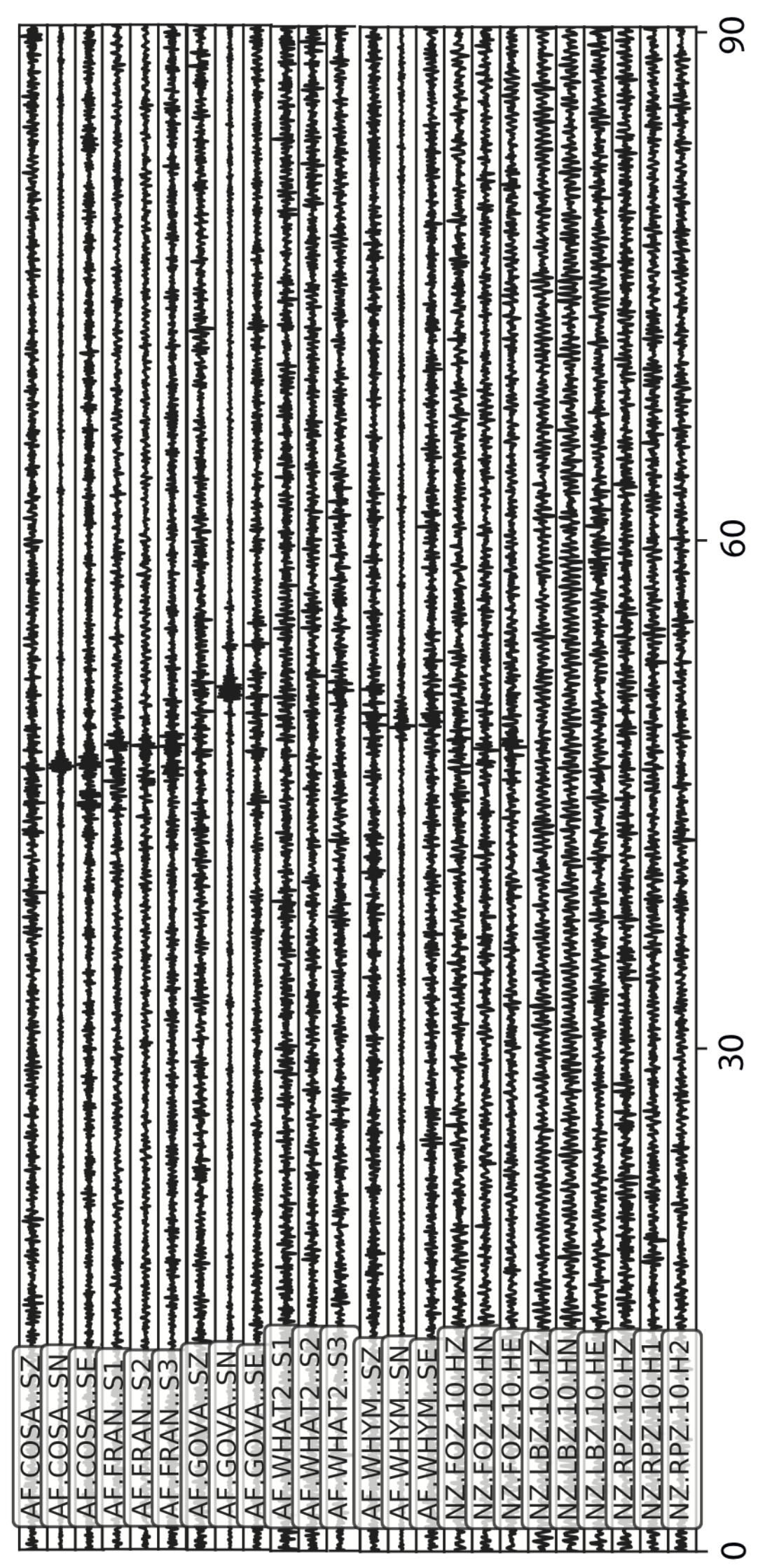

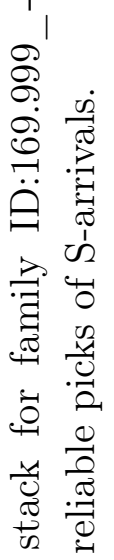

$$
\begin{aligned}
& \text { 苞范 } \\
& \therefore \stackrel{\circ}{\circ}
\end{aligned}
$$

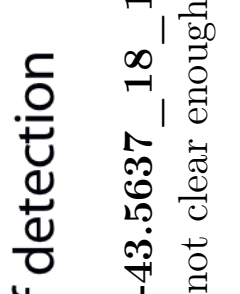

$$
\begin{aligned}
& \text { प) 1, } \\
& \text { (1) g } \\
& \text { E } \\
& \text { ह } \ddot{\theta} \stackrel{n}{.} \\
& \text { 늘 } \\
& \text { 으 릴 } \\
& \text { 일 ๘ } \\
& \text { ज } \\
& \text { 苍岁 } \\
& \text { ซี ڤ్ } \\
& \because \quad 0 \\
& 4 \\
& \text { 를 }
\end{aligned}
$$

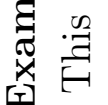

$$
\begin{aligned}
& \ddot{\sim} \stackrel{\sim}{\sim} \\
& \text { « }
\end{aligned}
$$

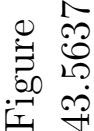




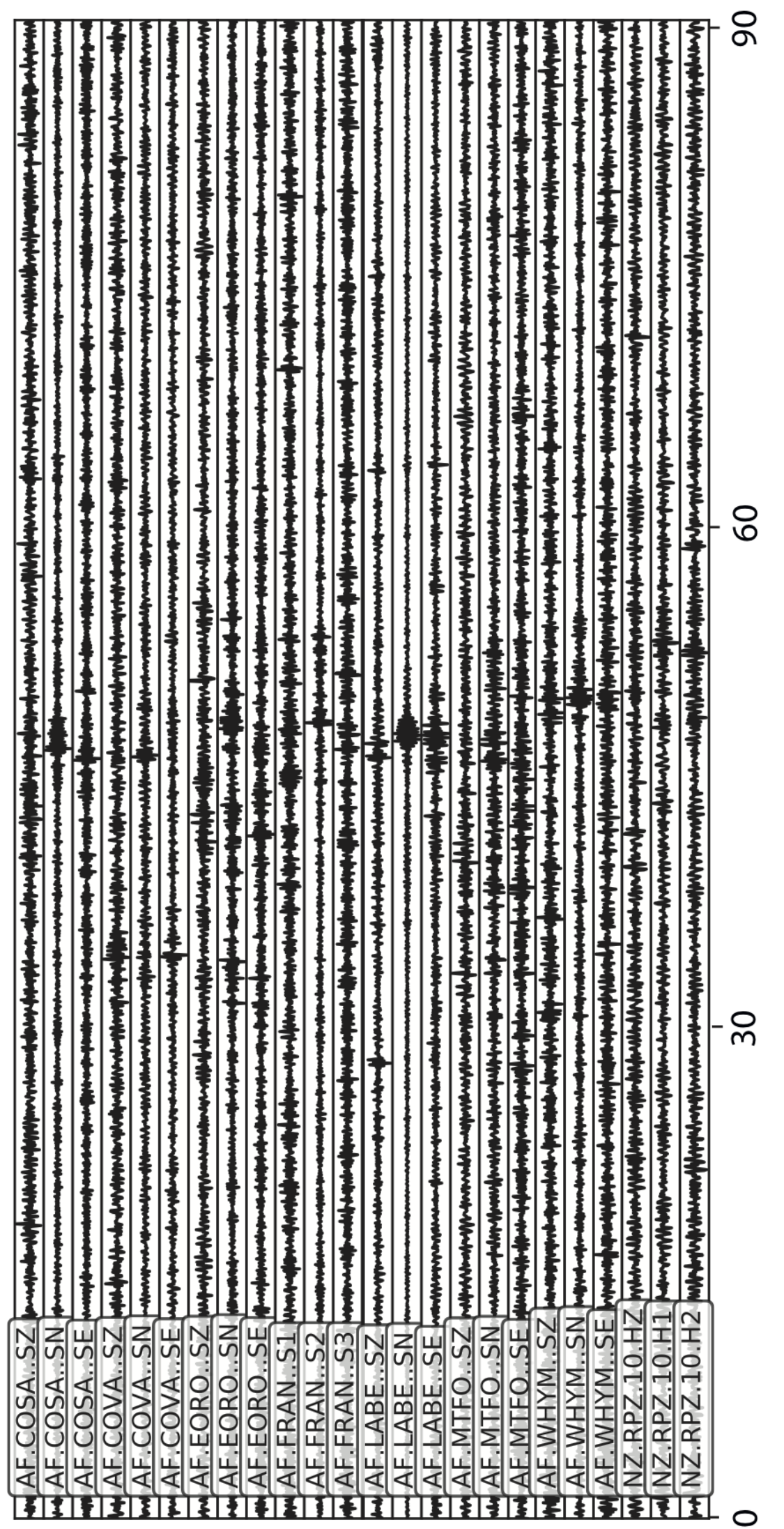

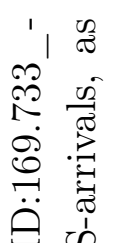

$\Rightarrow \overrightarrow{0}$

灵

宅

幽

急荨

. 웅

$1 \frac{9}{80}$

ำ

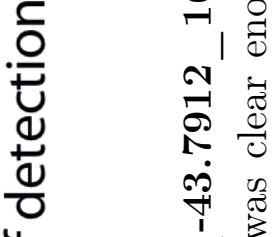

पे

\&

ह

$\varepsilon \quad 0^{0}$.

은 $\ddot{\theta}$

능 흘

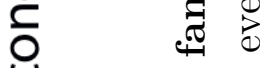

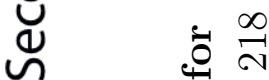

$+\frac{1}{0}$

匍

is

Ðే

4

올

藏

.. 다

స

\& 1

$\because \stackrel{0}{0}$

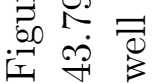




\section{A.3.2 Locations}

The preliminary locations for the continuous LFE catalogue were obtained with the SEISAN software (Havskov and Ottemoller, 1999). Here, we first show examples of picks made on the LFE stacked waveforms. Then, we show an example of how we visualised the preliminary locations using Google Earth.

Fig. A.23 gives a clear example of how the P- and S- picks were made. This figure shows a zoomed version of the manual $\mathrm{P}$ - and S-picks made in SEISAN, at the EORO station for all three channels for LFE family ID:169.837_-43.6483_36_1. We managed to pick at least one P-pick and 5 S-picks for each of the located families in this catalogue.

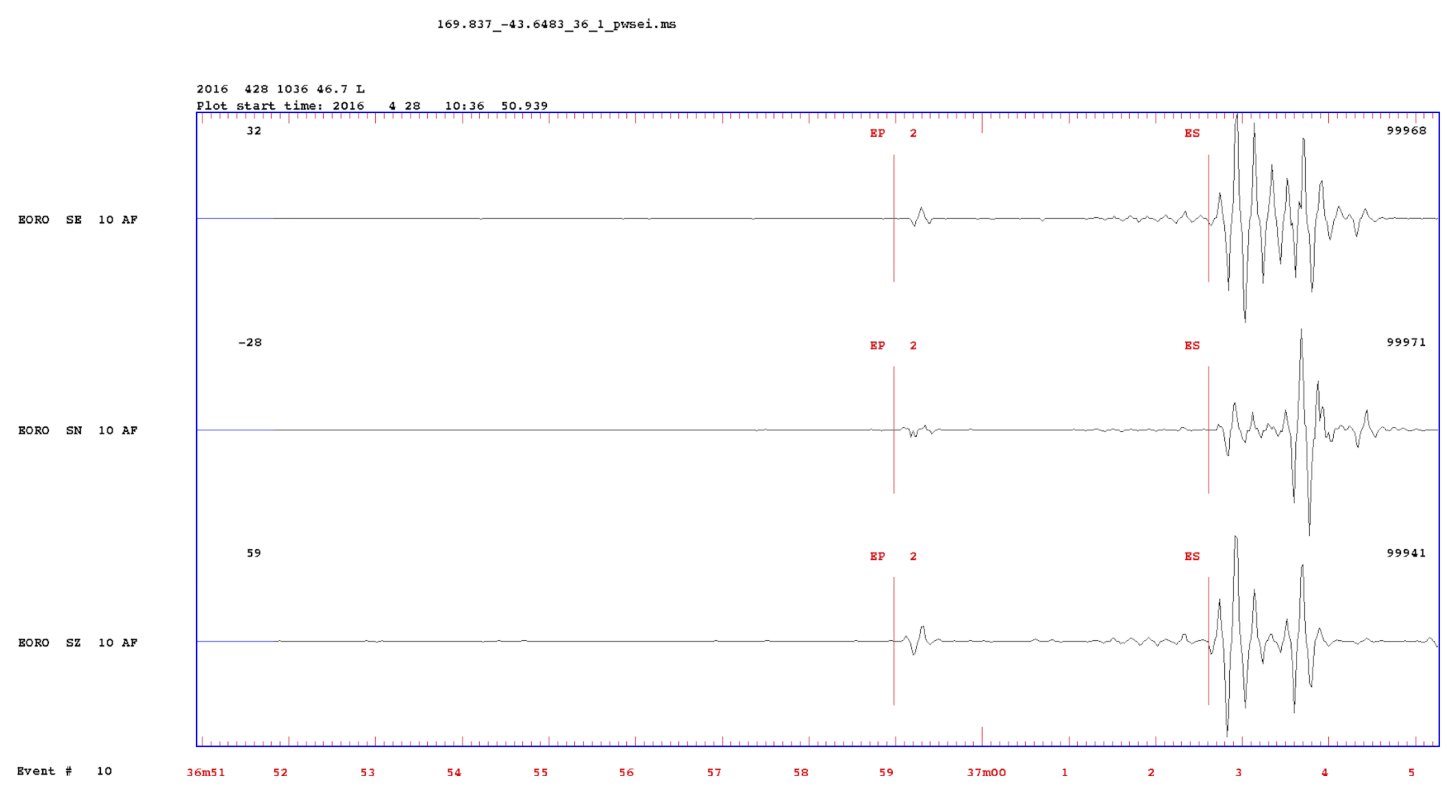

Figure A.23: Example of P- and S-picks. Screenshot from the SEISAN software (Havskov and Ottemoller, 1999) used for picking wave onsets. Here, we show a zoomed-in view of an example of P- and S-picks for LFE template 169.837_$43.6483 \_36 \_1$ at the FRAN station. 


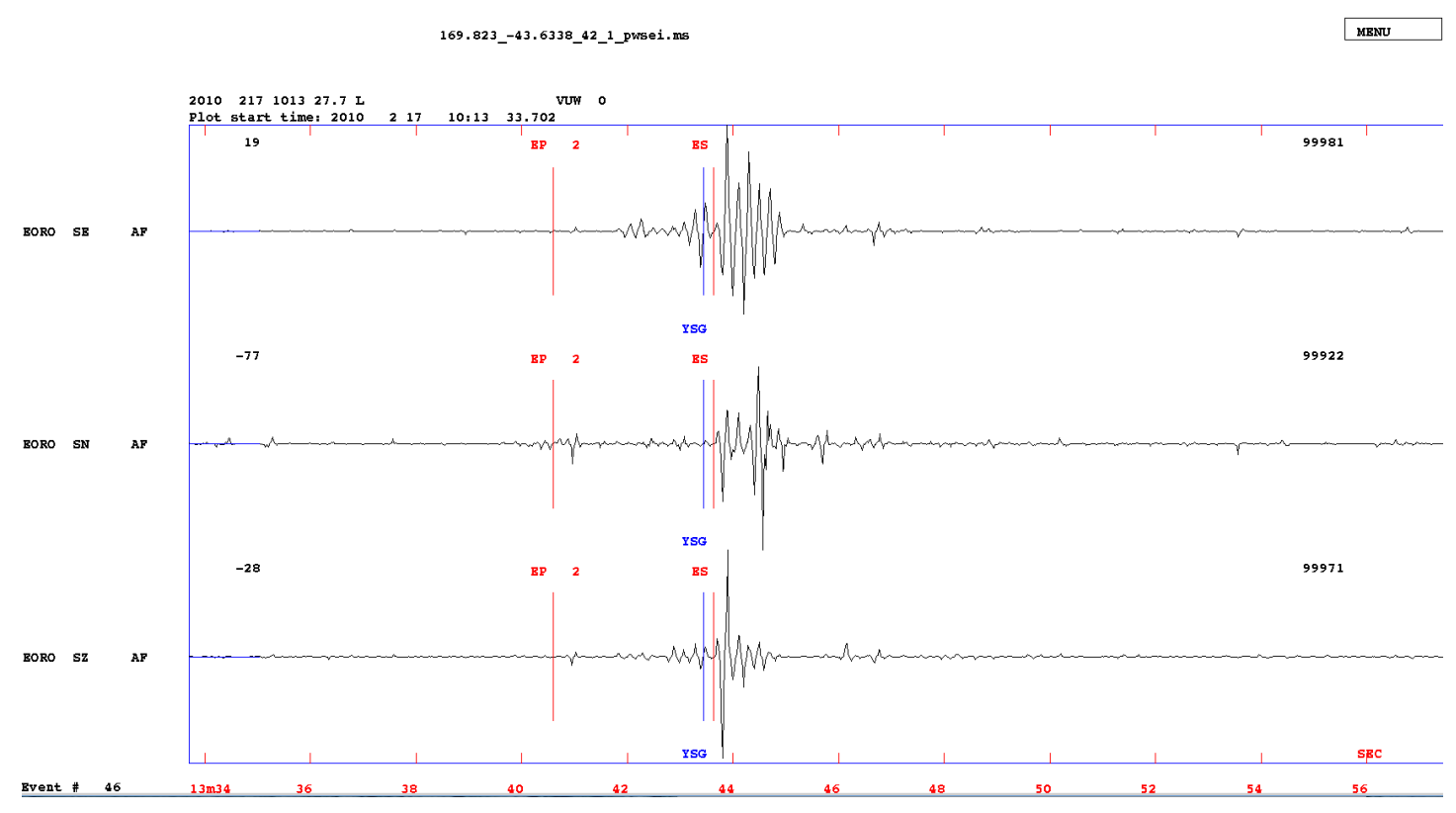

Figure A.24: Another example of P- and S-picks. Screenshot from the SEISAN software (Havskov and Ottemoller, 1999) used for picking wave onsets. Here, we show a zoomed-in view of an example of P- and S-picks for LFE template 169.823_-43.6338_42_1 at the FRAN station.

Figs. A.25 and A.26 show screenshots from SEISAN of the manual P- and Spicks on the linear and the phase-weighted stacks of LFE Family ID:170.224_43.5122_30_1. The picks were made on the phase-weighted stacks for all families. The picking times were then just transferred to the linear stacks in order to check if the arrivals seen on both stacks were coherent. 


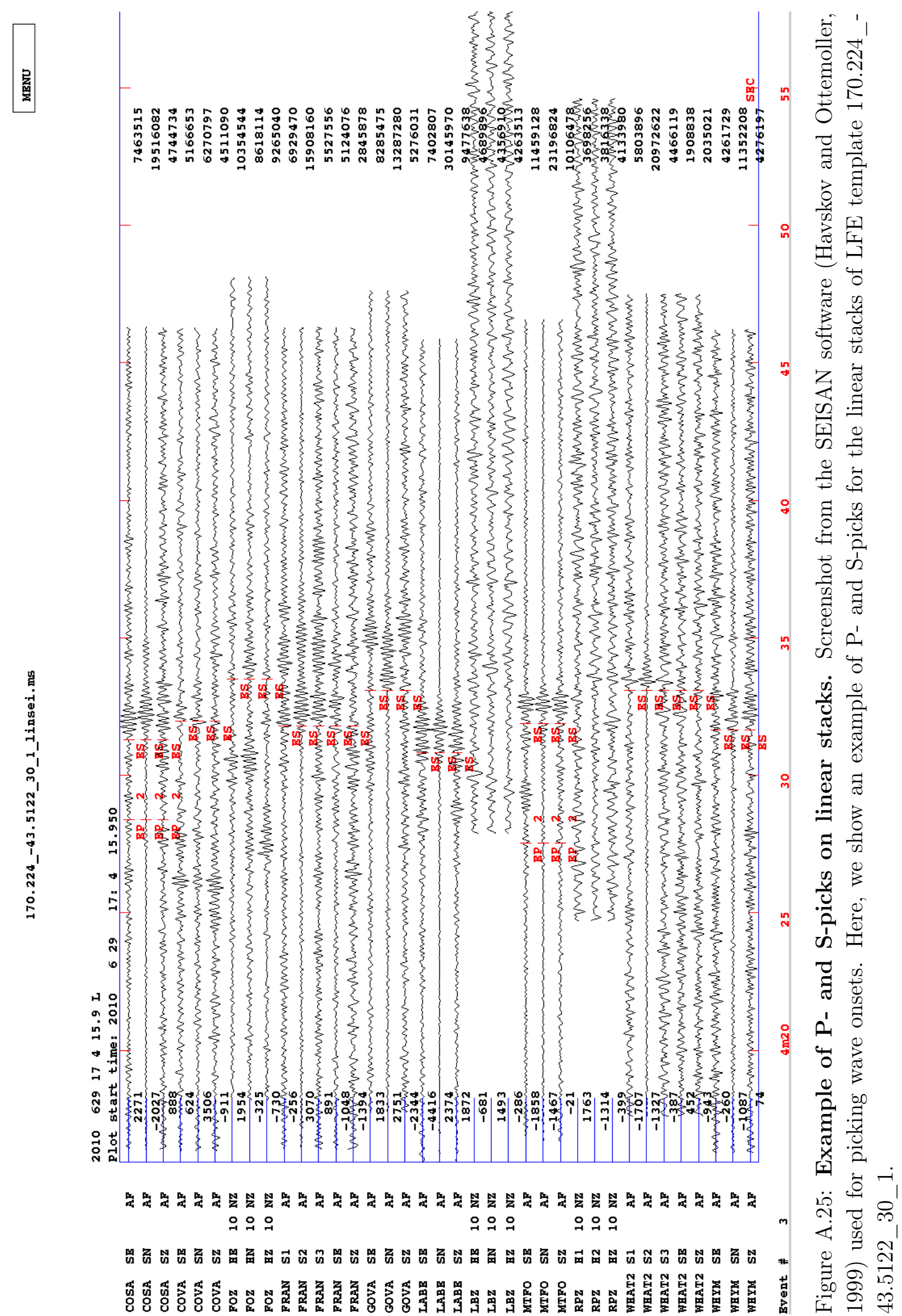




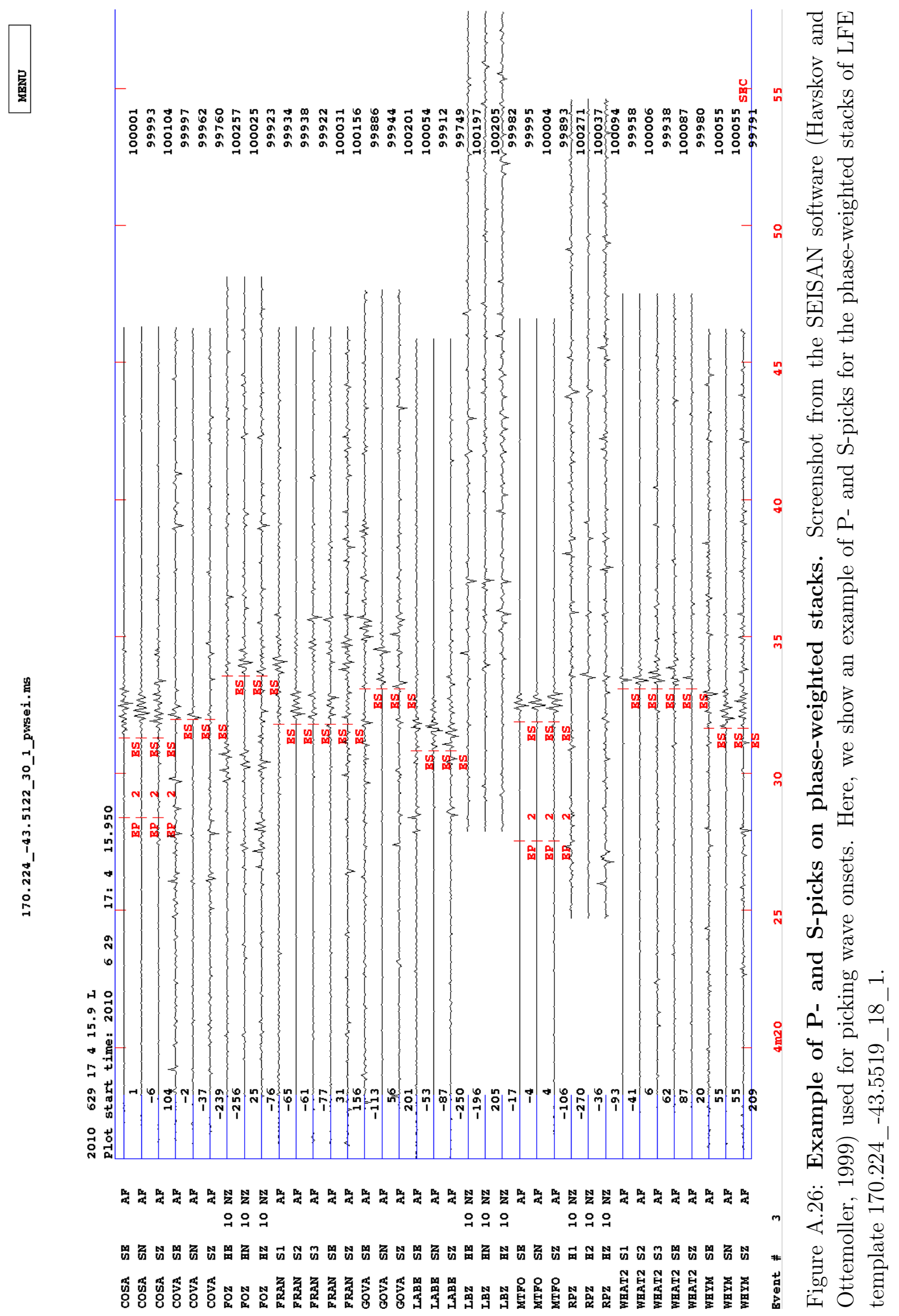




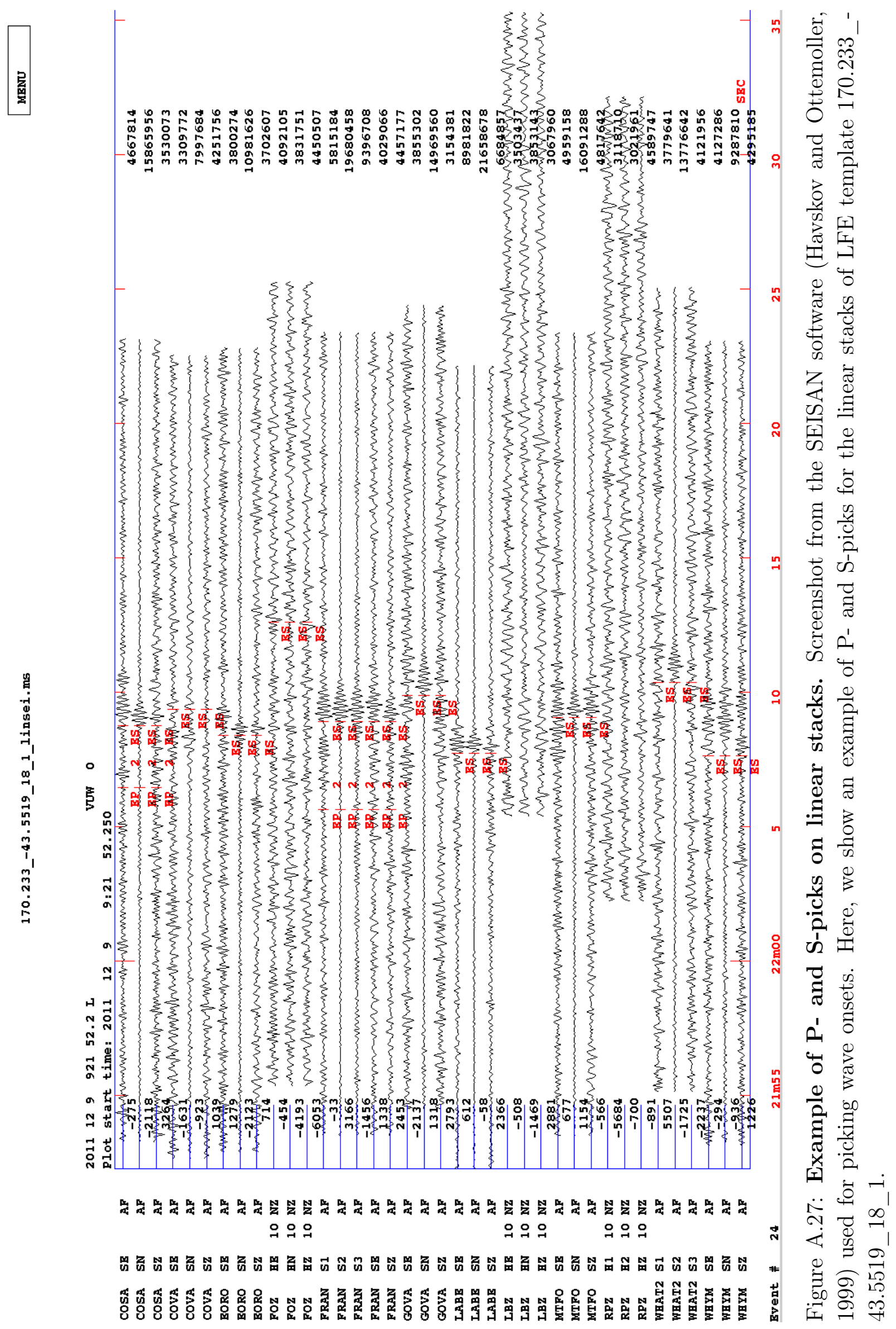




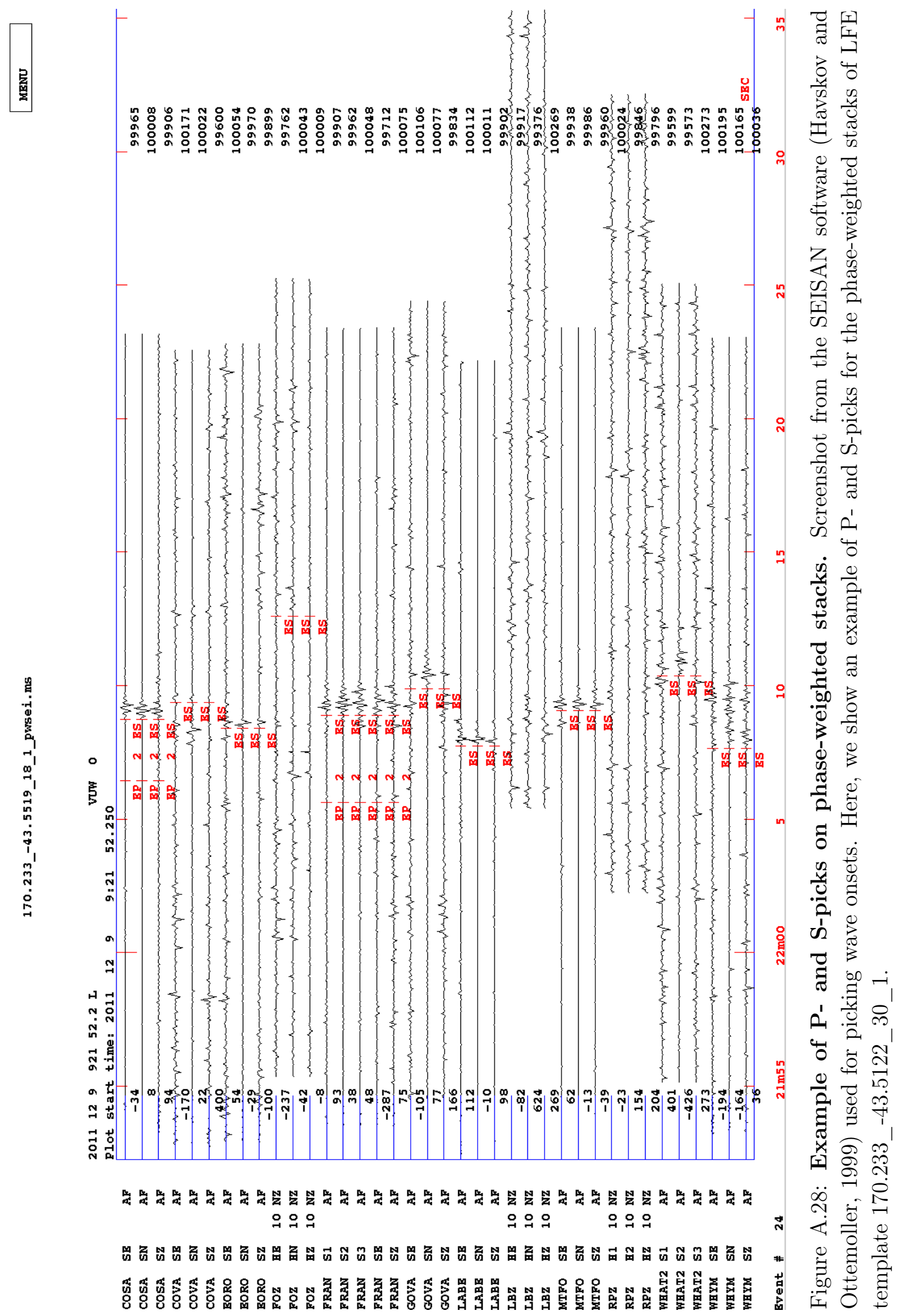


An example of how the preliminary locations were visualised in Google Earth can be seen in Fig. A.29. Stations are located by red pins. The colour of the triangles correspond to the degree of uncertainty in the picks for the corresponding stations (with green representing a high degree of confidence and yellow a medium level of confidence).

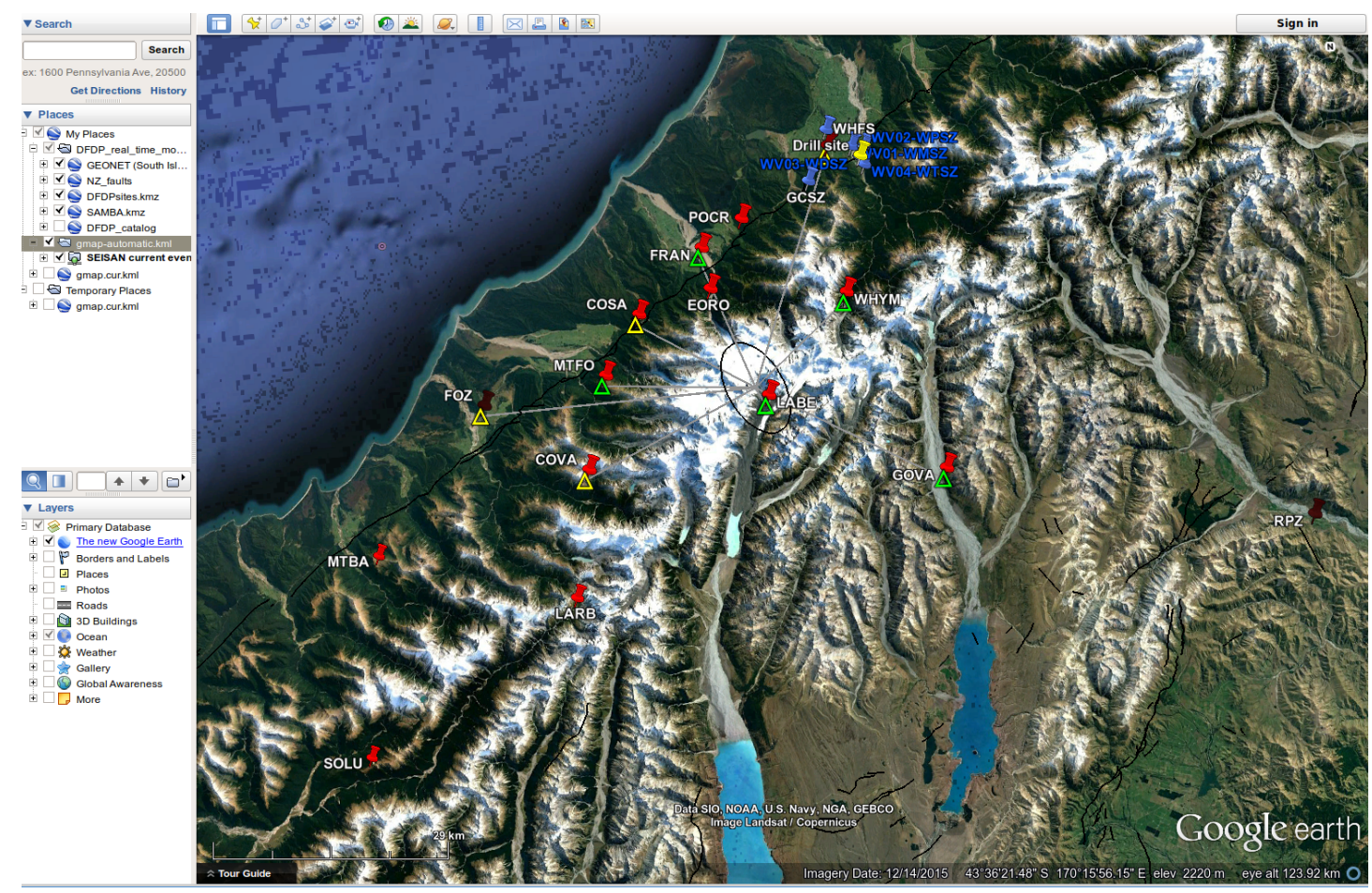

Figure A.29: Example of preliminary location in SEISAN. Location plotted with Google Earth.

In Fig. A.30, are plotted all 378 LFE families located with the NonLinLoc Software. This map is similar to Fig. 4.12 in Chapter 4, Section 4.3.3, with an additional information that is the uncertainties associated with each location (red clouds). 


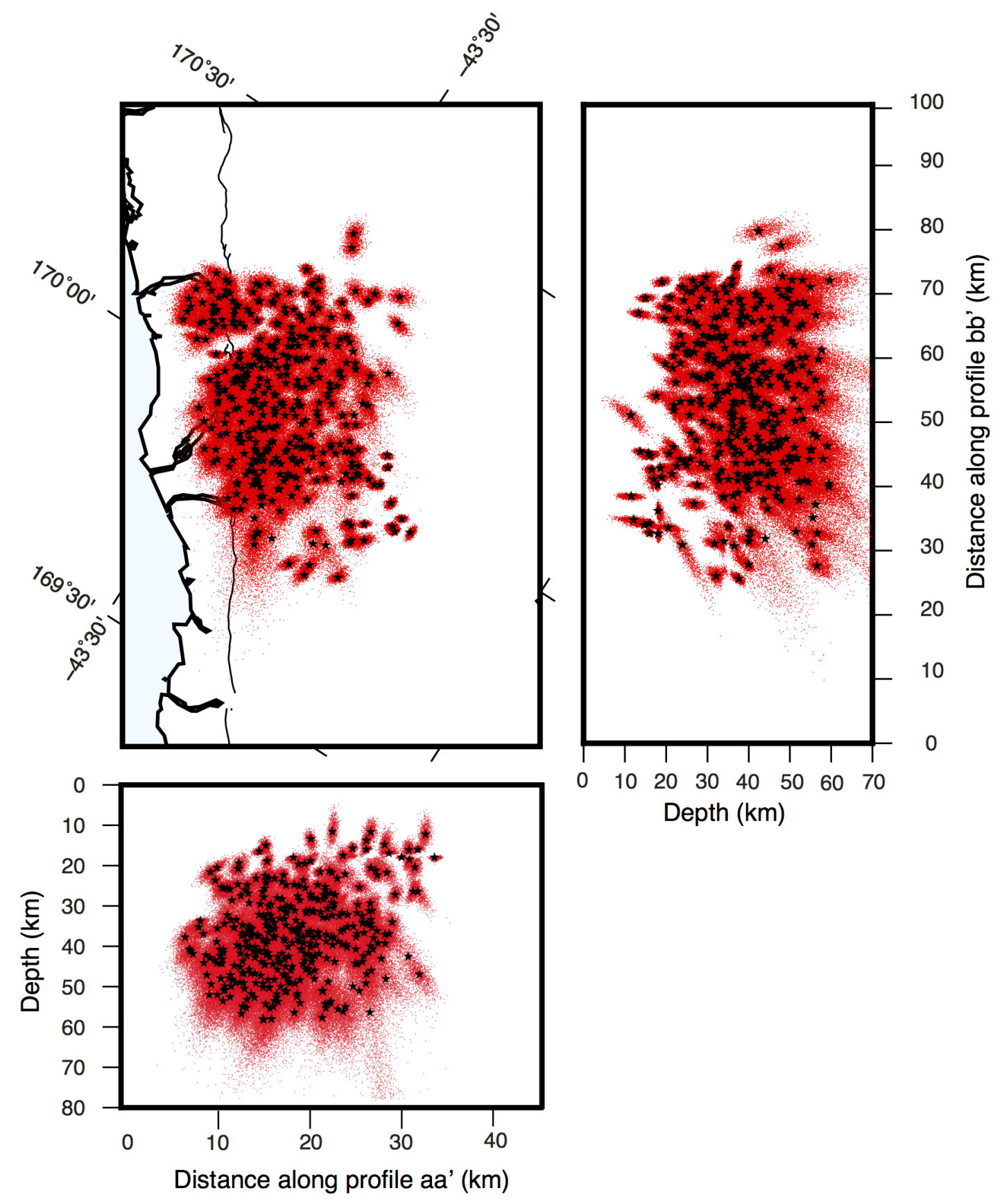

Figure A.30: NonLinLoc locations with clouds of uncertainties. On this map, we plot all 378 LFE families that were located in Chapter 4. Profile aa' and bb' are shown in Fig. 4.12 of Chapter 4. The uncertainties for the locations are plotted as red clouds. 
After obtaining all the SEISAN preliminary locations, the LFEs were relocated using the NonLinLoc software (Lomax et al., 2000). The 378 LFE families locations are described in Table A.1.

\begin{tabular}{|c|c|c|c|c|}
\hline Template ID & $\begin{array}{c}\text { Longitude } \\
{\left[^{\circ}\right]}\end{array}$ & $\begin{array}{c}\text { Latitude } \\
{\left[^{\circ}\right]}\end{array}$ & $\begin{array}{c}\text { Depth } \\
{[\mathrm{km}]}\end{array}$ & $\mathrm{COVr}$ \\
\hline $169.638 \_-43.6641 \_36 \_1$ & 169.914 & -43.5987 & 45.997 & 0.559775578 \\
\hline $170.273 \_-43.5307 \_40 \_1$ & 170.167 & -43.5236 & 28.9570 & 0.522266930 \\
\hline $169.742 \_-43.6761 \_16 \_1$ & 170.026 & -43.6696 & 20.7539 & 0.689268996 \\
\hline 169.739_-43.6112_44_1 & 169.911 & -43.5553 & 51.2715 & 0.602167894 \\
\hline $170.204 \_-43.5227 \_18 \_1$ & 170.192 & -43.5062 & 31.7402 & 0.652501106 \\
\hline $170.08 \_-43.5214 \_18 \_1$ & 170.120 & -43.4921 & 38.7715 & 0.727105703 \\
\hline $169.678 \_-43.643 \_42 \_1$ & 170.071 & -43.5356 & 37.6972 & 0.648624089 \\
\hline $170.054 \_-43.5571 \_18 \_1$ & 170.086 & -43.530 & 36.5253 & 0.569439302 \\
\hline $170.184 \_-43.5333 \_18 \_1$ & 170.191 & -43.5272 & 31.1542 & 0.839258266 \\
\hline 169.892_-43.4869_34_1 & 170.056 & -43.4065 & 43.7520 & 0.587512826 \\
\hline $170.102 \_-43.4208 \_18 \_1$ & 170.096 & -43.3996 & 39.6503 & 0.510892334 \\
\hline 170.082_-43.4314_32_1 & 170.121 & -43.4346 & 43.165 & 0.499104213 \\
\hline $169.932-43.4657 \_34 \_1$ & 170.011 & -43.448 & 54.7383 & 0.479295252 \\
\hline $170.065 \_-43.5068 \_18 \_1$ & 170.081 & -43.5033 & 33.5957 & 0.986042195 \\
\hline $170.099 \_-43.356 \_40 \_1$ & 170.175 & -43.3842 & 48.1465 & 0.518234682 \\
\hline 169.99_-43.524_18_1 & 169.950 & -43.5285 & 37.8926 & 0.836403118 \\
\hline $170.099 \_-43.356 \_16 \_1$ & 170.143 & -43.3757 & 23.9277 & 0.600888082 \\
\hline $170.187 \_-43.5982 \_18 \_1$ & 170.203 & -43.5441 & 32.2284 & 0.674859952 \\
\hline $170.198 \_-43.5479 \_18 \_1$ & 170.229 & -43.515 & 38.3808 & 0.943451731 \\
\hline 169.814_-43.7489_34_1 & 170.019 & -43.6366 & 32.7167 & 0.562030016 \\
\hline 170.1_-43.5108_30_1 & 170.158 & -43.4808 & 40.9198 & 1.384926740 \\
\hline $170.071 \_-43.4817 \_18 \_1$ & 170.116 & -43.4711 & 30.4707 & 0.602031876 \\
\hline $169.892 \_-43.4869 \_18 \_1$ & 170.04 & -43.5328 & 32.3262 & 0.688456673 \\
\hline 169.626_-43.7144_42_1 & 169.916 & -43.6079 & 39.1133 & 0.546217847 \\
\hline 169.783_-43.6549_28_1 & 169.855 & -43.5553 & 54.3965 & 0.565542341 \\
\hline $170.117 \_-43.4354 \_28 \_1$ & 170.116 & -43.4262 & 46.0957 & 0.551305127 \\
\hline 169.936_-43.6854_44_1 & 169.985 & -43.7531 & 26.7597 & 0.716761504 \\
\hline $169.655 \_-43.7435 \_40 \_1$ & 169.882 & -43.7348 & 33.9863 & 0.551014864 \\
\hline 169.661_-43.7184_42_1 & 170.002 & -43.7081 & 16.9941 & 0.737934101 \\
\hline 169.846_-43.5332_32_1 & 169.937 & -43.5818 & 49.8066 & 0.561207192 \\
\hline $170.079 \_-43.3665 \_42 \_1$ & 169.991 & -43.5581 & 43.3613 & 0.463381162 \\
\hline $170.132 \_-43.6048 \_18 \_1$ & 170.149 & -43.5904 & 38.3808 & 0.751842852 \\
\hline 169.886_-43.512_16_1 & 169.864 & -43.5103 & 43.165 & 0.728092179 \\
\hline $170.053 \_-43.4023 \_16 \_1$ & 170.107 & -43.4108 & 32.8145 & 0.573765064 \\
\hline
\end{tabular}




\begin{tabular}{|c|c|c|c|c|}
\hline $169.759 \_-43.6007 \_40 \_1$ & 169.920 & -43.5686 & 54.7383 & 0.615444646 \\
\hline $169.814 \_-43.5941 \_22 \_1$ & 169.892 & -43.5658 & 45.3633 & 0.761004306 \\
\hline $170.065 \_-43.5068 \_44 \_1$ & 170.157 & -43.5041 & 45.3633 & 0.520595033 \\
\hline $170.118 \_-43.5902 \_18 \_1$ & 170.196 & -43.4992 & 13.2832 & 0.573449218 \\
\hline $169.952 \_-43.4552 \_28 \_1$ & 170.049 & -43.4724 & 40.9198 & 0.533009544 \\
\hline $169.852 \_-43.5081 \_26 \_1$ & 169.92 & -43.5041 & 45.7539 & 0.591171311 \\
\hline 169.846_-43.5332_24_1 & 169.942 & -43.5454 & 35.3534 & 0.640947499 \\
\hline $170.099 \_-43.356 \_28 \_1$ & 170.114 & -43.3742 & 43.0683 & 0.456949196 \\
\hline 169.8_-43.5795_26_1 & 169.912 & -43.6296 & 44.8262 & 0.601171683 \\
\hline $169.967 \_-43.4697_{-} 18_{-} 1$ & 170.026 & -43.4851 & 37.5996 & 0.637348769 \\
\hline $170.129 \_-43.5399 \_18 \_1$ & 170.122 & -43.5285 & 37.7948 & 0.545651859 \\
\hline $169.823 \_-43.6338 \_42 \_1$ & 169.907 & -43.6086 & 53.6152 & 0.589409763 \\
\hline 169.699_-43.6324_16_1 & 169.836 & -43.5623 & 37.4042 & 0.572115332 \\
\hline $170.074 \_-43.5465 \_18 \_1$ & 170.138 & -43.5146 & 23.9277 & 0.684467963 \\
\hline $169.901 \_-43.5266 \_24 \_1$ & 169.930 & -43.5497 & 45.5097 & 0.540379954 \\
\hline $170.099 \_-43.356 \_20 \_1$ & 170.14 & -43.3898 & 41.6034 & 0.627609815 \\
\hline 169.932_-43.4657_26 & 170.061 & -43.4620 & 37.5508 & 1.157185992 \\
\hline 169.886_-43.512_26_1 & 170.032 & -43.5174 & 34.7676 & 0.605744481 \\
\hline $170.089 \_-43.5611 \_18 \_1$ & 170.200 & -43.488 & 19.6309 & 0.627701131 \\
\hline $170.193 \_-43.573 \_18 \_1$ & 170.199 & -43.5356 & 27.6387 & 0.711986802 \\
\hline $169.725 \_-43.5967 \_38 \_1$ & $16 !$ & -43.5658 & 39.3085 & 0.495851121 \\
\hline $169.652_{-}-43.6787_{-} 18 \_1$ & 169.916 & -43.4977 & 25.9785 & 0.500780315 \\
\hline 169.664_-43.6284_24_1 & 169.911 & -43.5405 & 42.042 & 0.517286012 \\
\hline $170.111_{-}-43.4605 \_18 \_1$ & 170.157 & -43.6330 & 49.0741 & 0.530118102 \\
\hline $169.947 \_-43.4803 \_42 \_1$ & 169.986 & -43.6071 & 42.6777 & 0.582479190 \\
\hline $170.076 \_-43.4565_{-} 18 \_1$ & 170.116 & -43.5174 & 25.3926 & 0.531554097 \\
\hline $169.852 \_-43.5081 \_22 \_1$ & 169.997 & -43.690 & 21.3887 & 0.538566011 \\
\hline $170.179 \_-43.7133{ }_{-} 16 \_1$ & 170.187 & -43.5441 & 23.4394 & 0.558699147 \\
\hline $170.207 \_-43.5876 \_30 \_1$ & 170.187 & -43.5686 & 51.0272 & 0.556897031 \\
\hline $170.195 \_-43.483 \_18 \_1$ & 170.175 & -43.4388 & 14.9434 & 0.710311170 \\
\hline 169.956_-43.52_18_1 & 170.113 & -43.494 & 22.5605 & 0.582610297 \\
\hline $169.912 \_-43.4763 \_36 \_1$ & 170.038 & -43.4844 & 47.7070 & 0.954117180 \\
\hline $170.355 \_-43.6432 \_26 \_1$ & 170.334 & -43.6043 & 47.9512 & 0.875023878 \\
\hline $169.944 \_-43.5703 \_34 \_1$ & 169.887 & -43.5033 & 44.0448 & 0.656682748 \\
\hline 169.936_-43.6854_36_1 & 169.836 & -43.6921 & 55.3241 & 0.450943329 \\
\hline $170.128 \_-43.3851 \_34 \_1$ & 170.158 & -43.3996 & 50.0020 & 0.518203324 \\
\hline $170.224 \_-43.5122 \_30 \_1$ & 170.258 & -43.4908 & 28.7129 & 0.763797748 \\
\hline $169.935 \_-43.5306 \_26 \_1$ & 169.983 & -43.4626 & 34.9628 & 0.488107261 \\
\hline $169.962 \_-43.6497 \_38 \_1$ & 169.990 & -43.6107 & 59.0352 & 0.659826688 \\
\hline $169.952 \_-43.4552 \_34 \_1$ & 169.992 & -43.4577 & 52.1015 & 0.578041283 \\
\hline $169.872{ }^{-}-43.4975 \_40 \_1$ & 169.888 & -43.5321 & 58.0585 & 0.513140617 \\
\hline
\end{tabular}




\begin{tabular}{|c|c|c|c|c|}
\hline $170.126 \_-43.4751 \_18 \_1$ & 170.158 & -43.4780 & 25.0995 & 0.658617985 \\
\hline $170.073 \_-43.3917 \_26 \_1$ & 170.08 & -43.4065 & 45.0215 & 0.654313345 \\
\hline $169.725 \_-43.5967 \_42 \_1$ & 169.973 & -43.494 & 56.2520 & 0.489988863 \\
\hline $170.059 \_-43.3771 \_32 \_1$ & 170.084 & -43.3898 & 48.1465 & 0.517687468 \\
\hline $170.007 \_-43.4486 \_40 \_1$ & 170.119 & -43.4423 & 52.9804 & 0.573652814 \\
\hline 169.872_-43.4975_36_1 & 169.97 & -43.5384 & 43.5566 & 0.495500248 \\
\hline $170.178 \_-43.5585 \_18 \_1$ & 170.187 & -43.4585 & 24.2207 & 0.681337108 \\
\hline 169.652_-43.6787_38_1 & 169.958 & -43.7986 & 18.0195 & 0.597471284 \\
\hline 169.912_-43.4763_40_1 & 169.965 & -43.4697 & 25.9785 & 0.521353158 \\
\hline $169.716 \_-43.7118 \_16 \_1$ & 169.914 & -43.5623 & 23.3417 & 0.478463316 \\
\hline 169.678_-43.643_32_1 & 169.877 & -43.4894 & 21.7792 & 0.543239668 \\
\hline 169.939_-43.7503_44_1 & 169.978 & -43.5343 & 51.2715 & 0.602167894 \\
\hline $170.06_{-}-43.5319 \_18 \_1$ & 170.038 & -43.5048 & 39.3573 & 0.596262637 \\
\hline 169.783_-43.6549_24_1 & 169.771 & -43.6225 & 23.9277 & 0.600888082 \\
\hline 170.186_-43.4433_38_1 & 170.202 & -43.4234 & 59.5722 & 0.497111903 \\
\hline $169.675 \_-43.733 \_38 \_1$ & 169.847 & -43.7081 & 36.5253 & 0.551667594 \\
\hline $170.233 \_-43.5519 \_18 \_1$ & 170.275 & -43.494 & 29.5917 & 0.618967657 \\
\hline $170.151 \_-43.4393 \_42 \_1$ & 170.216 & -43.4375 & 53.0292 & 0.976126527 \\
\hline $170.059 \_-43.3771 \_28 \_1$ & 170.113 & -43.3967 & 41.6034 & 0.579533606 \\
\hline $170.048 \_-43.5822 \_18 \_1$ & 170.079 & -43.5553 & 31.9355 & 0.572535290 \\
\hline 169.866_-43.5226_38_1 & 169.937 & -43.4515 & 45.0215 & 0.521157050 \\
\hline $169.658 \_-43.6536 \_44 \_1$ & 169.797 & -43.7040 & 56.5448 & 0.637393188 \\
\hline $170.02 \_-43.7079 \_38 \_1$ & 169.985 & -43.6773 & 37.3066 & 0.695912698 \\
\hline $170.093 \_-43.3811 \_24 \_1$ & 170.083 & -43.3631 & 41.5058 & 0.551161071 \\
\hline $170.169 \_-43.5188 \_18 \_1$ & 170.186 & -43.4808 & 34.1816 & 0.730364497 \\
\hline 169.693_-43.6575_32_1 & 170.017 & -43.5595 & 36.3301 & 0.685422254 \\
\hline 169.947_-43.4803_38_1 & 170.047 & -43.4823 & 50.1972 & 0.409941993 \\
\hline $170.131 \_-43.4499 \_18 \_1$ & 170.137 & -43.4178 & 27.834 & 0.546952326 \\
\hline $170.079 \_-43.3665 \_18 \_1$ & 170.15 & -43.3954 & 26.1738 & 0.596648563 \\
\hline 170.097_-43.4459_38_1 & 170.151 & -43.4326 & 53.8594 & 0.534181016 \\
\hline $170.16 \_-43.479 \_40 \_1$ & 170.193 & -43.475 & 50.2460 & 0.966973627 \\
\hline $169.78 \_-43.5901 \_36 \_1$ & 170.021 & -43.4724 & 47.5604 & 0.517550044 \\
\hline $170.099 \_-43.356 \_36 \_1$ & 170.151 & -43.3659 & 50.4902 & 0.562712895 \\
\hline $169.739 \_-43.6112 \_16 \_1$ & 169.853 & -43.5553 & 39.5527 & 0.52607704 \\
\hline 169.597_-43.6853_28_1 & 169.775 & -43.7053 & 32.0332 & 0.483254658 \\
\hline 169.927_-43.4909_30_1 & 170.080 & -43.4872 & 36.1835 & 0.581645324 \\
\hline $169.788 \_-43.6298 \_16 \_1$ & 169.876 & -43.5790 & 42.4823 & 0.768927880 \\
\hline 170.094_-43.5359_18_1 & 170.095 & -43.4767 & 34.1816 & 0.900625530 \\
\hline 169.754_-43.6258_30_1 & 169.930 & -43.5636 & 38.0878 & 0.443030784 \\
\hline $170.117 \_-43.4354 \_18 \_1$ & 170.078 & -43.387 & 37.5996 & 0.625873110 \\
\hline $169.658 \_-43.6536 \_16 \_1$ & 170.005 & -43.5244 & 36.1347 & 0.483286382 \\
\hline
\end{tabular}




\begin{tabular}{|c|c|c|c|c|}
\hline $170.122 \_-43.4102 \_24 \_1$ & 170.203 & -43.4283 & 18.9960 & 0.701818916 \\
\hline 170.346_-43.6035_38_1 & 170.388 & -43.5005 & 42.3847 & 0.583643220 \\
\hline 169.906_-43.5015_28_1 & 170.060 & -43.4767 & 23.4394 & 0.583618352 \\
\hline $170.08 \_-43.5214 \_30 \_1$ & 170.086 & -43.5139 & 48.9765 & 0.635316711 \\
\hline $170.079 \_-43.3665 \_26 \_1$ & 170.109 & -43.393 & 20.7051 & 0.532041042 \\
\hline $170.151 \_-43.4393 \_18 \_1$ & 170.145 & -43.4164 & 35.3534 & 0.599561022 \\
\hline 169.733_-43.6364_36_1 & 169.854 & -43.6274 & 18.0195 & 0.589709124 \\
\hline $169.722 \_-43.6867 \_38 \_1$ & 169.857 & -43.5932 & 33.8887 & 0.678727340 \\
\hline $169.952 \_-43.4552 \_18 \_1$ & 169.928 & -43.5285 & 47.6582 & 0.482271096 \\
\hline $170.131 \_-43.4499 \_28 \_1$ & 170.128 & -43.4206 & 50.0020 & 0.505907942 \\
\hline $169.932 \_-43.4657 \_18 \_1$ & 170.029 & -43.4739 & 26.3691 & 0.552244359 \\
\hline 169.783_-43.6549_32_1 & 169.924 & -43.5595 & 36.4277 & 0.622860737 \\
\hline $170.008 \_-43.6034 \_40 \_1$ & 169.958 & -43.5876 & 27.9316 & 0.586329681 \\
\hline $170.011 \_-43.6682 \_16 \_1$ & 170.116 & -43.5707 & 30.8613 & 0.823302661 \\
\hline $169.725 \_-43.5967 \_34 \_1$ & 169.928 & -43.5525 & 42.9707 & 0.527562906 \\
\hline $169.725 \_-43.5967 \_20 \_1$ & 169.82 & -43.5553 & 33.9863 & 0.655898584 \\
\hline $169.915 \_-43.5412 \_40 \_1$ & 170.015 & -43.4724 & 42.5801 & 0.553423902 \\
\hline $170.16 \_-43.479 \_18 \_1$ & 170.205 & -43.4570 & 31.9355 & 0.639992038 \\
\hline $169.912 \_-43.4763 \_28 \_1$ & 169.922 & -43.4675 & 53.1758 & 0.599087923 \\
\hline $170.122 \_-43.4102 \_40 \_1$ & 170.134 & -43.5413 & 21.7792 & 0.457474771 \\
\hline $170.014 \_-43.5782 \_44 \_1$ & 170 & -43.4752 & 56.1542 & 1.015301688 \\
\hline $170.042_{-}-43.4525_{-} 18 \_1$ & 170.044 & -43.4305 & 25.6855 & 0.570047442 \\
\hline $170.039 \_-43.5425 \_18 \_1$ & 170.07 & -43.494 & 32.9121 & 0.671986246 \\
\hline $170.186 \_-43.4433 \_16 \_1$ & 170.185 & -43.4262 & 29.9823 & 0.596018040 \\
\hline 170.288_-43.5453_40_1 & 170.279 & -43.4724 & 37.1113 & 0.582127525 \\
\hline $169.892_{-}-43.4869 \_38 \_1$ & 169.985 & -43.5174 & 48.2441 & 1.034271951 \\
\hline $170.068{ }_{-}-43.5717{ }_{-18}{ }_{-1}$ & 170.121 & -43.53138 & 31.6426 & 0.712390341 \\
\hline $170.079 \_-43.3665 \_30 \_1$ & 170.038 & -43.4795 & 36.3301 & 0.417610890 \\
\hline $170.008 \_-43.6034 \_28 \_1$ & 170.087 & -43.5476 & 39.9921 & 0.664171834 \\
\hline $169.713 \_-43.647 \_22 \_1$ & 169.776 & -43.6786 & 39.9433 & 0.639452891 \\
\hline $169.944 \_-43.5703 \_42 \_1$ & 170.036 & -43.5469 & 57.7167 & 0.548277978 \\
\hline $169.693 \_-43.6575 \_44 \_1$ & 169.877 & -43.7348 & 31.7402 & 0.623457027 \\
\hline $169.91 \_-43.5663{ }^{2} 44 \_1$ & 169.925 & -43.5257 & 27.2480 & 0.672936085 \\
\hline $170.109 \_-43.5505_{-} 18 \_1$ & 170.133 & -43.5103 & 29.0059 & 1.287492797 \\
\hline $170.097 \_-43.4459 \_18 \_1$ & 170.110 & -43.4318 & 38.0878 & 0.427597365 \\
\hline $170.063{ }_{-}-43.5968 \_18 \_1$ & 170.085 & -43.5608 & 37.4042 & 0.717373392 \\
\hline $169.979 \_-43.5743 \_16 \_1$ & 170.031 & -43.5497 & 31.6426 & 0.660600921 \\
\hline $169.933 \_-43.7754 \_18 \_1$ & 169.926 & -43.6212 & 18.9472 & 0.757615064 \\
\hline $169.733{ }_{-}-43.6364 \_16 \_1$ & 170.005 & -43.7263 & 17.9707 & 0.639589498 \\
\hline $169.841 \_-43.868 \_26 \_1$ & 169.907 & -43.7166 & 16.0176 & 0.542558062 \\
\hline 169.626_-43.7144_38_1 & 169.950 & -43.6296 & 33.4003 & 0.652658709 \\
\hline
\end{tabular}




\begin{tabular}{|c|c|c|c|c|}
\hline $170.014 \_-43.5782 \_18 \_1$ & 169.943 & -43.7796 & 16.0176 & 0.573274276 \\
\hline 169.618_-43.6747_38_1 & 169.949 & -43.7727 & 20.5097 & 0.683222224 \\
\hline 169.785_-43.5649_44_1 & 169.806 & -43.7475 & 37.5996 & 0.671910056 \\
\hline $170.088 \_-43.4062 \_18 \_1$ & 170.061 & -43.7124 & 16.1152 & 0.521810275 \\
\hline 169.664_-43.6284_32_1 & 169.999 & -43.7194 & 27.541 & 0.655522798 \\
\hline 169.71_-43.737_42_1 & 170.041 & -43.7222 & 18.5566 & 0.800532355 \\
\hline $170.052 \_-43.8019 \_44 \_1$ & 170.097 & -43.52368 & 52.9804 & 0.757615064 \\
\hline 169.863_-43.6126_38_1 & 170.185 & -43.5230 & 52.1503 & 0.609338217 \\
\hline $169.73 \_-43.7264 \_16 \_1$ & 169.955 & -43.7012 & 11.5253 & 0.757615064 \\
\hline 169.973_-43.4446_16_1 & 169.923 & -43.7376 & 14.747 & 0.555536158 \\
\hline 169.661_-43.7184_22_1 & 170.003 & -43.6786 & 29.7870 & 0.558539412 \\
\hline $169.719 \_-43.7767 \_40 \_1$ & 169.874 & -43.5328 & 24.9042 & 0.677293871 \\
\hline $170.045 \_-43.5174 \_40 \_1$ & 169.972 & -43.7783 & 12.1113 & 0.470580791 \\
\hline $170.143 \_-43.5545 \_42 \_1$ & 169.938 & -43.5202 & 35.7441 & 0.573274276 \\
\hline $170.077 \_-43.6114 \_18 \_1$ & 170.043 & -43.6058 & 44.7284 & 0.593720856 \\
\hline 169.932_-43.4657_30_1 & 169.997 & -43.4543 & 42.3847 & 0.445032660 \\
\hline 169.932_-43.4657_38_1 & 170.026 & -43.4536 & 51.0272 & 0.552582844 \\
\hline $169.82 \_-43.7238 \_28 \_1$ & 169.895 & -43.7503 & 40.040 & 0.651695118 \\
\hline 169.976_-43.5094_18_1 & 169.989 & -43.4795 & 40.5292 & 0.677123613 \\
\hline 169.996_-43.4988_42_1 & 170.047 & -43.4634 & 50.1484 & 0.592321678 \\
\hline 170.335_-43.6538_38_1 & 17 & -43.56928 & 37.7948 & 0.491529979 \\
\hline 169.791_-43.6946_38_1 & 169.906 & -43.7229 & 35.0116 & 0.568182519 \\
\hline $170.213 \_-43.5624 \_18 \_1$ & 170.233 & -43.5062 & 37.7948 & 0.842414372 \\
\hline $170.097 \_-43.4459 \_42 \_1$ & 170.09 & -43.494 & 53.8104 & 0.499158635 \\
\hline $169.73 \_-43.7264 \_40 \_1$ & 169.86 & -43.6856 & 40.4316 & 0.737093513 \\
\hline 169.812_-43.8389_36_1 & 170.05 & -43.5707 & 28.4198 & 0.510984732 \\
\hline $169.638{ }_{-}-43.6641{ }_{-} 16 \_1$ & 170.014 & -43.5448 & 52.2969 & 0.498223273 \\
\hline $169.67 \_-43.75811_{-} 40 \_1$ & 170.032 & -43.5441 & 36.3301 & 0.644562321 \\
\hline $169.964 \_-43.5597 \_18 \_1$ & 170.038 & -43.6730 & 36.8183 & 0.424666674 \\
\hline $170.085 \_-43.4962 \_44$ & 170.116 & -43.4872 & 44.5820 & 0.637781071 \\
\hline $169.84 \_-43.5583 \_40 \_1$ & 170.008 & -43.5118 & 28.2245 & 0.524808695 \\
\hline $170.132 \_-43.6048 \_42 \_1$ & 169.956 & -43.6563 & 40.2363 & 0.536063073 \\
\hline $170.289 \_-43.7001 \_38 \_1$ & 169.889 & -43.5595 & 16.5059 & 0.606802829 \\
\hline $169.9211_{-}-43.516{ }_{-} 18 \_1$ & 170.026 & -43.4731 & 47.7070 & 0.561184236 \\
\hline $169.95_{-}-43.5451_{-} 18_{-} 1$ & 170.04 & -43.53 & 33.790 & 0.598560926 \\
\hline 170.19_-43.663_30_1 & 170.173 & -43.631 & 37.5996 & 0.648144660 \\
\hline $169.84 \_-43.5583 \_44 \_1$ & 170.035 & -43.5636 & 42.6777 & 0.528122767 \\
\hline $170.181 \_-43.6233 \_18 \_1$ & 170.145 & -43.5910 & 45.167 & 0.582660994 \\
\hline $169.942 \_-43.6603 \_26 \_1$ & 170.079 & -43.5497 & 42.7753 & 0.835194781 \\
\hline $170.079 \_-43.3665 \_22 \_1$ & 170.095 & -43.3631 & 42.6777 & 0.668230014 \\
\hline $170.167{ }_{-}-43.6087 \_18 \_1$ & 170.121 & -43.5987 & 35.6465 & 0.652939921 \\
\hline
\end{tabular}




\begin{tabular}{|c|c|c|c|c|}
\hline $170.059 \_-43.37711_{-} 16 \_1$ & 170.049 & -43.3954 & 34.2792 & 0.580586115 \\
\hline $169.97 \_-43.5346 \_18 \_1$ & 170.077 & -43.5230 & 30.5684 & 0.654466243 \\
\hline $170.218 \_-43.5373 \_18 \_1$ & 170.239 & -43.4900 & 25.0507 & 1.034974606 \\
\hline $170.247 \_-43.5664 \_18 \_1$ & 170.258 & -43.5013 & 27.1992 & 0.737715505 \\
\hline 169.889_-43.5769_16_1 & 170.017 & -43.5413 & 30.8613 & 0.572242318 \\
\hline $170.099 \_-43.356 \_32 \_1$ & 170.175 & -43.3898 & 36.0371 & 0.546798753 \\
\hline $170.193 \_-43.573 \_42 \_1$ & 170.140 & -43.5974 & 30.0801 & 0.597205333 \\
\hline $170.076 \_-43.4565 \_42 \_1$ & 170.145 & -43.4191 & 51.6621 & 0.477654733 \\
\hline 169.904 -43.5914_40_1 & 169.984 & -43.5566 & 45.9003 & 0.625879662 \\
\hline 169.661_-43.7184_38_1 & 169.890 & -43.610 & 39.8457 & 0.583026241 \\
\hline $169.895 \_-43.5517_{-} 16_{-} 1$ & 169.982 & -43.5497 & 31.1542 & 0.541081632 \\
\hline $169.84 \_-43.5583 \_22 \_1$ & 169.952 & -43.6015 & 25.8809 & 0.504781012 \\
\hline $169.967 \_-43.4697 \_26 \_1$ & 169.996 & -43.4682 & 49.3183 & 0.572284900 \\
\hline $169.739 \_-43.6112 \_38 \_1$ & 169.807 & -43.5707 & 44.5332 & 0.417610890 \\
\hline 169.829_-43.6086_32_1 & 169.972 & -43.5454 & 38.5762 & 0.557940966 \\
\hline $169.965 \_-43.7145 \_44 \_1$ & 170.067 & -43.6394 & 43.2637 & 0.626176026 \\
\hline $169.872 \_-43.6523 \_38 \_1$ & 169.997 & -43.6935 & 39.9921 & 0.531784259 \\
\hline 169.904_-43.5914_36_1 & 170.115 & -43.5187 & 39.6503 & 0.644571275 \\
\hline 169.953_-43.61_42_1 & 170.077 & -43.6507 & 38.6738 & 0.539332688 \\
\hline $170.149 \_-43.5293 \_18 \_1$ & 170.143 & -43.4529 & 22.7559 & 0.572509796 \\
\hline $170.164 \_-43.5439 \_18 \_1$ & 170 & -43.5371 & 33.4003 & 0.544142875 \\
\hline $170.093 \_-43.3811_{-} 28 \_1$ & 170.130 & -43.3560 & 35.8417 & 0.638410739 \\
\hline $170.079 \_-43.3665 \_34 \_1$ & 170.121 & -43.3890 & 53.1758 & 0.594297355 \\
\hline $169.89 \_-43.7317 \_44 \_1$ & 170.012 & -43.6856 & 22.5605 & 0.753411343 \\
\hline $169.704 \_-43.7621 \_44 \_1$ & 170.045 & -43.5187 & 45.1191 & 0.589041519 \\
\hline $169.834 \_-43.5835 \_44 \_1$ & 170.025 & -43.6633 & 13.6738 & 0.649403697 \\
\hline 169.837_-43.6483_36_1 & 169.908 & -43.5608 & 24.6113 & 0.700964221 \\
\hline $169.941 \_-43.5054 \_16 \_1$ & 169 & -43.4816 & 36.1835 & 0.653223230 \\
\hline $170.099 \_-43.356 \_44 \_1$ & 170.176 & -43.3680 & 45.0703 & 0.633208722 \\
\hline $169.967 \_-43.4697 \_32 \_1$ & 169.995 & -43.4423 & 44.3866 & 0.530579530 \\
\hline $169.834 \_-43.5835 \_40 \_1$ & 170.024 & -43.5033 & 38.9667 & 0.596417878 \\
\hline $169.742_{-}-43.67611_{-} 28 \_1$ & 169.997 & -43.6338 & 33.0097 & 0.486282009 \\
\hline $169.952 \_-43.4552 \_42 \_1$ & 170.061 & -43.4816 & 41.8477 & 0.473818978 \\
\hline $170.059 \_-43.3771 \_36 \_1$ & 170.117 & -43.3975 & 45.167 & 0.511454521 \\
\hline 169.626_-43.7144_34_1 & 170.008 & -43.6492 & 15.7246 & 0.544263735 \\
\hline $170.059 \_-43.3771 \_44 \_1$ & 170.105 & -43.3547 & 41.9941 & 0.503884019 \\
\hline $170.085 \_-43.4962 \_18 \_1$ & 170.050 & -43.5005 & 30.4707 & 0.608440501 \\
\hline $170.113{ }_{-}-43.3705 \_16 \_1$ & 170.158 & -43.3926 & 34.0840 & 0.567358463 \\
\hline $169.947 \_-43.4803 \_18 \_1$ & 170.070 & -43.4585 & 25.0995 & 0.540054075 \\
\hline 169.774_-43.6152_32_1 & 169.932 & -43.5917 & 36.8183 & 0.450333557 \\
\hline $170.059{ }_{-}-43.37711_{-} 20 \_1$ & 170.066 & -43.3645 & 38.4784 & 0.726865348 \\
\hline
\end{tabular}




\begin{tabular}{|c|c|c|c|c|}
\hline $170.073 \_-43.3917 \_18 \_1$ & 170.149 & -43.4087 & 20.9492 & 0.512167095 \\
\hline $170.097 \_-43.6008 \_18 \_1$ & 170.122 & -43.6507 & 21.876 & 0.709647582 \\
\hline $169.876 \_-43.872 \_44 \_1$ & 170.116 & -43.5882 & 22.3164 & 0.302580157 \\
\hline $169.838 \_-43.8032 \_16 \_1$ & 170.104 & -43.5608 & 27.6387 & 0.345473589 \\
\hline $169.667 \_-43.6933 \_16 \_1$ & 169.860 & -43.595 & 36.2323 & 0.362832392 \\
\hline $170.118 \_-43.5902 \_42 \_1$ & 170.148 & -43.5630 & 56.8866 & 0.629275188 \\
\hline $169.87 \_-43.7423 \_38 \_1$ & 169.988 & -43.4844 & 40.8710 & 0.464413681 \\
\hline $169.98 \_-43.7291 \_36 \_1$ & 170.194 & -43.5910 & 57.667 & 0.860885574 \\
\hline 169.834_-43.5835_36_1 & 169.970 & -43.4690 & 50.9296 & 0.565262192 \\
\hline 169.661_-43.7184_28_1 & 170.092 & -43.595 & 17.5801 & 0.537417071 \\
\hline $170.195 \_-43.483 \_40 \_1$ & 170.148 & -43.5062 & 41.4082 & 0.483522811 \\
\hline $170.157 \_-43.4142 \_44 \_1$ & 170.178 & -43.4410 & 49.3671 & 0.523358204 \\
\hline $170.031+-43.6577 \_40 \_1$ & 170.098 & -43.5938 & 57.4727 & 0.470323084 \\
\hline 169.872_-43.4975_32_1 & 170.086 & -43.4838 & 30.4707 & 0.536808406 \\
\hline 169.965_-43.7145_36_1 & 169.967 & -43.677 & 52.1991 & 0.327623192 \\
\hline $170.049 \_-43.7371 \_26 \_1$ & 170.086 & -43.6058 & 41.0176 & 0.497451802 \\
\hline $170.152 \_-43.5942 \_42 \_1$ & 170.029 & -43.6471 & 45.3633 & 0.372607756 \\
\hline $170.259 \_-43.5161 \_40 \_1$ & 170.258 & -43.5013 & 54.9335 & 0.656717887 \\
\hline $170.047 \_-43.4274 \_40 \_1$ & 170.092 & -43.4072 & 48.9765 & 0.442632501 \\
\hline $170.167 \_-43.6087 \_42 \_1$ & 170.228 & -43.5384 & 56.2520 & 0.622037897 \\
\hline $170.15 \_-43.6842 \_40 \_1$ & 170 & -43.5595 & 29.8847 & 0.521974577 \\
\hline $169.84 \_-43.7132 \_42 \_1$ & 170.020 & -43.5855 & 46.9258 & 0.525492778 \\
\hline 169.754_-43.6258_38_1 & 169.932 & -43.5869 & 50.1484 & 0.573639758 \\
\hline $169.927_{-}-43.4909 \_16_{-} 1$ & 169.96 & -43.5131 & 35.9395 & 0.539648334 \\
\hline 169.699_-43.6324_34_1 & 169.943 & -43.5630 & 46.7304 & 0.599045582 \\
\hline $170.047 \_-43.4274 \_36 \_1$ & 170.098 & -43.4178 & 48.9277 & 0.575722780 \\
\hline 169.612_-43.6999_36_1 & 169.994 & -43.5328 & 45.4121 & 0.455760885 \\
\hline $169.719 \_-43.6218 \_36 \_1$ & 170.026 & -43.4808 & 29.6894 & 0.5216901 \\
\hline $170.075 \_-43.7013{ }_{-} 16 \_1$ & 170.214 & -43.5131 & 25.5879 & 0.556504223 \\
\hline $169.62 \_-43.7396 \_44 \_1$ & 170.031 & -43.5876 & 38.3808 & 0.389361055 \\
\hline $169.635 \_-43.7541{ }^{2} 36 \_1$ & 170.055 & -43.5630 & 41.0664 & 0.41475751 \\
\hline $170.114 \_-43.5253 \_18 \_1$ & 170.132 & -43.4921 & 30.4707 & 0.419664898 \\
\hline $169.577 \_-43.6959 \_32 \_1$ & 169.800 & -43.6388 & 43.9960 & 0.497017048 \\
\hline 169.829_-43.7635_22_1 & 170.055 & -43.5118 & 28.4198 & 0.604715038 \\
\hline $170.295 \_-43.675 \_16 \_1$ & 170.288 & -43.6225 & 26.7597 & 0.659175665 \\
\hline 169.618_-43.6747_34_1 & 169.785 & -43.6163 & 40.0897 & 0.505084862 \\
\hline $169.913 \_-43.786 \_42 \_1$ & 170.196 & -43.65 & 43.4102 & 0.432642293 \\
\hline $169.95 \_-43.5451 \_38 \_1$ & 169.982 & -43.6051 & 40.4804 & 0.604715038 \\
\hline 170.113_-43.7702_36_1 & 170.169 & -43.5917 & 34.8652 & 0.715886016 \\
\hline 169.684_-43.6178_38_1 & 170.012 & -43.5125 & 42.042 & 0.536556261 \\
\hline $169.838 \_-43.8032 \_24 \_1$ & 170.011 & -43.6758 & 40.7246 & 0.457684725 \\
\hline
\end{tabular}




\begin{tabular}{|c|c|c|c|c|}
\hline $169.872 \_-43.4975 \_28 \_1$ & 169.98 & -43.4985 & 41.0664 & 0.539212330 \\
\hline 170.34_-43.6287_38_1 & 170.363 & -43.509 & 47.8534 & 0.715886016 \\
\hline $169.765 \_-43.5755 \_24 \_1$ & 169.795 & -43.6163 & 51.417 & 0.642218716 \\
\hline $169.641 \_-43.729 \_44 \_1$ & 170.151 & -43.5125 & 31.6914 & 0.578433520 \\
\hline 169.759_-43.6007_44_1 & 170.115 & -43.5187 & 36.1347 & 0.514000600 \\
\hline $169.699 \_-43.7872 \_36 \_1$ & 170.125 & -43.5602 & 21.5352 & 0.321240198 \\
\hline $169.785 \_-43.5649 \_16 \_1$ & 169.835 & -43.576 & 43.6054 & 0.609040711 \\
\hline $169.597 \_-43.6853 \_40 \_1$ & 169.958 & -43.5630 & 42.6289 & 0.521004166 \\
\hline $169.82 \_-43.8786 \_36 \_1$ & 170.091 & -43.64157 & 35.9883 & 0.468426287 \\
\hline 170.135_-43.6696_30_1 & 170.205 & -43.5797 & 50.2460 & 0.445080185 \\
\hline $170.105 \_-43.4856 \_40 \_1$ & 170.146 & -43.4403 & 38.1854 & 0.583917918 \\
\hline $170.092 \_-43.6259 \_18 \_1$ & 170.109 & -43.5932 & 40.4316 & 0.420108199 \\
\hline 169.803_-43.7992_30_1 & 170.021 & -43.6584 & 44.7772 & 0.436075822 \\
\hline $169.733 \_-43.7912 \_16 \_1$ & 170.101 & -43.5236 & 19.7773 & 0.262552074 \\
\hline $170.083 \_-43.5862 \_18 \_1$ & 169.994 & -43.5966 & 49.0741 & 0.530201684 \\
\hline $169.837 \_-43.6483 \_20 \_1$ & 169.924 & -43.6135 & 44.3866 & 0.511021527 \\
\hline $169.635 \_-43.7541 \_32 \_1$ & 170.191 & -43.4570 & 28.7129 & 0.501485005 \\
\hline 169.736_-43.7012_22_1 & 169.955 & -43.6015 & 32.2284 & 0.574132694 \\
\hline 169.664_-43.6284_40_1 & 169.869 & -43.5741 & 59.4258 & 0.518464480 \\
\hline $170.123 \_-43.5651 \_18 \_1$ & 170.125 & -43.5356 & 38.4784 & 0.935107095 \\
\hline 169.606_-43.725_44_1 & 16 & -43.6507 & 52.247 & 0.584346798 \\
\hline $169.638 \_-43.66411_{-} 42 \_1$ & 169.979 & -43.6246 & 49.8554 & 0.503565884 \\
\hline $169.597 \_-43.6853 \_44 \_1$ & 169.845 & -43.6009 & 56.2030 & 0.621228047 \\
\hline $169.577_{-}-43.6959 \_40 \_1$ & 169.888 & -43.5735 & 32.5215 & 0.569056679 \\
\hline $169.62 \_-43.7396 \_36 \_1$ & 169.941 & -43.4936 & 29.0059 & 0.561061685 \\
\hline 169.829_-43.6086_36_1 & 169.938 & -43.5658 & 54.9335 & 0.512409216 \\
\hline $169.745 \_-43.5861 \_22 \_1$ & 169.88 & -43.5777 & 37.3066 & 0.685929115 \\
\hline $169.592 \_-43.7104 \_42 \_1$ & 169.978 & -43.6409 & 39.5527 & 0.414220625 \\
\hline $170.101 \_-43.6656 \_30 \_1$ & 170.168 & -43.6079 & 41.4570 & 0.741590726 \\
\hline $169.713 \_-43.8018 \_42 \_1$ & 170.264 & -43.4908 & 26.4667 & 0.519266477 \\
\hline $170.1 \_-43.5108 \_18 \_1$ & 170.084 & -43.4992 & 43.4590 & 0.552290605 \\
\hline $169.699+-43.6324 \_44 \_1$ & 169.872 & -43.5855 & 59.4258 & 0.531049133 \\
\hline $170.254{ }_{-}-43.69611_{-} 16{ }_{-} 1$ & 170.312 & -43.57209 & 34.5722 & 0.567719086 \\
\hline $169.632 \_-43.6893 \_44 \_1$ & 170.057 & -43.5946 & 21.876 & 0.564437162 \\
\hline $169.618 \_-43.6747 \_42 \_1$ & 169.925 & -43.5805 & 48.3417 & 0.664402758 \\
\hline $170.069 \_-43.7265 \_38 \_1$ & 170.246 & -43.5566 & 34.5722 & 0.572758314 \\
\hline $169.704 \_-43.6073 \_40 \_1$ & 170.102 & -43.5658 & 39.5039 & 0.484322105 \\
\hline 169.913_-43.786_38_1 & 169.926 & -43.5264 & 46.7304 & 0.599045582 \\
\hline $169.716 \_-43.7118 \_24 \_1$ & 169.889 & -43.6079 & 46.9258 & 0.634390730 \\
\hline $169.62 \_-43.7396 \_40 \_1$ & 170.008 & -43.5461 & 35.4022 & 0.489372962 \\
\hline 169.99_-43.524_28_1 & 170.045 & -43.4816 & 40.8710 & 0.428265599 \\
\hline
\end{tabular}




\begin{tabular}{|c|c|c|c|c|}
\hline $169.647 \_-43.7038 \_36 \_1$ & 169.958 & -43.4894 & 35.9395 & 0.568649536 \\
\hline 169.901_-43.5266_30_1 & 169.93 & -43.5208 & 56.4960 & 0.493210955 \\
\hline 169.658_-43.6536_30_1 & 170.003 & -43.5153 & 26.0273 & 0.528096153 \\
\hline $170.036 \_-43.4777 \_18 \_1$ & 170.084 & -43.475 & 26.8085 & 0.615669445 \\
\hline 169.592_-43.7104_38_1 & 169.959 & -43.6135 & 43.2147 & 0.461228008 \\
\hline $170.138 \_-43.5796 \_18 \_1$ & 170.162 & -43.5371 & 27.834 & 0.875334292 \\
\hline $170.019 \_-43.5531 \_44 \_1$ & 170.013 & -43.6051 & 55.5195 & 0.461228008 \\
\hline 169.976_-43.6643_38_1 & 169.950 & -43.6107 & 28.3710 & 0.538994391 \\
\hline 169.852_-43.5081_34_1 & 170.019 & -43.48728 & 38.3320 & 0.596911411 \\
\hline 169.687_-43.6827_32_1 & 169.816 & -43.6023 & 55.5195 & 0.580165603 \\
\hline 169.716_-43.7118_32_1 & 170.003 & -43.5069 & 32.667 & 0.533202574 \\
\hline $169.684 \_-43.6178 \_18 \_1$ & 169.878 & -43.5587 & 42.7265 & 0.569370991 \\
\hline 169.987_-43.4591_36_1 & 170.097 & -43.5041 & 52.3945 & 0.451809950 \\
\hline 170.053_-43.4023_34_1 & 170.115 & -43.4247 & 19.5332 & 0.411080351 \\
\hline $169.774 \_-43.6152 \_28 \_1$ & 169.961 & -43.5328 & 33.0097 & 0.527176877 \\
\hline 169.739_-43.7661_38_1 & 170.014 & -43.6388 & 35.792 & 0.507067485 \\
\hline $170.274 \_-43.6855 \_16 \_1$ & 170.200 & -43.5664 & 41.5058 & 0.659501981 \\
\hline 169.704_-43.7621_40_1 & 169.984 & -43.6886 & 35.0604 & 0.545797415 \\
\hline $170.209 \_-43.4976 \_18 \_1$ & 170.263 & -43.5399 & 32.2284 & 0.931736801 \\
\hline 169.612_-43.6999_32_1 & 169.863 & -43.610 & 47.8534 & 0.514851292 \\
\hline 169.993_-43.434_32_1 & 17 & -43.4626 & 37.4042 & 0.497912909 \\
\hline 169.817_-43.6589_34_1 & 169.924 & -43.6079 & 41.6522 & 0.569975044 \\
\hline 170.109_-43.5505_38_1 & 170.074 & -43.5904 & 38.4784 & 0.610453006 \\
\hline $170.105 \_-43.4856 \_18 \_1$ & 170.115 & -43.5103 & 25.001 & 0.345726232 \\
\hline 169.664_-43.6284_36_1 & 169.942 & -43.534 & 42.042 & 0.669968200 \\
\hline 169.904_-43.5914_44_1 & 169.962 & -43.588 & 55.2753 & 0.536176835 \\
\hline $170.158 \_-43.569 \_42 \_1$ & 170.163 & -43.515 & 43.9472 & 0.503986606 \\
\hline 169.597_-43.6853_36_1 & 170.050 & -43.5987 & 11.4277 & 0.650753079 \\
\hline $169.988 \_-43.7688 \_42 \_1$ & 170.12 & -43.6430 & 43.8984 & 0.527738303 \\
\hline 169.716_-43.7118_44_1 & 169.991 & -43.4675 & 53.7616 & 0.595962278 \\
\hline $169.69 \_-43.7475 \_44 \_1$ & 169.991 & -43.616 & 43.8496 & 0.598752465 \\
\hline $170.245 \_-43.6564 \_40 \_1$ & 170.295 & -43.5469 & 46.9746 & 0.478817192 \\
\hline $170.205 \_-43.6776 \_28 \_1$ & 170.178 & -43.5538 & 36.915 & 0.576149330 \\
\hline 169.704_-43.6073_36_1 & 169.902 & -43.5763 & 49.3183 & 0.572740516 \\
\hline $170.161 \_-43.6339 \_18 \_1$ & 170.176 & -43.6030 & 31.6426 & 0.646087057 \\
\hline $170.14 \_-43.4896 \_18 \_1$ & 170.169 & -43.4992 & 38.2832 & 0.441162102 \\
\hline $169.733{ }^{2}-43.7912 \_40 \_1$ & 169.946 & -43.5664 & 51.0762 & 0.577198117 \\
\hline $169.84 \_-43.7132 \_26 \_1$ & 169.978 & -43.5966 & 47.5116 & 0.588567016 \\
\hline $169.875 \_-43.7172 \_28 \_1$ & 170.044 & -43.6563 & 34.9628 & 0.594625650 \\
\hline 169.678_-43.643_38_1 & 169.872 & -43.5131 & 51.7597 & 0.566566806 \\
\hline $170.147 \_-43.6193{ }_{-} 18 \_1$ & 170.078 & -43.6282 & 25.6855 & 0.333961369 \\
\hline
\end{tabular}




\begin{tabular}{||c|c|c|c|c||}
\hline $170.002 \_-43.4737 \_18 \_1$ & 170.086 & -43.488 & 30.0801 & 0.950063133 \\
\hline $169.704 \_-43.6073 \_16 \_1$ & 170.014 & -43.5257 & 23.4394 & 0.592682044 \\
\hline $169.632 \_-43.6893 \_40 \_1$ & 170.026 & -43.5720 & 46.4863 & 0.713029405 \\
\hline $169.745 \_-43.7409 \_20 \_1$ & 169.992 & -43.5882 & 37.1602 & 0.611981914 \\
\hline $169.91 \_-43.7211 \_22 \_1$ & 169.977 & -43.5861 & 35.0604 & 0.375218404 \\
\hline $169.858 \_-43.6377 \_44 \_1$ & 170.145 & -43.5215 & 27.4434 & 0.631124616 \\
\hline $169.852 \_-43.5081 \_30 \_1$ & 169.881 & -43.5741 & 39.3085 & 0.665910870 \\
\hline $169.684 \_-43.6178 \_34 \_1$ & 169.816 & -43.5790 & 36.5253 & 0.626325142 \\
\hline $169.597 \_-43.6853 \_32 \_1$ & 169.943 & -43.6471 & 32.8633 & 0.570130847 \\
\hline $169.892 \_-43.6417 \_24 \_1$ & 170.048 & -43.5664 & 36.915 & 0.537327395 \\
\hline $169.696 \_-43.7224 \_42 \_1$ & 169.892 & -43.6296 & 46.6816 & 0.618644100 \\
\hline $169.652 \_-43.6787 \_30 \_1$ & 169.900 & -43.5574 & 44.7772 & 0.693277494 \\
\hline $169.906 \_-43.5015 \_42 \_1$ & 170.068 & -43.4838 & 50.3926 & 1.14607093 \\
\hline $169.632 \_-43.6893 \_36 \_1$ & 169.794 & -43.6184 & 56.6426 & 0.565045193 \\
\hline $170.14 \_-43.4896 \_44 \_1$ & 170.223 & -43.4697 & 49.415 & 1.341339727 \\
\hline $170.202 \_-43.6127 \_18 \_1$ & 170.211 & -43.5720 & 33.0097 & 0.542011396 \\
\hline
\end{tabular}

Table A.1: LFE family locations. The first column indicates the LFE family ID. The second, third and fourth columns correspond respectively to the longitude, latitude and depth of the source for the LFE family. The final column represent the values obtained for the LFE family covariance (COVr). 


\section{A.3.3 LFE families redundancy}

With a cross-channel coherency threshold of 0.2, we observe 57 LFE families that are redundant, which can then be grouped into 17 new families. Those families are highlighted in Table A.2 below.

\begin{tabular}{|c|c|c|}
\hline Family ID & Redundant families & Cross-channel coherence \\
\hline \multirow{2}{*}{$169.655-43.7435{ }_{-} 40 \_1$} & 169.841_-43.868_26_1 & 0.25770486273 \\
\hline & $169.626 \_-43.7144 \_34 \_1$ & 0.29999146628 \\
\hline \multirow{2}{*}{ 169.618_-43.6747_38_1 } & $169.638-43.6641 \quad 36 \quad 1$ & 0.295987810099 \\
\hline & $169.71 \_-43.737 \_42 \_1$ & 0.248513727998 \\
\hline \multirow{2}{*}{$169.8 \_-43.5795 \_26 \_1$} & 169.814_-43.7489_34_1 & 0.243964271134 \\
\hline & $170.052-43.8019-44 \_1$ & 0.203818857321 \\
\hline \multirow{4}{*}{$169.823-43.6338 \_42 \_1$} & 169.837_-43.6483_36_1 & 0.300396074682 \\
\hline & $169.814-43.7489 \_34 \_1$ & 0.254252154478 \\
\hline & $169.921-43.516 \_18 \_1$ & 0.233363219718 \\
\hline & $170.179 \_-43.7133 \_16 \_1$ & 0.20144866636 \\
\hline $169.852 \_-43.5081 \_22 \_1$ & $169.785 \_-43.5649 \_44 \_1$ & 0.209074825782 \\
\hline \multirow{2}{*}{ 169.638_-43.6641_36_1 } & 169.618_-43.6747_38_1 & 0.295987813992 \\
\hline & 169.89_-43.7317_44_1 & 0.222486902628 \\
\hline \multirow{2}{*}{ 169.837_-43.6483_36_1 } & $169.823-43.6338 \_42 \_1$ & 0.300396013215 \\
\hline & 169.846_-43.5332_32_1 & 0.239976247462 \\
\hline \multirow{3}{*}{ 169.846_-43.5332_32_1 } & $169.837 \_-43.6483 \_36 \_1$ & 0.239976233436 \\
\hline & $169.814 \_-43.7489 \_34 \_1$ & 0.222823482644 \\
\hline & $170.052 \_-43.8019 \_44 \_1$ & 0.216422292643 \\
\hline $169.742 \_-43.6761 \_16 \_1$ & $169.733 \_-43.6364 \_16 \_1$ & 0.222196560353 \\
\hline \multirow{3}{*}{$169.626 \_-43.7144 \_34 \_1$} & $169.655 \_-43.7435 \_40 \_1$ & 0.299991427484 \\
\hline & $169.936-43.6854{ }^{-} 44-1$ & 0.2373173206 \\
\hline & $169.704-43.7621 \_44 \_1$ & 0.28195404553 \\
\hline \multirow{2}{*}{ 169.814_-43.7489_34_1 } & $169.823 \_-43.6338 \_42 \_1$ & 0.254252153375 \\
\hline & $170.052 \_-43.8019 \_44 \_1$ & 0.204340198853 \\
\hline \multirow{4}{*}{$169.91-43.5663{ }^{4}{ }^{4}{ }_{-} 1$} & $169.89 \_-43.7317 \_44 \_1$ & 0.2106624048 \\
\hline & $169.84 \_-43.5583 \_40 \_1$ & 0.254378123623 \\
\hline & $169.912-43.4763 \_40 \_1$ & 0.239718086735 \\
\hline & $170.06 \_-43.5319 \_18 \_1$ & 0.223649000296 \\
\hline \multirow{3}{*}{$169.921 \_-43.516 \_18 \_1$} & $169.823-43.6338 \_42 \_1$ & 0.233363226211 \\
\hline & 169.837_-43.6483_36_1 & 0.223510141795 \\
\hline & $170.052-43.8019-44 \_1$ & 0.217042058491 \\
\hline \multirow{2}{*}{$170.052-43.8019 \_44 \_1$} & 169.846_-43.5332_32_1 & 0.216422360746 \\
\hline & 169.814_-43.7489_34_1 & 0.204340239418 \\
\hline \multirow{2}{*}{$170.122 \_-43.4102 \_40 \_1$} & $169.89 \_-43.7317_{-} 44_{-} 1$ & 0.202553079943 \\
\hline & $170.085 \_-43.4962 \_44 \_1$ & 0.328647188635 \\
\hline
\end{tabular}




\begin{tabular}{||c|c|c||}
\hline $170.085 \_-43.4962 \_44 \_1$ & $170.193 \_-43.573 \_42 \_1$ & 0.230412397534 \\
& $169.863 \_-43.6126 \_38 \_1$ & 0.216271373151 \\
\hline $170.179 \_-43.7133 \_16 \_1$ & $169.936 \_-43.6854 \_44 \_1$ & 0.211023899236 \\
& $169.823 \_-43.6338 \_42 \_1$ & 0.201448742766 \\
& $170.193 \_-43.573 \_42 \_1$ & 0.22855846682 \\
\hline
\end{tabular}

Table A.2: LFE family redundancy. The first column indicates the LFE family ID that was used as a reference in the cross-correlation calculation. The second column indicates all the LFE families that are supposed to be redundant with the LFE family mentioned in the first column. The final column represents the values obtained for the cross-channel coherency, calculated for each LFE family pair.

The detections for the redundant families are then combined together and a new stack can be generated for each new grouped family (Fig. A.31). Those new stacks are less emergent and possess a higher signal-to-noise ratio than the original stacks for each individual family (Fig. A.32). Regrouping redundant LFE families allow the generation of stacks with signal-to-noise ratios that are $\sim 7 \%$ higher. 


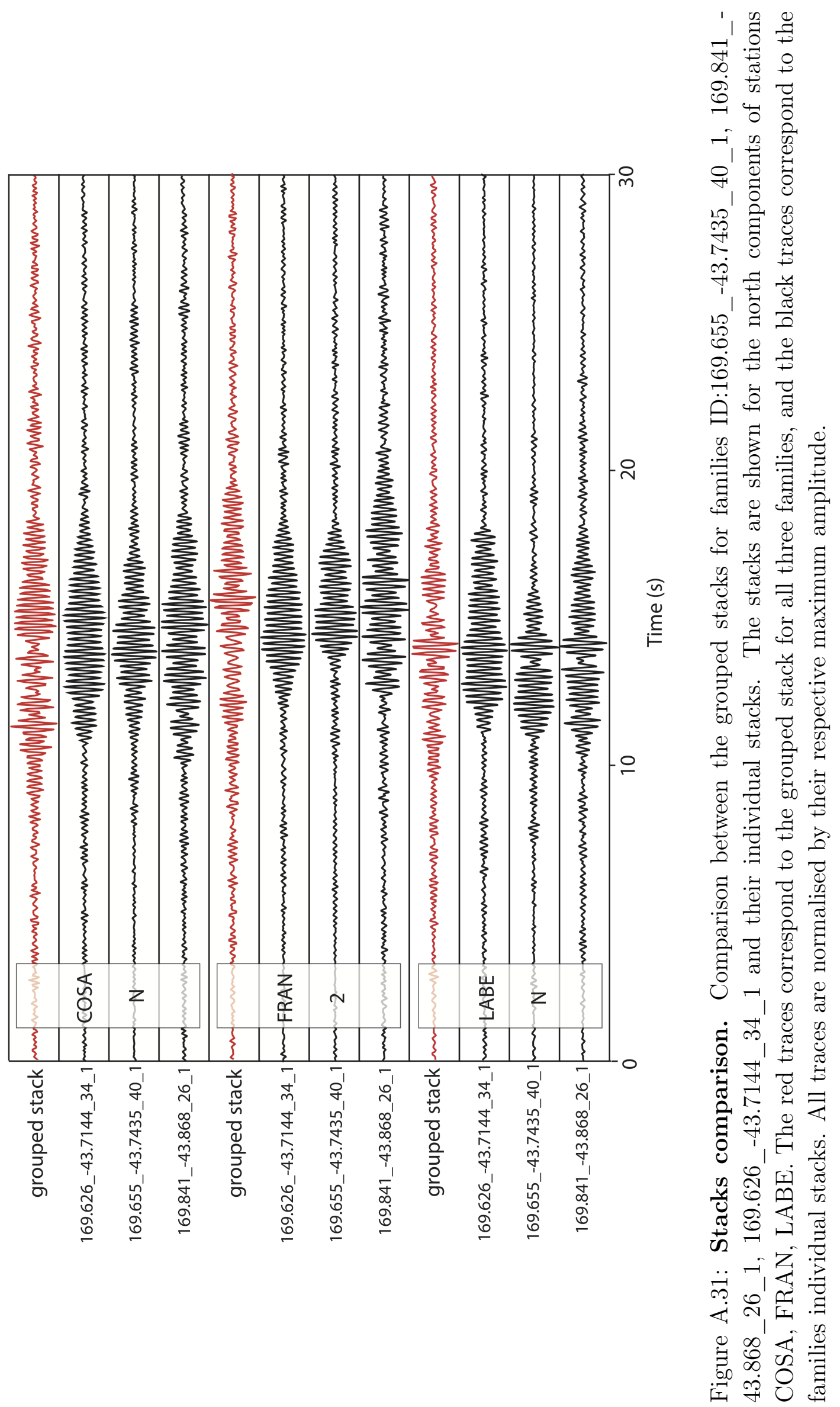


Average SNR $=7.07$

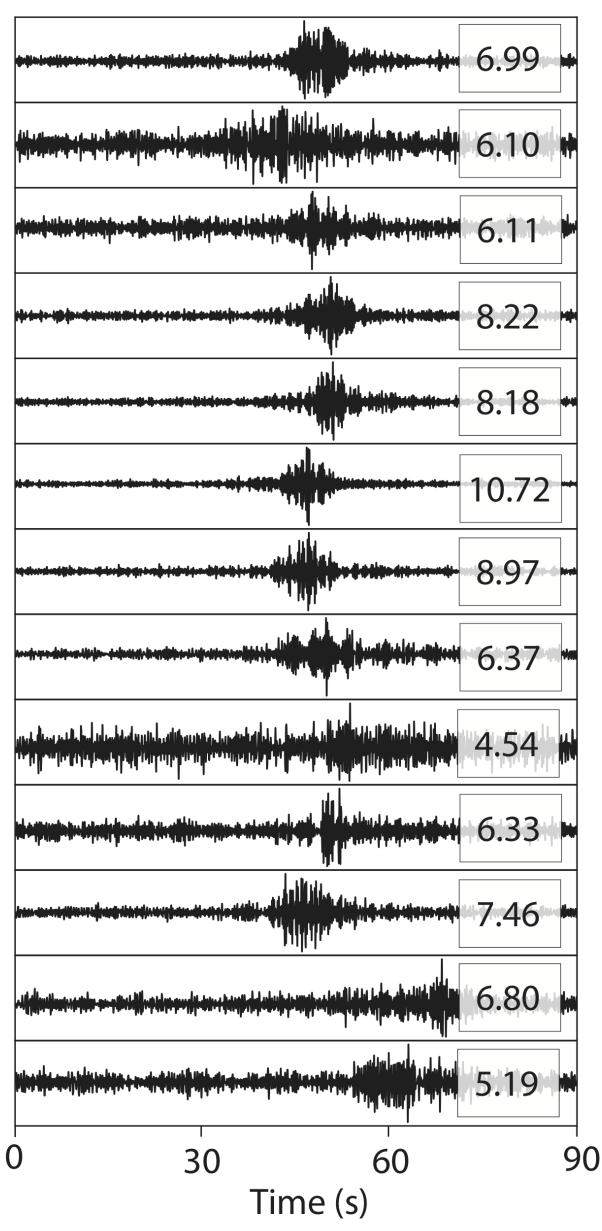

Average SNR $=6.65$

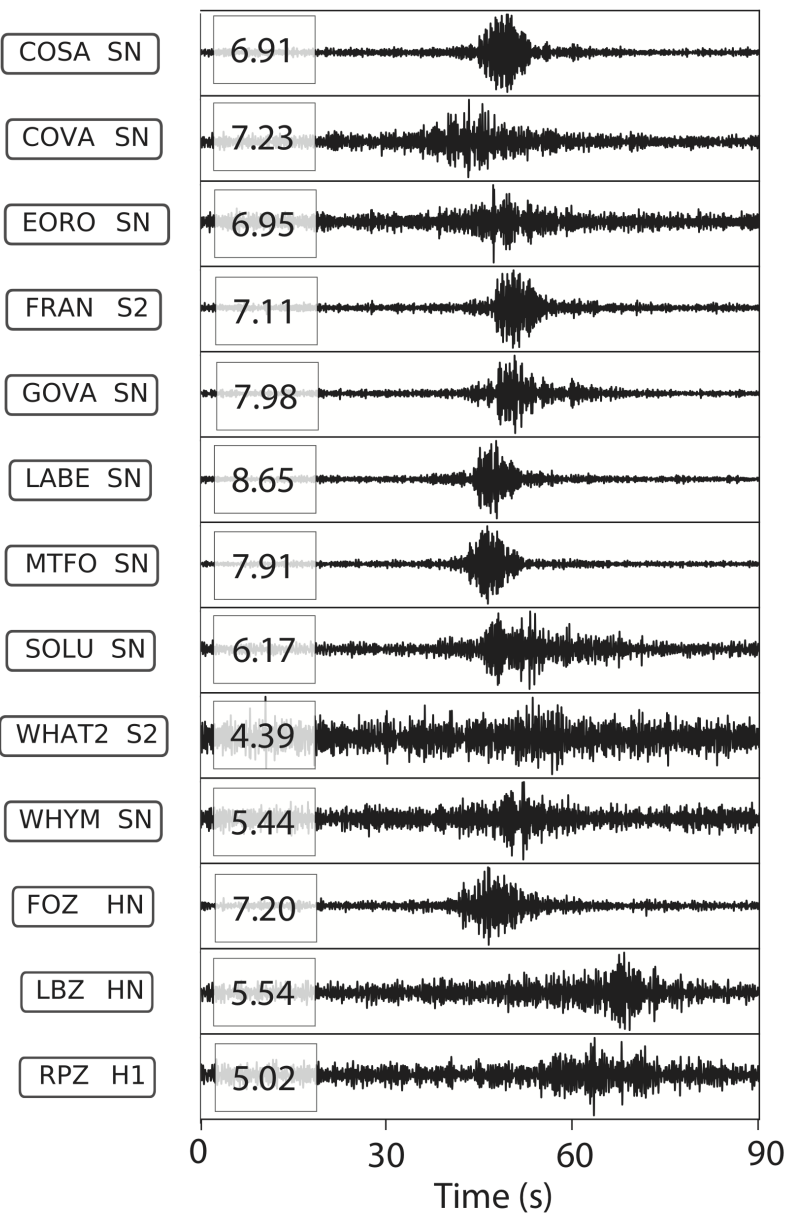

Figure A.32: Stack signal-to-noise ratios comparison. Comparison between the signal-to-noise ratios of the grouped stack for families ID:169.655_43.7435_40_1, 169.841_-43.868_26_1, 169.626_-43.7144_34_1 and the individual stack for LFE family ID: 169.841_-43.868_26_1. The stacks are shown for the north components for all stations. The signal-to-noise ratio value is indicated in a white box on the trace. 


\section{A.3.4 Anisotropy}

Here we show an example of an individual detection (made on 2015/01/17 at 01:49:19 UTC time) for LFE family ID: 169.641_-43.729_44_1 at station FRAN, at all three channels (Fig A.33). If we look at the horizontal channels, we can see a slight delay in the arrival of the S-phase in channel 2 when compared to channel 1. We also see a slight delay when looking at the linear stack for that same family (Fig. A.34). Since this delay appears on the stack, it is a feature that is consistent with the majority of the others individual detections originating from this LFE source. This delay in S-arrival across channels could indicate crustal anisotropy in this LFE source area. A systematic analysis of crustal anisotropy from LFEs could potentially be done for the New Zealand Southern Alps in order to provide useful ray-paths for analysis of splitting directions in the crust.

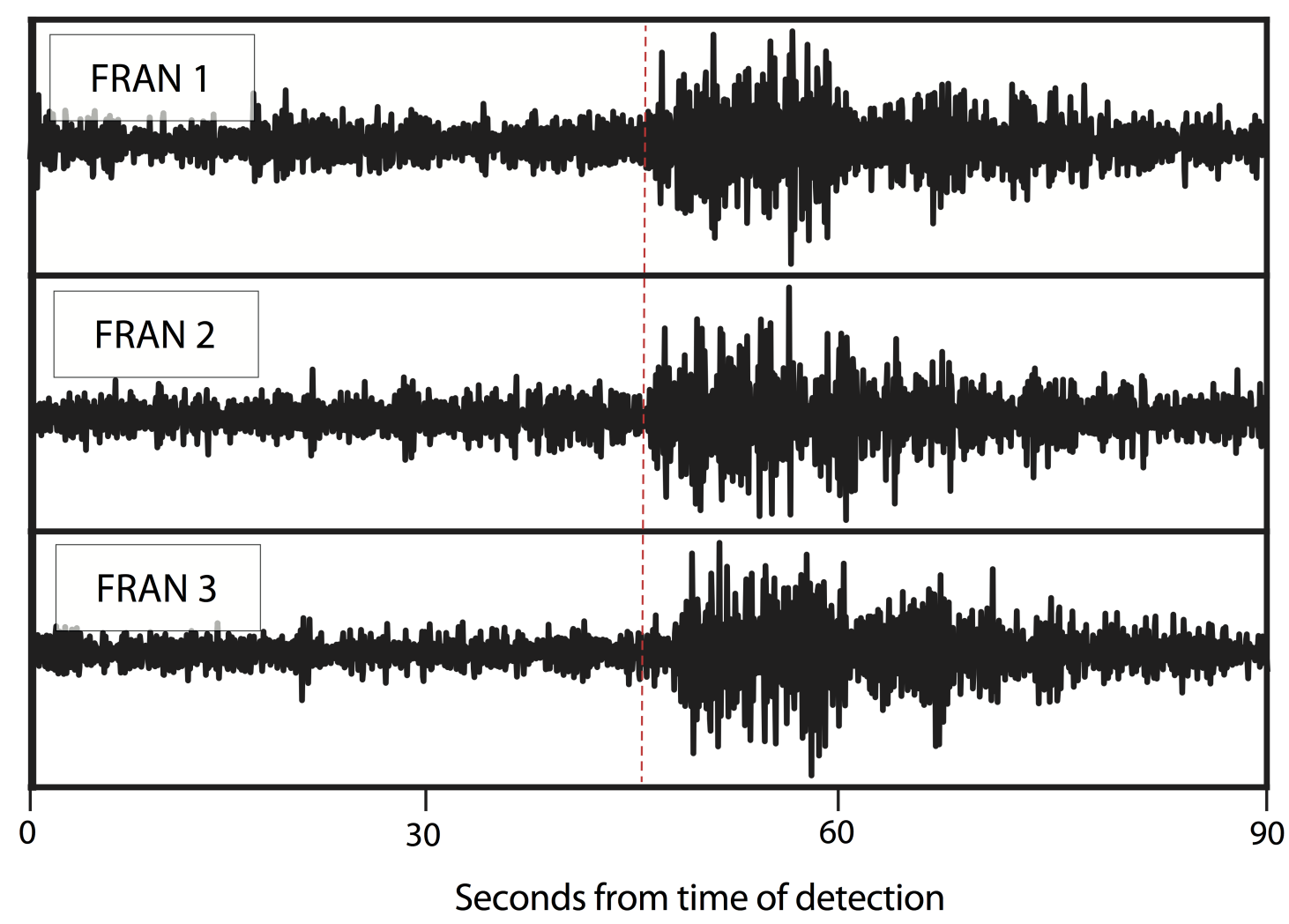

Figure A.33: LFE individual detection example. Example of an individual detection for family ID:169.641_-43.729_44_1. The waveforms of three components are shown for station FRAN. The dashed red line indicates the S-arrival on channel 1. 


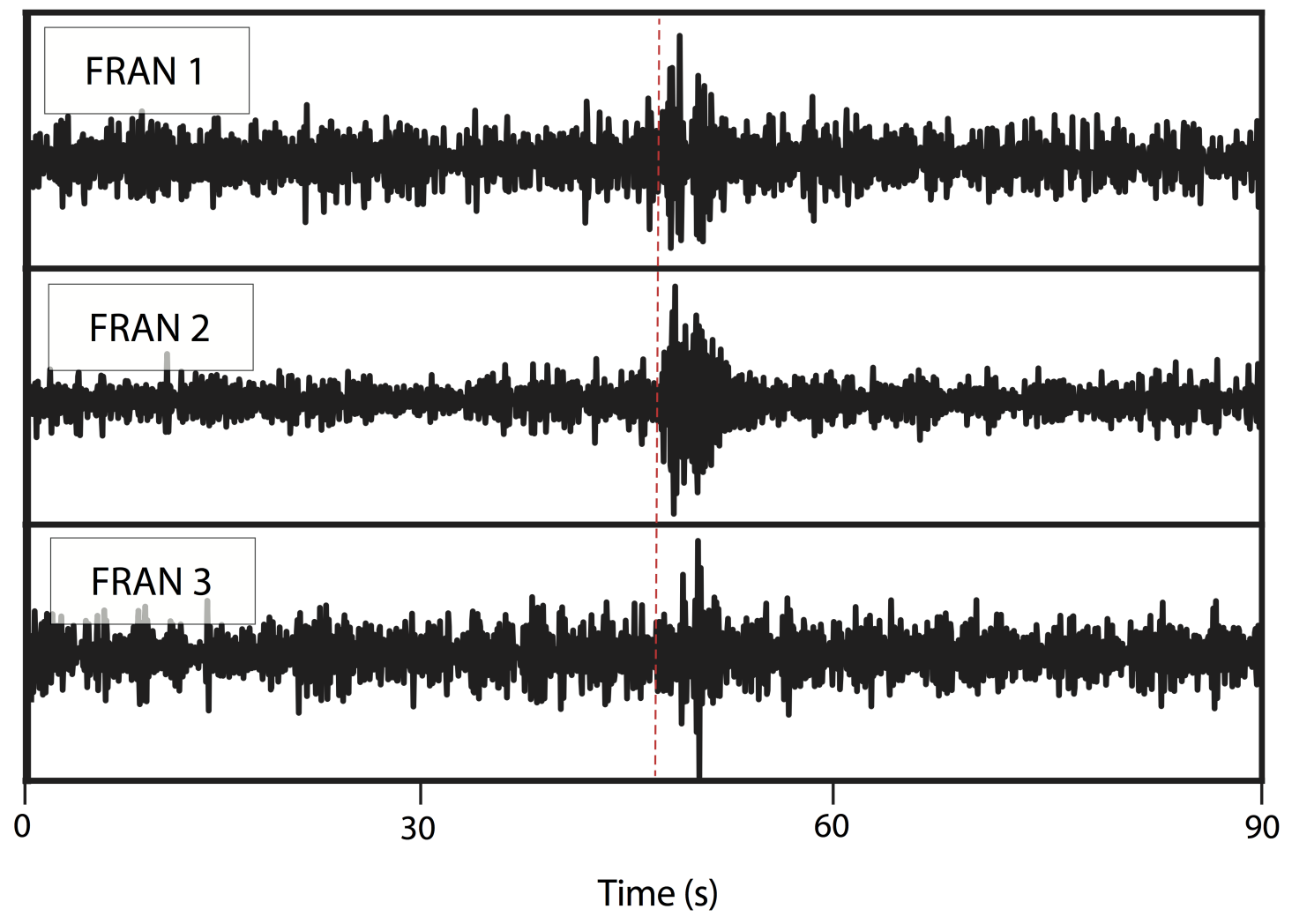

Figure A.34: LFE stack example. Linear stack for family ID:169.641_43.729_44_1. The stacks of three components are shown for station FRAN. The dashed red line indicates the S-arrival on channel 1. 


\section{B. Deep Fault Drilling Project - 2}

At the beginning of this PhD project, I participated in the Deep Fault Drilling Project - Phase 2 (DFDP-2) effort in Whataroa (West Coast of New Zealand). This project started in August 2014 and was completed in January 2015. The drill site is located in the Whataroa valley, an area that is part of the study area surveyed during this $\mathrm{PhD}$ project. The aim of the DFDP-2 project was to sample and evaluate the in-situ conditions of the central part of the Alpine fault, prior to a large earthquake $\left(M_{w} 7-8\right.$, Sutherland et al., 2007).

As part of this collaboration, I co-authored several publications during this $\mathrm{PhD}$ : Sutherland et al. (2017); Chamberlain et al. (2017a); Toy et al. (2017); Townend et al. (2017). I took part in this project between October and November 2014. During this time, I mainly helped with the real time seismic monitoring effort.

\section{B.1 Real time seismic monitoring}

A detailed overview of the monitoring effort has been published in Chamberlain et al. (2017a). Here, I only summarise the involvement of the duty seismologist (i.e. role that I played) in the real time seismic monitoring effort. The duty seismologist worked in a team comprising several seismologists, including several senior seismologists. Each duty seismologist worked a daily 12-hour shift. I covered the 4pm-4am shift between October $16^{\text {th }}, 2014$ and November, $26^{\text {th }} 2014$ ).

The real-time seismic monitoring was based on a traffic light system (detailed in Fig. B.1). A response plan was indeed put in place for earthquakes that were assumed to necessitate changes in the drilling operations. This response plan was based on a traffic light system (green/orange/red) that took into account the background seismicity in the Whataroa region, prior to drilling. In essence, if more than one small local earthquakes of magnitudes $1 \leq \mathrm{M} \leq 3$ was recorded within a 12-hour period and within $3 \mathrm{~km}$ of the drill site, then the orange light system would have been implemented. This step would have implied a more cautious approach to the drilling operations. If now, an earthquake of $\mathrm{M} \geq 3$ was recorded within $3 \mathrm{~km}$ of the drill site, the red light scheme would have been put in place, 
hence stopping all drilling activity. During the entirety of the drilling project, no events required a change of the light system from the green light.

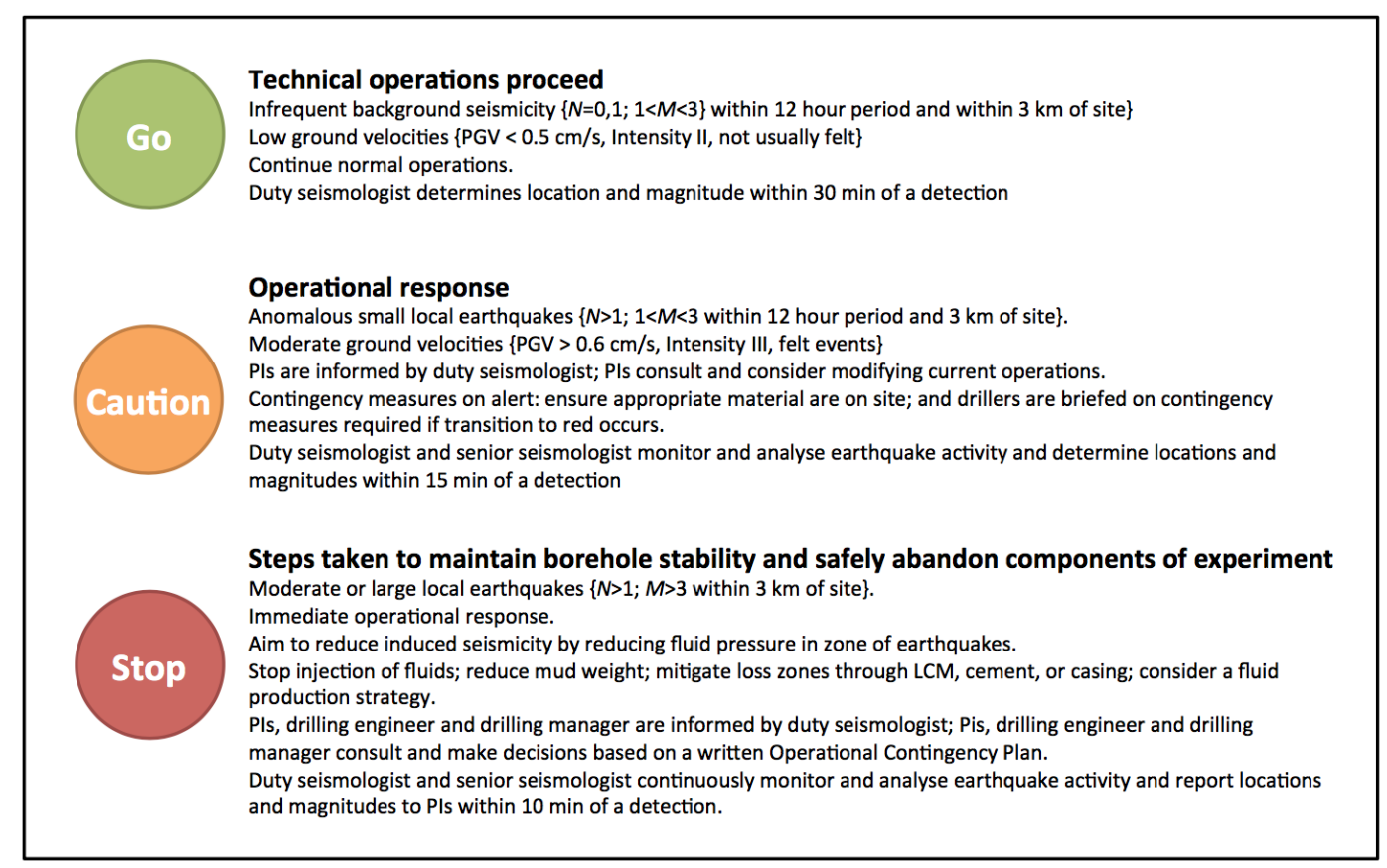

Figure B.1: Traffic light system used to react to the seismicity happening near the DFDP-2 drill site. From Sutherland et al. (2015). Traffic light highlighting the different steps taken to respond to the seismicity occurring in the DFDP-2 drill site area. This traffic light is divided into 3 status: (1) the Green/Go light, corresponding to a background seismicity and leading to a continuation of the drilling operations; (2) the Orange/Caution light, equivalent to an anomalous activity corresponding to small magnitude earthquakes, this status can lead to a change in operational response; and (3) the Red/Stop light, which coincides with the occurrence of moderate or large earthquakes near the drill site, this light scheme is automatically accompanied by a stop in the drilling operations.

The real-time seismic analysis was performed using the open-source software Rtquake integrated within Seisan (Utheim et al., 2014; Havskov and Ottemoller, 1999). This was done to facilitate P- and S-arrivals picking and the waveforms graphical output. All the steps involved in the processing of the data are detailed in Fig. B.2. Rtquake was configured to identify peaks on all the channels of the seismograms. When the number of identified peaks exceeded a certain threshold, in a specific time interval, the system issued a detection alert via email. This email alert contained (1) an estimate of the P- and possible S-arrivals, as well as (2) an estimate of the magnitude and finally (3) an estimate of the preliminary location. 
All the events detected by Rtquake were then verified by the duty seismologist. The duty seismologist then had two tasks. The first one was to check the emailed information (i.e.initial location, image file containing the waveforms and initial automatic picks). The duty seismologist was only interested in events occurring close to the drill site, so he manually discarded any events that was deemed too far ( $\geq 30 \mathrm{~km}$ distance from the drill site). Since the automatic picks on the Swave were unreliable, the duty seismologist would visually check the S-P times to estimate the distance to the drill site. Hence, any event that presented a S-P times $\geq 3 \mathrm{~s}$, was not kept by the duty seismologist for further analysis. If, however, the earthquake was considered relevant to the drilling operations, it was added to a catalogue.

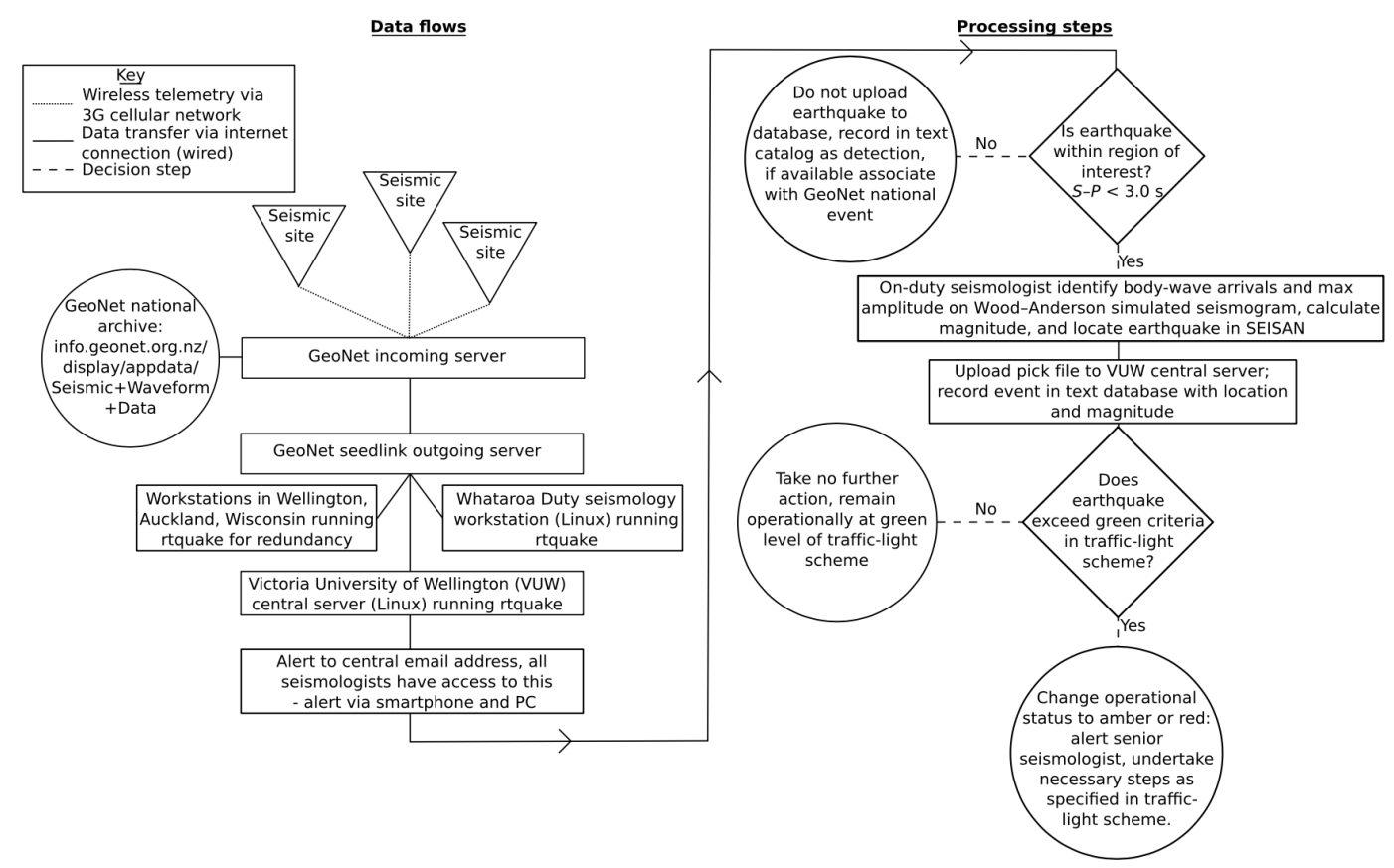

Figure B.2: Flow diagram of the steps undertaken by seismologists during real-time monitoring. From Chamberlain et al. (2017a). Flow diagram of the data and processing steps undertaken during the real-time seismic monitoring.

The second task involved obtaining the waveform data and uploading the data in SEISAN in order to repick the event. Once the arrival times were correctly picked on the event, the duty seismologist had to pick the amplitudes in order to estimate a magnitude for the event. Finally, the duty seismologist computed the event location and its magnitude. The correctly picked earthquakes were then uploaded to a server and recorded in a text database with basic information, such as: time of detection, magnitude, duty seismologist name and notes.

An overview of the seismicity recorded during the entirety of the project can be 
viewed in Fig. B.3. A total of 1,121 events were detected during the drilling operations. However, only 494 events were deemed of interest, and were subsequently manually picked and located. Of these, 494 picked events, 137 local magnitudes were generated, ranging between 0.6 and 4.2 .

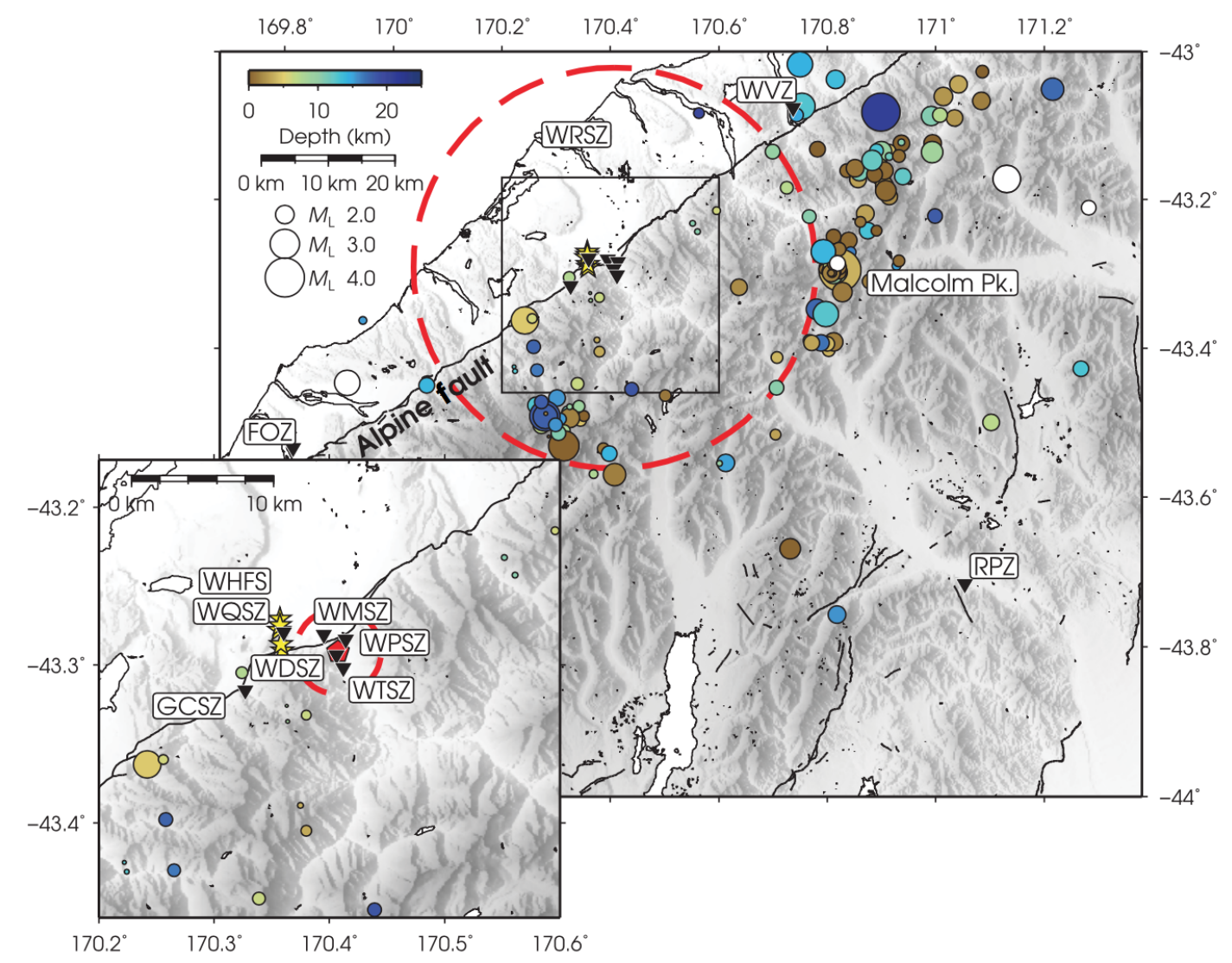

Figure B.3: Seismicity recorded during DFDP-2. From Chamberlain et al. (2017a). Main map: Earthquakes detected and located during the real-time seismic monitoring. The red dashed circle indicates a $30 \mathrm{~km}$ area surrounding the drill site. White circles represent earthquakes that are deeper than $25 \mathrm{~km}$. Inset: Zoomed view surrounding the drill site (shown in a red pentagon). The dashed red circle highlight an area of $3 \mathrm{~km}$ surrounding the drill site. Black triangles correspond to the seismic stations used to monitor the seismicity in the area. Finally, the yellow stars indicate explosions at the Whataroa Quarry (WQSZ site).

An interesting earthquake sequence occurred during one of my shifts: the Malcom Peak sequence. We recorded this sequence between October 22, 2014, 04:17-23 and October 23, 2014, 21:12:00 UTC. The preliminary locations and magnitudes were estimated in hours following the event, on site. However, Chamberlain (2016), did additional work as part of his $\mathrm{PhD}$, relocating the events and updating the magnitudes calculations, at a later stage. Overall 26 events were detected as 
part of this sequence, with magnitudes ranging from $M_{L} 1.0$ to 4.2. We also detected two earthquakes of $M_{L}$ 1.9, 13 and 6 minutes prior to the largest $\left(M_{L} 4.2\right)$ earthquake in the sequence. Unfortunately, we were not able to determine whether this sequence constituted a swarm or a mainshock-aftershock sequence as our catalogue was incomplete. This sequence occurred $30 \mathrm{~km}$ east of the drill site, $\sim 20 \mathrm{~km}$ southeast of the Alpine Fault surface trace. 
Appendix B. Deep Fault Drilling Project - 2 


\section{Field work reports}

This section gathers all the field reports that I have written for the period that I spent leading the maintenance of the SAMBA network (September 2015-October 2016). The servicing trips were bi-annual and generally lasted 2 weeks. It is worth noting that I participated in two others field trips (October 2014 and March 2015) when Calum Chamberlain was still in charge on the network maintenance. He spent those trips teaching me how to take over the maintenance and data collection for this network. As leader over that period, he wrote the reports himself and those are not featured here. All photographs are mine, unless otherwise stated.

\section{C.1 September 2015}

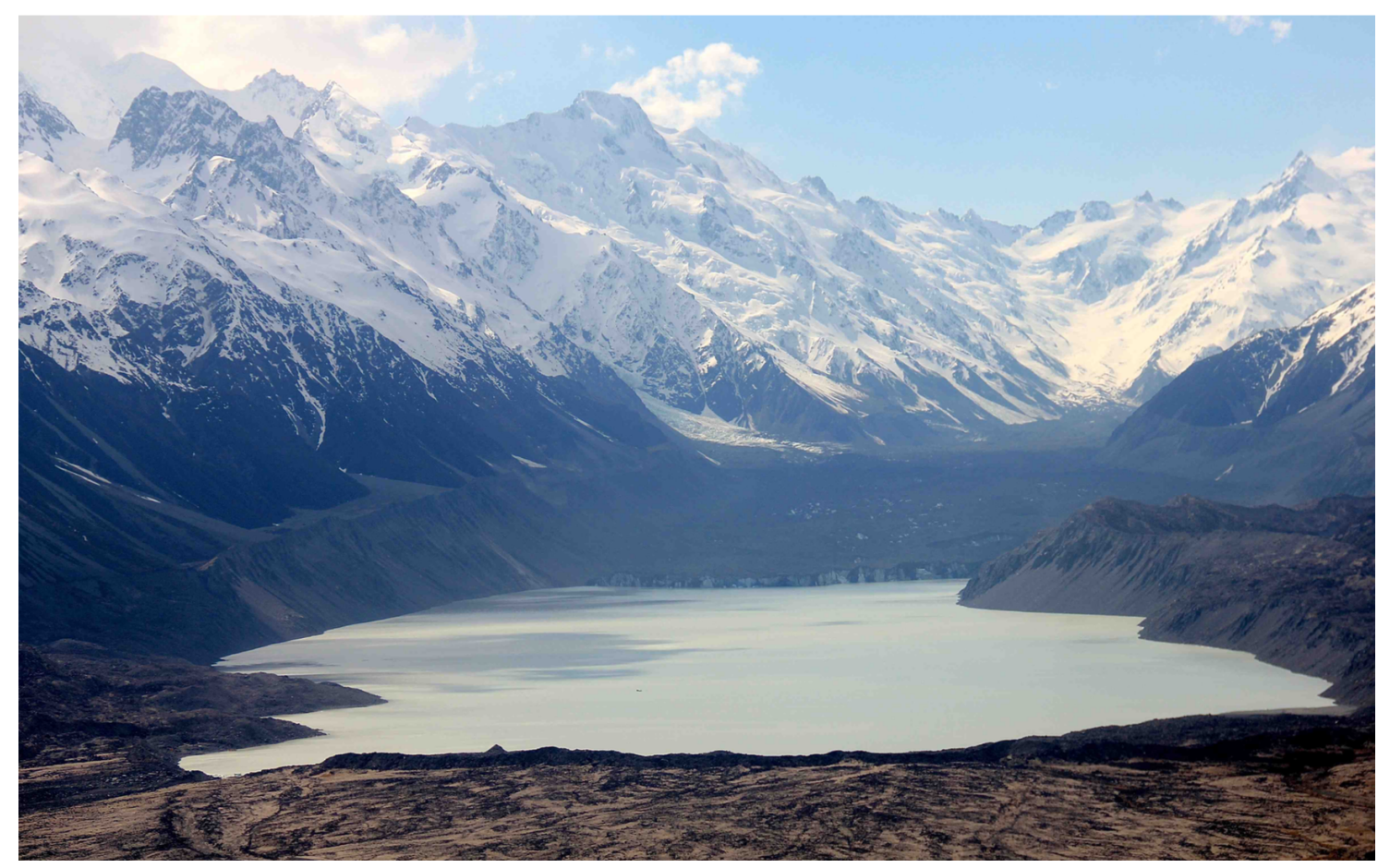

View of the Tasman Lake while flying to our De La Bêche site. 


\section{C.1.1 Summary}

This report summarises the service run for the SAMBA, the DFDP and the Japanese seismic networks. For this service trip the field work team consisted of:

- Laura-May Baratin, VUW;

- Adrian Benson, VUW;

- Steve Jenkins, VUW;

- Hubert Zal, VUW.

This trip began on the West Coast with the servicing of most of the SAMBA stations, the DFPD seismic sites, the Japanese array and the installation of two solar panels on the DFDP-2 container. We swapped all of the DFDP seismic sites' DAS, serviced successfully the Japanese seismometers and all of the SAMBA sites on the West Coast with the exception of the COVA site as the station was entirely covered with snow. We replaced the DAS at SOLU and MTBA with new ones and had to fly back to those two sites as we realised that the DAS hadn't been configured properly. We then drove to the East Coast and serviced LABE and GOVA. LABE wasn't powered on arrival, the site had probably been buried by snow during winter. As for the GOVA site, we had to reinstall part of it because the site had been colonised by lizards.

In total, 20 sites were serviced, two solar panels were installed on the DFDP-2 container and three Distributed Temperature Sensing (DTS) measurements were performed for Lucie Janku-Capova at the DFDP-2 borehole.

\section{C.1.2 Daily log}

\section{Tuesday 22 September}

We took the 8AM Bluebridge ferry to Picton, the ride was turbulent and three of us got sea sick. We arrived in Picton just after 1pm and split into two teams. Steve and Hubert rented a car and drove straight to the West Coast. Adrian and I took the Geophysics car and stopped in Blenheim to buy some tools and gear to install the solar panels on the DFDP-2B container. Adrian didn't get much sleep 
the day before and was too tired to drive at night, so we stopped in Murchison for the night.

\section{Wednesday 23 September}

Spent the day mostly driving to Franz Josef. We arrived in Whataroa around 5pm where we stopped because Steve and Hubert had some issues with their gravity measurements; their GPS wasn't working and Adrian fixed it. We got the DFDP keys from the White heron and went to the DFDP-2 container where Adrian took some measurements in order to build the solar panels frame. We arrived in Franz and stopped at the Fox and Franz Josef Heliservices office to arrange the flight to Mt Fox (MTFO site) and to the Southern sites with James.

\section{Thursday 24 September}

Adrian and I flew from Fox Glacier to the MTFO site and the servicing went smoothly. Nothing to report there. We couldn't fly with James on that day as he was busy, but arranged to fly with him the next day. We then serviced EORO; the site was working and the servicing went smoothly. However after checking the data, I realised that we were missing one month of data for the EORO site (batteries discharged during winter). We might need to add another battery there to allow the site to last through winter without powering down. We serviced the FRAN site, where everything was running great, we just noticed that the power cables (connected to the solar panel) needed to be changed preventively. The stability of the box could also be improved as it is wobbly. We finished the day by servicing the COSA site where everything went smoothly. One of the locks couldn't be put back on so we put a cable tie in one of the hole and kept a lock in the other. We noticed that the GPS was covered by a lot of trees so did a bit of gardening. Adrian suggested that he could make a longer GPS cable in order to put the GPS near the solar panels instead of under the tree cover.

\section{Friday 25 September}

Adrian, Hubert and I flew with James in the morning to the COVA site but when we landed on site, we saw that the station had entirely been buried by snow and ice (Picture C.3). Adrian got out of the helicopter and realised that it was too icy and dangerous to stay and dig out the station (also would take a lot of time). 


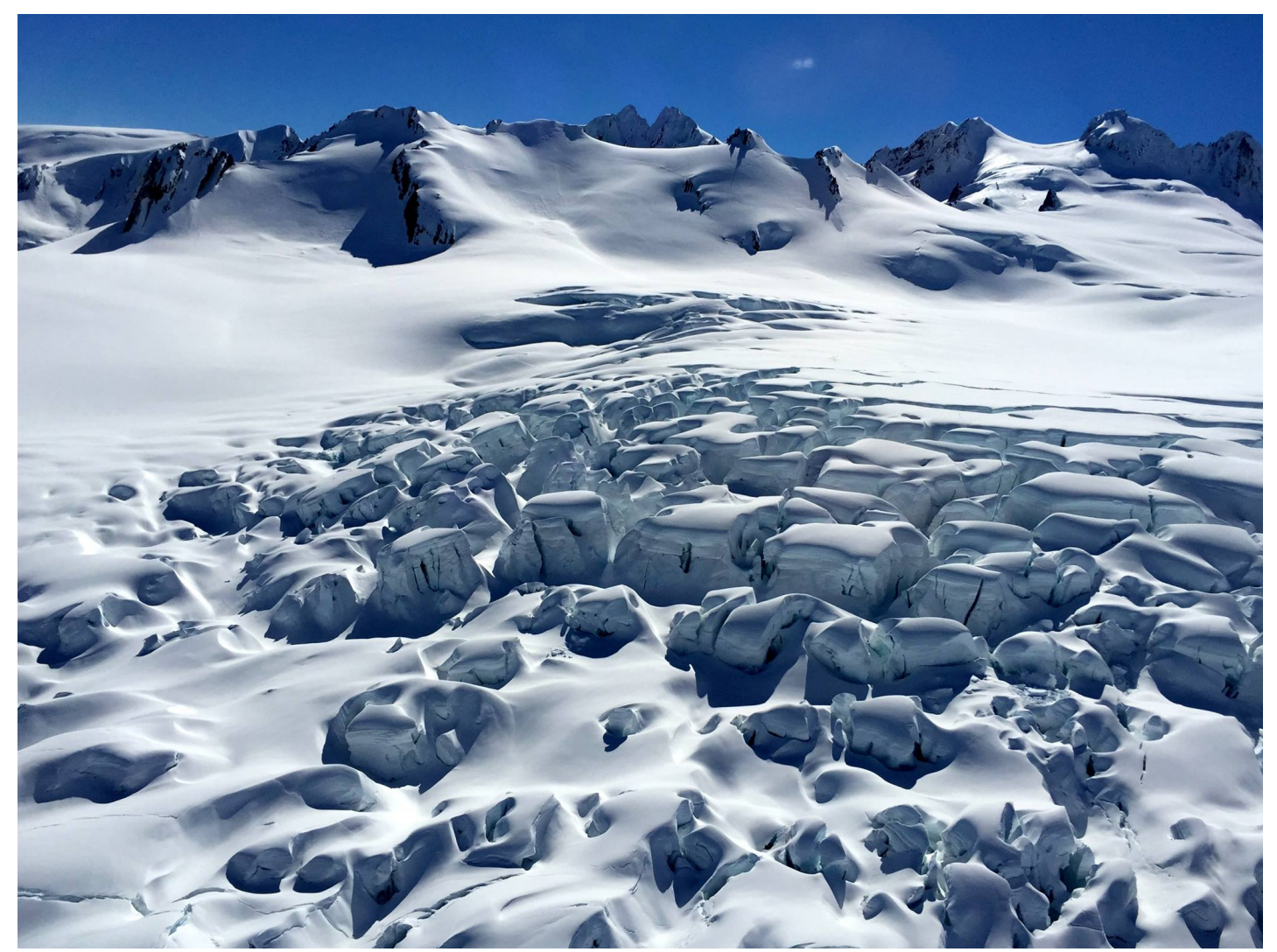

Figure C.2: View of the Fox Glacier, taken from the helicopter while flying to the Southern sites.

We then serviced the MTBA, SOLU and LARB site. When arriving at the MTBA site, we realised that the DAS has suffered a write error again (it wasn't dumping). We swapped the DAS at MTBA and reinstalled the same configuration files that were already on the DAS. When we arrived at SOLU where the DAS was working, both cards were full and it didn't seem stuck like the one at MTBA.

We swapped the DAS there too and went to service the LARB site where everything went smoothly. We then drove to Whataroa and flew from there to service WHYM. On our way there, we dropped Steve and Hubert on top of Mount Price (I think) where they did some gravity measurements. Everything went smoothly at the WHYM site and on our way back we picked up Steve and Hubert and flew to Mount Adams (I think...) so that they could do more measurements (Picture C.4). After checking the data for the SOLU and MTBA sites in the evening, we realised that too many files had been written for each day and that the SOLU site had only recorded a couple of days of data. As for the MTBA site, the cards seem corrupted and we couldn't read them.

We then checked both configuration files and noticed that the recording length 


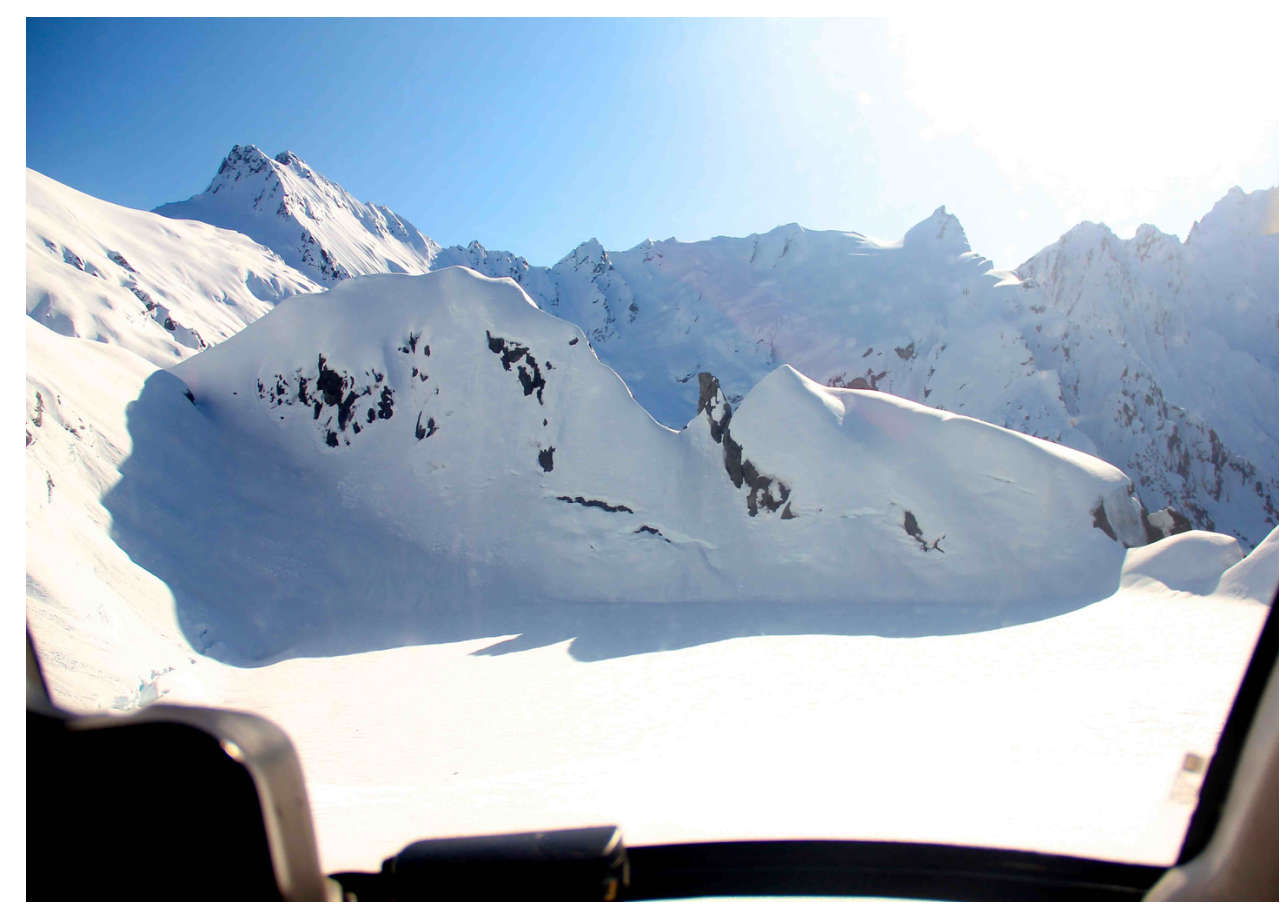

Figure C.3: Copland Valley station (COVA) entirely covered by snow.

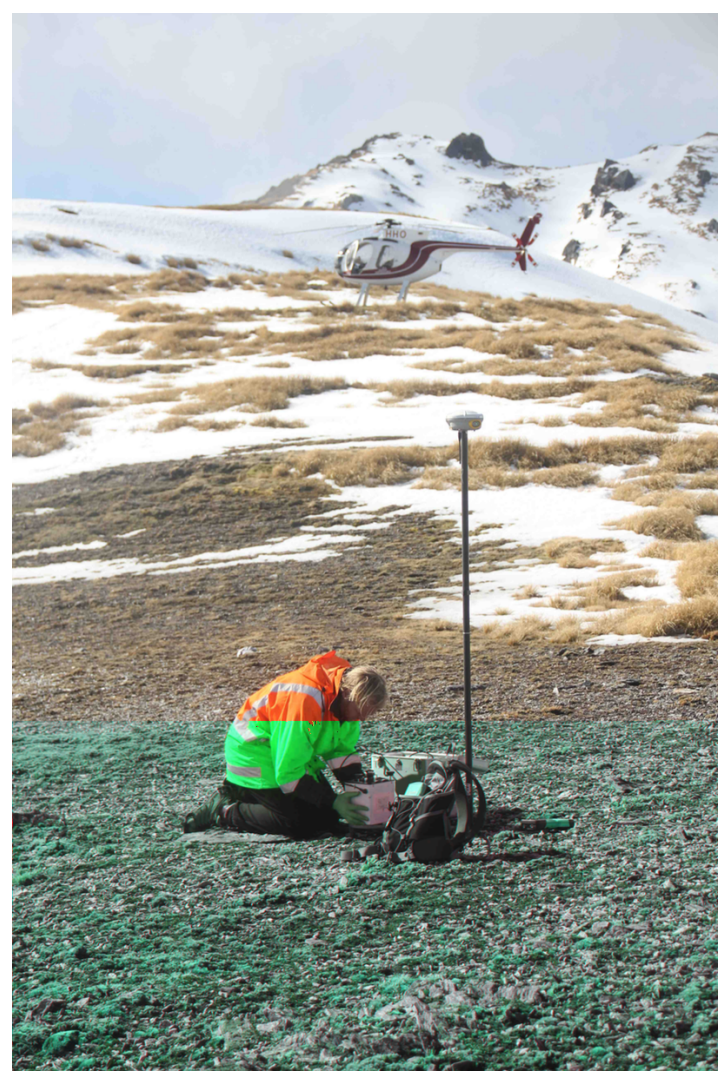

Figure C.4: Steve making some gravity measurements on top of Mount Adams.

was of .3600 seconds instead of 3600s. For SOLU, the gain was set on "unity" instead of "high", so we also updated that into the new configuration files. 


\section{Saturday 26 September}

We waited in Franz all morning to see if we would be able to go back to the MTBA and SOLU sites to fix the configuration files but James was already out flying and unreachable. We decided that we weren't going to wait all day as there was a chance we would be able to fly with him on Sunday. We then drove to Whataroa and serviced the quarry site where everything went smoothly.

\section{Sunday 27 September}

We drove to Whataroa in the morning to service some of the DFDP seismic sites. We went to the WMSZ site, where the servicing went smoothly; we swapped the DAS and reinstalled the settings for telemetry. We then went to the WPSZ site to swap the DAS and the GPS as they were IRIS equipment, we finally went to the WTSZ site where we had to swap the DAS and GPS twice as the one set up weren't working. We had to fly with James in the afternoon so didn't have the time to finish setting up this site. Steve, Hubert, Adrian and I flew back with James in the late afternoon to the SOLU and MTBA sites to fix the configuration files. There was a lot of clouds but James managed to get us to both sites where we updated the DAS' configuration files. Hubert made a cool short movie during this flight that you can see with the following link: http: //www . youtube. com/watch?v=KzBRpsMp8UA.

\section{Monday 28 September}

We went back to the WTSZ site to finish reinstalling the new gear and set up the telemetry. We then drove from Franz to Greymouth so that Adrian could buy what he would need to build the solar panels frame.

\section{Tuesday 29 September}

We serviced the WDSZ site and swapped the DAS and GPS as they were also IRIS equipment. We tried to service the Japanese seismometer in the container. When we arrived there, all the lights were turned off and we thought that the battery had died, so we turned the power off, changed the cards and then changed the batteries. When we powered the data logger again, there was an issue with the mother card as we hadn't stopped acquisition properly. We turned the data logger off as we wanted to try to service another Japanese seismometer before taking any 
action on that one. Late afternoon, we went to see if we could borrow a generator from Gordon Kelly for the next day but his wife asked us for an invoice number. So we called Kosta and figured that out.

\section{Wednesday 30 September}

We serviced successfully the Japanse seismometer in Whataroa. We then went to get the generator from Gordon Kelly. Adrian started building the solar panels frame outside of the container. In the meantime I swapped the DAS for the WBSZ site (borehole seismometer) and made 3 DTS measurements of $45 \mathrm{~min}$ each. The telemetry and DAS were set up for the WBSZ site but the DAS was not powered as we were waiting for the solar panels to be set up before starting data acquisition.

\section{Thursday 1 October}

We started the day by servicing the Japanese seismometer in Gaunt Creek but forgot to change the battery although we had already started acquisition (with the new cards in the data logger). Since data had been written on the cards, we couldn't stop and start acquisition again without reformatting the cards. So we had to go back to the container to get the DTS computer that was running on windows. I installed the Japanese software on that computer and reformatted all of the memory cards from the DFDP-1 and DFDP-2 Japanese seismometers. This process was relatively slow as for each memory card that had to be formatted, it would take 5-10 minutes. I also put the right settings in their "mother cards". Once the cards were ready for use, we successfully started acquisition on the DFDP-2 Japanese seismometer. We then went back to do their last seismometer at Gaunt Creek and everything went smoothly.

\section{Friday 2 October}

We finished installing the solar panels on the DFDP-2 container (Picture C.5) and then gave back the DFDP keys to the White Heron. As it was already late, we stopped in Wanaka for the night. 


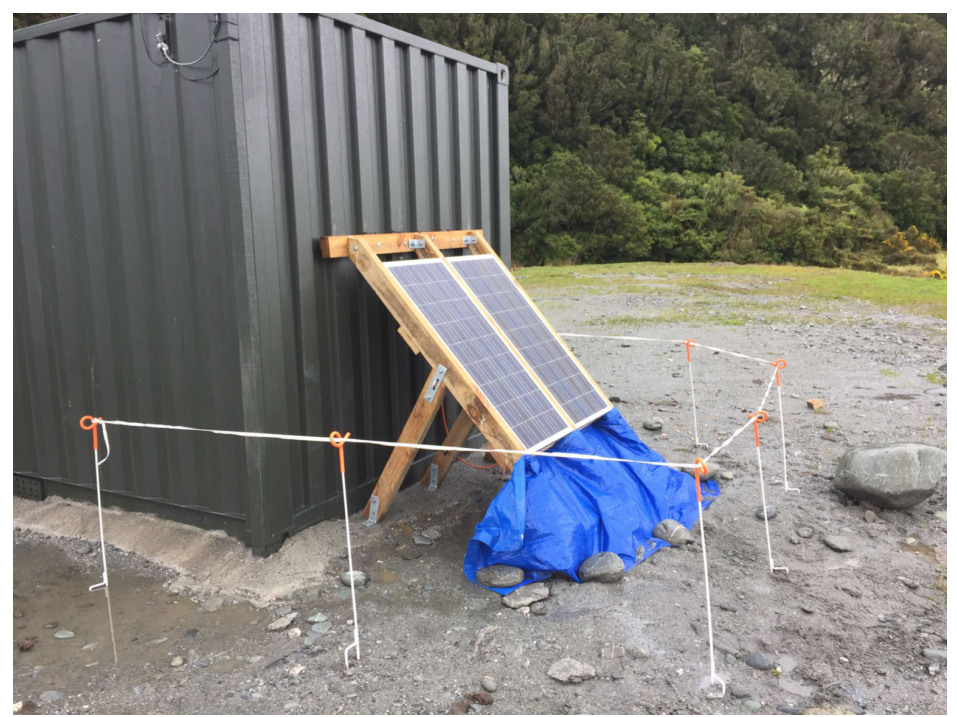

Figure C.5: Solar panels frame installed on the outside of the DFDP-2 container.

\section{Saturday 3 October}

We drove to Mt. Cook despite the Mt. Cook Helicopter Line telling us that it would be too windy to fly. We arrived there just after noon and they told us we might be able to fly to the LABE site at $3 \mathrm{pm}$. At $2 \mathrm{pm}$, their lead pilot told us he would be able to fly us and land at the old De La Bêche hut site.

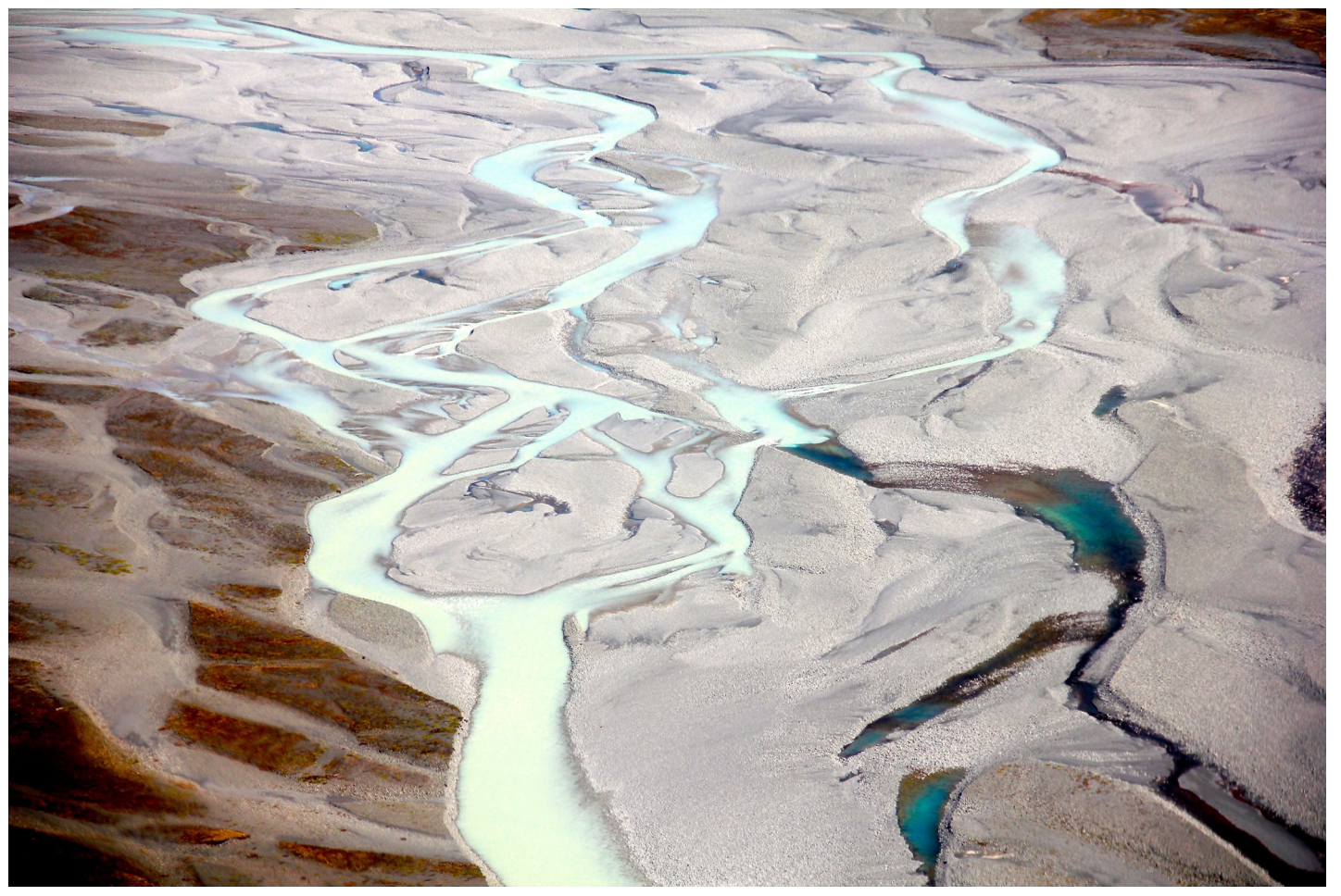

Figure C.6: View of the Tasman River, taken from the helicopter while flying to the LABE site. 


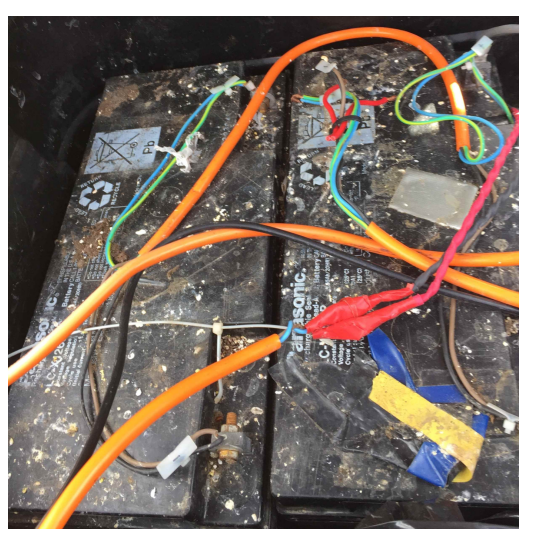

(a)

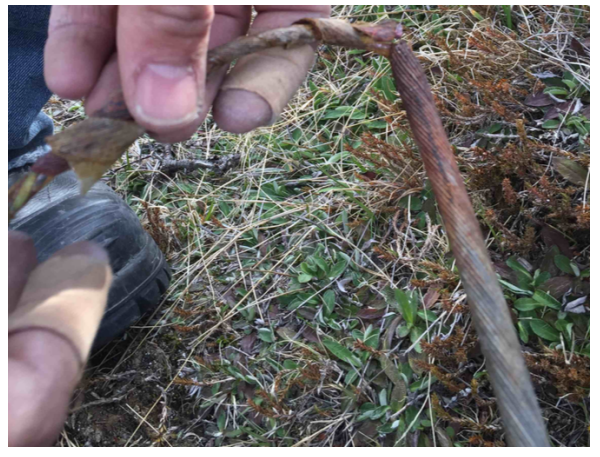

(b)

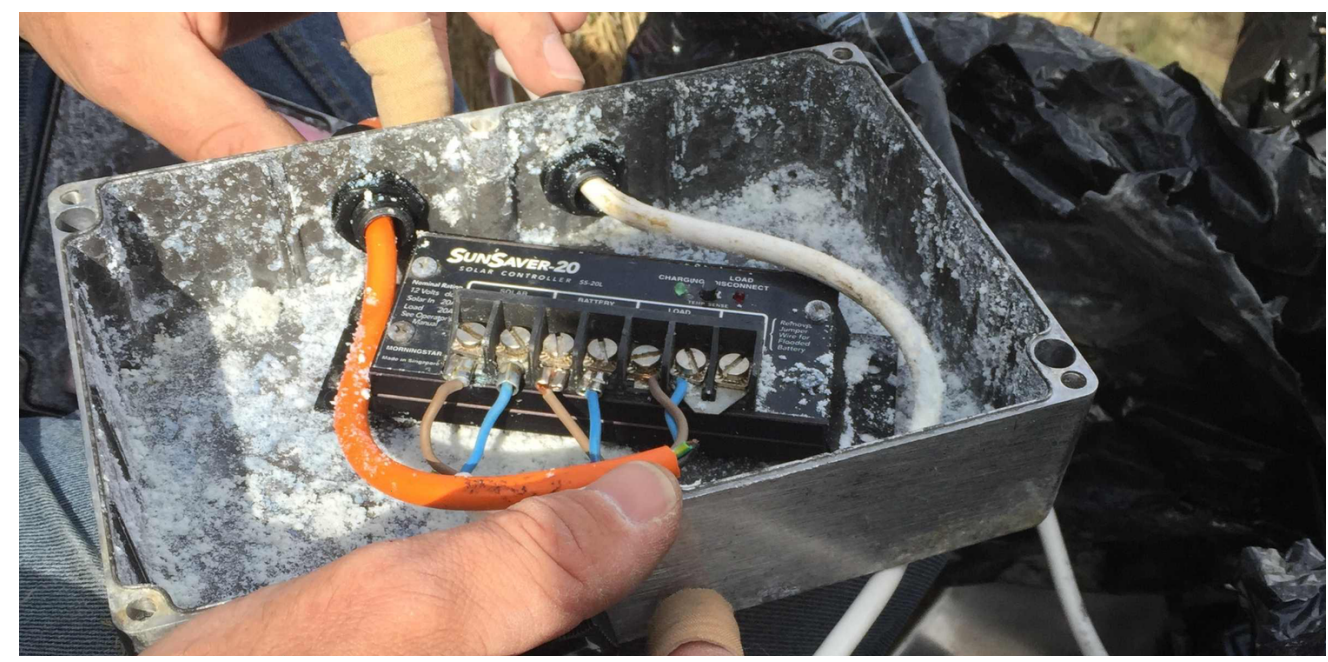

(c)

Figure C.7: Inside of the GOVA site box: (a) batteries covered in poo, (b) corroded sensor cable and (c) corroded solar controller box

We arrived there after a very windy flight and noticed that the DAS was off and that there was some snow at the bottom of the solar panel. Both batteries were discharged so we took them off, put another battery instead and swapped the DAS. The site was running smoothly when we left. We then drove to GOVA as it wasn't too late and arrived on site just before $6 \mathrm{pm}$. The DAS was off, the solar controller had died and everything was covered in what we thought to be rat poo (Picture C.7). Two batteries had died and the other two were discharged (but could be charged again). Since we had already replaced batteries at the LABE site, we didn't have any spare/charged batteries for GOVA and it looked like the site was in bad shape. So we decided to leave and come back in the morning to fix everything. 


\section{Sunday 4 October}

Fixing the GOVA site took about 3-4 hours as the conditions were a bit difficult (working on a slope under $100 \mathrm{kmh}$ winds and rain). We changed the batteries, solar controller, DAS and box. We fixed the sensor cable and the solar panel A-frame that was wobbly. We realised that it was lizards that had nested in the box and not rats. They had probably entered the box through the hole that was cut for the cables. We put two new batteries (that we had taken from the LABE site and charged overnight) in the bottom box and left two "dead" batteries in the upper box as a weight. We will need to swap the batteries between GOVA and LABE for the next servicing, as the batteries we took from LABE are bigger and sturdier and should be put back in the mountains. We then drove to Rangiora and stayed there for the night.

\section{Monday 5 October}

We drove back to Picton. We took the early afternoon Interislander ferry and arrived in Wellington around 6pm. I didn't get sick this time as I took some sea sickness medicine...

\section{C.1.3 Memo for next servicing}

- Swap GOVA and LABE batteries - meaning that GOVA needs to be serviced first - and take out two unused batteries at GOVA (need to charge them overnight so that they can be used at other sites);

- Change locks at COSA and change position of GPS by installing longer GPS cable (that Adrian needs to make beforehand);

- Change box for MTBA and MTFO;

- Bring two extra batteries and a GPS for COVA;

- Buy name tags for keys and sort out all the keys on SAMBA keychains;

- Install extra battery for EORO;

- Reformat all Japanese data cards and buy batteries for their seismometers. 


\section{C.2 April 2016}

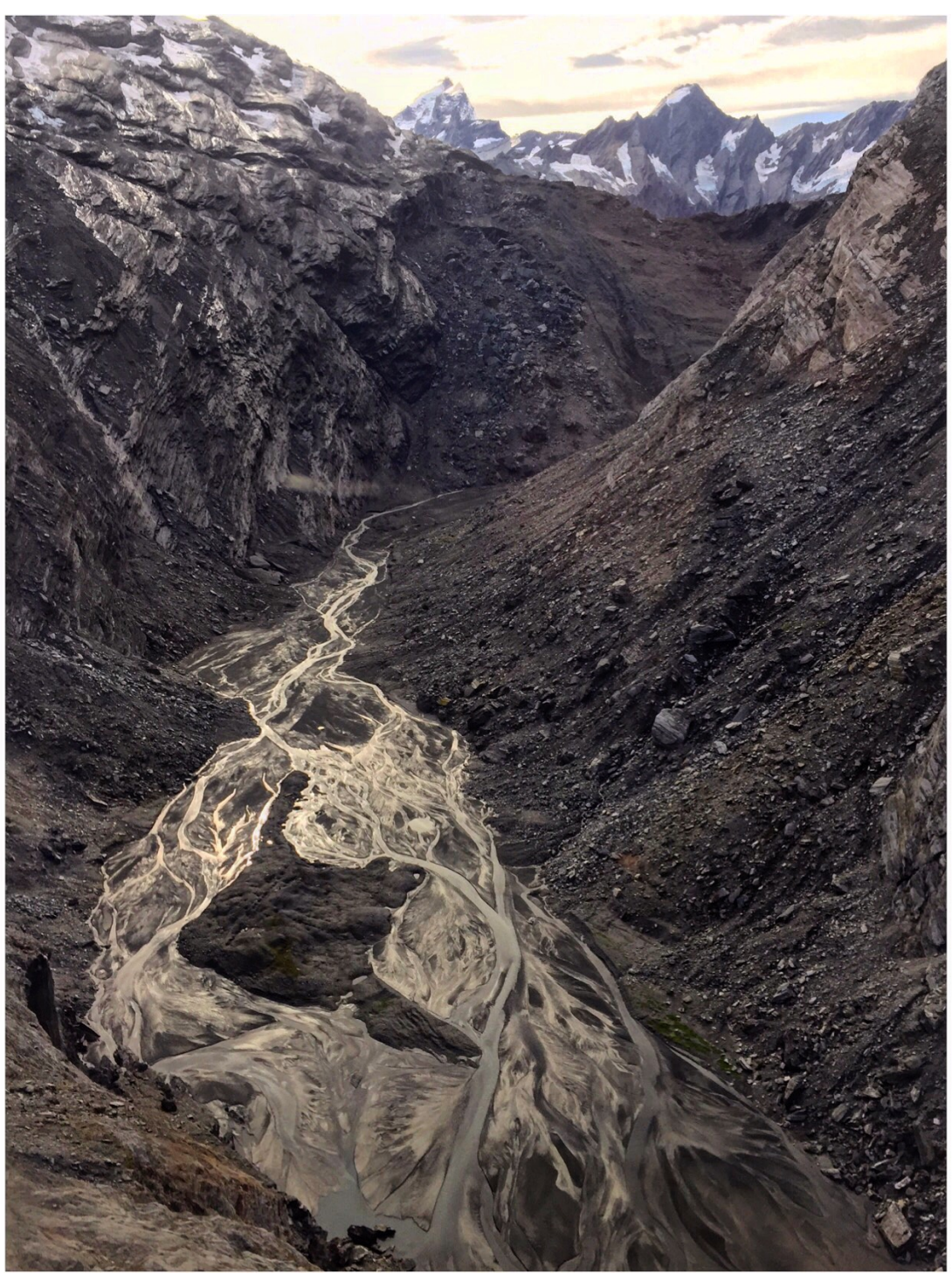

Southern Alps from the helicopter (while flying to our Landsborough site).

\section{C.2.1 Summary}

This report summarises the service run for the SAMBA, DFDP-13, Japanese and COSA networks. For this service trip the field work team consisted of:

- Laura-May Baratin, VUW;

- Calum Chamberlain, VUW;

- Konstantinos (Kostas) Michailos, VUW. 
- Emily Warren-Smith, VUW

This trip began on the West Coast with the servicing of most of the SAMBA stations, the DFPD seismic sites and the Japanese array. We serviced successfully the Japanese seismometers and all of the SAMBA sites on the West Coast. We then drove to central Otago to service the COSA network and were supposed to finish this servicing trip with two SAMBA sites : LABE and GOVA. Unfortunately, because of the weather, we weren't comfortable with crossing the river with the car, so we weren't able to service GOVA.

In total, 26 sites were serviced.

\section{C.2.2 Daily $\log$}

\section{Friday 1st April}

We took the 9AM Interislander ferry to Picton, the ride was smooth, quite a change from last servicing! We arrived in Picton just after $1 \mathrm{pm}$ and drove to Greymouth where we stayed at the Kiwi Holiday Park. All went well, except Kostas was scared of "big" waves, that were very far from us and not that big in retrospect!

\section{Saturday 2nd April}

The day started with a stop in Hokitika to buy groceries. We then drove to Whataroa and serviced all of the DFDP seismic sites. We showed Kostas how to service a station and he did quite well. On arrival at the WMSZ site, we realised that the glass of one of the two solar panels powering the modem had been shattered most probably by a cow (Picture C.9). However, we didn't have the keys to the Hari Hari container at that time, so we couldn't stop to the Hari Hari container on the way from Hokitika to Whataroa to grab a spare solar panel. And since everything was working, we suspected that the solar panel was still powering the site even though the glass had been broken. So we decided to leave it there. Nothing to report for all the others DFDP seismic sites. We then went on to service the Quarry site (WHAT) where telemetry had been down for a couple of weeks. After a long call with Mark (from GeoNet) he figured out what was 
wrong. One of the software that GeoNet used to identify data had to be reset. So telemetry is now up and running at WHAT. Kostas was scared of birds there, but besides that, the servicing went smoothly. We then drove back to Fox, to the Top 10 holiday where we stayed for 5 nights.

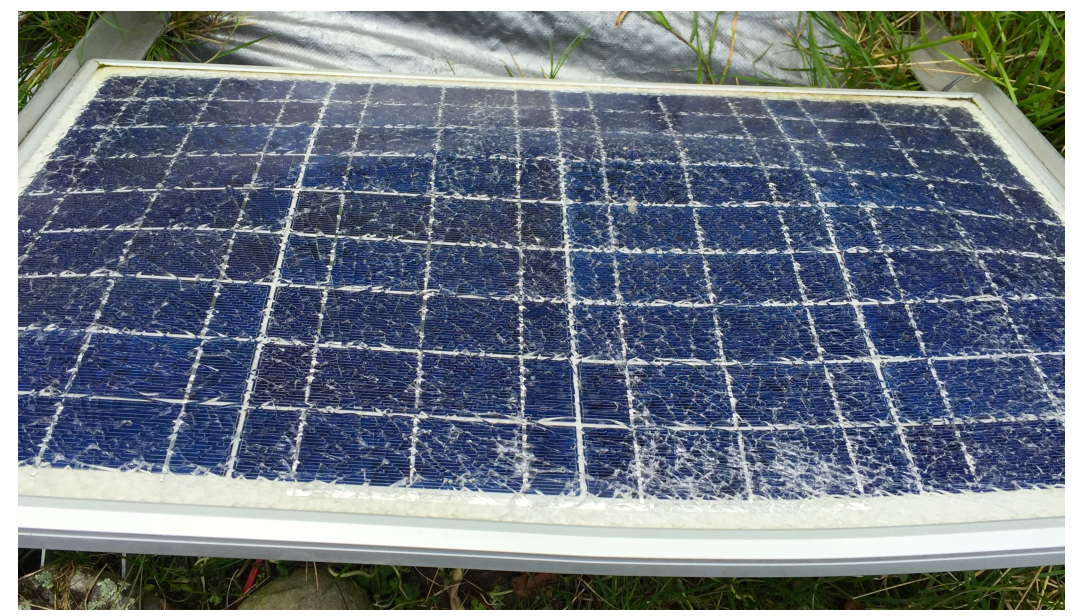

Picture C.9: Solar panel, most probably shattered by a cow, at the WMSZ site.

\section{Sunday 3rd April}

Sunday was a very wet day. We weren't able to fly to any of the SAMBA sites, so we ended up servicing COSA first (where we changed one lock on the box). We then went on to service the EORO site. The tourist path to access the site had to be diverted because its end had been swept away by the river (Picture C.10). We realised that there was no way we could get to our station by following the new track. So we took the old path (that is now closed to tourists) and found a safe way to the station. Once there, the servicing went smoothly. The station didn't go down this time. So we didn't think it necessary to put a second battery there. Would be cool if DOC allowed us to put a slightly bigger solar panel there though. We finished the day by servicing POCR where everything went smoothly.

\section{Monday 4th April}

We drove from Fox to Whataroa in order to service the Whymper Hutt station. The pilot was 30 minutes late, so we waited around a bit and fed a lot of sandflies... It was Kostas first helicopter flight and he loved it! We were all quite pleasantly surprised to find out that he was not scared of helicopters. After the flight, we went to service the two Japanese seismometers. We started with the one in Ian Coles' field (WAIT). All went well, we installed the new GPS and let the station run. We 


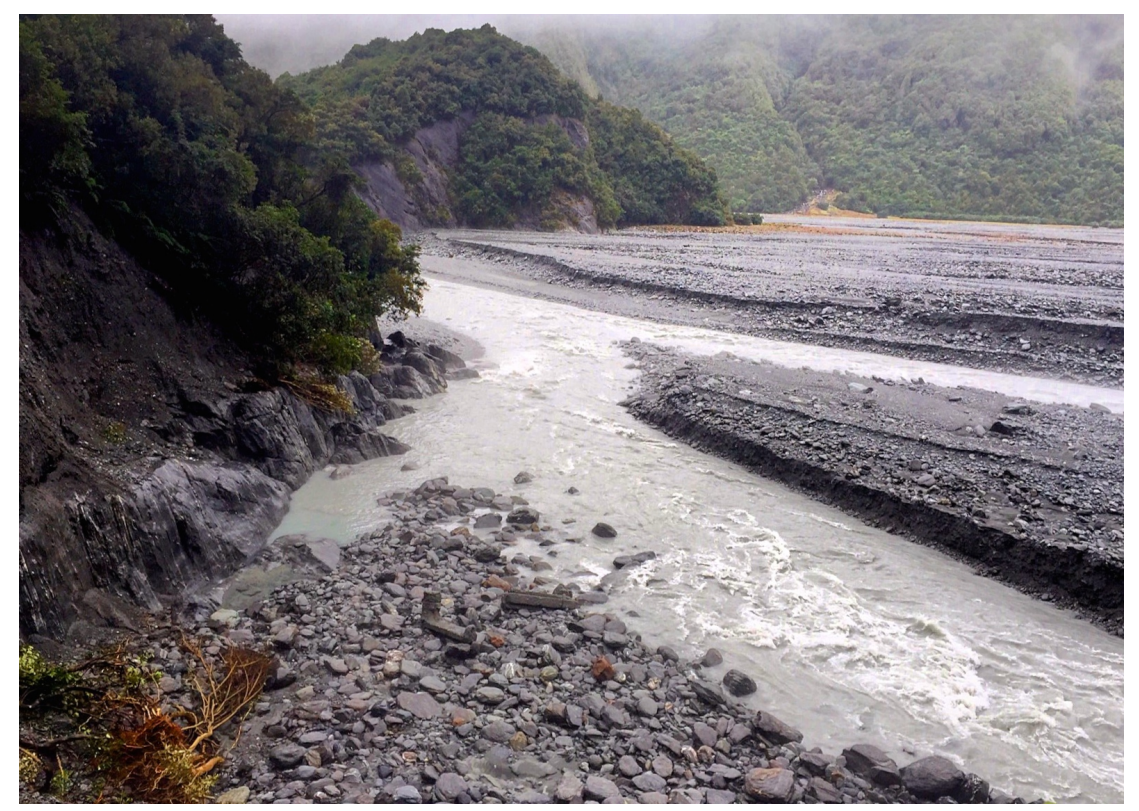

Picture C.10: River viewed from the new diverted tourist path.

then went on to the DFDP-2 container to reinstall their station there. Once on site, we realised that something was wrong with the sensor (not recording on the vertical component). We did the installation anyway and let the station running on two channels. We then went to the Franz Josef Transfer Station (FJTS) to get the keys to access the old Franz Josed dump site. John (the guy in charge of the FJTS) gave us a key, but it turns out it was the wrong one. So we ended up entering the site through a hole in the gate and walked to the site. Once there, the servicing went well. But there are a lot of scratchy bushes to cross. And the box is almost covered by trees. Site will need some gardening next time.

\section{Tuesday 5th of April}

We all flew from Fox to MTFO and the servicing went smoothly. Nothing to report there. We just swapped the lid of the box as it was a bit old. If we want to install a sturdier box, we need to get a metal one. We couldn't fly with James on that day as he was busy, but arranged to fly with him the next day. We then took care of switching the Japanese sensors, we took the sensor from the WAIT site and put it in the DFDP-2 container. That site is now up and running, as for WAIT, there's no sensor there, so Ryota and Tomomi will need to send a replacement. 


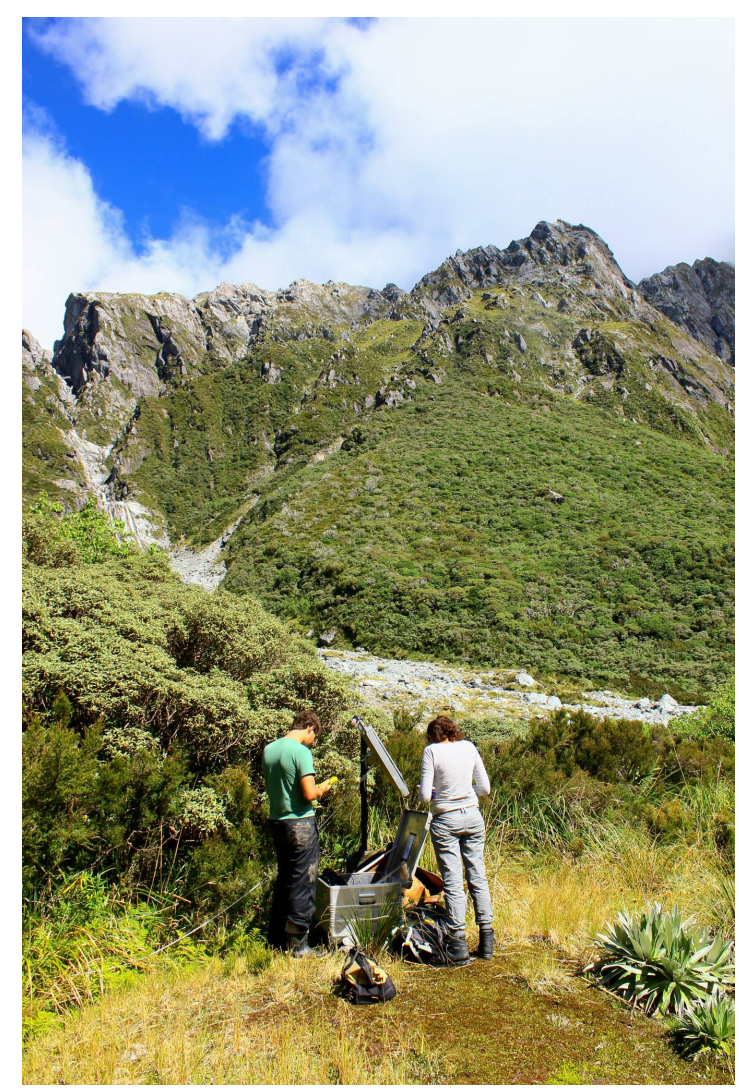

Picture C.11: Calum and I servicing WHYM (photograph by Emily WarrenSmith).

\section{Wednesday 6th of April}

We flew with James to all of our Southern sites. First we went to Mount Baird, where the servicing went smoothly. The Das was on, not stuck in writing mode, but both cards were full. We then went on to Solution Ranges, everything was well and working there. Then, we flew to Landsborough, again all went well.

Finally, James dropped us to Copland Valley (COVA). We were quite happy to be able to see the station this time! We knew we were going to need at least one hour at this site, so James left us to work there and arranged to come back in one hour. On arrival, the DAS was on and the site looked good. However, after closer inspection, the GPS had died. We then worked on replacing the GPS, installing a new elevated metal box for the DAS that we fixed on the pole, and on installing some earthing (Picture C.13). It took us 2 hours, so we had to call James from the satellite phone to let him know we would need a bit more time. When we got back to Fox, we took a look at the data and everything looked good except for the MTBA site. We could look at the data, but there are some chunks where there's a big jump in the data (Picture C.14). We're thinking that something might be 


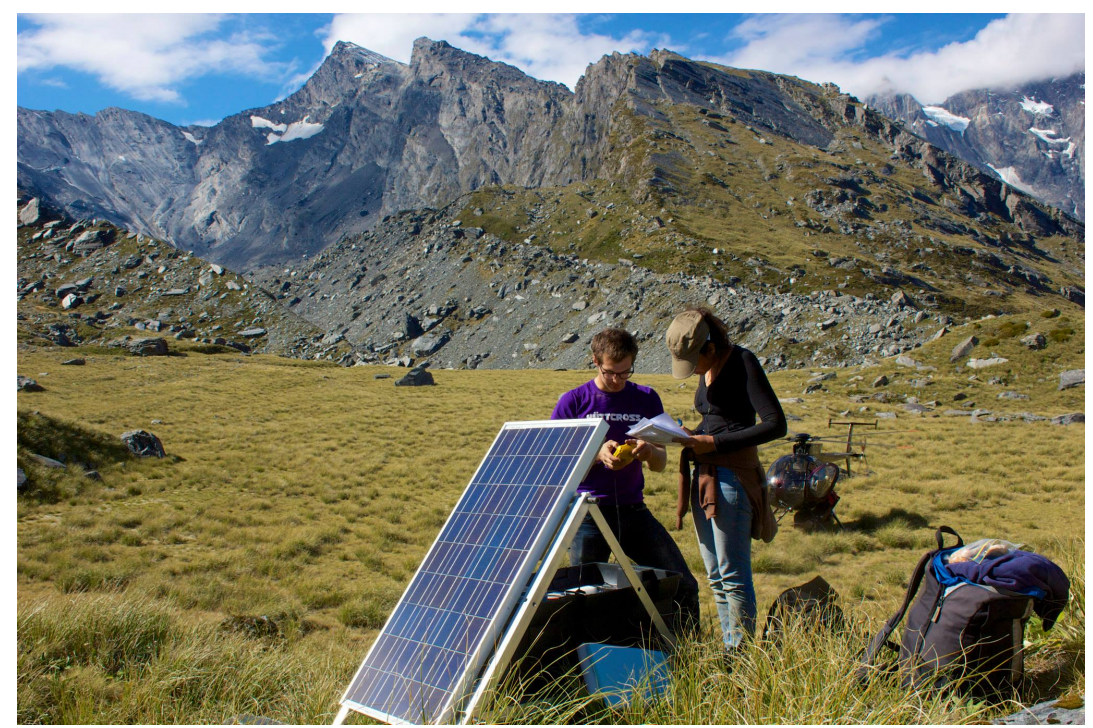

Picture C.12: Calum and I servicing LARB (photograph by Emily Warren-Smith).

wrong with the sensor or the sensor cable (as Emily experienced something similar for Kingston and it turns out the sensor or its cable was damaged). I think it would be really good to have an efficient field laptop, that would allow one of us, to look at the data while on site to determine if everything looks good. It shouldn't take more than 5-10min to have a look at the data if we have an efficient computer, and that's something that can be done by one person while the other one or two persons just take care of regular servicing.

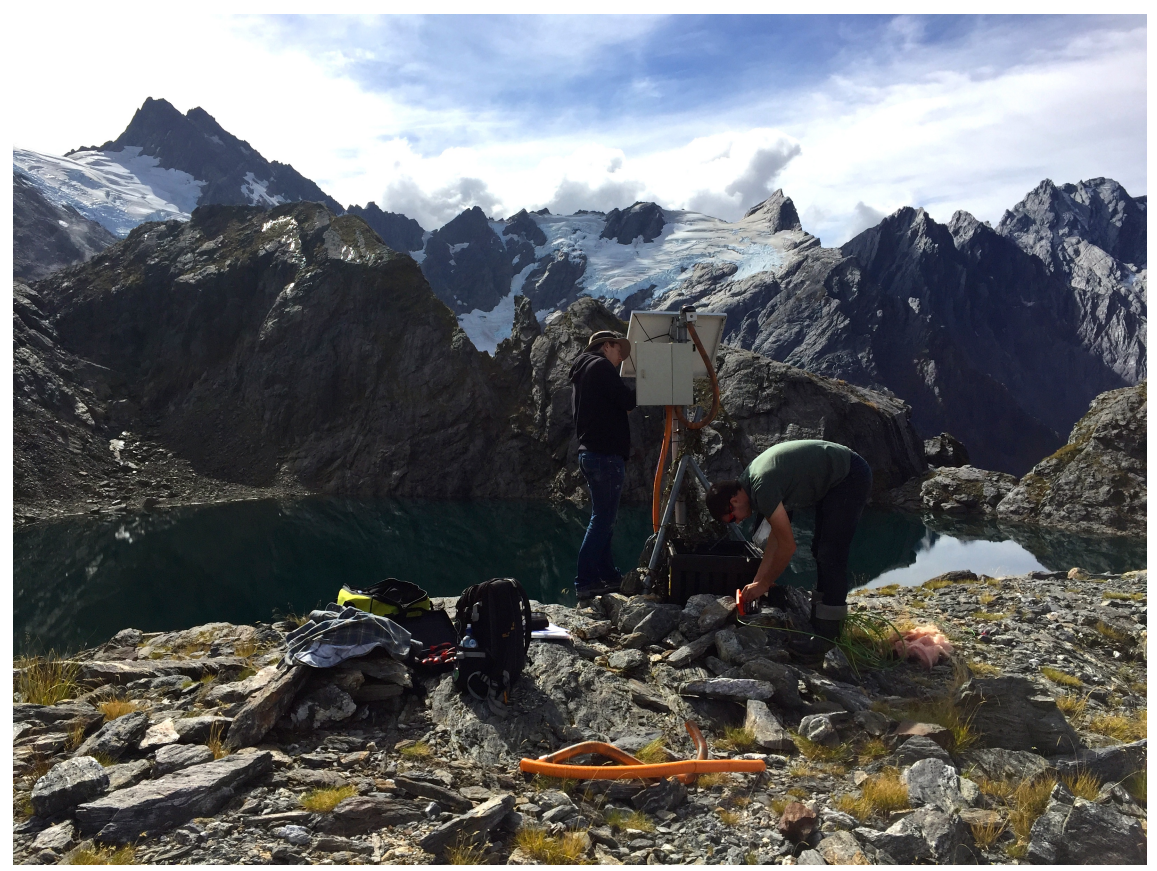

Picture C.13: New box \& earthing being installed at Copland Valley by Calum and Kostas. 


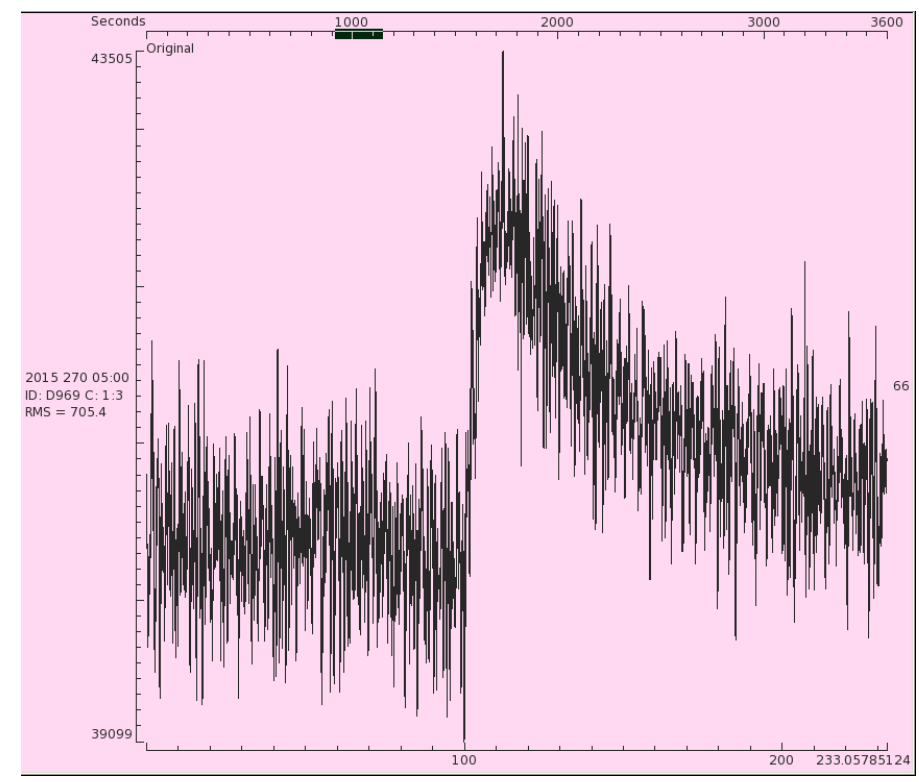

Picture C.14: Weird looking data at the MTBA station.

\section{Thursday 7th of April}

We drove from Fox to Central Otago and serviced the first COSA station on the way (the Haast station). All went well. We then went to the Lake Hawea station to service it. The servicing went smoothly. We then drove to Wanaka and stayed at the holiday park for the night.

\section{Friday 8th of April}

We serviced the Mt Aspiring, Lake Dunstan and the St Bathans stations. The DAS was off at the Mt Aspiring station and it turns out that the solar controller had died, it only had a $2.4 \mathrm{~V}$ output. So we changed it and everything else was working fine. Everything went well for the Lake Dunstan and St Bathans stations. We stayed in Arrowtown for two nights.

\section{Saturday 9th of April}

We drove to the Glenorchy station in the morning. All was running on arrival. Servicing went well. We spent the afternoon reinstalling the Kingston station (Picture C.15). We installed a new sensor and a new cable. We also put a new DAS there. The station seemed to be working well when we were done. 


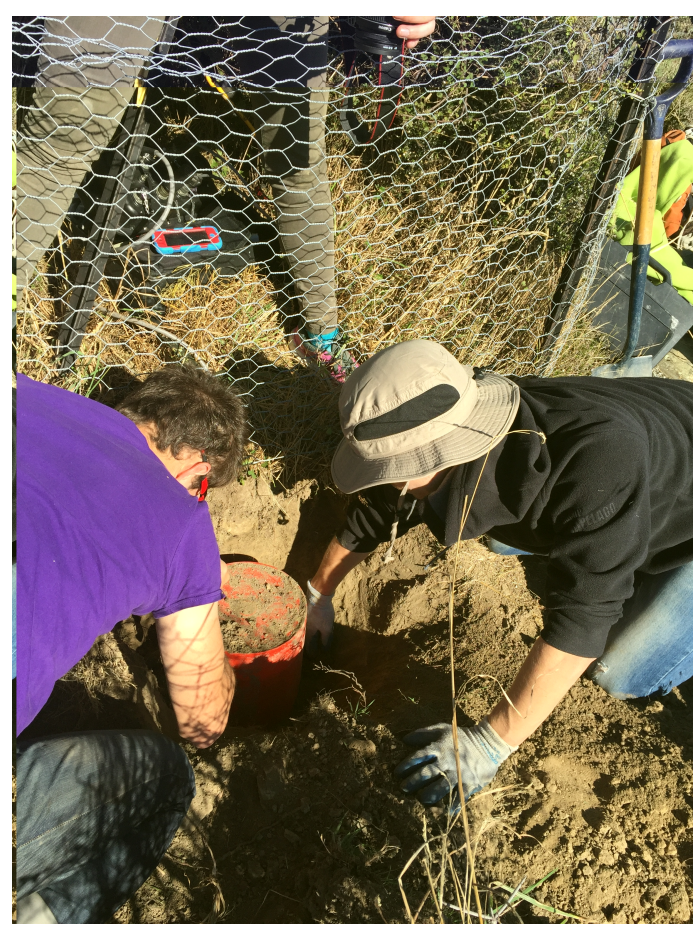

Picture C.15: Calum and Kostas digging out a sensor at Kingston.

\section{Sunday 10th of April}

We serviced the last COSA site near Lindis Pass and then drove to Mt. Cook where we managed to fly to De La Beche. We were supposed to go to Godley Valley first, as some batteries from Godley were meant to be put back at De La Beche. However, the weather forecast was not good at all for the next couple of days so we decided to prioritize the De La Beche site. We flew there from the Mt. Cook Airport and the servicing went really well (Picture C.16). We installed a spare battery (as there was only one there). We then drove back to Twizel where we spent the night at the Kiwi Holiday Park.

\section{Monday 11th of April}

We woke up at 6 am to be able to drive to Godley Valley and avoid the rain. The weather was fine around Lake Tekapo when we got there, but some dark menacing clouds were over the Southern Alps. And the weather forecast was predicting heavy rains to reach us by midday, so we decided to not risk going to the station. We had already payed for a second night in Twizel but decided to leave anyway to get a head start on the driving. The fees wouldn't be cancelled so we found a really cheap place to stay for the night in Christchurch so as to not waste to much money on accommodation. 


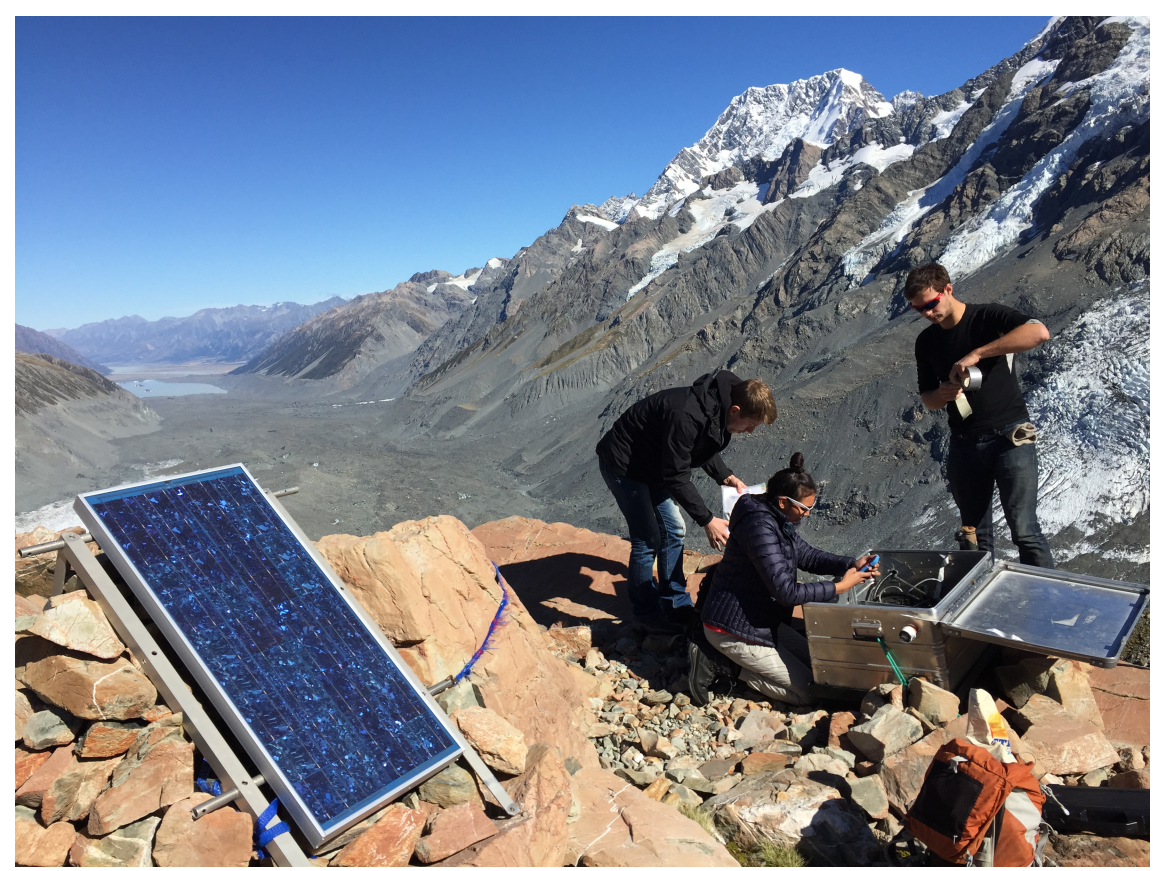

Picture C.16: Smooth servicing of LABE (photograph by Emily Warren-Smith).

\section{Tuesday 12th of April}

We drove from Christchurch to Picton in the morning, then took the 2pm Interislander ferry back to Wellington. The sea was pretty calm so everybody enjoyed the trip.

\section{C.2.3 Update on Godley Valley}

Huw and Sam had to do some field work around the Tasman Glacier so we asked them if they would be able to go check on Godley Valley. On arrival, they discovered that the site had been vandalised (both solar panels had been shot, box was opened, batteries were disconnected and solar controller was missing). They changed one solar panel (put a $40 \mathrm{~W}$ panel there) and added a battery, they also replaced the flash disks. I had a look at the data to try to determine when was the last day of recorded data that we had and pinpointed it to the 11th of February 2016. However, half of the data is now corrupted. And unfortunately, after looking at the data that is not corrupted, I realised that most of it is spiked. I am currently trying to figure out how to salvage the data by: (1) Looking into unspiking routine; (2) trying restore the raw data on the reftek flash disks. 


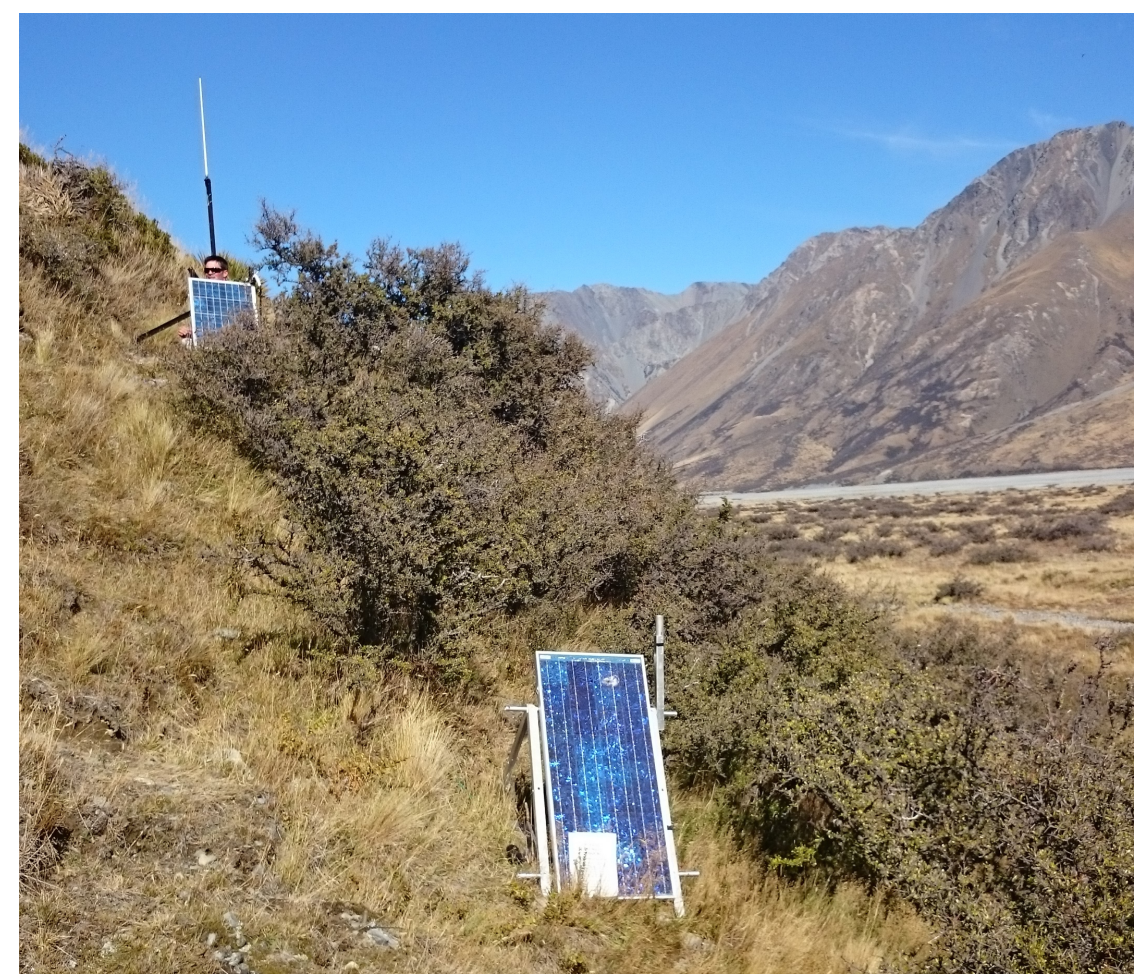

Picture C.17: Huw reinstalling our GOVA station (photograph by Sam TaylorOfford).

\section{C.2.4 Memo for next servicing}

- Add another battery and a bigger solar panel at GOVA;

- Bring some gardening tools at FRAN;

- Change position of GPS by installing longer GPS cable (that Adrian needs to make beforehand);

- Change box for MTBA and MTFO, with sturdy steel box;

- Reformat all Japanese data cards and get batteries for their seismometers;

- Bring sensor and sensor cable for MTBA (will need time there). 


\section{C.3 October 2016}

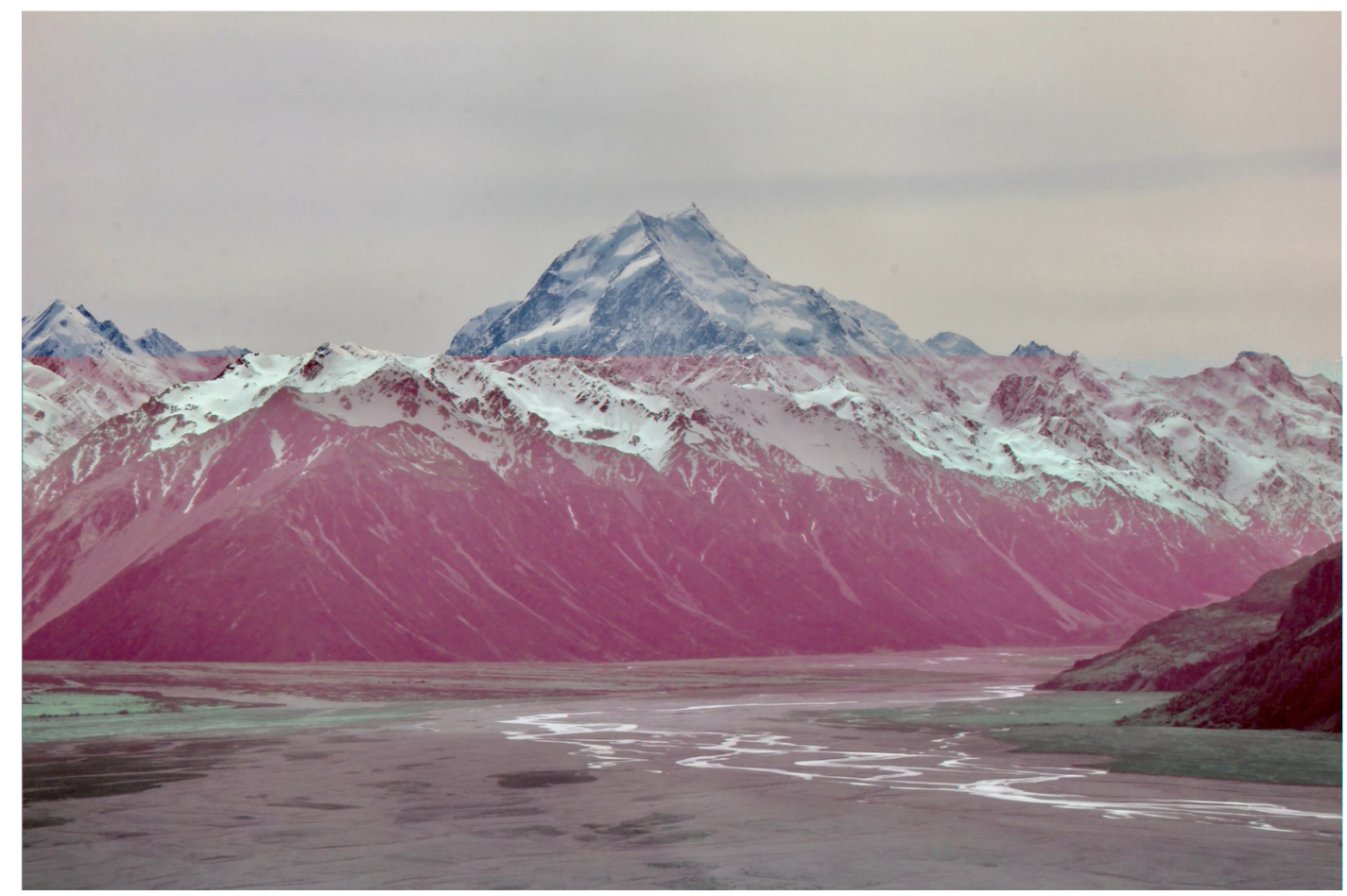

Mt. Cook viewed from an helicopter (while servicing our De La Bêche station).

\section{C.3.1 Summary}

This report summarises the service run for the SAMBA, DFDP-13, Japanese and COSA networks. For this service trip, the field team consisted of:

- Laura-May Baratin, VUW;

- Adrian Benson, VUW;

- Konstantinos (Kostas) Michailos, VUW.

This trip began on the West Coast with the servicing of most of the SAMBA stations, the DFPD seismic sites and the Japanese array. Next, we serviced COSA network and the two remaining SAMBA sites: LABE and GOVA. In total, 25 sites were serviced. 


\section{C.3.2 Daily log}

\section{Monday 17 October}

We took the 8:00am Bluebridge ferry to Picton. We arrived in Picton just after 12:00am and drove to Reefton where we stayed for the night.

\section{Tuesday 18 October}

We drove to Franz Josef glacier and serviced the ground sites: EORO, FRAN and POCR, as the weather did not allow us to fly to the mountain sites. To reach EORO we took the old path (that is now closed to tourists). Once there, the servicing went smoothly. The station was down for a couple of months. Would be good if DOC allowed us to put a bigger solar panel there. Then, the FRAN and POCR sites' servicing were also done with no problems. Boxes in POCR are getting quite old, especially the one containing the batteries, so will need changing at next servicing. We then drove back to Franz Josef glacier, to the Top 10 holiday where we stayed for 4 nights.

\section{Wednesday 19 October}

The weather was again pretty bad so we continued with the servicing of the ground sites. We drove to Whataroa to service the WHAT station (Picture C.19) and all the DFDP seismic sites. On our way back we serviced COSA as well.

The locks in the Quarry site (WHAT) were in terrible shape, we were barely able to open them. So we removed them and put cable ties instead. Other than that the servicing went smoothly. Next we did the DFDP seismic sites. Servicing of WTSZ site went well. The electric fence was down so we fixed it and put it back on. But as it created spikes on the signal we removed it. Next, for WDSZ, everything went well, the only thing to be noted is that the box containing the batteries needs to be replaced. At WPSZ we found out that the telemetry was down. Solar controller for modem got fried and resulted in battery dying. Besides that, nothing to note. Then, at WMSZ the telemetry was also down for the same reasons. Also, on arrival, we found the box containing the gear for telemetry open... We finished the day by servicing the COSA station. The locks there were in bad condition. We cut the box on one side to open it, so keys are no longer needed for this site. Servicing in itself went smoothly. We also did some gardening 
at the site.

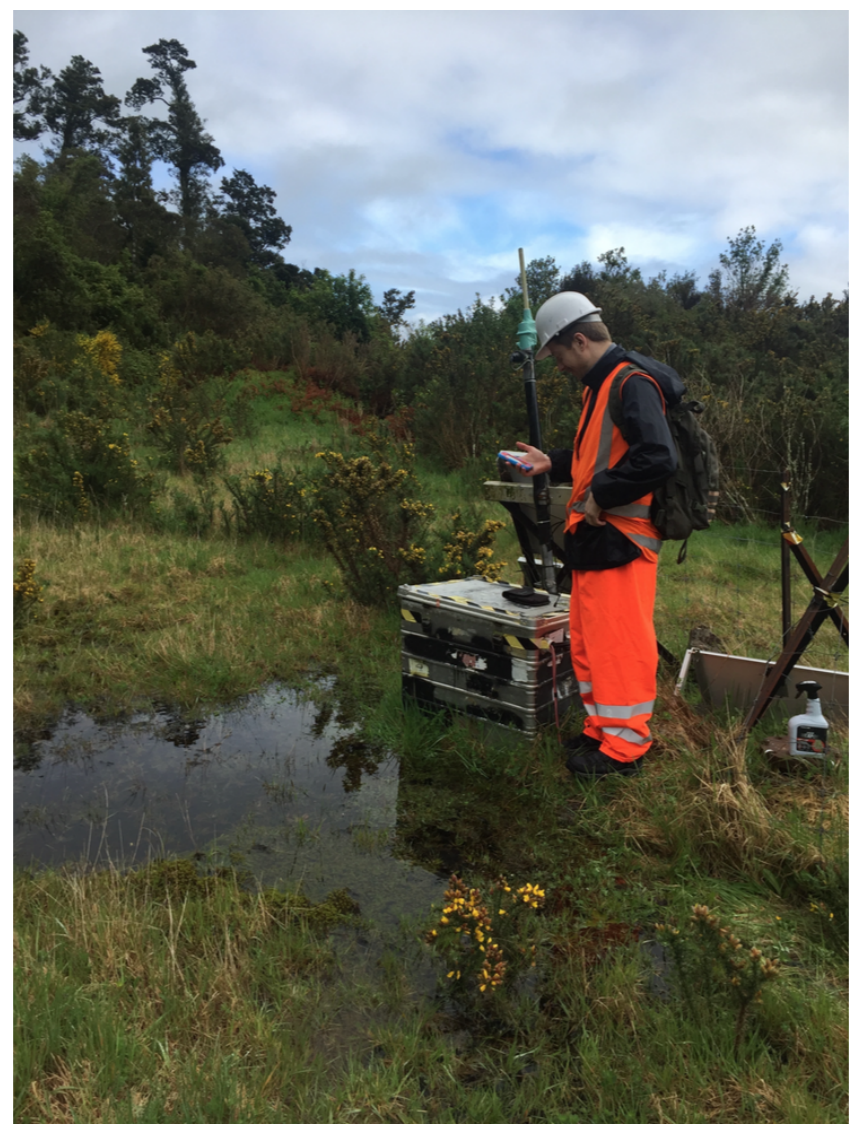

Picture C.19: A bit of a pond at the Quarry site while Kostas was servicing WHAT.

\section{Thursday 20 October}

The weather was once again bad, so we went to service the two Japanese seismometers. We re-installed the seismometer from DFDP-2 to DFDP-1b and serviced the seismometer in DFDP-1. We were quite proud to have successfully installed one Japanese seismometer at the first try as they are quite arduous to install (Pictures C.20 and C.21)!

After that, we picked up two batteries from the DFDP-2 container and went to fix the telemetry at WMSZ and WPSZ. At these two sites we replaced the dead batteries and the solar controllers. 


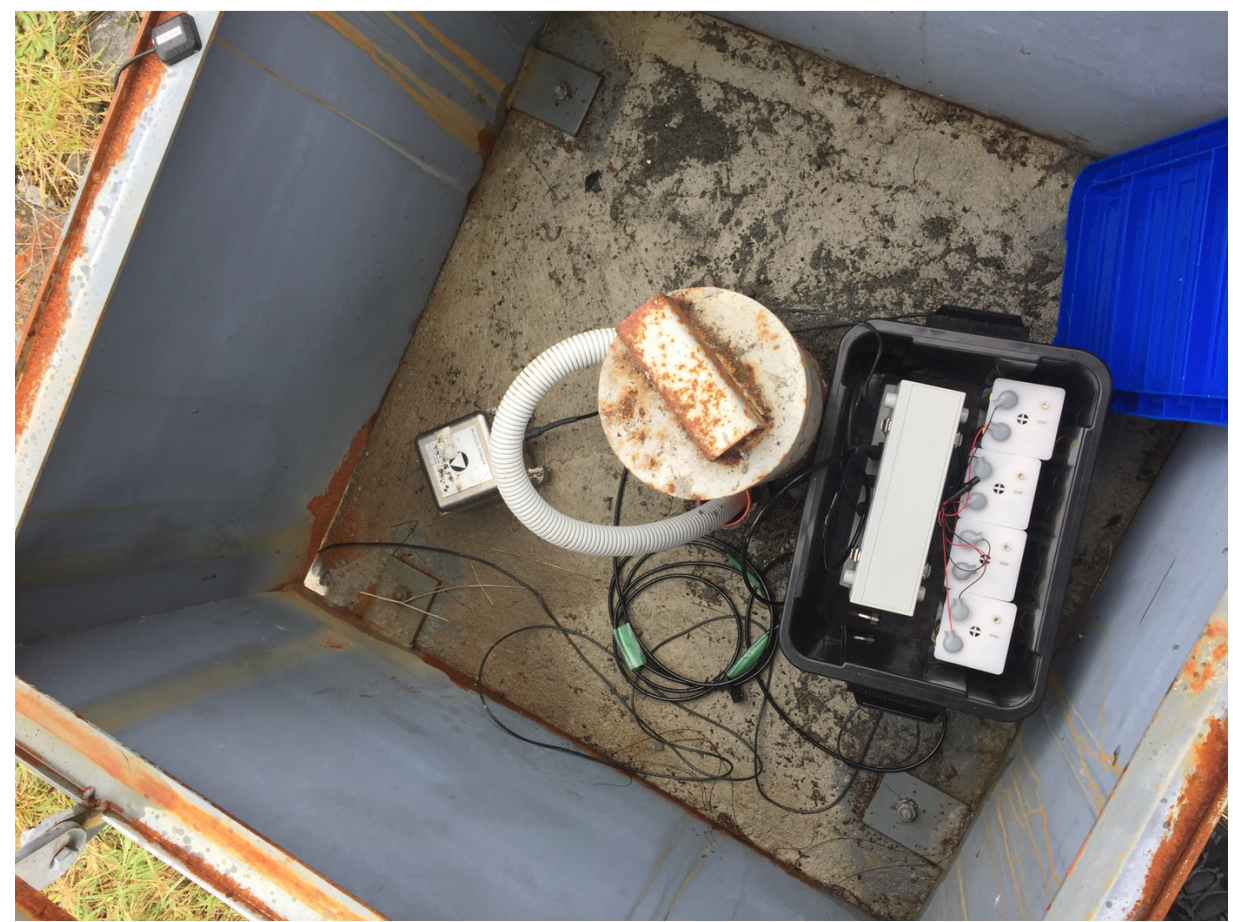

Picture C.20: GCS2 : the new Japanese station, installed at the DFDP-1A location.

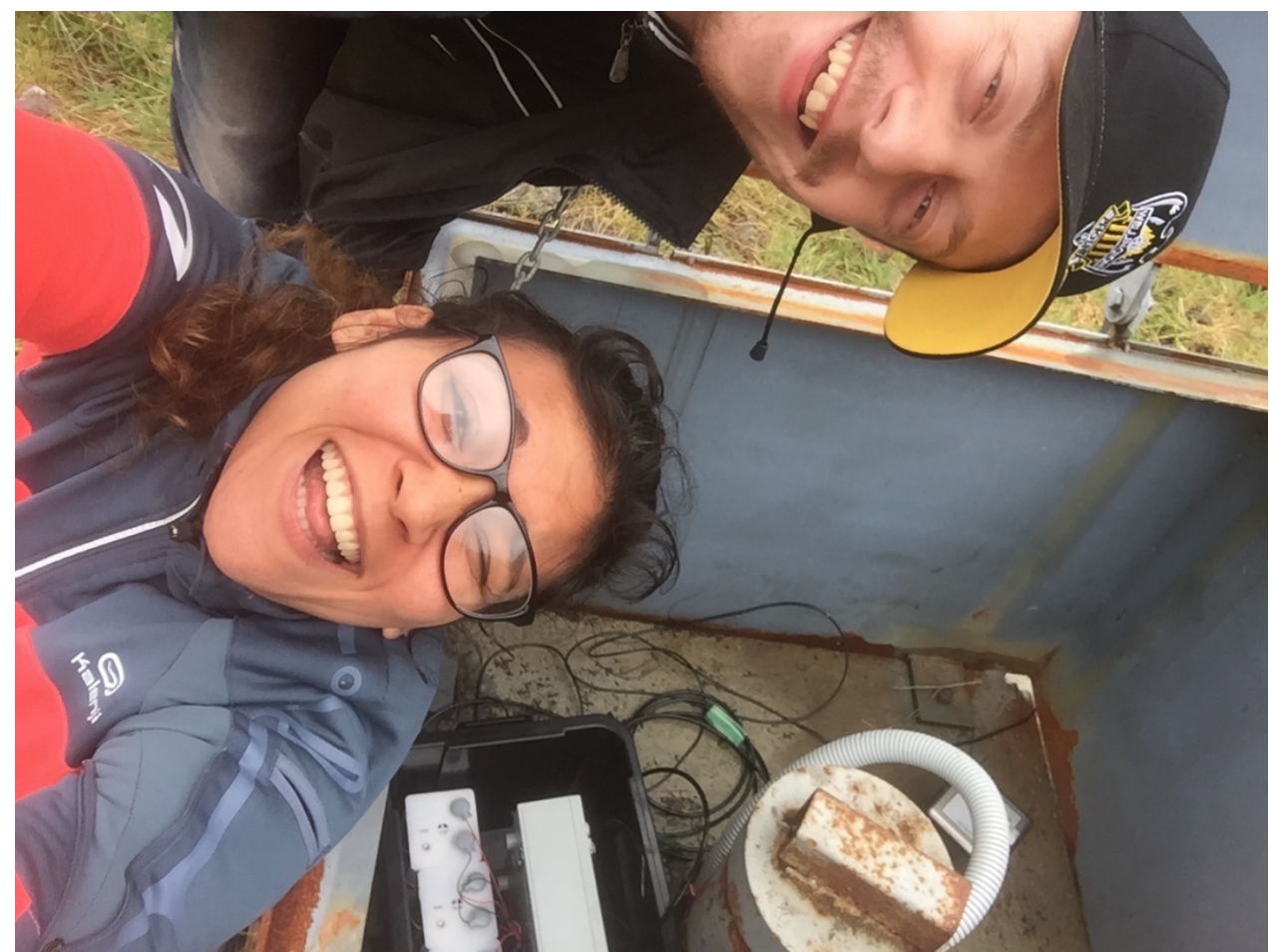

Picture C.21: Proud PhD Students after successfully installing the Japanese seismometers on the first try. 


\section{Friday 21 October}

On Friday the weather was finally good so we got a chance to fly to Mount Fox, Mount Baird, Landsborough, Copland Valley and Whymper's Hutt.

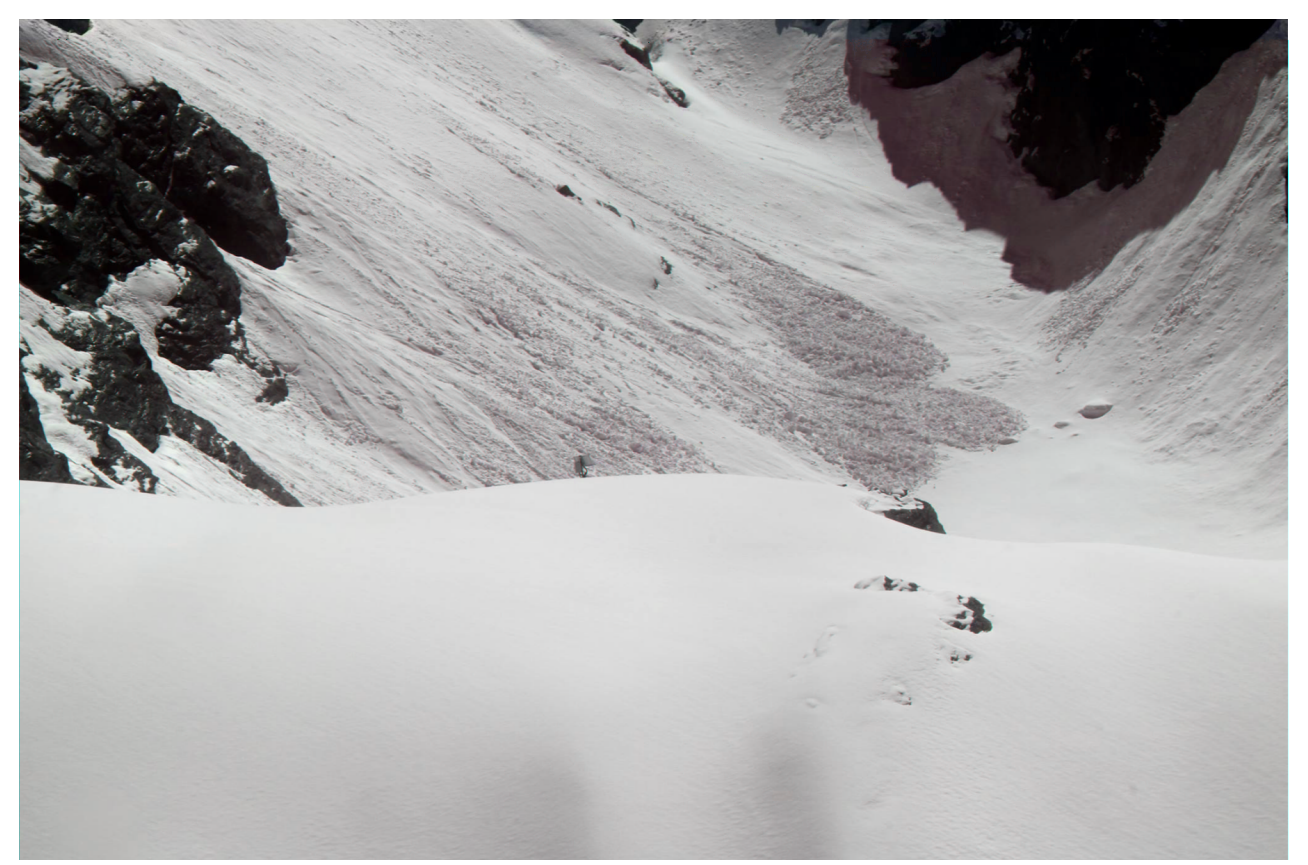

Picture C.22: We can see COVA above the snow! What a beautiful sight!!

Early in the morning, we flew from Fox to the MTFO site. Servicing went smoothly. The rubber ring seal of the DAS needs replacing. Later that same morning, we flew with James to all the Southern SAMBA sites (MTBA, SOLU, LARB and COVA). First we went to Mount Baird, where James left us to work there and arranged to come back two hours later. We replaced both the sensor and the sensor cable. Servicing went well. Later, James picked us up and dropped us to to Solution Ranges, everything was well and working there. Then, we flew to Landsborough, again all went well. Finally, we serviced Copland Valley. We were overjoyed to see part of the station (elevated metal box for the DAS) over the snow surface (Picture C.23).

Later the same day, we drove to Whataroa and from there flew to Whymper's Hutt. We were left there for about an hour and a half as we intended to install the grounding to protect the station from lightnings. On arrival though, there was no power on the DAS. Solar controller got fried and cables from solar panel to solar controller were degrading. We changed the solar controller to solar panel cable and the solar controller. Started acquisition but GPS was down, so we ended up replacing the GPS also. Grounding rod was installed but we didn't have 


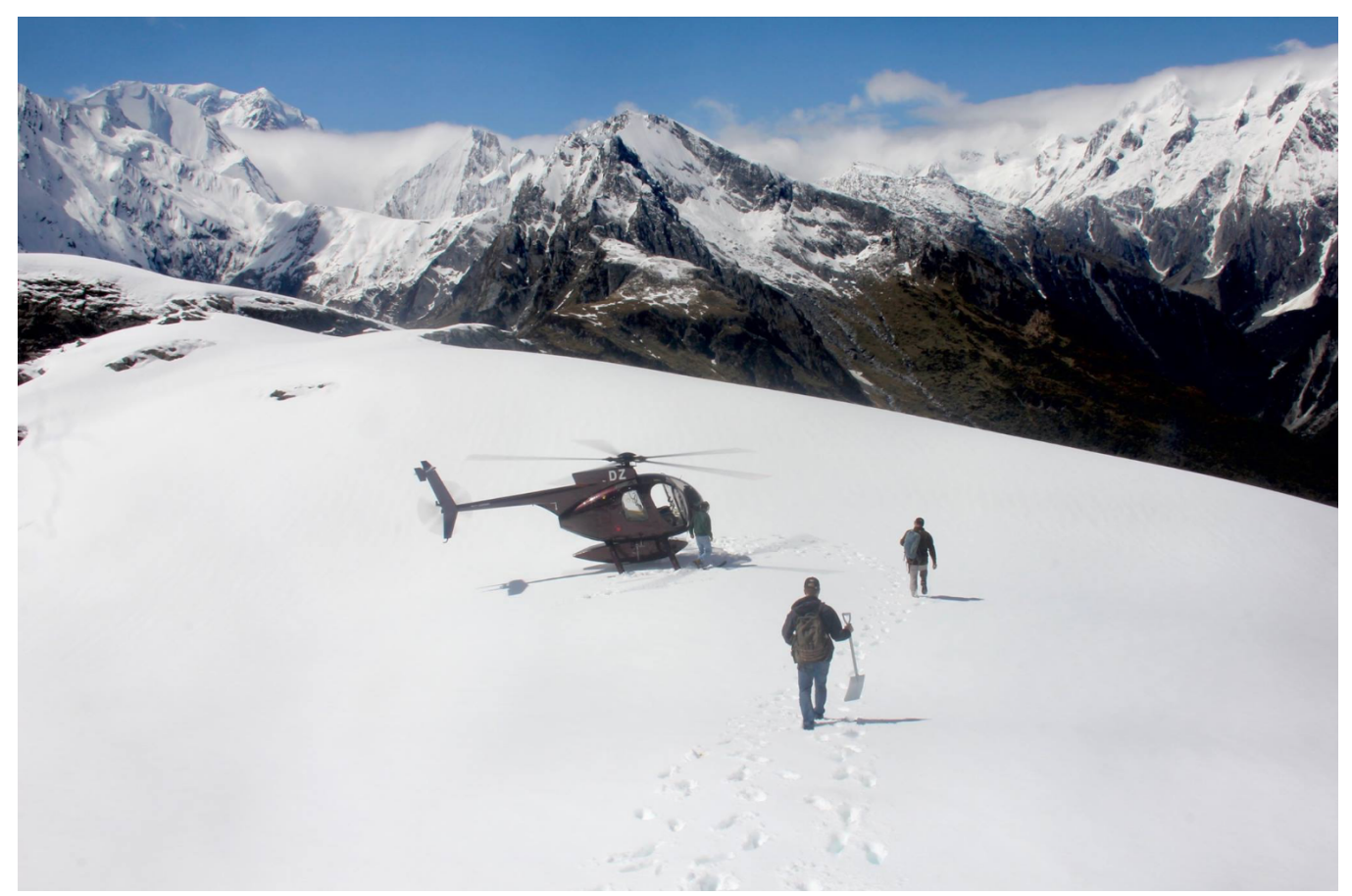

Picture C.23: Field team walking back to the Helicopter after successfully servicing COVA.

enough time to connect it.

\section{Saturday 22 October}

We drove from Franz Joseph glacier to Central Otago and serviced the first COSA network station on the way near Haast (HAAS). All went well. We then went to the Lake Hawea station (HUVA) to service it (Picture C.24). Nothing to report there. We then drove to Wanaka and stayed there for the night.

\section{Sunday 23 October}

We drove to Mt. Cook to fly to De La Bêche site (LABE), as the weather forecast was not good at all for the next couple of days. Thus, we decided to prioritise this site. We flew to LABE from the Glentanner Aerodrome (Picture C.25) and the servicing went really well. Next we serviced the COSA network site near Lindis Pass (MORV). We first changed the DAS with a newer version. We also noticed on real time monitoring that the 3 rd channel was barely recording the stamp test (might have been o level). We locked and unlocked the sensor to fix this issue. We then drove to Cromwell and stayed there for the night. 


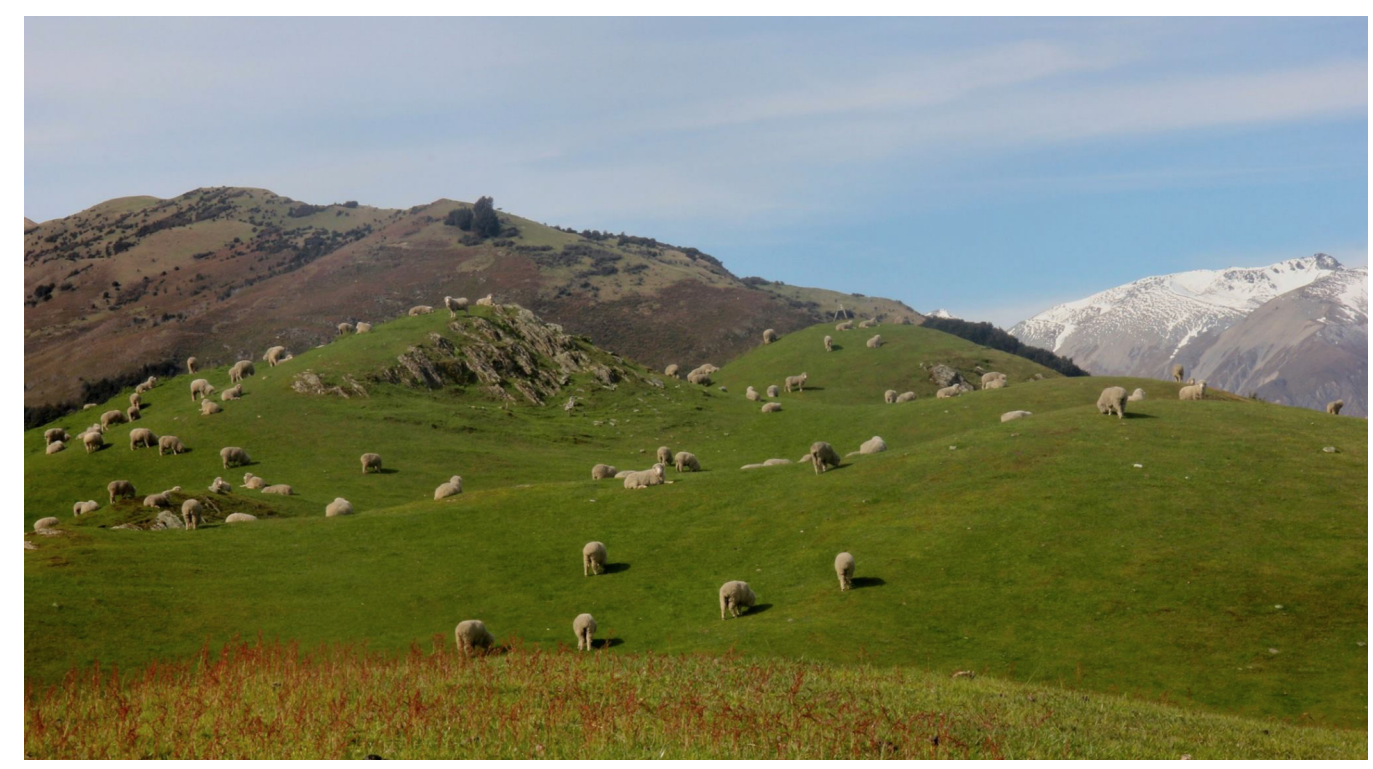

Picture C.24: Free range sheep at our site near Lake Hawea.

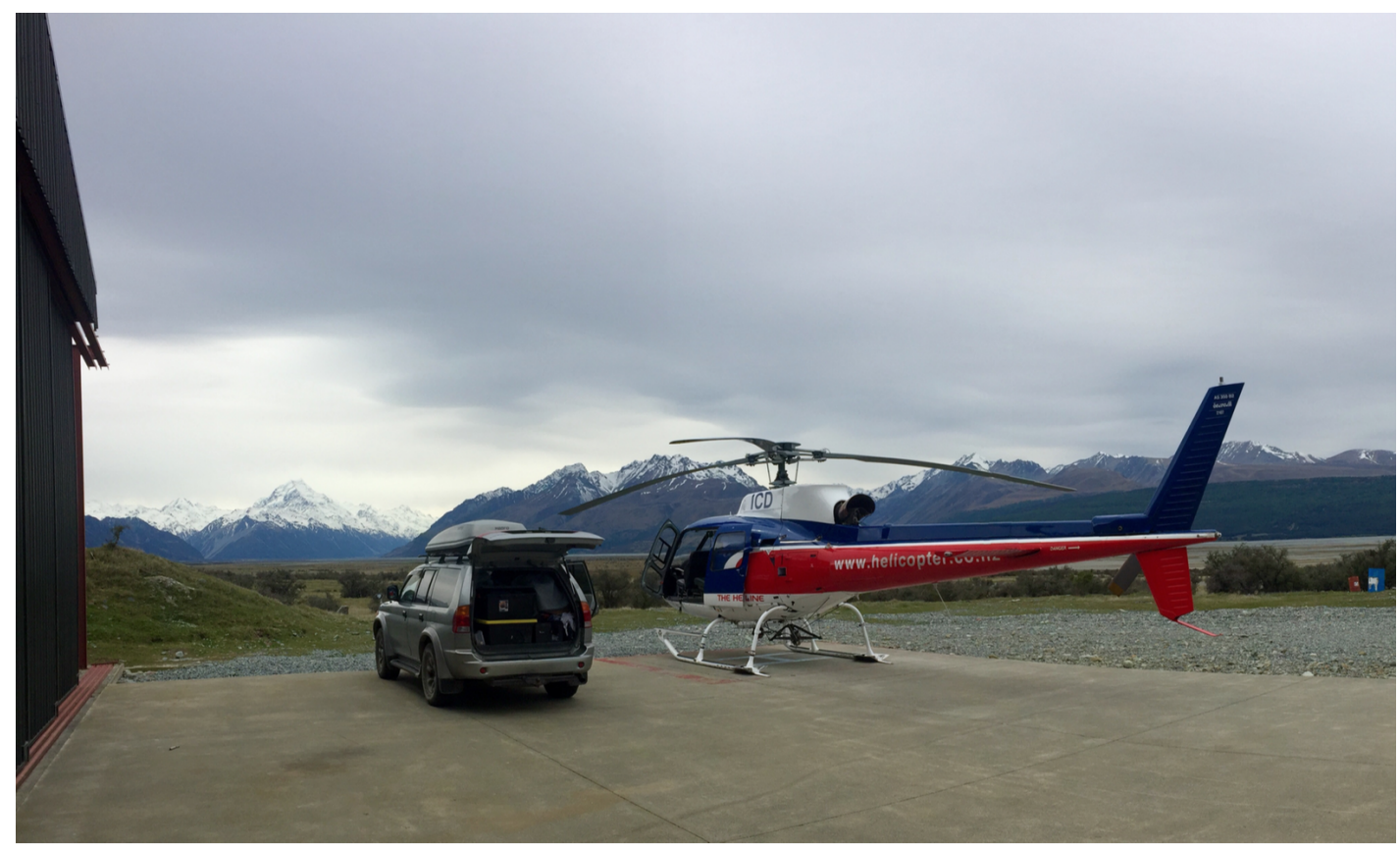

Picture C.25: Our means of transportation - Glentanner Aerodrome.

\section{Monday 24 October}

We drove to the COSA network station in Kingston (KING station) in the morning. All was running on arrival. Servicing went well. We put the solar controller into a small box to protect it from water. We then drove to the Glenorchy (TEPE) where servicing went well. Box is getting wore o new one is needed. We drove back to Cromwell and stayed there for the night. 


\section{Tuesday 25 October}

We serviced the St. Bathans (STBA), Lake Dunstan (NOBU) and the Mt. Aspiring (ASPR) stations. At St. Bathans and Lake Dunstan sites we re-leveled the sensors as they had the same issue as at the MORV site. Rather than that servicing went well for all three sites. New boxes are needed for St. Bathans and Mt. Aspiring stations.

\section{Wednesday 26 October}

We drove to Godley Valley to assess the site (GOVA) after it had been shot a couple of months ago. On arrival, the acquisition was on and the servicing went well. We replaced the battery and the solar controller. We also installed a new box. On site, there were also two extra grey batteries. The batteries were not dead so we connected them to the old solar panel, that had been previously shot (Picture C.26), in order to keep them fed and happy. We then drove to Methven where we stayed for the night.

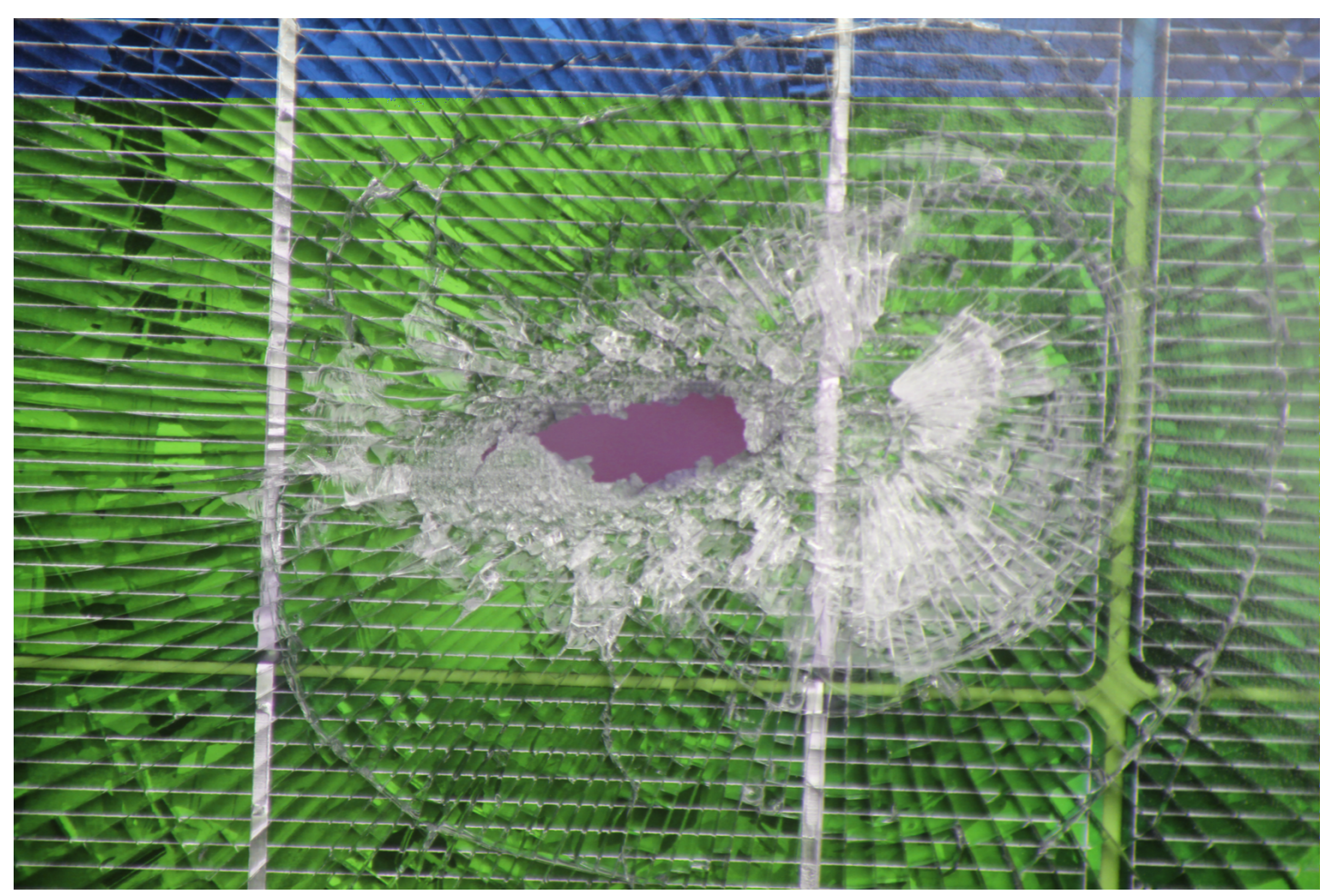

Picture C.26: Shot solar panel at Godley Valley (still working so far). 


\section{Thursday 27 October}

We drove from Methven to Picton, then took the $7 \mathrm{pm}$ Interislander ferry back to Wellington.

\section{C.3.3 Memo for next servicing}

- New box for batteries in POCR and WDSZ;

- New locks for WHAT?;

- Rubber ring seal of the DAS's well for MTFO and WHYM;

- Connect grounding rod, replace solar panel cable and install flexible contour at WHYM;

- Reformat all Japanese data cards and get batteries for their seismometers;

- New boxes for TEPE, STBA, ASPR;

- Pick up the two extra batteries from GOVA?; 


\section{Bibliography}

Aguiar, A. and Beroza, G. (2014). PageRank for earthquakes. Seismological Research Letters, 85(2):344-350.

Aguiar, A., Melbourne, T., and Scrivner, C. (2009). Moment release rate of Cascadia tremor constrained by GPS. Journal of Geophysical Research: Solid Earth, 114(B7).

Aiken, C., Peng, Z., and Chao, K. (2013). Tremors along the Queen Charlotte margin triggered by large teleseismic earthquakes. Geophysical Research Letters, 40(5):829-834.

Aki, K. and Richards, P. (2002). Quantitative seismology, volume 1. University Science Books.

Anderson, H. (1997). Southern Alps passive seismic experiment. Institute of Geological and Nuclear Sciences.

Anderson, H., Webb, T., and Jackson, J. (1993). Focal mechanisms of large earthquakes in the South Island of New Zealand: implications for the accommodation of Pacific-Australia plate motion. Geophysical journal international, 115(3):1032-1054.

Ando, R., Nakata, R., and Hori, T. (2010). A slip pulse model with fault heterogeneity for low-frequency earthquakes and tremor along plate interfaces. Geophysical Research Letters, 37(10).

Arnold, R. and Townend, J. (2007). A Bayesian approach to estimating tectonic stress from seismological data. Geophysical Journal International, 170(3):13361356.

Audet, P. and Burgmann, R. (2014). Possible control of subduction zone slowearthquake periodicity by silica enrichment. Nature, 510(7505):389-392.

Bannister, S. and Gledhill, K. (2012). Evolution of the 2010-2012 Canterbury earthquake sequence. New Zealand Journal of Geology and Geophysics, 55(3):295-304. 
Baratin, L.-M., Chamberlain, C., Townend, J., and Savage, M. (2018). Focal mechanisms and inter-event times of low-frequency earthquakes reveal quasicontinuous deformation and triggered slow slip on the deep alpine fault. Earth and Planetary Science Letters, 484:111-123.

Barnes, P., Sutherland, R., and Delteil, J. (2005). Strike-slip structure and sedimentary basins of the southern Alpine Fault, Fiordland, New Zealand. Geological Society of America Bulletin, 117(3-4):411-435.

Barth, N., Toy, V., Langridge, R., and Norris, R. (2012). Scale dependence of oblique plate-boundary partitioning: New insights from LiDAR, central Alpine fault, New Zealand. Lithosphere, 4(5):435-448.

Beavan, J., Ellis, S., Wallace, L., and Denys, P. (2007). Kinematic constraints from GPS on oblique convergence of the Pacific and Australian plates, central South Island, New Zealand. A Continental Plate Boundary: Tectonics at South Island, New Zealand, pages 75-94.

Beavan, J., Samsonov, S., Denys, P., Sutherland, R., Palmer, N., and Denham, M. (2010). Oblique slip on the Puysegur subduction interface in the 2009 july Mw 7.8 Dusky Sound earthquake from GPS and InSAR observations: Implications for the tectonics of southwestern New Zealand. Geophysical Journal International, 183(3):1265-1286.

Beavan, J., Tregoning, P., Bevis, M., Kato, T., and Meertens, C. (2002). Motion and rigidity of the Pacific plate and implications for plate boundary deformation. Journal of Geophysical Research: Solid Earth (1978-2012), 107(B10):ETG-19.

Beeler, N., Thomas, A., Bürgmann, R., and Shelly, D. (2013). Inferring fault rheology from low-frequency earthquakes on the San Andreas. Journal of Geophysical Research: Solid Earth, 118(11):5976-5990.

Beroza, G. and Ide, S. (2011). Slow earthquakes and nonvolcanic tremor. Annual review of Earth and planetary sciences, 39:271-296.

Berryman, K., Beanland, S., Cooper, A., Cutten, H., Norris, R., and Wood, P. (1992). The Alpine Fault, New Zealand: variation in Quaternary structural style and geomorphic expression. In Annales tectonicae, volume 6, pages 126163. 
Berryman, K., Cooper, A., Norris, R., Sutherland, R., and Villamor, P. (1998). Paleoseismic investigation of the Alpine Fault at Haast and Okuru. In Geological Society of NZ, Joint Annual Conference: programme $\&$ abstracts, volume 101, page 44 .

Berryman, K., Cooper, A., Norris, R., Villamor, P., Sutherland, R., Wright, T., Schermer, E., Langridge, R., and Biasi, G. (2012). Late Holocene rupture history of the Alpine Fault in South Westland, New Zealand. Bulletin of the Seismological Society of America, 102(2):620-638.

Bletery, Q., Thomas, A., Hawthorne, J., Skarbek, R., Rempel, A., and Krogstad, R. (2017). Characteristics of secondary slip fronts associated with slow earthquakes in Cascadia. Earth and Planetary Science Letters, 463:212-220.

Blick, G., Read, S., and Hall, P. (1989). Deformation monitoring of the Ostler fault zone, South Island, New Zealand. Tectonophysics, 167(2-4):329-339.

Boese, C. (2012). Microseismicity in the central Southern Alps, Westland, New Zealand. PhD thesis, Victoria University of Wellington.

Boese, C., Jacobs, K., Smith, E., Stern, T., and Townend, J. (2014). Background and delayed-triggered swarms in the central Southern Alps, South Island, New Zealand. Geochemistry, Geophysics, Geosystems, 15(4):945-964.

Boese, C., Stern, T., Townend, J., Bourguignon, S., Sheehan, A., and Smith, E. (2013). Sub-crustal earthquakes within the Australia-Pacific plate boundary zone beneath the Southern Alps, New Zealand. Earth and Planetary Science Letters, 376:212-219.

Boese, C., Townend, J., Smith, E., and Stern, T. (2012). Microseismicity and stress in the vicinity of the Alpine Fault, central Southern Alps, New Zealand. Journal of Geophysical Research: Solid Earth (1978-2012), 117(B2).

Boese, C. M., Stern, T. A., Michailos, K., Townend, J., and Chamberlain, C. J. (2018). Implications of upper-mantle seismicity for deformation in the continental collision zone beneath the alpine fault, south island, new zealand. New Zealand Journal of Geology and Geophysics, 61(3):283-308.

Bormann, P. (2012). New Manual of Seismological Observatory Practice (NMSOP2). German Research Centre for Geosciences. 
Bostock, M. and Christensen, N. (2012). Split from slip and schist: Crustal anisotropy beneath northern Cascadia from non-volcanic tremor. Journal of Geophysical Research: Solid Earth, 117(B8).

Bostock, M., Royer, A., Hearn, E., and Peacock, S. (2012). Low frequency earthquakes below southern Vancouver Island. Geochemistry, Geophysics, Geosystems, 13(11).

Bott, M. (1959). The mechanics of oblique slip faulting. Geological Magazine, 96(2):109-117.

Boulton, C., Moore, D., Lockner, D., Toy, V., Townend, J., and Sutherland, R. (2014). Frictional properties of exhumed fault gouges in DFDP-1 cores, Alpine Fault, New Zealand. Geophysical Research Letters, 41(2):356-362.

Bourguignon, S. (2009). Lithospheric deformation at the South Island oblique collision, New Zealand. PhD thesis, Victoria University of Wellington.

Bourguignon, S., Bannister, S., Henderson, C., Townend, J., and Zhang, H. (2015). Structural heterogeneity of the midcrust adjacent to the central Alpine Fault, New Zealand: Inferences from seismic tomography and seismicity between Harihari and Ross. Geochemistry, Geophysics, Geosystems, 16(4):1017-1043.

Bourguignon, S., Stern, T., and Savage, M. (2007). Crust and mantle thickening beneath the southern portion of the Southern Alps, New Zealand. Geophysical Journal International, 168(2):681-690.

Brown, J., Beroza, G., and Shelly, D. (2008). An autocorrelation method to detect low-frequency earthquakes within tremor. Geophysical Research Letters, 35(16).

Brown, J., Prejean, S., Beroza, G., Gomberg, J., and Haeussler, P. (2013). Deep low-frequency earthquakes in tectonic tremor along the Alaska-Aleutian subduction zone. Journal of Geophysical Research: Solid Earth, 118(3):1079-1090.

Chamberlain, C. (2016). Improved earthquake detection as a probe for active fault structures in New Zealand's central Southern Alps. PhD thesis, Victoria University of Wellington.

Chamberlain, C., Boese, C., Eccles, J., Savage, M., Baratin, L.-M., Townend, J., Gulley, A., Jacobs, K., Benson, A., Taylor-Offord, S., et al. (2017a). RealTime Earthquake Monitoring during the Second Phase of the Deep Fault Drilling Project, Alpine Fault, New Zealand. Seismological Research Letters, 88(6):1443-1454. 
Chamberlain, C., Boese, C., and Townend, J. (2017b). Cross-correlation-based detection and characterisation of microseismicity adjacent to the locked, lateinterseismic Alpine Fault, South Westland, New Zealand. Earth and Planetary Science Letters, 457:63-72.

Chamberlain, C., Hopp, C., Boese, C., Warren-Smith, E., Chambers, D., Chu, S., Michailos, K., and Townend, J. (2017c). EQcorrscan: Repeating and nearrepeating earthquake detection and analysis in Python. Seismological Research Letters.

Chamberlain, C., Shelly, D., Townend, J., and Stern, T. (2014). Low-frequency earthquakes reveal punctuated slow slip on the deep extent of the Alpine Fault, New Zealand. Geochemistry, Geophysics, Geosystems, 15(7):2984-2999.

Cochran, U., Clark, K., Howarth, J., Biasi, GPand Langridge, R., Villamor, P., Berryman, K., and Vandergoes, M. (2017). A plate boundary earthquake record from a wetland adjacent to the Alpine fault in New Zealand refines hazard estimates. Earth and Planetary Science Letters, 464:175-188.

Cowan, H. (1991). The north Canterbury earthquake of September 1, 1888. Journal of the Royal Society of New Zealand, 21(1):1-12.

Cowan, H., Nicol, A., and Tonkin, P. (1996). A comparison of historical and paleoseismicity in a newly formed fault zone and a mature fault zone, North Canterbury, New Zealand. Journal of Geophysical Research: Solid Earth, 101(B3):6021-6036.

Cox, S. and Barrell, D. (2007). Geology of the Aoraki area. Institute of Geological Er Nuclear Sciences.

Cox, S. and Sutherland, R. (2007). Regional geological framework of South Island, New Zealand, and its significance for understanding the active plate boundary. A continental plate boundary: tectonics at South Island, New Zealand, pages $19-46$.

Cullen, L., Duncan, R., Wells, A., and Stewart, G. (2003). Floodplain and regional scale variation in earthquake effects on forests, Westland, New Zealand. Journal of the Royal Society of New Zealand, 33(4):693-701.

Dahm, T. and Krüger, F. (2014). Topic Moment tensor inversion and moment tensor interpretation. Helmholtz Centre Potsdam GFZ German Research Centre for Geosciences. 
Davey, F., Eberhart-Phillips, D., Kohler, M., Bannister, S., Caldwell, G., Henrys, S., Scherwath, M., Stern, T., and Van Avendonk, H. (2007). Geophysical structure of the Southern Alps orogen, South Island, New Zealand. A Continental Plate Boundary: Tectonics at South Island, New Zealand, pages 47-72.

Davey, F., Henyey, T., Holbrook, W., Okaya, D., Stern, T., Melhuish, A., Henrys, S., Anderson, H., Eberhart-Phillips, D., McEvilly, T., et al. (1998). Preliminary results from a geophysical study across a modern, continent-continent collisional plate boundary - the Southern Alps, New Zealand. Tectonophysics, 288(1):221235.

Davey, F., Henyey, T., Kleffmann, S., Melhuish, A., Okaya, D., Stern, T., Woodward, D., and Transect, S. I. G. (1995). Crustal reflections from the Alpine Fault zone, South Island, New Zealand. New Zealand Journal of Geology and Geophysics, 38(4):601-604.

Delahaye, E., Townend, J., Reyners, M., and Rogers, G. (2009). Microseismicity but no tremor accompanying slow slip in the Hikurangi subduction zone, New Zealand. Earth and Planetary Science Letters, 277(1):21-28.

DeMets, C., Gordon, R., Argus, D., and Stein, S. (1994). Effect of recent revisions to the geomagnetic reversal time scale on estimates of current plate motions. Geophysical Research Letters, 21(20):2191-2194.

Doser, D., Webb, T., and Maunder, D. (1999). Source parameters of large historical (1918-1962) earthquakes, South Island, New Zealand. Geophysical Journal International, 139(3):769-794.

Douglas, A., Beavan, J., Wallace, L., and Townend, J. (2005). Slow slip on the northern Hikurangi subduction interface, New Zealand. Geophysical Research Letters, 32(16).

Dragert, H., Wang, K., and James, T. (2001). A silent slip event on the deeper Cascadia subduction interface. Science, 292(5521):1525-1528.

Dragert, H., Wang, K., and Rogers, G. (2004). Geodetic and seismic signatures of episodic tremor and slip in the northern cascadia subduction zone. Earth, planets and space, 56(12):1143-1150.

Eberhart-Phillips, D. (1995). Examination of seismicity in the central Alpine fault region, South Island, New Zealand. New Zealand journal of Geology and Geophysics, 38(4):571-578. 
Eberhart-Phillips, D. and Bannister, S. (2002). Three-dimensional crustal structure in the Southern Alps region of New Zealand from inversion of local earthquake and active source data. Journal of Geophysical Research: Solid Earth (1978-2012), 107(B10):ESE-15.

Eberhart-Phillips, D., Chadwick, M., and Bannister, S. (2008). Three-dimensional attenuation structure of central and southern South Island, New Zealand, from local earthquakes. Journal of Geophysical Research: Solid Earth, 113(B5).

Eberhart-Phillips, D., Reyners, M., and Bannister, S. (2015). A 3D QP Attenuation Model for All of New Zealand. Seismological Research Letters, 86(6):1655.

Evison, F. (1971). Seismicity of the alpine fault, new zealand. Bulletin of the Royal Society of New Zealand, 9:161-165.

Fagereng, A. and Diener, J. F. (2011). Non-volcanic tremor and discontinuous slab dehydration. Geophysical Research Letters, 38(15).

Feenstra, J., Thurber, C., Townend, J., Roecker, S., Bannister, S., Boese, C., Lord, N., Bourguignon, S., and Eberhart-Phillips, D. (2016). Microseismicity and $\mathrm{P}$-wave tomography of the central Alpine fault, New Zealand. New Zealand Journal of Geology and Geophysics, 59(4):483-495.

Frank, W. (2016). Slow slip hidden in the noise: The intermittence of tectonic release. Geophysical Research Letters, 43(19).

Frank, W., Radiguet, M., Rousset, B., Shapiro, N., Husker, A., Kostoglodov, V., Cotte, N., and Campillo, M. (2015). Uncovering the geodetic signature of silent slip through repeating earthquakes. Geophysical Research Letters, 42(8):27742779 .

Frank, W. and Shapiro, N. (2014). Automatic detection of low-frequency earthquakes (LFEs) based on a beamformed network response. Geophysical Journal International, 197(2):1215-1223.

Frank, W., Shapiro, N., Husker, A., Kostoglodov, V., Gusev, A., and Campillo, M. (2016). The evolving interaction of low-frequency earthquakes during transient slip. Science advances, 2(4):e1501616.

Frank, W., Shapiro, N., Husker, A., Kostoglodov, V., Romanenko, A., and Campillo, M. (2014). Using systematically characterized low-frequency earthquakes as a fault probe in Guerrero, Mexico. Journal of Geophysical Research: Solid Earth, 119(10):7686-7700. 
Frank, W., Shapiro, N., Kostoglodov, V., Husker, A., Campillo, M., Payero, J., and Prieto, G. (2013). Low-frequency earthquakes in the Mexican Sweet Spot. Geophysical Research Letters, 40(11):2661-2666.

Geiger, L. (1910). Determination of seismic centres. Nachrichten von der Gesellschaft der Wissenschaften zu Gottingen, Mathematisch-Physikalische Klasse, 1910:331-349.

Gephart, J. and Forsyth, D. (1984). An improved method for determining the regional stress tensor using earthquake focal mechanism data: application to the San Fernando earthquake sequence. Journal of Geophysical Research: Solid Earth, 89(B11):9305-9320.

Ghosh, A., Vidale, J., and Creager, K. (2012). Tremor asperities in the transition zone control evolution of slow earthquakes. Journal of Geophysical Research: Solid Earth, 117(B10).

Ghosh, A., Vidale, J., Peng, Z., Creager, K., and Houston, H. (2009). Complex nonvolcanic tremor near Parkfield, California, triggered by the great 2004 Sumatra earthquake. Journal of Geophysical Research: Solid Earth, 114(B12).

Ghosh, A., Vidale, J., Sweet, J., Creager, K., Wech, A., Houston, H., and Brodsky, E. (2010). Rapid, continuous streaking of tremor in Cascadia. Geochemistry, Geophysics, Geosystems, 11(12).

Gledhill, K., Ristau, J., Reyners, M., Fry, B., and Holden, C. (2011). The Darfield (Canterbury, New Zealand) Mw 7.1 earthquake of September 2010: A preliminary seismological report. Seismological Research Letters, 82(3):378-386.

Gledhill, K., Robinson, R., Webb, T., Abercrombie, R., Beavan, J., Cousins, J., and Eberhart-Phillips, D. (2000). The Mw 6.2 cass, New Zealand, earthquake of 24 November 1995: Reverse faulting in a strike-slip region. New Zealand Journal of Geology and Geophysics, 43(2):255-269.

Gomberg, J., Rubinstein, J., Peng, Z., Creager, K., Vidale, J., and Bodin, P. (2008). Widespread triggering of nonvolcanic tremor in California. Science, 319(5860):173-173.

Gonzalez-Huizar, H. and Velasco, A. (2011). Dynamic triggering: Stress modeling and a case study. Journal of Geophysical Research: Solid Earth, 116(B2).

Goossens, M., Mittelbach, F., and Samarin, A. (1994). The ETTEX Companion. Addison-Wesley. 
Guilhem, A. and Nadeau, R. (2012). Episodic tremors and deep slow-slip events in Central California. Earth and Planetary Science Letters, 357:1-10.

Hamling, I., Hreinsdóttir, S., Clark, K., Elliott, J., Liang, C., Fielding, E., Litchfield, N., Villamor, P., Wallace, L., Wright, T., et al. (2017). Complex multifault rupture during the 2016 Mw 7.8 Kaikōura earthquake, New Zealand. Science, 356(6334):71-94.

Hardebeck, J. and Shearer, P. (2003). Using S/P amplitude ratios to constrain the focal mechanisms of small earthquakes. Bulletin of the Seismological Society of America, 93(6):2434-2444.

Havskov, J. and Ottemoller, L. (1999). SEISAN earthquake analysis software. Seismological Research Letters, 70(5):532-534.

Hawthorne, J. and Rubin, A. (2010). Tidal modulation of slow slip in Cascadia. Journal of Geophysical Research: Solid Earth, 115(B9).

Hill, D. (2012). Surface-wave potential for triggering tectonic (nonvolcanic) tremor - corrected. Bulletin of the Seismological Society of America, 102(6):2337-2355.

Hirose, H. and Obara, K. (2006). Short-term slow slip and correlated tremor episodes in the Tokai region, central Japan. Geophysical Research Letters, $33(17)$.

Holden, C. (2011). Kinematic source model of the 22 february $2011 \mathrm{Mw} 6.2$ Christchurch earthquake using strong motion data. Seismological research letters, 82(6):783-788.

Holt, W. and Haines, A. (1995). The kinematics of northern South Island, New Zealand, determined from geologic strain rates. Journal of Geophysical Research: Solid Earth, 100(B9):17991-18010.

Houlié, N. and Stern, T. (2012). A comparison of GPS solutions for strain and SKS fast directions: Implications for modes of shear in the mantle of a plate boundary zone. Earth and Planetary Science Letters, 345:117-125.

Houston, H., Delbridge, B., Wech, A., and Creager, K. (2011). Rapid tremor reversals in Cascadia generated by a weakened plate interface. Nature Geoscience, $4(6): 404$. 
Howarth, J., Fitzsimons, S., Norris, R., and Jacobsen, G. (2014). Lake sediments record high intensity shaking that provides insight into the location and rupture length of large earthquakes on the Alpine Fault, New Zealand. Earth and Planetary Science Letters, 403:340-351.

Howarth, J., Fitzsimons, S., Norris, R., Langridge, R., and Vandergoes, M. (2016). A 2000 yr rupture history for the Alpine Fault derived from Lake Ellery, South Island, New Zealand. Geological Society of America Bulletin, 128(3-4):627-643.

Ide, S. (2008). A Brownian walk model for slow earthquakes. Geophysical Research Letters, 35(17).

Ide, S., Beroza, G., Shelly, D., and Uchide, T. (2007a). A scaling law for slow earthquakes. Nature, 447(7140):76.

Ide, S., Shelly, D., and Beroza, G. (2007b). Mechanism of deep low frequency earthquakes: Further evidence that deep non-volcanic tremor is generated by shear slip on the plate interface. Geophysical Research Letters, 34(3).

Ito, Y., Hino, R., Kido, M., Fujimoto, H., Osada, Y., Inazu, D., Ohta, Y., Iinuma, T., Ohzono, M., Miura, S., et al. (2013). Episodic slow slip events in the Japan subduction zone before the 2011 Tohoku-Oki earthquake. Tectonophysics, 600:14-26.

Ito, Y., Obara, K., Shiomi, K., Sekine, S., and Hirose, H. (2007). Slow earthquakes coincident with episodic tremors and slow slip events. Science, 315(5811):503506.

Johnson, P., Carpenter, B., Knuth, M., Kaproth, B., Le Bas, P.-Y., Daub, E., and Marone, C. (2012). Nonlinear dynamical triggering of slow slip on simulated earthquake faults with implications to Earth. Journal of Geophysical Research: Solid Earth, 117(B4).

Johnson, P., Ferdowsi, B., Kaproth, B., Scuderi, M., Griffa, M., Carmeliet, J., Guyer, R., Le Bas, P.-Y., Trugman, D., and Marone, C. (2013). Acoustic emission and microslip precursors to stick-slip failure in sheared granular material. Geophysical Research Letters, 40(21):5627-5631.

Johnston, M., Borcherdt, R., Linde, A., and Gladwin, M. (2006). Continuous borehole strain and pore pressure in the near field of the 28 September 2004 M6.0 Parkfield, California, earthquake: Implications for nucleation, fault response, earthquake prediction, and tremor. Bulletin of the Seismological Society of America, 96(4B):S56-S72. 
Kaneko, L., Ide, S., and Nakano, M. (2018). Slow earthquakes in the microseism frequency band (0.1-1.0 hz) off Kii Peninsula, Japan. Geophysical Research Letters.

Kao, H., Wang, K., Dragert, H., Kao, J. Y., and Rogers, G. (2010). Estimating seismic moment magnitude $(\mathrm{Mw})$ of tremor bursts in northern Cascadia: Implications for the "seismic efficiency" of episodic tremor and slip. Geophysical Research Letters, 37(19).

Kato, A., Obara, K., Igarashi, T., Tsuruoka, H., Nakagawa, S., and Hirata, N. (2012). Propagation of slow slip leading up to the $2011 \mathrm{Mw} 9.0$ Tohoku-Oki earthquake. Science, 335(6069):705-708.

Katsumata, A. and Kamaya, N. (2003). Low-frequency continuous tremor around the Moho discontinuity away from volcanoes in the southwest Japan. Geophysical Research Letters, 30(1):20-1.

Kleffmann, S., Davey, F., Melhuish, A., Okaya, D., Stern, T., Team, S., et al. (1998). Crustal structure in the central South Island, New Zealand, from the Lake Pukaki seismic experiment. New Zealand Journal of Geology and Geophysics, 41(1):39-49.

Koons, P., Norris, R., Craw, D., and Cooper, A. (2003). Influence of exhumation on the structural evolution of transpressional plate boundaries: An example from the Southern Alps, New Zealand. Geology, 31(1):3-6.

Lamb, S. and Smith, E. (2013). The nature of the plate interface and driving force of interseismic deformation in the New Zealand plate-boundary zone, revealed by the continuous GPS velocity field. Journal of Geophysical Research: Solid Earth, 118(6):3160-3189.

Lamb, S., Smith, E., Stern, T., and Warren-Smith, E. (2015). Continent-scale strike-slip on a low-angle fault beneath New Zealand's Southern Alps: Implications for crustal thickening in oblique collision zones. Geochemistry, Geophysics, Geosystems, 16(9):3076-3096.

Leitner, B., Eberhart-Phillips, D., Anderson, H., and Nabelek, J. (2001). A focused look at the Alpine Fault, New Zealand: Seismicity, focal mechanisms, and stress observations. Journal of Geophysical Research, 106(B2):2193-2220.

Lengliné, O., Frank, W., Marsan, D., and Ampuero, J.-P. (2017). Imbricated slip rate processes during slow slip transients imaged by low-frequency earthquakes. Earth and Planetary Science Letters, 476:122-131. 
Lienert, B., Berg, E., and Frazer, L. (1986). HYPOCENTER: An earthquake location method using centered, scaled, and adaptively damped least squares. Bulletin of the Seismological Society of America, 76(3):771-783.

Little, T. (2004). Transpressive ductile flow and oblique ramping of lower crust in a two-sided orogen: Insight from quartz grain-shape fabrics near the Alpine Fault, New Zealand. Tectonics, 23(2).

Little, T., Cox, S., Vry, J., and Batt, G. (2005). Variations in exhumation level and uplift rate along the obliqu-slip Alpine Fault, central Southern Alps, New Zealand. Geological Society of America Bulletin, 117(5-6):707-723.

Little, T., Holcombe, R., and Ilg, B. (2002). Ductile fabrics in the zone of active oblique convergence near the Alpine Fault, New Zealand: identifying the neotectonic overprint. Journal of Structural Geology, 24(1):193-217.

Little, T. and Jones, A. (1998). Seven million years of strike-slip and related off-fault deformation, Northeastern Marlborough Fault System, South Island, New Zealand. Tectonics, 17(2):285-302.

Liu, Y. and Rice, J. (2005). Aseismic slip transients emerge spontaneously in three-dimensional rate and state modeling of subduction earthquake sequences. Journal of Geophysical Research: Solid Earth, 110(B8).

Lockner, D., Morrow, C., Moore, D., and Hickman, S. (2011). Low strength of deep San Andreas fault gouge from SAFOD core. Nature, 472(7341):82.

Lomax, A., Virieux, J., Volant, P., and Berge-Thierry, C. (2000). Probabilistic earthquake location in $3 \mathrm{D}$ and layered models. In Advances in seismic event location, pages 101-134. Springer.

Lowen, S. and Teich, M. (2005). Fractal-based point processes, volume 366. John Wiley \& Sons.

Luyendyk, B. (1995). Hypothesis for Cretaceous rifting of east Gondwana caused by subducted slab capture. Geology, 23(4):373-376.

Marone, C. (1998). Laboratory-derived friction laws and their application to seismic faulting. Annual Review of Earth and Planetary Sciences, 26(1):643696.

Matharu, G., Bostock, M., Christensen, N., and Tromp, J. (2014). Crustal anisotropy in a subduction zone forearc: Northern Cascadia. Journal of Geophysical Research: Solid Earth, 119(9):7058-7078. 
Mazzotti, S. and Adams, J. (2004). Variability of near-term probability for the next great earthquake on the Cascadia subduction zone. Bulletin of the Seismological Society of America, 94(5):1954-1959.

Metropolis, N., Rosenbluth, A., Rosenbluth, M., Teller, A., and Teller, E. (1953). Equation of state calculations by fast computing machines. The journal of chemical physics, 21(6):1087-1092.

Miyazawa, M. and Brodsky, E. (2008). Deep low-frequency tremor that correlates with passing surface waves. Journal of Geophysical Research: Solid Earth, 113(B1).

Miyazawa, M., Brodsky, E., and Mori, J. (2008). Learning from dynamic triggering of low-frequency tremor in subduction zones. Earth, Planets and Space, 60(10):e17-e20.

Miyazawa, M. and Mori, J. (2006). Evidence suggesting fluid flow beneath Japan due to periodic seismic triggering from the 2004 Sumatra-Andaman earthquake. Geophysical research letters, 33(5).

Mortimer, N. (2004). New Zealand's geological foundations. Gondwana Research, $7(1): 261-272$.

Nadeau, R. and Dolenc, D. (2005). Nonvolcanic tremors deep beneath the San Andreas Fault. Science, 307(5708):389-389.

Nadeau, R. and Guilhem, A. (2009). Nonvolcanic tremor evolution and the San Simeon and Parkfield, California, earthquakes. science, 325(5937):191-193.

Nakata, R., Suda, N., and Tsuruoka, H. (2008). Non-volcanic tremor resulting from the combined effect of Earth tides and slow slip events. Nature Geoscience, $1(10): 676$.

Norris, R. and Cooper, A. (1995). Origin of small-scale segmentation and transpressional thrusting along the Alpine Fault, New Zealand. Geological Society of America Bulletin, 107(2):231-240.

Norris, R. and Cooper, A. (2001). Late Quaternary slip rates and slip partitioning on the Alpine Fault, New Zealand. Journal of Structural Geology, 23(2):507520 .

Norris, R. and Cooper, A. (2003). Very high strains recorded in mylonites along the Alpine Fault, New Zealand: implications for the deep structure of plate boundary faults. Journal of Structural Geology, 25(12):2141-2157. 
Norris, R. and Cooper, A. (2007). The Alpine Fault, New Zealand: surface geology and field relationships. A Continental Plate Boundary: Tectonics at South Island, New Zealand, pages 157-175.

Norris, R. and Toy, V. (2014). Continental transforms: A view from the Alpine Fault. Journal of Structural Geology, 64:3-31.

Obara, K. (2002). Nonvolcanic deep tremor associated with subduction in southwest Japan. Science, 296(5573):1679-1681.

Obara, K. (2010). Phenomenology of deep slow earthquake family in southwest Japan: Spatiotemporal characteristics and segmentation. Journal of Geophysical Research: Solid Earth, 115(B8).

Obara, K. and Hirose, H. (2006). Non-volcanic deep low-frequency tremors accompanying slow slips in the southwest Japan subduction zone. Tectonophysics, 417(1):33-51.

Obara, K., Hirose, H., Yamamizu, F., and Kasahara, K. (2004). Episodic slow slip events accompanied by non-volcanic tremors in southwest Japan subduction zone. Geophysical Research Letters, 31(23).

Oestreicher, N. (2018). Geodetic, hydrologic and seismological signals associated with precipitation and infiltration in the central Southern Alps, New Zealand.

Ohta, Y., Freymueller, J., Hreinsdóttir, S., and Suito, H. (2006). A large slow slip event and the depth of the seismogenic zone in the south central Alaska subduction zone. Earth and Planetary Science Letters, 247(1):108-116.

Okaya, D., Henrys, S., and Stern, T. (2002). Double-sided onshore-offshore seismic imaging of a plate boundary:'super-gathers' across South Island, New Zealand. Tectonophysics, 355(1):247-263.

Okaya, D., Stern, T., Davey, F., Henrys, S., and Cox, S. (2007). Continentcontinent collision at the Pacific/Indo-Australian plate boundary: Background, motivation, and principal results. A Continental Plate Boundary: Tectonics at South Island, New Zealand, pages 1-18.

O'Keefe, B. (2008). Microseismicity of the central Alpine Fault region, New Zealand. Master's thesis, Victoria University of Wellington.

Ozawa, S., Miyazaki, S., Hatanaka, Y., Imakiire, T., Kaidzu, M., and Murakami, M. (2003). Characteristic silent earthquakes in the eastern part of the Boso peninsula, Central Japan. Geophysical research letters, 30(6). 
Peng, Y., Rubin, A., Bostock, M., and Armbruster, J. (2015). High-resolution imaging of rapid tremor migrations beneath southern Vancouver Island using cross-station cross correlations. Journal of Geophysical Research: Solid Earth, 120(6):4317-4332.

Peng, Z. and Chao, K. (2008). Non-volcanic tremor beneath the Central Range in Taiwan triggered by the $2001 \mathrm{Mw} 7.8$ Kunlun earthquake. Geophysical Journal International, 175(2):825-829.

Peng, Z., Vidale, J., Wech, A., Nadeau, R., and Creager, K. (2009). Remote triggering of tremor along the San Andreas Fault in central California. Journal of Geophysical Research: Solid Earth, 114(B7).

Plourde, A., Bostock, M., Audet, P., and Thomas, A. (2015). Low-frequency earthquakes at the southern Cascadia Margin. Geophysical Research Letters.

Quigley, M., Van Dissen, R., Litchfield, N., Villamor, P., Duffy, B., Barrell, D., Furlong, K., Stahl, T., Bilderback, E., and Noble, D. (2012). Surface rupture during the $2010 \mathrm{Mw} 7.1$ Darfield (Canterbury) earthquake: Implications for fault rupture dynamics and seismic-hazard analysis. Geology, 40(1):55-58.

Radiguet, M., Cotton, F., Vergnolle, M., Campillo, M., Valette, B., Kostoglodov, V., and Cotte, N. (2011). Spatial and temporal evolution of a long term slow slip event: the 2006 guerrero slow slip event. Geophysical Journal International, $184(2): 816-828$.

Ranalli, G. (1995). Rheology of the Earth. Springer Science \& Business Media.

Reyners, M. (1988). Reservoir-induced seismicity at Lake Pukaki, New Zealand. Geophysical Journal International, 93(1):127-135.

Ristau, J. (2008). Implementation of routine regional moment tensor analysis in New Zealand. Seismological Research Letters, 79(3):400-415.

Rivera, L. and Cisternas, A. (1990). Stress tensor and fault plane solutions for a population of earthquakes. Bulletin of the Seismological Society of America, 80(3):600-614.

Robinson, R. and McGinty, P. (2000). The enigma of the Arthur's Pass, new zealand, earthquake: 2. the aftershock distribution and its relation to regional and induced stress fields. Journal of Geophysical Research: Solid Earth, 105(B7):16139-16150. 
Rogers, G. and Dragert, H. (2003). Episodic tremor and slip on the Cascadia subduction zone: The chatter of silent slip. Science, 300(5627):1942-1943.

Rousset, B., Jolivet, R., Simons, M., Lasserre, C., Riel, B., Milillo, P., Çakir, Z., and Renard, F. (2016). An aseismic slip transient on the north anatolian fault. Geophysical Research Letters, 43(7):3254-3262.

Royer, A. and Bostock, M. (2014). A comparative study of low frequency earthquake templates in northern Cascadia. Earth and Planetary Science Letters, 402:247-256.

Royer, A., Thomas, A., and Bostock, M. (2015). Tidal modulation and triggering of low-frequency earthquakes in northern Cascadia. Journal of Geophysical Research: Solid Earth, 120(1):384-405.

Rubin, A. (2011). Designer friction laws for bimodal slow slip propagation speeds. Geochemistry, Geophysics, Geosystems, 12(4).

Rubin, A. and Armbruster, J. (2013). Imaging slow slip fronts in Cascadia with high precision cross-station tremor locations. Geochemistry, Geophysics, Geosystems, 14(12):5371-5392.

Rubinstein, J., Gomberg, J., Vidale, J., Wech, A., Kao, H., Creager, K., and Rogers, G. (2009a). Seismic wave triggering of nonvolcanic tremor, episodic tremor and slip, and earthquakes on Vancouver Island. Journal of Geophysical Research: Solid Earth, 114(B2).

Rubinstein, J., La Rocca, M., Vidale, J., Creager, K., and Wech, A. (2008). Tidal modulation of nonvolcanic tremor. Science, 319(5860):186-189.

Rubinstein, J., Shelly, D., and Ellsworth, W. (2009b). Non-volcanic tremor: A window into the roots of fault zones. In New Frontiers in Integrated Solid Earth Sciences, pages 287-314. Springer.

Rubinstein, J., Vidale, J., Gomberg, J., Bodin, P., Creager, K., and Malone, S. (2007). Non-volcanic tremor driven by large transient shear stresses. Nature, 448(7153):579-582.

Ruiz, S., Metois, M., Fuenzalida, A., Ruiz, J., Leyton, F., Grandin, R., Vigny, C., Madariaga, R., and Campos, J. (2014). Intense foreshocks and a slow slip event preceded the 2014 Iquique Mw 8.1 earthquake. Science, 345(6201):1165-1169. 
Samuelson, J., Elsworth, D., and Marone, C. (2009). Shear-induced dilatancy of fluid-saturated faults: Experiment and theory. Journal of Geophysical Research: Solid Earth, 114(B12).

Savage, M. (1999). Seismic anisotropy and mantle deformation: what have we learned from shear wave splitting? Reviews of Geophysics, 37(1):65-106.

Savard, G. and Bostock, M. (2015). Detection and Location of Low-Frequency Earthquakes Using Cross-Station Correlation. Bulletin of the Seismological Society of America.

Schimmel, M. and Gallart, J. (2007). Frequency-dependent phase coherence for noise suppression in seismic array data. Journal of Geophysical Research: Solid Earth, 112(4):1-14.

Schimmel, M. and Paulssen, H. (1997). Noise reduction and detection of weak, coherent signals through phase-weighted stacks. Geophysical Journal International, 130(2):497-505.

Scholz, C. (2002). The mechanics of earthquakes and faulting. Cambridge University Press.

Scholz, C., Rynn, J., Weed, R., and Frohlich, C. (1973). Detailed seismicity of the Alpine fault zone and Fiordland region, New Zealand. Geological Society of America Bulletin, 84(10):3297-3316.

Schwartz, S. and Rokosky, J. (2007). Slow slip events and seismic tremor at circum-Pacific subduction zones. Reviews of Geophysics, 45(3).

Segall, P. and Bradley, A. (2012). Slow-slip evolves into megathrust earthquakes in 2D numerical simulations. Geophysical Research Letters, 39(18).

Segall, P., Rubin, A., Bradley, A., and Rice, J. (2010). Dilatant strengthening as a mechanism for slow slip events. Journal of Geophysical Research: Solid Earth, 115(B12).

Shelly, D. (2009). Possible deep fault slip preceding the 2004 Parkfield earthquake, inferred from detailed observations of tectonic tremor. Geophysical Research Letters, 36(17).

Shelly, D. (2015). Complexity of the deep San Andreas Fault zone defined by cascading tremor. Nature Geoscience, 8(2):145. 
Shelly, D. (2017). A 15 year catalog of more than 1 million low-frequency earthquakes: Tracking tremor and slip along the deep San Andreas Fault. Journal of Geophysical Research: Solid Earth, 122(5):3739-3753.

Shelly, D., Beroza, G., and Ide, S. (2007). Non-volcanic tremor and low-frequency earthquake swarms. Nature, 446(7133):305-307.

Shelly, D., Beroza, G., Ide, S., and Nakamula, S. (2006). Low-frequency earthquakes in Shikoku, Japan, and their relationship to episodic tremor and slip. Nature, 442(7099):188-191.

Sibson, R., White, S., and Atkinson, B. (1979). Fault rock distribution and structure within the Alpine Fault zone: a preliminary account. The Origin of the Southern Alps, 18:55-65.

Sibson, R., White, S., and Atkinson, B. (1981). Structure and distribution of fault rocks in the Alpine Fault zone, New Zealand. Geological Society, London, Special Publications, 9(1):197-210.

Solano, E., Hjörleifsdóttir, V., and Liu, Q. (2017). Full-waveform detection of nonimpulsive seismic events based on time-reversal methods. Geophysical Journal International, 211(3):1396-1413.

Stein, S. and Wysession, M. (2009). An introduction to seismology, earthquakes, and earth structure. John Wiley \& Sons.

Stern, T., Kleffmann, S., Okaya, D., Scherwath, M., and Bannister, S. (2001). Low seismic-wave speeds and enhanced fluid pressure beneath the Southern Alps of New Zealand. Geology, 29(8):679-682.

Stern, T., Molnar, P., Okaya, D., and Eberhart-Phillips, D. (2000). Teleseismic $\mathrm{P}$ wave delays and modes of shortening the mantle lithosphere beneath South Island, New Zealand. Journal of Geophysical Research, 105(B9):21615-31.

Stern, T., Okaya, D., Kleffmann, S., Scherwath, M., Henrys, S., and Davey, F. (2007). Geophysical exploration and dynamics of the Alpine fault zone. A Continental Plate Boundary: Tectonics at South Island, New Zealand, pages 207-233.

Sutherland, R., Berryman, K., and Norris, R. (2006). Quaternary slip rate and geomorphology of the Alpine Fault: Implications for kinematics and seismic hazard in southwest New Zealand. Geological Society of America Bulletin, 118(3-4):464-474. 
Sutherland, R., Davey, F., and Beavan, J. (2000). Plate boundary deformation in South Island, New Zealand, is related to inherited lithospheric structure. Earth and Planetary Science Letters, 177(3):141-151.

Sutherland, R., Eberhart-Phillips, D., Harris, R., Stern, T., Beavan, J., Ellis, S., Henrys, S., Cox, S., Norris, R., Berryman, K., et al. (2007). Do great earthquakes occur on the Alpine Fault in central South Island, New Zealand? Geophysical Monograph-American Geophysical Union, 175:237.

Sutherland, R., Townend, J., Toy, V., Upton, P., Coussens, J., Allen, M., Baratin, L.-M., Barth, N., Becroft, L., Boese, C., et al. (2017). Extreme hydrothermal conditions at an active plate-bounding fault. Nature, 546(7656):137-140.

Sutherland, R., Townend, J., Toy, V., Upton, P., Coussens, J., Allen, M., Boles, A., et al. (2015). Deep Fault Drilling Project (DFDP), Alpine Fault boreholes DFDP-2A and DFDP-2B technical completion report. GNS Science, Te Pū Ao.

Sutherland, R., Toy, V., Townend, J., Cox, S., Eccles, J., Faulkner, D., Prior, D., Norris, R., Mariani, E., Boulton, C., et al. (2012). Drilling reveals fluid control on architecture and rupture of the Alpine Fault, New Zealand. Geology, 40(12):1143-1146.

Tarantola, A. and Valette, B. (1982). Generalized nonlinear inverse problems solved using the least squares criterion. Reviews of Geophysics, 20(2):219-232.

Thomas, A., Bürgmann, R., Shelly, D. R., Beeler, N. M., and Rudolph, M. (2012). Tidal triggering of low frequency earthquakes near Parkfield, California: Implications for fault mechanics within the brittle-ductile transition. Journal of Geophysical Research: Solid Earth, 117(B5).

Thomas, A., Nadeau, R., and Bürgmann, R. (2009). Tremor-tide correlations and near-lithostatic pore pressure on the deep San Andreas fault. Nature, 462(7276):1048-1051.

Thurber, C. (1985). Nonlinear earthquake location: theory and examples. Bulletin of the Seismological Society of America, 75(3):779-790.

Thurber, C., Zeng, X., Thomas, A., and Audet, P. (2014). Phase-Weighted Stacking applied to low-frequency earthquakes. Bulletin of the Seismological Society of America.

Townend, J., Sutherland, R., and Toy, V. (2009a). Deep Fault Drilling ProjectAlpine Fault, New Zealand. Scientific drilling, 8:75-82. 
Townend, J., Sutherland, R., Toy, V., Doan, M.-L., Célérier, B., Massiot, C., Coussens, J., Jeppson, T., Janku-Capova, L., Remaud, L., et al. (2017). Petrophysical, Geochemical, and Hydrological Evidence for Extensive FractureMediated Fluid and Heat Transport in the Alpine Fault's Hanging-Wall Damage Zone. Geochemistry, Geophysics, Geosystems.

Townend, J., Sutherland, R., Toy, V., Eccles, J., Boulton, C., Cox, S., and McNamara, D. (2013). Late-interseismic state of a continental plate-bounding fault: Petrophysical results from DFDP-1 wireline logging and core analysis, Alpine Fault, New Zealand. Geochemistry, Geophysics, Geosystems, 14(9):3801-3820.

Townend, J., Sutherland, R., Toy, V., et al. (2009b). Deep Fault Drilling ProjectAlpine Fault, New Zealand. Scientific drilling, 8:75-82.

Toy, V., Boulton, C., Sutherland, R., Townend, J., Norris, R., Little, T., Prior, D., Mariani, E., Faulkner, D., Menzies, C., et al. (2015). Fault rock lithologies and architecture of the central alpine fault, New Zealand, revealed by DFDP-1 drilling. Lithosphere, pages L395-1.

Toy, V., Sutherland, R., Townend, J., Allen, M., Becroft, L., Boles, A., Boulton, C., Carpenter, B., Cooper, A., Cox, S., et al. (2017). Bedrock geology of DFDP2B, central Alpine Fault, New Zealand. New Zealand Journal of Geology and Geophysics, pages 1-22.

Trugman, D., Wu, C., Guyer, R., and Johnson, P. (2015). Synchronous low frequency earthquakes and implications for deep San Andreas Fault slip. Earth and Planetary Science Letters, 424:132-139.

Utheim, T., Havskov, J., Ozyazicioglu, M., Rodriguez, J., and Talavera, E. (2014). RTQUAKE, a real-time earthquake detection system integrated with SEISAN. Seismological Research Letters, 85(3):735-742.

Walcott, R. (1978). Present tectonics and late Cenozoic evolution of New Zealand. Geophysical Journal International, 52(1):137-164.

Wallace, L. and Beavan, J. (2010). Diverse slow slip behavior at the Hikurangi subduction margin, New Zealand. Journal of Geophysical Research: Solid Earth, 115(B12).

Wallace, R. E. (1951). Geometry of shearing stress and relation to faulting. The journal of Geology, 59(2):118-130. 
Walsh, D., Arnold, R., and Townend, J. (2009). A Bayesian approach to determining and parametrizing earthquake focal mechanisms. Geophysical Journal International, 176(1):235-255.

Wang, C.-Y. and Manga, M. (2010). Hydrologic responses to earthquakes and a general metric. Geofluids, 10(1-2):206-216.

Wannamaker, P., Jiracek, G., Stodt, J., Caldwell, G., Gonzalez, V., McKnight, D., and Porter, A. (2002). Fluid generation and pathways beneath an active compressional orogen, the New Zealand Southern Alps, inferred from magnetotelluric data. Journal of Geophysical Research: Solid Earth, 107(B6).

Warren-Smith, E., Lamb, S., and Stern, T. (2017). Stress field and kinematics for diffuse microseismicity in a zone of continental transpression, South Island, New Zealand. Journal of Geophysical Research: Solid Earth, 122(4):2798-2811.

Wech, A. and Bartlow, N. (2014). Slip rate and tremor genesis in Cascadia. Geophysical Research Letters, 41(2):392-398.

Wech, A., Boese, C., Stern, T., and Townend, J. (2012). Tectonic tremor and deep slow slip on the Alpine Fault. Geophysical Research Letters, 39(10).

Wech, A. and Creager, K. (2011). A continuum of stress, strength and slip in the Cascadia subduction zone. Nature Geoscience, 10(1038):624-628.

Wech, A., Sheehan, A., Boese, C., Townend, J., Stern, T., and Collins, J. (2013). Tectonic tremor recorded by ocean bottom seismometers. Seismological Research Letters, 84(5):752-758.

Wech, A. G. and Creager, K. C. (2007). Cascadia tremor polarization evidence for plate interface slip. Geophysical Research Letters, 34(22).

Wellman, H. (1953). Data for the study of recent and late Pleistocene faulting in the South Island of New zealand. New Zealand Journal of Science and Technology, 34:270-288.

Wells, A., Yetton, M., Duncan, R., and Stewart, G. (1999). Prehistoric dates of the most recent Alpine Fault earthquakes, New Zealand. Geology, 27(11):995-998.

Yamashita, T. (2013). Generation of slow slip coupled with tremor due to fluid flow along a fault. Geophysical Journal International, 193(1):375-393.

Yamashita, T. and Suzuki, T. (2011). Dynamic modeling of slow slip coupled with tremor. Journal of Geophysical Research: Solid Earth, 116(B5). 
Yetton, M. (1998). Progress in understanding the paleoseismicity of the central and northern Alpine Fault, Westland, New Zealand. New Zealand Journal of Geology and Geophysics, 41(4):475-483.

Zhang, H. and Thurber, C. (2003). Double-difference tomography: The method and its application to the Hayward Fault, California. Bulletin of the Seismological Society of America, 93(5):1875-1889. 